U. S. DEPARTMENT OF THE INTERIOR

U. S. GEOLOGICAL SURVEY

\title{
MINERAL AND ENERGY REBOURCE ASSESSMENT \\ OF THE HELENA NATIONAL FOREST, \\ WEST-CENTRAL MONTANA
}

Edited by

R.G. Tysdal ${ }^{1}$, Steve Ludington ${ }^{2}$, and A.E. McCafferty ${ }^{1}$

Open-File Report 96-683-A

This report is preliminary and has not been reviewed for conformity with U.S. Geological Survey editorial standards or with the North American Stratigraphic Code. Any use of trade, product, or firm names is for descriptive purposes only and does not imply endorsement by the U.S. Government.

${ }^{1}$ Denver, Colorado

${ }^{2}$ Menlo Park, California 


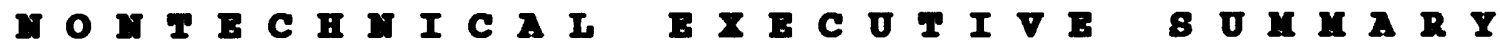

\section{MINERAL AND ENERGY RESOURCE ASSESSMENT \\ OF THE HELENA NATIONAL FOREST, WEST-CENTRAL MONTANA}

\section{U.8. GEOLOGICAL SURVEY OPEN-FILE REPORT 96-683-A}

* The Helena National Forest (the Forest) covers about 975,000 acres $(1,520$ square miles) in west-central Montana. It lies in the vicinity of Helena, the capitol city of Montana, and includes Federal lands within parts of five counties: Broadwater, Jefferson, Meagher, Powe11, and Lewis and Clark.

* Gold was discovered in the region in Powell County in 1852. Mining began in the $1860^{\prime} \mathrm{s}$, and since then the Forest has produced gold, silver, copper, lead, zinc, molybdenum, and phosphate minerals.

* At the time of this study, the only mineral production within the Forest is gold from small-scale placer operations, although exploration continues. Minerals are produced from areas adjacent to the Forest. Properties where significant production is most probable in the immediate future are on land adjacent to the Forest.

* Estimates of undiscovered resources contained in seven types of mineral deposits are made. Statistical descriptions of the estimates are given for each deposit type (table G3). Gold and copper are the commodities most likely to be present in the Forest in significant quantities.

* Geologic maps, and maps that show areas where undiscovered deposits may exist, are presented for a variety of types of mineral deposits. These are provided in hard copy and are available in digital form (on a CD-ROM of companion report: U.S. Geological Survey Open-File Report 96-683-B) for use in land management by hydrologists, soils scientists, biologists, botanists and other scientists of the Forest Service and other entities.

* The distribution of carbonate rocks (limestone and dolomite) is shown on a separate map (also in digital form). These rock types act to neutralize acid, thus fix (chemically bind) potentially hazardous chemical elements present in surface and ground waters.

* Only part of the Forest is considered to have potential for oil and gas. 


\section{CONTENTS}

\section{SUMMLARY}

\section{CHAPTER}

A. The Helena National Forest study area by R.G. Tysdal and Steve Ludington

Introduction

Importance of the study

Location

Previous and concurrent studies

Methodology

Undiscovered resources

Digital products

Acknowledgments

References cited

B. Geologic setting of the Helena National Forest study area by R.G. Tysdal Geologic mapping

Regional geologic setting

Stratigraphy

Volcanic rocks

Plutonic rocks

Structure

Lewis and Clark line

Sapphire thrust terrane

North of St. Marys-Helena Valley fault

Big Belt Mountains

South of St. Marys-Helena Valley fault

Great Falls tectonic zone

Extensional faulting

References cited

C. Geophysics of the Helena National Forest study area

Aeromagnetic and gravity studies by A.E. McCafferty

Aeromagnetic and gravity data

Previous aeromagnetic and gravity studies

Magnetization and densities of rocks

Terrace maps

Sources of aeromagnetic anomalies

Sources for magnetic and gravity anomalies in Big Belt Mountains

Gamma-ray radioactivity by J.A. Pitkin

Introduction

Discussion

Interpretation

References cited

D. Geochemistry of the Helena National Forest by H.V. Alminas

Introduction

Sample types

Analytical procedures 
Statistical summary

Data sets

Basic statistics

Geochemical exploration

Geochemical models based on statistical analysis

Geochemical Association model methodology

La-association mode1

$\mathrm{Pb}$-association model

Au-association model

Element plots

Heavy-metal analyses from stream sediments and outcrops Geochemical-geophysical association models

References cited

Geochemical-geophysical association model summary

E. Mines and prospects in and adjacent to the Helena National Forest by

Christopher Osterman, R.G. Tysdal, J.E. Elliott, and Steve Ludington

Introduction

References cited

F. Mineral resource geology of mining districts/areas

Lincoln Region by R.G. Tysdal, J.E. Elliott, E.A. du Bray, and J.W. Whipple

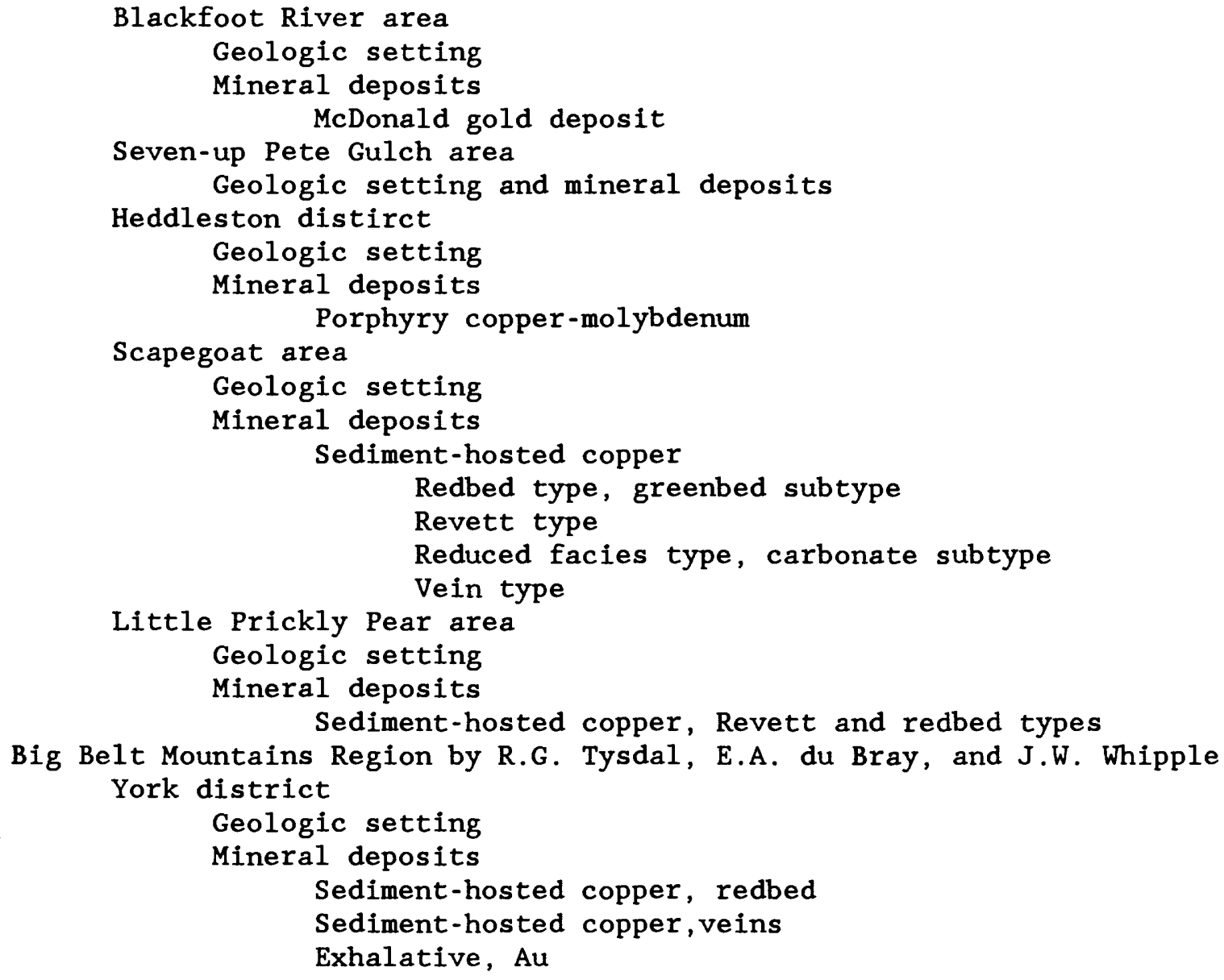

Big Belt Mountains Region by R.G. Tysdal, E.A. du Bray, and J.W. Whipple York district

Geologic setting

Mineral deposits

Sediment-hosted copper, redbed

Sediment-hosted copper, veins

Exhalative, Au 
Vein, Au-bearing

Confederate Gulch district and Southern Big Belt area

Geologic setting

Mineral deposits

Exhalative(?), gold

Cretaceous gold-bearing veins

Northern part of Big Belt Mountains

Geologic setting

Mineral deposits

Lower Belt Supergroup strata in Big Belt Mountains

Geologic setting

Mineral deposits

Massive sulfide deposits

Continental Divide Region by J.E. Elliott

Geologic setting

Subregions

Granite Butte-Ogden Mountain subregion

Geologic setting

Mineral deposits

Lincoln Gulch area

Little Prickly Pear area

Big Blackfoot (Ogden Mountain) district

McClellan Gulch district

Stemple-Gould district

Finn district

Marysville (Silver Creek) district

Black Mountain-Stemwinder Hill subregion

Geologic setting

Mineral deposits

Ophir (Showshoe-Carpenter Creek) distirct

Dog Creek area

Austin district

Stemwinder Hill area

North Boulder Mountains subregion

Geologic setting

Mineral deposits

Helena (Last Chance) district

E11iston district

Rimini (Vaughn) district

Clancy (Lump Gulch) district

Basin (Cataract) district

Wickes (Colorado) district

Boulder (Comet) district

Amazon distict

Elkhorn Mountains Region by Steve Ludington, F.E. Mutschler, and Marta

Franchini

Introduction

Geologic setting

Mineral deposits

Montana City district

McClellan-Mitchell district

Warm Springs district 


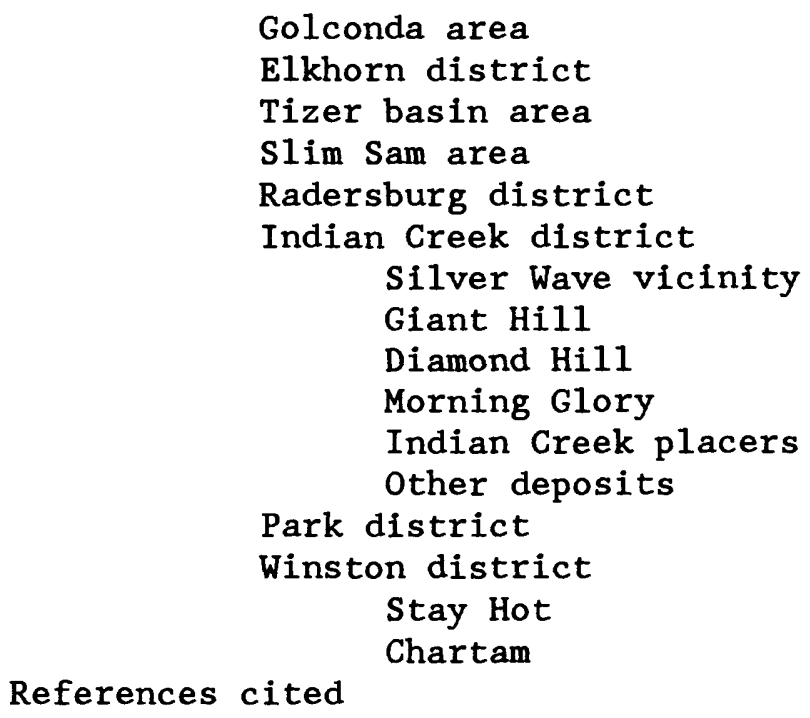

G. Mineral resource assessment for locatable minerals by Steve Ludington, J.E. E1liott, Christopher Osterman, and J.W. Whipple

Introdution

Delineation of permissive areas

Grade and tonnage models

Estimation of number of undiscovered deposits

Simulation

Undiscovered deposits in the Forest

Porphyry copper deposits

Choice of mode1

Known examples

Tract delineation

Estimate of undiscovered deposits

Gold skarn deposits

Choice of model

Known examples

Tract delineation

Estimate of undiscovered deposits

Tungsten-(molybdenum) skarn deposits

Choice of model

Known examples

Tract delineation

Estimate of undiscovered deposits

Polymetallic replacement deposits

Choice of mode1

Known examples

Tract delineation

Estimate of undiscovered deposits

Polymetallic vein deposits

Uranium-bearing polymetallic vein deposits

Climax molybdenite deposits

Choice of model

Known examples

Tract delineation 
Estimate of undiscovered deposits

York-type exhalative(?) gold deposits (and gold-bearing veins)

Tract delineation

Estimate of undiscovered deposits

Cretaceous gold-bearing vein deposits

Quartz-adularia and hot-spring gold-silver deposits

Choice of mode1

Known examples

Tract delineation

Estimate of undiscovered deposits

Sedimentary exhalative zinc-lead deposits

Known examples

Tract delineation

Estimate of undiscovered deposits

Sediment-hosted copper deposits, redbed type

Choice of model

Known examples

Tract delineation

Estimate of undiscovered deposits

Computer simulation results and estimated contained metal

Nature of distributions

Meaning of the estimates

Environmental considerations

Summary

References cited

H. Placer gold

Geology of placer gold deposits by R.G. Tysda1

Introduction

Placers in and adjacent to the Forest

Permissive tracts

Composition of gold grains from placers by W.H. Raymond and G.A.

Desborough

Introduction

Placer gold collected in 1970

Placer gold collected in 1990-94

References cited

I. Nonmetallic mineral deposits by R.G. Tysdal

Barite

Building stone

Clay

Limestone

Phosphate

Sapphires in placer deposits

Silica

Shoreline placer deposits

References cited

J. Petroleum potential of the Helena National Forest by W.J. Perry, Jr., M.J. Pawlewicz, T.A. Daws, T.S. Dyman, and G.A. Desborough

Introduction 
$0 i 1$ and gas plays

Drillhole data

Potential source rocks for hydrocarbons

Methods and terminology

Precambrian

Upper Devonian-Lower Mississippian

Upper Mississippian

Conclusions

Cretaceous

References cited

K. Coal resources of the Helena National Forest area by C.W. Holmes

Geology

References cited

APPENDIX

Digital data products

\section{ILLUSTRATIONS}

\section{PLATES}

1. Map A. Geologic map of Helena National Forest

2. Map B. Map of mines, prospects, and mining districts/area boundaries

3. Map C. Map showing formations mainly composed of limestone and dolomite Map D. Map showing streams with reported placer operations

Map E. Map showing sample locations of placers sampled for analysis of gold

Map F. Map showing contours of lead distribution in stream sediments

4. Map G. Map showing contours of gold distribution in stream sediments

Map H. Map showing lanthanum-geophysical association model

Map I. Map showing geochemical-geophysical model for lead

Map J. Map showing geochemical-geophysical model for gold

5. Map K. Map showing permissive and favorable tracts for porphyry coppermolybdenum deposits

Map L. Map showing permissive and favorable tracts for gold and tungsten skarn deposits

Map M. Map showing permissive tracts for polymetallic replacement deposits

Map N. Map showing permissive tracts for Climax molybdenum deposits

6. Map 0. Map showing permissive tracts for York-type exhalative(?) gold deposits and Cretaceous gold-bearing vein deposits

Map P. Map showing permissive tracts for hot-spring gold-silver deposits and quartz-adularia deposits

Map Q. Map showing permissive tracts for sedimentary exhalative zinclead deposits

Map R. Map showing permissive and favorable tracts for redbed sedimenthosted copper deposits 


\section{FIGURES}

A1. Index map of Helena National Forest and vicinity

A2. Map of Helena National Forest, showing regional subdivisions of Forest, and $1^{\circ} \times 2^{\circ}$ quadrangles.

B1. Map of major geologic features in western Montana and area of Helena National Forest.

C1. Aeromagnetic map of the Helena National Forest.

C2. Index map of aeromagnetic surveys.

C3. Bouguer gravity map of the Helena National Forest.

C4. Terrace-magnetization map of the Helena National Forest.

C5. Terrace-density map of the Helena National Forest.

C6. Potassium aeroradioactivity map of the Helena National Forest.

C7. Uranium aeroradioactivity map of the Helena National Forest.

C8. Thorium aeroradioactivity map of the Helena National Forest.

C9. Uranium:Thorium aeroradioactivity map of the Helena National Forest.

C10. Potassium:Thorium aeroradioactivity map of the Helena National Forest.

D1. Study area and Helena National Forest boundary.

D2. Plot of RASS outcrop sample sites within the study area.

D3. Plot of combined RASS and NURE stream-sediment sites of samples analyzed spectrographically for 31 elements.

D4. Plot of combined RASS and NURE stream-sediment sites of samples analyzed for gold by flameless atomic absorption.

D5. Plot of NURE stream-sediment sample sites.

D6. Graphs showing the relationship of La content classes and elements of the La-association.

D7. Plot showing the La-association distribution within the study area.

D8. Graphs showing the relationship of $\mathrm{Pb}$ content classes and elements of the $\mathrm{Pb}$-association in stream sediments.

D9. Plot showing the $\mathrm{Pb}$-association distribution relative to mines and prospect pits within the Helena National Forest.

D10. Graphs showing relationship of Au content classes and elements of the Auassociation in stream sediments.

D11. Plot showing the Au-association distribution relative to mines and prospect pits within the Helena National Forest.

D12. Plot showing gold distribution in RASS and NURE stream sediments within the study area.

D13. Plot showing silver distribution in RASS and NURE stream sediments within the study area.

D14. Plot showing lead distribution in RASS and NURE stream sediments within the study area.

D15. Plot showing bismuth distribution in RASS and NURE stream sediments within the study area.

D16. P1ot showing copper distribution in RASS and NURE stream sediments within the study area.

D17. Plot showing molybdenum distribution in RASS and NURE stream sediments within the study area.

D18. Plot showing tin distribution in RASS and NURE stream sediments within the study area.

D19. Plot showing zinc distribution in RASS and NURE stream sediments within the study area.

D20. P1ot showing iron distribution in RASS and NURE stream sediments within the study area. 
D21. Plot showing manganese distribution in RASS and NURE stream sediments within the study area.

D22. Graphs showing the SEM-EDS determined major composition of heavy minera1 concentrates from the study area.

D23. Graphs showing the SEM-EDS determined major composition of slabbed outcrop samples from the study area.

D24. Graphs showing the SEM-EDS determined major composition of heavy mineral concentrates from the Elliston mining district.

D25. Graphs showing the SEM-EDS determined major composition of heavy mineral concentrates from the Marysville mining district.

D26. Graphs showing the SEM-EDS determined major composition of heavy mineral concentrates from the Stemple-Gould mining district.

F1. Regions, subregions, and mining districts and areas, Elkhorn Mountains.

F2. Geology and mining districts and areas in E1khorn Mountains.

F3. Selected mining and exploration properties in Elkhorn Mountains.

G1. Simulated metal content in undiscovered porphyry copper deposits.

G2. Simulated metal content in undiscovered skarn gold deposits exclusive of Elkhorn Mountains.

G3. Simulated metal content in undiscovered skarn gold deposits in Elkhorn Mountains only.

G4. Simulated metal content in undiscovered polymetallic replacment deposits .

G5. Simulated metal content in undiscovered Climax molybdenite deposits.

G6. Simulated metal content in undiscovered hot-spring gold deposits.

G7. Simulated metal content in undiscovered epithermal quartz-adularia deposits.

G8. Arsenic and antimony content of mineralized samples from Elkhorn Mountains

J1. Map showing drillholes, sample sites, and petroleum plays in vicinity of Helena National Forest.

J2. Graphic presentation of data shown in table J6.

K1. Coal fields and potentially coal-bearing rocks near the Helena National Forest.

\section{TABLES}

Al. Mineral deposit types in the Helena National Forest.

Cl. Flight specifications for aeromagnetic surveys.

C2. Densities of rock samples.

C3. Summary of aeromagnetic anomalies.

D1. List of the lower limits of determination for the six-step semiquantitative emission spectrographic analytical method.

D2. Table listing the NURE analytical techniques and elements analyzed.

D3. Minima, maxima, means, and standard deviations of the RASS outcrop samples.

D4. Correlation table for the spectrographic analytical data from the RASS outcrop sample analyses.

D5. Percent frequency distributions of elements determined by spectrographic analyses of analytical data from the RASS ouctrop samples.

D6. Minima, maxima, means, and standard deviations of the combined NURE and RASS stream sediments determined by spectrographic methods.

D7. Correlation table for the combined NURE and RASS stream sediment analytical data analyzed by spectrographic methods. 
D8. Percent frequency distributions of elements determined by spectrographic analyses of combined NURE and RASS stream sediments.

D9. Minima, maxima, means, and standard deviations of the gold values of the combined NURE and RASS stream sediments.

D10. Correlation table of spectrographic analyses of NURE and RASS streamsediments analyzed for gold.

D11. Percent frequency distributions of elements determined by spectrographic analyses of the NURE and RASS stream sediments analyzed for gold.

D12. Minima, maxima, means, and standard deviations of the NURE analyses of stream sediment sample analyses.

D13. Correlation matrix for the NURE analyses.

D14. Percent frequency distributions of elements in stream sediments analyzed by NURE.

D15. Minima, maxima, means and standard deviation of Au in NURE and RASS stream sediments analyzed by atomic absorption spectrographic methods.

D16. Correlation of gold and other elements in NURE and RASS stream sediments as determined by atomic absorption spectrographic methods.

D17. Percent frequency distribution of gold values in NURE and RASS stream sediments as determined by atomic absorption spectrographic methods.

E1. Location and description of mines and prospects in the Helena National Forest.

F1. Mineral deposits and correlative magmatic events in Elkhorn Mountains.

F2. Chemical analyses of mineralized rocks from E1khorn Mountains.

G1. Mineral deposit models used in the assessment.

G2. Summary of mineral deposit types analyzed.

G3. Summaries of simulated metal in undiscovered deposits.

G4. Mean and median estimated metal endowment for undiscovered metallic deposits.

H1. Production from placer deposits in and adjacent to the Helena National Forest.

H2. Composition of gold grains collected from placers in the Helena National Forest.

J1. Drillholes in the vicinity of Helena National Forest

J2. Rock-Eval analyses of samples from the Helena National Forest and vicinity.

J3. Vitrinite reflectance of samples from the Helena National Forest and vicinity.

J4. Rock-Eval analyses for samples from a drillhole.

J5. XRD relative peak heights of samples from the Helena National Forest and vicinity.

J6. Summary of previously published or available source-rock data.

\section{APPENDIX}

Resource/reserve classification diagram.

Geologic time chart. 


\title{
THE HELENA NATIONAL FOREST STUDY AREA
}

\author{
By R.G. Tysdal and Steve Ludington
}

\section{INTRODUCTION}

The geology and mineral and energy resources of the Helena National Forest (the Forest) $(975,088$ acres; fig. Al) were studied by the U.S. Geological Survey (USGS) during 1993-1995. The study was conducted to assess the undiscovered mineral resources of the Forest and to provide minerals information to the U.S. Forest Service (USFS) for land-use planning in the management of Forest lands. The study did not assess the mineral resource potential of the few large tracts of private land that exist within the Forest boundary, mainly in the southern part of the Big Belt Mountains (fig. A2). Mineral resources of lands adjacent to the Forest were considered in the assessment process, however, because the resource potential of the Forest lands were evaluated within a regional geologic context. The study used published geologic mapping that was supplemented with new mapping where needed. The study integrated existing geochemical data with newly obtained data; conducted local detailed gravity, magnetic, and gamma-ray aeroradiometric geophysical studies for specific areas of the Forest, augmenting a Forest-wide database that had been acquired previously; and assembled preexisting mineral occurrence and current exploration data.

\section{Importance of the Study}

Mining and exploration activity and other lines of evidence from geologic, geochemical, and geophysical data indicate the Forest has significant potential for the occurrence of undiscovered resources. The Forest contains mining districts/areas that historically have produced metals from both lode mines and placer deposits. No lode mines were in production during the course of this study, small-scale placering operations exist, mainly in Confederate Gulch in the Big Belt Mountains. Properties where exploration/development is concentrated currently are on land adjacent to the Forest--at McDonald Meadows, near Lincoln; the Elkhorn mine, on the southern flank of the Elkhorn Mountains; and the Diamond Hill prospect, on the eastern flank of the Elkhorn Mountains. The McDonald Meadows hot-spring gold deposit contains about 8.2 million ounces of gold within 414 million tons of rock, of which about 5.2 million ounces of gold can be mined economically at present (Canyon Resources Corporation, 1993). At a price of $\$ 375$ per ounce, economically recoverable gold of the deposit has a value of about $\$ 2$ billion.

This study of the Helena National Forest is primarily concerned with mineral and energy resources. However, the geologic, geochemical, and geophysical components that make up parts of the report also constitute fundamental building blocks that pertain to other, related scientific studies that may be undertaken--for example, mine remediation. The foundation of the mineral resource assessment is a geologic map (scale 1:126,720, where 1 in. 
equals $2 \mathrm{mi}$ ), shown on plate 1 . A derivative of the geologic map shows the geologic formations that are largely composed of limestone and (or) dolomite (plate 3, map C). The derivative map is important because calcareous rocks act to fix (chemically bind) potentially hazardous chemical elements present in streams, thus the rocks are naturally occurring remediation substances. The geologic map also can be a fundamental building block for USFS hydrologists, soils scientists, biologists, and botanists to use in conjunction with other scientific studies conducted for effective management of Federal lands. Rock chemistry is a primary control of the types of soils and plants that exist in an area, the quality of water, and ultimately the health of wildlife in an area. Maps of this report show the distribution of tracts of rock permissive for different types of mineral deposits in the Forest, but they also give clues to the distribution of naturally occurring chemical elements.

\section{Location}

The Helena National Forest is comprised of 975,088 acres that 1ie within parts of seven counties: Broadwater, Cascade, Gallatin, Jefferson, Meagher, Powe11, and Lewis and Clark (fig. Al). No Federal land is present in the parts of Cascade and Gallatin Counties that lie within the Forest boundary. The Forest includes virtually all of the Big Belt Mountains; most of the Elkhorn Mountains; and, west of $112^{\circ}$ longitude, mountainous terrain that extends from about $46^{\circ} 20^{\prime}$ $\mathrm{N}$. latitude, southwest of Helena, to about $47^{\circ} 15^{\prime} \mathrm{N}$., north of Lincoln, Mont. (fig. A2). The Gates of the Mountains Wilderness lies within the Forest in the northern extent of the Big Belt Mountains, and the southern part of the Scapegoat Wilderness lies within the Forest in the mountains north of Lincoln (fig. A2).

\section{Previous and Concurrent Studies}

Mineral resource assessment studies were conducted previously in three regions of the Forest under consideration for Wilderness status, as mandated by the Wilderness Act of 1964 (public law 88-577). Two of the three regions were designated as wilderness and their boundaries are shown in figure Al. The Gates of the Mountains Wilderness (about $45 \mathrm{sq} \mathrm{mi} ; 28,600$ acres), and proposed additions thereto (about $16 \mathrm{sq} \mathrm{mi} ; 10,000$ acres), in the northern part of the Big Belt Mountains, were examined in 1976 (Reynolds and Close, 1984; Close and Rigby, 1984). The Scapegoat Wilderness (375 sq mi; 240,000 acres), which includes about $130 \mathrm{sq} \mathrm{mi}(83,000$ acres) in the Helena National Forest north of Lincoln, was studied in the early 1970's (Mudge and others, 1974). Two proposed additions to the Scapegoat (totaling $84 \mathrm{sq} \mathrm{mi} ; 53,750$ acres), which includes about $60 \mathrm{sq} \mathrm{mi}$ (38,000 acres) within the Helena National Forest, were examined in the mid-1970's (Earhart and others, 1977). Neither of the two proposed additions is recommended for wilderness status by the Forest Service, and their boundaries are not shown in figure Al; one area adjoined the wilderness to the south and the other adjoined to the southeast. A large part of the Elkhorn Mountains also was studied under the Wilderness Act (Greenwood and others, 1978, 1990), although the area has not been designated a wilderness. This study encompassed about $135 \mathrm{sq}$ 


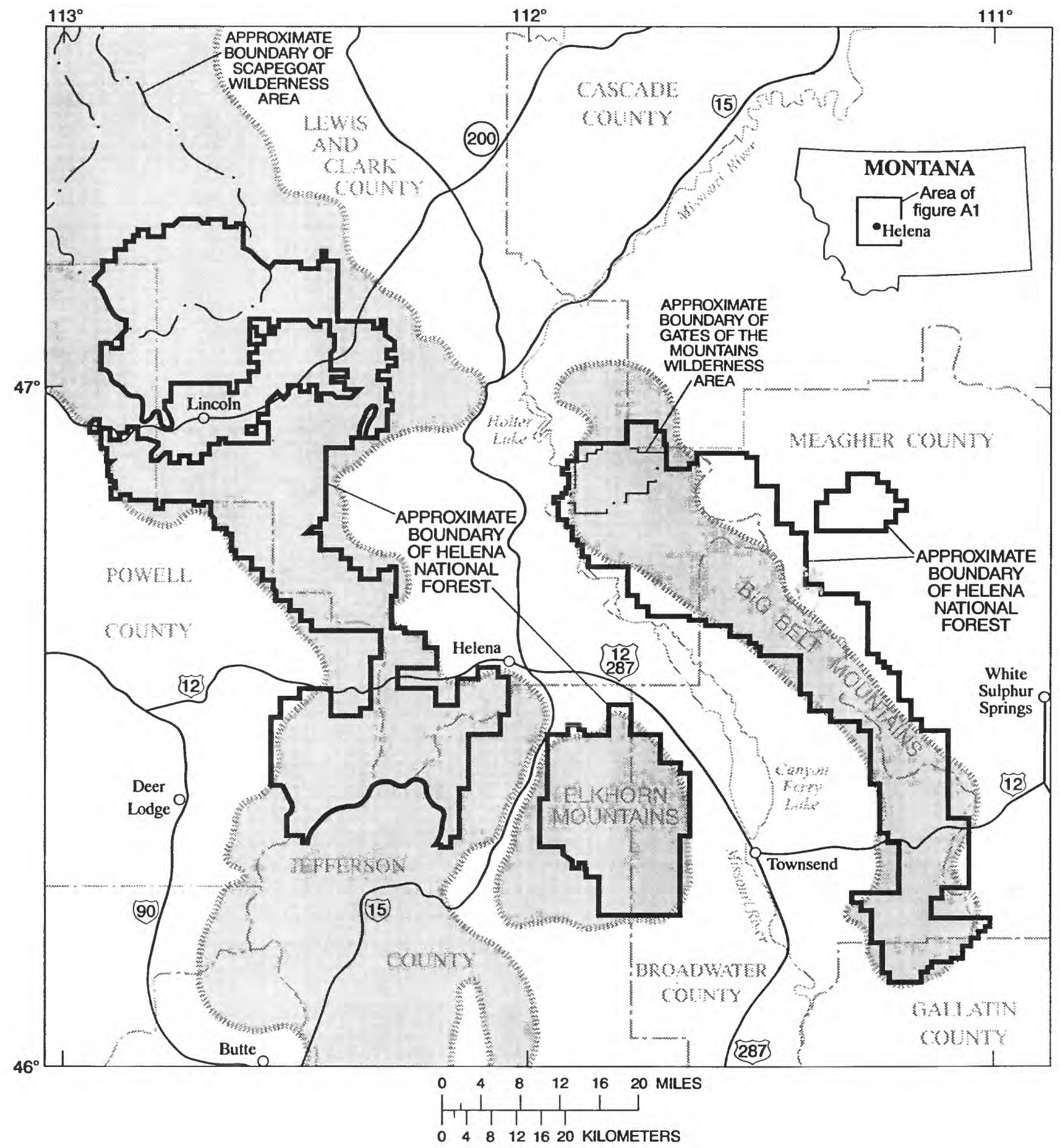

Figure A1. Index map of Helena National Forest and vicinity, showing Gates of the Mountains Wilderness, Scapegoat Wilderness, principal towns, county boundaries, and highways. Shading shows mountainous terrain. 
mi (87,000 acres), which mostly lies in the Helena National Forest. No new data were acquired from the two wildernesses during the present study, or proposed additions thereto, but new data were obtained from the Elkhorn study area. The previously collected data from all three regions were incorporated into the present study.

The Forest lies within parts of three $1^{\circ} \times 2^{\circ}$ quadrangles (fig. A2). Two of the quadrangles have been examined under Geological Survey CUSMAP (Continental United States Mineral Resource Appraisal Program) projects, in which delineated terranes were rated as having a low, medium, high, and locally very high, resource potential for specific types of mineral deposits. The Choteau $1^{\circ} \times 2^{\circ}$ quadrangle was examined in the late 1970's (Earhart and others, 1981; Mudge and others, 1982). The Butte $1^{\circ} \times 2^{\circ}$ quadrangle was the focus of extensive study in the 1980's (Elliott and others, 1992; Elliott and others, 1993a,b,c; Wallace and others, 1986). Publications of the two CUSMAP projects provide extensive citation to previous topical and areal geologic work conducted within the respective quadrangles. Results from the CUSMAP studies were incorporated into the present study, supplemented by new data obtained during the 1993 and 1994 field seasons.

In addition to previous wilderness and CUSMAP studies, topical and areal studies conducted by the U.S. Geological Survey, either within the Forest or including areas within the Forest, contributed data to this report. The first comprehensive compllation of mining and mineral exploration in the Forest region was by Pardee and Schrader (1933), who visited mines and prospects when they were actively being examined, mined, and (or) developed. Many of the mine workings are no longer accessible. Detailed mineral resource and map data were obtained from reports on the Elkhorn Mountains by Klepper and others $(1957,1971)$. Several geologic maps, in addition to those cited previously, were used to compile the geologic map of this report; the area and reference for each is shown in the "Index map showing sources of geologic data" on plate 1.

An extensive compilation of historical data on placer gold operations was made by Lyden (1948) of the Montana Bureau of Mines and Geology. These data form the basis of maps D and E (plate 3), which show the distribution of deposits that historically produced placer gold in and adjacent to the Forest.

The Forest overlies parts of several regional "plays," which are geologic provinces that are evaluated for their petroleum potential. The petroleum plays are discussed in Chapter $\mathrm{J}$, which makes use of the previous assessment of Perry (1989) and the more recently completed study of Perry (1995).

\section{Methodology}

The assessment of mineral resources makes use of all of the previous studies as well as data collected during this and concurrent studies within the Forest and adjacent areas. Geophysical (Chapter C) and geochemical (Chapter D, plates 3,4$)$ data were compiled from a variety of sources. These data are shown in a variety of figures and tables. Mines, prospects, and mineral occurrences data are presented in a lengthy table in Chapter E, with locations shown on plate 2. Mineral resource data are presented and discussed in Chapter $F$, which is arranged by regions and by mining district or mining area. This arrangement permits discussion of the geology in a geographic scheme designed for ease of use by USFS personnel and, simultaneously, for convenience in discussing geology. 


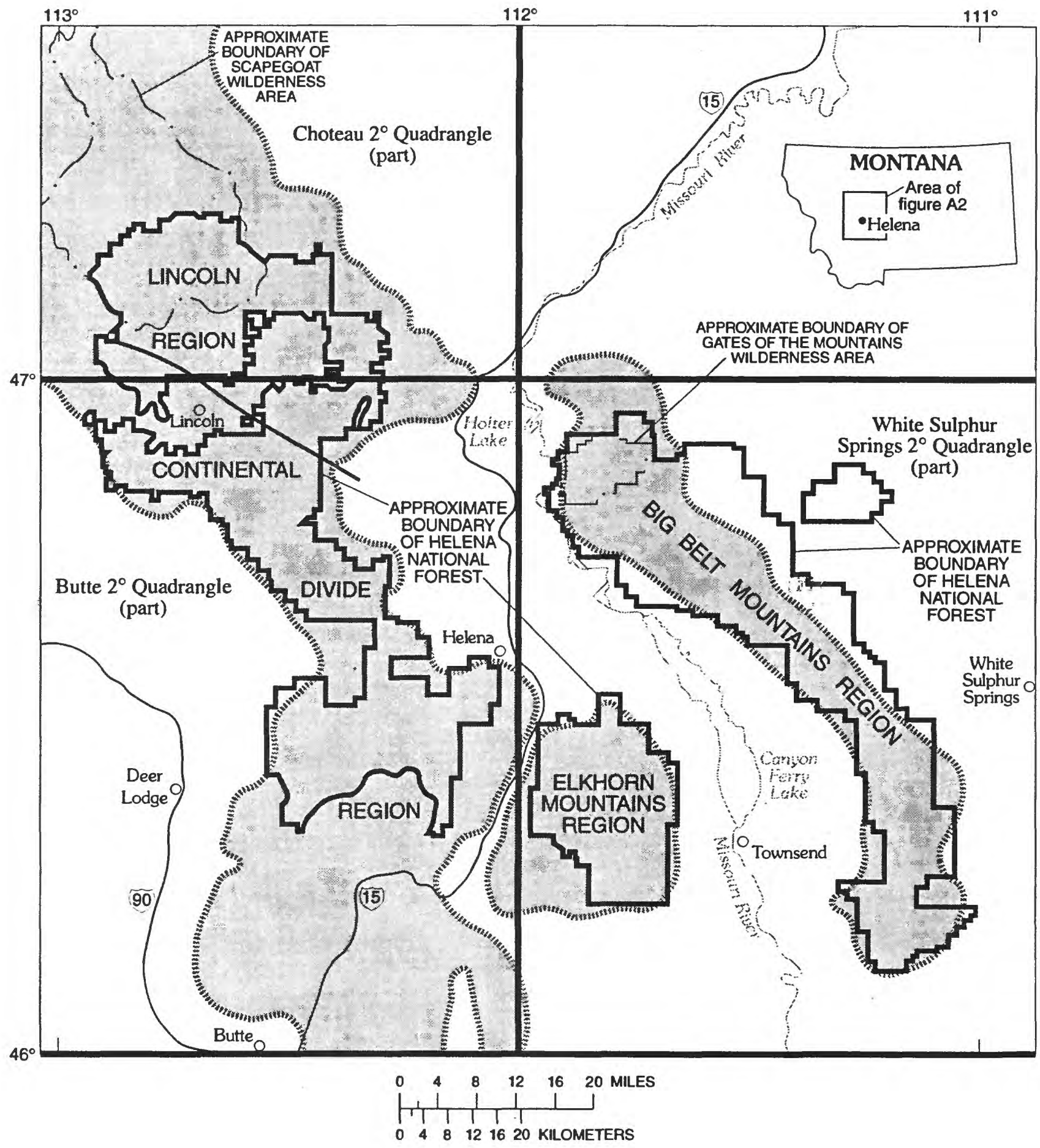

Figure A2. Map of Helena National Forest, showing regional subdivisions of the Forest used for discussion of geologic characteristics, and boundaries of the Choteau, Butte, and White Sulphur Springs $1^{\circ} \times 2^{\circ}$ quadrangles. Shading shows mountainous terrain. 
In the assessment process, mineral deposits of the Forest were classified into types (table Al) based on mineral deposit models (Cox and Singer, 1986), which describe the essential characteristics of a class of mineral deposits. Each of the mines, prospects, and mineral occurrences listed in table E1 are classified as to deposit type, except for a few that are 1 isted as unclassified. The study region was delineated into tracts (areas) of land that contain attributes typical of deposits of a particular type. A permissive tract may contain known deposits of the type, and (or), based on geologic evidence, deposits can reasonably be inferred to exist within the tract even though none have been found (tracts are "permissive" for their existence). Terranes deemed permissive for the occurrence of one or more mineral deposit types are shown as mineral resource assessment tracts (plates 4, 5, 6, maps $\mathrm{K}$ through $\mathrm{R}$ ). Although the tracts are delimited to include all rocks considered likely to host the deposit type(s), the boundaries could change in the future if new data became available. Some areas within tracts are shown as favorable, meaning that a combination of criteria suggest that deposits are more likely to be present in this part of the permissive tract. The criteria used to define each tract and favorable area are discussed in Chapter $\mathrm{G}$.

For several deposit types present in the Forest, a subjective, quantitative estimate was made of the number of undiscovered deposits of a particular type that may lie beneath a tract. The estimate was made only for those types of deposits for which grade and tonnage data exist. The quantitative methodolgy, explained in Chapter G, has been described in Singer and Cox (1988), Menzie and Singer (1990), and Ludington and others (1992).

\section{Undiscovered Resources}

Definitions for resource classification for minerals are as follows, based on the scheme devised jointly by the U.S. Bureau of Mines and the U.S. Geological Survey (1980). A diagram of the resource/reserve classification scheme is presented in the Appendix.

Resource--A concentration of naturally occurring minerals in or on the Earth's crust in such form and amount that economic extraction of a commodity from the concentration is currently or potentially feasible.

Identified Resources--Resources whose location, grade, quality, and quantity are known or estimated from specific geologic evidence. Identified resources may be economic, marginally economic, or subeconomic. To reflect varying degrees of geologic certainty, these economic divisions can be subdivided into demonstrated (measured and indicated) and inferred resources.

Undiscovered Resources--Resources whose existence is only postulated; these postulated resources may be in deposits that are economic, marginally economic, or subeconomic. 
Table Al. Mineral deposit types known or suspected to occur within the Helena National Forest, and principal commodities expected.

\begin{tabular}{ll}
\hline \multicolumn{1}{c}{ Deposit type } & Commodities expected \\
\hline Porphyry copper-molybdenum & Copper, molybdenum \\
Climax molybdenum & Molybdenum \\
Skarn gold & Gold \\
Skarn tungsten & Tungsten \\
Breccia pipe, tourmaline & Gold \\
Breccia pipe, polymetallic & Gold, silver, lead, zinc \\
Vein, gold-bearing & Gold \\
Vein and desseminated, gold-bearing & Gold \\
Vein, polymetallic & Gold, silver, copper, lead, zinc \\
Vein, polymetallic, uranium-bearing & Uranium \\
Polymetallic replacement & Gold, silver, copper, lead, zinc \\
Hot-spring gold-silver & Gold, silver \\
Epithermal vein, quartz-adularia & Gold, silver \\
Epithermal manganese & Manganese \\
York-type exhalative(?) gold & Gold \\
Sedimentary exhalative zinc-lead & Zinc, lead \\
Redbed sediment-hosted copper & Copper, silver \\
Sediment-hosted copper, veins & Copper \\
Quartz veins & High-purity silica \\
Vein, barite & Barite \\
Bog copper & Copper \\
Bog manganese & Manganese \\
Shoreline placer & Titanium, iron, zircon \\
Limestone & Limestone \\
Phosphate, upwe1ling type & Phosphate \\
Placer gold & Gold \\
Placer sapphire & Sapphires \\
Stone, building & Building stone \\
& \\
&
\end{tabular}

\section{Digital Products}

The geologic map (plate 1) also was prepared in digital form (ARC/INFO) at the request of the USFS. Several other maps, of plates 2-6, also are on the same disk. Many figures, some of which contain closely spaced patterns (shading) that does not reproduce we 11 in black-and-white, also are contained on the same CD-ROM (Green and Tysdal, 1996). A listing of the contents of the disk is provided in the Appendix of this report.

\section{ACKNOWLEDGMENTS}

Many individuals in private industry, in the U.S. Forest Service, and within the U.S. Geological Survey provided essential information for this study or assisted the authors in various aspect of data compilation and mineral 
resource assessment. The initial meeting concerning the mineral resource assessment of the Forest benefited from input of representatives of the Montana Bureau of Mines and Geology, Butte; personnel from the U.S. Bureau of Mines, Spokane; U.S. Forest Service representatives from the Deer Lodge and Helena National Forests, and from the Region 1 Office, Missoula; and representatives from the mining industry, including Noranda Mining Co., Phelps Dodge Corporation, and Pegasus Gold, Incorporated.

U.S. Forest Service geologists Beth Ihle of the Townsend Ranger District and Bill Straley of the Helena Supervisors Office were especially helpful in providing information on active prospecting and exploration. Beth also very kindly arranged for our use of Forest Service storage facilities, facilitated arrangements with other USFS personnel and with private property owners, and provided lively discussions of geologic exploration activities and needs of the USFS. Several owners of private property permitted USGS personnel to cross their land to gain access to Forest land that otherwise was not readily accesible. We thank these persons for their cooperation.

Information of much significance to the mineral resource assessment of the Forest was gained through discussions and visits to mines and exploration projects. Geologists of the Phelps Dodge Corporation provided access and shared information on the McDonald Meadows gold property adjacent to the Forest, near Lincoln. Mike Maslowski, Greg Wittman, and Steve Petroni of Pegasus Resources provided access to exploration properties, shared information, and conducted field excursions to several properties on or adjacent to the Forest. Jeff Brooks of Santa Fe Minerals Corporation conducted a field excursion to the Elkhorn mine, adjacent to the Forest, on the south flank of the Elkhorn Mountains. Operators of the French Bar and Lovestone placer sapphire mines, on alluvial bars adjacent to the Missouri River, kindly provided access to their producing properties.

The assessment of oil and gas resources was aided by David W. Ballard, Bradford R. Burton, and John R. Warne, geologists from industry, who provided source-rock samples. Subsurface data was provided by Melody R. Holm (U.S. Forest Service) and James W. Halvorson, Montana Board of Oil and Gas Conservation.

G.N. Green of the USGS worked carefully and patiently to digitize maps shown on plates in this report. These maps and a digital geologic map are available on compact disk (Green and Tysdal, 1996). G.I. Selner of the USGS provided advice on use of GSPOST and GSMAP used in compilation of some maps and files. C.A. Wallace provided unpublished descriptions of Proterozoic and Phanerozoic strata of the Butte $1^{\circ} \times 2^{\circ}$ quadrangle, from which shortened descriptions of rock units were derived for the geologic map; he also provided an advance copy of a map that was subsequently published. Timothy Hall, a seasonal geologist with the USGS, performed excellent field and laboratory assistance to the geochemical part of this study. Steven S. Smith donated several days of time to revise digital geochemical maps (plate 3 and 4, maps $F$, G, H, I, J), and Gregory Lee provided help with the digital files of geochemical data. Several of the figures were drafted by W.R. Stephens and later revised by D.A. Lindsey of the U.S.G.S.

\section{REFERENCES CITED}

Canyon Resources Corporation, 1993, McDonald gold project in Montana moves forward with final feasibility study: News Release, 1 p. 
Close, T.J., and Rigby, J.G., 1984, Mineral investigation of the Gates of the Mountains Wilderness Study Area, Lewis and Clark County, Montana: U.S. Bureau of Mines Open-File Report MLA 3-84, 20 p.

Cox, D.P., and Singer, D.A., eds., 1986, Mineral deposit models: U.S. Geological Survey Bulletin 1693, 379 p.

Earhart, R.L., Grimes, D.J., Leinz, R.W., and Marks, L.Y., 1977, Mineral resources of the proposed additions to the Scapegoat Wilderness, Powell and Lewis and Clark Counties, Montana, with a section on Geophysical surveys by D.L. Peterson: U.S. Geological Survey Bulletin 1430, 62 p.

Earhart, R.L., Mudge, M.R., Whipple, J.W., and Connor, J.J., 1981, Mineral resources of the Choteau $1^{\circ} \times 2^{\circ}$ quadrangle, Montana: U.S. Geological Survey Miscellaneous Field Investigations Map MF-858A, scale 1:250,000.

Elliott, J.E., Loen, J.S., Wise, K.K., and Blaskowski, M.J., 1992, Maps showing locations of mines and prospects in the Butte $1^{\circ} \times 2^{\circ}$ quadrangle, western Montana: U.S. Geological Survey Miscellaneous Investigations Series Map I-2050-C, scale 1:250,000.

E1liott, J.E., Wallace, C.A., Lee, G.K., Antweller, J.C., Lidke, D.J., Rowan, L.C., Hanna, W.F., Trautwein, C.M., Dwyer, J.L., and Mo11, S.H., 1993a, Maps showing mineral resource assessment for vein and replacement deposits of gold, silver, copper, lead, zinc, manganese, and tungsten in the Butte $1^{\circ} \times 2^{\circ}$ quadrangle, Montana: U.S. Geological Survey Miscellaneous Investigations Series Map I-2050-D, scale 1:250,000.

Elliott, J.E., Wallace, C.A., Lee, G.K., Antweiler, J.C., Lidke, D.J., Rowan, L.C., Hanna, W.F., Trautwein, C.M., Dwyer, J.L., and Mo11, S.H., 1993b, Maps showing mineral resource assessment for skarn deposits of gold, silver, copper, tungsten and iron in the Butte $1^{\circ} \times 2^{\circ}$ quadrangle, Montana: U.S. Geological Survey Miscellaneous Investigations Series Map I-2050-E, scale $1: 250,000$.

Elliott, J.E., Wallace, C.A., Lee, G.K., Antweiler, J.C., Lidke, D.J., Rowan, L.C., Hanna, W.F., Trautwein, C.M., Dwyer, J.L., and Mol1, S.H., 1993c, Maps showing mineral resource assessment for porphyry and stockwork deposits of copper, molybdenum, and tungsten and for stockwork and disseminated deposits of gold and silver in the Butte $1^{\circ} \times 2^{\circ}$ quadrangle, Montana: U.S. Geological Survey Miscellaneous Investigations Series Map I-2050-F, scale 1:250,000.

Green, G.R., and Tysdal, R.G., 1996, Digital maps and figures on CD-ROM for mineral and energy resource assessment of the Helena National Forest, west-central Montana: U.S. Geological Survey Open-File Report 96-683-B, 1 CD-ROM disk.

Greenwood, W.R., Ludington, S.D., Miller, W.R., and Hanna, W.F., 1978, Mineral resources of the Elkhorn Wilderness study area, Montana, Chapter A, Mineral resource assessment, with a section on Geophysical exploration by W.F. Hanna, a section on Geochemical exploration by W.R. Miller, Steve Ludington, and W.R. Greenwood, a section on Geological and geochemical evaluation by Steve Ludington and W.R. Greenwood, and a section on Uranium and thorium potential by K.J. Wenrich-Verbeck, W.R. Miller V.J. Suits, and John McHugh: U.S. Geological Survey Open File Report 78-325, 362 p.

Greenwood, W.R., Ludington, Steve, Miller, W.R., and Hanna, W.F., 1990, Mineral resources of the Elkhorn Wilderness study area, Broadwater and Jefferson Counties, Montana, with a section on Uranium and thorium potential by K.J. Wenrich, W.R. Miller, V.J. Suits, and John McHugh: U.S. Geological Survey Bulletin 1805, 37 p. 
Klepper, M.R., Weeks, R.A., and Ruppel, E.T., 1957, Geology of the southern Elkhorn Mountains, Jefferson and Broadwater Counties, Montana: U.S. Geological Survey Professional Paper 292, 82 p.

Klepper, M.R., Ruppe1, E.T., Freeman, V.L., and Weeks, R.A., 1971, Geology and mineral deposits, east flank of the Elkhorn Mountains, Broadwater County, Montana: U.S. Geological Survey Professional Paper 665, 66 p.

Ludington, Steve, Orris, G.J., and Cox, D.P., 1992, Methods of resource assessment, in U.S. Geological Survey and Servicio Geologico de Bolivia, Geology and mineral resources of the Altiplano and Cordillera Occidental, Bolivia: U.S. Geological Survey Bulletin 1975, p. 218-219.

Lyden, C.J., 1948, The gold placers of Montana: Montana Bureau of Mines and Geology Memoir 26, $152 \mathrm{p}$.

Menzie, W.D., and Singer, D.A., 1990, A course on mineral resource assessment; Proceedings of the International symposium on mineral exploration--The case for intelligence: Tokyo and Tsukuba, Japan, Mining and Material Processing Institute of Japan, p. 177-188.

Mudge, M.R., Earhart, R.L., Watts, K.C., Tuchek, E.T., and Rice, W.L. 1974, Mineral resources of the Scapegoat Wilderness, Powell and Lewis and Clark Counties, Montana with a section on Geophysical surveys by D.L. Peterson: U.S. Geological Survey Bulletin 1385-B, 82 p.

Mudge, M.R., Earhart, R.L., Whipple, J.W., and Harrison, J.E., 1982, Geologic and structure map of the Choteau $1^{\circ} \times 2^{\circ}$ quadrangle, western Montana: U.S. Geological Survey Miscellaneous Investigations Series Map I-1300, scale $1: 250,000$.

Pardee, J.T., and Schrader, F.C., 1933, Metalliferous deposits of the greater Helena mining region, Montana: U.S. Geological Survey Bulletin 842, 318 p.

Perry, W.J., Jr., 1989, A review of the geology and petroleum resource potential the Montana thrust belt, with a section on geology of potential Mississippian treservoir rocks, disturbed belt sector of the Montana thrust belt by K.M. ichols: U.S. Geological Survey Open-File Report 88450C, $28 \mathrm{p}$.

Perry, W.J., Jr., 1995, Montana thrust belt province (027), in Gautier, D.L., Dolton, G.L., Takahashi, K.I., and Varnes, K.L., eds., 1995 National Assessment of United States oil and gas resources--Results, methodology, and supporting data: U.S. Geological Survey Digital Data Series DDS-30 [CD-ROM] .

Reynolds, M.W., and Close, T.J., 1984, Gates of the Mountains Wilderness and additions, Montana, in Marsh, S.P., Kropschot, S.J., and Dickinson, R.G., Wilderness mineral potential, Assessment of mineral-resource potential in U.S. Forest Service lands studied, 1964-1984: U.S. Geological Survey Professional Paper 1300, p. 705-708.

Singer, D.A., and Cox, D.P., 1988, Applications of mineral deposit models to mineral resource assessments: U.S. Geological Survey Yearbook, Fiscal Year 1987, p. 55-57.

U.S. Bureau of Mines and U.S. Geological Survey, 1980, Principles of a resource/reserve classification for minerals: U.S. Geological Survey Circular $831,5 \mathrm{p}$.

Wallace, C.A., Schmidt, R.G., Lidke, D.J., Waters, M.R., Elliott, J.E., French, A.B., Whipple, J.W., Zarske, S.E., Blaskowski, M.J., Heise, B. A., Yeoman, R.A., O'Neil1, J.M., Lopez, D.A., Robinson, G.D., and Klepper, M.R., 1986, Preliminary geologic map of the Butte $1^{\circ} \times 2^{\circ}$ quadrangle, western Montana: U.S. Geological Survey Open-File Report 86-292, scale 1:250,000, text. 


\title{
GEOLOGIC SETTING OF THE HELENA NATIONAL FOREST STUDY AREA
}

\author{
By R.G. Tysdal
}

\section{GEOLOGIC MAPPING}

The geologic map compiled for this study ( $p 1.1$ ) is at a scale of $1: 126,720$ ( 1 in. equals $2 \mathrm{mi}$ ), in accord with the scale of the "Forest Use Map of the Helena National Forest", published by the U.S. Forest Service. No geologic map with the appropriate geologic detail was available for the entire Helena National Forest (the Forest). The database of maps used to compile the geologic map, and the area covered by each map, is shown on plate 1 . A geologic time chart is given in the Appendix.

\section{REGIONAL GEOLOGIC SETTING}

Strata of Middle Proterozoic and younger age form a package of rocks tens of thousands of feet thick in western Montana and an area of central Montana that is delimited by the Wagner Gulch-Volcano Valley fault on the north and by faults of the southwestern Montana Transverse Zone on the south (fig. B1). The Forest overlies this thick package of strata and the eastern part of the Forest lies within the fault-bounded area. The faults at the north and south margins of the fault-bounded area were active intermittently from the Proterozoic to the present and influenced the distribution, facies, and thickness of sediments. Strata of the Middle Proterozoic Belt Supergroup, and some Paleozoic and younger stratigraphic units as we11, accumulated to a much greater thickness in the fault-bounded area of west-central Montana than in regions to the north and south.

The rocks of western Montana and the fault-bounded area of west-central Montana lie within the Cordilleran thrust belt terrane, in which large-scale thrust faults displaced strata eastward during the Late Cretaceous to early Tertiary. The Wagner Gulch-Volcano Valley fault and faults of the southwestern Montana Transverse Zone, which delimit the north and south margins of the central Montana area, also delimit the north and south margins of thrust terrane within central Montana. This eastern protrusion of thrusted terrane is known as the Helena structural salient, or simply the Helena salient (fig. Bl).

\section{STRATIGRAPHY}

Sedimentary rocks exposed in the Forest and directly adjacent areas include Precambrian, Paleozoic, Mesozoic, and Cenozoic strata. Unconsolidated rock materials such as gravels are present locally in the mountains, where they mainly form veneers, and on mountain flanks and within intermontane valleys where they form thick deposits. Individual rock units present in the Forest are described on plate 1 and the descriptions are not repeated here. Where appropriate, 
however, map symbols from plate 1 (for example, Kk, for Cretaceous Kootenai Formation) appear in parentheses in the text that follows. Carbonate (limestone and dolomite) rock units are shown on a derivative geologic map (p1. 3, map C). This derivative map was prepared at the request of the Forest Service for applications in their ongoing botanical, biological, hydrologic, and soil studies. Rocks shown as calcareous or dolomitic generally contain a small percentage of calcite or dolomite.

Precambrian sedimentary rocks are comprised of the Middle Proterozoic Belt Supergroup that, in aggregate, is several tens of thousands of feet thick in the overthrust belt of western Montana, where these strata comprise the majority of rocks (Harrison, 1972). This sequence extends well eastward within the embayment into central Montana (McMannis, 1965). Belt strata constitute more than half of the areal extent of rocks in the Forest and underlie the younger rocks in the remainder of the Forest. The maximum thickness of the Belt strata exposed within or directly adjacent to the Forest is greater than 35,000 ft. The lower two units, the Newland and Greyson Formations, crop out only in the Big Belt Mountains, except for slivers of the Greyson north of Lincoln. The two formations total about 20,000 ft in combined thickness in the central to southern part of the Big Belt Mountains (Nelson, 1963; Skipp and Peterson, 1965). Belt Supergroup rocks only as young as the Helena Formation are present in the Big Belt Mountains, whereas younger formations of the Supergroup are present in the western part of the Forest, in the region north from U.S. Highway 12 to the northern extent of the Forest. Several formations of the Belt Supergroup contain mineral occurrences and the distribution of the minerals was determined by processes that took place during sedimentation or shortly thereafter. Throughout the region, rocks of the Belt Supergroup were metamorphosed to the lower greenschist facies prior to deposition of Paleozoic and younger strata.

Paleozoic rocks of the Forest range from Middle Cambrian through Permian. All systems except the Ordovician and Silurian are represented. Thickness of the Paleozoic sequence generally is in the range of 4,500-6,000 ft. Facies changes and erosional unconformities between and within formations account for part of the variability, and juxtaposition via thrust faults of rock sequences originally deposited distant from one another account for other thickness changes. Greater than three-fourths of the thickness of the sequence is limestone and dolomite (p1. 3, map C), and one-third to one-half of which is composed of the Mississippian Madison Group (Mm); the remainder of the sequence is largely sandstone and clay shale.

Mesozoic rocks are Jurassic and Cretaceous in age; no Triassic rocks are known in the Forest. Mesozoic sedimentary rocks are exposed mainly in the E1liston area of the southwestern part of the Forest, locally in the Elkhorn Mountains, and at the northernmost and southernmost parts of the Big Belt Mountains. The sedimentary rocks are chiefly sandstone, siltstone, mudstone, and shale. The only limestones are a thin unit in the upper part of the Cretaceous Kootenai Formation $(\mathrm{Kk})$, and the lower part of the Jurassic sequence, in which the 50-200 ft thick Jurassic E1lis Group (Sawtooth, Rierdon, and Swift Formations; part of unit Ju) is chiefly limestone, very calcareous shale, and calcareous sandstone. Dolomite is absent or uncommon in both Jurassic and Cretaceous rocks. Orogenic conglomerate, an outlier of the Golden Spike Formation (Kgs) that is widespread west of the Forest, crops out in the Priest Pass area northeast of Elliston. The maximum thickness of the preserved Mesozoic sedimentary rocks is about $4,000 \mathrm{ft}$, attained in the vicinity of Elliston and at the southern end of the Big Belt Mountains. 


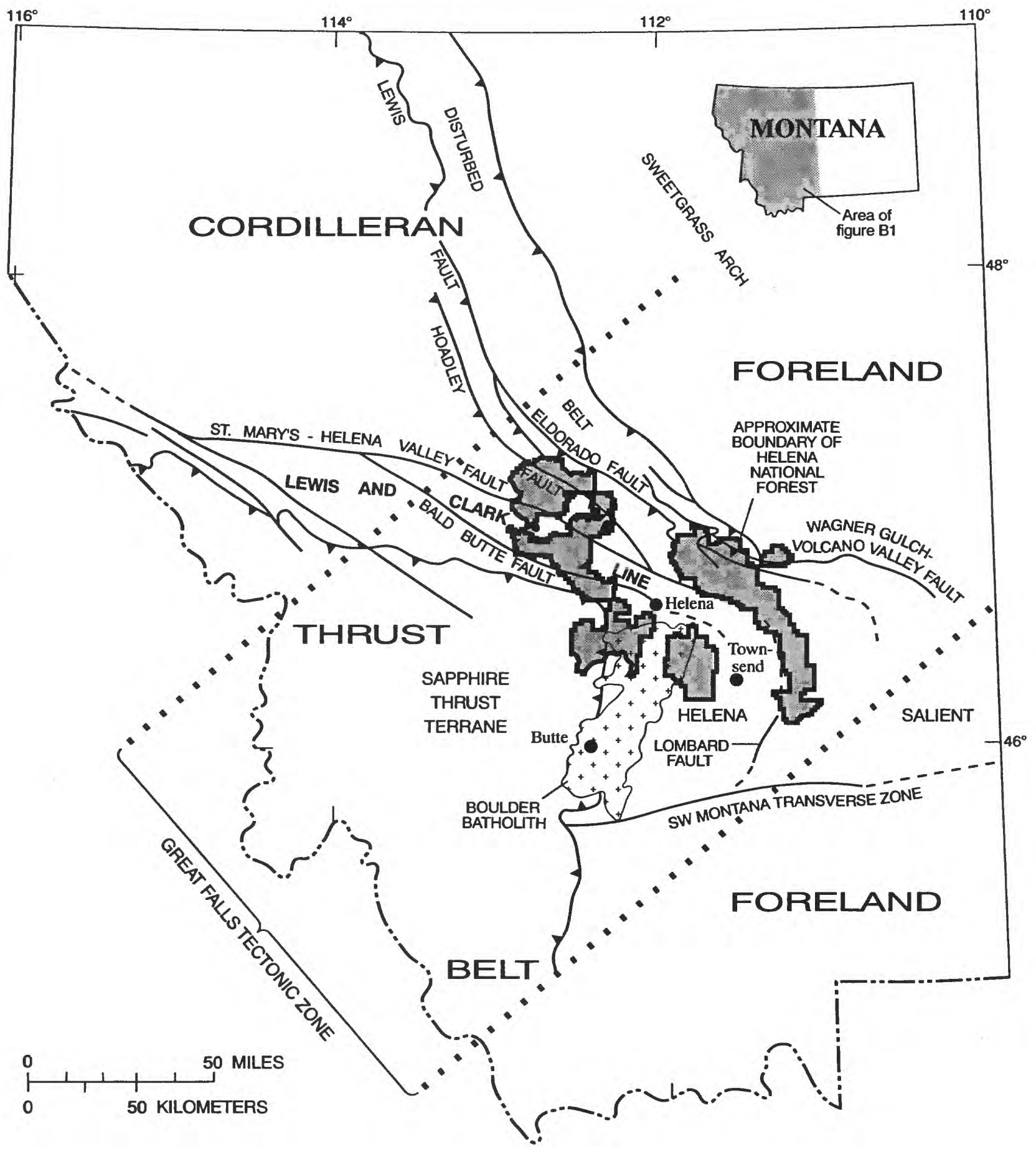

Figure B1. Map of major geologic features in western Montana and area of Helena National Forest. 
Cenozoic units include Tertiary sedimentary rocks and Quaternary unconsolidated (surficial) deposits. Miocene rocks of the Bozeman Group ( $\mathrm{Tbz}$ ) crop out on the flanks of the southernmost part of the Big Belt Mountains. The unit is comprised of conglomeratic strata representative of the middle to upper part of the group (Robinson, 1967; Skipp and Peterson, 1965), which is common within the nonmarine strata of the Tertiary basins of southwestern Montana. Mountain flanks in the northern and westernmost parts of the Forest contain isolated deposits and aprons of nonmarine rocks (Ts).

Quaternary unconsolidated deposits are widespread in the Forest. Due to the scale of the map, however, they are shown only where they cover extensive areas and (or) along stream drainages where they form narrow but thick deposits. The deposits are separated into terrace gravels, alluvium, colluvium, alluvium and colluvium undivided, landslides, and four types of glacial deposits. The unconsolidated materials were separated because the properties of each type of deposit shown are somewhat different and this distinction may prove useful in determining potential sources of road metal, potential for placer deposits, relationships to hydrologic properties, relationships to distribution of plants, erosion, or other correlations.

Quaternary and (or) Tertiary terrace gravels (QTg) are present mainly at the western edge of the Forest, northward from the vicinity of E11iston, where they form a veneer on terraces flanking the mountains. Unconsolidated gravels assigned to this unit also are present locally in the Big Belt Mountains.

Alluvial deposits ( $Q$ a) mainly consist of long, narrow deposits within stream channels and adjacent flood plains of modern stream courses. Colluvium (Qc) forms veneers on mountain flanks in some areas. Generally, however, alluvium and colluvium are combined in an undivided unit (Qac) because colluvium and alluvial fans commonly form a mixture of the two types of deposit. Only areally large landslide deposits (Q1s) are shown and are of the earth flow variety. Glacial deposits are grouped into four units: undivided deposits $(Q g)$, which includes all types of glacial deposits; till (Qgt), composed of unstratified deposits; outwash (Qgo), composed of stratified deposits; and glacial-lake sediments (Qgs). Glacial deposits are thickest and areally most extensive in and adjacent to the northwestern part of the Forest where they occupy broad valleys.

\section{VOLCANIC ROCKS}

Volcanic activity was widespread in western Montana in the Late Cretaceous and Tertiary (Chadwick, 1981). Late Cretaceous through 01igocene volcanic rocks form areally extensive fields that are concentrated in certain areas of the Forest. These fields are briefly described below.

Late Cretaceous volcanic and volcaniclastic rocks are extensive in the area south of U.S. Highway 12; and in the Elkhorn Mountains, where the Elkhorn Mountains Volcanics (Kev) are of widely variable thickness but in aggregate total as thick as $15,000 \mathrm{ft}$. These volcanic rocks are remnants of an extensive volcanic field that may have covered as much as 10,000 sq mi (Smedes, 1966). They formed between about 81 and 76 million years ago (Ma), partly coincident with intrusion of the Boulder batholith (described below), to which they are genetically related (Tilling, 1968; Rutland and others, 1989). The volcnic sequence constitutes more than half of the rocks at the surface in the E1khorn 
Mountains part of the Forest. Several large intrusive bodies (Ki), compositionally similar to the volcanics, intrude the sequence in the Elkhorn Mountains (Klepper and others, 1971; Greenwood and others, 1990). Only the larger intrusive bodies are shown on the geologic map (p1. 1).

Two areas of Cretaceous volcanic rocks (Kv) are present in the Big Belt Mountains. Volcanic rocks at the southernmost part of the range are several thousand feet thick, but mostly lie outside the Forest boundary. These rocks, part of the Maudlow Formation, are considered to be remnants of the Elkhorn Mountains Volcanics field (Skipp and Peterson, 1965; Skipp and McGrew, 1977). An area of andesitic volcanic rocks on the west flank of the range, northwest of Confederate Gulch (pl. 1) (Mertie and others, 1951; Gualtieri, 1975), is included within the $\mathrm{Kv}$ map unit because it probably also is an erosional remnant of the same volcanic fleld.

Late Cretaceous to Paleocene lava flows, breccias, tuff, and associated small intrusions of basalt to andesite composition (TKbv) lie unconformably on lower rocks of the Elkhorn Mountains Volcanics in the area south of Elliston (Ruppe1, 1963; Schmidt and others, 1994). Widespread, isolated patches of Eocene rhyolite ( $\mathrm{Tr}$ ) of 37-40 Ma (Schmidt and others, 1994, and references cited therein) occur in the area from Helena to west and south of Elliston and locally in the western part of the Elkhorn Mountains. These rocks are remnants of the Helena volcanic field and probably had multiple sources.

The Lowland Creek Volcanics (Tlv), in the southernmost part of the Forest west of the Elkhorn Mountains, are northern remnants of a large oligocene volcanic field that covered at least $600 \mathrm{sq} \mathrm{mi}$. The field consists mainly of extrusive rocks but contains related intrusives as well (Smedes, 1962; Becraft and others, 1963; Wallace and others, 1986). The Lowland Creek Volcanics probably had many sources.

The Lincoln volcanic field covers an extensive area of the Forest east of Lincoln. It consists of early Eocene or late Paleocene andesitic flows and tuffs (Ta) and late oligocene rhyolitic volcanic rocks ( $\mathrm{Tr}$ ). The volcanic centers for these rocks are within the areas where the field is preserved.

\section{PLUTONIC ROCKS}

Plutonic rocks that range from mafic to felsic in composition are abundant in the Forest. The oldest known plutonic rocks are Middle Proterozoic sills of andesite, basaltic andesite, and dacite that locally intrude strata of the Middle Proterozoic Belt Supergroup in the northwestern part of the Forest. Late Proterozoic sills of gabbro, microgabbro, diorite, and locally diabase (Zd) intrude Belt strata throughout the Forest. The sills, which range up to 1,500 ft thick, are present mainly in the northwestern part of the Forest and in the Big Belt Mountains. Radiometric dates on the Late Proterozoic sills are in the 750-850 Ma range. A sill in the northwestern part of the Forest yielded a date of $740 \pm 31 \mathrm{Ma}$ (Earhart and others, 1977). On the southwest flank of the central part of the Big Belt Mountains, two sills yielded dates of $826 \pm 41$ and $744 \pm 37$ Ma (Marvin and Dobson, 1979, p. 20).

The Cretaceous Boulder batholith is a granitic mass about $60 \mathrm{mi}$ long and $30 \mathrm{mi}$ wide, extending from south of Butte to directly south of Helena (fig. B1). It crops out extensively in the Elkhorn Mountains and in the southern part of the Forest south of U.S. Highway 12 (p1.1). The batholith is a composite of several 
intrusions, but the Butte Quartz Monzonite (Kmgd) makes up three-fourths of the mass (Knopf, 1963; Becraft and others, 1963; Ruppe1, 1963; Rutland and others, 1989). The thickness of the batholith is estimated to range from $3 \mathrm{mi}$ (Hamilton and Meyers, 1967) to $9 \mathrm{mi}$ (Klepper and others, 1974), based mainly on gravity and magnetic data; to $10-11 \mathrm{mi}$, based on seismic and gravity data (Schmidt and others, 1990). The batholith was intruded between about 80 and $70 \mathrm{Ma}$ (Tilling and others, 1968; Tilling, 1974; Rutland and others, 1989).

The origin of the Boulder batholith, associated rocks, and structures has been the subject of extensive study and debate over the years. The following publications, in addition to those cited above, provide a sampling of the literature: Knopf (1957), Klepper and others (1971), Tilling (1973), and Hyndman (1978).

In addition to the main mass of the Boulder batholith, several satellite intrusions (Kgd, $\mathrm{Kgdm}, \mathrm{Kgdo}, \mathrm{Kgdb}, \mathrm{Kgdd}$ ), generally more mafic and older than the Butte Quartz Monzonite, are present near the eastern, northern, and western margins of the batholith ( 1 1. 1). North of the batholith, these intrusions are mainly granodiorite in composition and most are of Late Cretaceous age (Schmidt and others, 1994); one, the Heddleston intrusive complex (Tmp) about $14 \mathrm{mi}$ northeast of Lincoln, is Eocene (Miller and others, 1973).

Granitic intrusions of similar age also are present in the central part of the Big Belt Mountains. One of these, centered near Boulder Baldy, has a range of compositions that form a concentric pattern of rock types ( $p 1$. 1) (Gualtieri, 1975; du Bray, 1995). The other major pluton of the Big Belt Mountains crops out in the Mount Edith area. It is compositionally similar to a phase of the Boulder Baldy pluton and to a third pluton (not shown on p1. 1) that lies west of the Forest and yielded radiometric dates of about 64 and $72 \mathrm{Ma}$ (Daniel and Berg, 1981, p. 80; du Bray, 1995).

\section{STRUCTURE}

The Forest overlies folds and thrust faults of the Cordilleran thrust belt of western Montana and the area of central Montana delimited by the Wagner GulchVolcano Valley fault on the north and faults of the Southwest Montana Transverse Zone on the south. This eastward protrusion of the Cordilleran thrust belt is known as the Helena structural salient. Across the north-south width of the salient, the general structural pattern is one of faults and folds that are convex to the east (fig. B1).

The faults that delimit the north and south boundaries of the salient separate it from the stable craton (foreland) of central and southwestern Montana, respectively. The craton is composed of Archean metamorphic rocks, which are widely exposed in southwestern Montana but in central Montana are largely concealed beneath a thin sequence of Middle Proterozoic and Paleozoic strata, and Mesozoic strata that are predominantly of Cretaceous age. During Late Cretaceous-early Tertiary deformation, plates of strata were thrust eastward within the Helena salient. As the plates moved eastward past the stable, resistent, cratonic areas north and south of the salient, complex patterns of faults and folds formed at the mutual edges of the salient and the cratonic areas (Bregman, 1976; Schmidt, 1977; Woodward, 1981; Schmidt and 0'Neill, 1982; Schmidt and others, 1988; Banowsky and others, 1989; and references cited in these publications). 
For the sequence of strata within the Helena salient to have moved eastward, it must be underlain by a fault(s) that separates ("decouples") the displaced sequence from rocks that lie still deeper. Such a fault(s) likely would be present deep within the Proterozoic strata, the oldest rocks of the displaced sequence, or at the base of the Proterozoic strata, at their contact with deeply buried Archean metamorphic rocks (Robinson, 1959; Woodward, 1981, 1983; Schmidt and O'Neill, 1982; Schmidt and others, 1990).

General descriptions of structures within the Forest are presented in the following order (fig. B1): Lewis and Clark line, a several-mile-wide belt of deformed rock that trends northwest across the Forest; Sapphire thrust terrane; region north of the St. Marys-Helena Valley fault, which is the northernmost fault of the Lewis and Clark line in the Forest; Big Belt Mountains; and the large area south of the St. Marys-Helena Valley fault (exclusive of Sapphire thrust terrane), including structures in the Elkhorn Mountains. The Forest also lies within a northeast-trending, diffuse zone of structural features known as the Great Falls tectonic zone, which is described briefly. Basins that separate the forest into eastern and western parts are described last.

\section{Lewis and Clark Line}

The Forest is transected by northwest-trending, steeply dipping strike-slip faults that are part of the Lewis and Clark line, a several-mile-wide belt of structural weakness that extends for about $250 \mathrm{mi}$ across west-central Montana into Idaho (Billingsley and Locke, 1939) (fig. B1). Reynolds (1979) considered the principal faults of the line to terminate in the vicinity of Townsend Valley, which lies southeast of Helena. Conversely, some workers (for example, Smith, 1965; Lorenz, 1984) considered the Lewis and Clark line to extend more than 100 mi farther east, into south-central Montana. Differing interpretations also exist concerning displacement of rocks along the line, and faults within it. Some believe it is right-lateral, others suggest left-lateral. Faults along this line have been active since the Middle Proterozoic (Hobbs and others, 1965; Harrison and others, 1974; Reynolds, 1979; Wallace and others, 1990).

Two principal faults of the Lewis and Clark line are exposed within the western part of the Forest. Farther east they are concealed beneath unconsolidated rocks of the Helena Valley, then, still farther east, the northern of the two faults is again exposed on the west flank of the Big Belt Mountains. One of these faults, which forms the northern boundary of the Lewis and Clark line in the study area, is the St. Marys-Helena Valley fault. A segment of this fault trends northwest across the western part of the Forest (p1. 1). Within the Forest it is a Tertiary right-slip fault that is downthrown on its south side. The St. Marys-Helena Valley fault is concealed beneath unconsolidated deposits of the Helena Valley directly east of the western part of the Forest, is exposed locally farther east (Bregman, 1976, 1981) (in an area of the Helena Valley for which rocks are not shown on $\mathrm{p} 1.1$ ), and is exposed again along the western margin of the Big Belt Mountains (Schmidt, 1977, 1986). The easternmost segment of the St. Mary's-Helena Valley fault is a right-slip feature that becomes a dipslip fault where it turns south along the west side of the Big Belt Mountains (Reynolds, 1979). Most of the right-slip offset along this fault took place between Late Cretaceous and early Eocene time (Wallace and others, 1990, p. 1026), but the fault may have experienced some recent movement because the 
epicenter of the 1935 Helena earthquake lies near the fault trace (Schmidt, 1986, p. 16).

The St. Mary's-Helena Valley fault truncates the thrust faults in the northern part of the Forest (p1. 1), shown by the maps of Mudge and others (1982) and Whipple and others (1987). It also truncates thrust faults at the northwestern margin of the Big Belt Mountains, as shown by the maps of Schmidt $(1977,1986)$.

The Bald Butte fault (fig. Bl, pl. 1) is the other principal fault of the Lewis and Clark line within the Forest area (Schmidt, 1986). Within the western part of the Forest, Schmidt and others (1994) concluded that sedimentary rocks on the north side of this steeply dipping strike-slip fault were displaced southeastward about $17 \mathrm{mi}$ relative to correlative rocks on the south side. Major movement on the fault is not well confined, but it occurred between about 97 and $47 \mathrm{Ma}$, during the Late Cretaceous to middle Eocene (Wallace and others, 1990; Schmidt and others, 1994). From the edge of the Forest about $10 \mathrm{mi}$ northwest of Helena, to the Helena Valley southeast of Helena, the Bald Butte fault is concealed by unconsolidated deposits. The southeastern limit of this concealed fault is uncertain; the fault is shown as ending in the Townsend Valley on the geologic map of plate 1 . The fault may continue along the south margin of the Helena Valley and join a fault along the northern front of the Elkhorn Mountains (Schmidt, 1986; Smedes, 1966), and then turn southward into the Townsend Valley (Reynolds, 1979).

\section{Sapphire Thrust Terrane}

Rocks west of the Boulder batholith and south of the Lewis and Clark line (fig. B1) form a distinctive sequence that here is termed the Sapphire thrust terrane. These rocks were called the Sapphire thrust plate by Wallace and others (1989). The Sapphire thrust terrane is a deformed package of thrust faults and associated folds (Hyndman, 1980; Ruppe1 and others, 1981; Sears, 1988; Wallace and others, 1989). The thrust faults delimit thrust plates, which were displaced eastward during Late Cretaceous compressional deformation (Ruppe1 and others, 1981; Wallace and others, 1989). The leading edge of the package of structures is at the western boundary of the Forest, in the vicinity of E11iston (p1. 1), where it consists of a zone of anastamosing, gently westward dipping imbricate thrust slices (Schmidt and others, 1994). The zone turns south near Elliston and is truncated by granitic rocks of the Boulder batholith (Wallace and others, 1989).

Rocks within the thrust slices have been displaced eastward over younger strata, and contain strata originally deposited west of their present location. The strata commonly are distinctly different from time-correlative strata east of the frontal zone of this thrust terrane, as described in the "Rock Unit Descriptions" that accompany the geologic map of Plate 1.

\section{North of the St. Mary's-Helena Valley Fault}

In the northern part of the Forest, northeast from the St. Mary's-Helena Valley fault, overthrust terrane is comprised of parts of three thrust plates (p1. 1; fig B1). From south to north, they are the Scapegoat, Hoadley, and 
Eldorado plates (Mudge and others, 1974, 1982; Earhart and others, 1977; Whipple and others, 1987). (The eastern edge of each of the three thrust plates is delimited by the fault of the same name; the Scapegoat fault is not labeled on figure B1, however.) Northward from the Forest, the Hoadley fault dies out and displacement is taken up by the Lewis thrust fault (fig. B1). In the Forest, the thrust plates are comprised mainly of Proterozoic rocks, but two small areas of Paleozoic carbonate strata also are present. Only the thrust faults at the base of the Scapegoat and Hoadley plates are exposed in the Forest. The Hoadley thrust is the westernmost fault of the Montana disturbed belt, a zone of closely spaced folds and imbricate thrust faults that extends from the Canadian border southward along the eastern front of the Rocky Mountains into the area north of Helena (Mudge and Earhart, 1980).

Eastward transport on the Hoadley thrust fault has been estimated to be as great as $45 \mathrm{mi}$ (Mudge and Earhart, 1980). Stratigraphic displacement of Proterozoic strata is greatest across the Hoadley thrust: 1ithofacies of Proterozoic rocks on the Hoadley and Scapegoat plates are from a more basinward facies than equivalent lithofacies on the Eldorado plate (Whipple and others, 1987). The relative displacement between the Hoadley and the Scapegoat plates is uncertain, but the Helena Formation is nearly twice as thick in the Scapegoat plate as in the Hoadley plate, suggesting significant transport on the Scapegoat thrust fault (Mudge and Earhart, 1980; Whipple and others, 1987).

An exploratory borehole for petroleum was drilled about 18 mi due east of Lincoln, less than $1 \mathrm{mi}$ east of the Forest boundary. The borehole went through $12,000 \mathrm{ft}$ of Proterozoic rocks of the thrust plates, through the Eldorado thrust fault at the base of the Eldorado plate, and then penetrated Cretaceous, Jurassic, and Paleozoic strata (Peterson and Sims, 1992). The findings demonstrated that the Proterozoic rocks are allochthonous--they have been thrust into their present position--displaced eastward during the Late Cretaceous to early Tertiary (Whipple and others, 1987).

\section{Big Belt Mountains}

The Big Belt Mountains lie in the northwestern part of the Helena structural salient. Here thrust faults and folds change orientations from the northwesterly trends typical of the northern part of the Helena salient to the northerly trends of the Montana disturbed belt and terrane present to the west and northwest. The gently westward dipping Eldorado thrust fault, the major discontunity penetrated by the borehole about $10 \mathrm{mi}$ to the west, comes to the surface in and adjacent to the westernmost part of the Forest in the Big Belt Mountains (p1. 1). Geologic studies of M.W. Reynolds in the Big Belt Mountains suggest that rocks above the Eldorado thrust fault have been displaced eastward a minimum distance of $20 \mathrm{mi}$ (Tysdal and others, 1991, p. 14).

Within the northern part of the Big Belt Mountains, rocks east of (and structurally below) the lower plate of the Eldorado thrust are deformed into complex geologic structures that include more thrust faults and intensely folded strata, thrust faults overriding other thrusts, and refolding of thrust plates. Folds beneath one of the thrust faults can be traced for at least $18 \mathrm{mi}$ southeast from the Gates of the Mountains Wilderness. Progressively older formations are exposed southeastward along the principal fold (Reynolds and Close, 1984). The oldest strata of the Big Belt Mountains, Middle Proterozoic strata, are exposed 
in the central area of the southern part of the range.

Compressional structures in the Big Belt Mountains are genetically related to associated thrust faults, folds, and plates that formed between the range and the stable craton north of the Helena salient, as shown by Banowsky and others (1989). The Volcano Valley segment of the Wagner Gulch-Volcano Valley fault forms the north edge of the Helena salient (fig. B1). This segment was active during deposition of Middle Proterozoic sediments and active intermittently in the Paleozoic, Mesozoic, and Tertiary as well (Godlewski and Zieg, 1984). The Wagner Gulch-Volcano Valley fault, which is $65 \mathrm{mi}$ long, was an active thrust fault during the Late Cretaceous-early Tertiary deformation. The Volcano Valley fault segment, which displays a left-slip component of movement, formed as the overlying thrust plate moved east-northeastward onto and south of the cratonic area north of the salient. Strata north of the fault are not severely deformed (Woodward, 1981; Banowsky and others, 1989).

\section{South of St. Marys-Helena Valley Fault, exclusive of Sapphire Thrust Terrane}

For the western part of the Forest exclusive of the Sapphire terrane, the entire region south of the St. Marys-Helena Valley fault is underlain by thrust faults ( $\mathrm{p} 1.1$ ). Within the Choteau and Butte $1^{\circ} \times 2^{\circ}$ quadrangles (fig. A2), the most intense development of thrusts and associated folds took place during the Late Cretaceous. Most Late Cretaceous granitic stocks and the Boulder batholith intruded the already deformed terrane (Ruppe1, 1963; Wallace and others, 1986; E11lott and others, 1992; Schmidt and others, 1994). Steep, northeast-trending gravity gradients at the northern, northeastern, and eastern margins of the Boulder batholith are evidence that emplacement of this intrusion was strongly influenced by steeply dipping faults that bordered these sides (Hanna and others, 1994). Some faulting and folding also took place concurrently with extrusion of the E1khorn Mountains Volcanics (Ruppe1, 1963).

In the Elkhorn Mountains, directly east of the Butte $1^{\circ} \times 2^{\circ}$ quadrangle, geologic mapping demonstrates again that deformation preceded intrusion of the Boulder batholith. It also shows that deformation preceded deposition of the Late Cretaceous Elkhorn Mountains Volcanics and that tectonism took place concurrently with volcanism and plutonism (Klepper and others, 1957; 1971; Freeman and others, 1958; Knopf, 1963; Smedes, 1966; Robinson and others, 1968; Greenwood and others, 1978, 1990; Hanna and others, 1994). Schmidt and others (1990) recently suggested that, based on data from an unpublished seismic line, a nearly horizontal detachment fault lies at a depth of 10-11 mi beneath the Boulder batholith, and that the batholith was emplaced as the sedimentary rocks were translated eastward several miles on the fault surface.

\section{Great Falls Tectonic Zone}

A broad belt of diverse types of geologic structures that trend northeast across central Idaho into western and central Montana has been named the Great Falls tectonic zone ( $0^{\prime} \mathrm{Neill}$ and Lopez, 1985). The zone ranges from about 90 to $150 \mathrm{mi}$ wide and, in west-central Montana, includes the entire area of the Forest (fig. B1).

The Great Falls tectonic zone is inferred to constitute a genetic 
association of structures that reflect some fundamental weakness of the earth's crust. The weakness served as a locus of high-angle faults, shear zones, depositional patterns of Paleozoic and Mesozoic sedimentary rocks, Late Cretaceous to Early Tertiary igneous intrusions and volcanic rocks, linear gravity and aeromagnetic anomalies in basement rocks, and associated mineralization (O'Neill and Lopez, 1985; Foster and Childs, 1993, and references cited therein). Some features of the zone formed as early as the Middle Proterozoic and have been active recurrently throughout Paleozoic, Mesozoic, and Tertiary time (O'Neill and Lopez, 1985).

\section{Extensional Faulting}

The two principal basins in the map area of plate 1 , the Helena and Townsend Valleys, separate the Forest area of the Big Belt Mountains from the Forest of the Elkhorn Mountains and the region to west and north. These basins are near the end of the Lewis and Clark Line, which ends in west-central Montana in a series of basins and ranges; the faults display dip-slip movment that developed during regional extension (Reynolds, 1979). The basins contain Tertiary sedimentary, volcanic, and volicaniclastic rocks up to several thousand feet thick. The rocks are largely concealed beneath unconsolidated sediments (Pardee, 1950; Mertie and others, 1951; Nelson, 1963; Reynolds, 1979; Schmidt, 1986). The structures that form the mutual boundaries of the basins and the ranges generally are steeply dipping normal faults along which the basins have been downdropped relative to the ranges.

The timing of fault movement coincident with basin development has been ascribed variously to middle Eocene and younger, 0ligocene and younger, and Miocene and younger ages (Reynolds, 1979; Fields and others, 1985; Ruppe1, 1993; and many references cited in these papers). Based on studies in the Butte $1^{\circ} \mathrm{x}$ $2^{\circ}$ sheet, in and adjacent to the western part of the Forest, Wallace and others (1990) and Schmidt and others (1994, p. 20-22) found that the earliest slip on normal faults took place in the Late Cretaceous, and they suggested that outlines of the main ranges and valleys in the region predate volcanic rocks of middle Eocene age.

\section{REFERENCES CITED}

Banowsky, B.R., Longden, M.R., and Woodward, L.A., 1989, Structure of Snedaker basin quadrangle--Implications for regional tectonics and hydrocarbon exploration along northern Helena salient, Montana thrust belt, in French, D.E., and Grabb, R.F., eds., Geologic resources of Montana: Montana Geological Society 1989 Field Conference and Guidebook--Montana Centennial edition, p. 271-278.

Becraft, G.E., Pickney, D.M., and Rosenblum, Sam, 1963, Geology and mineral deposits of the Jefferson City quadrangle, Jefferson and Lewis and Clark Counties, Montana: U.S. Geological Survey Bulletin 428, 101 p.

Billingsley, P.R., and Locke, A., 1939, Structure of ore deposits in the continental framework: New York, American Institute of Mining and Metallurgical Engineers, 51 p.

Becraft, G.E., Pinckney, D.M., and Rosenblum, Sam, 1963, Geology and mineral 
deposits of the Jefferson City quadrangle, Jefferson and Lewis and Clark Counties, Montana: U.S. Geological Survey Professional Paper 428, 101 p. Bregman, M.L., 1976, Change in tectonic style along the Montana thrust belt: Geology, v. 4, p. 775-778.

Bregman, M.L., 1981, Structural geology of the Sheep Creek and Rattlesnake Mountain quadrangles, Lewis and Clark County, Montana: Montana Bureau of Mines and Geology Geologic Map 26, scale 1:24,000, pamphlet.

Chadwick, R.A., 1981, Chronology and structural setting of volcanism in southwestern and central Montana, in Tucker, T.E., ed., Field conference and symposium guidebook to southwest Montana: Montana Geological Society, p. $301-310$.

Danie1, Faith, and Berg, R.B., 1981, Radiometric dates of rocks in Montana: Montana Bureau of Mines and Geology Bulletin 114, $136 \mathrm{p}$.

du Bray, E.A., 1995, Geologic map showing distribution of Cretaceous intrusive rocks in the central Big Belt Mountains, Broadwater and Meagher Counties, Montana: U.S. Geological Survey Miscellaneous Field Studies Map MF-2291, scale 1:50,000, with text.

Earhart, R.L., Grimes, D.J., Leinz, R.W., and Marks, L.Y., 1977, Mineral resources of the proposed additions to the Scapegoat Wilderness, Powell and Lewis and Clark Counties, Montana, with a section on Geophysical surveys by D.L. Peterson: U.S. Geological Survey Bulletin 1430, 62 p.

Elliott, J.E., Loen, J.S., Wise, K.K., and Blaskowski, M.J., 1992, Maps showing locations of mines and prospects in the Butte $1^{\circ} \times 2^{\circ}$ quadrangle, western Montana: U.S. Geological Survey Miscellaneous Field Investigations Map I-2050-C, scale 1:250,000, with pamphlet.

Fields, R.W., Rasmussen, D.L., Tabrum, A.R., and Nichols, Ralph, 1985, Cenozoic rocks of the intermontane basins of western Montana and eastern Idaho, in Flores, R.M., and Kaplan, S.S., eds., Cenozoic paleogeography of westcentral United States, Rocky Mountain paleogeography symposium 3: Denver, Rocky Mountain Section of the Society of Economic Paleontologists and Mineralogists, p. 9-30.

Foster, Fess, and Childs, J.F., 1993, An overview of significant lode gold systems in Montana, and their regional geologic setting: Exploration and Mining Geology, v. 2, p. 217-244.

Freeman, V.L., Ruppe1, E.T., and Klepper, 1958, Geology of part of the Townsend Valley, Broadwater and Jefferson Counties, Montana: U.S. Geological Survey Bulletin 1042-N, P. 481-556.

Godlewski, D.W., and Zieg, G.A., 1984, Stratigraphy and depositional setting of the Precambrian Newland Limestone, in Hobbs, S.W., ed., The Belt: Montana Bureau of Mines and Geology Special Publication 90, p. 2-4.

Greenwood, W.R., Ludington, S.D., Miller, W.R., and Hanna, W.F., 1978, Mineral resources of the Elkhorn Wilderness study area, Montana, Chapter A, Mineral resource assessment, with a section on Geophysical exploration by W.F. Hanna, a section on Geochemical exploration by W.R. Miller, Steve Ludington, and W.R. Greenwood, a section on Geological and geochemical evaluation by Steve Ludington and W.R. Greenwood, and a section on Uranium and thorium potential by K.J. Wenrich-Verbeck, W.R. Miller V.J. Suits, and John McHugh: U.S. Geological Survey Open-File Report 78-325, 362 p.

Greenwood, W.R., Ludington, S.D., Miller, W.R., and Hanna, W.F., 1990, Mineral resources of the Elkhorn Wilderness study area, Broadwater and Jefferson Counties, Montana: U.S. Geological Survey Bulletin 1805, 37 p.

Gualtieri, J.L., 1975, Preliminary unedited geologic map of the Confederate 
Gulch area, Broadwater and Meagher Counties, Montana: U.S. Geological Survey Open-File Report 75-211, scale 1:48,000.

Hamilton, Warren, and Myers, W.B., 1967, The nature of batholiths: U.S. Geological Survey Professional Paper 554-C, 30 p.

Hanna, W.F., Hassemer, E11lott, J.E., Wallace, C.A., and Snyder, S.L., 1994, Maps showing gravity and aeromagnetic anomalies in the Butte $1^{\circ} \times 2^{\circ}$ quadrangle, Montana: U.S. Geological Survey Miscellaneous Investigations Series Map I-2050-I, scale 1:250,000, pamphlet.

Harrison, J.E., 1972, Precambrian Belt basin of northwestern United States - Its geometry, sedimentation, and copper occurrences: Geological Society of America Bulletin, v. 83, p. 1215-1240.

Harrison, J.E., Griggs, A.B., Wells, J.D., 1974, Tectonic features of the Precambrian Belt basin and their influence on post-Belt structures: U.S. Geological Survey Professional Paper 866, 15 p.

Hobbs, S.W., Griggs, A.B., Wallace, R.W., and Campbe11, A.B., 1965, Geology of the Coeur d'Alene district, Shoshone County, Idaho: U.S. Geological Survey Professional Paper 478, 139 p.

Hyndman, D.W., 1978, Major tectonic elements and tectonic problems along the line of section from northeastern Oregon to west-central Montana: Geological Society of America Map and Chart Series, no. MC-28C.

1980, Bitterrot dome--Sapphire tectonic block, an example of a plutoniccore gneiss-dome complex with its detached suprastructure, in Crittenden, M.D., Jr., Coney, P.J., and Davis, G.H., eds., Cordilleran metamorphic core complexes: Geological Society of America Memoir 153, p. 427-443.

Klepper, M.R., Robinson, G.D., and Smedes, H.W., 1974, Nature of the Boulder batholith of Montana--Discussion: Geological Society of America Bulletin, v. 85, p. 1953-1960.

Klepper, M.R., Weeks, R.A., and Ruppe1, E.T., 1957, Geology of the southern Elkhorn Mountains, Jefferson and Broadwater Counties, Montana: U.S. Geological Survey Professional Paper 292, 82 p.

Klepper, M.R., Ruppe1, E.T., Freeman, V.L., and Weeks, R.A., 1971, Geology and mineral deposits, east flank of the Elkhorn Mountains, Broadwater County, Montana: U.S. Geological Survey Professional Paper 665, 66 p.

Knopf, Adolph, 1957, The Boulder bathylith of Montana: American Journal of Science, v. 255, p. 81-103.

1963, Geology of the northern part of the Boulder bathylith and adjacent area, Montana: U.S. Geological Survey Miscellaneous Geologic Investigations Map I-381, scale 1:48,000, pamphlet.

Lorenz, J.S., 1984, The function of the Lewis and Clark fault system during the Laramide orogeny, in McBane, J.S., and Garrison, P.B., eds., Northwest Montana and adjacent Canada: Billings, Montana Geological Society 1984 Field Conference Guidebook, p. 221-230.

Marvin, R.F., and Dobson, S.W., 1979, Radiometric ages--Compilation B, U.S. Geological Survey: Isochron/West, no. 26, p. 3-32.

McMannis, W.J., 1965, Resume of depositional and structural history of western Montana: American Association of Geologists Bulletin, v. 49, p. 1801 1823.

Mertie, J.B., Fischer, R.P., and Hobbs, S.W., 1951, Geology of the Canyon Ferry quadrangle, Montana: U.S. Geological Survey Bulletin 972, 97 p.

Miller, R.N., Shea, E.P., Goddard, C.C., Jr., Potter, C.W., and Brox, G.B., 1973, Geology of the Heddleston copper-molybdenum deposit, Lewis and Clark County, Montana: American Institute of Mining, Metallurgical, and 
Petroleum Engineers, Inc., 1973, Pacific Northwest metals and minerals conference, p. 1-33.

Mudge, M.R., and Earhart, R.L., 1980, The Lewis thrust fault and related structures in the disturbed belt, Northwestern Montana: U.S. Geological Survey Professional Paper 1174, $18 \mathrm{p}$.

Mudge, M.R., Earhart, R.L., Whipple, J.W., and Harrison, J.E., 1982, Geologic and structure map of the Choteau $1^{\circ} \times 2^{\circ}$ quadrangle, western Montana: U.S. Geological Survey Miscellaneous Series Investigations Map I-1300, scale $1: 250,000$.

Nelson, W. H., 1963, Geology of the Duck Creek Pass quadrangle, Montana: U.S. Geological Survey Bulletin $1121-\mathrm{J}, 55 \mathrm{p} .$, scale $1: 62,500$.

O'Neill, J.M., and Lopez, D.A., 1985, Character and regional significance of Great Falls tectonic zone, east-central Idaho and west-central Montana: American Association of Petroleum Geologists Bulletin, v. 69, p. 437-447.

Pardee, J.T., 1950, Late Cenozoic block faulting in western Montana: Geological Society of America Bulletin, v. 61, p. 359-406.

Peterson, C.H., and Sims, R.C., 1992, How Montana's deepest hole cut Mississippian carbonates underneath Precambrian belt: $0 i 1$ and Gas Journa1, v. 90, no. 31, August 3, 1992, p. 67-70.

Reynolds, M.W., 1979, Character and extent of basin-range faulting, western Montana and east-central Idaho, in Newman, G.W., and Goode, H.D., eds., RMAG-UGA--1979 basin and range symposium: Denver, Colorado, Rocky Mountain Association of Geologists, p. 185-193.

Reynolds, M.W., and Close, T.J., 1984, Gates of the Mountains Wilderness and additions, Montana, in Marsh, S.P., Kropschot, S.J., and Dickinson, R.G., Wilderness mineral potential, assessment of mineral-resource potential in U.S. Forest Service lands studied, 1964-1984: U.S. Geological Survey Professional Paper 1300, p. 705-708.

Robinson, G.D., 1959, The disturbed belt in the Sixteenmile area, Montana, in Hammond, C.R., and Trapp, Harry, Jr., eds., Tenth annunal field conference guidebook: Billings Geological Society, p. 34-40.

1967, Geologic map of the Toston quadrangle, southwestern Montana: U.S. Geological Survey Miscellaneous Investigations Map I-486, scale 1:24,000.

Robinson, G.S., Klepper, M.R., and Obradovich, J.D., 1968, Overlapping plutonism, volcanism, and tectonism in the Boulder batholith region, western Montana, in Coats, R.R., Hay, R.L., and Anderson, C.A., eds., Studies in volcanology--A memoir in honor of Howel Williams: Geological Society of America Memoir 116, p. 557-576.

Ruppe1, E.T., 1963, Geology of the Basin quadrangle, Jefferson, Lewis and Clark, and Powell Counties, Montana: U.S. Geological Survey Bulletin $1151,121 \mathrm{p}$.

1993, Cenozoic tectonic evolution of southwest Montana and east-central Idaho: Montana Bureau of Mines and Geology Memoir 65, $62 \mathrm{p}$.

Ruppel, E.T., Wallace, C.A., Schmidt, R.G., and Lopez, D.A., 1981, Preliminary interpretation of the thrust belt in southwest and west-central Montana and east-central Idaho, in Tucker, T.E., ed., Field conference and symposium guidebook to southwest Montana: Montana Geological Society, p. $139-159$.

Rutland, Carolyn, Smedes, H.W., Tilling, R.I., and Greenwood, W.R., 1989, Volcanism and plutonism at shallow crustal levels; the Elkhorn Mountains Volcanics and the Boulder batholith, southwestern Montana, in Hyndman, D.W., ed., Cordilleran volcanism, plutonism, and magma generation at 
various crustal levels, Montana and Idaho: 28th International Geological Congress, Field Trip Guidebook T337, p. 16-31.

Schmidt, C.J., and O'Neill J.M., 1982, Structural evolution of the southwest Montana transverse zone, in Geologic studies of the Cordilleran thrust belt, vol. 1: Denver, Colorado, Rocky Mountain Association of Geologists, p. 193-218.

Schmidt, C.J., O'Neill, J.M., and Brandon, W.C., 1988, Influence of Rocky Mountain foreland uplifts on the development of the frontal fold and thrust belt, southwestern Montana, in Schmidt, C.J., and Perry, W.J., Jr., eds., Interaction of the Rocky Mountain Foreland and the Cordilleran thrust belt: Geological Society of America Memoir 171, p. 171-201.

Schmidt, C.J., Smedes, H.W., and O'Neill, J.M., 1990, Syncompressional emplacment of the Boulder and Tobacco Root batholiths (Montana--USA) by pull-apart along old fault zones: Geological Journal, v. 25, p. 305-318.

Schmidt, R.G., 1977, Geologic map of the Craig quadrangle, Lewis and Clark and Cascade Counties, Montana: U.S. Geological Survey Geologic Quadrangle Map GQ-1141, scale: $1: 24,000$.

Schmidt, R.G., 1986, Geology, earthquake hazards, and land use in the Helena area, Montana--A review: U.S. Geological Survey Professional Paper 1316, $64 \mathrm{p}$.

Schmidt, R.G., Loen, J.S., Wallace, C.A., and Mehnert, H.H., 1994, Geology of the Elliston region, Powell and Lewis and Clark Counties, Montana: U.S . Geological Survey Bulletin 2045, 25 p.

Sears, J.W., 1988, Two major thrust slabs in the west-central Montana Cordillera, in Schmidt, C.W., and Perry, W.J., Jr., eds, Interaction of the Rocky Mountain foreland and Cordilleran thrust belt: Geological Society of America Memoir 171, p. 165-170.

Skipp, Betty, and McGrew, 1977, The Maudlow and Sedan Formations of the Upper Cretaceous Livingston Group on the west edge of the Crazy Mountains Basin, Montana: U.S. Geological Survey Bulletin 1422-B, 68 p.

Skipp, Betty, and Peterson, A.D., 1965, Geologic map of the Maudlow quadrangle, southwestern Montana: U.S. Geological Survey Miscellaneous Geologic Investigations Map I-452, scale 1:24,000.

Smedes, H.W., 1962, Lowland Creek Volcanics, an upper Oligocene Formation near Butte, Montana: Journal of Geology, v. 70, p. 255-266.

Smedes, H.W., 1966, Geology and igneous petrology of the northern Elkhorn Mountains, Jefferson and Broadwater Counties, Montana: U.S. Geological Survey Professional Paper 510 , 116 p.

Smith, J.G., 1965, Fundamental transcurrent faulting in northern Rocky Mountains: American Association of Petroleum Geologists Bulletin, v. 49, p. $1398-1409$.

Tilling, R.I., 1973, The Boulder batholith, Montana--A product of two contemporaneous but chemically distinct magma series: Geological Society of America Bulletin, v. 84, p. 3879-3900.

Tilling, R.I., 1974, Composition and time relations of plutonic and associated volcanic rocks, Boulder batholith region, Montana: Geological Society of America Bulletin, v. 85, p. 1925-1930.

Tilling, R.I., Klepper, M.R., and Obradovich, J.D., 1968, K-Ar ages and time span of emplacement of the Boulder batholith, Montana: American Journa1 of Science, v. 266, p. 671-689.

Tysdal, R. G., Reynolds, M.W., Carlson, R.R., Kleinkopf, M.d., Rowan, L.S., and Peters, T.J., 1991, Mineral resources of the Sleeping Giant Wilderness 
study aream, Lewis and Clark County, Montana: U.S. Geological Survey Bulletin 1724-E, $31 \mathrm{p}$.

Wallace, C.A., Lidke, D.J., and Schmidt, R.G., 1990, Faults of the central part of the Lewis and Clark line and fragmentation of the Late Cretaceous foreland basin in west-central Montana: Geological Society of America Bulletin, v. 102, p. 1021-1037.

Wallace, C.A., Lidke, D.J., Waters, M.R., and Obradovich, J.D., 1989, Rocks and structure of the southern Sapphire Mountains, Granite and Ravalli Counties, western Montanaa: U.S. Geolofical Survey Bulletin 1824, 29 p. Wallace, C.A., Schmidt, R.G., Lidke, D.J., Waters M.R., Elliott, J.E., French, A.B., Whipple, J.W., Zarske, S.E., Blaskowski, M.J., Heise, B. A., Yeoman, R.A., O'Neil1, J.M., Lopez, D.A., Robinson, G.D., and Klepper, M.R., 1986, Preliminary geologic map of the Butte $1^{\circ} \times 2^{\circ}$ quadrangle, western Montana: U.S. Geological Survey Open-File Report 86-292, scale 1:250,000, text.

Whipple, J.W., Mudge, M.R., and Earhart, R.L., 1987, Geologic map of the Rogers Pass area, Lewis and Clark County, Montana: U.S. Geological Survey Miscellaneous Investigations Series Map I-1642, scale 1:48,000.

Woodward, L.A., 1981, Tectonic framework of disturbed belt of west-central Montana: American Association of Petroleum Geologists Bulletin, v. 65, p. 291-300.

1983, Structural geology of the Helena salient--A synopsis, in Smith, D.L., compiler, Guidebook of the fold and thrust belt, west-central Montana: Montana Bureau of Mines and Geology Special Publication 86, p. 83-87. 


\title{
GEOPHYSICS OF THE HELENA NATIONAL FOREST
}

\author{
AEROMAGNETIC AND GRAVITY STUDIES
}

By Anne E. McCafferty

\section{Aeromagnetic and Gravity Data}

Aeromagnetic anomaly data are available for the Helena National Forest and surrounding area. Aeromagnetic anomaly maps show changes in the earth's magnetic field that reflect variations in the amounts of magnetic minerals in rocks, typically the mineral magnetite. Most sedimentary rocks have low to negligible amounts of magnetic minerals and therefore contribute little to a study of magnetic anomalies. A magnetic anomaly map primarily reflects abrupt lateral lithologic, hydrothermal, and structural changes related to the magnetic rock properties of volcanic and crystalline rocks that contain enough magnetic minerals to produce anomalies. Aeromagnetic anomaly maps are particularly useful in identifying buried geologic features such as intrusive bodies, which commonly have strong magnetization contrasts with the surrounding rock.

Figure $\mathrm{Cl}$ is a magnetic anomaly map of the Forest compiled from ten separate surveys, represented at a constant elevation of $1000 \mathrm{ft}$ (305 m) above the ground. The data are from a larger regional aeromagnetic compilation of Idaho and southwest Montana (McCafferty, 1992). Table Cl shows surveys used in the compilation and the index map (fig. C2) shows area of the original survey.

Gravity anomaly data were compiled for the Helena National forest and surrounding area (fig. C3) from regional compilations covering most of Idaho and southwest Montana (Bankey, 1992) and from a gravity study of the Choteau $1^{\circ} \times 2^{\circ}$ quadrangle (Kulik, 1983). In general, gravity anomalies are primarily a response to lateral density variations in rocks and provide useful information regarding the distribution and configuration of geologic features that have strong density contrasts. Because different rock types are often characterized by contrasting densities, gravity maps are useful in extending the geologic mapping into areas covered by surficial deposits and in determining the subsurface position and attitude of such density boundaries.

The gravity station distribution (see Bankey, 1992 and Kulik, 1983 for gravity station locations) and flight line spacing of the magnetic anomaly data (figure C2) does not allow a detailed interpretation of the structural and lithologic complexities of the Forest and adjacent area. However, the anomaly maps point to large geologic units that have strong magnetization or density contrasts. The aeromagnetic data are especially useful in determining the lateral extent of buried and partially exposed plutons, many of which have spatially and genetically associated mineralization.

\section{Previous Aeromagnetic and Gravity Studies}

Aeromagnetic and gravity data for the northern and western parts of the 
Forest have been previously studied as parts of U.S. Aeromagnetic data over the valleys east of the Big Belt Mountains were studied in the 1960's to delineate igneous rock masses and determine the configuration of the bedrock surface beneath the Cenozoic sedimentary rocks in the main valleys (Kinoshita and others, 1964, 1965; Davis and others, 1963).

\section{Magnetization and Densities of Rocks}

The physical properties that relate aeromagnetic and gravity anomalies to their sources are magnetization and density, respectively. Rocks of the Forest area exhibit a wide range of physical properties, reflecting the diverse geology. Magnetization values measured from rock samples collected within and around the study area (Hanna and others, 1994, and for this study, samples collected by E. du Bray and Steve Ludington) range over five orders of magnitude. There are too few measurements to permit average estimates of magnetization for different rocks units, but some qualitative generalizations can be made. The most magnetic rock units are, as expected, the more mafic igneous units. Induced magnetization is the main contributor to the overall magnetic anomaly patterns in the Forest area; remnant magnetization contributes only a minor amount to the overall total magnetization. Exceptions to this are seven rock units within the Elkhorn Mountain Volcanics and five units within the Lowland Creek Volcanics in which remnant magnetization is dominant. However, only two of these units have sufficient intensity to cause anomalies (Hanna and others, 1994).

Hanna and others (1994) summarized investigations from the past 30 years of measured densities of various rock types within the Butte $1^{0} \times 2^{0}$ quadrangle and they updated this information with their own study of additional rock samples. Although only about one-third of the Helena National Forest lies within the Butte $1^{0} \times 2^{0}$ quadrangle, the same rock types occur within the Forest adjacent to the quadrangle. Densities of the diverse suite of rocks in the Butte quadrangle are assumed to be characteristic of the surrounding region, including the remainder of the Forest; these densities are summarized in table $\mathrm{C} 2$.

\section{Terrace Maps}

"Terracing" is a data processing technique (Cordell and McCafferty, 1989) that converts potential field data into maps of physical properties. When applied to gravity data, the technique results in a "terrace-density" map showing large, sharply bounded domains of similar density. When applied to magnetic data, the result is a "terrace-magnetization" map. For this study, the aeromagnetic and gravity data were converted to terrace maps in order to (1) map physical property domains corresponding to both known and unknown geologic structures; and (2) objectively locate positions of boundaries between geologic units with differing physical properties. The terrace method assumes the physical property domain edges are steeply dipping to nearly vertical. If the boundaries are not steeply dipping, location of the domain edge will be offset slightly down dip from the contact (Grauch and Cordell, 1987). Terrace-density and terrace-magnetization maps were calculated from the aeromagnetic and gravity data of the Forest, leading directly to inferred physical-property (density and magnetization) maps. The terrace-magnetization was used in the resource assessment 

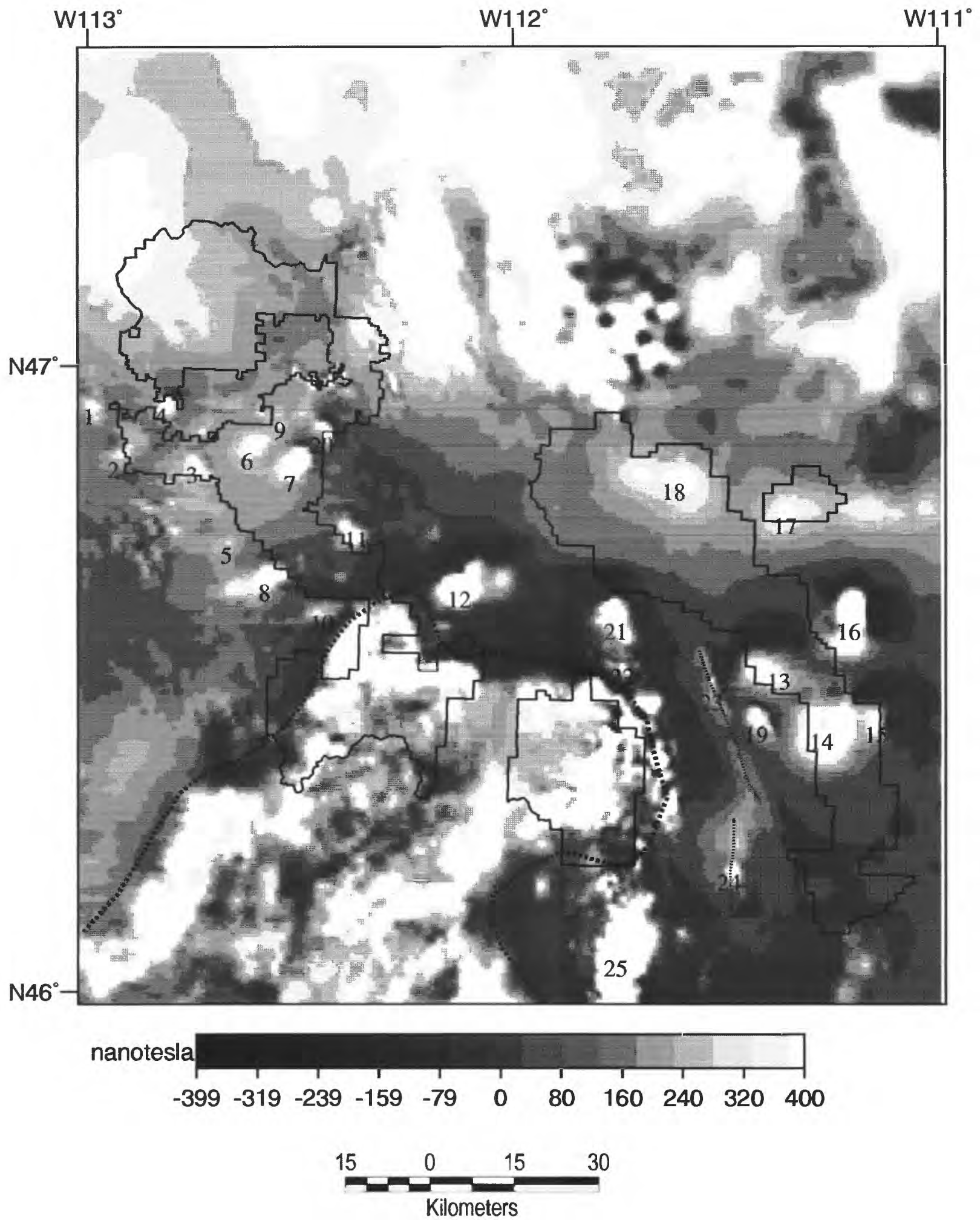

Figure C1. Merged aeromagnetic anomaly map of the Helena National Forest and vicinity. Map is a synthesis of surveys described in Table C1 and index map (fig. C2). Surveys have been corrected for the earth's regional geomagnetic reference field and represented at a constant datum of $1,000 \mathrm{ft}(305 \mathrm{~m})$ above the ground surface. Numbers refer to locations of exposed and buried igneous features described in text. Heavy dashed line shows extent of Boulder batholith. 


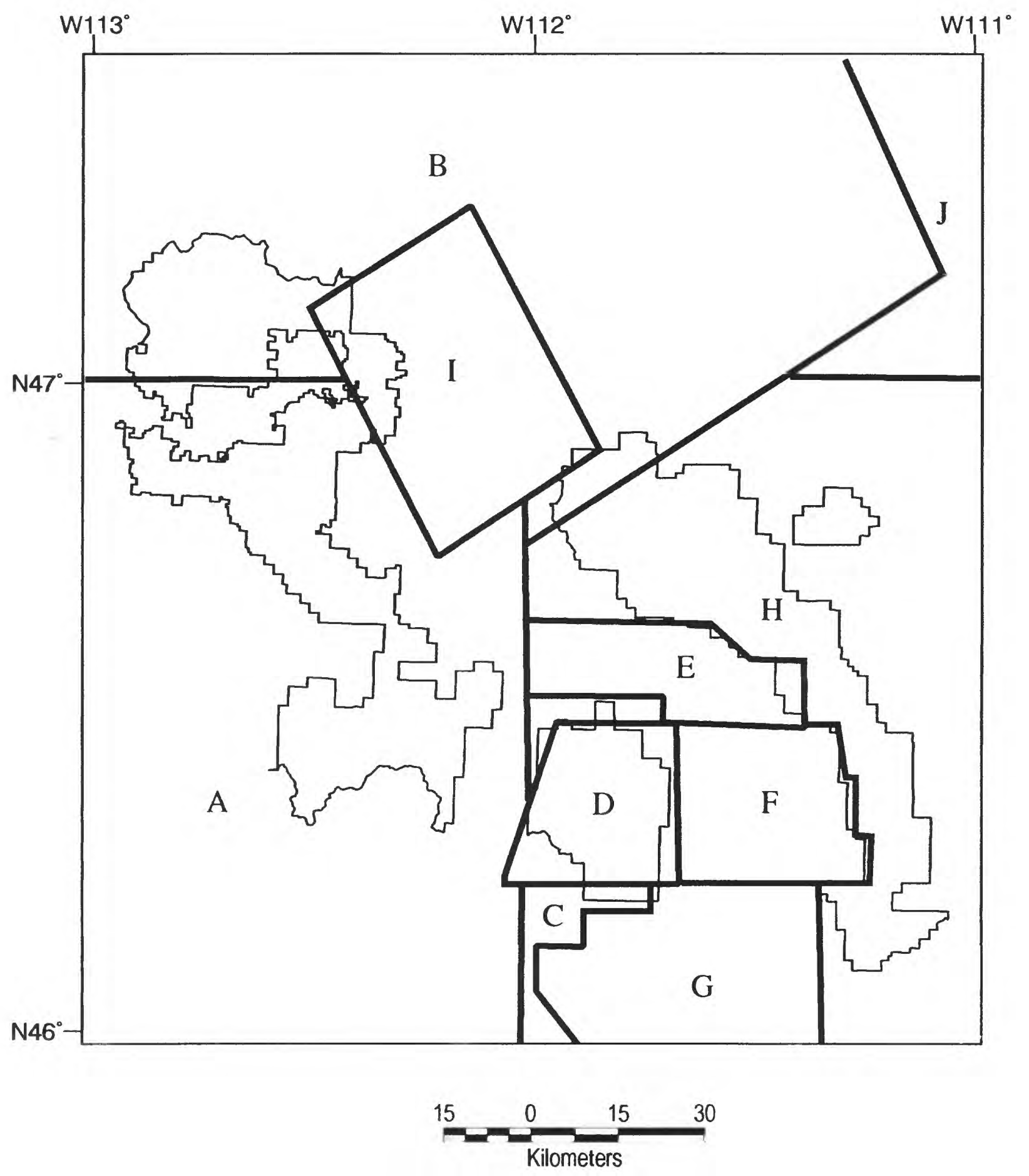

Figure C2. Index map of aeromagnetic surveys used in the compilation of data for the Helena National Forest. Survey specifications are summarized in table $\mathrm{C} 1$. 
Table C1. Flight specifications for aeromagnetic surveys used in the compilation of data for this study.

\begin{tabular}{|c|c|c|c|c|c|}
\hline Survey 1 & $\begin{array}{c}\text { Line } \\
\text { direction }\end{array}$ & $\begin{array}{c}\text { Flight altitude } \\
\text { ft (m) }\end{array}$ & $\begin{array}{c}\text { Line spacing } \\
\mathrm{mi}(\mathrm{km})\end{array}$ & Data type $^{3}$ & Reference cited \\
\hline A & E-W & $\begin{array}{c}9,000(2,740) \\
\text { B }\end{array}$ & $1(1.6)$ & D & USGS (1984) \\
\hline $\bar{B}$ & NE-SW & $\begin{array}{c}9,000(2,740) \\
\text { B }\end{array}$ & $2(3.2)$ & $\bar{A}$ & $\begin{array}{l}\text { Kleinkopf and Mudge } \\
\text { (1972) }\end{array}$ \\
\hline $\mathrm{C}$ & $\overline{E-W}$ & $\begin{array}{c}10,500(3200) \\
\text { B }\end{array}$ & $2(3.2)$ & $\bar{A}$ & $\begin{array}{l}\text { Johnson and others } \\
\text { (1965) }\end{array}$ \\
\hline $\bar{D}$ & E-W & $\begin{array}{c}9,000(2,740) \\
\text { B }\end{array}$ & $.5(0.8)$ & $\bar{D}$ & USGS (1978) \\
\hline $\mathrm{E}$ & E-W & $500(150) A G$ & $.5(0.8)$ & A & $\begin{array}{l}\text { Kinoshita and others } \\
\text { (1964) }\end{array}$ \\
\hline F & E-W & $500(150) A G$ & $.5(0.8)$ & $\bar{A}$ & $\begin{array}{l}\text { Davis and others } \\
\text { (1963) }\end{array}$ \\
\hline $\mathrm{G}$ & E-W & $500(150) A G$ & $.5(0.8)$ & $\bar{A}$ & $\begin{array}{l}\text { Kinoshita and others } \\
\text { (1965) }\end{array}$ \\
\hline $\mathrm{H}$ & E-W & $400(120) \mathrm{AG}$ & $3(4.8)$ & D & $\begin{array}{l}\text { Geodata International } \\
\text { (1979) }\end{array}$ \\
\hline I & NE-SW & $\begin{array}{c}7,000(2,134) \\
\text { B }\end{array}$ & $.75(1.2)$ & D & USGS (1980) \\
\hline $\mathrm{J}$ & E-W & $400(120) A G$ & $3(4.8)$ & D & $\begin{array}{l}\text { Geodata International } \\
\text { (1979) }\end{array}$ \\
\hline
\end{tabular}

${ }^{1}$ Surveys refer to figure $\mathrm{C} 2$.

2Flight-altitude: AG; survey originally flown "above ground" in a draped mode above the topographic surface. B; survey flown at a constant barometric elevation.

3Data type: A, data are in "analog" form only. Subsequently, maps were digitized from published versions; $\mathrm{D}$, data exist as original "digital" flight-line data. 


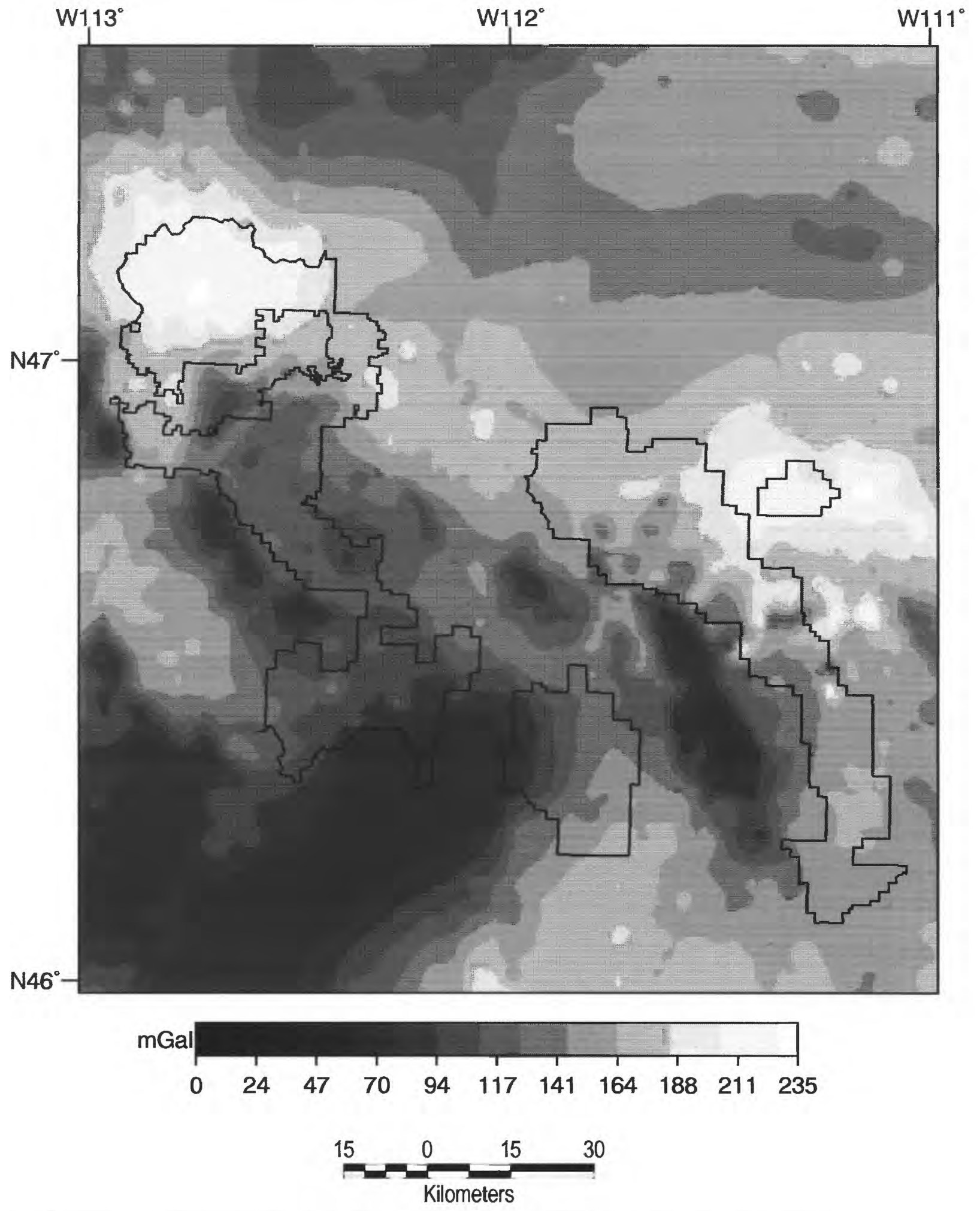

Figure C3. Bouguer gravity anomaly map of the Helena National Forest and vicinity. Data are from Bankey (1992) and Kulik (1983). Approximate forest boundary shown in black. 
Table C2. Densities $\left(\mathrm{g} / \mathrm{cm}^{3}\right)$ from samples within and surrounding the study area (Hanna and others, 1994)

Rock type

Density

Sedimentary and Metasedimentary Rocks

$\left(\mathrm{g} / \mathrm{cm}^{3}\right)$

shale

quartzite, sandstone, siltstone, siltite

hornfels (slightly metamorphosed rocks)

2.64

limestone (and slightly metamorphosed marble)

2.68

argillite

2.70

marble

2.80

dolomite

2.80

hornfels and skarn (intensely metamorphosed rocks)

2.97

phosphatic rock

3.40

Average

2.70

Basin fill (unconsolidated sediments)

Volcanic Rocks

Tertiary rhyolite and latite

Cretaceous and Tertiary rhyodacite

Cretaceous andesite

Cretaceous non-vesicular basalt

Average for Cretaceous volcanic rocks

\section{Plutonic Rocks}

syenite

alaskite, granite, quartz monzonite

quartz monzonite

2.65

granodiorite

2.70

mafic granodiorite, monzodiorite, and lamprophyre

2.78

monzogabbro, sulfide-enriched quartz monzonite

2.85

gabbro

3.10

pyroxenite

Average 

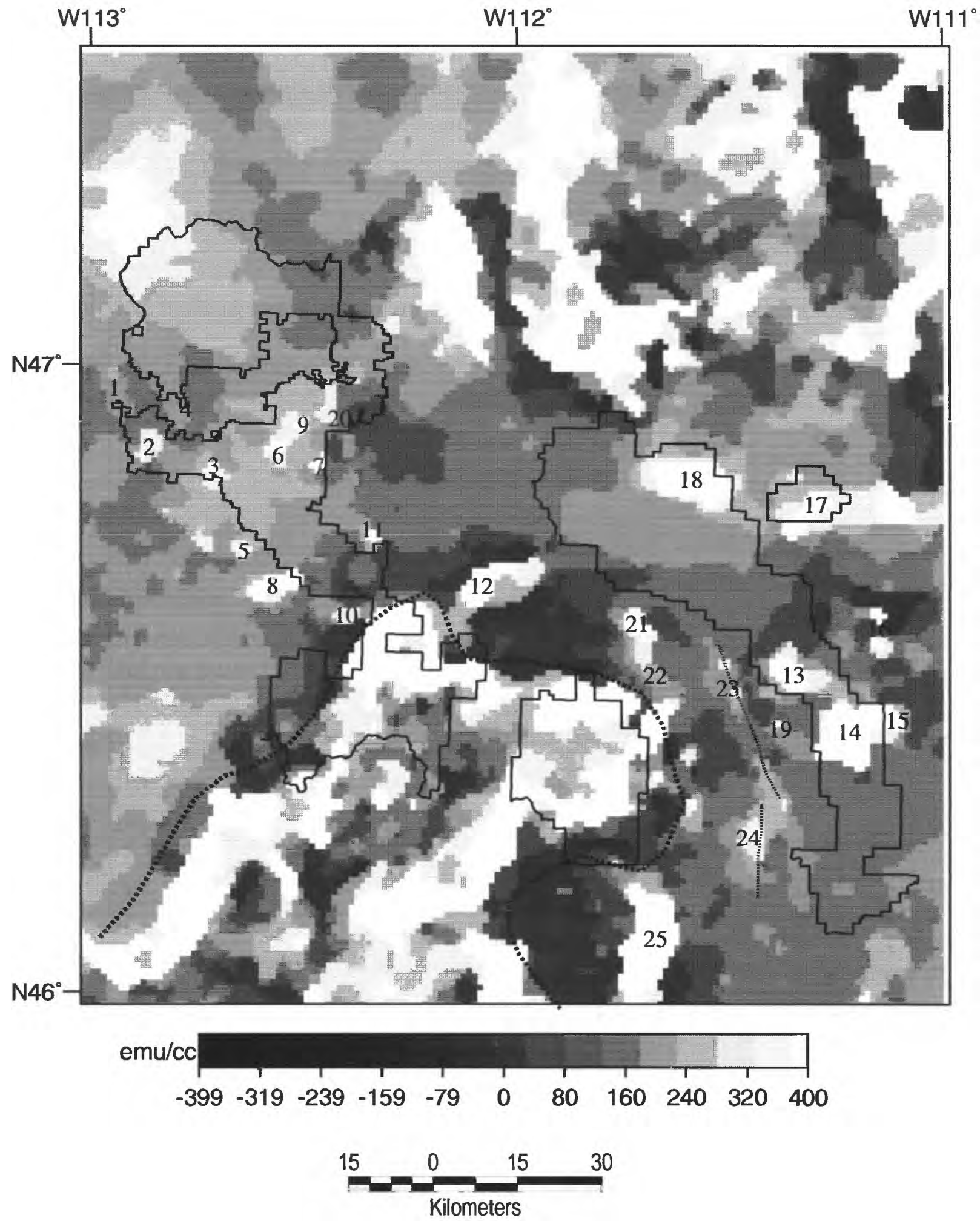

Figure C4. Terrace-magnetization map calculated from the aeromagnetic data (fig. C1). The map emphasizes large, sharply bounded domains of similar magnetization. The edges of the domains are inferred to mark steeply dipping lithologic and structural boundaries. Units are relative magnetization in electomagnetic units per centimeter-cubed (emu/cc). Data from this map were used as input to the geochemical-geophysical $\mathrm{Pb}$ and $\mathrm{Au}$ models (Chapter $\mathrm{D}$, this report), to map the lateral extent of buried and partially exposed plutons to refine favorable and permissive tracts for selected deposit models. Heavy dashed line outlines the Boulder batholith. Numbers correspond to features described in table C3. 


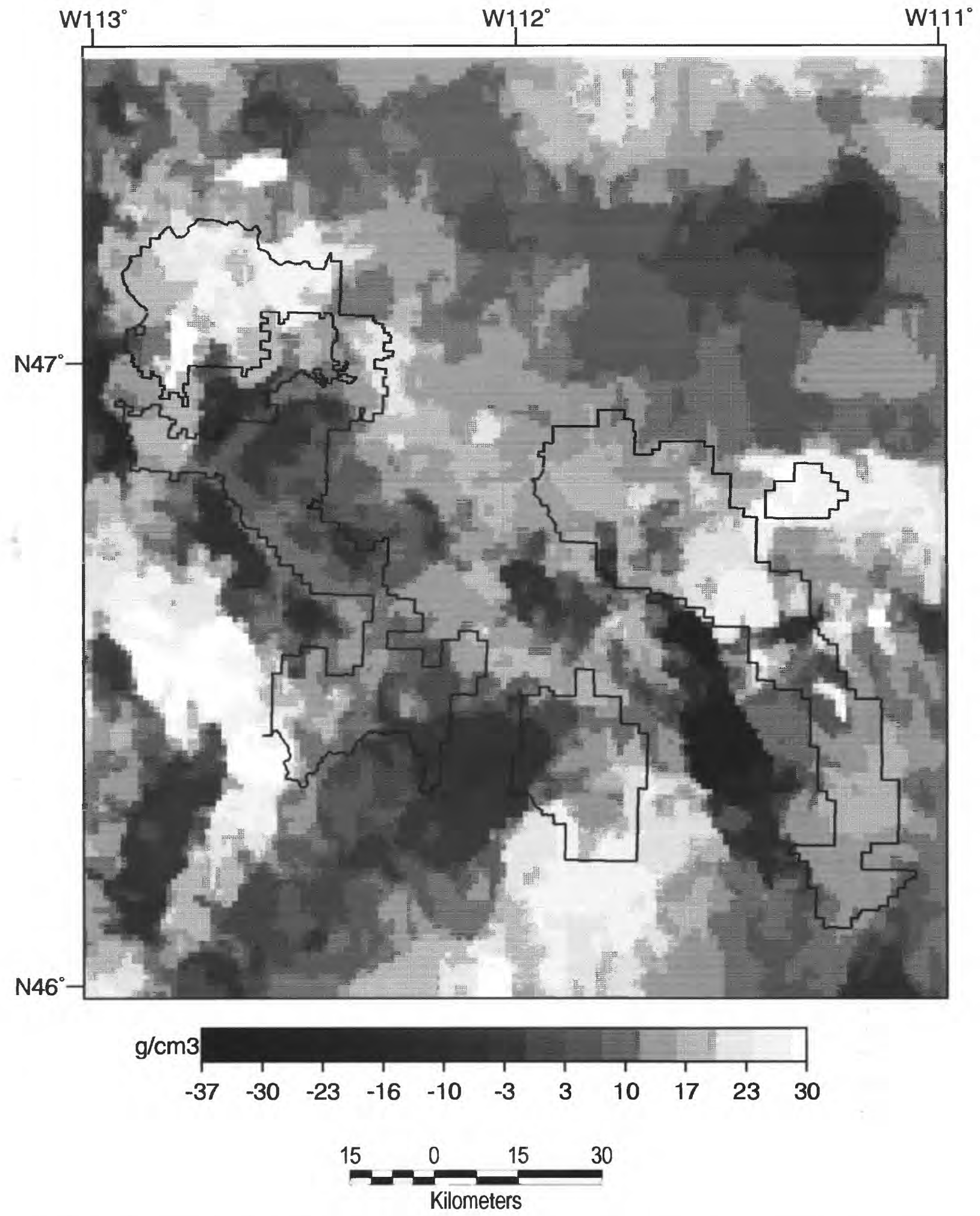

Figure C5. Terrace-density map of residual Bouguer gravity. Map shows areas of constant density separated by hard, steeply dipping edges. The edges of the density domains are inferred to represent geologic boundaries such as faults, lithologic contacts, and igneous intrusive bodies. Values within domains are relative densities in grams per centimeter-cubed. Data from this map were used in the geochemical-geophysical $\mathrm{Pb}$ and $\mathrm{Au}$ models described by Alminas (Chaper $\mathrm{D}$, this report). 
Table C3. Summary of the aeromagnetic anomalies for the Forest area. Numbered anomalies are indicated on figures $\mathrm{C} 1$ and $\mathrm{C} 4$. Refer to Plate 1 for geologic mapping and rock descriptions.

\begin{tabular}{|c|c|c|}
\hline Anomaly & $\begin{array}{l}\text { Source or location of } \\
\text { aeromagnetic anomaly }\end{array}$ & Composition of anomaly source \\
\hline 1 & \begin{tabular}{|l|} 
Mineral Hill stock \\
\end{tabular} & \begin{tabular}{|l} 
hornblende-biotite granodiorite \\
\end{tabular} \\
\hline 2 & Ogden Mountain stock & hornblende-biotite granodiorite \\
\hline 3 & Dalton Mountain stock & granodiorite \\
\hline 4 & \begin{tabular}{|l|} 
mostly buried \\
\end{tabular} & \begin{tabular}{|l|} 
granodiorite \\
\end{tabular} \\
\hline 5 & buried under Avon valley & $?$ \\
\hline 6 & Silver Bell stock & granodiorite \\
\hline 7 & Granite Butte stock & $\begin{array}{l}\text { hornblende-clinopyroxene-biotite } \\
\text { granodiorite }\end{array}$ \\
\hline 8 & Blackfoot City stock & hornblende-biotite granite/granodiorite \\
\hline 9 & $\begin{array}{l}\text { buried under Lincoln volcanic } \\
\text { field }\end{array}$ & $?$ \\
\hline 10 & mostly buried & granodiorite \\
\hline 11 & Marysville stock & quartz monzonite/mafic granodiorite \\
\hline 12 & \begin{tabular}{|l|} 
Scratch Gravel Hills stock \\
\end{tabular} & augite-hornblende monzonite \\
\hline 13 & Boulder Baldy intrusive suite & granodiorite-monzodiorite-monzonite \\
\hline$\overline{14}$ & Mount Edith stock & monzodiorite \\
\hline 15 & $\begin{array}{l}\text { buried source along eastern flank } \\
\text { of Big Belt Mts }\end{array}$ & $\begin{array}{l}\text { high magnetization, likely a different age } \\
\text { from } 13 \text { and } 14 \text {, or structurally rotated }\end{array}$ \\
\hline 16 & $\begin{array}{l}\text { buried source east of Big Belt } \\
\text { Mountains }\end{array}$ & $\begin{array}{l}\text { high magnetization, likely related to anomaly } \\
15\end{array}$ \\
\hline 17 & $\begin{array}{l}\text { buried source under } \\
\text { Mississippian sedimentary rocks }\end{array}$ & $?$ \\
\hline 18 & $\begin{array}{l}\text { buried source under Tertiary } \\
\text { Bozeman Group sedimentary } \\
\text { rocks }\end{array}$ & $?$ \\
\hline 19 & $\begin{array}{l}\text { buried source under Middle } \\
\text { Proterozoic Belt sedimentary } \\
\text { rocks }\end{array}$ & $?$ \\
\hline 20 & \begin{tabular}{|l|} 
buried source under Middle \\
Proterozoic sedimentary rocks \\
and Lincoln Volcanic field rocks
\end{tabular} & $?$ \\
\hline 21 & \begin{tabular}{|l|} 
Intrusives in Spokane Hills \\
\end{tabular} & monzonite \\
\hline 22 & Antelope Creek stock & monzonitic \\
\hline 23 & partly exposed intrusive bodies & monzonitic and lamprophyre \\
\hline 24 & $\begin{array}{l}\text { buried under valley-fill } \\
\text { sedimentary rocks }\end{array}$ & $\begin{array}{l}\text { coincides with axis of Deep Creek anticline, } \\
\text { dike? }\end{array}$ \\
\hline 25 & $\begin{array}{l}\text { partly exposed intrusive rocks } \\
\text { related to Elkhorn Mountain } \\
\text { Volcanics }\end{array}$ & monzodiorite and granodiorite \\
\hline
\end{tabular}


process to refine the lateral extent of known igneous intrusions and to locate inferred plutons in evaluating certain deposit types. Both terrace data sets were statistically integrated with geochemical data to produce the geochemicalgeophysical $\mathrm{Pb}$ and $\mathrm{Au}$ models described by Alminas (Chapter $\mathrm{D}$ of this report).

Before terracing, the aeromagnetic anomaly data were transformed to a form equivalent to a vertically incident magnetic field, often referred to as "having been reduced to the magnetic north pole." The reduction-to-pole transformation corrects for anomaly distortion caused at the geomagnetic latitude of the Forest and, assuming induced magnetization, shifts and centers anomalies over their source. Figure C4 shows a terrace-magnetization map of the reduced-to-pole magnetic field. Details on the steps involved to calculate terrace data from potential field grids are given in Cordell and McCafferty (1989).

A third-order regional field was analytically subtracted from the grid of Bouguer gravity data ( $f i g$. C3) before terracing to enhance shorter wavelength anomalies that map shallow crustal density sources. The resulting terracedensity map of the residual gravity field is shown in figure C5.

No attempt was made to calculate values of magnetization and density within domain boundaries. In order to obtain physical property values, one needs to make assumptions regarding the depth to and thickness of the anomaly sources. Such assumptions were not made for the Forest because the depth and thickness of density and magnetization sources are usually not known, due to the complex geology and lack of detailed drill hole information. Consequently, the grayscale bar on both the terrace-magnetization map (fig. C4) and terrace-density map (fig. C5) label the physical properties as "relative".

Data from both terrace maps were statistically integrated with geochemical data to determine associations between intrusive bodies, defined from the terrace data as areas of relatively high magnetization and low density, and high concentrations of select geochemical elements. The geochemical-geophysical models are described in Alminas (Chapter D of this report) and shown on plate 4 (maps I, J).

\section{Sources of Aeromagnetic Anomalies}

Many of the aeromagnetic anomalies in and surrounding the Forest can be directly correlated to known geologic features. Isolated, large amplitude aeromagnetic anomaly highs occur over many known igneous plutons. Additional plutons are inferred from the aeromagnetic data based on similarity in anomaly patterns and proximity to the exposed plutons. Table C3 summarizes aeromagnetic anomalies associated with known and inferred buried plutons in and around the Forest. Based on the lateral extent of the magnetization domains (fig. C4) in comparison with the mapped, exposed rocks, the subsurface horizontal cross section areas of many of the plutons (for example, the Marysville, Silver Belle, Dalton Mountain, Mineral Hill, Blackfoot City, and Scratch Gravel Hills plutons) are inferred to be twice their mapped surface exposures. The magnetic anomalies in the northwestern part of the study area are primarily the result of the magnetization contrast of the Cretaceous or Tertiary plutons in contact with the nonmagnetic metasediments of the Middle Proterozoic Belt Supergroup. Induced magnetization (normal polarization) is the primary contributor to the anomalies.

The aeromagnetic and gravity maps covering the southwest and Elkhorn Mountain parts of the Forest area (figs. $\mathrm{Cl}$ and $\mathrm{C} 4$ ) are dominated by physical properties of the northern part of the Boulder batholith. On a regional scale, the northeast trending horseshoe-shaped aeromagnetic high extends $25 \mathrm{mi}$ from the 
center of the study area southward into the adjacent Dillon $1^{\circ} \times 2^{\circ}$ quadrangle (McCafferty, 1992; Hanna and others, 1993). The Bouguer gravity map (fig. C3) shows a coincident large amplitude gravity low that extends to the Idaho-Montana border (Bankey, 1992).

On a local scale, the anomalies form a complicated pattern of highs and lows that reflect magnetic properties of the E1khorn Mountains Volcanics, Boulder batholith rocks, and the Lowland Creek Volcanics. Descriptions of individual anomalies for the northern batholith as outlined in figures $\mathrm{C} 1$ and $\mathrm{C} 3$ are provided in Hanna and others (1994) for the Butte $1^{\circ} \times 2^{\circ}$ quadrangle and by Hanna (1990) for the Elkhorn Mountains.

\section{Sources for magnetic and gravity anomalies in the Big Belt Mountains}

Table C3 includes anomalies and their known or inferred sources for the Big Belt Mountains region of the Forest. Two prominent, large amplitude magnetic anomaly highs occur in the central part of the Big Belt Mountains (anomalies 13 and 14, fig. C1). The northern anomaly corresponds to the Boulder Baldy pluton and the southern anomaly is located over the Mount Edith stock (plate 1; du Bray, 1995). The terrace-magnetization map (fig. C4) shows that the horizontal mapped exposure of the Boulder Baldy pluton is equivalent in diameter to that of the magnetization domain (13 on fig. C4). However, the Mount Edith stock is laterally more extensive in the subsurface ( 14 on fig. C4) than in its mapped outcrop. The terrace magnetization domain associated with Mount Edith pluton exceeds the mapped diameter by $2.5 \mathrm{mi}$. Additionally, from the terracemagnetization map, it appears that the smaller satellite stock 1 mi east of the Boulder Baldy pluton is connected at depth to the Mount Edith pluton (note the north projection of the Mount Edith pluton magnetization domain 14 on fig. C4).

A 2.5-dimensional model was constructed across each of the central Big Belt Mountains plutons to estimate their thickness and subsurface configuration. Magnetizations of samples were acquired by laboratory measurements (I. Judy, 1995, written commun.) from samples collected by E. du Bray from both plutons and average values were used for each model (Mount Edith: $0.002 \mathrm{emu} / \mathrm{cm}^{3}$; Boulder Baldy: $0.0025 \mathrm{emu} / \mathrm{cm}^{3}$ ). The magnetic property measurements show that the remanent magnetization component of the total magnetization is negligible.

Results of the models suggest both plutons are thick, extending to depths greater than $4 \mathrm{mi}$ below sea level. This suggests the plutons are rooted and were emplaced after thrusting ceased. The small satellite pluton to the east of Boulder Baldy is a relatively thin body, modeled with a thickness of less than $1.2 \mathrm{mi}$ and using an equivalent magnetization as the Mount Edith pluton. Results of the geophysical modeling support du Bray's (1995) geologic observations that both plutons are likely related to a single intrusive event.

Magnetic sources 15 and 16, located along the east flank of the Big Belt Mountains (figs. $\mathrm{Cl}$ and $\mathrm{C} 4$ ), are buried and appear to have radically different magnetic properties from the Big Belt plutons as evidenced by the distinct, large amplitude, dipole low associated with each anomaly. This observation suggests the following possibilities: (1) the east flank sources have a high component of remanent magnetization implying they were emplaced when the earth's magnetic field had a different polarity from the time when the Big Belt plutons were emplaced; or (2) the sources are similar in age to the Big Belt plutons but were structurally rotated after emplacement. Physical property information from a well located drill hole is needed to confirm either possibility presented. 
GAMMA-RAY RADIOACTIVITY

By James A. Pitkin

Introduction

Gamma-ray radioactivity data for the Helena National Forest consists of aerial spectrometer surveys flown during the U.S. Department of Energy National Uranium Resource Evaluation (NURE) program (ca 1974-1983). NURE surveys that include parts of the Forest are those conducted for the Butte (Texas Instruments, Inc., 1979), Choteau (Texas Instruments, Inc., 1979), and White Sulphur Springs (High Life Helicopters and Geodata International, Inc., 1979) $1^{\circ} x 2^{\circ}$ quadrangles. Aerial gamma-ray data (aeroradioactivity) from these surveys were used to prepare an aeroradioactivity database for the Forest. The database also includes the NURE survey for the Great Falls $1^{\circ} \times 2^{\circ}$ quadrangle (Texas Instruments, Inc., 1979), which was used to provide a rectangular database. Other compilations of NURE aerial gamma-ray data that include the Forest are those of Duval and others (1995) and Phillips and others (1993).

Aeroradioactivity is the measurement of terrestrial radioactivity with instruments operated in low-flying aircraft. The source of the radioactivity measured is the near-surface rock and soil (to 12-in depth) where the primary gamma-ray emitting isotopes are from the natural radioelements potassium (K), uranium (U), and thorium (Th). NURE aerial systems were quantitatively calibrated at sites of known radioelement concentrations, permitting quantitative reporting of survey data in percent for $K$ and parts per million (ppm) for $U$ and Th (assuming equilibrium in the respective decay series). The near-surface distribution of $\mathrm{K}, \mathrm{U}$, and Th generally corresponds to bedrock lithology and modifications due to weathering, erosion, rock transport, ground water movement, and hydrothermal alteration.

Aerial flight-1ine spacing for the Forest database is 3-mi for east-west lines except for the area of the Choteau quadrangle, which is 6-mi for east-west lines; all north-south flight lines are $12 \mathrm{mi}$ apart. A minimum-curvature algorithm (Webring, 1981) was applied to the flight line data, producing $\mathrm{K}, \mathrm{U}$, and Th $1.8 \mathrm{mi}$ square grids, which comprise the Forest aeroradioactivity database. The grids were used to prepare $\mathrm{K}, \mathrm{U}$, and Th color and black-and-white maps at 1:250,000-scale for use in the assessment and grey-scale maps at $1: 1,000,000-s c a l e$ for inclusion in this report. Grids of the ratios $U: T h$ and $\mathrm{K}$ : Th were also prepared and maps were made at several scales.

\section{Discussion}

Aeroradioactivity grey-scale contour of $\mathrm{K}, \mathrm{U}, \mathrm{Th}, \mathrm{U}: \mathrm{Th}$ and $\mathrm{K}: \mathrm{Th}$ are shown (respectively) in figures $\mathrm{C6}, \mathrm{C7}, \mathrm{C} 8, \mathrm{C} 9$, and $\mathrm{C10}$. Bodies of water, such as Canyon Ferry Lake, have no measureable aeroradioactivity. The grids used to make figures 66-10 were not masked to delete data for lakes and reservoirs, however, and the discernable grey-scale values for the water should be ignored.

The near-surface distribution patterns of $K, U$, and Th displayed by the aeroradioactivity maps are often similar, but discontinuities in the patterns can reflect significant mineralogic discontinuities, such as the contrasting 
properties of felsic and mafic igneous rocks. The distribution pattern for Th generally is more consistent than that for $\mathrm{K}$ or $\mathrm{U}$, 1ikely because $\mathrm{Th}$ is the least mobile of these elements. For this reason, Th is used as the stable denominator in $U: T h$ and $K: T h$ ratios, thereby highlighting subtle variations in $U$ and $K$ distribution. Of particular interest are variations from the 0.25 ratio on figure $C 9$. While the crustal ratio of $U: T h$ most commonly ranges from $1: 2$ to $1: 8$ (Hoover and others, 1992, fig. 25, p. 68-69), a value of $1: 4$, or 0.25 in the case of figure $C 9$, has been chosen arbitrarily for use in the following interpretive discussion. Values greater than 0.25 on figure $C 9$ may indicate relative enrichment of $U$, values less than 0.25 may indicate relative depletion of $U$.

\section{Interpretation}

Natural radioelement distribution for the area that includes the Forest has a diverse pattern that reflects the complex geology of the area, as demonstrated in the grey-scale contour maps (figs $\mathrm{C6-10}$ ). These maps show few linear features of more than about 30-mi length because of the paucity of geologic features with lithologic and thus radioelement continuity. Generally higher radioelemment concentrations are west of the east side of the Boulder batholith and its extension to the north-northeast; generally lower concentrations are to the east. Faults locally have radioelement expression as linear features 5- to 15 -mi long, and lithologies with distinct radioelement character occur throughout the area.

The Boulder batholith (fig. B1) underlies much of the Butte-Boulder-Helena region that is depicted on figures C6-10. The west side of the south-central part of the Forest (i.e., west half of the Elkhorn Mountains) displays prominent, higher radioelement concentrations ( 2 to 2.6 percent $\mathrm{K}, 2.6$ to $3.8 \mathrm{ppm} \mathrm{U,} 9$ to $14 \mathrm{ppm} \mathrm{Th}$ ) that correspond to the felsic rocks of the Butte quartz monzonite; this rock type comprises most of the batholith. The $U$ :Th ratio ranges between .22 and .28 , which does not indicate an excess of $U$ relative to Th (crustal ratio - 25). The batholith was determined favorable for $U$ vein deposits by the NURE program study of the Butte $1^{\circ} \times 2^{\circ}$ quadrangle (Sartoris and others, 1982, p. 15-18). The remainder of this part of the Forest has relatively lower radioelement concentrations ( 1 to 1.6 percent $\mathrm{K}, 1.4$ to $2 \mathrm{ppm} \mathrm{U,} 4$ to $6 \mathrm{ppm}$ Th) that are assoclated with less felsic, andesitic Elkhorn Mountain Volcanics and, at the southeast side of the Elkhorn Mountains, Paleozoic and Mesozoic sedimentary rocks, including the Permain Phosphoria Formation (p1. 1). The uraniferous character of the Phosphoria is not apparent in the aeroradioactivity data because the formation is thin and discontinuous (see Chapter $H$ for discussion of Phosphoria Formation). The E1khorn Mountain Volcanics have prominent expression in the $\mathrm{U}: \mathrm{Th}(>.31)$ and $\mathrm{K}: \mathrm{Th}(>.16)$ ratios, indicating relative enhancement of $U$ and $K$ compared to $T$.

The pattern of higher radioelement concentrations associated with felsic igneous rocks continues to the west to include an appreciable area in the southwest part of the Forest. Values of 1.8 to 2.6 percent $\mathrm{K}, 2.6$ to $3.8 \mathrm{ppm} \mathrm{U}$, and 11 to $14 \mathrm{ppm}$ Th relate to more rocks of the Cretaceous Boulder batholith and to Tertiary plutonic rocks. The U:Th ratio is relatively constant around .25 in this area, indicating a radioelement lithology different from the Th-dominant feature at the west side of the south-central part of the Forest. The K:Th ratio is relatively uniform, continuing the pattern from the south-central part. E1khorn Mountain Volcanics in this part of the Forest differ from those in the 
south-central part, as $\mathrm{K}$ is higher at 2 to 2.6 percent, and $U$ and $T$ are moderate at 2 to $2.8 \mathrm{ppm}$ and 7 to $10 \mathrm{ppm}$, respectively. The volcanic rocks have a distinctive $\mathrm{K}$ :Th pattern of .26 to .36 that reflects their more felsic character; the $U:$ Th ratio has no signficant character.

A pattern of somewhat lower aeroradioactivity values ( 1.4 to 1.8 percent $\mathrm{K}, 1.4$ to $2 \mathrm{ppm} \mathrm{U}, 5$ to $8 \mathrm{ppm}$ Th) occurs at the west side of the southwest part of the Forest, extending to the north across Avon Valley (directly west of the Forest), between $46^{\circ} 32^{\circ}$ and $46^{\circ} 50 \mathrm{~N}$.lat., and into the west-central part of the Forest. Source rocks for this feature are Paleozoic and Mesozoic sedimentary strata of the eastern part of the Sapphire thrust terrane and Cretaceous and Tertiary mafic volcanic rocks due southwest of the southwest part of the Forest. The Phosphoria Formation of the sedimentary sequence of rocks may be contributing to this aeroradioactivity feature; however its surface expression is limited.

A circular aeroradioactivity high (2 to 2.4 percent $\mathrm{K}, 2.6$ to $3.8 \mathrm{ppm} \mathrm{U}$, 9 to $13 \mathrm{ppm}$ Th) occurs directly west of the previously discussed sedimentary rock feature in the Sapphire terrane. The high is outside of the Forest centered at about $112^{\circ} 35^{\circ} \mathrm{W}$. long, and $46^{\circ} 35^{\circ} \mathrm{N} .1$ at. It is about $10-\mathrm{mi}$ in diameter, has a central area of lower values, and is best expressed in $U$ and $T h$, especially at its southeast side. Source rocks for the aeroradioactivity feature are not obvious. The feature lies outside the area of the geology shown on the geologic map (p1. 1). Tertiary rhyolites of the Helena volcanic field occur in the area, as do other volcanic rocks of less felsic composition (Wallace and others, 1986) that probably contain lesser radioelement concentrations. Tertiary sedimentary rocks and Quaternary sediments are also present.

Continuing to the north, aeroradioactivity data for the west-central part of the Forest, south of Lincoln, is characterized by moderate to low values ( 1.2

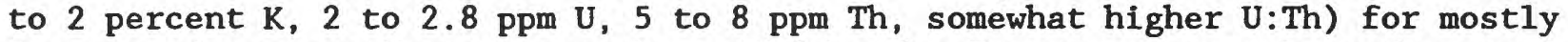
Proterozoic metasedimentary rocks. Within the Forest, east and southeast of Lincoln, an area of relatively higher values ( 1.6 to 2.2 percent $\mathrm{K}, 2.6$ to 3.2 ppm U, 10 to $13 \mathrm{ppm} \mathrm{Th}$ ) corresponds to Cretaceous granite and Tertiary rhyolite of the Lincoln volcanic field, which have a Th-distinctive lithology as indicated by relatively lower $\mathrm{U}: \mathrm{Th}$ and $\mathrm{k}$ :Th ratios. At Lincoln and in the surrounding valley eastward to about $112^{\circ} 30^{\circ} \mathrm{W}$. long., outside the Forest, a three-element relative high ( 1.8 to 2.2 percent $\mathrm{K}, 2.6$ to $3.2 \mathrm{ppm} \mathrm{U,} 9$ to $11 \mathrm{Th}$ ) suggests source rock of felsic or similar composition. Lack of expression in either ratio indicates a non-Th distinctive lithology for the Quaternary sediments in the valley as compared to the igneous rocks east and southeast of Lincoln.

Aeroradioactivity data for the northwest part of the Forest include a northwest-trending linear feature, most apparent in $\mathrm{K}$ and $\mathrm{U}$, which relates to the Hoadley thrust fault that bisects this part of the Forest (p1. 1). Rocks northeast of the fault have somewhat higher values of $\mathrm{K}$ ( 1.8 to 2.4 percent), U $(2.4$ to $3 \mathrm{ppm})$, and $\mathrm{Th}(7$ to $10 \mathrm{ppm})$, rocks on the southwest side have somewhat lower values ( 1.2 to 2 percent $\mathrm{K}, 1.8$ to $2.4 \mathrm{ppm} \mathrm{U}$, and 6 to $9 \mathrm{ppm} \mathrm{Th}$ ), and the ratios are not distinct for either side. Although Proterozoic Mount Shields Formation, Spokane Formation and associated metasedimentary rocks crop out on both sides of the fault, the strata of each side are from different depositional facies (see Chapter B of this report). The radioelement data reflect this by showing slightly enhanced $\mathrm{K}$ and $\mathrm{U}$ lithologies for the northeast side.

Noteworthy aeroradioactivity features in the north-central part of the study area, outside the Forest, include a prominent $\mathrm{K}$ ( 2 to 2.4 percent) and $\mathrm{K}: \mathrm{Th}$ $(>.30)$ feature east and northeast of Craig that reflects Cretaceous Adel Mountain Volcanics (not shown or described on p1. 1). These probable intermediate 
(andesitic) rocks (Schmidt, 1978) have slight expression in $U$ and none in Th. South of Craig and north of Helena is a large area of relatively higher aeroradioactivity ( 2 to 2.4 percent $\mathrm{K}, 2.8$ to $3.2 \mathrm{ppm} \mathrm{U,} 9$ to $11 \mathrm{ppm} \mathrm{Th}$ ) whose source rocks are primarily shale of the Proterozoic Spokane Formation. East of Helena, a strong aeroradioactivity feature $(2.2$ percent $\mathrm{K}, 2.6$ to $3.4 \mathrm{ppm} \mathrm{U,} 10$ to $13 \mathrm{ppm} \mathrm{Th}$ ) relates to tuffaceous sedimentary rocks of the Tertiary Bozeman Group in the Helena basin, considered favorable for the occurrence of ". Wyoming roll-type uranium deposits.." (Dodd and Wopat, 1982, p. 27). The elevated K and Th relate to felsic volcanic rocks. The Townsend basin to the southeast (west of the Big Belt Mountains and east of about $110^{\circ} 40^{\prime} \mathrm{W}$.long.) contains similar rocks, and is considered favorable for uranium deposits (Dodd and Wopat, 1982, p. 28). The basin has an aeroradioactivity expression only as a local U-high (3 ppm) north of Winston (not shown figs. C6-10) and as a prominent $U: T h$ (>.37) linear feature along the west side of Canyon Ferry Lake.

The western part of the Big Belt Mountains, in the eastern region of the Forest, includes a prominent northwest-trending linear area of relatively high radioelement concentrations ( 1.6 to 2.2 percent $\mathrm{K}, 1.8$ to $2.2 \mathrm{ppm} \mathrm{U,} 8$ to $10 \mathrm{ppm}$ $\mathrm{Th}$ ) that is most distinct in $\mathrm{K}$. The linear $\mathrm{K}$ anomaly reflects potassium feldspar zones, which are Au-bearing in the York mining district, in siltite and argillite of the lower part of the Proterozoic Greyson Formation. The slightly elevated $\mathrm{U}$ and Th possibly correspond to black siltite rather than the K-rich rocks. The feature extends northwest and southeast from York (not shown on figs. C6-10) for about $20 \mathrm{mi}$ as it follows the outcrop of the Greyson, and turns to a southerly trend outside of the Forest due east from Helena. Here the aeroradioactivity pattern associated with the Greyson becomes obscure, especially in $U$ and $T h$, where Tertiary Bozeman Group rocks of the Townsend Valley have similar values. An aeroradioactivity feature also occurs within the southern area of the eastern region of the Forest, northeast of Cedar Hi11, as indicated by locally elevated values of 1.6 to 2 percent $\mathrm{K}, 1.6$ to $2 \mathrm{ppm} \mathrm{U}$, and 8 to $9 \mathrm{ppm} \mathrm{Th;} \mathrm{this}$ feature reflects Greyson and upper Newland Formation strata. This feature is apparent in the aeroradioactivity data because most Proterzoic metasedimentary rocks of the Big Belt Mountains have low radioelement concentrations ( 0.8 to 1.2 percent $\mathrm{K}, 0.8$ to $1.2 \mathrm{ppm} \mathrm{U}, 3$ to $5 \mathrm{ppm} \mathrm{Th).} \mathrm{The} \mathrm{feature} \mathrm{has} \mathrm{no} \mathrm{expression} \mathrm{in}$ the ratios; however, there are a number of prominent $\mathrm{U}:$ Th features $(>.39)$ around the northern border, as well as at the south end of the Forest. These features are associated mainly with Paleozoic and Mesozoic sedimentary rocks at the northwest area of the Forest, with Paleozoic sedimentary rocks along the northern area where Tertiary volcanic rocks are ratio lows (Th-distinctive), and with Paleozoic and Mesozoic sedimentary rocks at the south end.

Near the east-central side of the Big Belt Mountains region of the Forest, southwest and south of Fort Logan, a distinctive aeroradioactivity relative high ( 1.8 to 2.4 percent $\mathrm{K}, 2.4$ to $3.2 \mathrm{ppm} \mathrm{U,} 9$ to $13 \mathrm{ppm} \mathrm{Th}$ ) has an arcuate shape open to the northwest. The feature occurs at the northeast side of the Boulder Baldy pluton and extends in an arcuate manner to Fort Logan, mostly in Newland Formation. It is most clearly expressed in $U$ and $T h$, and the area of highest $K$ occurs between two areas of higher $U$ and $T$ at each end of the feature.

The Boulder Baldy and Mount Edith plutons (plate 1) have aeroradioactivity expressions that reflect their intermediate composition. They have no expression in $\mathrm{K}$, have distinctly low $\mathrm{U}$ (each 1.2 to $1.6 \mathrm{ppm}$ ) and Th ( 4 to $5 \mathrm{ppm}$ and 5 to 6 $\mathrm{ppm}$, respectively). They have an expression in $\mathrm{K}$ : Th only because of the generally low $\mathrm{K}$ background levels of the intruded Proterozoic metasedimentary rocks, which include calcareous to dolomitic siltite and argillite. 


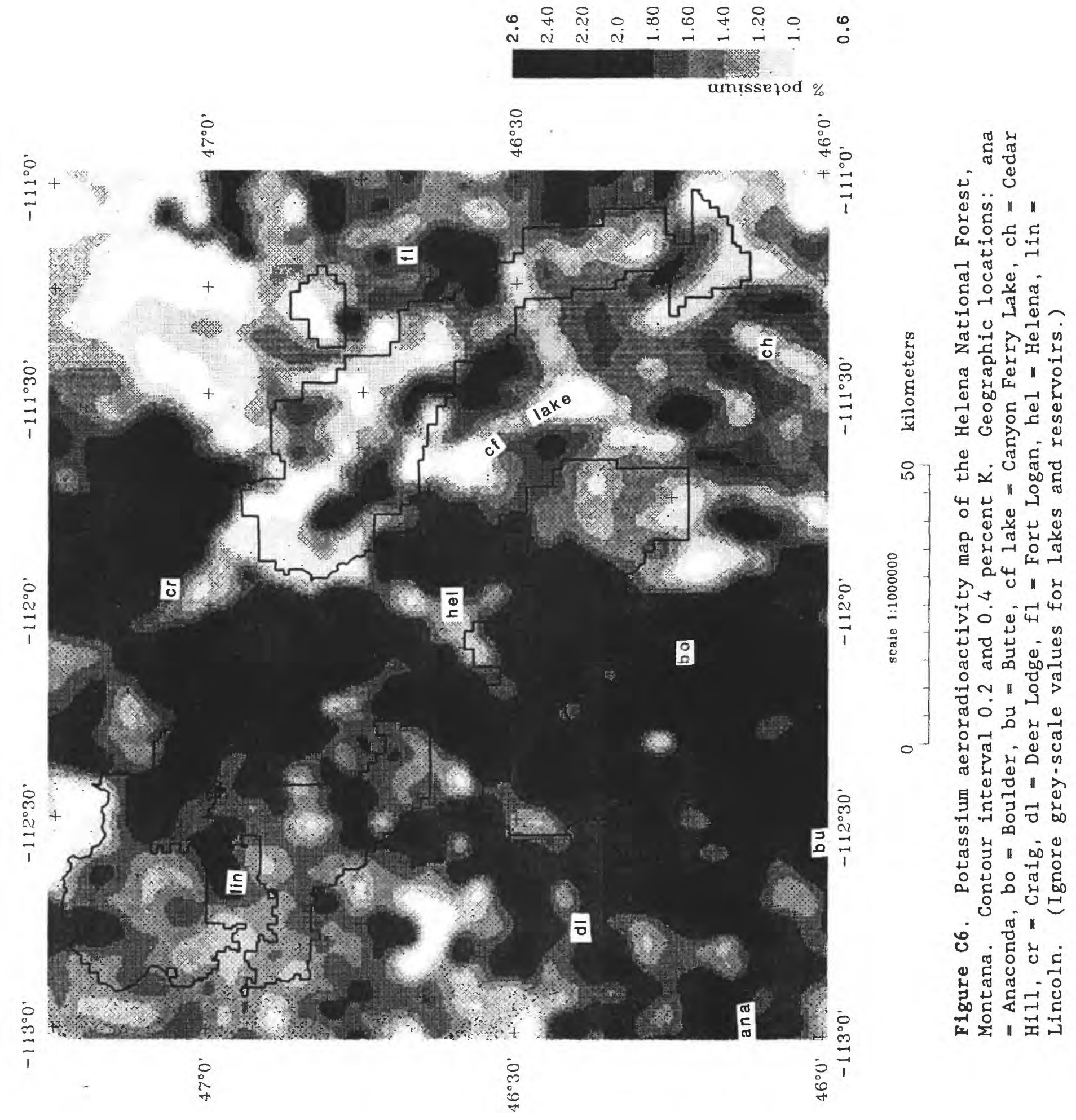




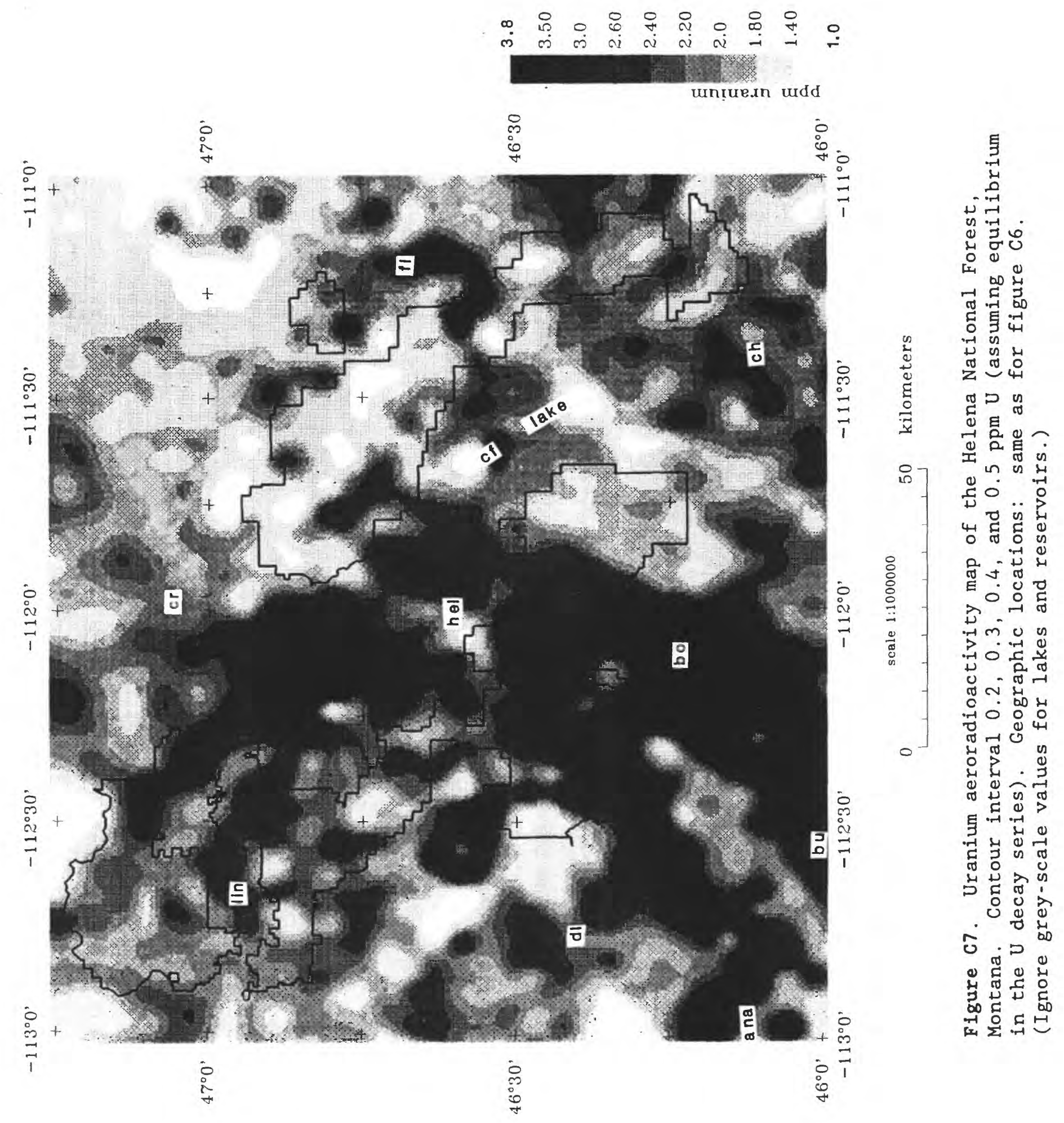



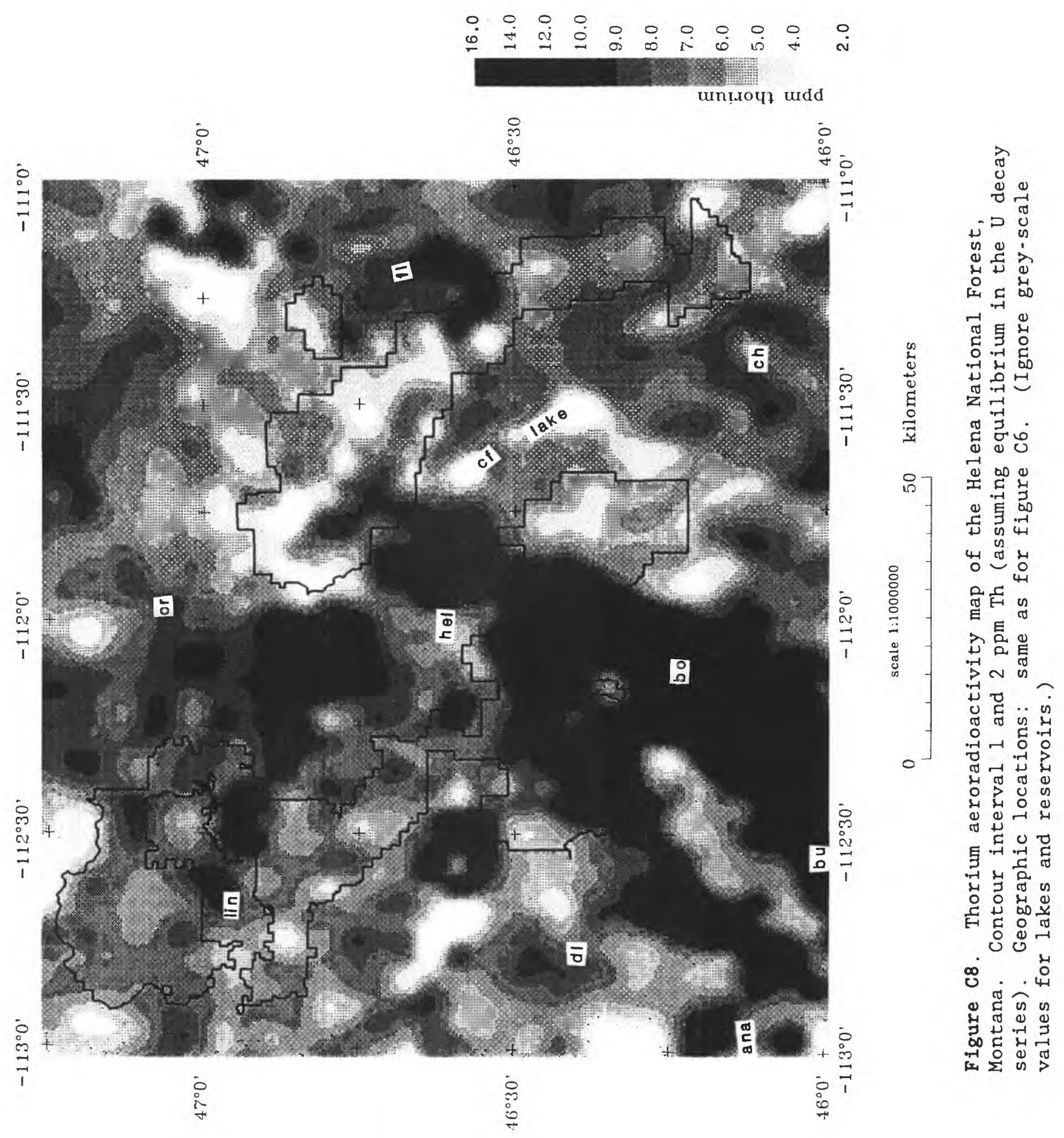


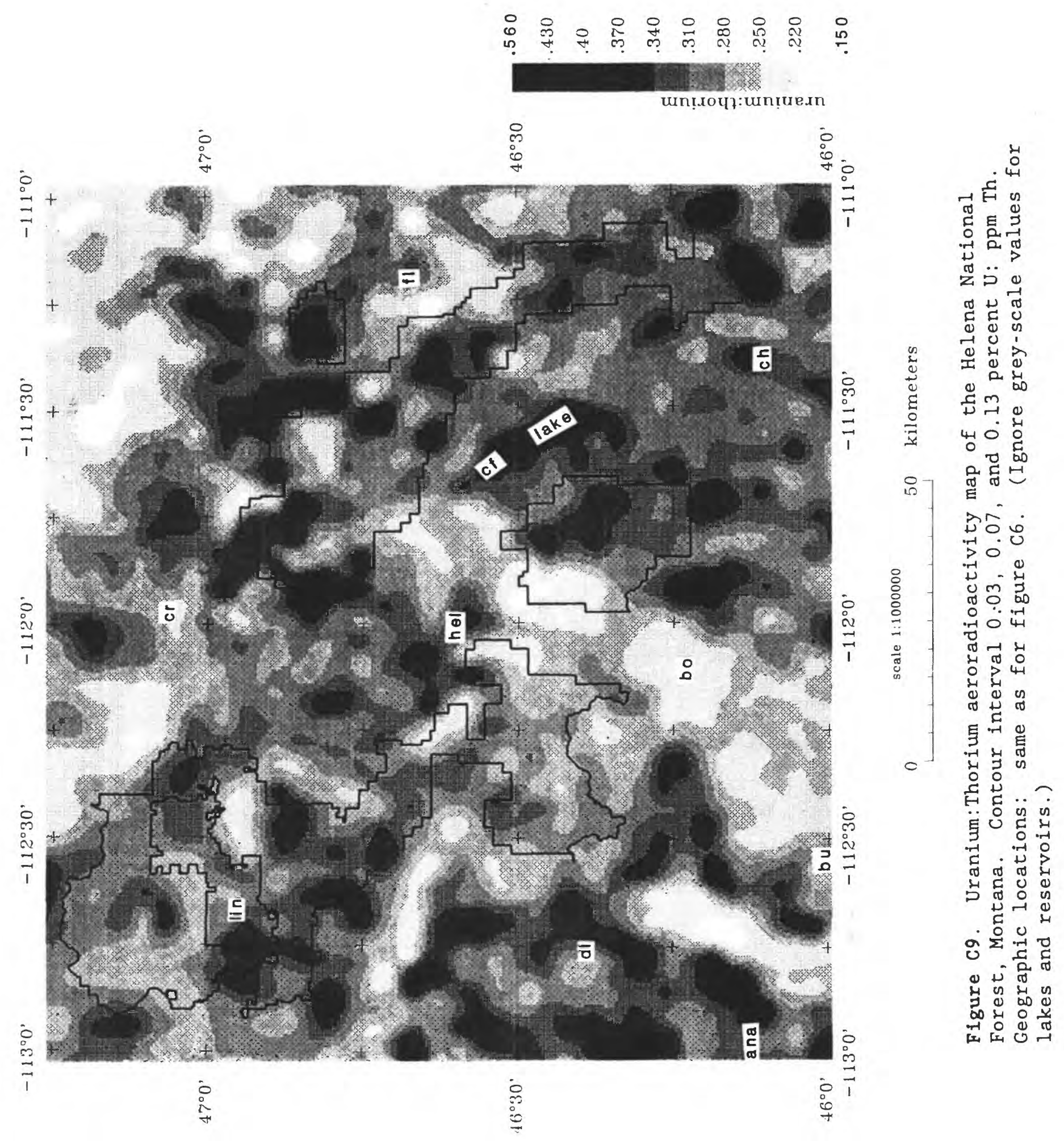




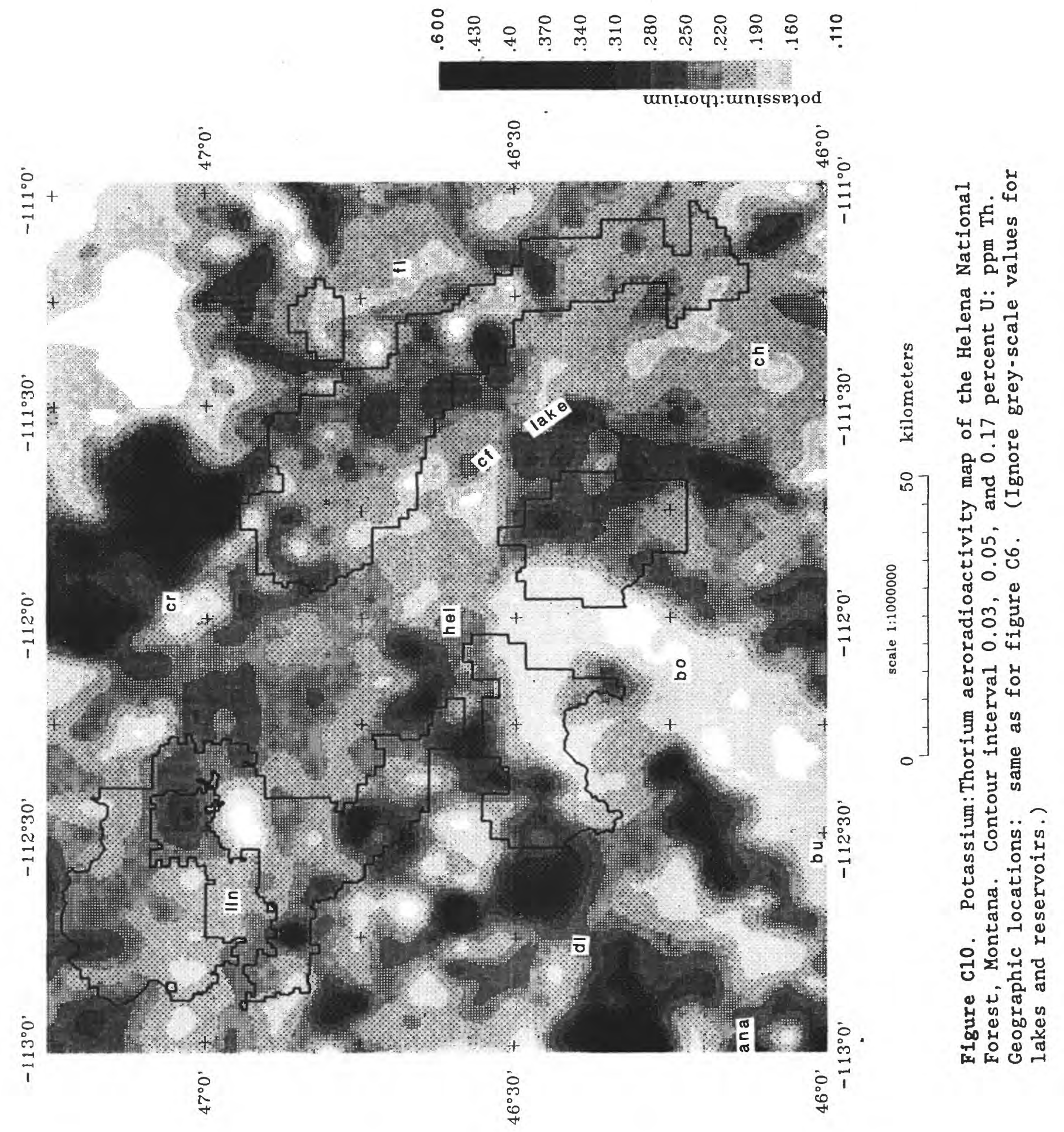




\section{REFERENCES CITED}

Bankey, Viki, 1992, Complete Bouguer gravity, isostatic residual gravity, and related geophysical maps centered on the Idaho batholith and Challis volcanic field, northwestern United States: U.S. Geological Survey Geophysical Investigations Map GP-995, scale 1:1,000,000.

Corde11, Lindrith, and McCafferty, A.E., 1989, A terracing operator for physical property mapping with potential field data: Geophysics, v. 54, no. 5, p. 621-634.

Davis, W.E., Kinoshita, W.T., and Smedes, H.W., 1963, Bouguer gravity, aeromagnetic, and generalized geology map of east Helena, Canyon Ferry quadrangle and part of the Diamond City quadrangle, Lewis and Clark, Broadwater, and Jefferson Counties, Montana: U.S. Geological Survey Geophysical Investigations Map GP-444, scale 1:62,500.

Dodd, S.P., and Wopat, M.A., 1982, Natural Uranium Resource Evaluation, White Sulphur Springs Quadrangle, Montana: U.S. Department of Energy, Grand Junction Office Report PGJ/F-136(82), 99 p., 15 plates, varied scales.

Duva1, J.S., Jones, W.J., Riggle, F.R., and Pitkin, J.A., 1995, Potassium, uranium, and thorium aerial gamma-ray maps of the conterminous United States: U.S. Geological Survey Geophysical Investigations Map GP-nnnn, 3 maps, scale $1: 2,500,000,7 \mathrm{p}$. text.

Geodata International, 1979, Aerial radiometric and magnetic survey, White Sulfur Springs national topographic map, Montana: U.S. Department of Energy Report GJBX 096-79, scale 1:250,000.

Grauch, V.J.S., and Corde11, Lindrith, 1987, Limitations of determining density or magnetic boundaries from the horizontal gradient of gravity or pseudogravity data: Geophysics, v. 52, p. 118-121.

Greenwood, W.R., Ludington, S., Miller, W.R., Hanna, W.F., Wenrich, K.J., Suits, V.J., and McHugh, 1990, Mineral resources of the E1khorn wilderness study area, Broadwater and Jefferson Counties, Montana: U.S. Geological Survey Bulletin 1805, $37 \mathrm{p}$.

Hanna, W.R., 1990, Geophysical exploration, in Greenwood, W.R., Ludington, Steve, Miller, W.R., and Hanna, W.F., 1990, Mineral resources of the E1khorn Wilderness study area, Broadwater and Jefferson Counties, Montana, with a section on Uranium and thorium potential by K.J. Wenrich, W.R. Miller, V.J. Suites, and John McHugh: U.S. Geological Survey Bulletin $1805,37 \mathrm{p}$.

Hanna, W.F., Hassemer, J.H., Elliott, J.E., Wallace, C.A., and Snyder, S.L., 1994, Maps showing gravity and aeromagnetic anomalies in the Butte $1^{\circ} \times 2$ - quadrangle, Montana: U.S. Geological Survey Miscellaneous Investigations Series Map I-2050-I, scale 1:250,000.

Hanna, W.F., Kaufmann, H.E., Hassemer, J.H., Ruppe1, B.D., Pearson, R.C., and Ruppe1, E.T., 1993, Maps showing gravity and aeromagnetic anomalies in the Dillon $1^{\circ} \times 2^{\circ}$ quadrangle, Idaho and Montana: U.S. Geological Survey Miscellaneous Investigations Map I-1803-I, scales 1:250,000 and 1:500,000, with pamphlet, $27 \mathrm{p}$.

High Life Helicopters and Geodata International, Inc., 1979, Aerial radiometric and magnetic survey, White Sulphur Springs National Topographic Map, Montana: U.S. Department of Energy, Grand Junction Office Report GJBX-096(79), 2 vols.

Hoover, D.B., Heran, W.D., and Hill, P.L. eds, 1992, The geophysical expression of selected ore deposit models: U.S. Geological Survey Open-File Report 92-257, 129p. 
Johnson, R.W., Jr., Henderson, J.R., and Tyson, N.S., 1965, Aeromagnetic map of the Boulder batholith area, southwestern Montana: U.S. Geological Survey Geophysical Investigations Map GP-538, scale 1:250,000.

Kinoshita, W.T., Davis, W.E., and Robinson, G.D., 1965, Aeromagnetic, Bouguer gravity, and generalized geology map of the Toston and Radersburg quadrangles and part of the Devils Fence quadrangle, Gallitan, Broadwater, and Jefferson Counties, Montana: U.S. Geological Survey Geophysical Investigations Map GP-496, scale $1: 62,500$.

Kinoshita, W.T., Davis, W.E., Smedes, H.W., and Nelson, W.H., 1964, Bouguer gravity, aeromagnetic, and generalized geology map of Townsend and Duck Creek Pass quadrangles, Broadwater County, Montana: U.S. Geological Survey Geophysical Investigations Map GP-439, scale 1:62,500.

Kleinkopf, M.D., 1981, The aeromagnetic map of the Choteau $1^{\circ} \times 2^{\circ}$ quadrangle, Montana: U.S. Geological Survey Miscellaneous Field Studies Map MF-856-G, scale 1:250,000.

Kleinkopf, M.D., and Mudge, M.R., 1972, Aeromagnetic, Bouguer gravity, and generalized geologic studies of the Great Falls-Mission Range area, northwestern Montana: U.S. Geological Survey Professional Paper 726-A, scale $1: 250,000,19 \mathrm{p}$.

Kulik, D.M., 1983, Complete Bouguer gravity and generalized geology and structure map of the Choteau $1^{\circ} \times 2^{\circ}$ quadrangle, Montana: U.S. Geological Survey Miscellaneous Field Investigations Map MF-1489, scale 1:250,000.

McCafferty, A.E., 1992, Aeromagnetic maps and terrace-magnetization map centered on the Idaho batholith and Challis volcanic field, northwestern United States: U.S. Geological Survey Geophysical Investigations Map GP994, scale $1: 1,000,000$.

Phillips, J.D., Duval, J.S., and Ambroziak, R.A., 1993, National geophysical data grids--Gamma-ray, gravity, magnetic, and topographic data for the conterminous United States: U.S. Geological Survey Digital Data Series DDS-9, 1 CD-ROM.

Sartoris, D.J., Gilmour, E.H., Salisbury, W.G., and McKee, C.J., 1982, National Uranium Resource Evaluation, Butte Quadrangle, Montana: U.S. Department of Energy, Grand Junction Office Report PGJ/F-064(82), 43 p., 16 plates, varied scales.

Schmidt, R.G., 1978, Rocks and mineral resources of the Wolf Creek area, Lewis and Clark and Cascade Counties, Montana: U.S. Geological Survey Bulletin 1441, $91 \mathrm{p}$.

Texas Instruments, Inc., 1979, Aerial radiometric and magnetic reconnaissance survey of portions of Arizona, Idaho, Montana, New Mexico, South Dakota, and Washington: U.S. Department of Energy, Grand Junction Office Report GJBX-126(79), vol. 1 and individual volumes for 12 quadrangles. [Butte quadrangle, v. 2G; Choteau quadrangle, v. 2D; Great Falls quadrangle, v. 2E]

U.S. Geological Survey, 1978, Mineral resources of the Elkhorn wilderness study area, Montana: U.S. Geological Survey and U.S. Bureau of Mines, Open-File Report 78-0325, aeromagnetic map scale 1:48,000, 362 p.

1980, Aeromagnetic map of Rogers Pass, Montana: U.S. Geological Survey Open-File Report 80-244, scale 1:62,500.

1984, Aeromagnetic map of the Butte $1^{\circ} \times 2^{\circ}$ quadrangle, Montana: U.S. Geological Survey Open-File Report 84-278, 7 sheets, scales 1:62,500 and $1: 250,000$.

Wallace, C.A., Schmidt, R.G., Lidke, D.J., Waters, M.R., Elliott, J.E., French, A.B., Whipple, J.W., Zarske, S.E., Blaskowski, M.J., Heise, B.A., 
Yeoman, R.A., O'Neill, J.M., Lopez, D.A., Robinson, G.D., and Klepper, M.R., 1986, Preliminary geologic map of the Butte $1^{\circ} \times 2^{\circ}$ quandrangle, western Montana: U.S. Geological Survey Open-File Report 86-292, scale $1: 250,000$, text.

Webring, M.W., 1981, MINC--A gridding program based on minimum curvature:

U.S. Geological Survey Open-File Report 81-1224, 41 p. 


\section{H A $\mathbf{P}$ T E R $\mathrm{R}$ \\ GEOCHEMISTRY OF THE HELENA NATIONAL FOREST}

By H.V. Alminas

\section{INTRODUCTION}

A geochemical survey of the Helena National Forest was designed to determine the broad regional mineral trends that impinge on or cross the Forest. The study area includes the western half of the White Sulphur Springs, eastern half of the Butte, and the southeastern corner of the Choteau $1^{\circ} \times 2^{\circ}$ quadrangles (fig. D1). The study area encompasses approximately 4,623,360 acres of which the Forest constitutes 975,088 acres.

\section{Sample Types}

This study is based (1) primarily on stream sediment samples collected under U.S. Geological Survey (USGS) mineral resource projects that cover the Butte $1^{\circ} \times 2^{\circ}$ quadrangle (McDanal and others, 1985), the E1khorn Wilderness Study Area (Greenwood and others, 1990), the Choteau $1^{\circ} \times 2^{\circ}$ quadrangle (Grimes and Leinz, 1980a, 1980b; Leinz and Grimes, 1980a, 1980b, 1980c), and (2) 151 samples collected during the Department of Energy NURE (National Uranium Resource Evaluation) studies within the Butte, Choteau, and White Sulphur Springs $1^{\circ} \times 2^{\circ}$ quadrangles. USGS and NURE data are augmented by samples collected for this study, chiefly within the Big Belt Mountains. The USGS sample types are sieved $<80$-mesh and $<100$-mesh stream-sediments and were collected predominantly from 2 nd and 3rd order streams. The NURE samples are sieved $<100$-mesh stream-sediments.

Results of rock and stream sediment samples analyzed within USGS geochemistry laboratories or labatories under contract to the USGS are retained as part of the RASS (rock analysis storage system) data base. Descriptors of "USGS" and "RASS" describe the source of the geochemical data bases and are used interchangeably throughout this report.

In addition to the USGS and NURE stream-sediment samples, data from spectrographic analyses of 1,916 outcrop samples collected during the course of the previous USGS studies were incorporated. The sample site distribution of these outcrop samples is extremely non-uniform and the data were used only for statistical purposes. The sample site distribution for the outcrop samples is shown in figure D2.

A data set consisting of selected elements from the USGS and NURE streamsediment samples was created to investigate base-metal distribution within the study area. For this study, the NURE samples were reanalyzed in the USGS laboratories to bring the analyses into conformance with the existing USGS spectrographic analyses (Arbogast, 1996). The resultant data set incorporates 3,282 samples. Sample-site distribution is relatively uniform throughout the study area (fig. D3).

Taking care to maintain relatively uniform sample-site distribution, 1,901 samples were selected from the combined USGS/NURE sample collection to study gold 
distribution. These samples were analyzed for gold spectrographi-cally and by flameless atomic absorption. Sample-site distribution for these data set is shown in figure D4.

The original NURE data from all of the 2,151 stream-sediments within the study area are incorporated into one data set. This data set was used to investigate the distribution of those elements not analyzed for by spectrographic means. Figure 5 shows the distribution of the NURE sample-sites.

\section{Analytical Procedures}

Al1 of the outcrop and stream-sediment samples (fig. D3) were analyzed for 31 elements using a six-step semi-quantitative spectrographic technique described by Grimes and Maranzino (1968). In this technique a $10 \mathrm{mg}$ powdered sample is mixed with a buffer and placed into a cup-shaped graphite electrode. A directcurrent arc volatizes the sample and the resultant spectra are recorded on a photographic film. Results are obtained by a visual comparison of spectra derived from the sample and that derived from standards made from pure oxides and carbonates. Results of these spectrographic analyses are read within geometric intervals having the boundaries of $1200 ; 830 ; 560 ; 380 ; 260 ; 120$; and so on in parts per million, but are reported by approximate geometric midpoints, such as $1000 ; 700 ; 500 ; 300 ; 200 ; 150$; and 100 . Precision of a reported value is approximately plus or minus one reporting interval at the 83 percent confidence level, and plus or minus two reporting intervals at the 96 percent confidence level (Motooka and Grimes, 1976). Values determined for the major elements (calcium, iron, magnesium, sodium, phosphorus, and titanium) are given in weight percent. Table D1 lists the elements analyzed and the lower limits of detection.

Gold content of the outcrop and stream-sediment samples (fig. D4) was determined using an atomic-absorption spectrographic method. In this method a 10g sample is roasted for 1 hour at $700^{\circ} \mathrm{C}$; gold is then extracted with hydrobromic acid-0.5 percent bromine solution and MIBK (methyl isobutyl ketone). Electrothermal atomic-absorption spectroscopy, using background correction, is used to determine gold to $0.001 \mathrm{ppm}(1 \mathrm{ppb})$ ( $0^{\prime}$ Leary and Meier, 1986). In the case of the $<100$ mesh NURE stream-sediments, due to a shortage of sample material, only $5 \mathrm{~g}$ of sample was used for the gold analysis and the lower limit of determination is $0.002 \mathrm{ppm}$ or $2 \mathrm{ppb}$.

Table D2 1ists the types of NURE analytical techniques and the elements analyzed for by each method in the analysis of the 2,151 NURE <100-mesh streamsediment samples (fig. D5).

\section{Statistical Summary}

\section{$\underline{\text { Data }} \underline{\text { Sets }}$}

Four distinct data sets were utilized for the purposes of this study. The data sets include the following sample types and analyses:

1. Spectrographic analyses of 1,916 USGS (RASS) outcrop samples. 


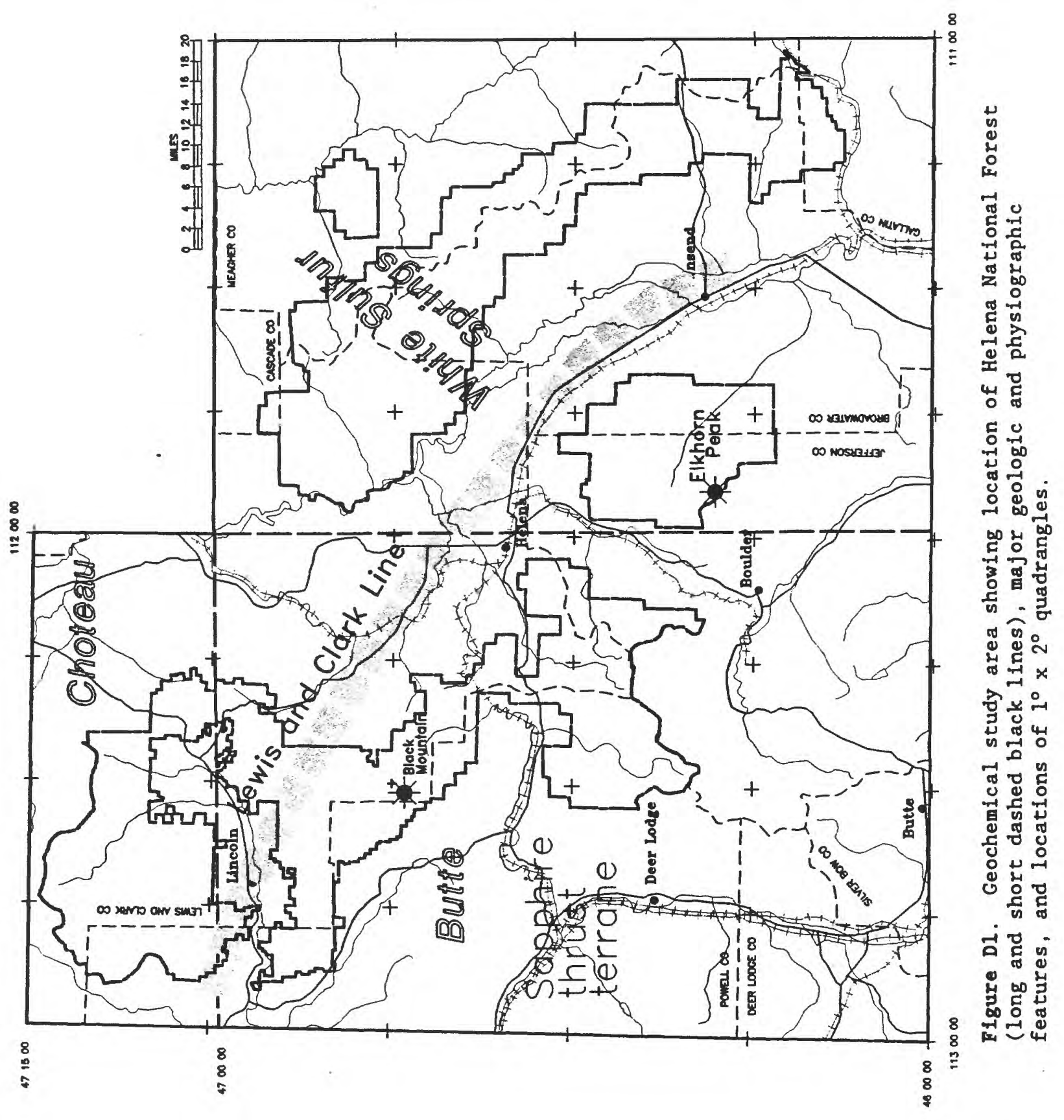


2. Spectrographic analyses of 3,282 USGS (RASS) and NURE streamsediments

3. Gold and spectrographic analyses of 1,901 USGS (RASS) and NURE stream-sediments

4. Various NURE analyses of 2,151 NURE stream-sediments.

The geochemical data that comprise these four sets are contained on the companion CD-ROM release (Green and Tysdal, 1996).

\section{Basic Statistics}

A statistical summary of each of the four data sets was calculated using the STATPAC statistical evaluation 1ibrary (Van Trump and Miesch, 1977; Grundy and Miesch, 1988) and is given in table format. A statistical summary of the 30 elements analyzed in the outcrop data set is given in table D3. The corresponding correlation table is given in table D4 and frequency distributions for all analyzed elements are given in table D5.

Table D6 summarizes the combined NURE and RASS spectrographically analyzed stream-sediment data set consisting of 3,282 sample analyses. Table D7 shows the correlations for this data set and table D8 shows the frequency distributions for the respective elements.

Table D9 summarized the spectrographic data of all the USGS and NURE samples analyzed for gold. Table D10 shows the corresponding correlation coefficients and table D11 shows the percent frequency distributions of all the analyzed elements.

The original NURE analyses are summarized in table D12. The corresponding correlation table is shown in table D13. The percent frequency distributions of the NURE analyzed elements are shown in table D14. For purposes of this report, the data has been grouped into spectrographic reporting classes.

Gold values, determined by the USGS on selected NURE and RASS streamsediments, are summarized in table D15. Gold correlation coefficients are shown in table D16 and the gold value frequency distribution is shown in table D17.

\section{GEOCHEMICAL EXPLORATION}

Geochemical anomalies are extensive and intense within the study area. Anomalous values of many geochemical elements are far more extensive within the eastern part of the Butte $1^{\circ} \times 2^{\circ}$ quadrangle than in the western part of the White Sulfur Springs $1^{\circ} \times 2^{\circ}$ quadrangle or the southeastern corner of the Chotoeau $1^{\circ} \times 2^{\circ}$ quadrangles. The anomaly-rich area is bounded on the north east by the Lewis and Clark line and dies out on the west in the Sapphire thrust terrane (fig. D1). Nearly all the geochemical anomalies occur over, or immediately adjacent to, Tertiary rhyolites (porphyry flows, porphyry plugs, breccia plugs, dikes) or Tertiary to Late Cretaceous plutonic rocks-predominantly of monzonitic to monzodioritic composition (plate 1). Some geochemical anomalies occur over exposed sedimentary units that have distinct aeromagnetic highs (fig. Cl) and gravity lows (fig. C3), which is suggestive of intrusives emplaced at some depth. 
Mineralized areas are often defined on the basis of an assemblage of geochemical elements as well as the relative amounts of individual elements within the assemblage. The REM (relative element magnitude) method of Van Trump and Alminas (1978) mathematically combines individual elements to form multielement geochemical anomalies. Results are elemental assemblages referred to as "association" models. The individual elements that make up the assemblage are defined on the basis of one principal element that forms an association with other elements in the suite. Associations can be determined either by a: (1) statistical correlation with other geochemical elements that may characterize the expected mineralization; or (2) by geological/geochemical knowledge of processes that associate one element with another; or (3) by a combination of (1) and (2). The REM method also calculates the relative magnitude or contribution of each individual element within the overall association.

For the study area, three association models based on three principal elements were derived with the REM computer program (Van Trump and Alminas, 1978) using data from NURE stream sediment analyses: one model based on lanthanum ( $\mathrm{La}$, a second based on lead $(\mathrm{Pb})$, and a third on gold ( $\mathrm{Au}$ ). The La-association model combines many rare earth elements to map locations of exposed and shallowly buried intermediate-composition plutons that can act as hosts to metal sulfide minerals. The $\mathrm{Pb}$-association model is based on a geochemical assemblage that combines a suite of metals for the purpose of mapping metal-rich areas related to sulfide mineralization. The Au-association model combines elements commonly associated with gold.

\section{Geochemica1 Association Mode1 Methodology}

REM values for the $\mathrm{La}-\mathrm{Pb}-$, and $\mathrm{Au}$-association models were derived by calculating two parameters for each element in the assemblage. The first parameter is called the "intensity factor" (Ii) and is calculated by dividing the mean of all anomalous values for a given element by its threshold value. "Anomalous value" is defined as being equal to or greater than a subjectively defined threshold value. For this study, the threshold value was defined to be any value that exceeded the $75^{\text {th }}$ percentile within the data set. Thus,

Ii $=$ mean of al1 anomalous values

threshold value of the sample

The second parameter is termed the "area factor" (c) and is a constant value defined by dividing the number of anomalous sample elements that exceed the threshold value by the total number of samples:

$c=$ Number of anomalous samples greater than the threshold value Total number of samples within study area

The intensity factor (Ii) is multiplied by the area factor to obtain the "element magnitude" (EI):

$$
E i=c(I i)
$$


The "anomaly magnitude" (A) is calculated by summing all the element magnitudes in the assemblage:

$$
A=E_{1}+E_{2}+E_{3}+\ldots \ldots E_{j} \text {, where } \mathbf{j} \text { - total number of samples }
$$

Finally, the element magnitude (Ei) is divided by the anomaly magnitude (A) and expressed in percent to obtain the "relative element magnitude" (REM) value for each sample location:

$$
R E M=E I \frac{*}{A} \underline{100}
$$

REM values for the three association models of this study were gridded onto rectangular 1 - by $1-\mathrm{km}$ grids using the commercial software package EarthVision ${ }^{1}$ and presented as gray-level contour plots.

\section{La-Association Mode1}

The La-association model was derived from the NURE stream sediment data set and consists of the following geochemical elements: La, Ce, Dy, Eu, Hf, $\mathrm{Lu}, \mathrm{Sm}, \mathrm{U}$, and $\mathrm{Yb}$. Figure D6 contains graphs showing lanthanum against the contribution of each individual element and its magnitude (Ei) within the overall association.

A contour plot of the distribution of the derived La-association values is shown in figure D7. The La-association model is composed of a suite of rare earth elements that characterize the nearly all of the mentioned intrusive types, many of which are mineralized. Many of the La-association anomalies correspond to areas delineated by terrace-magnetization domains with relatively high values (fig. C4) and low value terrace-density domains (fig. C5).

\section{$\underline{\mathrm{Pb}-\text { Association }} \underline{\text { Model }}$}

Lead is the most abundant ore-forming element within the study area. It has the most intense and extensive anomalies in comparison with other base metals and apparently occurs in most every type of mineral deposit within the study area. $\mathrm{A} \mathrm{Pb}$-based geochemical association derived from the combined NURE and USGS data set consisting of $\mathrm{Ag}, \mathrm{As}, \mathrm{B}, \mathrm{Be}, \mathrm{Bi}, \mathrm{Cu}, \mathrm{Mn}, \mathrm{Mo}, \mathrm{Sb}, \mathrm{Sn}, \mathrm{W}$, and $\mathrm{Zn}$ (fig. D8) characterizes most areas of known past mineral exploitation within the study area, as well as some areas that were not developed. The $\mathrm{Pb}$-association values were calculated in the same manner as the La-association values.

A contour map of $\mathrm{Pb}$-association value distribution relative to known mine and prospect pits within the Forest boundary is shown in figure D9. High value $\mathrm{Pb}$-association anomalies occur to the west of a line defined by Wolf Creek, Helena, and Townsend, which roughly colncides with the St. Mary's-Helena Valley fault (plate 1 and fig. B1). Although every mining district is characterized by

\footnotetext{
${ }^{1}$ Any use of trade names is for descriptive purposes only and does not imply endorsements by the USGS.
} 
this association to some degree, $\mathrm{Pb}$-association anomalies with high REM values occur over the following districts (plate 2 and fig. F1) and are ranked according to highest (1) to lowest REM values:
1. Marysville
2. Winston - Indian Creek-Park
3. Stemple-Gould
4. Wickes
5. North Boulder Mountain (west of Helena National Forest)
6. E1khorn
7. Heddleston
8. Warm Springs
9. Montana City

In addition, numerous high amplitude $\mathrm{Pb}$-association anomalies occur outside mining district boundaries and are interpreted as possible areas of unknown or unmapped mineralization. The anomalous concentration of $\mathrm{Pb}$ in rocks and (or) stream sediments is not necessarily indicative of anthropomorphic, mining-related contamination. $\mathrm{Pb}$ occurs naturally in several different forms (chemical compounds, or minerals) and the mobility of $\mathrm{Pb}$ is dependent on mineralogic siting and a wide variety of local geochemical conditions.

\section{$\underline{\text { Au-Association Mode1 }}$}

Gold is also widespread within the study area. A Au-association was determined and calculated in the same manner as described for the La-association and includes $\mathrm{Ag}, \mathrm{As}, \mathrm{Cd}, \mathrm{Cu}, \mathrm{Mn}, \mathrm{Pb}, \mathrm{Sn}, \mathrm{Sr}$, and $\mathrm{Zn}$ (fig. D10). A contour plot of the Au-association ( $f i g$. D11) tends to delineate the same general areas as the $\mathrm{Pb}$-association ( $\mathrm{fig}$. $\mathrm{D} 9$ ). As in the case of the $\mathrm{Pb}$-association, the $\mathrm{Au}$ association characterizes all defined mining districts to some degree, and in addition, delineates other areas falling outside the known districts (plate 2 and fig. F1). Major Au-association anomalies with relatively high REM values occur over the following known mining districts and are ranked from high (1) to low (9) based on REM values:

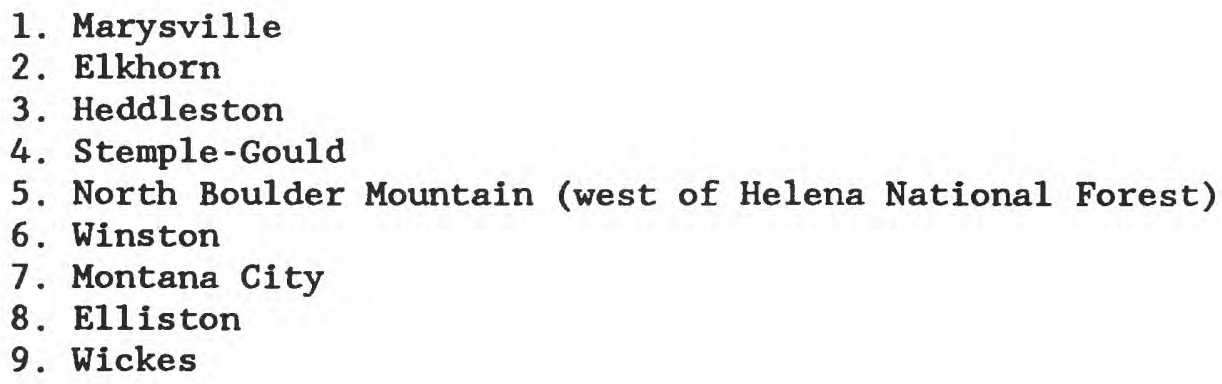

2. E1khorn

3. Heddleston

4. Stemple-Gould

5. North Boulder Mountain (west of Helena National Forest)

6. Winston

7. Montana City

8. E11iston

9. Wickes

Although the ranking varies somewhat, the areas defined are essentially the same ones as were defined by the $\mathrm{Pb}$-association. Generally, the Au-association anomalies occur over or near the mapped intrusives (Plate 1). Positive spatial relationships can be seen between the Au-association, distributions of terracemagnetization domains with relatively high values (fig. $\mathrm{C} 4$ ), and distributions of low values of terrace-density domains (fig. C5). 


\section{Element P1ots}

Selected individual element plots are shown in figures D12 through D21 and at a scale of 1:250,000 on maps F and G (plates 3 and 4). These elements were selected on the basis of their relationship with the $\mathrm{Pb}$ - or $\mathrm{Au}$ - associations as well as on the basis of widespread occurrence. Below is a list of the elements shown and the corresponding figure numbers.

$\begin{array}{llcc}\text { Figure } & \frac{\text { Element }}{\text { Dold }} & \frac{\text { Map }}{\text { silver }} & \frac{\text { Plate }}{4} \\ \text { D13 } & & \text { s } & \\ \text { D14 } & \begin{array}{l}\text { lead } \\ \text { bismuth }\end{array} & \text { F } & 3 \\ \text { D15 } & \text { copper } & & \\ \text { D16 } & \text { molybdenum } & & \\ \text { D17 } & \text { tin } & & \\ \text { D18 } & \text { zinc } & \\ \text { D19 } & \text { iron } & \\ \text { D20 } & \text { manganese } & \end{array}$

Heavy-Metal Analyses from Stream Sediments and Outcrop Samples

Forty-four heavy-mineral concentrates from stream-sediments collected within the Forest were examined by scanning electron microscope (SEM) and concurrently analyzed by energy dispersive spectrometry (EDS). Only minerals incorporating elements with atomic weights equal to or greater than that of iron were examined. The results are summarized in the graphs in figure D22. It can be seen that barite is a very wide-spread mineral in stream sediments followed immediately by galena, then zircon, pyrite, monazite, scheelite, and gold and silver.

Slabs from 53 outcrops from the Forest were also investigated in this manner. The results (fig. D23) show that the major "heavy metal" mineralogy is similar to that seen in the heavy-mineral concentrates derived from streamsediments. The weathering and transport processes operating in the surficial environment have had minor effect on the relative abundance of these minerals in the stream-sediments. This lends substantial credibility to the geochemical and derivative plots base on the analyses of stream-sediments collected within the area.

Heavy-mineral concentrates were also collected from the E1liston, Marysville, and the Stemple-Gould districts (plate 2, fig. F1). The results of these SEM-EDS studies are shown in figures D24, D25, and D26, respectively. It is immediately apparent that the similarity in geochemical signatures seen within the study area as a whole is reflected by the essentially identical "heavy metal" mineralogy seen between the various mining districts.

\section{Geochemical-Geophysical Association Models}

Grids of the three defined geochemical associations ( $\mathrm{La}-, \mathrm{Pb}-$, and $\mathrm{Au}-$ ) 
were mathematically combined with grids of the terrace-magnetization and terracedensity data. In general, terrace-density and terrace-magnetization data emphasize large, sharply bounded domains of density and magnetization. The domains and their edges often delineate known geologic features such as faults, contacts, or intrusive igneous bodies. In this study the terrace data are used primarily to define magnetization and density domains associated with igneous plutonic bodies. A description of the derivation of terrace maps is given in McCafferty (Chapter $\mathrm{C}$ of this report).

The resulting integrated products are referred to as the La-terrace, $\mathrm{Pb}$ terrace, and Au-terrace association models and plots are shown on maps H, I, and J (plate 4), respectively.

The La-terrace association model (map $\mathrm{H}$, plate 4 ) delineates the outer margins of the Boulder batholith (fig. Bl,plate 1 ) and numerous mapped intrusives throughout the study area. In addition, three to four northwesterly trends are delineated in the west-central part of the study area along which a number of intrusives occur. These lineaments are parallel to a line extending from Black Mountain in the northwest over Elkhorn Peak in the southeast (plate 1). These same northwesterly trends occur in a plot of the $\mathrm{Pb}$-terrace association model (map I, plate 4). Essentially every major $\mathrm{Pb}$-terrace association anomaly occurs along the lineaments which appear to transect the Boulder batholith. A plot of the Au-terrace association model (map J, plate 4) shows essentially the same northwesterly linear pattern as that of the $\mathrm{Pb}$-terrace association model. Here again all the major anomalies occur along the northwesterly trends seeming to transect the Boulder batholith.

\section{Geochemical-Geophysical Association Mode1 Summary}

Areas delineated by these 3 geochemical-terrace models (maps H, I, and J, plate 4) are deemed to be the most affected by mineralizing processes. The intense geochemical anomalies are due in part to natural processes acting within the weathering environment and in part to past and present mineral exploitation activities. All of the delineated areas, and especially those outside known mining districts, may be potential areas of future mineral exploration. Time was not available to conduct the necessary follow-up studies to determine the possible resource potential of the identified anomalous areas. The results are reported for future reference.

Modified versions of the La-terrace and $\mathrm{Pb}$-terrace association models presented in this report have been calculated and extended to include data covering the state of Montana as part of a regional mineral-environmental study (McCafferty and others, 1996).

\section{REFERENCES CITED}

Arbogast, B.F., ed., 1996, Analytical Methods Manual for the Mineral Resource program of the U.S. Geological Survey: U.S. Geological Survey Open-File Report 96-525, $248 \mathrm{p}$.

Green, G.N., and Tysdal, R.G., 1996, Digital maps and figures on CD-ROM for mineral and energy resource assessment of the Helena National Forest, west-central Montana: U.S. Geological Survey Open-File Report 96-683-B, 
1 CD-ROM disk.

Grimes, D.J., and Marranzino, A.P., 1968, Direct current are and alternating spark emission spectrographic field methods for the semi-quantitative analyses of geologic materials: U.S. Geologic Survey Circular 591, 12p. Grimes, D.J., and Leinz, R.W., 1980a, Geochemical and generalized geologic maps showing the distribution and abundance of copper of the Choteau $1^{\circ} \mathrm{x}$ $2^{\circ}$ quadrangle, Montana: U.S. Geological Survey Miscellaneous Field Studies Map MF-858-B, scale 1:250,000.

1980b, Geochemical and generalized geologic maps showing the distribution and abundance of silver in the Choteau $1^{\circ} \times 2^{\circ}$ quadrangle, Montana: U.S. Geological Survey Miscellaneous Field Studies Map MF-858-C, scale 1:250,000.

Grimes, J.G., 1984, NURE HSSR Geochemical Sample Archives Transfer Report; Geochemical Analyses; NURE Report Number: K TR-500, 82 p.

Grundy, W.D., and Miesch, A.T., 1988, Brief description of STATPAC and related statistical programs for the IBM personal computer: U.S. Geological Survey Open-File Report 87-411-A.

Leinz, R.W., and Grimes, D.J., 1980a, Geochemical and generalized geologic maps showing the distribution and abundance of lead in the Choteau $1^{\circ} \times 2^{\circ}$ quadrangle, Montana: U.S. Geological Survey Miscellaneous Field Studies Map MF-858-D, scale $1: 250,000$.

1980b, Geochemical and generalized geologic maps showing the distribution and abundance of zinc in the Choteau $1^{\circ} \times 2^{\circ}$ quadrangle, Montana: U.S. Geological Survey Miscellaneous Field Studies Map MF-858-E, scale $1: 250,000$.

1980c, Geochemical and gneralized geologic maps showing the distribution and abundance of mercury arsenic and molybdenum in the Choteau $1^{\circ} \times 2^{\circ}$ quadrangle, Montana: U.S. Geological Survey Miscellaneous Field Studies Map MF-858-F, scale 1:250,000.

McCafferty, A.E., Knepper, D.H., and Alminas, H.V., 1996, Integration of geochemical and geophysical data for the Montana mineral-environmental study: Environmental and Engineering Geophysical Society, Proceedings from the 1996 Symposium on the Application of Geophysics to Engineering and Environmental Problems, p. 877-886.

McDanal, S.K., Campbe11, W.L., Fox, J.P., and Lee, G.K., 1985, Magnetic tape containing analytical results from rocks, soils, stream sediments, and heavy-mineral concentrate samples: U.S. Geological Survey Report USGS-GD85-006. [Available only from U.S. Department of Commerce, National Technical Information Service, Springfield, Virginia 22151 as NTIS Report PB86-119484] .

Motooka, J.M., and Grimes, D.J., 1976, Analytical precision of one-sixth order semi-quantitative spectrographic analysis: U.S. Geologic Survey Circular $738,25 p$.

O'Leary, R.M., Meier, A.L., 1984, Analytical methods used in geochemical exploration: U.S. Geological Survey Circular 984, 120 p.

Van Trump, George Jr., and Miesch, A.T., 1977, The U.S. Geological Survey RASS-STATPAC system for management and data reduction of geochemical data: Computers and Geoscience, v. 3, p. 475-488.

Van Trump, George, Jr., and Alminas, H.V., 1978, REM program explanation and computer program 1isting: U.S. Geological Survey Open-File Report 78-1014, $18 \mathrm{p}$. 


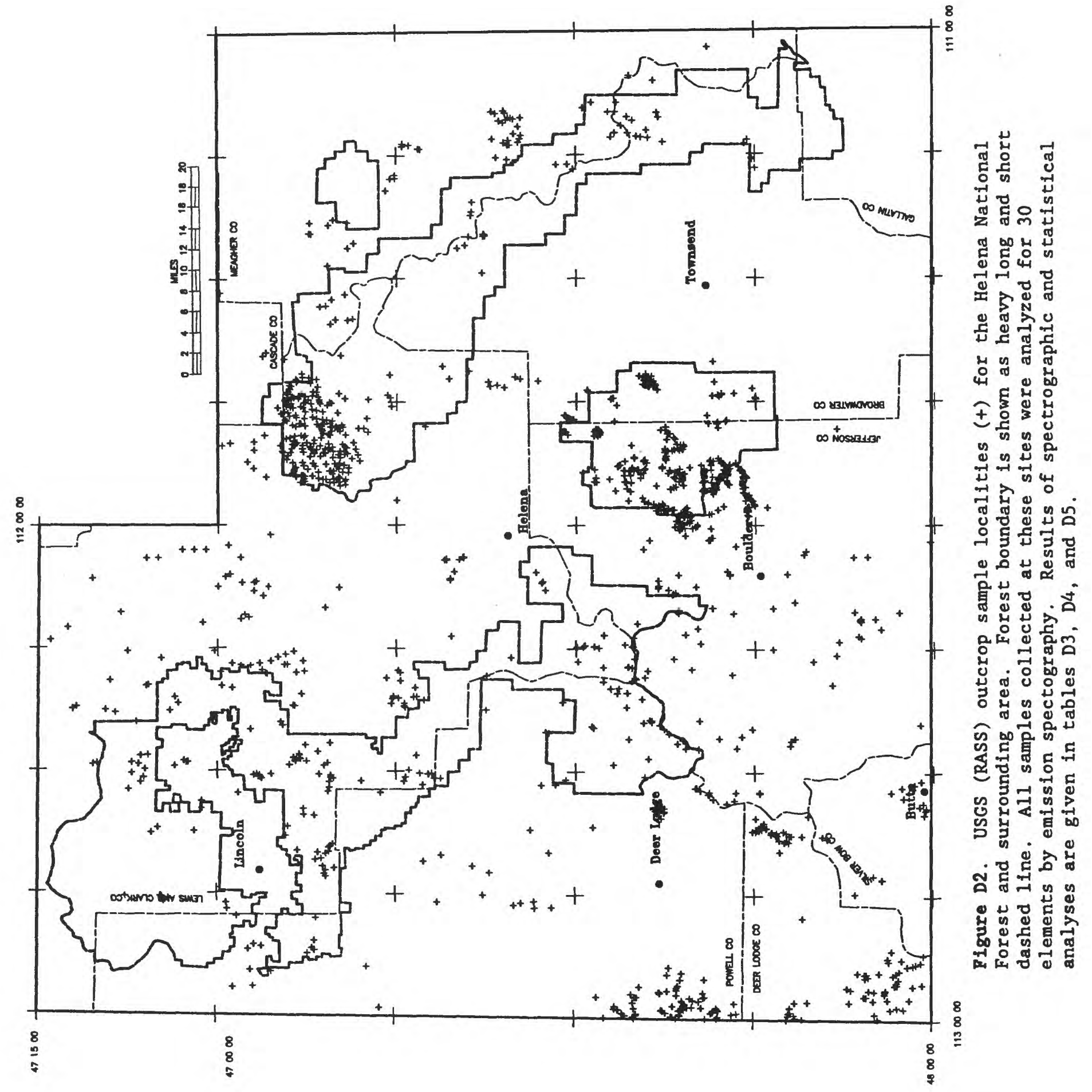




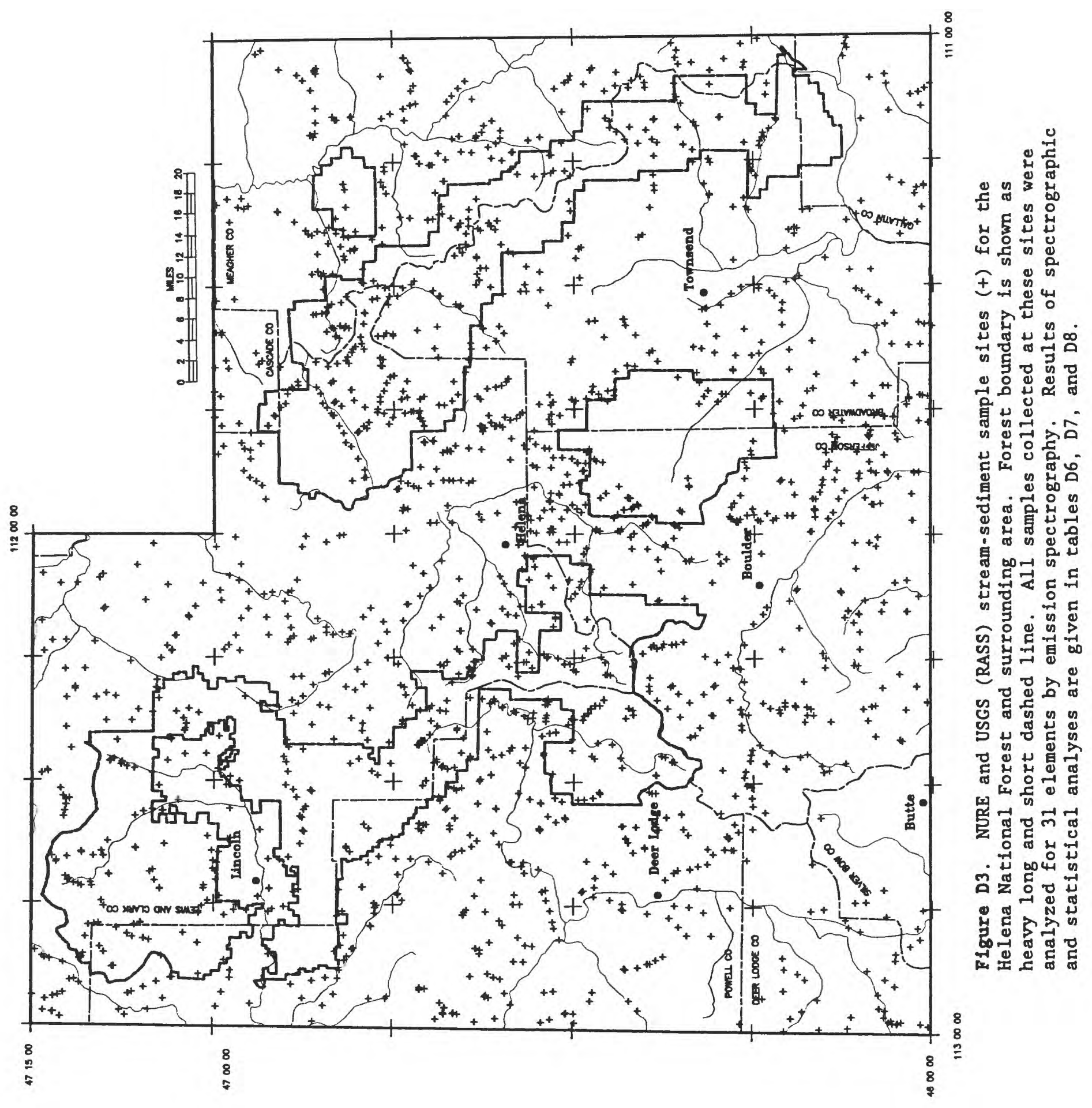




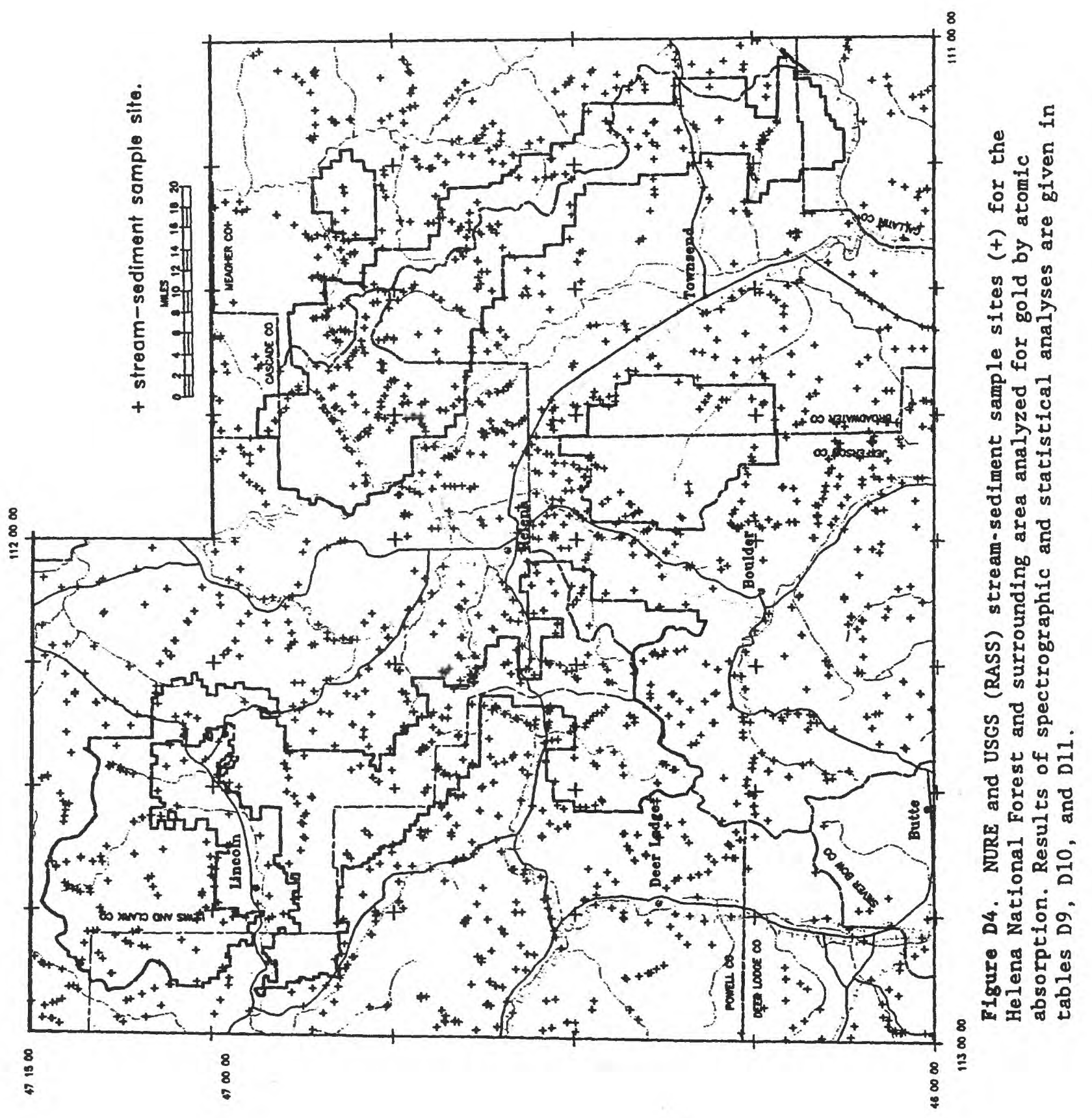




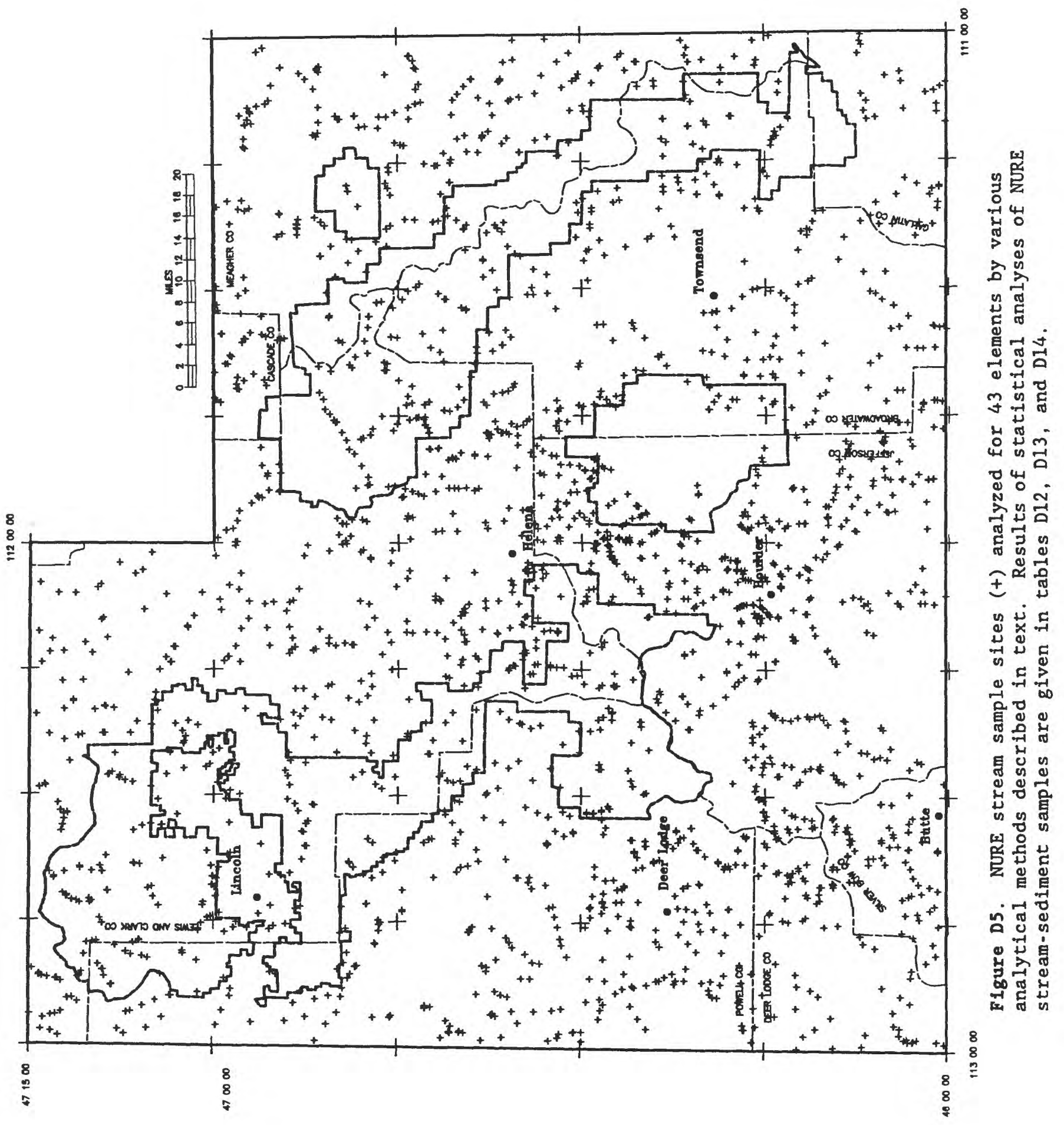



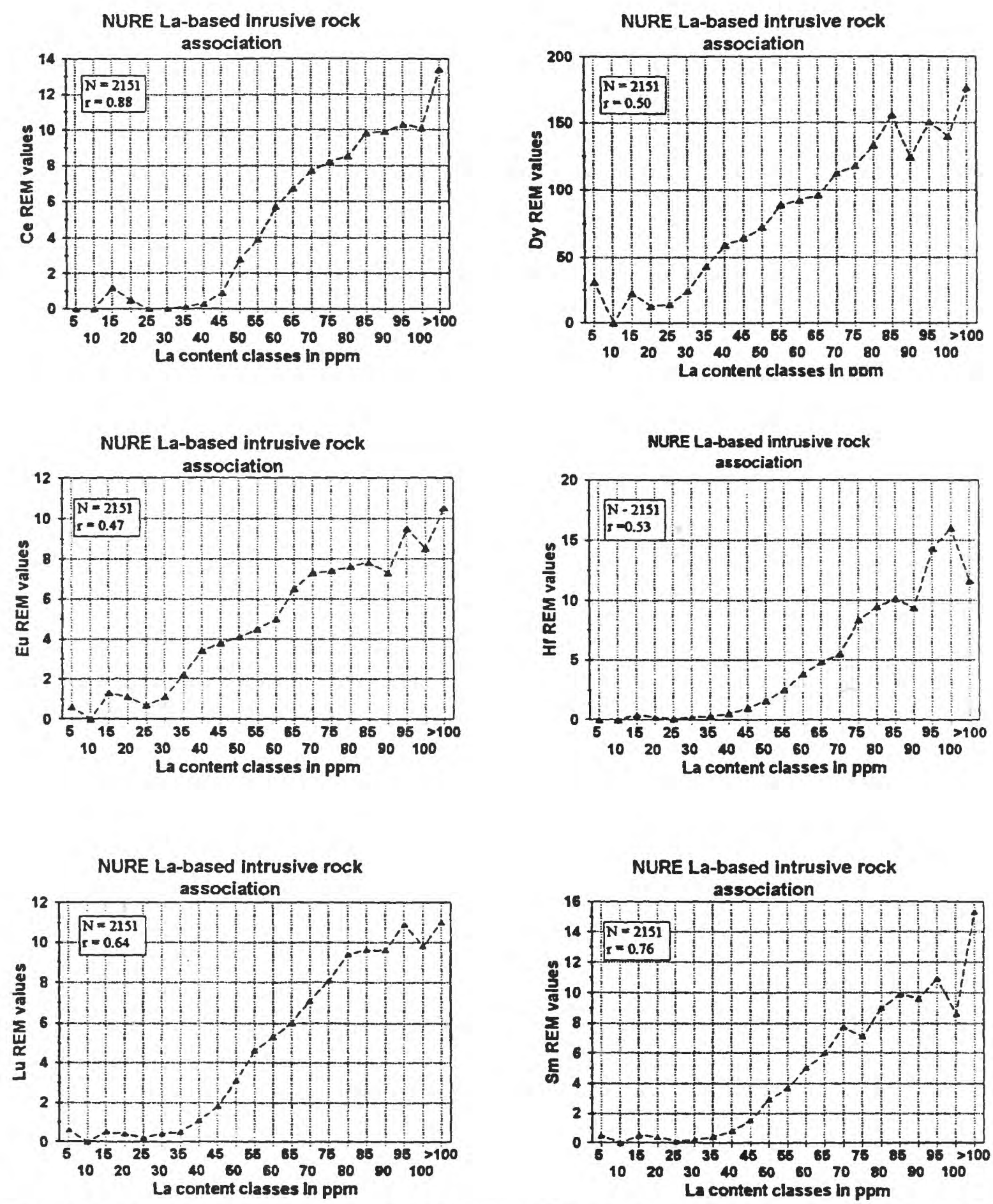

Figure D6. Graphs showing the relationship between lanthanum and suite of individual elements used in the La-association model for the study area. Values for the individual elements (on the vertical axis) are presented as "element magnitudes" and were calculated using the REM (relative element magnitude) method of Van Trump and Alminas (1978) using NURE stream sediments. The element magnitude values indicate the relative amount of each element within the overall elemental assemblage that define the La-association model. This particular suite of elements tends to map locations of plutons with intermediate compositions. Many of the plutons are host to major mineral deposits in the study area. 


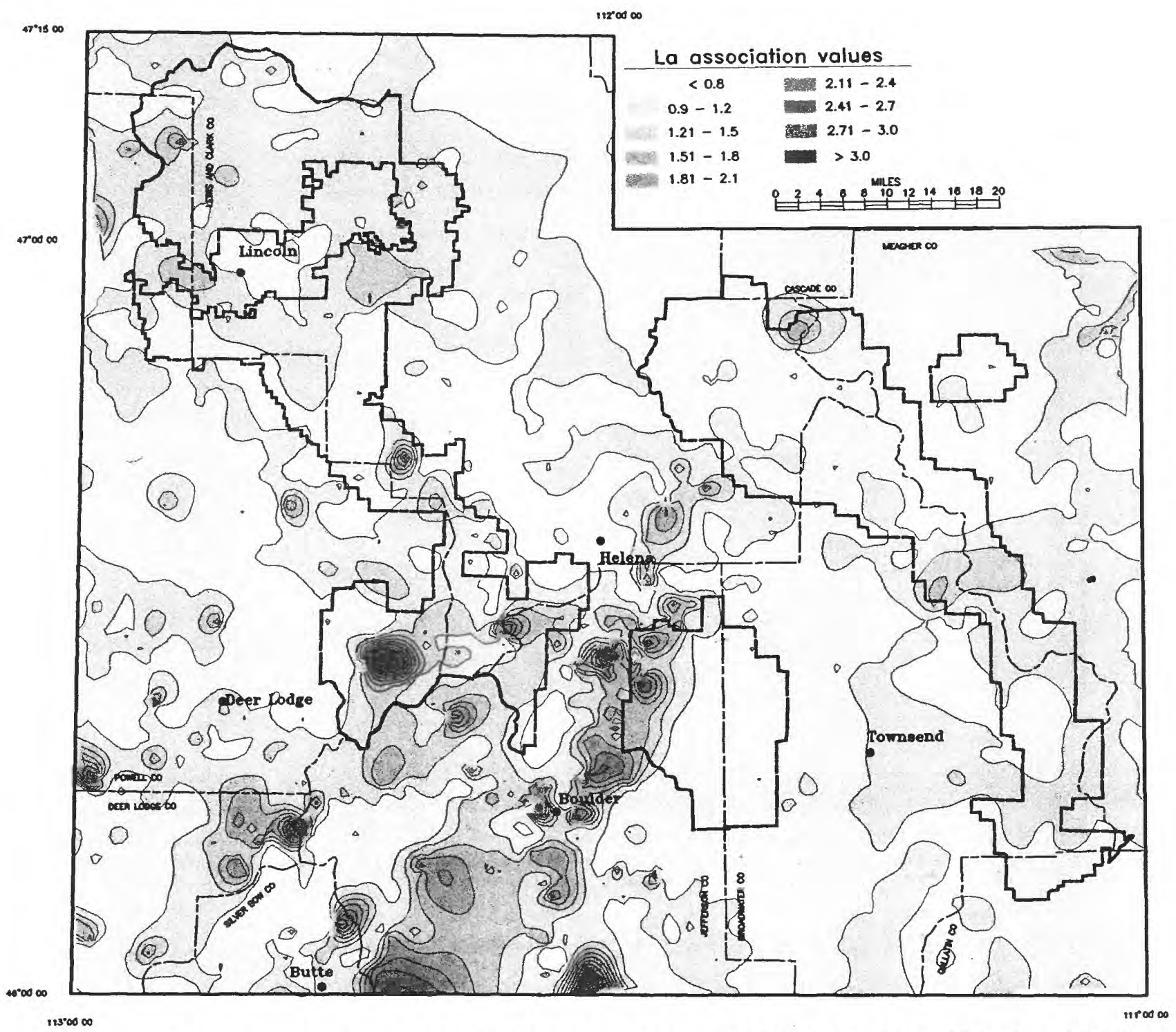

F1gure D7. Map showing distribution of La-association anomalies for the study area. Values are in "relative element magnitudes" (unitless) and represent high concentrations of an assemblage of rare earth elements (Ce, Dy, Eu, Hf, $\mathrm{La}, \mathrm{Lu}, \mathrm{Sm}, \mathrm{U}$, and $\mathrm{Yb}$ ) in stream sediments. 

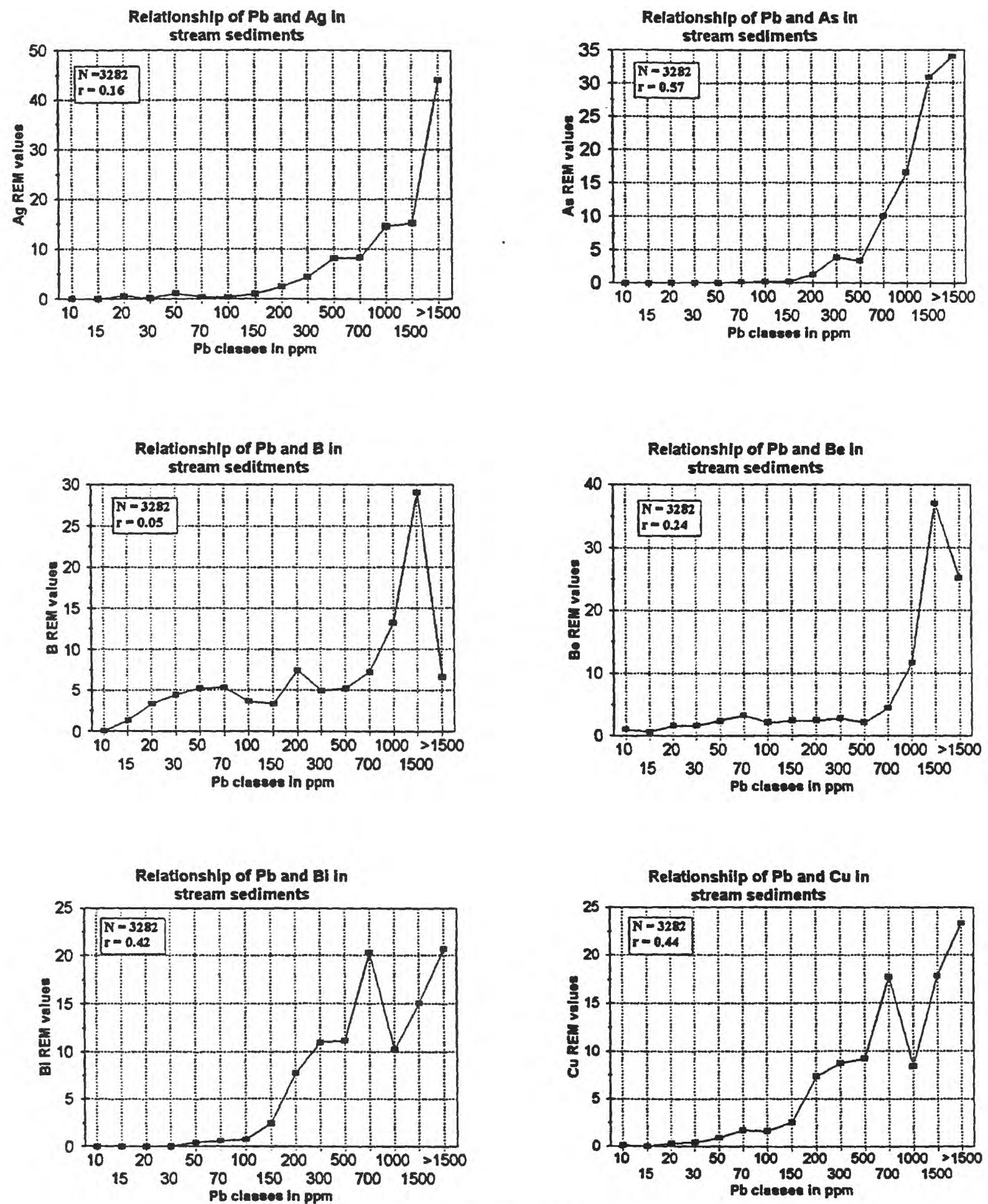

Figure D8. Graphs showing the relationship between lead and suite of individual elements used in the $\mathrm{Pb}$-association model for the study area. Values for the Individual elements (on the vertical axis) are presented as "element magnitudes" and were calculated using the REM (relative element magnitude) method of Van Trump and Alminas (1978) using USGS and NURE stream sediments. The element magnitude values indicate the relative amount of each element within the overall elemental assemblage that define the $\mathrm{Pb}$-association model. This particular elemental assemblage characterizes many of the known deposit types in the study area, the majority of which relate to sulfide mineralization. 

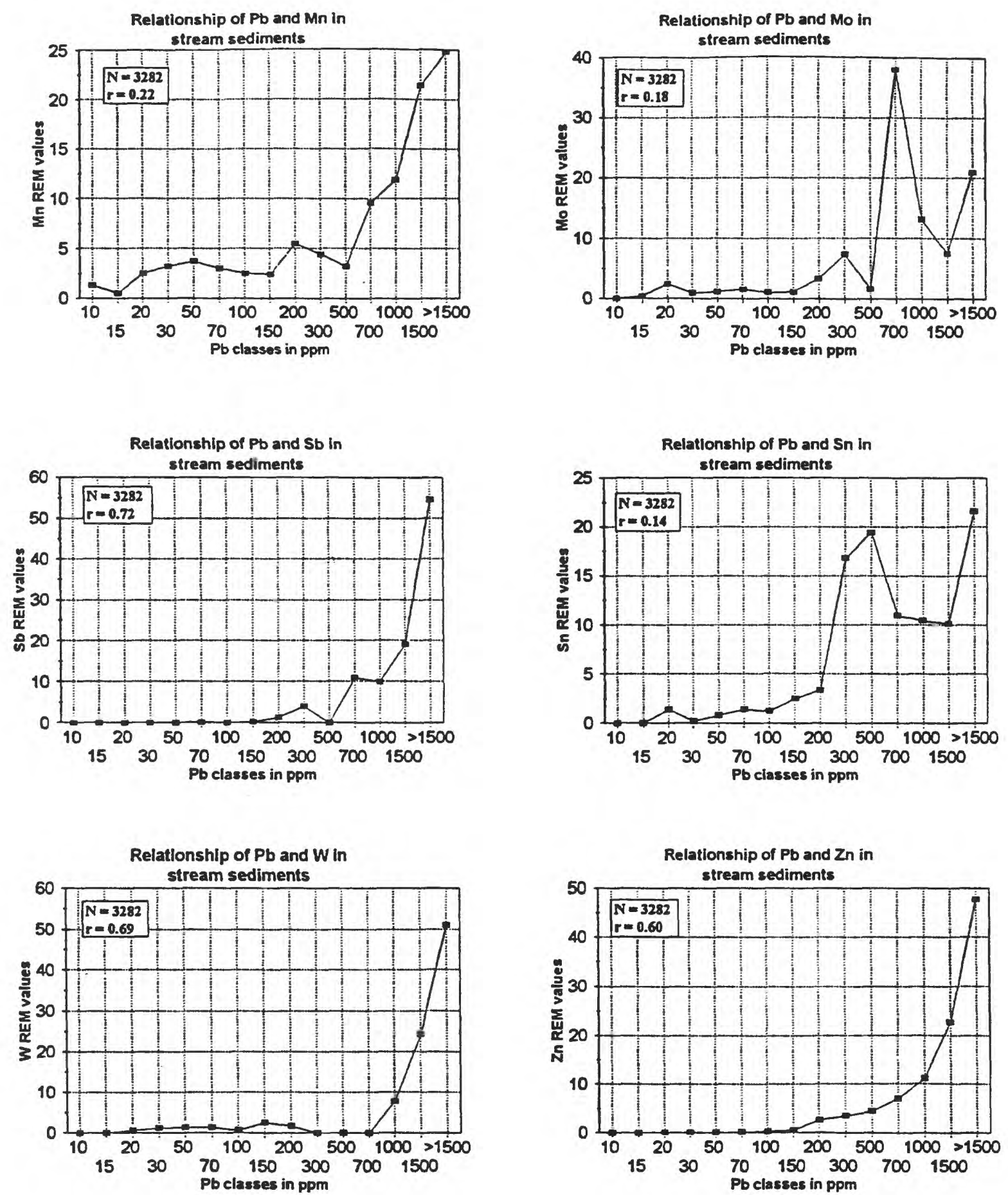

Figure D8 (continued). Graphs showing the relationship between lead and suite of individual elements used in the $\mathrm{Pb}$-association model for the study area. Values for the individual elements (on the vertical axis) are presented as "element magnitudes" and were calculated using the REM (relative element magnitude) method of Van Trump and Alminas (1978) using USGS and NURE stream sediments. The element magnitude values indicate the relative amount of each element within the overall elemental assemblage that define the $\mathrm{Pb}$-association mode1. This particular elemental assemblage characterizes many of the known deposit types in the study area, the majority of which relate to sulfide mineralization. 


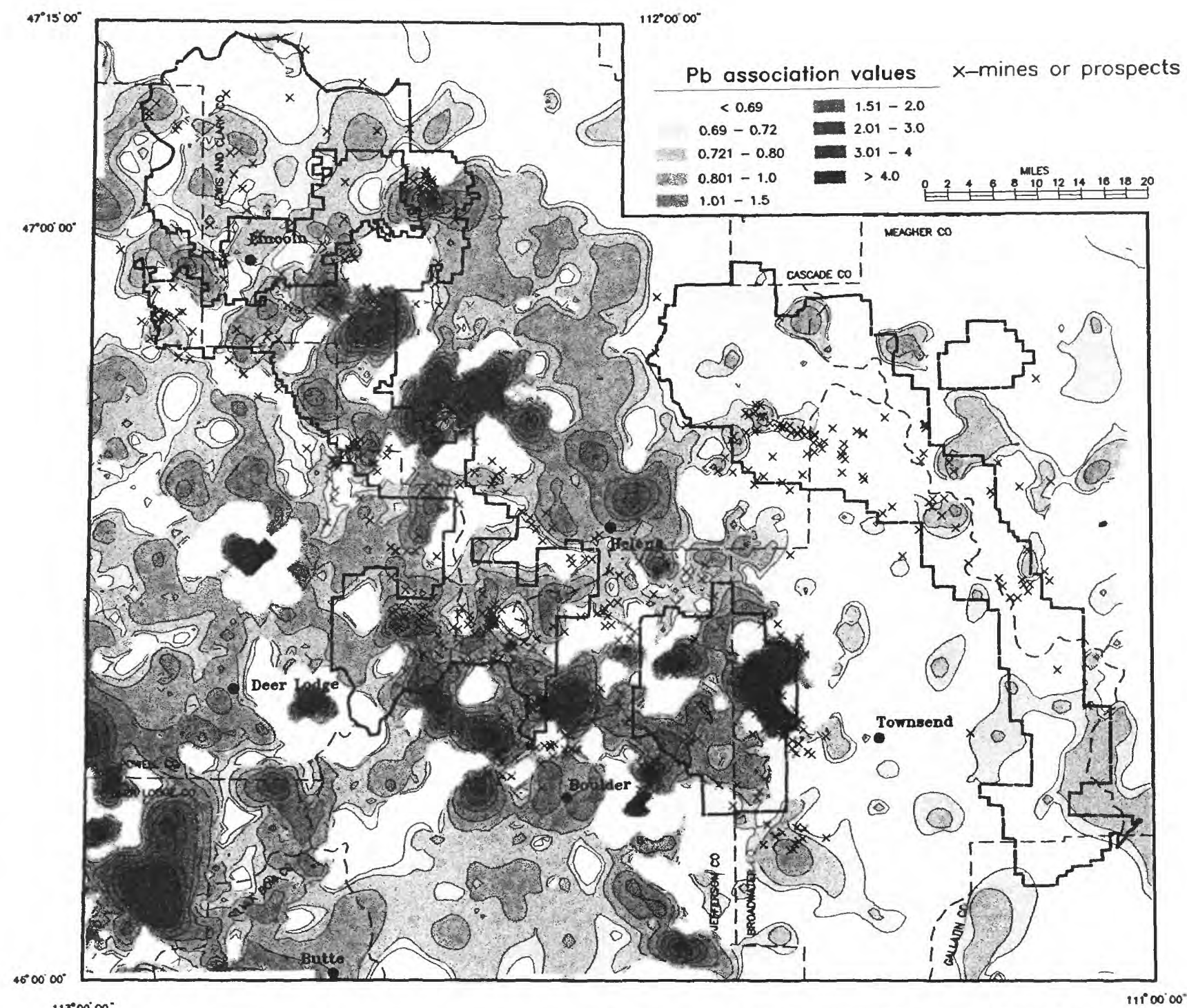

$113^{\circ} 00^{\circ} 00^{\circ}$

Figure D9. Map showing distribution of $\mathrm{Pb}$-association anomalies with values indicating relatively high concentrations of a metal suite (Ag, As, B, Be, Bi, $\mathrm{Cu}, \mathrm{Mn}, \mathrm{Mo}, \mathrm{Pb}, \mathrm{Sb}, \mathrm{Sn},-\mathrm{W}$, and $\mathrm{Zn}$ ) within stream sediment samples. Values are in "relative element magnitudes" (unitless). Mines and prospects (x) are plotted within forest boundary. 

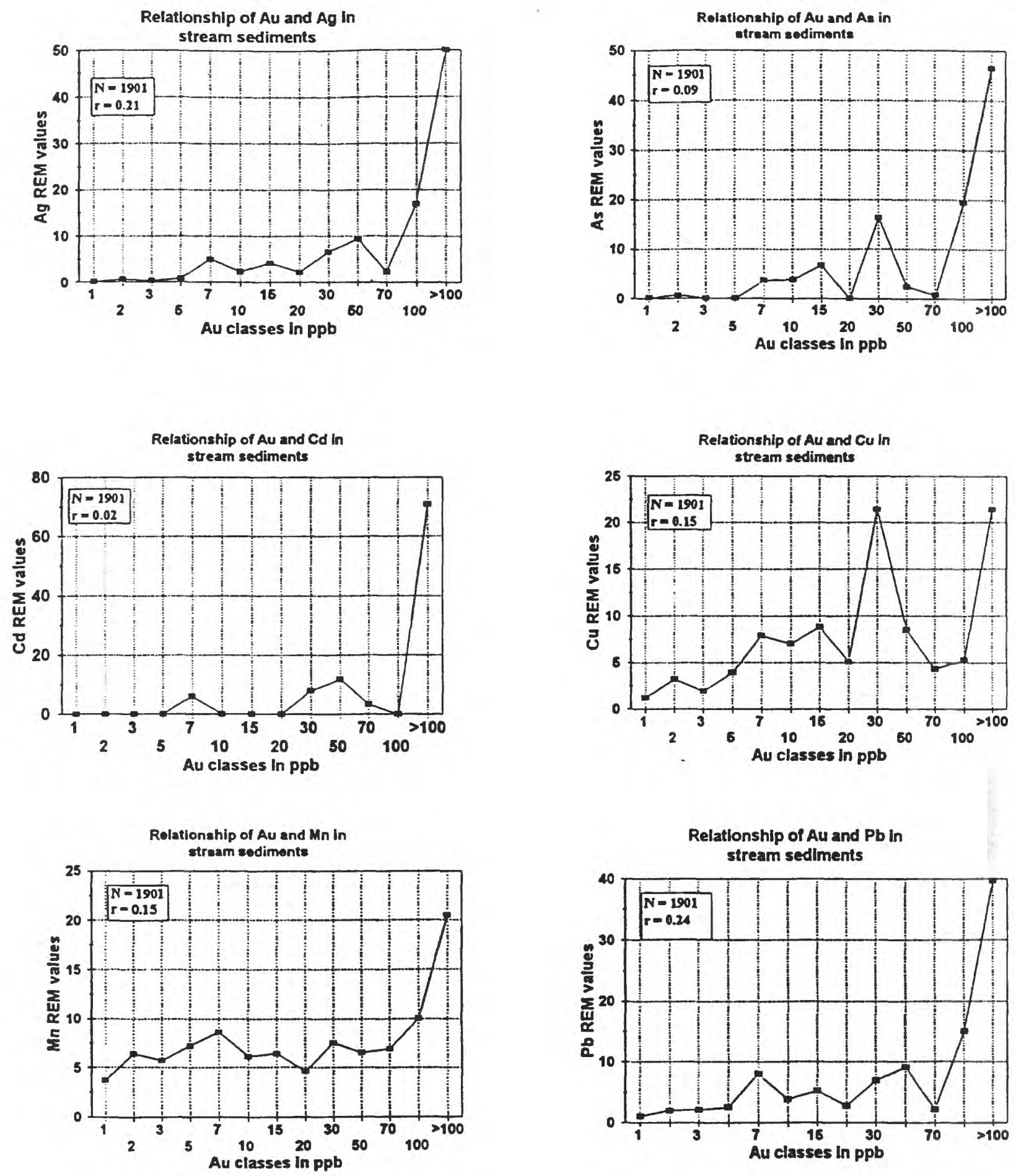

Figure D10. Graphs showing the relationship between gold and sulte of individual elements used in the Au-association model for the study area. Values for the individual elements (on the vertical axis) are presented as "element magnitudes" and were calculated using the REM (relative element magnitude) method of Van Trump and Alminas (1978) using USGS and NURE stream sediments. The element magnitude values indicate the relative amount of each element within the overall elemental assemblage that define the Au-association model. This particular elemental assemblage characterizes many of the known deposit types hosting gold within the study area. 

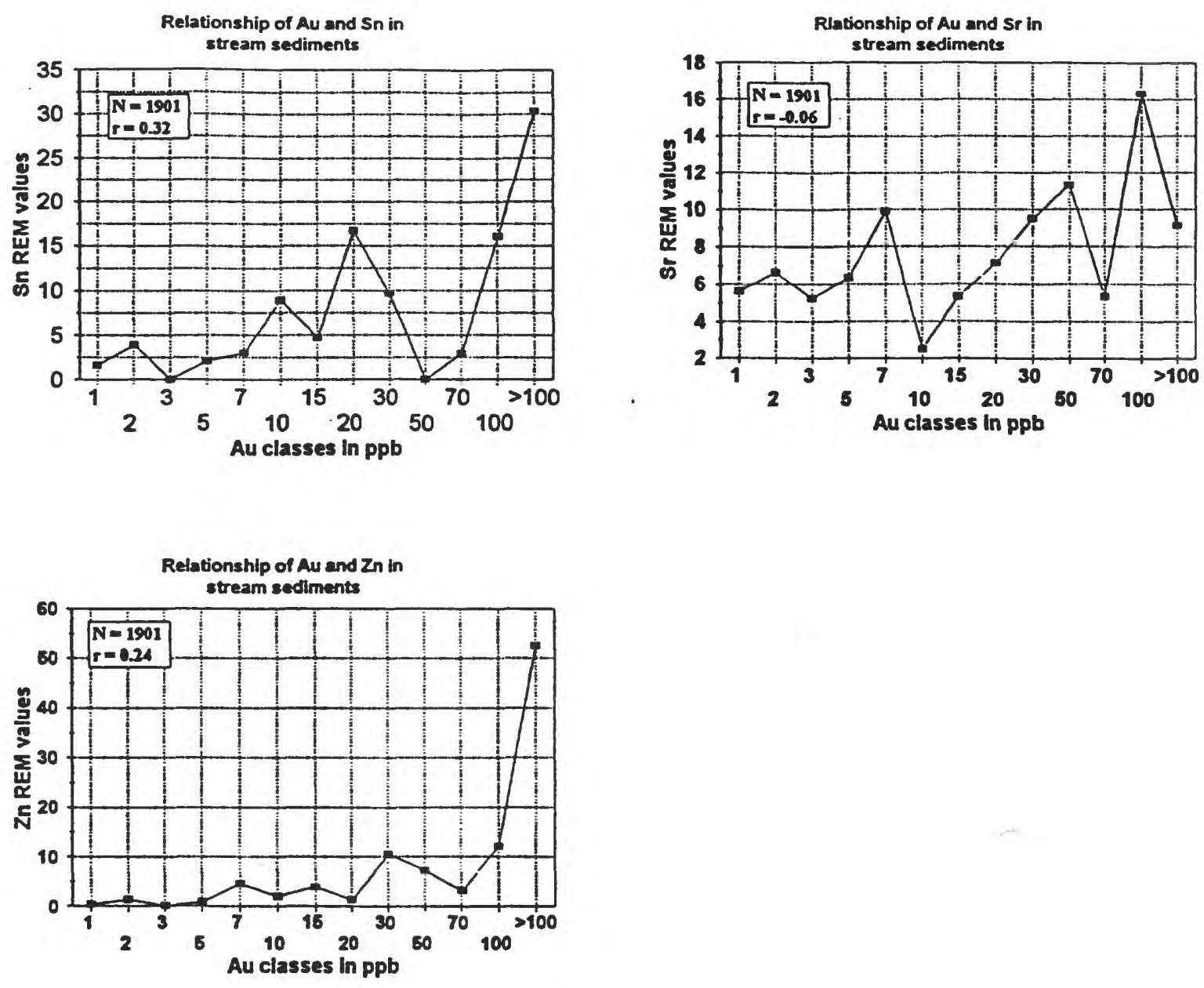

Figure D10 (continued). Graphs showing the relationship between gold and suite of individual elements used in the Au-association model for the study area. Values for the individual elements (on the vertical axis) are presented as "element magnitudes" and were calculated using the REM (relative element magnitude) method of Van Trump and Alminas (1978) using USGS and NURE stream sediments. The element magnitude values indicate the relative amount of each element within the overall elemental assemblage that define the Au-association mode1. This particular elemental assemblage characterizes many of the known deposit types hosting gold within the study area. 


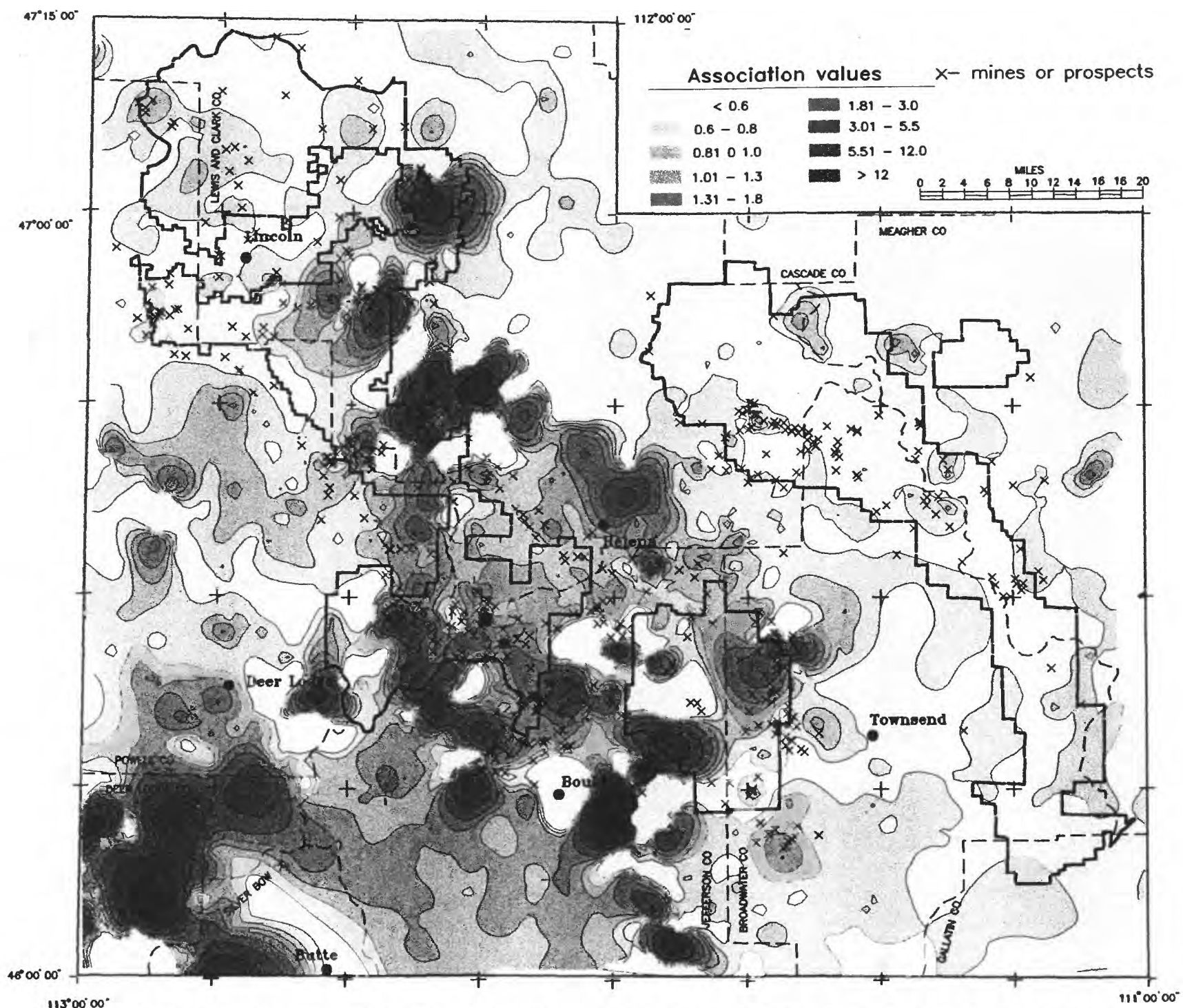

$113^{\circ} 00^{\circ} 00^{\circ}$

Figure D11. Map showing distribution of Au-association anomalies with values indicating relatively high concentrations of elements ( $\mathrm{Ag}, \mathrm{As}, \mathrm{Cd}, \mathrm{Cu}, \mathrm{Mn}, \mathrm{Pb}$, $\mathrm{Sn}, \mathrm{Sr}$, and $\mathrm{Zn}$ ) within stream sediment samples. Values are in "relative element magnitudes" (unitless) and are from an assemblage of elements expected to occur with gold mineralization. Mines and prospect pits (x) within forest boundary are plotted. 


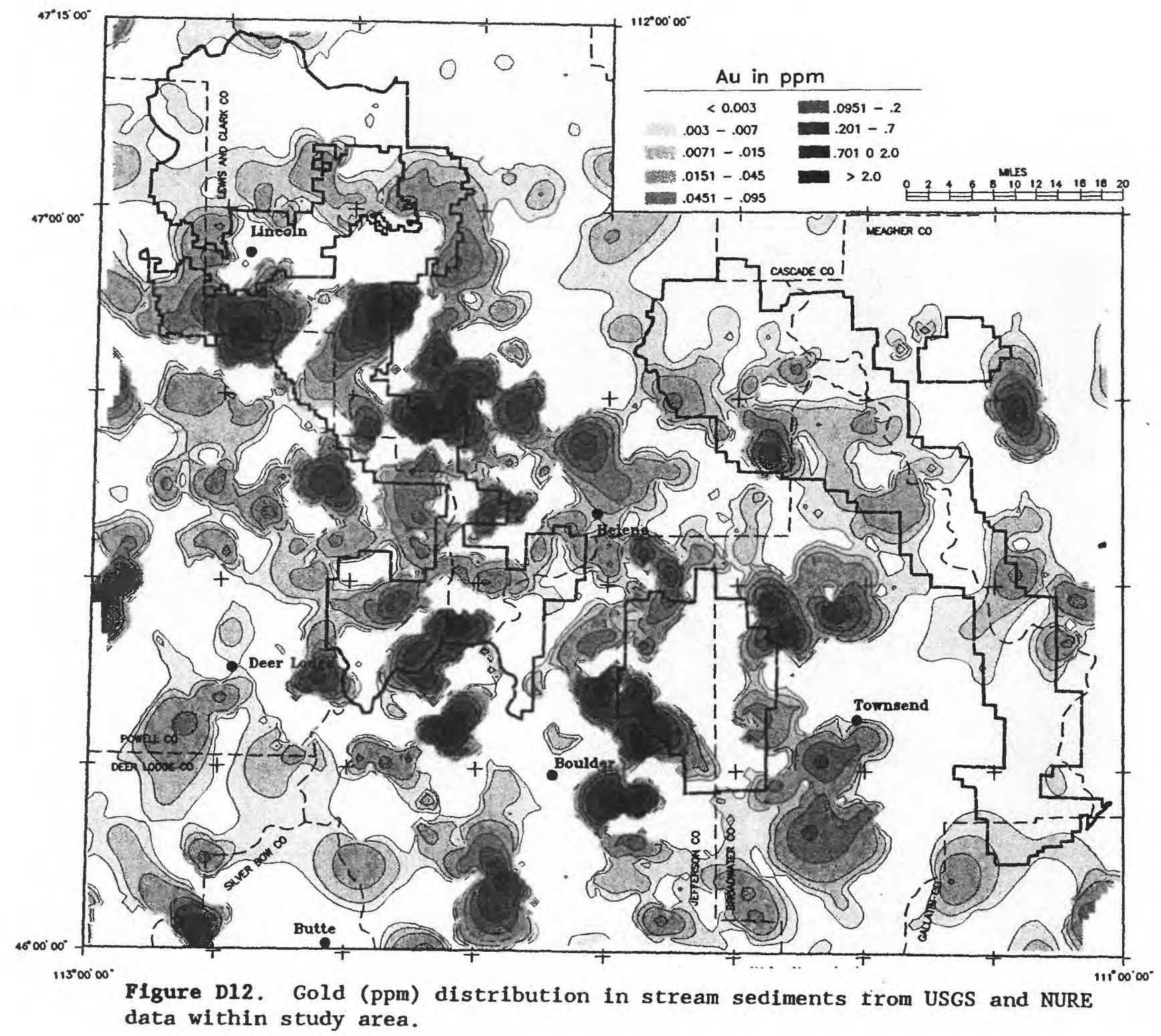




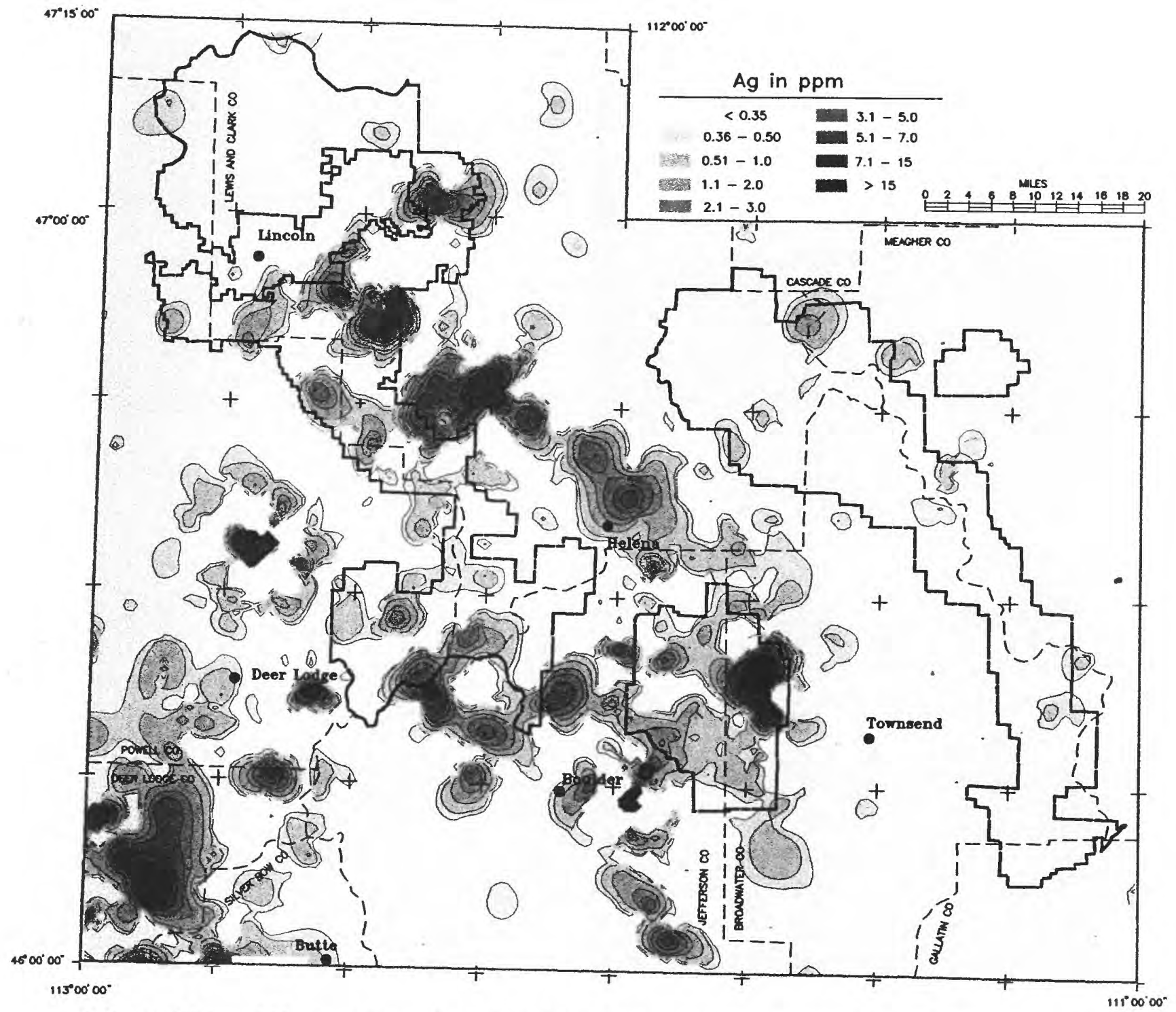

Plgure D13. Silver (ppm) distribution in stream sediments from USGS and NURE
data within study area. 


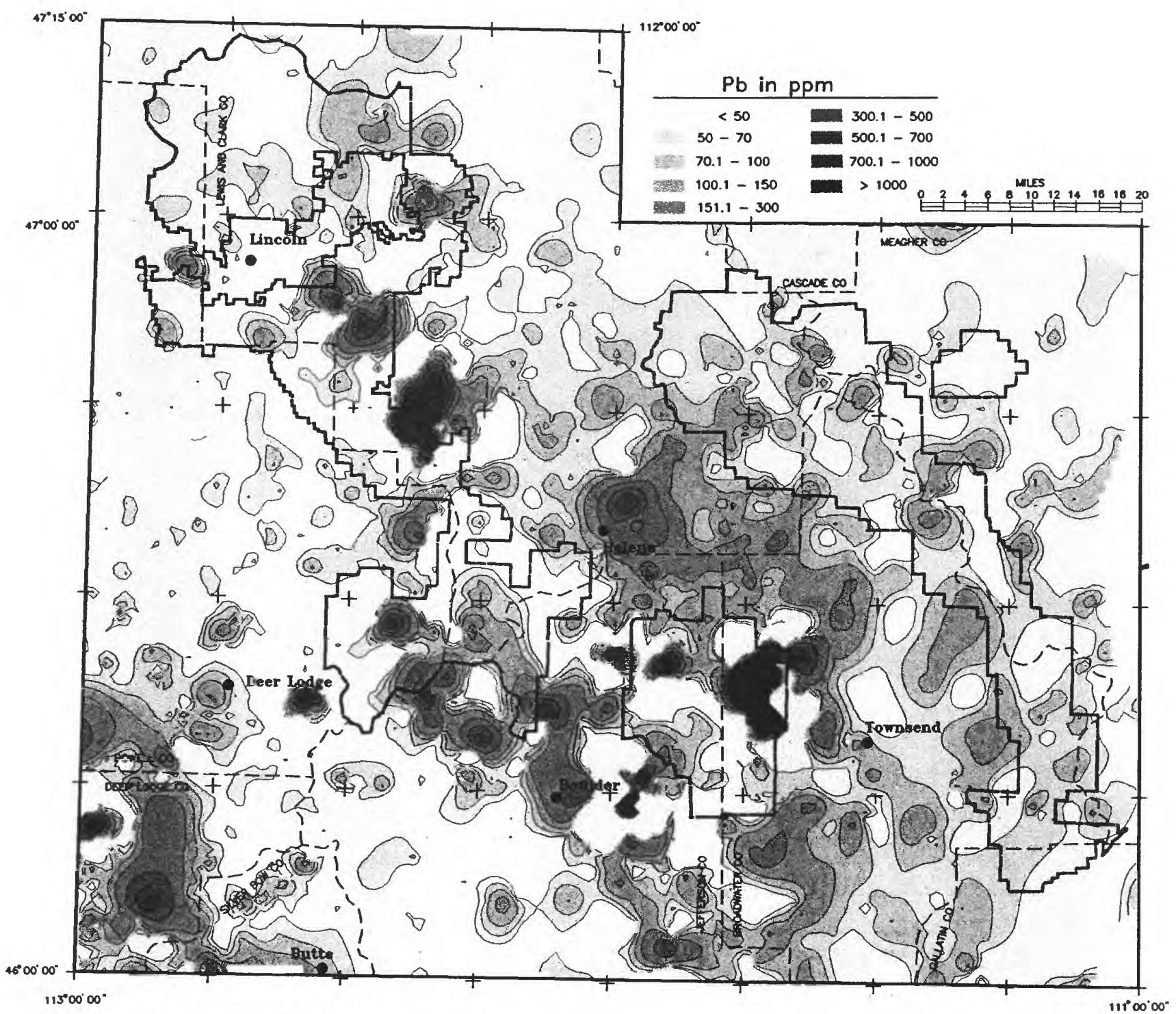

Figure D14. Lead (ppm) distribution in stream sediments from USGS and NURE data within study area. 


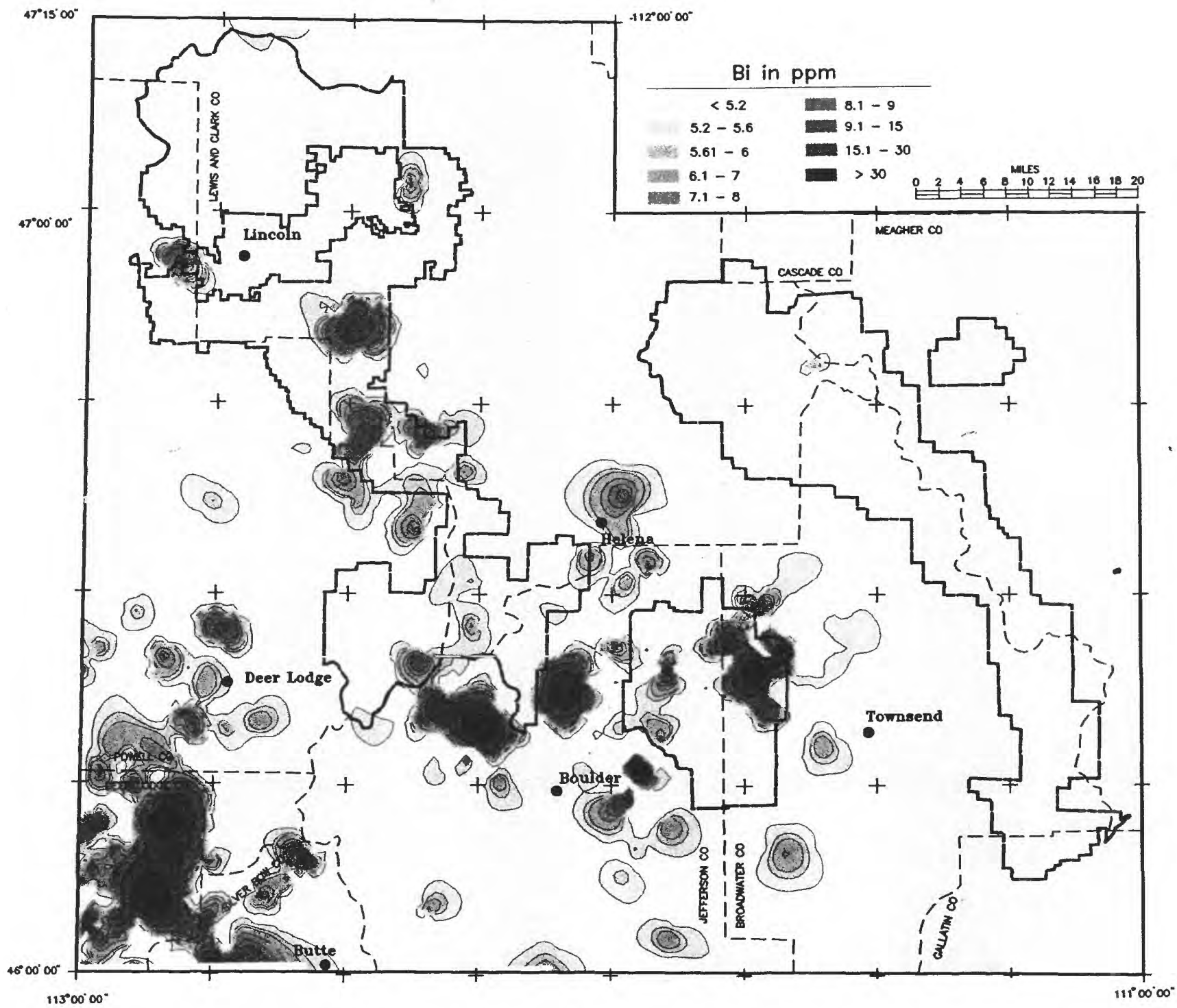

Figure D15. Bismuth (ppm) distribution in stream sediments from USGS and NURE data within study area. 


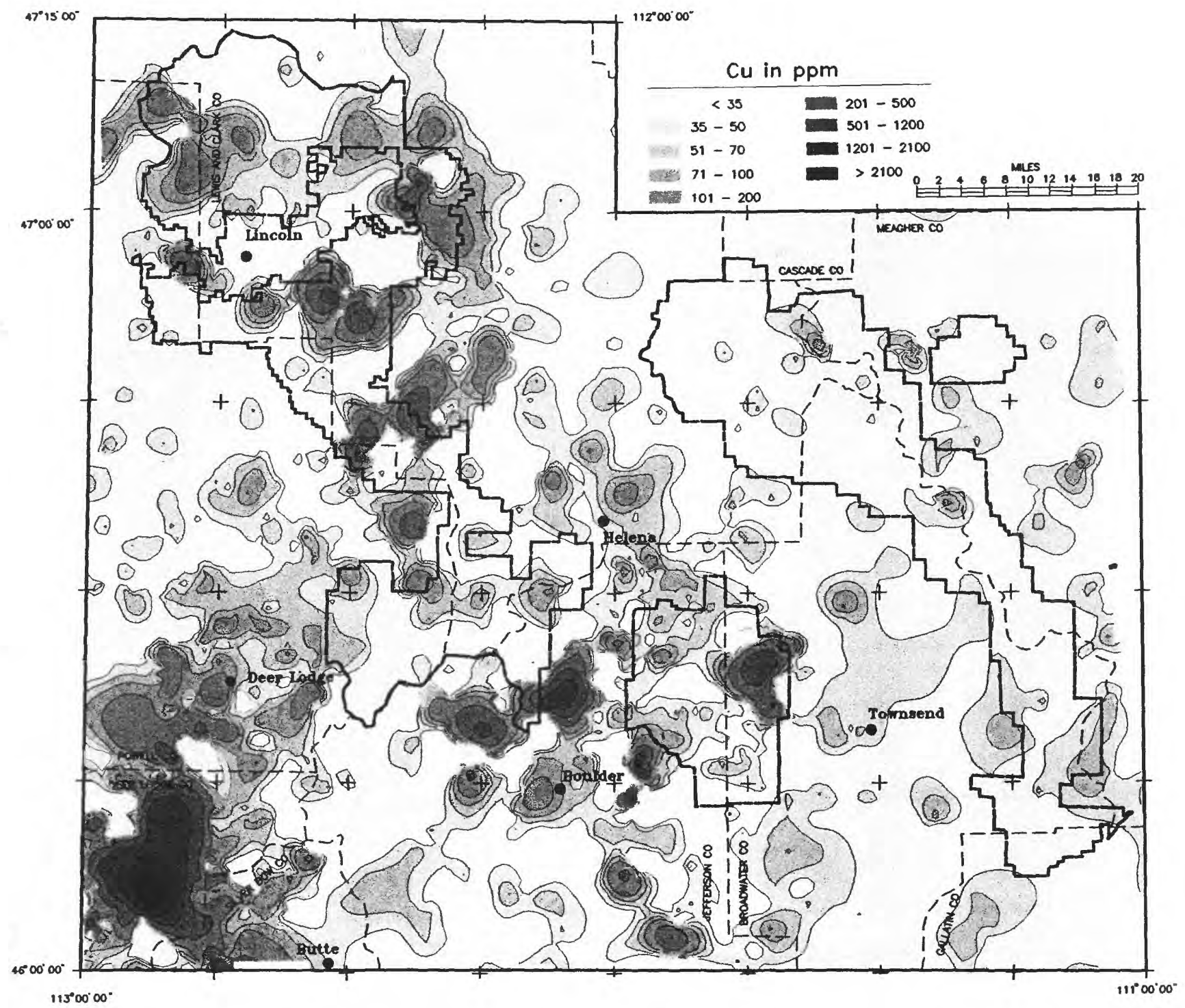

Figure D16. Copper (ppm) distribution in stream sediments from USGS and NURE data within study area. 


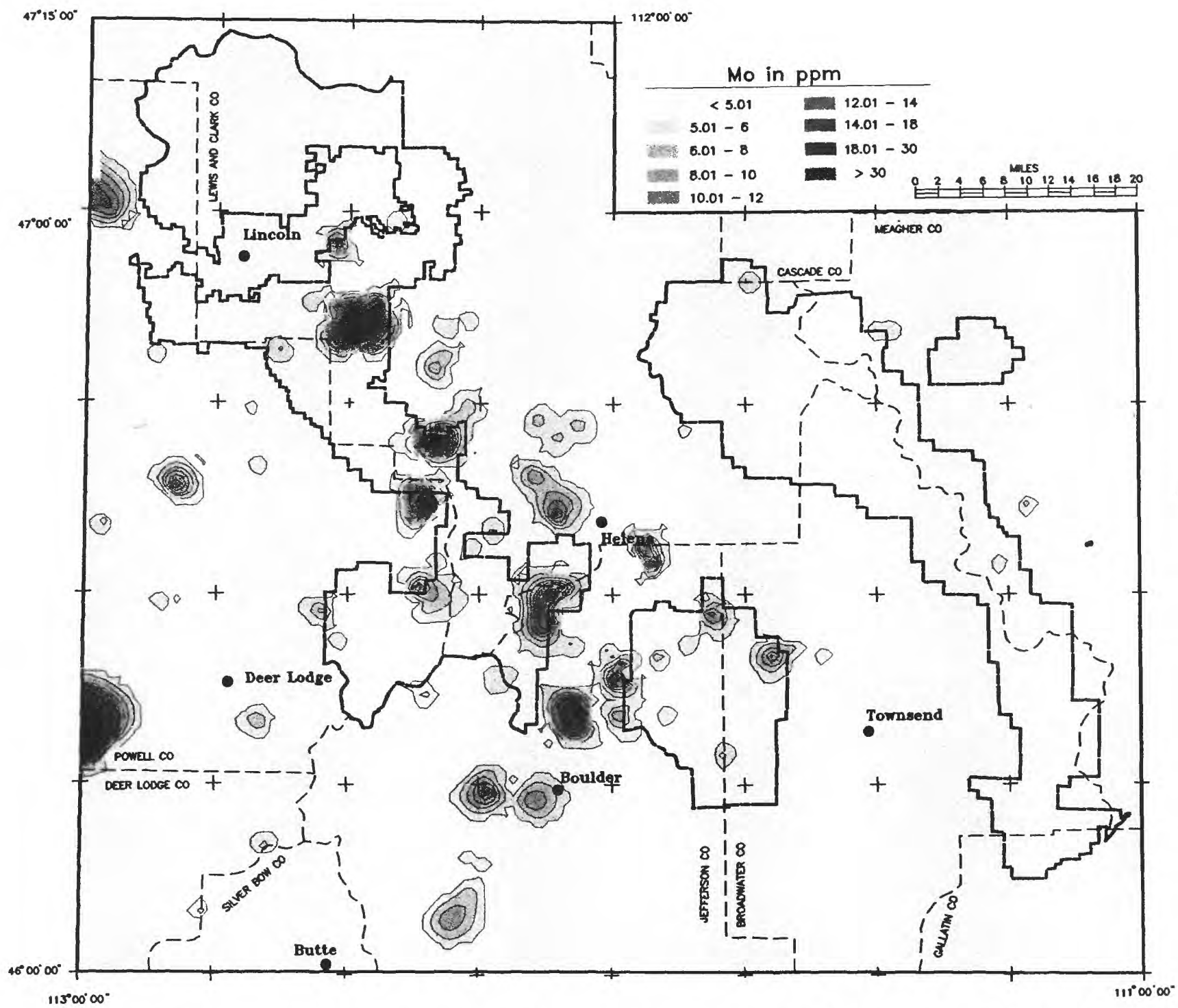

Figure D17. Molybdenum (ppm) distribution in stream sediments from USGS and NURE data within study area. 


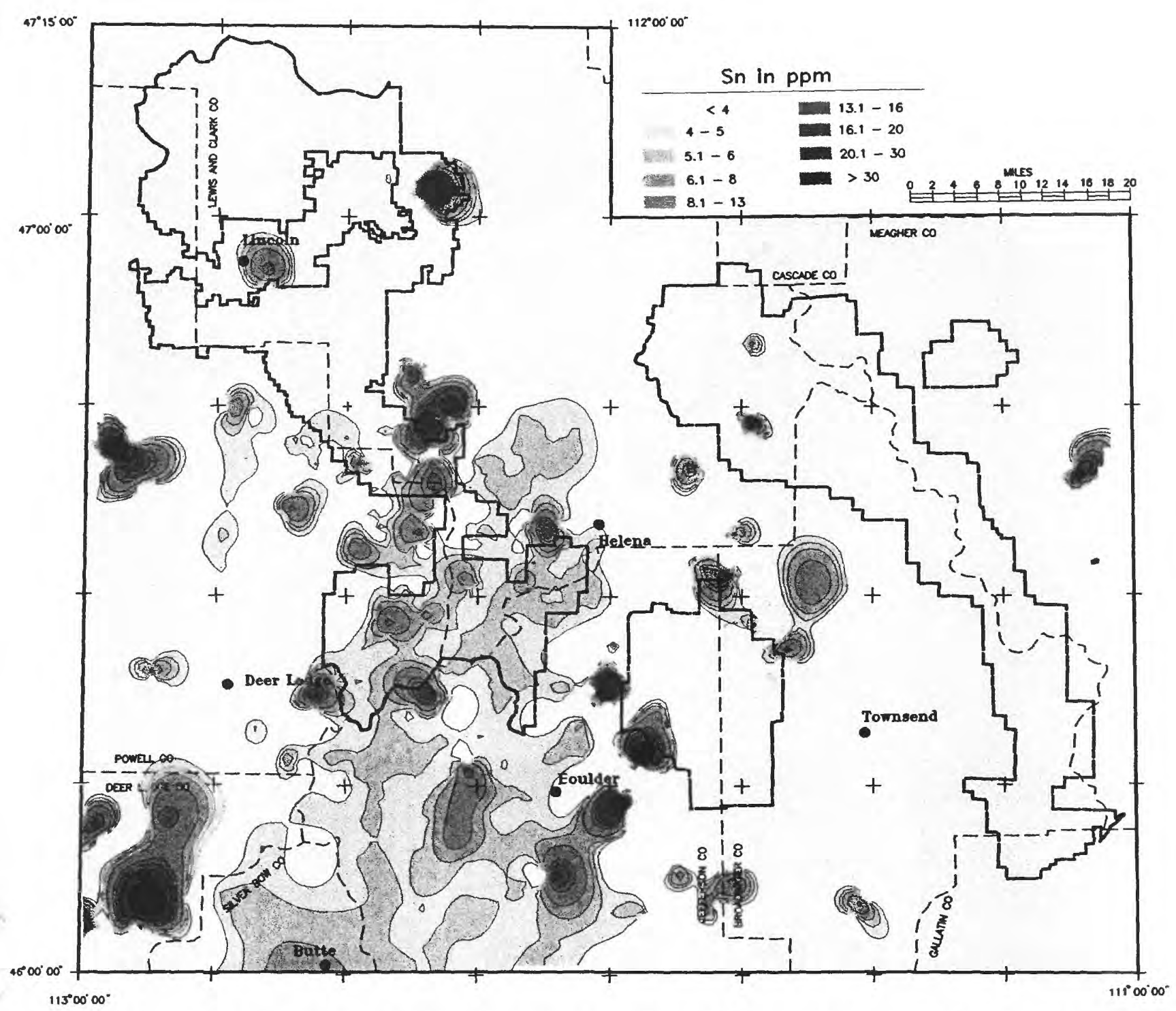

FIgure D18. Tin (ppm) distribution in stream sediments from USGS and NURE data within study area. 


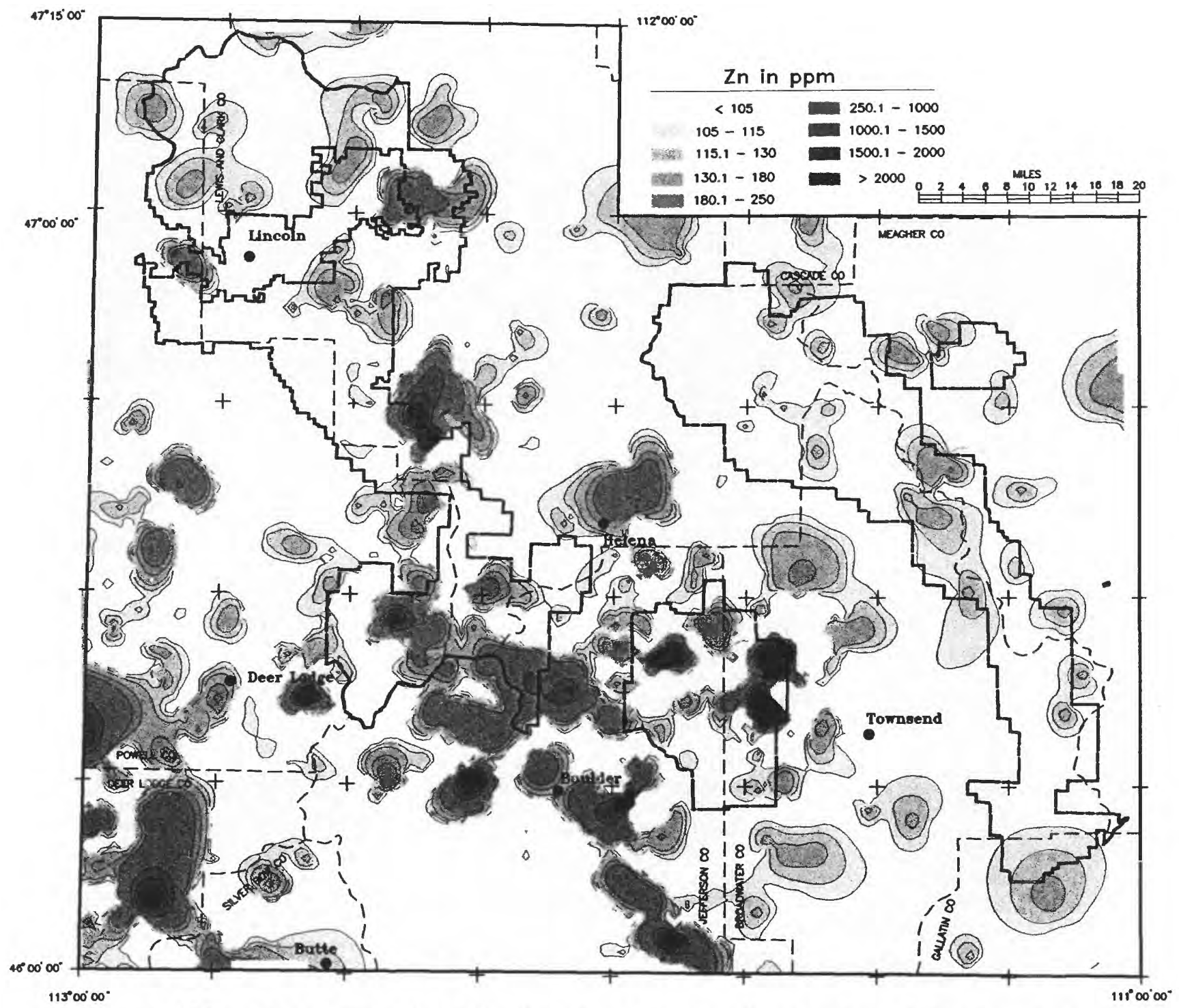

Figure D19. Zinc (ppm) distribution in stream sediments from USGS and NURE data within study area. 


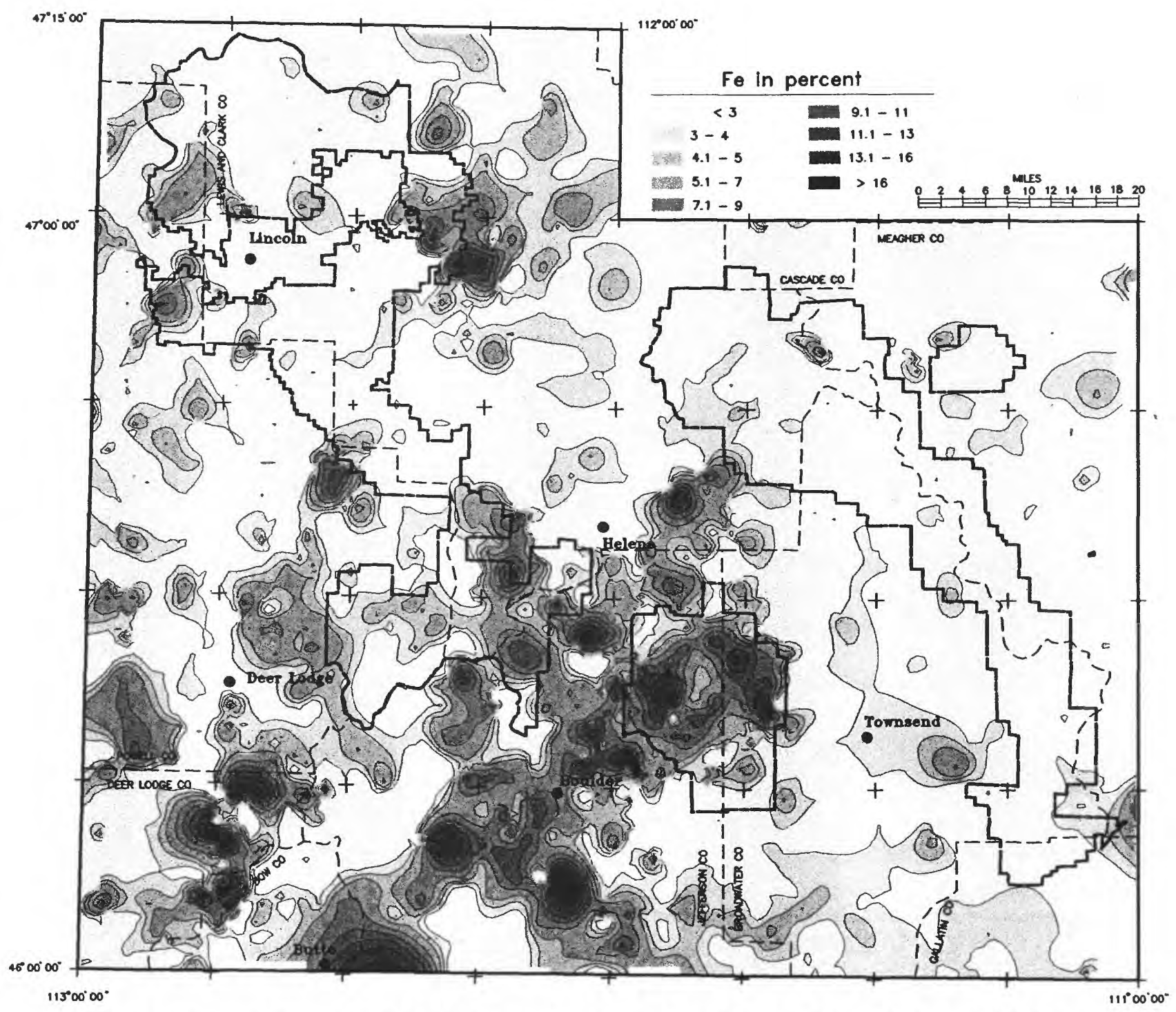

Figure D20. Iron ( 8 ) distribution in stream sediments from USGS and NURE data within study area. 


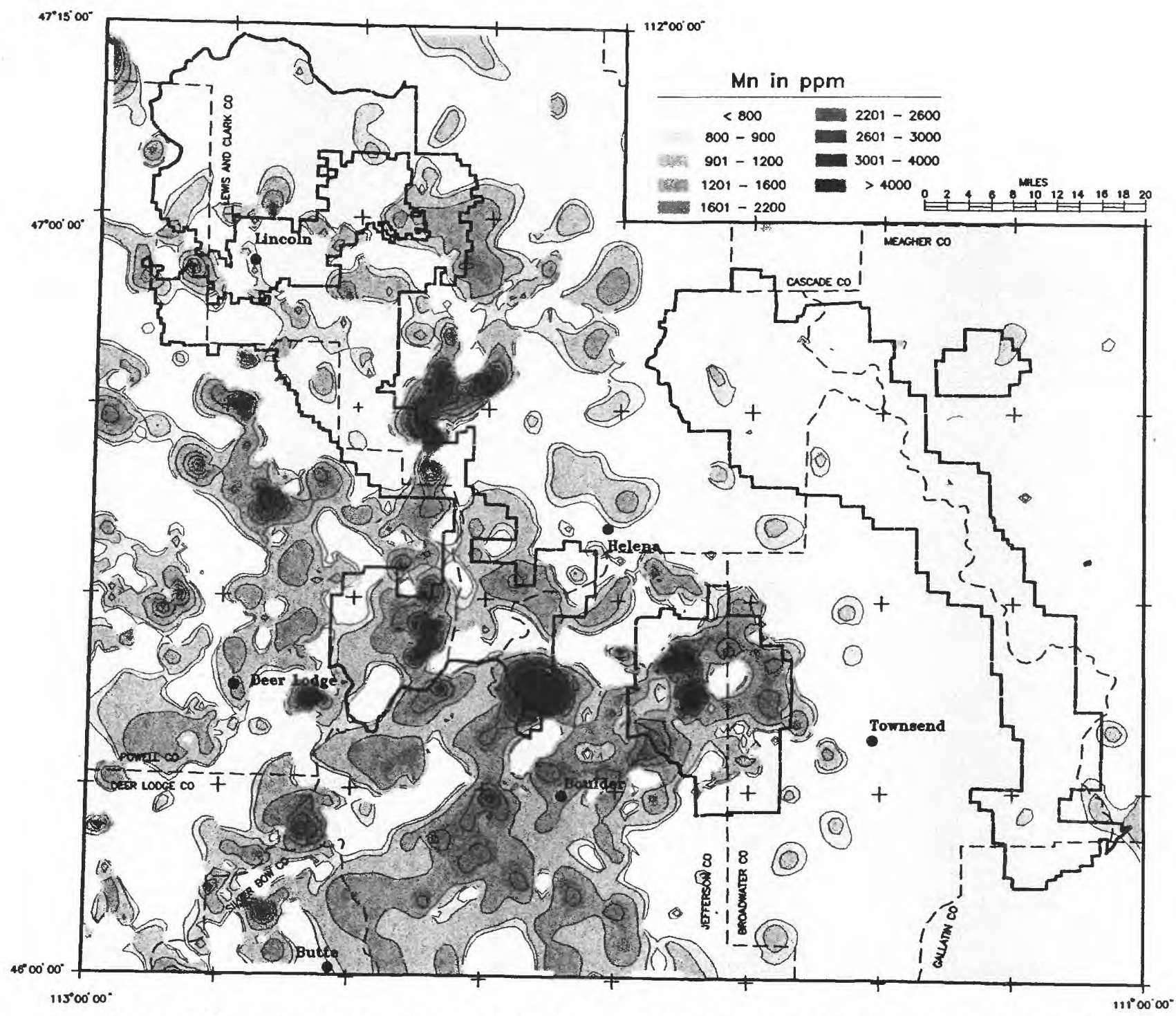

F1gure D21. Manganese (ppm) distribution in stream sediments from USGS and NURE data within study area. 
Stream sediment concentrates

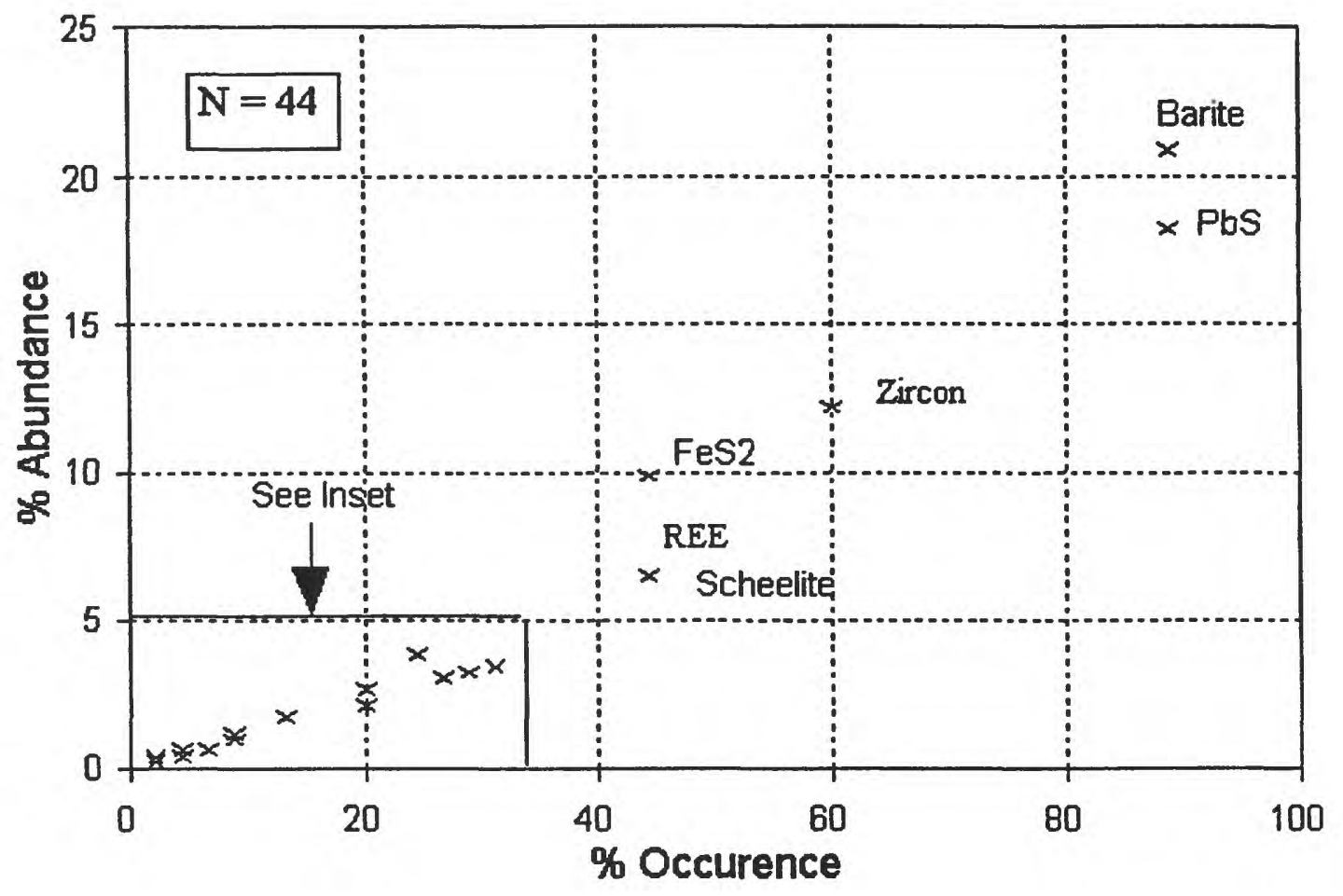

Helena SEM mineral scan (inset) Stream sediment concentrates

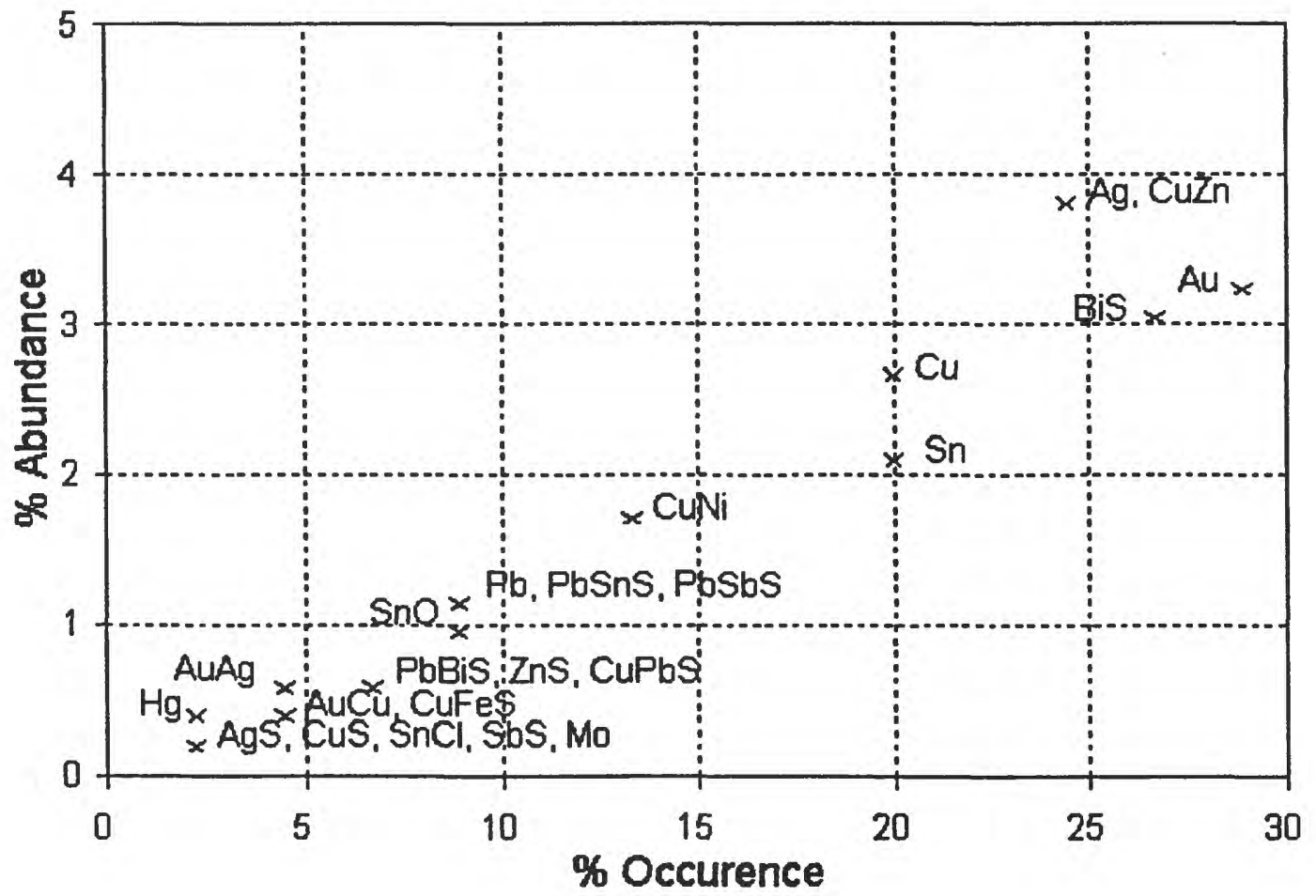

Figure D22. Graphs showing the SEM-EDS determined major composition of heavy mineral concentrates from the study area. 
Outcrop samples

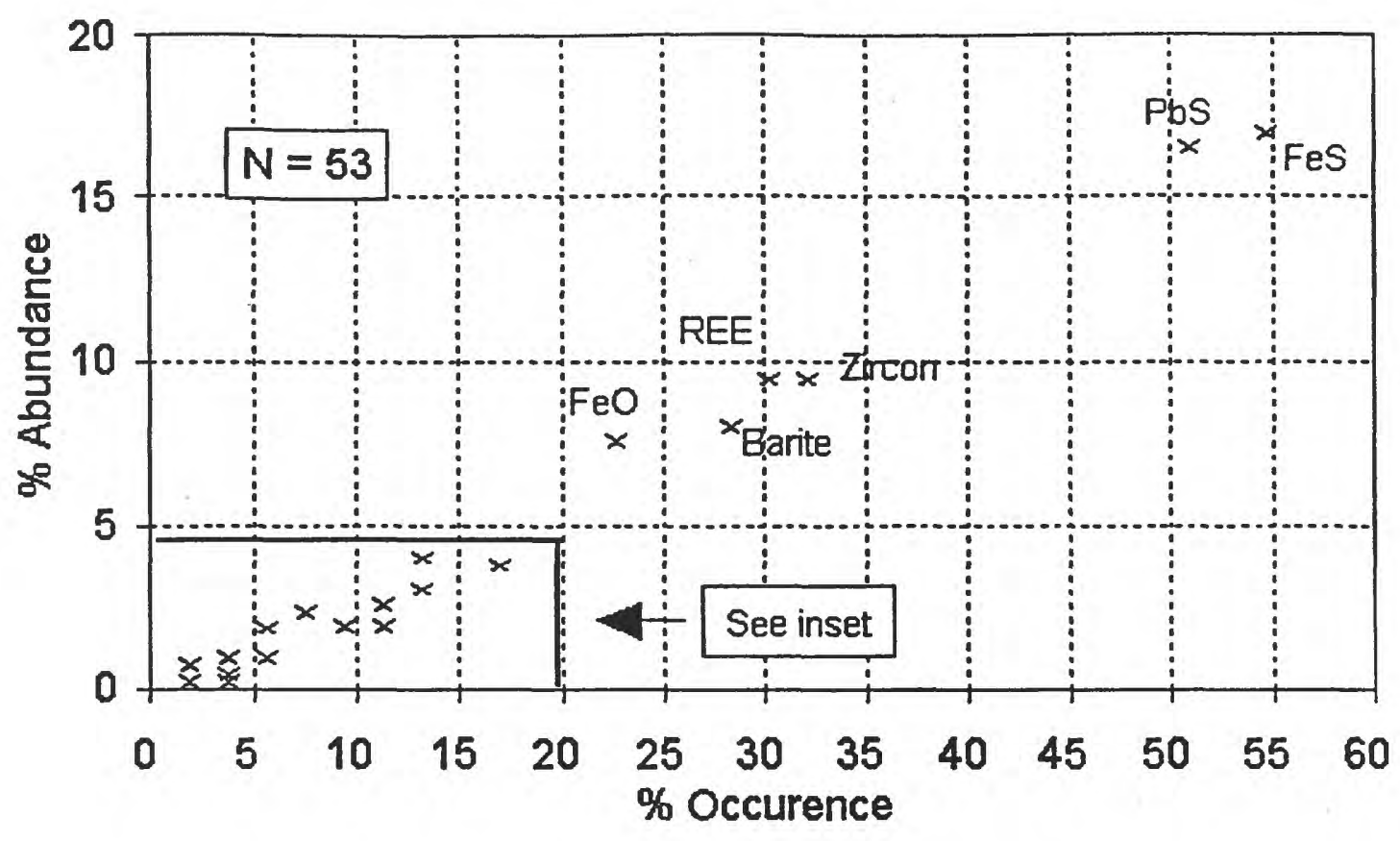

Helena SEM mineral scan

Outcrop samples

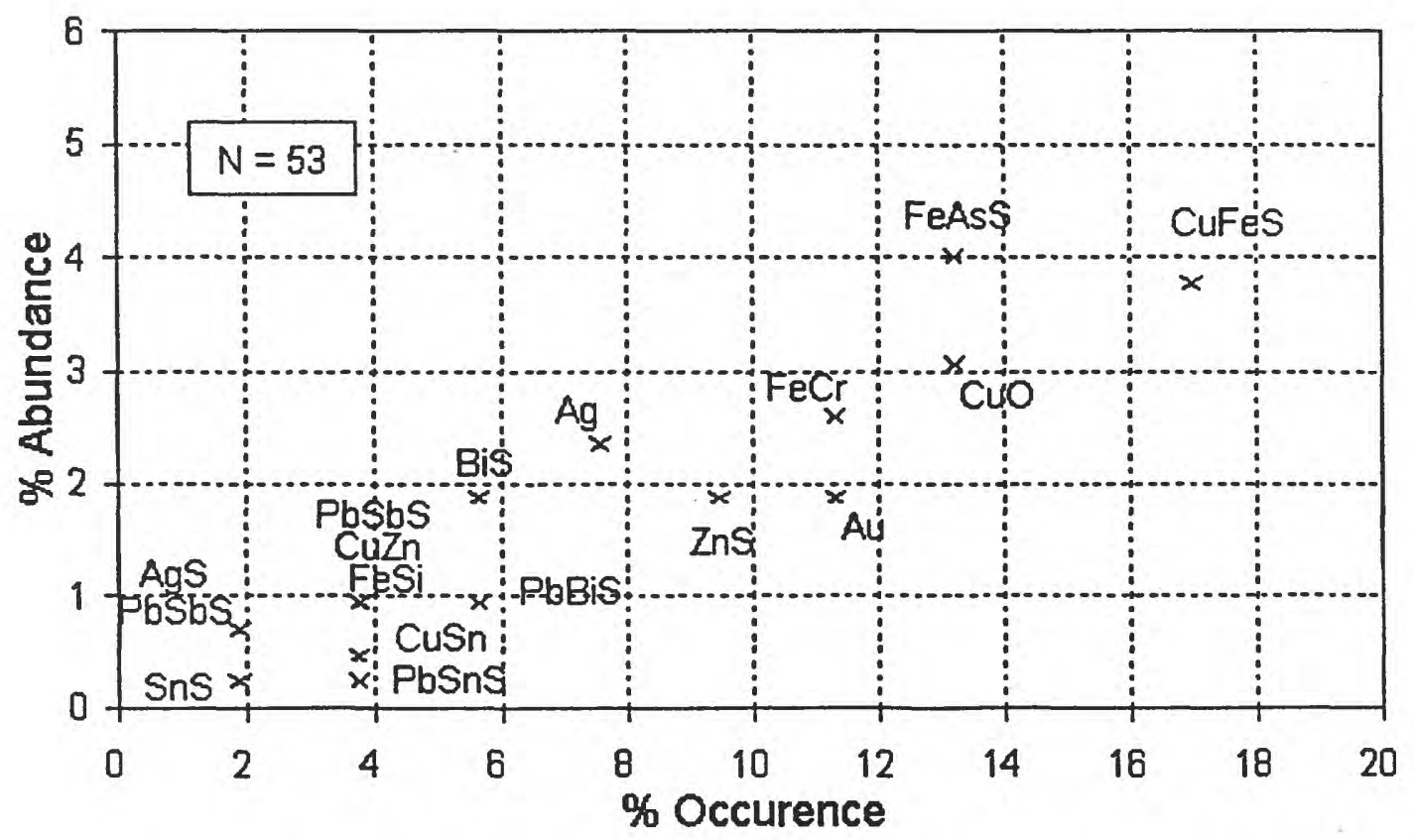

Figure D23. Graphs showing the SEM-EDS determined major composition of slabbed outcrop samples from the study area.

$$
\begin{array}{r}
\text { D } 34 \\
84
\end{array}
$$




\section{Elliston district concentrates}

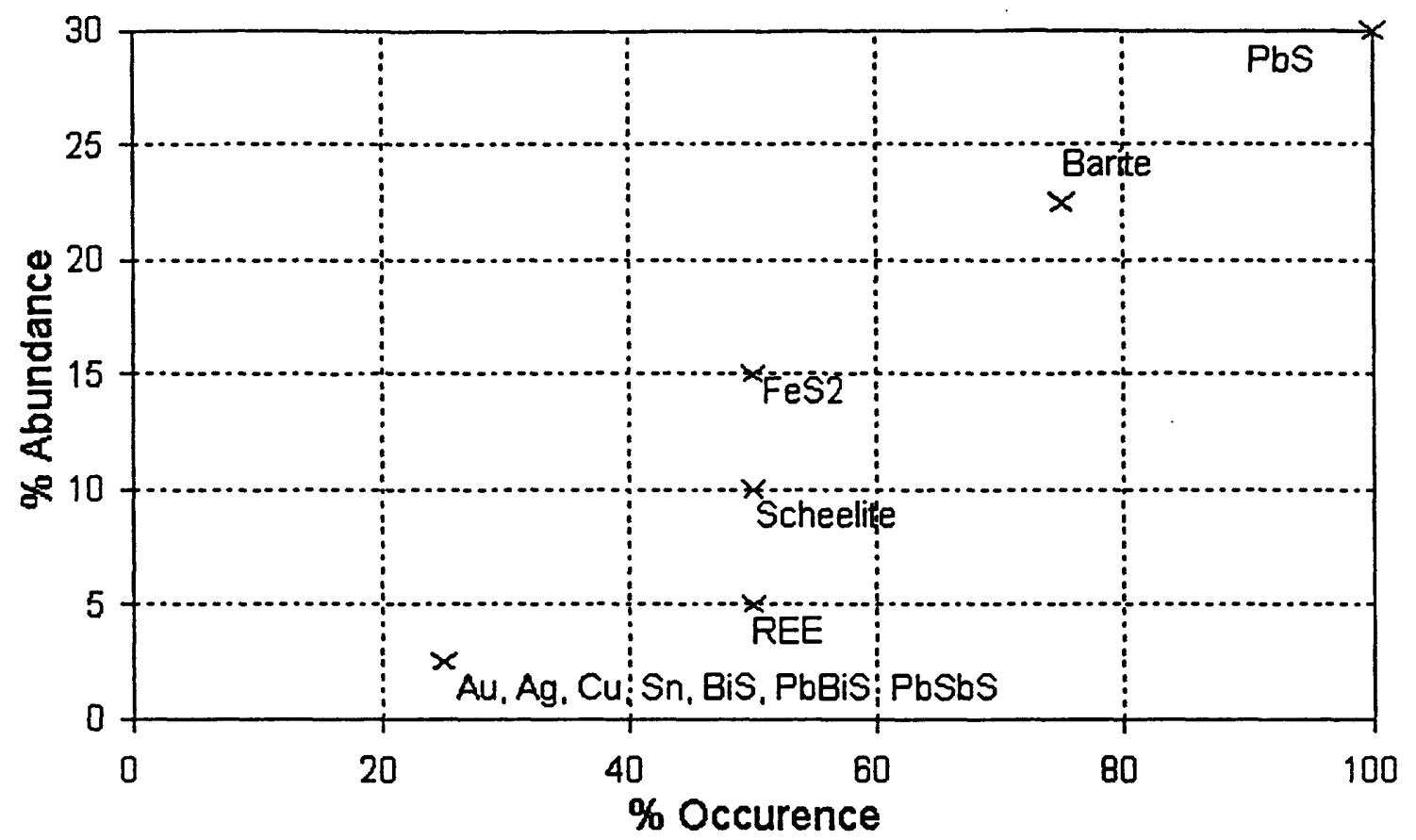

Figure D24. Graphs showing the SEM-EDS determined major composition of heavy mineral concentrates from the Elliston mining disrict.

Helena SEM mineral scan

Marysville district concentrates

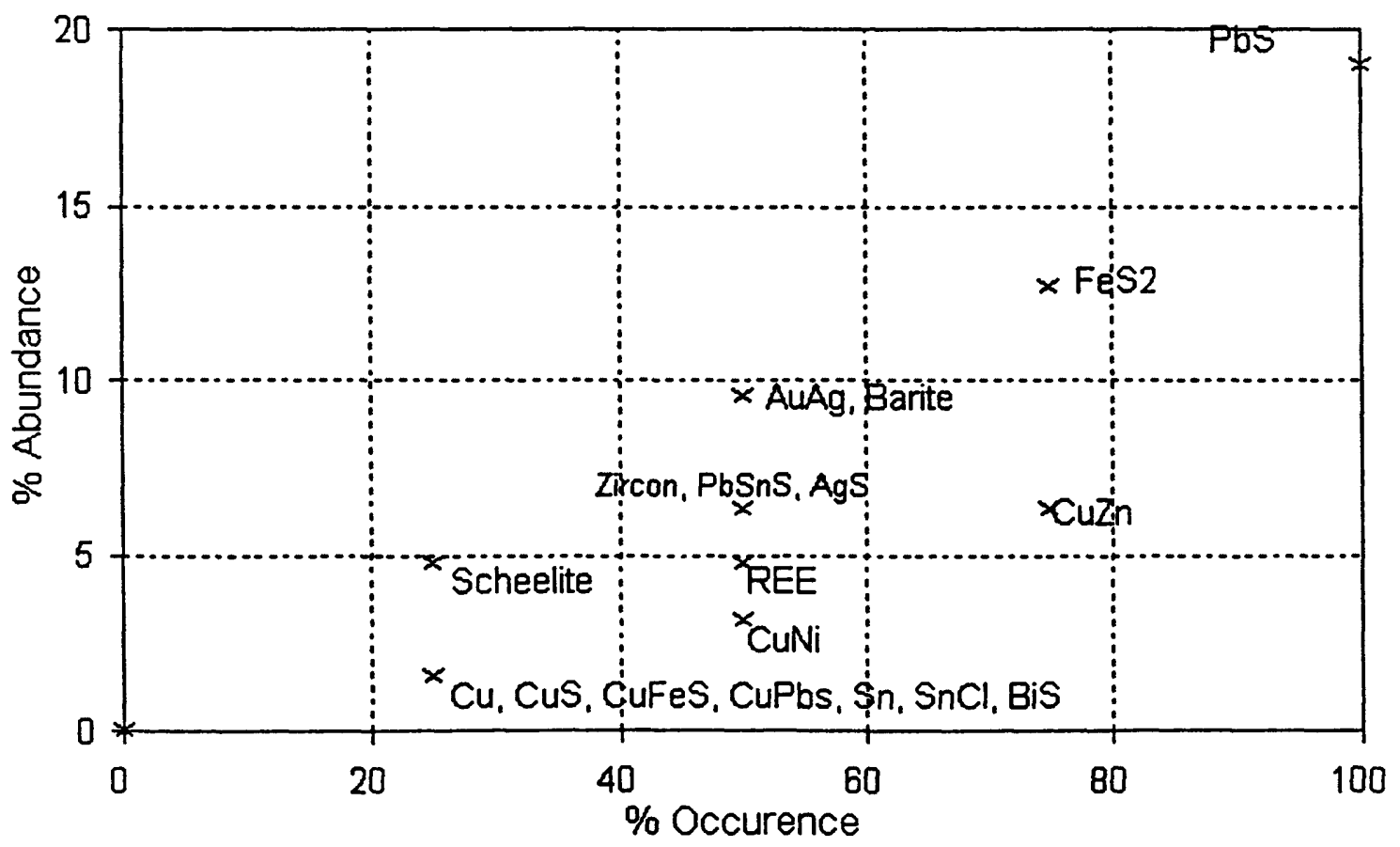

Figure D25. Graphs showing the SEM-EDS determined major composition of heavy mineral concentrates from the Marysville mining district. 


\section{Helena SEM mineral scan \\ Stemple-Gould district concentrates}

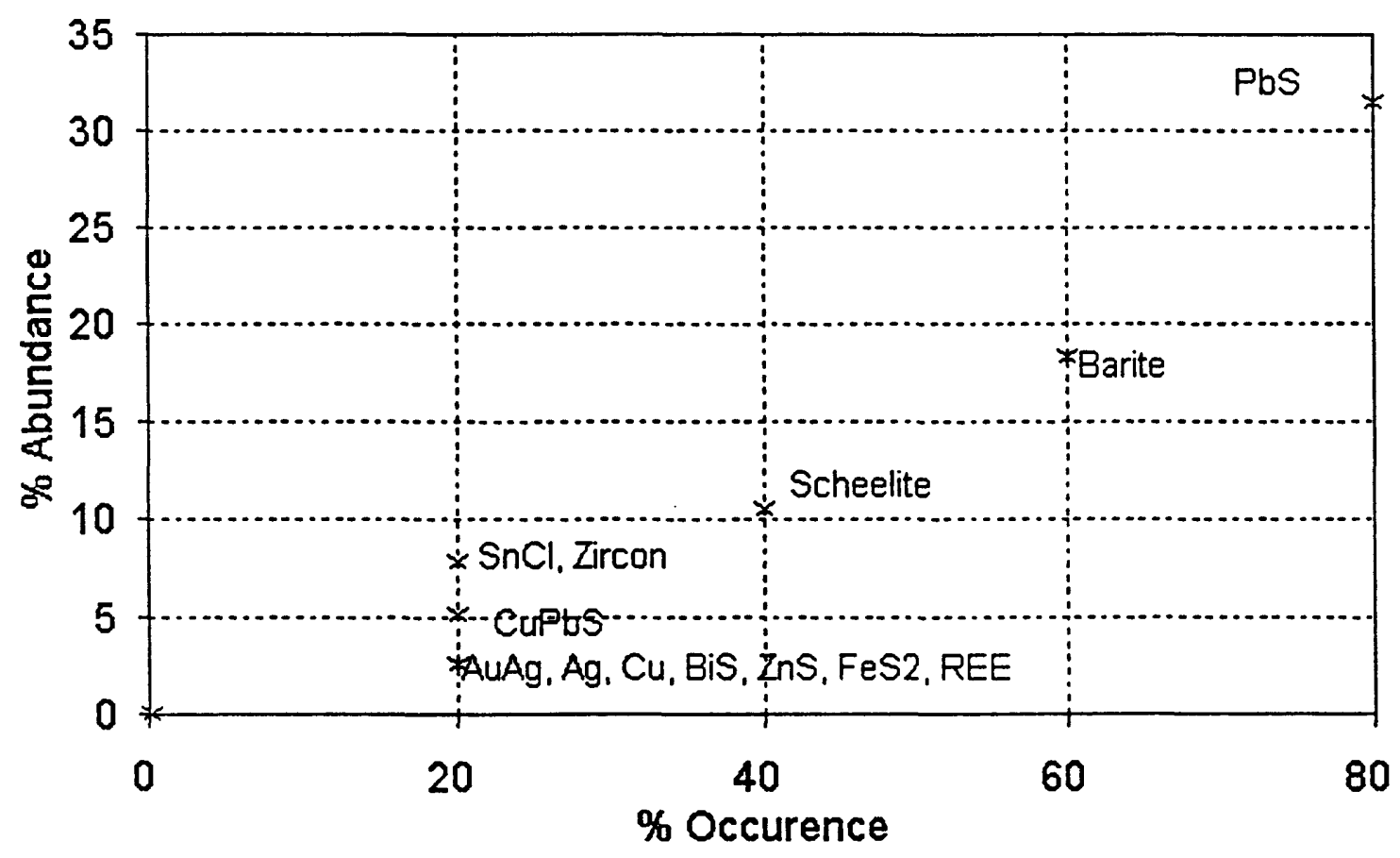

Figure D26. Graphs showing the SEM-EDS determined major composition of heavy mineral concentrates from the Stemple-Gould mining district. 
Table D1. Lower limits of determination for the six-step semi-quantitative emission spectrographic analytical method (8, weight percent; ppm, parts per million).

\begin{tabular}{|c|c|c|c|c|c|}
\hline $\mathrm{Ca}$ & 8 & 0.05 & $\mathrm{Ge}$ & ppm & 10.0 \\
\hline $\mathrm{Fe}$ & 8 & 0.05 & La & ppm & 50.0 \\
\hline $\mathrm{Mg}$ & 8 & 0.02 & Mn & ppm & 10.0 \\
\hline $\mathrm{Na}$ & 8 & 0.20 & Mo & ppm & 5.0 \\
\hline $\mathbf{P}$ & 8 & 0.20 & $\mathrm{Nb}$ & ppm & 10.0 \\
\hline $\mathrm{Ti}$ & 8 & 0.02 & Ni & ppm & 5.0 \\
\hline $\mathrm{Ag}$ & ppr & 0.50 & $\mathrm{~Pb}$ & ppm & 10.0 \\
\hline As & ppr & 100.00 & $\mathrm{Sb}$ & ppm & 100.0 \\
\hline $\mathrm{Au}$ & ppr & 10.00 & Sc & ppm & 5.0 \\
\hline B & ppr & 5.00 & Sn & ppm & 5.0 \\
\hline $\mathrm{Ba}$ & ppr & 50.00 & Sr & ppm & 100.0 \\
\hline $\mathrm{Be}$ & $\mathrm{ppr}$ & 1.00 & V & ppm & 10.0 \\
\hline $\mathrm{Bi}$ & ppr & 10.00 & W & ppm & 20.0 \\
\hline Cd & ppr & 20.00 & $Y$ & ppm & 10.0 \\
\hline Co & $\mathrm{ppr}$ & 10.00 & $\mathrm{Zn}$ & ppm & 200.0 \\
\hline $\mathrm{Cr}$ & ppr & 10.00 & $\mathrm{Zr}$ & ppm & 10.0 \\
\hline $\mathrm{Cu}$ & ppr & 5.00 & Th & ppm & 100.0 \\
\hline Ga & $\mathrm{ppr}$ & 5.00 & & & \\
\hline
\end{tabular}

Table D2. A list of the NURE analytical techniques and elements analyzed for the 2,151 stream sediments used in this report (Grimes, 1984).

Delayed-Neutron Counting

Energy Dispersive X-ray

Flourescence

Neutron Activation Analysis
$\mathrm{U}$

$\mathrm{Ag}, \mathrm{As}, \mathrm{Bi}, \mathrm{Cd}, \mathrm{Cu}, \mathrm{Nb}$,

$\mathrm{Ni}, \mathrm{Pb}, \mathrm{Se}, \mathrm{Sn}, \mathrm{W}, \mathrm{Zr}$

$\mathrm{Al}, \mathrm{Au}, \mathrm{Ba}, \mathrm{Ca}, \mathrm{Ce}, \mathrm{CI}, \mathrm{Co}$,

$\mathrm{Cr}, \mathrm{Cs}, \mathrm{Dy}, \mathrm{Eu}, \mathrm{Fe}, \mathrm{Hf}, \mathrm{K}$,

$\mathrm{La}, \mathrm{Lu}, \mathrm{Mg}, \mathrm{Mn}, \mathrm{Na}, \mathrm{Rb}, \mathrm{Sb}$,

$\mathrm{Sc}, \mathrm{Sm}, \mathrm{Sr}, \mathrm{Sr}, \mathrm{Ta}, \mathrm{Tb}, \mathrm{Th}, \mathrm{Ti}$,

$\mathrm{V}, \mathrm{Yb}, \mathrm{Zn}$. 
Table D3. Statistical summary of the spectrographic analysis of USGS (RASS) outcrop samples collected within the study area. The 1 ist below gives the minimum, maximum, and mean values (in 8 , weight percent; all other values are in ppm, parts per million) as well as the standard deviations of all unqualified values in addition to the numbers of qualified values.

[Qualifiers on data include: Valid, number of samples used in statistical analysis; B, number of samples not analyzed; L, number of samples detected with spectrographic analysis but measured below lowest standard; $N$, number of samples not detected; $G$, number of samples with values greater than the highest standard]

Element

\begin{tabular}{llllllllll}
\hline & Min. & Max & Mean & Dev & Valid & B & L & $\mathbf{N}$ & $\mathbf{G}$ \\
$\mathrm{Fe} \%$ & 0.02 & 50 & 4.755227 & 5.111292 & 1833 & 0 & 19 & 29 & 35 \\
$\mathrm{Mg} \%$ & 0.03 & 10 & 1.502993 & 1.850895 & 1862 & 0 & 50 & 0 & 4 \\
$\mathrm{Ca} \%$ & 0.01 & 20 & 4.013549 & 5.318001 & 1696 & 1 & 148 & 11 & 60 \\
$\mathrm{Ti} \%$ & 0.005 & 1 & 0.291861 & 0.253625 & 1852 & 0 & 33 & 28 & 3 \\
$\mathrm{Mn}$ & 5 & 10000 & 676.2856 & 829.7076 & 1845 & 10 & 11 & 2 & 48 \\
$\mathrm{Ag}$ & 0.5 & 5000 & 142.8114 & 435.6688 & 721 & 0 & 111 & 1080 & 4 \\
$\mathrm{As}$ & 50 & 10000 & 2132.5 & 2684.14 & 240 & 0 & 11 & 1611 & 54 \\
$\mathrm{Au}$ & 10 & 150 & 26.66667 & 26.45121 & 51 & 7 & 20 & 1838 & 0 \\
$\mathrm{~B}$ & 10 & 2000 & 91.11324 & 226.7479 & 1307 & 0 & 171 & 376 & 62 \\
$\mathrm{Ba}$ & 20 & 5000 & 823.6401 & 830.1956 & 1728 & 0 & 66 & 108 & 14 \\
$\mathrm{Be}$ & 1 & 150 & 3.718049 & 11.25167 & 1025 & 0 & 443 & 448 & 0 \\
$\mathrm{Bi}$ & 10 & 10000 & 154.5561 & 699.767 & 214 & 0 & 49 & 1642 & 11 \\
$\mathrm{Cd}$ & 20 & 500 & 136.4835 & 132.5257 & 91 & 0 & 16 & 1780 & 29 \\
$\mathrm{Co}$ & 5 & 1500 & 20.42153 & 52.18093 & 1217 & 0 & 205 & 494 & 0 \\
$\mathrm{Cr}$ & 7 & 5000 & 151.294 & 322.8122 & 1439 & 0 & 284 & 193 & 0 \\
$\mathrm{Cu}$ & 5 & 20000 & 761.5003 & 2711.267 & 1599 & 0 & 211 & 97 & 9 \\
$\mathrm{La}$ & 20 & 700 & 40.32441 & 41.88604 & 1233 & 68 & 243 & 372 & 0 \\
$\mathrm{Mo}$ & 5 & 5000 & 62.46192 & 290.6244 & 407 & 0 & 66 & 1440 & 3 \\
$\mathrm{Nb}$ & 10 & 150 & 41.58333 & 27.19354 & 120 & 0 & 447 & 1349 & 0 \\
$\mathrm{Ni}$ & 5 & 700 & 34.48748 & 66.0005 & 1477 & 0 & 333 & 106 & 0 \\
$\mathrm{~Pb}$ & 10 & 50000 & 749.6122 & 2875.64 & 1599 & 10 & 119 & 114 & 74 \\
$\mathrm{Sb}$ & 30 & 10000 & 1248.049 & 2349.765 & 164 & 1 & 42 & 1706 & 3 \\
$\mathrm{Sc}$ & 5 & 70 & 13.08982 & 8.050104 & 1258 & 0 & 229 & 429 & 0 \\
$\mathrm{Sn}$ & 10 & 1000 & 49.54546 & 113.5765 & 143 & 0 & 36 & 1737 & 0 \\
$\mathrm{Sr}$ & 100 & 5000 & 544.0618 & 406.4169 & 1263 & 1 & 136 & 516 & 0 \\
$\mathrm{~V}$ & 10 & 5000 & 125.0593 & 236.9372 & 1772 & 0 & 102 & 37 & 5 \\
$\mathrm{~W}$ & 50 & 1000 & 120.3571 & 151.5132 & 56 & 0 & 77 & 1783 & 0 \\
$\mathrm{Y}$ & 7 & 1000 & 28.2159 & 44.54991 & 1459 & 1 & 175 & 281 & 0 \\
$\mathrm{Zn}$ & 200 & 20000 & 2282.449 & 3098.476 & 245 & 0 & 57 & 1553 & 61 \\
$\mathrm{Zr}$ & 10 & 1000 & 127.967 & 105.6292 & 1697 & 0 & 46 & 172 & 1
\end{tabular}




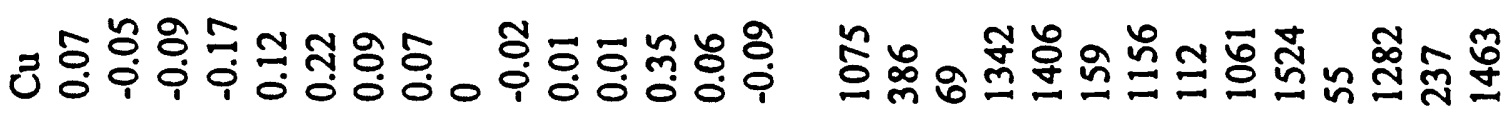

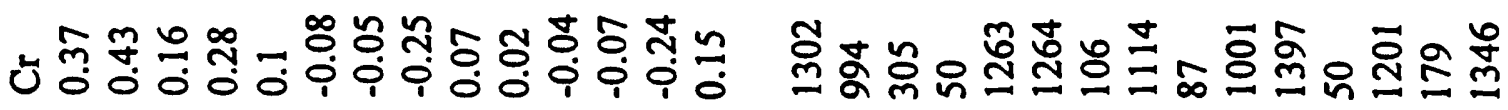

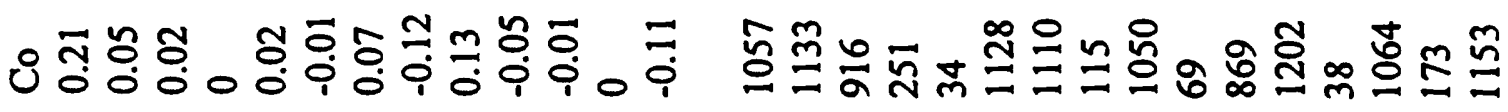

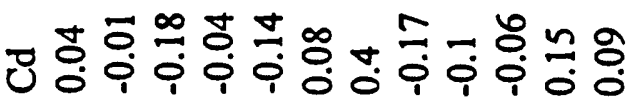

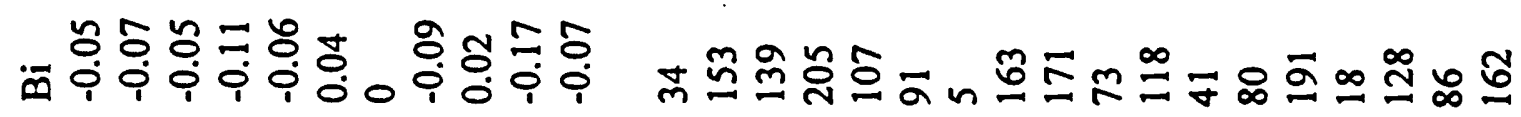

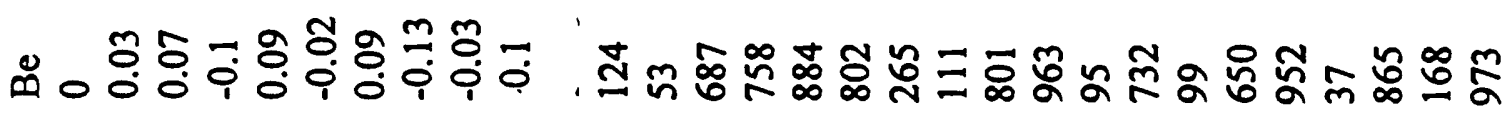

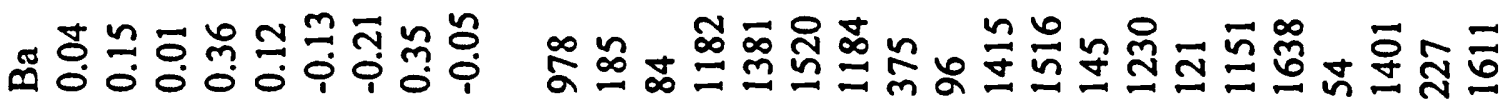
๓ ₹

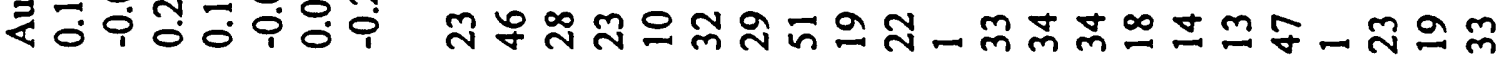

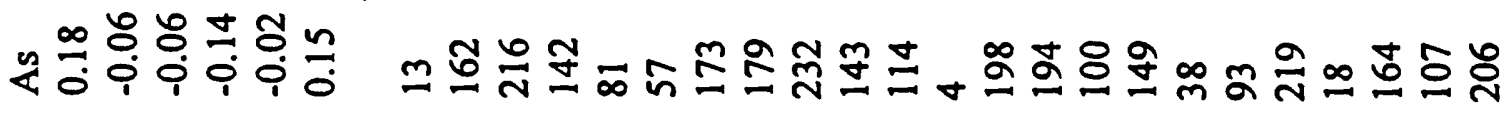

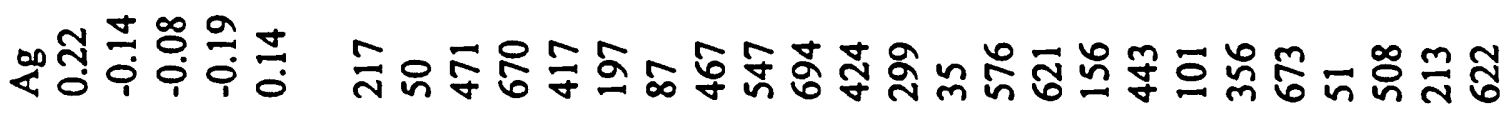

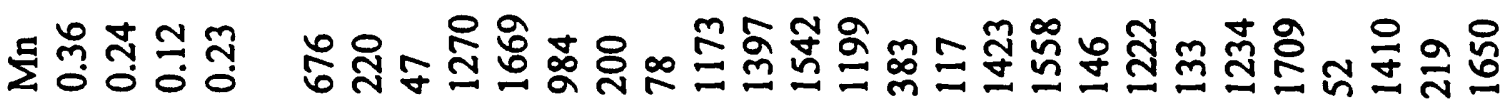

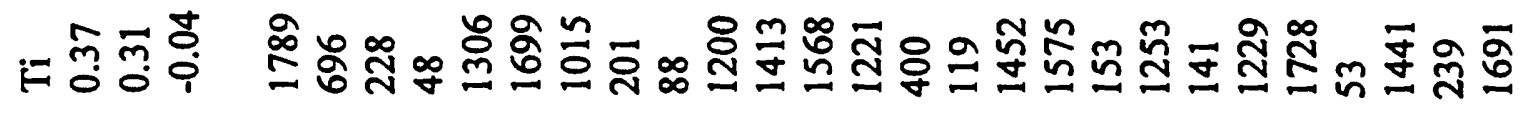

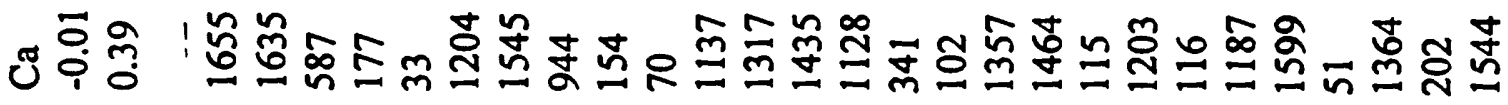

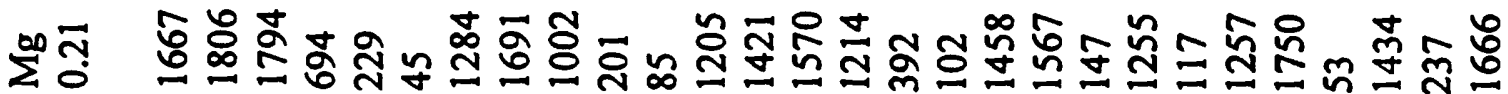

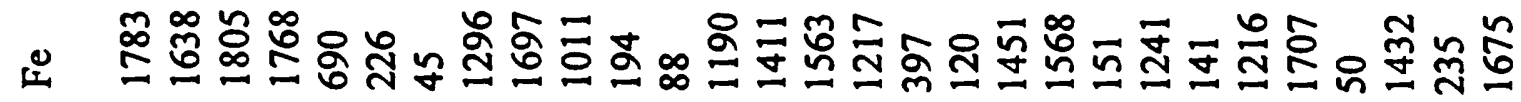

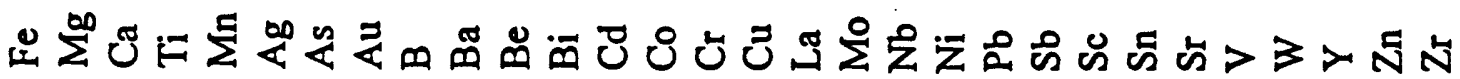




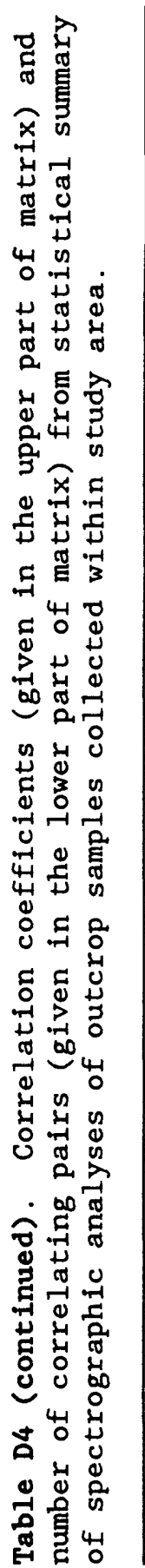

సँ

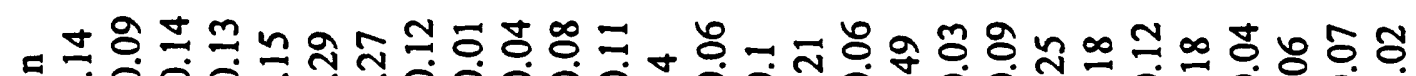
Nี >

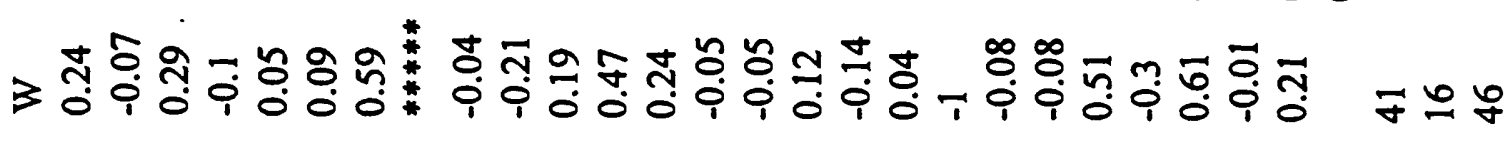

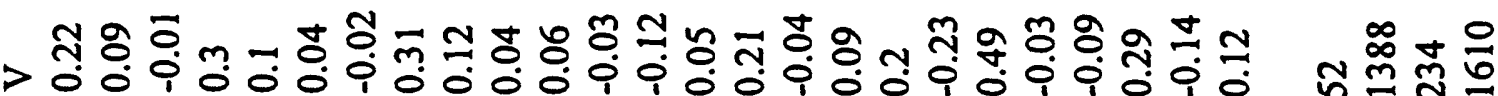

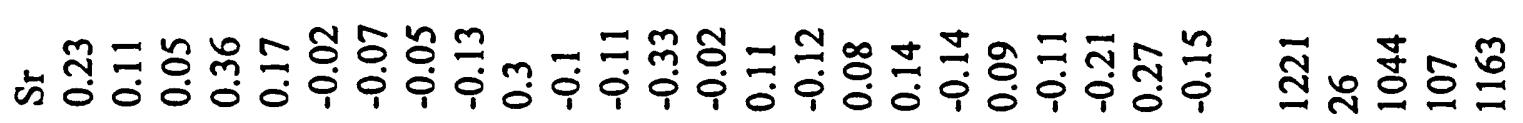
ש ن レ 응 궁 -

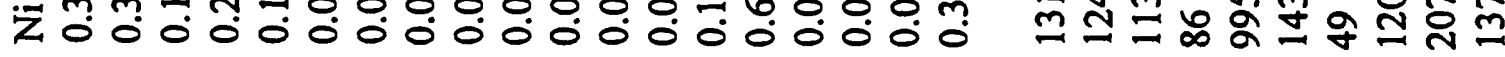

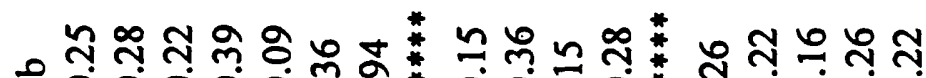
Z 造品

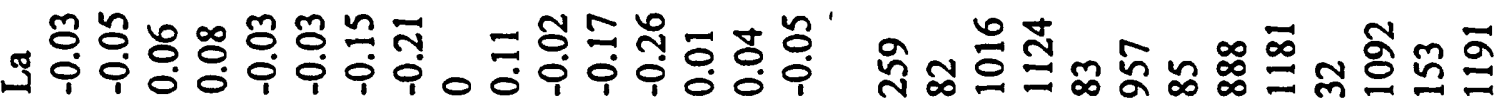

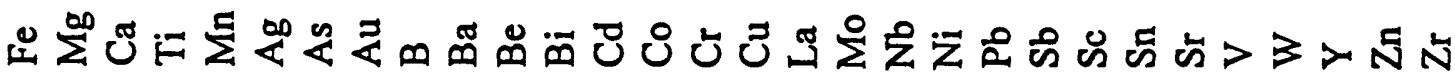


Table D5. Percent frequency distributions of spectrographic analyses of USGS (RASS) outcrop samples. Scale: reported spectrographic intervals in \& (weight percent) or ppm (parts per million) by element.

\begin{tabular}{|c|c|c|c|c|}
\hline & $\mathrm{Fe}$ & $\mathrm{Mg}$ & $\mathrm{Ca}$ & $\pi$ \\
\hline Scale & $\%$ & $\%$ & $\%$ & $\%$ \\
\hline & & & & N1.5 \\
\hline & & & & L 1.7 \\
\hline 0.002 & & & & 1.15 \\
\hline 0.003 & & & & 0.78 \\
\hline 0.005 & & & & 1.2 \\
\hline 0.007 & & & & 0.89 \\
\hline 0.01 & & & & 1.98 \\
\hline 0.015 & & & & 3.03 \\
\hline 0.02 & N1.5 & L2.6 & N0.6 & 2.3 \\
\hline 0.03 & $L 1.0$ & 2.77 & L7.7 & 4.65 \\
\hline 0.05 & 0.99 & 3.29 & 5.06 & 4.59 \\
\hline 0.07 & 0.68 & 3.91 & 4.44 & 5.74 \\
\hline 0.1 & 84 & 4.44 & 4.28 & 6.78 \\
\hline 0.15 & 0.94 & 3.08 & 2.71 & 7.52 \\
\hline 0.2 & 1.83 & 5.43 & 3.76 & 11.22 \\
\hline 0.3 & 3.44 & 7.83 & 3.97 & 14.04 \\
\hline 0.5 & 3.29 & 6.89 & 3.71 & 14.77 \\
\hline 0.7 & 5.58 & 6.58 & 3.65 & 13.83 \\
\hline 1 & 6.05 & 8.87 & 5.17 & 1.98 \\
\hline 1.5 & 9.5 & 11.59 & 6.11 & G 0.2 \\
\hline 2 & 10.59 & 11.33 & 7.67 & \\
\hline 3 & 11.43 & 8.14 & 9.34 & \\
\hline 5 & 12.47 & 4.91 & 8.87 & \\
\hline 7 & 9.13 & 3.91 & 6.21 & \\
\hline 10 & 11.12 & 0.78 & 5.01 & \\
\hline 15 & 5.79 & G 0.2 & 3.65 & \\
\hline 20 & 1.62 & & 4.85 & \\
\hline 30 & 0.16 & & G 3.1 & \\
\hline 50 & 0.21 & & & \\
\hline 70 & G 1.8 & & & \\
\hline$N=$ not de & atected & & & \\
\hline \multicolumn{5}{|c|}{$\mathrm{L}=$ detected but below lowest standard } \\
\hline \multicolumn{4}{|c|}{ G = above highest standard } & \\
\hline
\end{tabular}




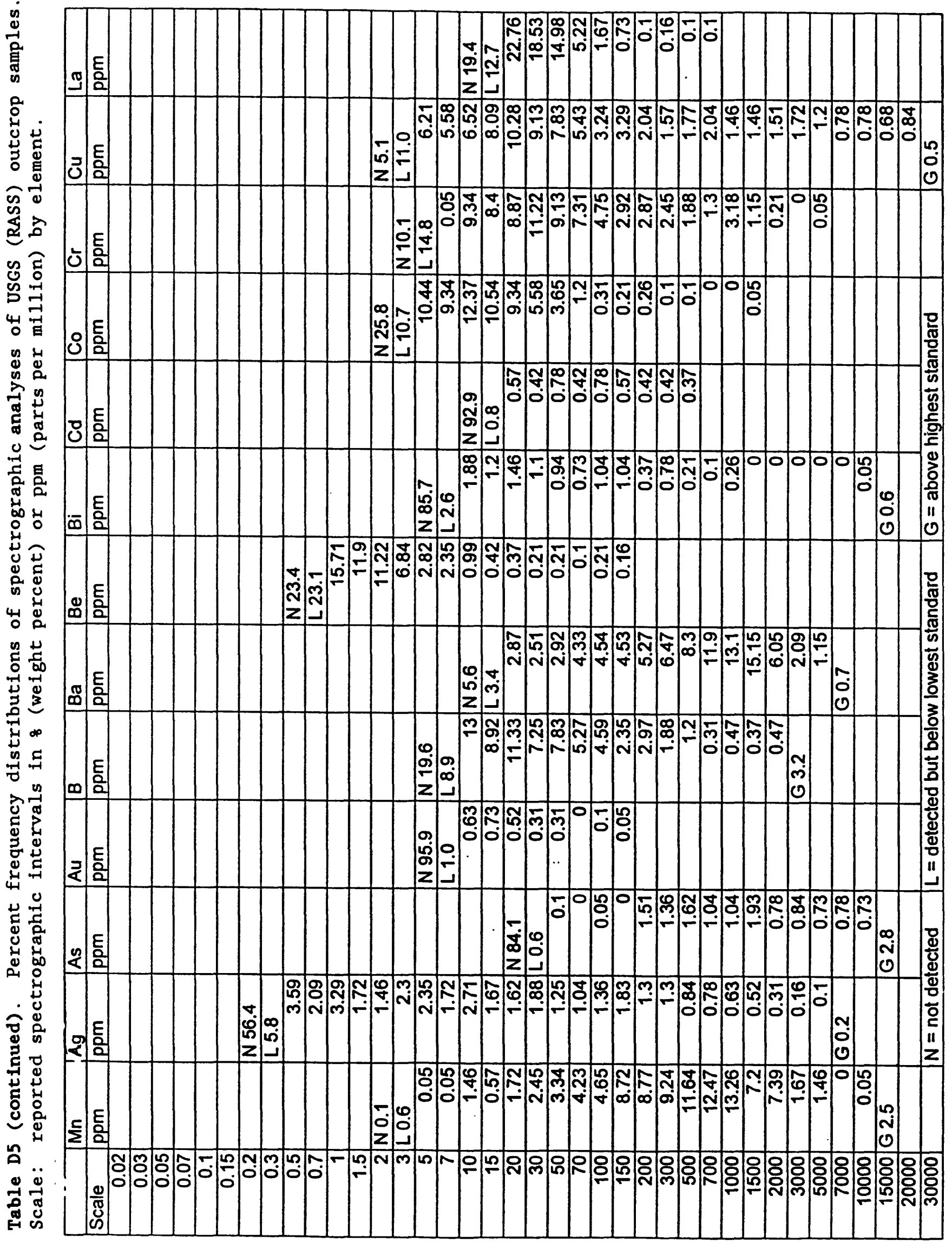




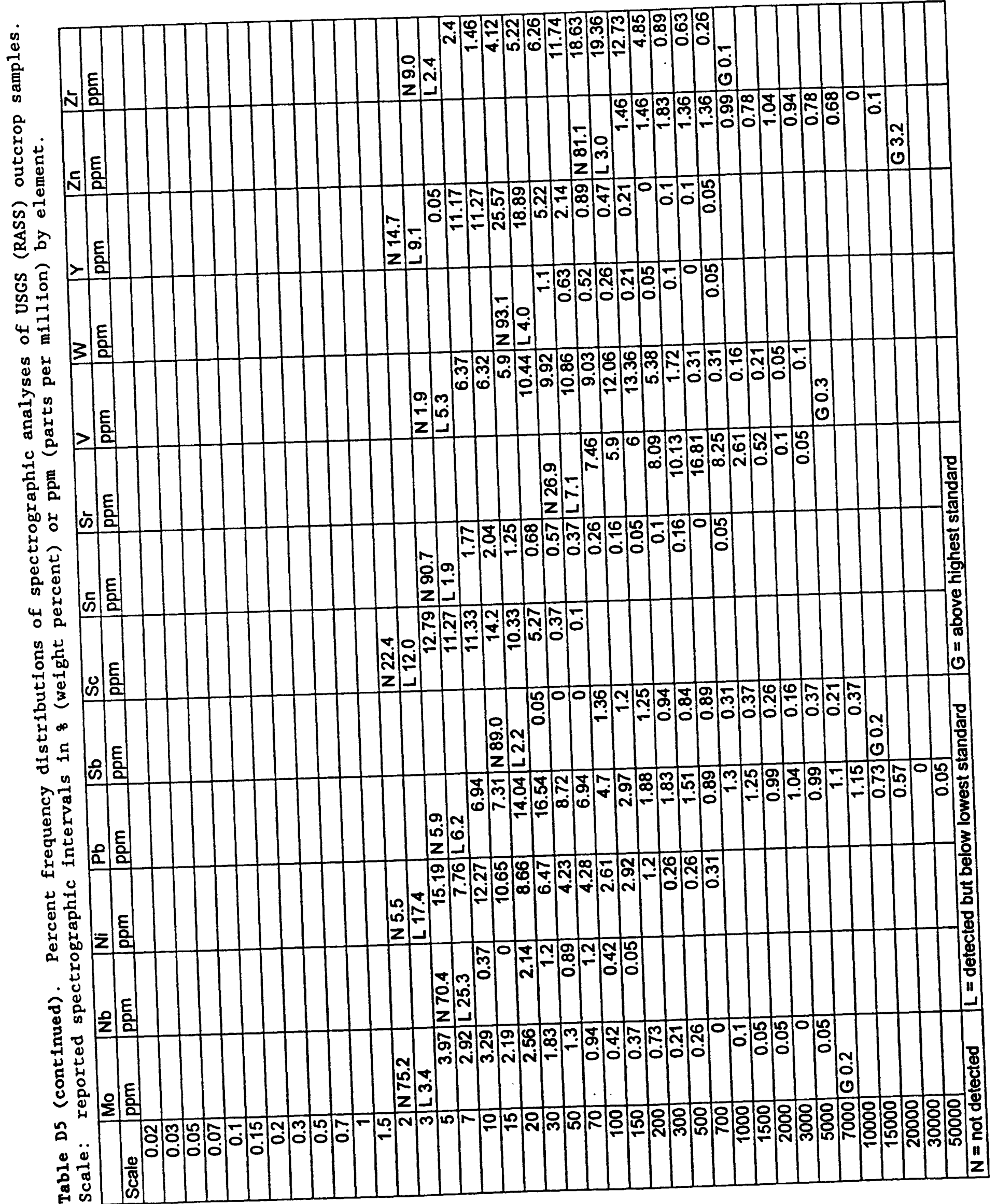


Table D6. Statistical summary of combined NURE and USGS (RASS) streamsediment samples collected within the study area. The list below gives the minimum, maximum, and mean values (in 8 , weight percent; all other values are in $\mathrm{ppm}$, parts per million) as well as the standard deviations of all unqualified values in addition to the numbers of qualified values.

[Qualifiers on data include: Valid, number of samples used in statistical analysis; $B$, number of samples not analyzed; $L$, number of samples detected with spectrographic analysis but measured below lowest standard; $N$, number of samples not detected; $G$, number of samples with values greater than the highest standard]

\begin{tabular}{|c|c|c|c|c|c|c|c|c|c|}
\hline \multicolumn{4}{|c|}{ Element } & \multicolumn{6}{|l|}{ Standard } \\
\hline & Min. & Max. & Mean. & Dev. & Valid & B. & $\underline{\mathbf{L}}$ & $\underline{\text { N. }}$ & G. \\
\hline $\mathrm{Ca} \%$ & 0.05 & 20 & 1.916317 & 2.346737 & 3280 & 1 & 1 & 0 & \\
\hline $\mathrm{Fe} \%$ & 0.05 & 30 & 3.636581 & 2.832488 & 3279 & 1 & 0 & 0 & \\
\hline Mg\% & 0.07 & 10 & 1.17808 & 0.891853 & 3281 & 1 & 0 & 0 & \\
\hline Ti\% & 0.02 & 1.428 & 0.394584 & 0.210705 & 3223 & 3 & 0 & 0 & \\
\hline $\mathrm{Ag}$ & 0.25 & 700 & 681 & 591 & 895 & 1 & 293 & 2093 & \\
\hline As & 100 & 5000 & 218 & 71 & 424 & 1 & 24 & 2832 & \\
\hline $\mathrm{Au}$ & 5 & 30 & 5.08 & 04 & 373 & 1 & 1 & 2907 & \\
\hline B & 5 & 1500 & 48.8 & 5 & 3189 & 1 & 7 & 65 & \\
\hline $\mathrm{Ba}$ & 50 & 3000 & 678 & 28 & 3281 & 1 & 0 & 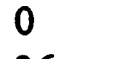 & \\
\hline $\mathrm{Be}$ & 0.5 & 200 & 2.18 & 732 & 3101 & 1 & 144 & 36 & c \\
\hline $\mathrm{Bi}$ & 5 & 100 & 9.5 & 543 & 463 & 1 & 85 & 2733 & 0 \\
\hline $\mathrm{Cd}$ & 10 & 150 & 11.76166 & 11. & 386 & 1 & 4 & 2891 & 0 \\
\hline Co & 2.5 & 200 & 12.54923 & 9.800403 & 2783 & 1 & 377 & 119 & 2 \\
\hline $\mathrm{Cr}$ & 7 & 5000 & 91.84223 & 153.6821 & 3277 & 1 & 4 & 0 & \\
\hline $\mathrm{Cu}$ & 5 & 3000 & 59.17993 & 136 & 3279 & 1 & 2 & 0 & 0 \\
\hline $\mathrm{La}$ & 14 & 700 & 49.52067 & 44.61053 & 2395 & 1 & 815 & 71 & 0 \\
\hline $\mathrm{Mn}$ & 30 & 7142.8 & 895.9095 & 637.4252 & 3263 & 1 & 0 & 0 & 18 \\
\hline Mo & 2.5 & 100 & 5.90689 & 9.80 & 537 & 1 & 147 & 2597 & 0 \\
\hline $\mathrm{Nb}$ & 10 & 200 & 16.83667 & 898 & 600 & 1 & 1487 & 1194 & 0 \\
\hline $\mathrm{Ni}$ & 3.5 & 200 & 23.92787 & 722 & 3244 & 1 & 33 & 4 & 0 \\
\hline $\mathrm{Pb}$ & 10 & 10000 & 98.83547 & 363 & 3276 & 1 & 4 & 0 & 1 \\
\hline $\mathrm{Sb}$ & 50 & 1500 & 62.34694 & 88.69674 & 392 & 2 & 5 & 2883 & 0 \\
\hline Sc & 3.5 & 50 & 9.352137 & 4.200612 & 3182 & 1 & 96 & 3 & 0 \\
\hline Sn & 5 & 150 & 10.65217 & 17.08012 & 460 & 2 & 27 & 2793 & 0 \\
\hline $\mathrm{Sr}$ & 50 & 1500 & 351.6862 & 207.9662 & 2651 & 1 & 185 & 445 & 0 \\
\hline Th & 50 & 500 & 53.29787 & 33.53448 & 376 & 162 & 5 & 273 & 0 \\
\hline V & 7 & 1500 & 125.0997 & 119.219 & 3280 & 1 & 1 & 0 & 0 \\
\hline W & 20 & 150 & 26.05943 & 8.106421 & 387 & 2 & 39 & 2854 & 0 \\
\hline Y & 5 & 1500 & 30.24885 & 39.98839 & 3251 & 1 & 27 & 3 & 0 \\
\hline $\mathrm{Zn}$ & 100 & 10000 & 446.5549 & 1134.97 & 537 & 2 & 208 & 2532 & 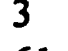 \\
\hline $\mathrm{Zr}$ & 15 & 1428.5 & 225.4268 & 169.511 & 3220 & 1 & 0 & 0 & \\
\hline
\end{tabular}




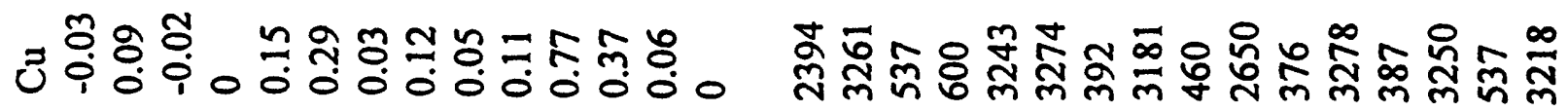

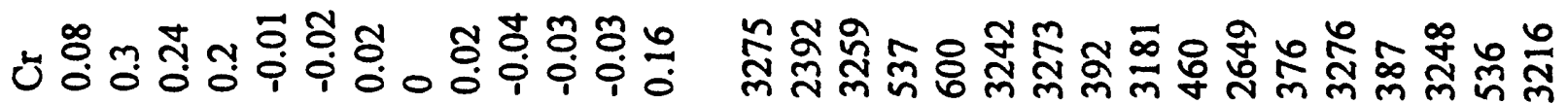

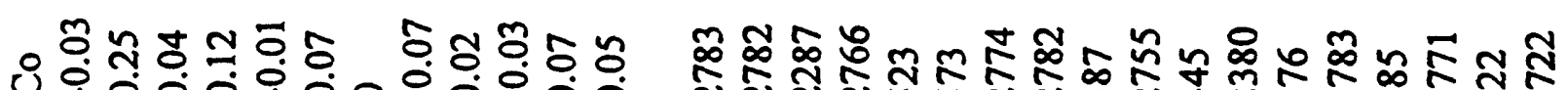

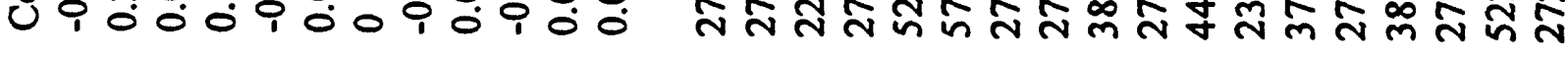

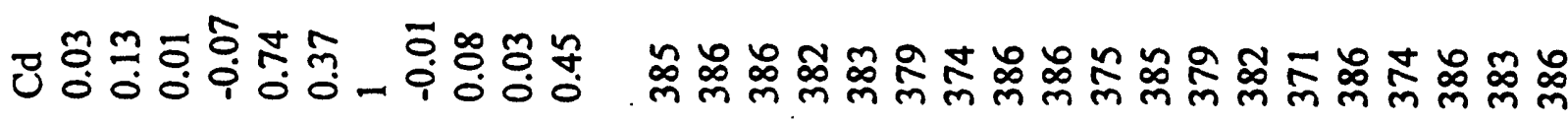

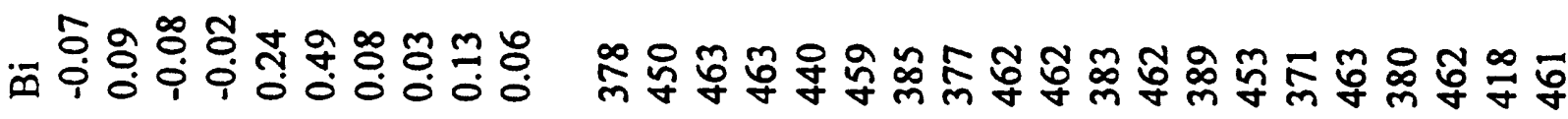

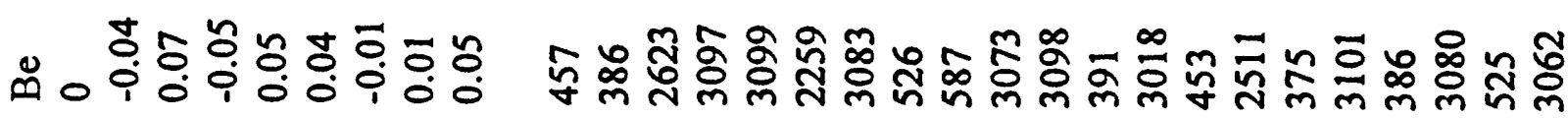

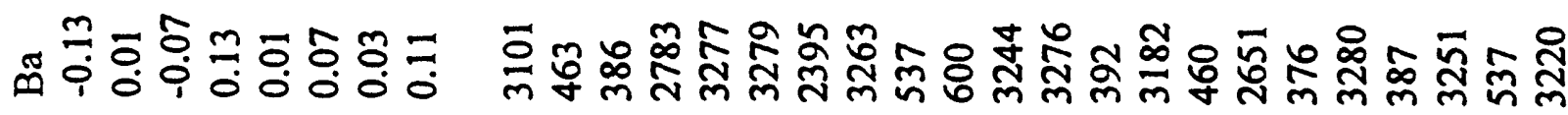

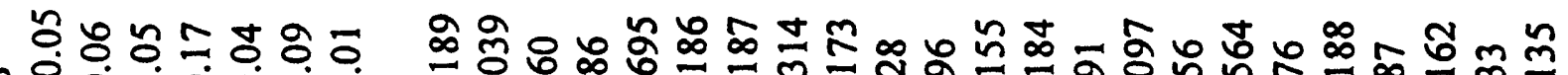

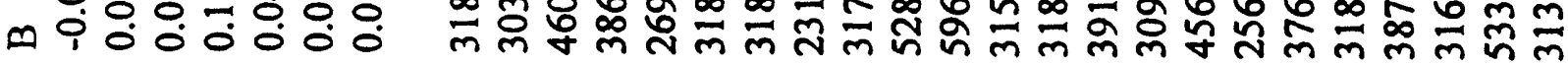

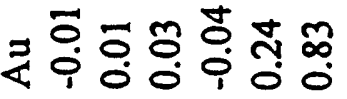

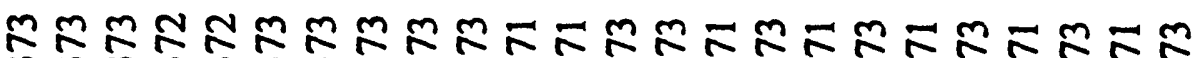
ת ชิ ชุ

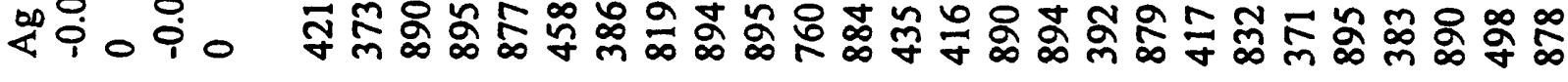

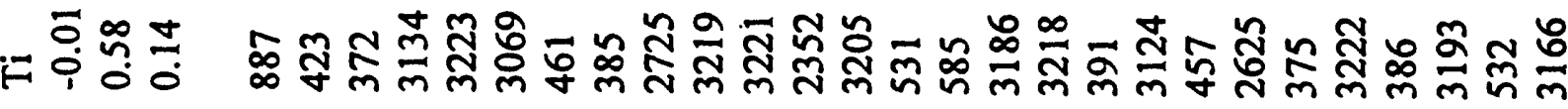
舟 ¿0ல0

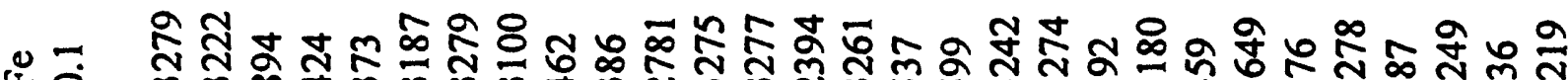

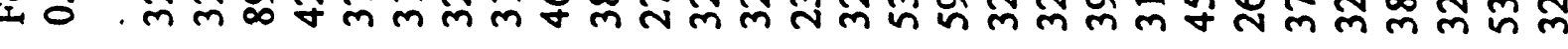

๙ U. . స్ స్ల

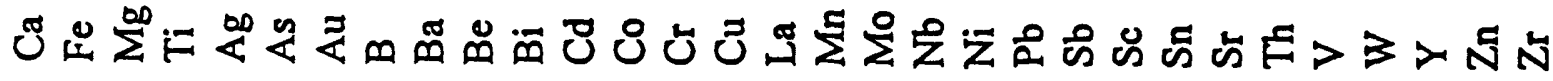


山 స̃

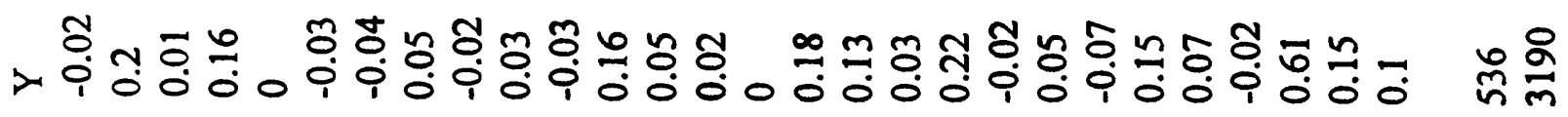
३

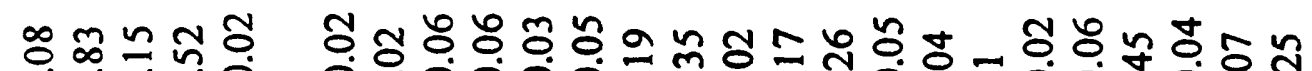
$>$

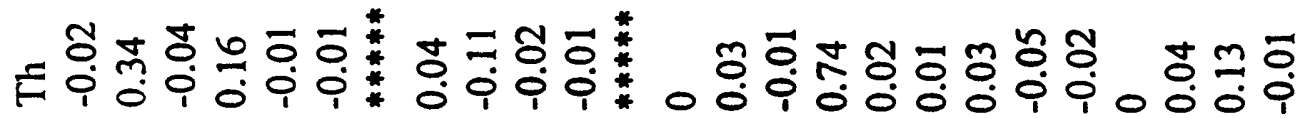

$\stackrel{2}{2} \because \approx n$ দ ๘ 饮

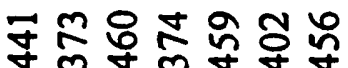

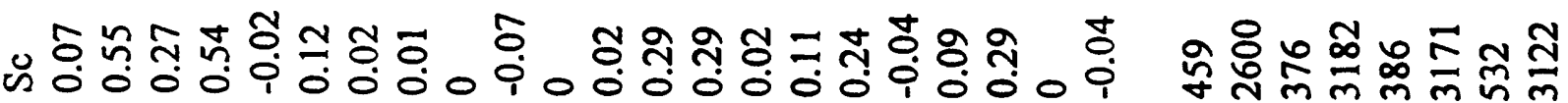
灾 ㅇํㅇㅇํำ

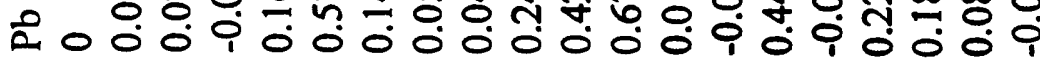

후유ำก z

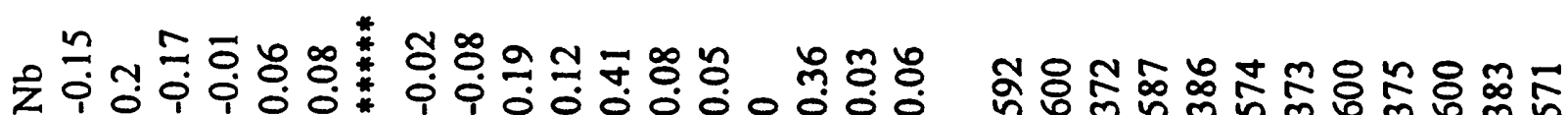
Z

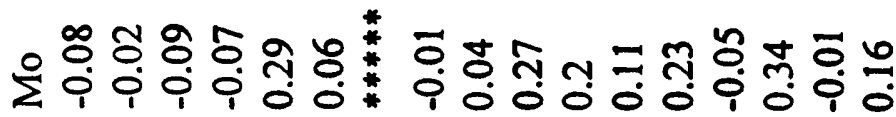

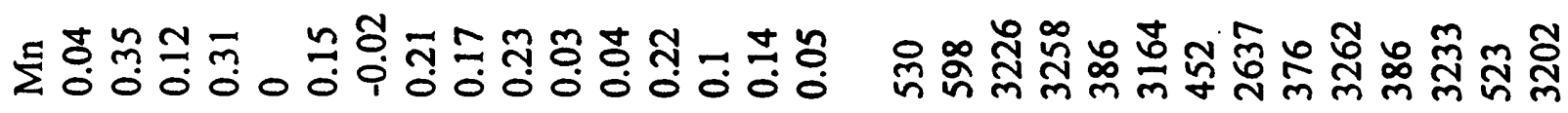

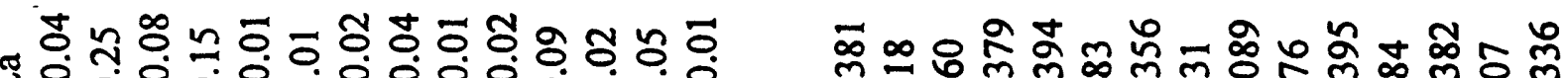
品

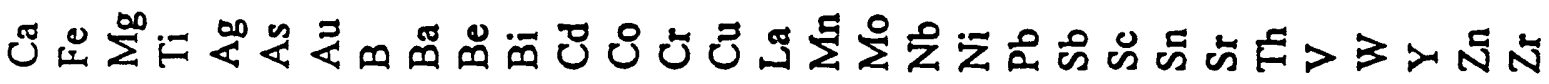


ㄱ.

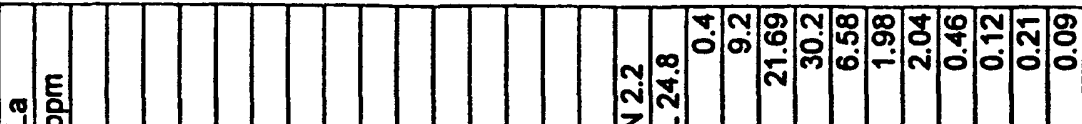

荈

怘

물

它

ठ

点

4

o

o

-

ह్ర

r

ह

م.

感

兵

出

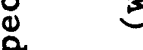

4

4.

ป

기흠

$\div$

z.

ㅇ․을

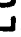

능

- 0 :

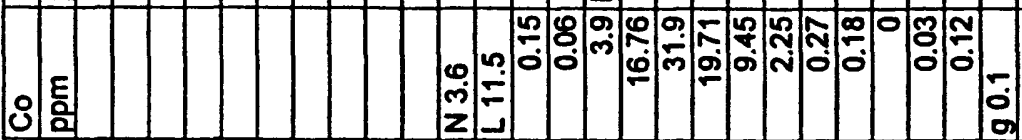

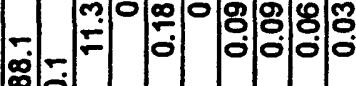

기흠

z.

궁

条

E

$\infty$

-

z-

틍

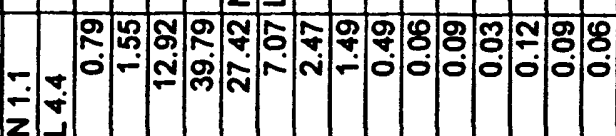

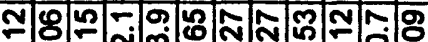

○.0

\$등

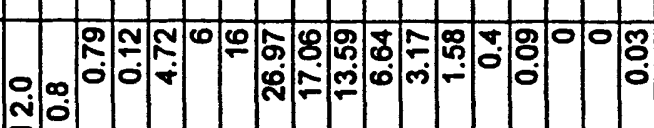

틍

z.

쿠흥

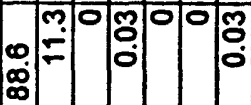

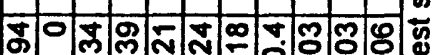

m

웅임

z:

zo

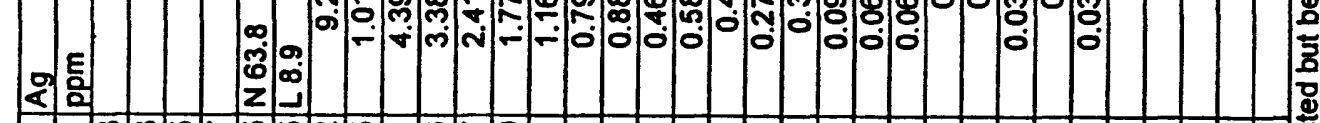

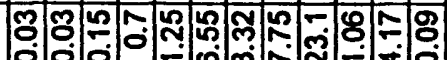

F.8 0

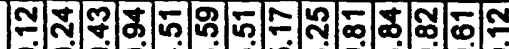

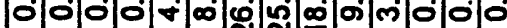

กำ

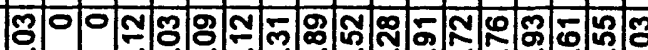

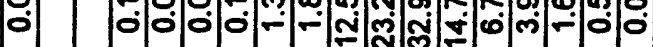

48

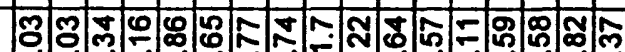

$4 \rightarrow$ H

0. ค.

窃

宫 岂.

o

章 曾

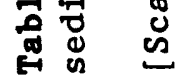

ง๊ะ

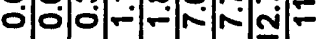

० न m N

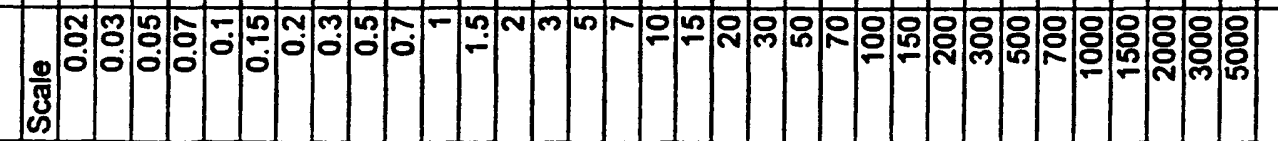




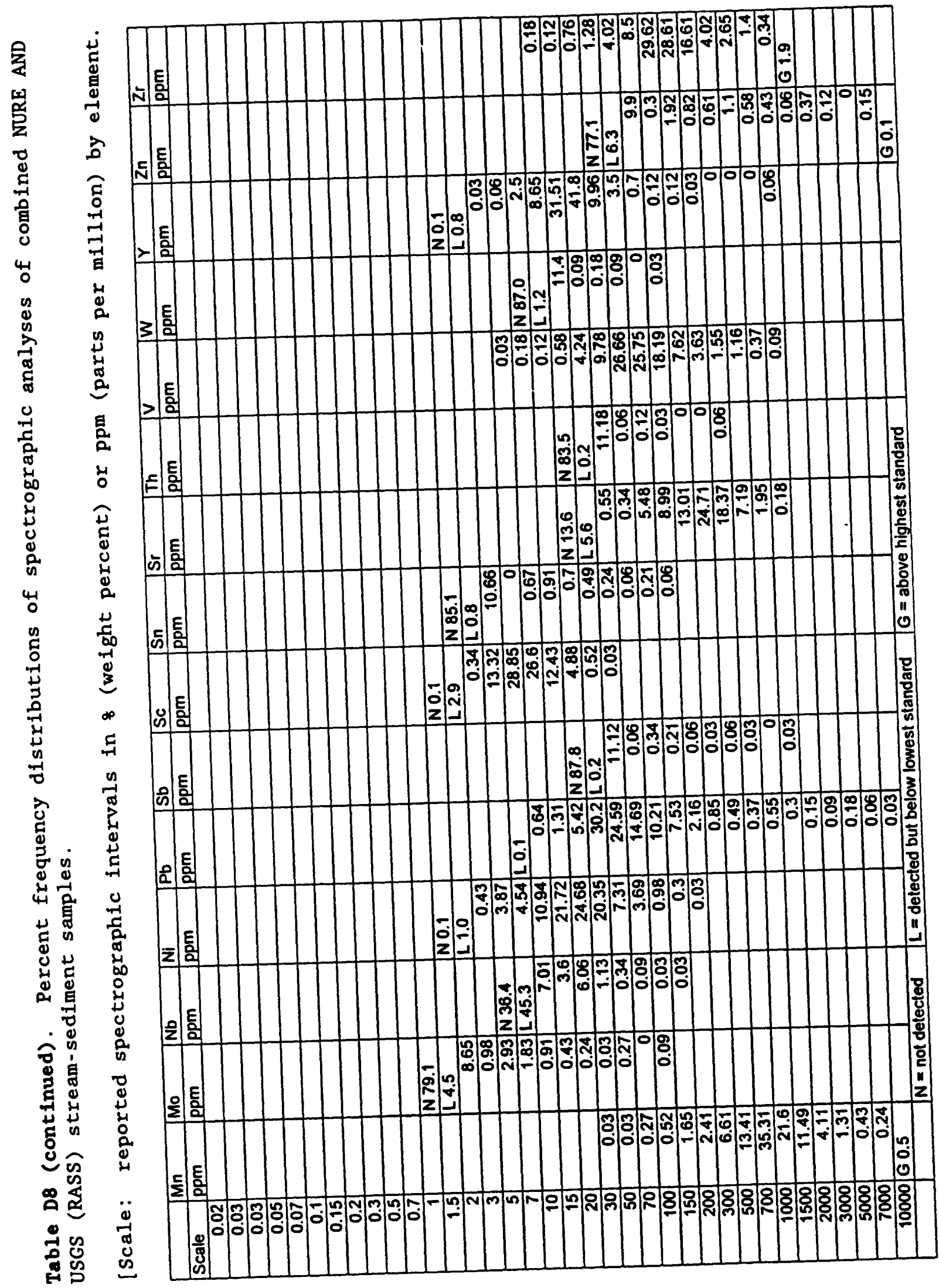


Table D9. Statistical summary of spectrographic analyses of combined NURE and USGS (RASS) stream sediments analyzed for gold. The 1ist below gives the minimum, maximum, and mean values (in 8 , weight percent; all other values are in ppm, parts per million) as well as the standard deviations of all unqualified values in addition to the numbers of qualified values.

[Qualifiers on data include: Valid, number of samples used in statistical analysis; B, number of samples not analyzed; $L$, number of samples detected with spectrographic analysis but measured below lowest standard; $N$, number of samples not detected; $G$, number of samples with values greater than the highest standard]

\section{ELEMENT}

\begin{tabular}{lrrrrrrrrr} 
& \multicolumn{1}{c}{ MIN. } & \multicolumn{1}{c}{ MAX. } & \multicolumn{1}{c}{ MEAN } & DEV & VALID & B & I & N & G \\
CA & 0.050 & 20.000 & 1.713 & 2.277 & 1899 & 1 & 1 & 0 & 0 \\
FE & 0.050 & 20.000 & 3.082 & 2.155 & 1900 & 1 & 0 & 0 & 0 \\
MG & 0.070 & 10.000 & 1.025 & 0.788 & 1900 & 1 & 0 & 0 & 0 \\
TI & 0.020 & 1.428 & 0.347 & 0.186 & 1880 & 2 & 0 & 0 & 19 \\
AG & 0.250 & 100.000 & 1.947 & 6.558 & 641 & 1 & 149 & 1110 & 0 \\
AS & 100.000 & 3000.000 & 163.092 & 272.092 & 401 & 1 & 22 & 1477 & 0 \\
AU & 5.000 & 5.000 & 5.000 & $\star * *$ & 371 & 1 & 1 & 1528 & 0 \\
B & 5.000 & 500.000 & 38.400 & 30.742 & 1896 & 1 & 3 & 1 & 0 \\
BA & 50.000 & 3000.000 & 611.994 & 293.187 & 1900 & 1 & 0 & 0 & 0 \\
BE & 0.500 & 100.000 & 1.915 & 3.247 & 1861 & 1 & 38 & 1 & 0 \\
BI & 5.000 & 70.000 & 8.002 & 9.734 & 419 & 1 & 34 & 1447 & 0 \\
Cd & 10.000 & 150.000 & 10.765 & 8.618 & 379 & 1 & 4 & 1517 & 0 \\
CO & 2.500 & 200.000 & 14.094 & 9.313 & 1435 & 1 & 358 & 105 & 2 \\
CR & 7.000 & 2000.000 & 83.618 & 85.722 & 1899 & 1 & 1 & 0 & 0 \\
CU & 5.000 & 3000.000 & 56.112 & 126.270 & 1899 & 1 & 1 & 0 & 0 \\
IA & 14.000 & 700.000 & 52.867 & 38.889 & 1040 & 1 & 793 & 67 & 0 \\
MN & 30.000 & 7142.857 & 760.761 & 631.421 & 1896 & 1 & 0 & 0 & 4 \\
MO & 2.500 & 100.000 & 4.240 & 6.841 & 403 & 1 & 77 & 1420 & 0 \\
NB & 10.000 & 70.000 & 14.733 & 6.587 & 499 & 11008 & 393 & 0 \\
NI & 3.500 & 150.000 & 23.476 & 15.832 & 1881 & 1 & 15 & 4 & 0 \\
PB & 10.000 & 7000.000 & 101.031 & 310.170 & 1896 & 1 & 4 & 0 & 0 \\
SB & 50.000 & 1500.000 & 59.267 & 85.849 & 382 & 1 & 3 & 1515 & 0 \\
SC & 3.500 & 30.000 & 9.037 & 3.815 & 1846 & 1 & 52 & 2 & 0 \\
SN & 5.000 & 100.0000 & 8.289 & 11.232 & 415 & 1 & 12 & 1473 & 0 \\
SR & 50.000 & 1000.000 & 297.317 & 171.563 & 1465 & 1 & 135 & 300 & 0 \\
TH & 50.000 & 500.000 & 51.586 & 23.641 & 372 & 1 & 1 & 1527 & 0 \\
V & 10.000 & 1500.000 & 110.826 & 91.198 & 1899 & 1 & 1 & 0 & 0 \\
W & 20.000 & 70.000 & 25.250 & 3.082 & 380 & 1 & 13 & 1507 & 0 \\
Y & 5.000 & 200.000 & 27.781 & 13.201 & 1887 & 1 & 11 & 2 & 0 \\
ZN & 100.000 & 10000.000 & 326.773 & 869.953 & 437 & 1 & 168 & 1295 & 0 \\
ZR & 15.000 & 1428.571 & 219.906 & 175.282 & 1884 & 1 & 0 & 0 & 16
\end{tabular}

\section{STANDARD}

$1110 \quad 0$ 西 


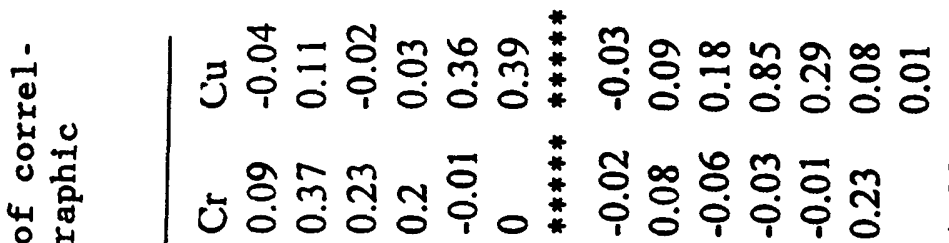

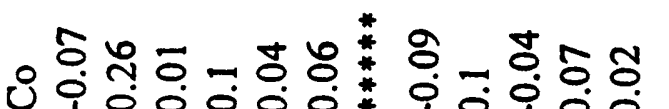

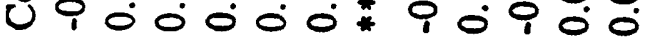

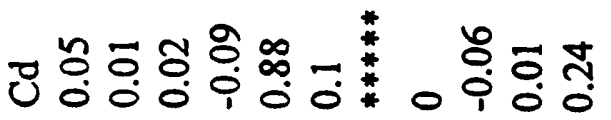
年每 己 品. 范嵌 क्षे 出窟 䗆 फल $+\rightarrow \frac{1}{3}$ $\infty$ o

क.

40

i t

类

ว 青它 站出 n 象華 出帛 5 $\sum_{-1}^{\infty} \underset{1}{\infty}$ Do 4 出

舟 $+4$ a a. म 斗 出 ब 80 되 造 동

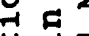
車 동 ? 品

क 4 . 움 궁 口 0 品 곡ㄱ

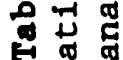

윙 $\infty$ 每 ㅇำ $\infty$ 向

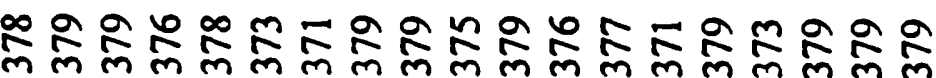

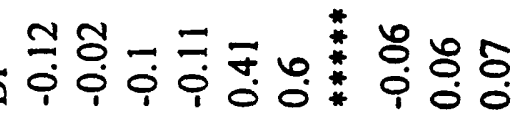

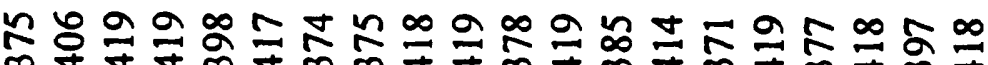

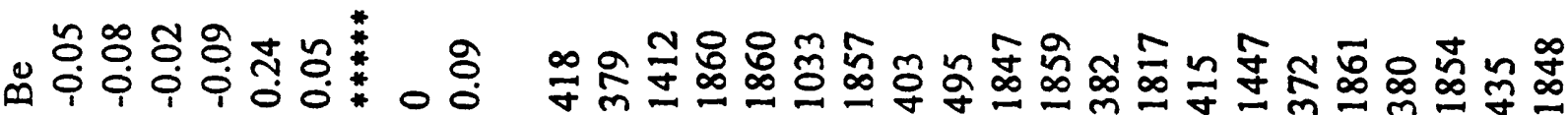
ตீ

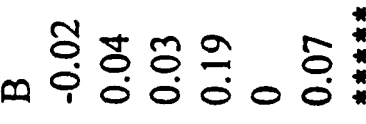
융

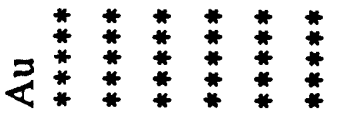

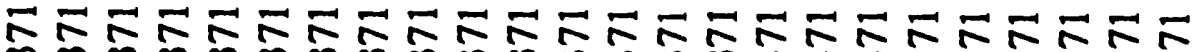

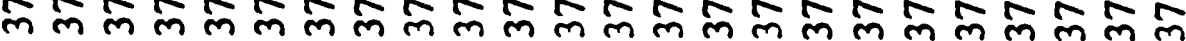

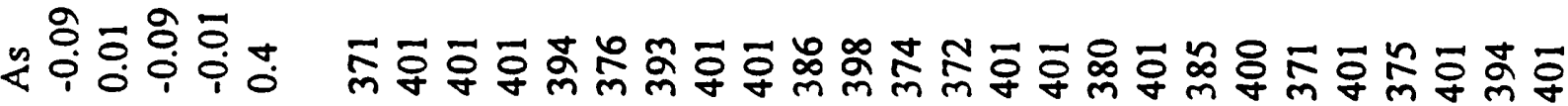

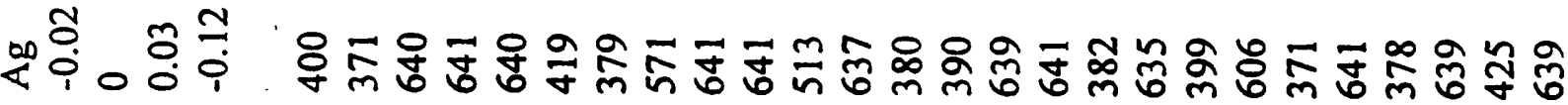

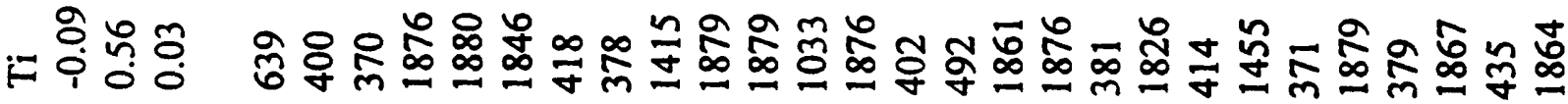

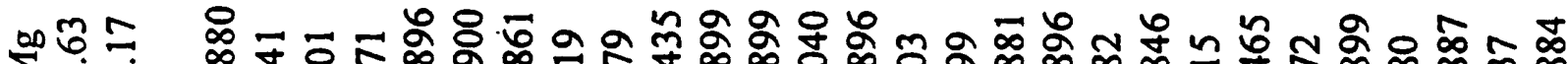

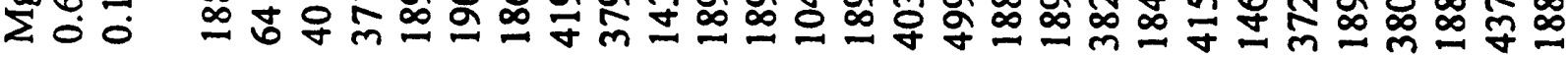

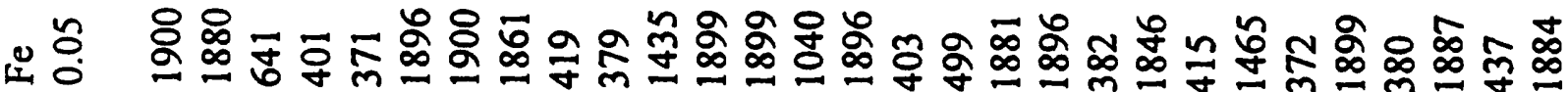
i

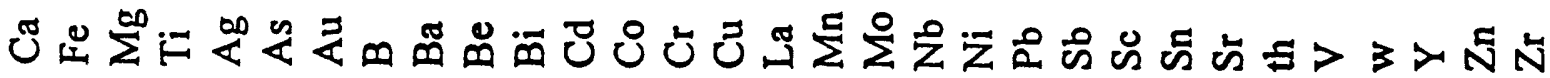




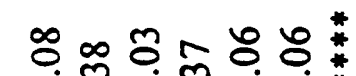

సै

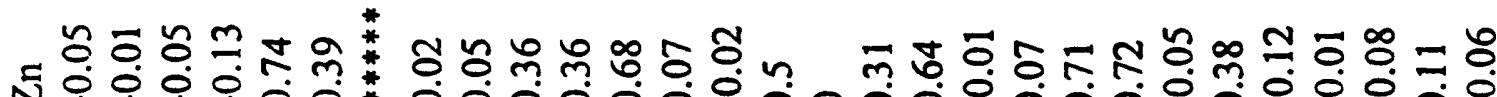

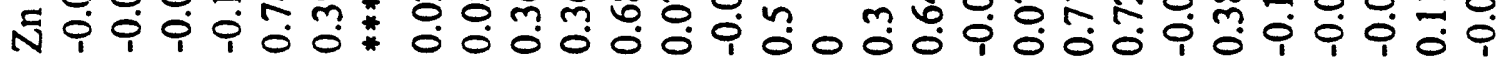
= ㅇํㅇㅇํ.

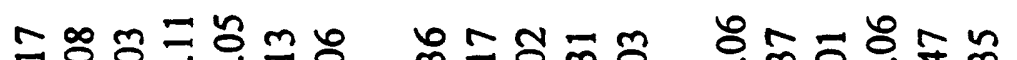
$\nu$ > ३ ț $>$ >

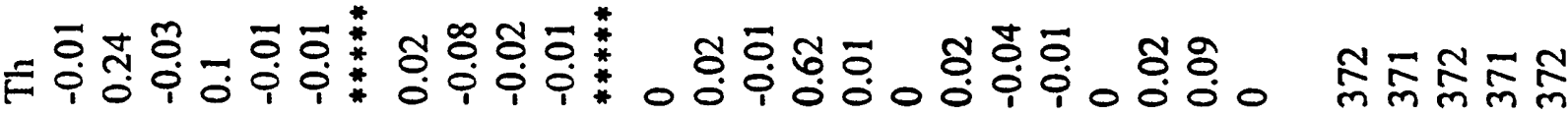
山

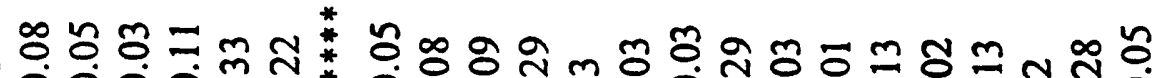
ઈี ن ๘ :

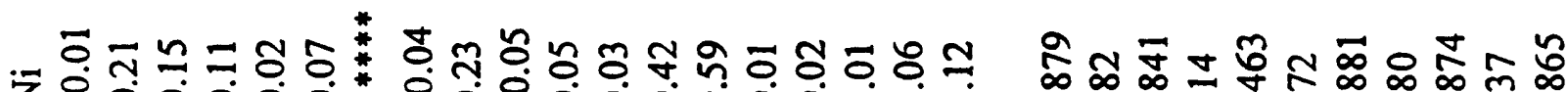
Z

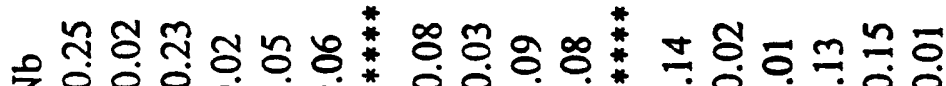
Z

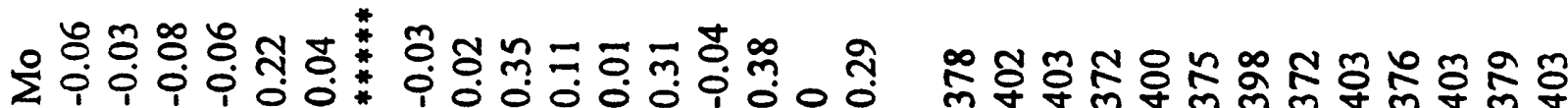
동 ஸุ

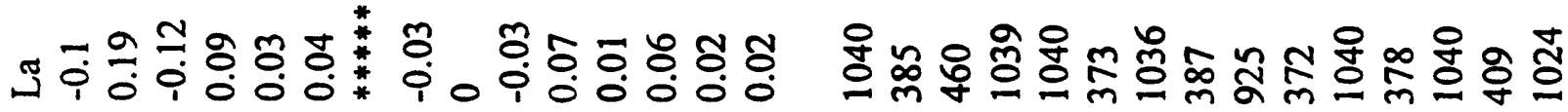

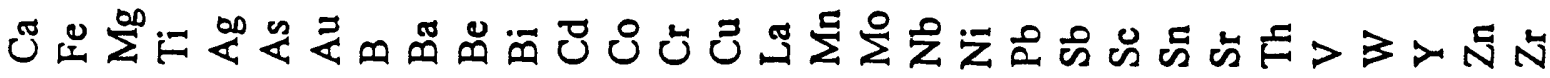




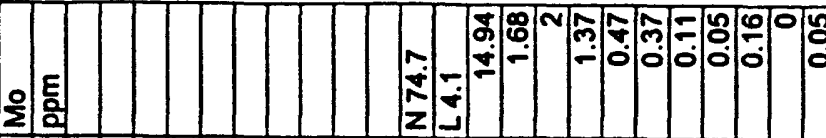

돈통

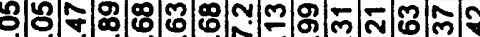
- 0 O 0 N m N

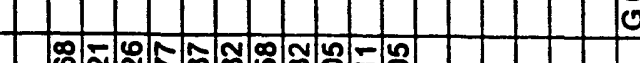

w

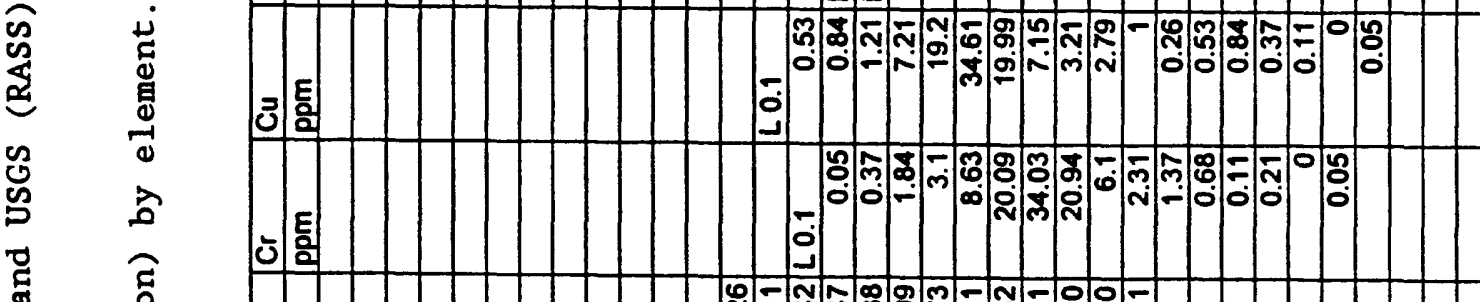

电

임음

$\omega$

స్ํ:

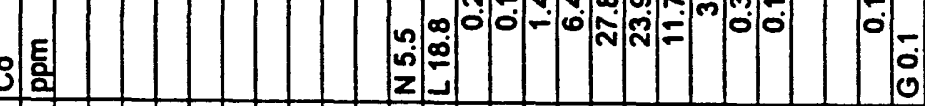

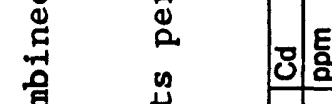

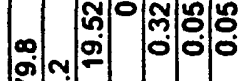

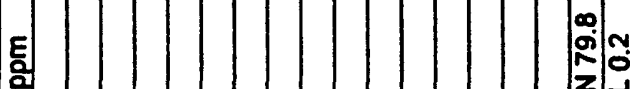

틍

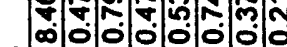

$\infty$

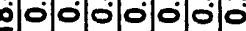

ำ.

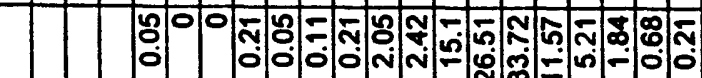

i.

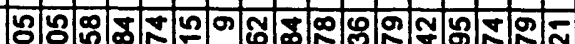

$0 \%$

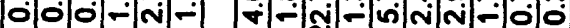

告 
(s)

官

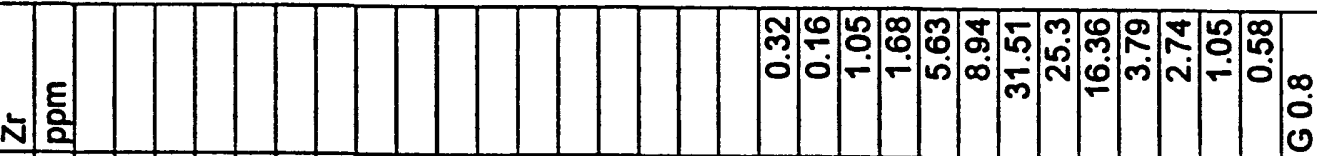

00

虫 定

(2)

o

사맘

๓

क् :

둥

0 .

당

2.

낭

0 용

웅

岁

O.

$\ddot{0}$

$0 \underset{\pi}{0}$

40

m.

\%)

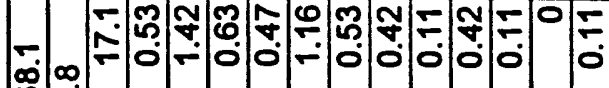

도응

틍

을

z:

3 틍

ஸั

$>$ 틍

o 0 0 0 -

○े
타응
மே
ふั ํㅡㅇ

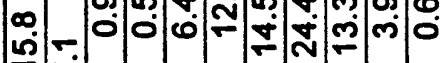
ב-1

है

+ o

0

40

iो 4

त् 0

足 2

त) 0

덛다

总 焉

on

4) 4

䗆 م

㲾 0

0

मे ते

का क

. 긍

- 宁

$\partial$ o

릉

है 㿟

足

\&

U

ผ 틍

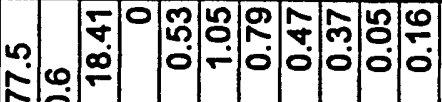

$2-1$

ऊ) 응

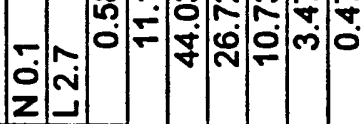

ڤุ

옹 팅

テ

क्ष

인 응

으름

\begin{tabular}{lll}
2 & 0 \\
\hline & 1 & \\
\hline
\end{tabular}

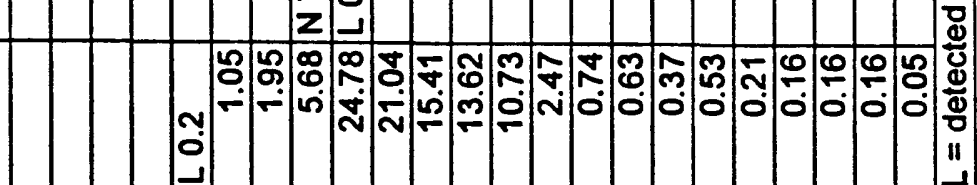

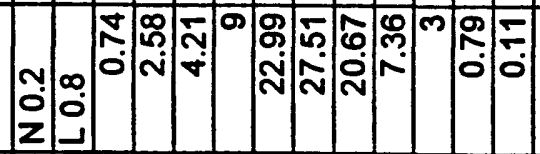

능 용

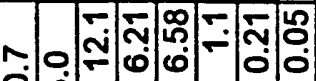

กิ่

2

(1) है

os

욜

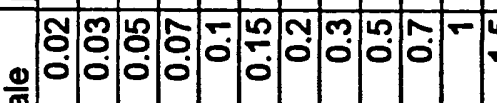

$\frac{1}{8}$

क 
Table D12. Statistical summary of spectrographic analyses of NURE stream sediments. The list below gives the minimum, maximum, and mean values (in ppm, parts per million) as well as the standard deviations of all unqualified values in addition to the numbers of qualified values.

[Qualifiers include: Valid, number of samples used in statistical analysis; $B$, number of samples not analyzed; $N$, number of samples not detected]

\begin{tabular}{|c|c|c|c|c|c|c|c|}
\hline & \multicolumn{6}{|c|}{ STANDARD } \\
\hline \multicolumn{2}{|c|}{ ELEMENT } & MAX. & MEAN & DEV. & VALID & $B$ & $\mathbf{N}$ \\
\hline Ag & 5.00 & 69.00 & 11.95 & 11.39 & 98 & 22 & 2031 \\
\hline Al & 11390.00 & 114200.00 & 62843.35 & 11246.82 & 2151 & 0 & 0 \\
\hline $\mathrm{Au}$ & 0.06 & 9.07 & 0.85 & 1.32 & 102 & 0 & 2049 \\
\hline $\mathrm{Ba}$ & 197.00 & 3572.00 & 662.95 & 266.01 & 1963 & 0 & 188 \\
\hline $\mathrm{Bi}$ & 5.00 & 143.00 & 9.15 & 9.53 & 511 & 22 & 1618 \\
\hline $\mathrm{Ca}$ & 2310.00 & 264400.00 & 26846.90 & 24025.95 & 2135 & 0 & 16 \\
\hline$C d$ & 5.00 & 75.00 & 8.33 & 8.27 & 188 & 22 & 1941 \\
\hline $\mathrm{Ce}$ & 16.00 & 307.00 & 75.77 & 29.79 & 2140 & 0 & 11 \\
\hline $\mathrm{Cl}$ & 64.00 & 1194.00 & 195.95 & 89.77 & 451 & 0 & 1700 \\
\hline Co & 1.70 & 51.50 & 11.77 & 4.99 & 2144 & 0 & 7 \\
\hline $\mathrm{Cr}$ & 9.00 & 947.00 & 62.052 & 59.81 & 2077 & 0 & 74 \\
\hline Cs & 1.50 & 592.30 & 8.12 & 14.59 & 2088 & 0 & 63 \\
\hline Cy & 10.00 & 3617.00 & 69.66 & 137.83 & 2114 & 22 & 15 \\
\hline Dy & 1.00 & 40.00 & 4.79 & 2.13 & 2090 & 0 & 61 \\
\hline Eu & 0.30 & 3.90 & 1.19 & 0.32 & 2137 & 0 & 14 \\
\hline $\mathrm{Fe}$ & 4186.00 & 387100.00 & 29909.82 & 19769.24 & 2150 & 0 & 1 \\
\hline Hf & 0.90 & 229.10 & 11.33 & 14.07 & 2140 & 0 & 11 \\
\hline $\mathrm{K}$ & 4370.00 & 51720.00 & 18471.23 & 4554.96 & 2103 & 0 & 48 \\
\hline La & 9.00 & 254.00 & 43.25 & 18.44 & 2109 & 0 & 42 \\
\hline Lu & 0.10 & 3.60 & 0.42 & 0.22 & 2089 & 0 & 62 \\
\hline $\mathrm{Mg}$ & 2093.00 & 108800.00 & 15963.79 & 10377.38 & 2084 & 0 & 67 \\
\hline $\mathrm{Na}$ & 408.00 & 36760.00 & 11123.03 & 5137.46 & 2150 & 0 & 1 \\
\hline $\mathrm{Nb}$ & 20.00 & 83.00 & 32.53 & 11.54 & 263 & 22 & 1866 \\
\hline $\mathrm{Ni}$ & 15.00 & 153.00 & 27.72 & 18.11 & 893 & 22 & 1236 \\
\hline $\mathrm{Pb}$ & 5.00 & 6896.00 & 60.75 & 292.66 & 2000 & 22 & 129 \\
\hline $\mathrm{Rb}$ & 22.00 & 231.00 & 77.83 & 30.57 & 1641 & 0 & 510 \\
\hline $\mathrm{Sb}$ & 2.00 & 804.00 & 19.26 & 74.15 & 344 & 4 & 1803 \\
\hline Sc & 1.90 & 34.30 & 10.35 & 4.14 & 2151 & 0 & 0 \\
\hline $\mathrm{Sm}$ & 1.00 & 42.60 & 5.93 & 2.84 & 2100 & 0 & 51 \\
\hline $\mathrm{Sn}$ & 10.00 & 344.00 & 28.45 & 47.16 & 60 & 22 & 2069 \\
\hline Sr & 219.00 & 1246.00 & 609.88 & 205.29 & 294 & 10 & 1847 \\
\hline $\mathrm{Ta}$ & 1.00 & 10.00 & 2.04 & 1.39 & 182 & 26 & 1943 \\
\hline $\mathrm{Tb}$ & 1.00 & 6.00 & 1.40 & 0.81 & 147 & 21 & 1983 \\
\hline Th & 1.70 & 213.00 & 14.85 & 15.90 & 2145 & 0 & 6 \\
\hline $\mathrm{Ti}$ & 727.00 & 32410.00 & 3759.69 & 2251.01 & 2063 & 0 & 88 \\
\hline $\mathrm{Nu}$ & 0.84 & 153.00 & 6.20 & 7.51 & 2151 & 0 & 0 \\
\hline $\mathrm{v}$ & 13.00 & 791.00 & 98.19 & 74.25 & 2136 & 0 & 15 \\
\hline W & 15.00 & 166.00 & 29.64 & 20.75 & 141 & 22 & 1988 \\
\hline $\mathrm{Yb}$ & 1.10 & 26.80 & 4.29 & 2.02 & 1844 & 0 & 307 \\
\hline $\mathrm{Zn}$ & 23.00 & 9536.00 & 171.97 & 426.75 & 1350 & 6 & 795 \\
\hline & & & $\begin{array}{r}\text { D5 } 4 \\
04\end{array}$ & & & & \\
\hline
\end{tabular}




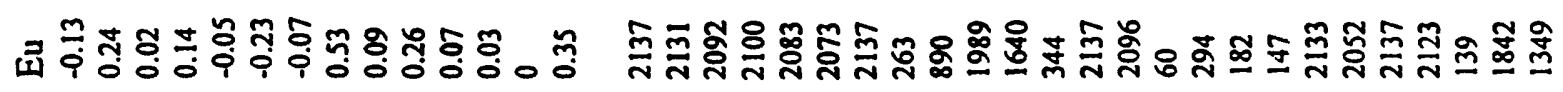

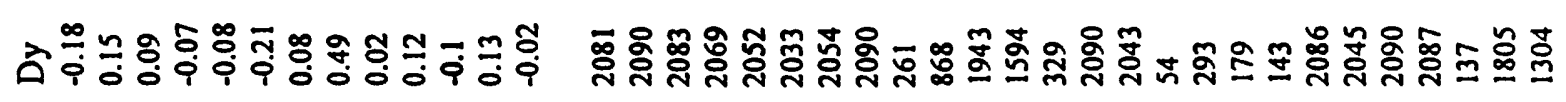

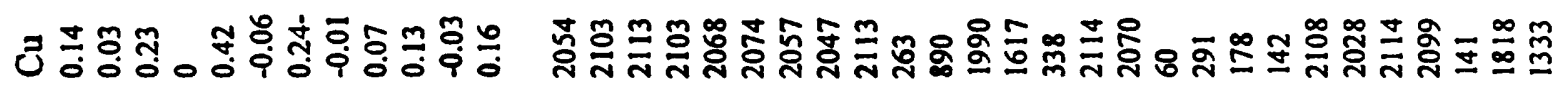

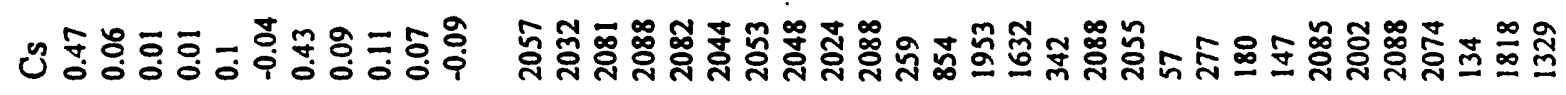

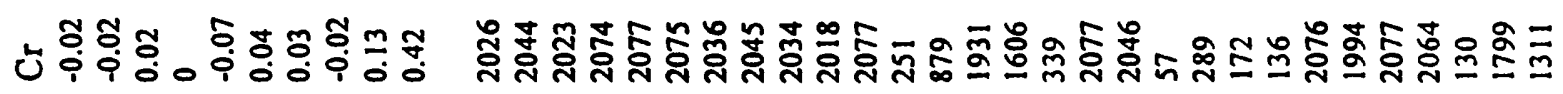

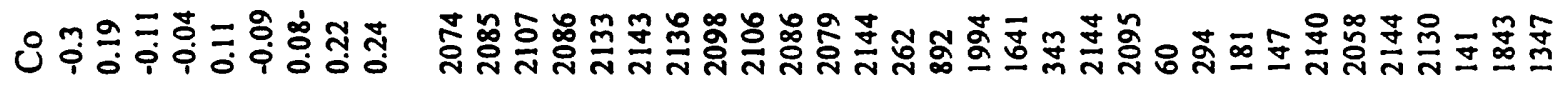

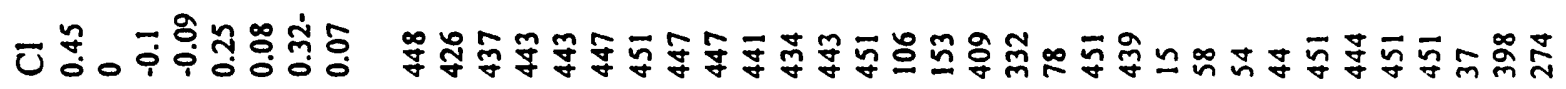

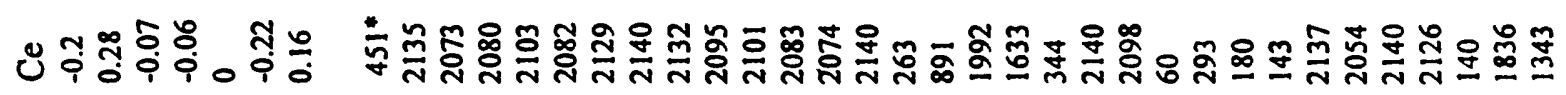

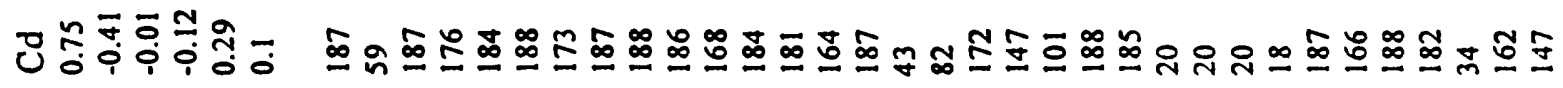

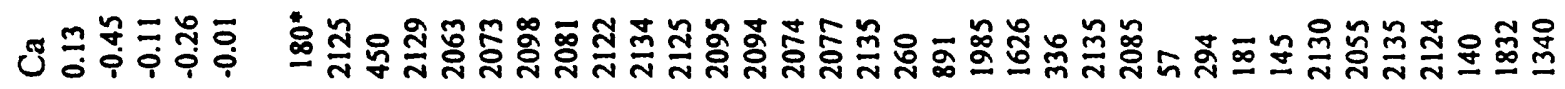

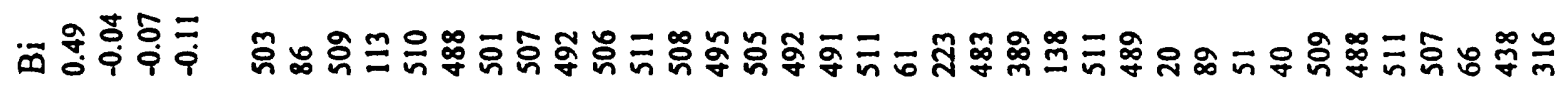

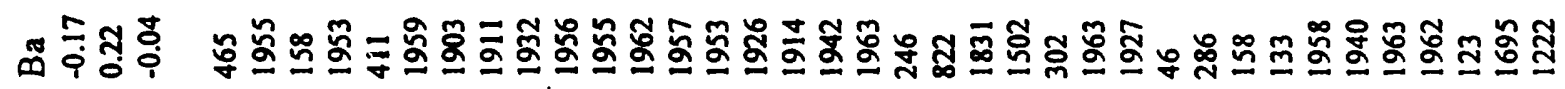

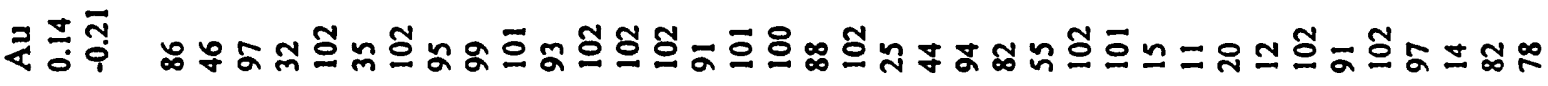

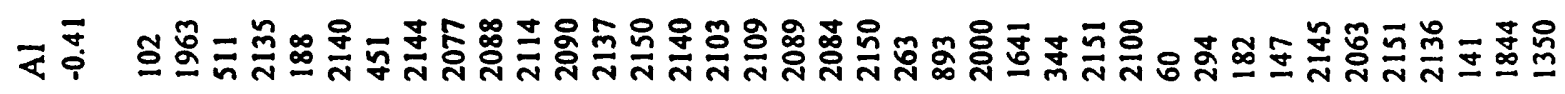

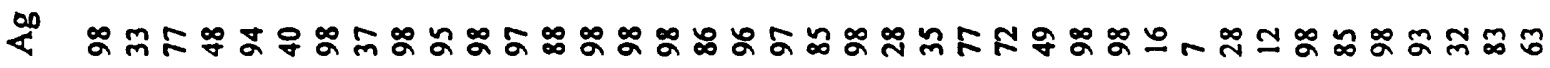

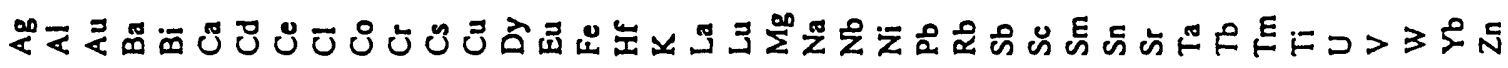


Table D13 (continued). Correlation coefficients (given in the upper part of matrix) and number of correlating pairs (given in the lower part of matrix) from statistical summary of NURE analyses of stream sediments within study area.

\begin{tabular}{|c|c|c|c|c|c|c|c|c|c|c|}
\hline & $\mathrm{Sr}$ & $\mathrm{Ta}$ & $\mathrm{Tb}$ & Th & $\mathrm{Ti}$ & $\mathbf{U}$ & V & W & $\mathrm{Yb}$ & $\mathrm{Zn}$ \\
\hline $\mathrm{Ag}$ & -0.1 & 0.09 & 0.57 & 0.04 & -0.18 & -0.08 & 0.08 & 0.32 & 0.14 & 0.56 \\
\hline $\mathrm{Al}$ & 0.47 & -0.24 & 0.28 & 0.19 & 0.06 & 0.18 & 0.03 & -0.06 & 0.17 & -0.13 \\
\hline $\mathrm{Au}$ & -0.23 & -0.12 & 0.26 & -0.06 & -0.14 & -0.03 & 0 & 0.23 & -0.03 & 0.13 \\
\hline $\mathrm{Ba}$ & 0.26 & 0.16 & -0.03 & -0.22 & 0.04 & -0.12 & -0.19 & -0.15 & -0.15 & -0.04 \\
\hline $\mathrm{Bi}$ & -0.16 & -0.18 & 0.13 & 0.07 & 0.01 & 0.12 & 0.03 & -0.06 & 0.04 & 0.33 \\
\hline $\mathrm{Ca}$ & -0.04 & -0.13 & -0.07 & -0.08 & -0.11 & -0.11 & -0.02 & -0.08 & -0.17 & 0.03 \\
\hline $\mathrm{Cd}$ & -0.14 & 0.91 & 0.11 & -0.09 & -0.2 & -0.07 & -0.06 & 0.2 & -0.03 & 0.86 \\
\hline $\mathrm{Ce}$ & -0.1 & 0.02 & 0.25 & 0.69 & 0.16 & 0.41 & 0.39 & 0.26 & 0.67 & -0.02 \\
\hline $\mathrm{Cl}$ & 0.08 & 0.32 & 0.16 & 0.06 & 0.11 & .0 .08 & 0.19 & -0.02 & 0.07 & 0.2 \\
\hline Co & 0.06 & 0.05 & 0.15 & 0.26 & 0.47 & 0.15 & 0.55 & 0.17 & 0.28 & 0.09 \\
\hline $\mathrm{Cr}$ & 0.03 & -0.01 & 0.2 & -0.02 & 0.11 & -0.09 & 0.32 & 0.18 & -0.01 & 0.02 \\
\hline Cs & 0.01 & 0.3 & 0.24 & 0.11 & 0.02 & 0.12 & 0.03 & 0.26 & 0.35 & 0.61 \\
\hline $\mathrm{Cu}$ & -0.06 & -0.01 & 0.09 & 0.01 & 0.06 & 0.05 & 0.06 & 0.02 & 0.05 & 0.33 \\
\hline Dy & -0.38 & 0.25 & 0.62 & 0.43 & 0.14 & 0.43 & 0.21 & 0.29 & 0.71 & -0.01 \\
\hline $\mathrm{Eu}$ & -0.08 & 0 & 0.38 & 0.35 & 0.19 & 0.14 & 0.3 & 0.35 & 0.45 & 0.01 \\
\hline $\mathrm{Fe}$ & -0.22 & 0.02 & 0.08 & 0.53 & 0.39 & 0.13 & 0.75 & $* * * * *$ & 0.48 & 0.12 \\
\hline Hf & -0.19 & -0.01 & 0.01 & 0.82 & 0.14 & 0.35 & 0.65 & 0.65 & 0.66 & 0 \\
\hline K & -0.22 & 0.02 & -0.07 & -0.02 & -0.08 & -0.09 & -0.2 & -0.09 & 0.06 & -0.03 \\
\hline $\mathrm{La}$ & -0.06 & 0.02 & 0.35 & 0.66 & 0.09 & 0.54 & 0.32 & 0.24 & 0.62 & 0.02 \\
\hline $\mathrm{Lu}$ & -0.19 & 0.23 & 0.69 & 0.71 & 0.17 & 0.55 & 0.41 & 0.52 & 0.84 & 0.03 \\
\hline $\mathrm{Mg}$ & 0.09 & -0.21 & 0.01 & 0 & 0.04 & 0 & 0.09 & -0.24 & -0.08 & 0.05 \\
\hline $\mathrm{Na}$ & 0.49 & -0.24 & -0.02 & 0.33 & 0.08 & 0.28 & 0.19 & 0.02 & 0.21 & -0.08 \\
\hline $\mathrm{Nb}$ & 0.06 & 0.23 & -0.01 & 0.16 & -0.06 & 0.22 & 0.07 & 0.06 & 0.25 & 0.11 \\
\hline $\mathrm{Ni}$ & 0.11 & 0.22 & 0.02 & -0.03 & 0.09 & -0.05 & 0.22 & 0.24 & -0.05 & 0.03 \\
\hline $\mathrm{Pb}$ & -0.04 & 0.08 & 0.13 & 0 & -0.04 & 0.04 & -0.01 & 0.19 & 0.02 & 0.53 \\
\hline $\mathrm{Rb}$ & -0.4 & 0.4 & 0.38 & 0.11 & -0.02 & 0.02 & -0.03 & 0.18 & 0.3 & 0.04 \\
\hline $\mathrm{Sb}$ & -0.41 & 0.62 & 0.6 & -0.05 & -0.04 & 0 & -0.06 & 0.07 & 0 & 0.8 \\
\hline Sc & -0.1 & -0.07 & 0.24 & 0.41 & 0.47 & 0.18 & 0.62 & 0.14 & 0.45 & 0.05 \\
\hline Sm & -0.16 & 0.34 & 0.6 & 0.59 & 0.13 & 0.59 & 0.27 & 0.2 & 0.75 & -0.02 \\
\hline $\mathrm{Sn}$ & -0.19 & -0.19 & 0.74 & -0.15 & -0.13 & -0.17 & -0.18 & -0.13 & -0.15 & -0.03 \\
\hline $\mathrm{Sr}$ & & 0 & 0.06 & -0.14 & -0.16 & 0.01 & -0.13 & -0.07 & -0.15 & -0.1 \\
\hline $\mathrm{Ta}$ & 21 & & 0.29 & -0.01 & 0.05 & 0.12 & -0.1 & -0.03 & 0.24 & 0.37 \\
\hline $\mathrm{Tb}$ & 13 & 37 & & 0.2 & -0.05 & 0.36 & -0.06 & -0.24 & 0.72 & 0.36 \\
\hline Th & 293 & 180 & 146 & & 0.12 & 0.5 & 0.55 & 0.54 & 0.74 & 0 \\
\hline $\mathrm{Ti}$ & 294 & 176 & 140 & 2058 & & 0.06 & 0.61 & -0.06 & 0.17 & -0.02 \\
\hline $\mathrm{U}$ & 294 & 182 & 147 & 2145 & 2063 & & 0.22 & 0.21 & 0.51 & 0.02 \\
\hline V & 294 & 180 & 147 & 2131 & 2063 & 2136 & & 0.42 & 0.44 & -0.01 \\
\hline $\mathbf{w}$ & 13 & 26 & 13 & 141 & 136 & 141 & 140 & & 0.5 & 0.21 \\
\hline $\mathrm{Yb}$ & 212 & 177 & 139 & 1841 & 1778 & 1844 & 1833 & 128 & & 0.07 \\
\hline $\mathrm{Zn}$ & 201 & 127 & 105 & 1349 & 1282 & 1350 & 1338 & 82 & 1175 & \\
\hline
\end{tabular}




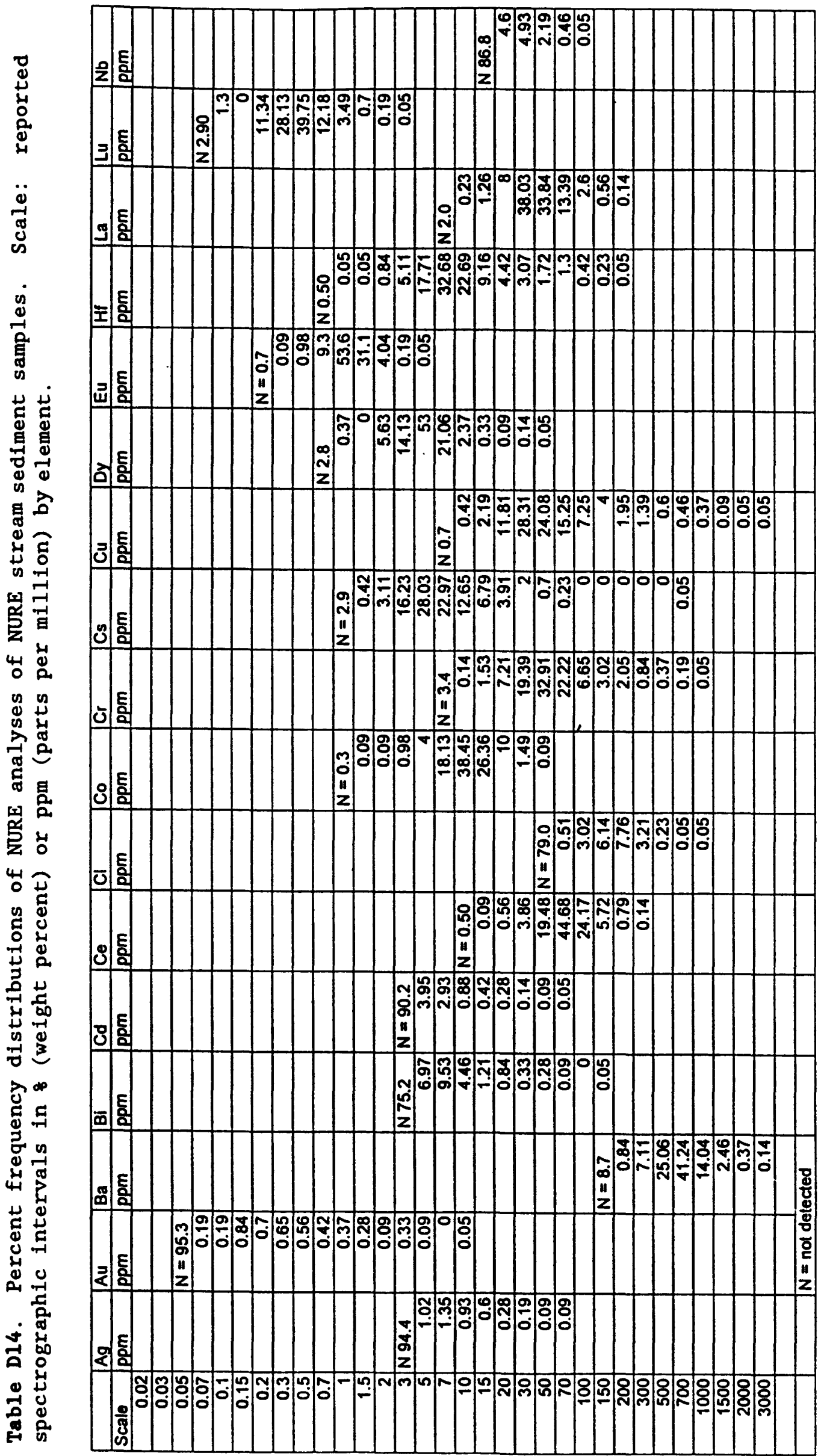




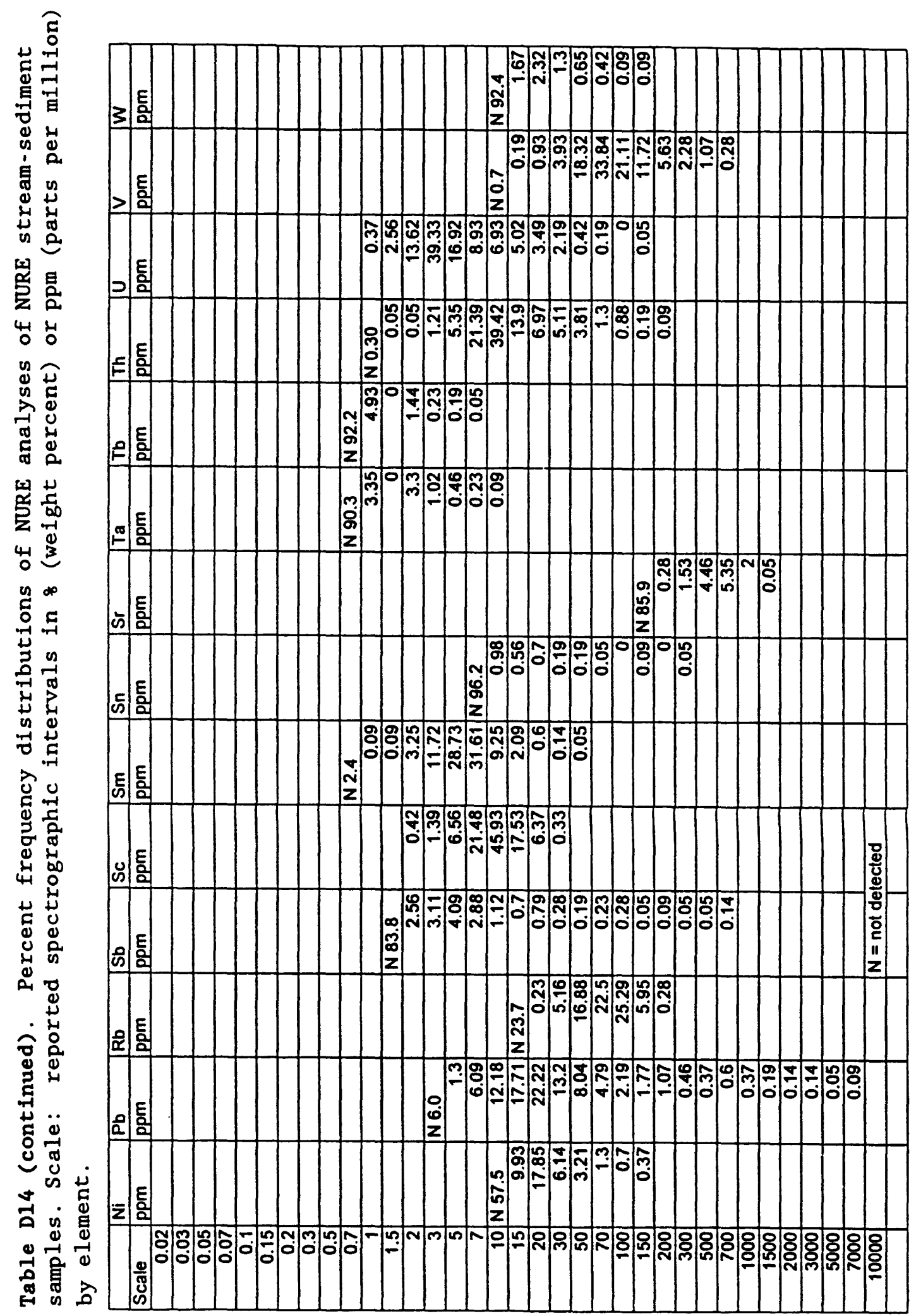


Table D14 (continued). Percent frequency distributions of NURE analyses of NURE stream sediment samples. Scale: reported spectrographic intervals in \& (weight percent) or ppm (parts per million) by element.

\begin{tabular}{|c|c|c|c|c|c|c|c|}
\hline & $\mathrm{Al}$ & $\mathrm{Ca}$ & $\mathrm{Fe}$ & $\mathbf{K}$ & $\mathrm{Mg}$ & $\mathrm{Na}$ & $\pi i$ \\
\hline Scale & $\%$ & $\%$ & $\%$ & $\%$ & $\%$ & $\%$ & $\%$ \\
\hline 0.05 & & & & & & 0.05 & N4.1 \\
\hline 0.07 & & & & & & 0.09 & 0.05 \\
\hline 0.1 & & & & & & 0.23 & 0.23 \\
\hline 0.15 & & N 0.7 & & & N 3.1 & $\overline{0.46}$ & 1.86 \\
\hline 0.2 & & 0.05 & & & 0.09 & 0.74 & 17.02 \\
\hline 0.3 & & 0.93 & & N2.2 & 0.33 & 3.39 & 44.72 \\
\hline 0.5 & & 1.91 & 0.09 & 0.09 & 2.19 & 8.51 & 25.38 \\
\hline 0.7 & & 5.16 & 0.79 & 0.37 & 13.11 & 17.99 & 4.56 \\
\hline 1 & 0.05 & 10.83 & 5.81 & 4.93 & 25.66 & 31.57 & 1.07 \\
\hline 1.5 & 0.09 & 17.02 & 15.48 & 41.1 & 27.01 & 26.5 & 0.46 \\
\hline 2 & 0.56 & 30.03 & 24.78 & 45.84 & 17.62 & 9.53 & 0.37 \\
\hline 3 & 1.63 & 18.64 & 34.77 & 5.3 & 7.72 & 0.88 & $\overline{0.19}$ \\
\hline 5 & 22.59 & 7.25 & 11.95 & 0.14 & 2.09 & & \\
\hline 7 & 71.22 & 3.58 & 4.14 & & 0.74 & & \\
\hline 10 & 3.86 & 2.42 & 1.49 & & 0.33 & & \\
\hline 15 & & 1.21 & 0.51 & & & & \\
\hline 20 & & 0.19 & 0.09 & & & & \\
\hline 30 & & 0.05 & 0 & & & & \\
\hline 50 & & & 0.05 & & & & \\
\hline 70 & & & & & & & \\
\hline 100 & & & $N=$ not de & etected & & & \\
\hline
\end{tabular}


Table D15. Statistical summary of atomic absorption spectrographic analyses of combined NURE and USGS (RASS) stream sediments. For qualified values.

[Valid, number of samples used in statistical analysis; B, number of samples not analyzed; L, number of samples detected with spectrographic analysis, but measured below lowest standard; ppb, parts per billion]

\begin{tabular}{cccccccc}
\hline $\begin{array}{c}\text { Element } \\
(\mathrm{ppb})\end{array}$ & $\begin{array}{c}\text { Minimum } \\
(\mathrm{ppb})\end{array}$ & $\begin{array}{r}\text { Maximum } \\
(\mathrm{ppb})\end{array}$ & $\begin{array}{c}\text { Mean } \\
(\mathrm{ppb})\end{array}$ & $\begin{array}{l}\text { Standard } \\
\text { deviation }\end{array}$ & Valid & B & L \\
\hline $\mathrm{Au}$ & 0.001 & 14.0 & 0.70 & 0.58 & 1683 & 94 & 124 \\
\hline
\end{tabular}

Table D16. Correlation coefficients ("Au") and numbers of correlating pairs ("Pairs") of stream-sediment gold values as determined by atomic-absorption spectrographic methods.

[N.D., not determined]

\begin{tabular}{|c|c|c|c|c|c|}
\hline Element & $\mathrm{Au}$ & Pairs & Element & $\mathrm{Au}$ & Pairs \\
\hline $\mathrm{Ca}$ & 0 & 1682 & Mn & 0.15 & 1679 \\
\hline $\mathrm{Fe}$ & 0.06 & 1683 & Mo & 0.19 & 350 \\
\hline $\mathbf{M g}$ & 0.04 & 1683 & $\mathrm{Nb}$ & 0.02 & 440 \\
\hline $\mathrm{Ti}$ & 0.01 & 1664 & $\mathrm{Ni}$ & -0.03 & 1667 \\
\hline $\mathrm{Ag}$ & 0.21 & 581 & $\mathrm{~Pb}$ & 0.24 & 1679 \\
\hline As & 0.09 & 355 & $\mathrm{Sb}$ & 0.09 & 336 \\
\hline $\mathrm{Au}$ & N.D. & 325 & Sc & -0.02 & 1653 \\
\hline B & 0.01 & 1680 & $\mathrm{Sn}$ & 0.32 & 367 \\
\hline $\mathrm{Ba}$ & 0.03 & 1683 & $\mathrm{Sr}$ & -0.06 & 1347 \\
\hline $\mathrm{Be}$ & 0.22 & 1655 & Th & -0.01 & 326 \\
\hline $\mathrm{Bi}$ & 0.09 & 373 & V & 0.04 & 1682 \\
\hline Cd & 0.02 & 333 & $\mathbf{W}$ & -0.05 & 334 \\
\hline Co & -0.04 & 1306 & $Y$ & 0.01 & 1677 \\
\hline $\mathrm{Cr}$ & 0 & 1682 & $\mathrm{Zn}$ & 0.24 & 390 \\
\hline $\mathrm{Cu}$ & 0.15 & 1682 & $\mathrm{Zr}$ & 0.04 & 1667 \\
\hline $\mathrm{La}$ & -0.01 & 963 & & & \\
\hline
\end{tabular}


Table D17. Percent frequency distribution of gold values in NURE and USGS (RASS) stream-sediments as determined by atomic-absorption spectrographic analyses.

\begin{tabular}{lcll}
\hline Au class & $\begin{array}{l}\text { Percent } \\
\text { frequency }\end{array}$ & Au class & $\begin{array}{l}\text { Percent } \\
\text { frequency }\end{array}$ \\
\hline 0.001 & 6.5 & 0.15 & 0.26 \\
0.001 & 22.09 & 0.2 & 0.68 \\
0.002 & 25.09 & 0.3 & 0.05 \\
0.003 & 5.21 & 0.5 & 0.63 \\
0.005 & 10.99 & 0.7 & 0.32 \\
0.007 & 8.15 & 1 & 0.42 \\
0.01 & 4.58 & 1.5 & 0.21 \\
0.015 & 2.42 & 2 & 0.11 \\
0.02 & 2.31 & 3 & 0.11 \\
0.03 & 1.26 & 5 & 0.42 \\
0.05 & 1 & 7 & 0.05 \\
0.07 & 1.05 & 10 & 0.05 \\
0.1 & 1 & 15 & 0.05 \\
\hline
\end{tabular}




\section{MINE8 AND PROBPECTS IN AND ADJACENT TO THE HELENA NATIONAL FORE8T}

By Christopher Osterman, R.G. Tysda1, J.E. E11iott, and Steve Ludington

\section{INTRODUCTION}

A wide variety of sources were used to compile information on deposit types, mineral commodities, and production history of the mines and prospects in and adjacent to the Helena National Forest (the Forest). These sources include the U.S. Geological Survey Mineral Resource Data System (MRDS); the Anaconda Documents Collection maintained by the American Heritage Center at the University of Wyoming, Laramie, Wyo.; publications from previous mineral resource assessment studies; county records; research reports; theses; data of private industry; and records of current and recent activity maintained by the U.S. Forest Service. Several mining companies allowed us access to their properties and company geologists conducted tours for members of the assessment team, providing information on geology, exploration, and mine and prospect development.

Mines and prospects in and adjacent to the Forest are 1isted in table E1. Site locations of the properties are shown on plate 2 (map scale 1:126,720). Site numbers start from the northwest corner of the map and are grouped by mining district/area. Some sites include more than one property, and other sites have had several names over their history; alternate names are shown in parentheses. The table was compiled from the geologic literature. It is not a compilation of mining claims. Small exploration pits (1-2 ft deep) are widespread in the Forest, but are not cited in this tabulation.

Assignment to a probable deposit type is made for sites where sufficient data exist. Other sites are left as unclassified. Patterns of the distribution of the different deposit types can indicate areas where different types of mineralizing processes were operative. Publically available tonnage and grade data for production, reserves, and identified resources for individual mines and prospects are also included in table E1. These data provide a basis for comparing grades and tonnages of identified resources in and adjacent to the Forest with models developed from a worldwide database.

Analysis of the type and distribution of identified resources is an essential, fundamental step in the process of assessesing for undiscovered mineral resources within a region. Identified resources provide clues to the types and sizes of mineral deposits that may be present but are yet to be discovered. These data are useful in the determination of areas (tracts) that are permissive or favorable for deposit types. The data also are fundamental to understanding the distribution of chemical elements that occur naturally in geologic terranes, whether or not disturbed by humans. From undisturbed areas, such data provide the background (baseline) geochemical characteristics needed for comparison with characteristics that potentially could be produced by disturbance or development. 


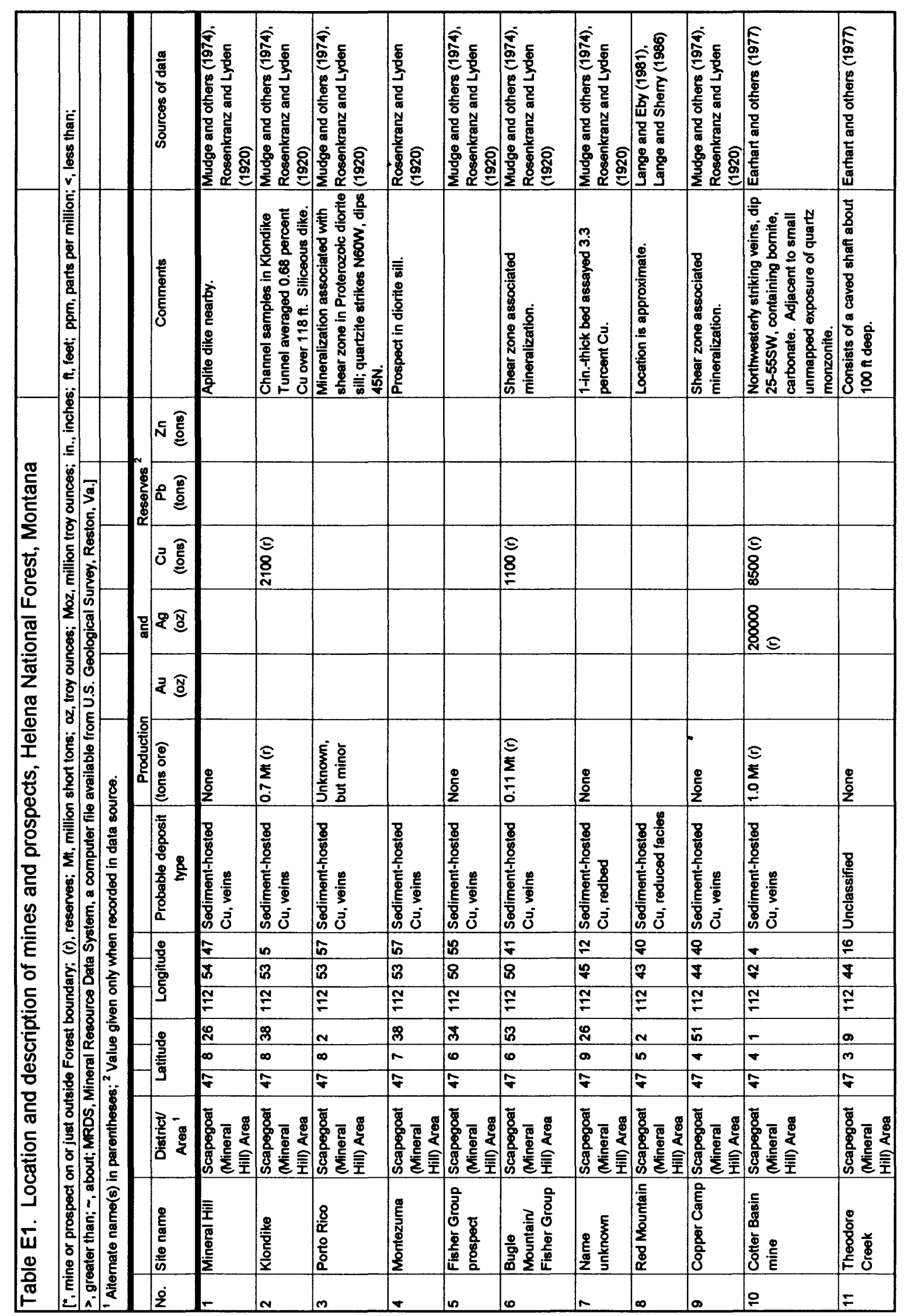




\begin{tabular}{|c|c|c|c|c|c|c|c|c|c|c|c|c|}
\hline 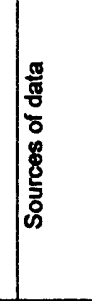 & 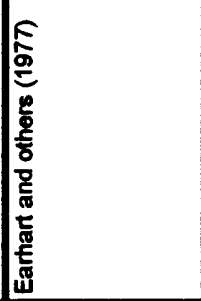 & 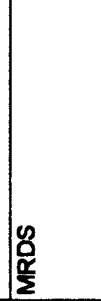 & 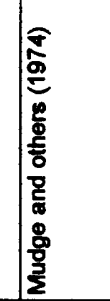 & 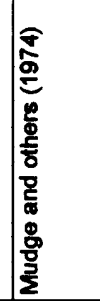 & 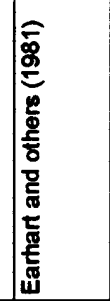 & 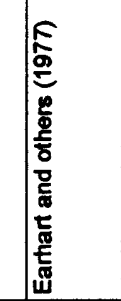 & 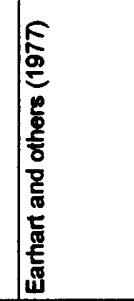 & 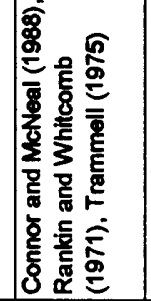 & 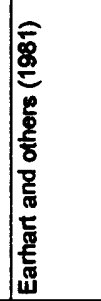 & 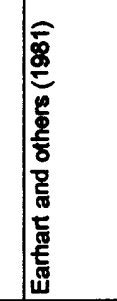 & 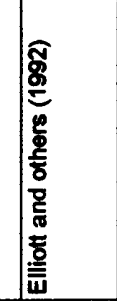 & 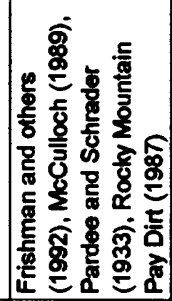 \\
\hline 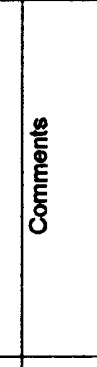 & 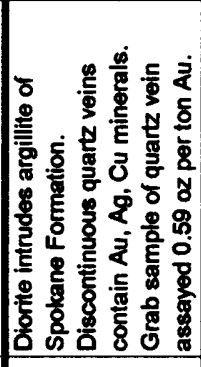 & & 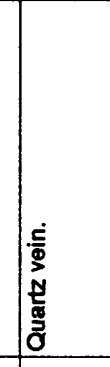 & 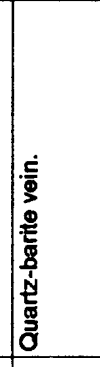 & 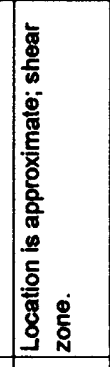 & 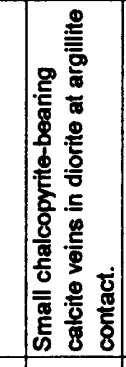 & & 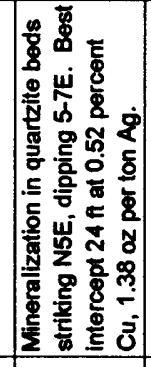 & & & 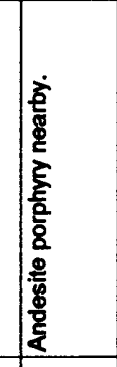 & \\
\hline \multicolumn{13}{|l|}{ 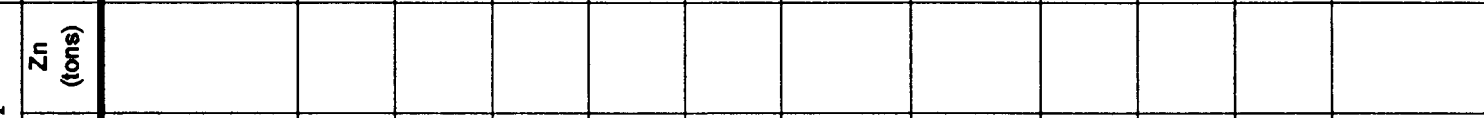 } \\
\hline 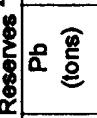 & & & & & & & & & & & & \\
\hline $\overrightarrow{0}$ & & & & & & & & జ్ & & & & \\
\hline 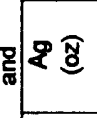 & & & & & & & & $\begin{array}{l}8 \\
\\
\end{array}$ & & & & \\
\hline ₹ तु & & & & & & & & & & & & 8 \\
\hline & & & & & & 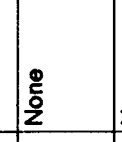 & 旁 & & & & & \\
\hline 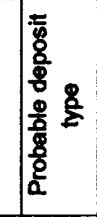 & 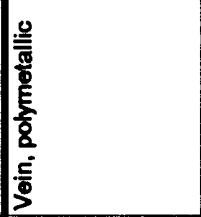 & & 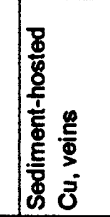 & 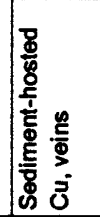 & 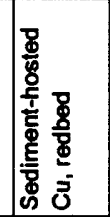 & 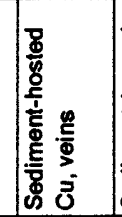 & 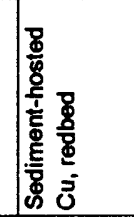 & 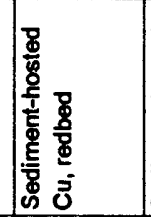 & 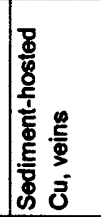 & 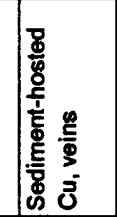 & 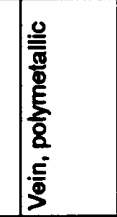 & 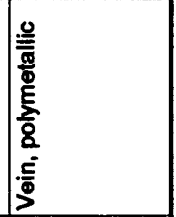 \\
\hline-8 & 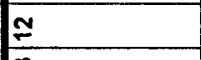 & 9 & I & $F$ & is & $\infty$ & $\%$ & $\tilde{\infty}$ & $\%$ & $\bar{m}$ & 8 & $\infty$ \\
\hline 茞 & 8 & F & / & $\%$ & $\bar{m}$ & ని & 8 & $\hat{\wedge}$ & $\pi$ & $\bar{m}$ & 9 & 18 \\
\hline$\overline{0}$ & $\underline{\underline{F}}$ & $\cong$ & $\cong$ & $\cong$ & $\cong$ & $\stackrel{N}{=}$ & $\cong$ & $\cong$ & $\cong$ & $\cong$ & $\cong$ & $\cong$ \\
\hline & a & 2 & 9 & I & $\div$ & $\mid \begin{array}{l}0 \\
0 \\
\end{array}$ & 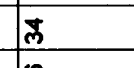 & in & 9 & $\%$ & $\infty$ & 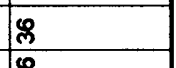 \\
\hline 嘻 & $N$ & 0 & $\stackrel{m}{m}$ & $\simeq$ & $\infty$ & $\div$ & $\infty$ & 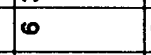 & 10 & 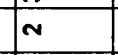 & 8 & 8 \\
\hline & $F$ & $F$ & 7 & 7 & $F$ & $F$ & $F$ & F & 7 & $F$ & 9 & 9 \\
\hline 童- & 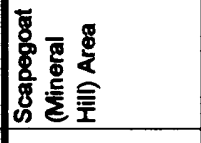 & 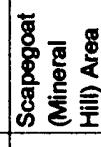 & 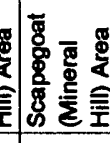 & 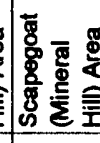 & 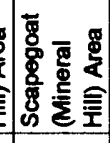 & 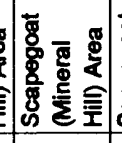 & 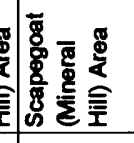 & 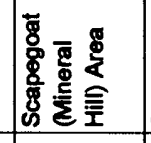 & 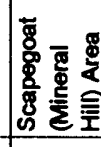 & 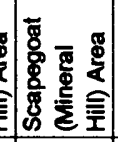 & 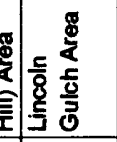 & 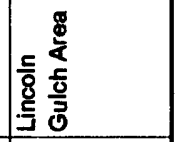 \\
\hline 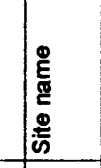 & 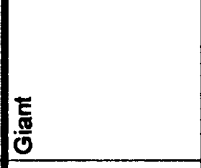 & 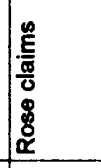 & 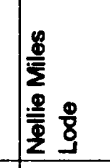 & 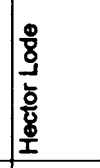 & 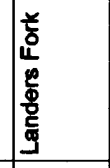 & 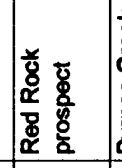 & 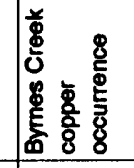 & 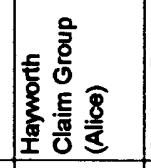 & 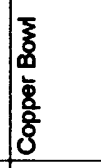 & \begin{tabular}{|l}
$\overline{\overline{\underline{T}}}$ \\
$\underline{\underline{\underline{\underline{z}}}}$ \\
\end{tabular} & 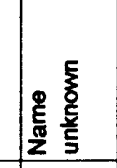 & 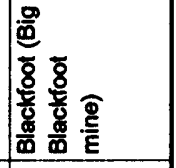 \\
\hline$\dot{i}$ & $\underline{\simeq}$ & $\cong$ & $I$ & $\underline{0}$ & $\dddot{0}$ & $E$ & $\Phi$ & $\Phi$ & 8 & $\bar{\Lambda}$ & $\mathbb{N}$ & \\
\hline
\end{tabular}

辛 


\begin{tabular}{|c|c|c|c|c|c|c|c|c|c|c|c|}
\hline 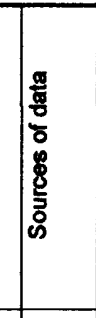 & 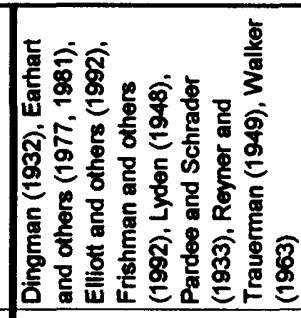 & 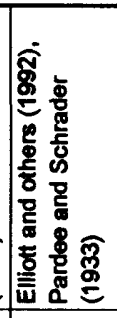 & 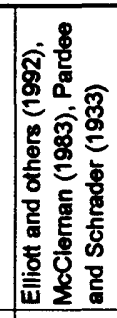 & 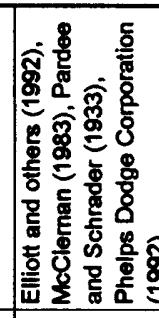 & 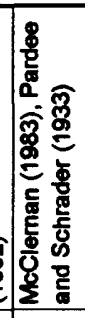 & 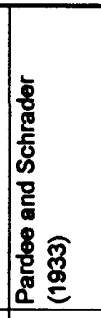 & 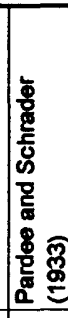 & 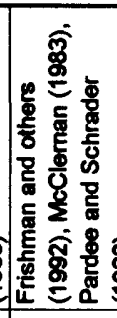 & 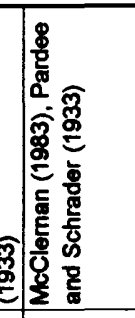 & 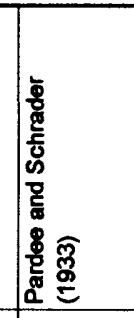 & 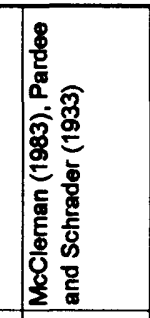 \\
\hline 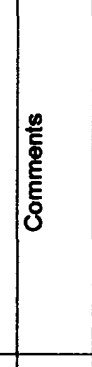 & 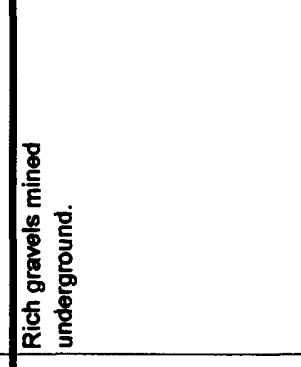 & 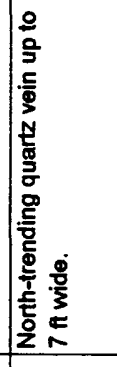 & & 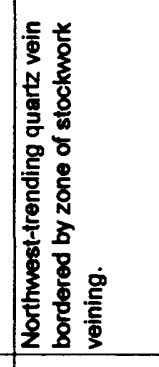 & 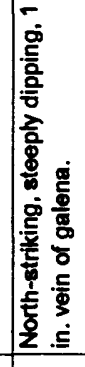 & 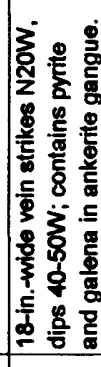 & 递 & 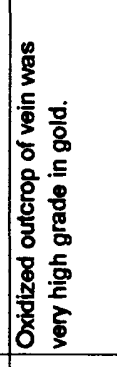 & 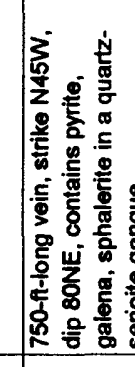 & 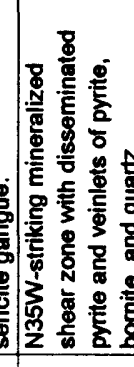 & 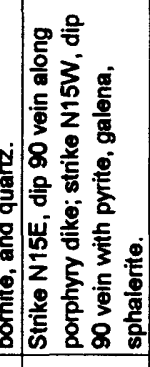 \\
\hline 要 & & & & & & & & & & & \\
\hline 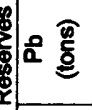 & & & & & & & & & & & \\
\hline 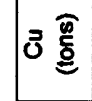 & & & & & & & & & & & \\
\hline 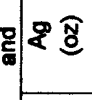 & & & & $\frac{5}{3}$ & & & & & & & \\
\hline ₹ $\bar{\sigma}$ & 8 & & & 8 & & & & 通 & & & \\
\hline 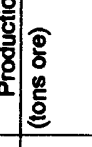 & & 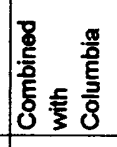 & 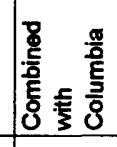 & 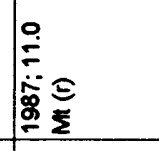 & & & & & & & \\
\hline 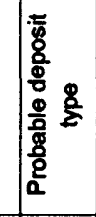 & 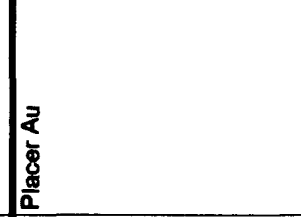 & 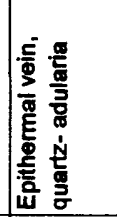 & 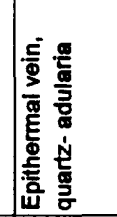 & 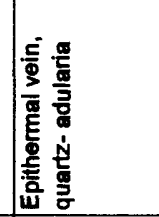 & 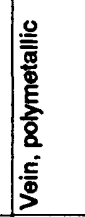 & 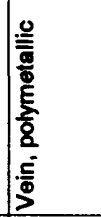 & 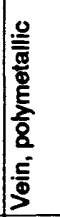 & 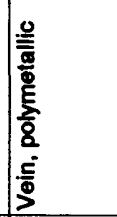 & 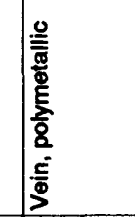 & 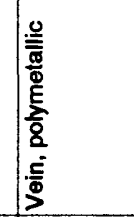 & 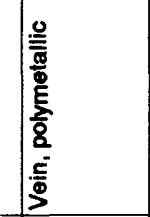 \\
\hline $4 \frac{8}{3}$ & $\infty$ & $\hat{n}$ & $\bar{\pi}$ & 8 & $E$ & & $\%$ & $\infty$ & 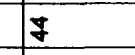 & $\infty$ & $\bar{m}$ \\
\hline 73 & 8 & $\bar{m}$ & 8 & 8 & $\mathbb{N}$ & $\tilde{\mathbf{N}}$ & $\boldsymbol{N}$ & $\mathbb{N}$ & $\tilde{N}$ & $\tilde{N}$ & $\bar{\lambda}$ \\
\hline 3 & $\underline{F}$ & $\cong$ & $\cong$ & $\cong$ & $\cong$ & $\cong$ & $\cong$ & $\cong$ & $\cong$ & $\cong$ & $\cong$ \\
\hline 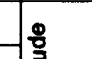 & 8 & $\underset{\sim}{\infty}$ & $\infty$ & $\begin{array}{l}\phi \\
0\end{array}$ & N & $\tilde{\boldsymbol{N}}$ & $\infty$ & $N$ & F & $\bar{n}$ & $\bar{s}$ \\
\hline$-f^{\frac{2}{2}}$ & \begin{tabular}{|l}
8 \\
8
\end{tabular} & $\frac{88}{6}$ & $\frac{18}{10}$ & 18 & $m$ & $\frac{m}{2}$ & $\frac{m}{n}$ & $\frac{m}{n}$ & $\frac{N}{N}$ & $\frac{N}{n}$ & N \\
\hline 总 & 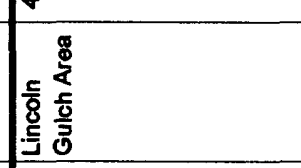 & 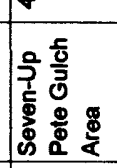 & 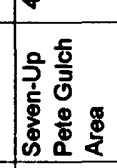 & 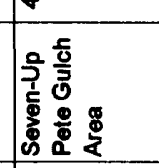 & 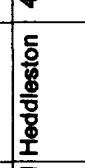 & \begin{tabular}{|l} 
\\
$\frac{5}{8}$ \\
$\frac{8}{8}$ \\
$\mathbf{x}$ \\
\end{tabular} & 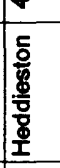 & 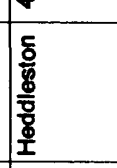 & 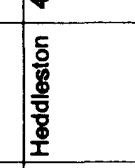 & 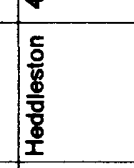 & 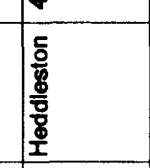 \\
\hline 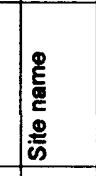 & 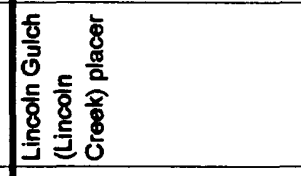 & 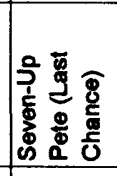 & 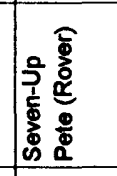 & 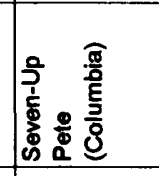 & 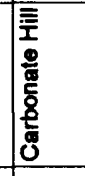 & 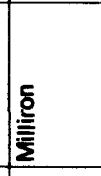 & 烹 & 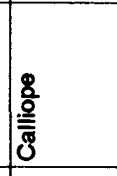 & 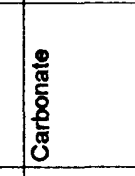 & 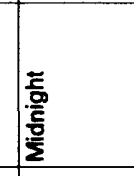 & 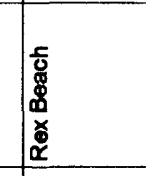 \\
\hline io & 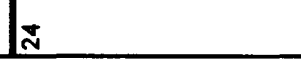 & W & $\stackrel{2}{2}$ & $\pi$ & $\mathbb{\infty}$ & I & \% & in & m & m & 要 \\
\hline
\end{tabular}

똔 


\begin{tabular}{|c|c|c|c|c|c|c|c|c|c|c|c|c|c|c|c|c|}
\hline 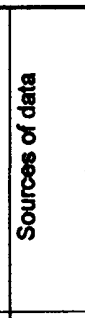 & 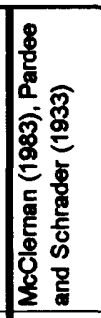 & 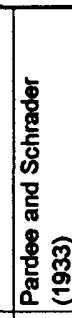 & 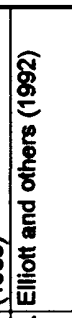 & 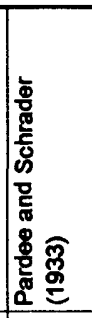 & 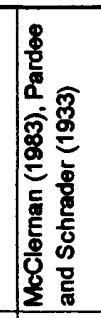 & 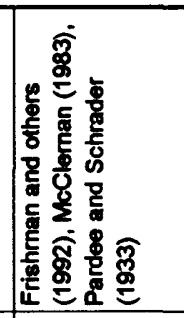 & 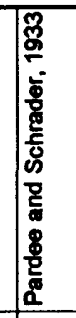 & 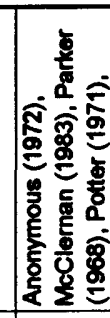 & & 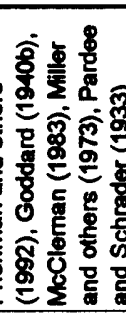 & 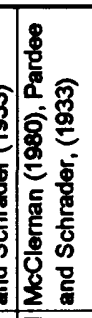 & 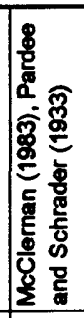 & 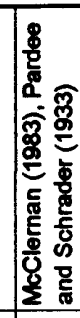 & 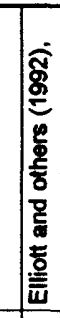 & & 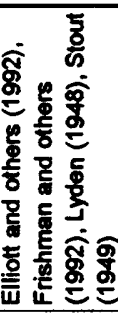 \\
\hline 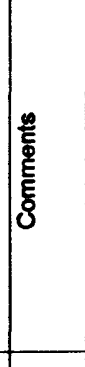 & 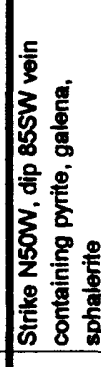 & 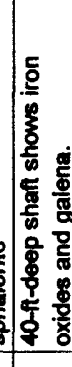 & 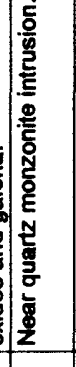 & 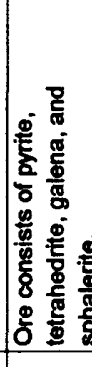 & 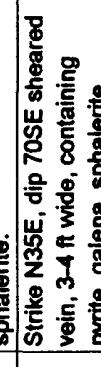 & 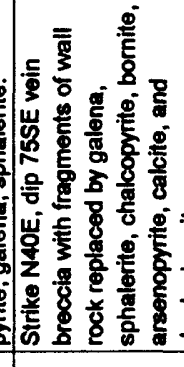 & 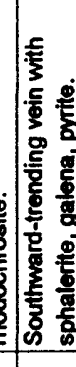 & 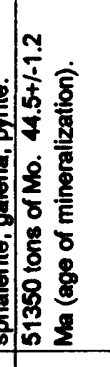 & & 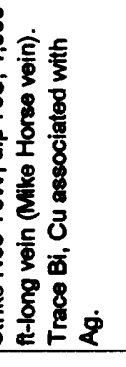 & 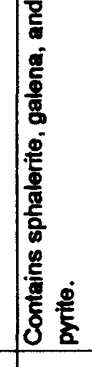 & 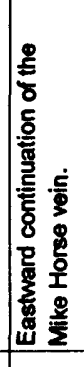 & 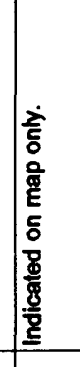 & 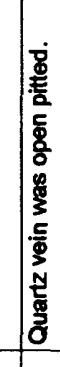 & & \\
\hline N & & & & & & & & & ఫ్ & & & & & & & \\
\hline 要 & & & & & & $\stackrel{\infty}{\stackrel{\infty}{\infty}}$ & & & 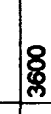 & & & & & 6 & & \\
\hline$\overline{0}$ 要 & & & & & & ֻُ & & 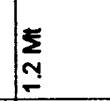 & & & & & & & & \\
\hline $2 \pi \bar{g}$ & & & & & & 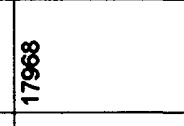 & & 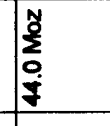 & $\frac{8}{8}$ & & & & & జ్ & & \\
\hline ₹ & & & & & & $\frac{9}{7}$ & & & & & & & & 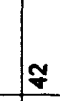 & & \\
\hline 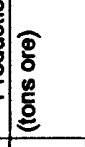 & & & 产 & 8 & & 迢 & & 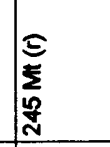 & & 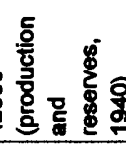 & & & & 8 & & \\
\hline 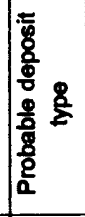 & 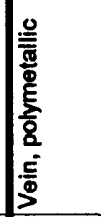 & 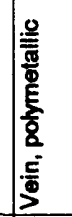 & 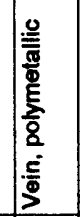 & 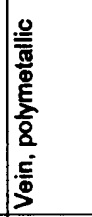 & 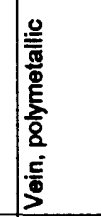 & 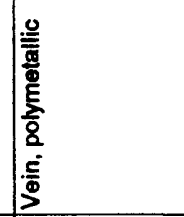 & 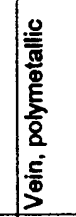 & 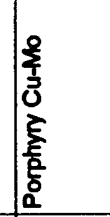 & 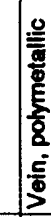 & & 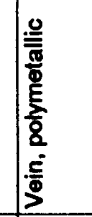 & 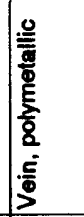 & 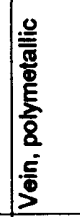 & 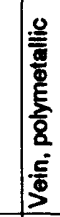 & & \\
\hline$\%$ & 8 & 2 & 9 & \pm & 5 & 8 & $\approx$ & 7 & 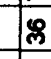 & & $\%$ & $\hat{a}$ & $\bar{\pi}$ & $\infty$ & & $\overline{5}$ \\
\hline 誩 & $\bar{\Lambda}$ & $\bar{\lambda}$ & $\pi$ & $\approx$ & $\bar{\AA}$ & $\bar{\lambda}$ & $\bar{\Lambda}$ & $\tilde{\pi}$ & $\bar{\AA}$ & & $\bar{\Lambda}$ & $\bar{\Lambda}$ & $\stackrel{\infty}{\infty}$ & 8 & & 8 \\
\hline $\bar{s}$ & $\cong$ & $\cong$ & $\cong$ & $\cong$ & $\stackrel{\underline{N}}{=}$ & $\cong$ & $\cong$ & $\cong$ & $\cong$ & & $\cong$ & $\cong$ & $\cong$ & $\stackrel{2}{F}$ & & $\cong$ \\
\hline$\frac{8}{3}$ & 7 & ñ & $\cong$ & $\pi$ & 9 & $a$ & 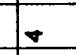 & 8 & 8 & & $m$ & 9 & $\tilde{m}$ & $\infty$ & & 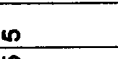 \\
\hline 韵 & $\frac{N}{\sigma}$ & \begin{tabular}{|l}
$N$ \\
\end{tabular} & $\frac{N}{f}$ & $\frac{N}{a}$ & $\frac{N}{F}$ & $\frac{N}{a}$ & $\begin{array}{l}\alpha \\
F\end{array}$ & $\frac{F}{F}$ & $\bar{a}$ & & $\frac{F}{5}$ & $\bar{F}$ & $\frac{1}{5}$ & \begin{tabular}{|l|}
5 \\
$g$ \\
\end{tabular} & & $\begin{array}{l}\frac{6}{6} \\
\frac{6}{9}\end{array}$ \\
\hline 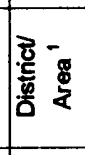 & 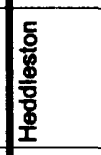 & & 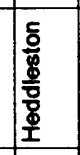 & & 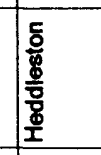 & 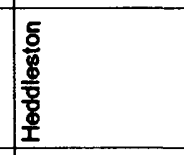 & 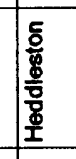 & \begin{tabular}{|l|}
$\frac{5}{2}$ \\
$\frac{\underline{\underline{z}}}{\mathbf{8}}$ \\
\end{tabular} & $\mid \frac{\vec{\phi}}{\underline{\underline{\phi}}}$ & & 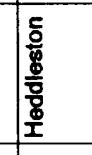 & 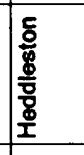 & 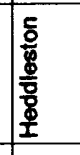 & & & 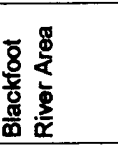 \\
\hline 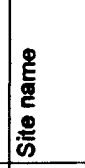 & 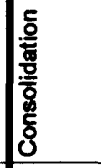 & 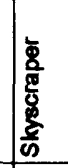 & 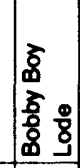 & & \begin{tabular}{|l}
$\overline{\overline{\mathbf{z}}}$ \\
$\underline{\underline{\underline{\underline{\varepsilon}}}}$ \\
\end{tabular} & 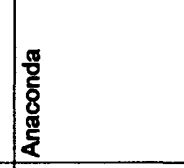 & 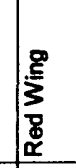 & 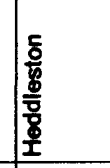 & 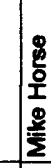 & & 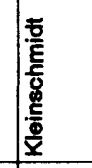 & 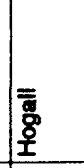 & 产 & 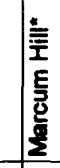 & & 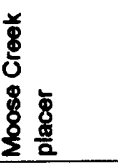 \\
\hline is & $\%$ & $\Phi$ & in & $\infty$ & g & 9 & $F$ & I & $g$ & & $\$$ & 28 & 9 & F & & \\
\hline
\end{tabular}




\begin{tabular}{|c|c|c|c|c|c|c|c|c|c|c|c|}
\hline 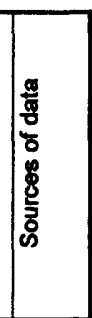 & 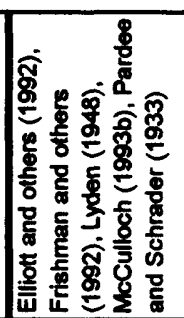 & 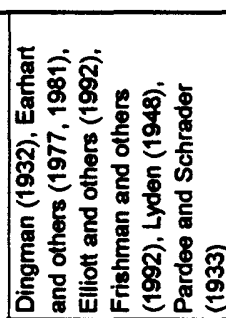 & 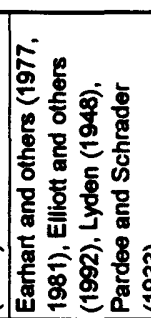 & 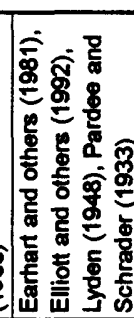 & 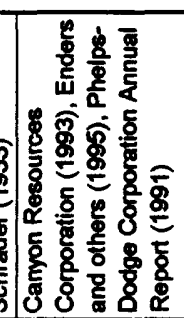 & 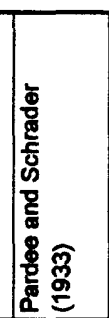 & 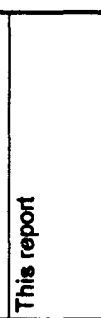 & 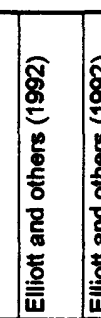 & 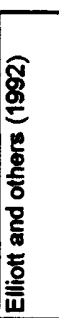 & 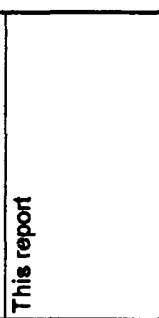 & 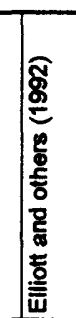 \\
\hline 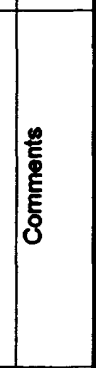 & 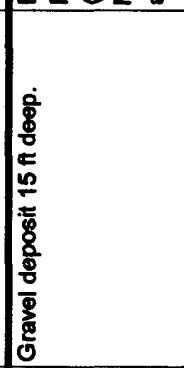 & 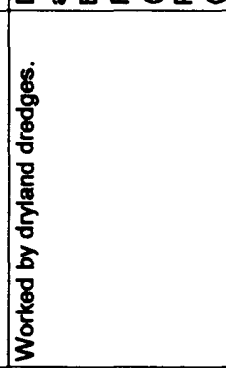 & 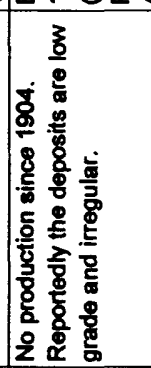 & 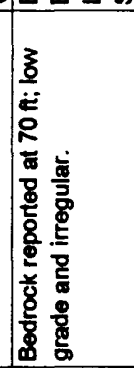 & & & 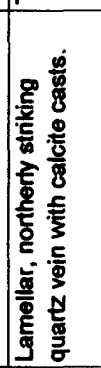 & 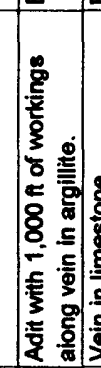 & & 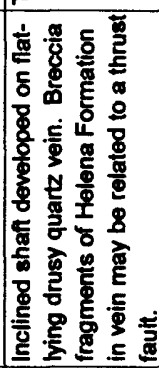 & 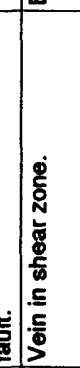 \\
\hline 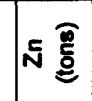 & & & & & & & & & & & \\
\hline$\circ \frac{\bar{g}}{\mathrm{~g}}$ & & & & & & & & & & & \\
\hline ¿ ¿ & & & & & & & & & & & \\
\hline $2 \pi$ & & & & & & & & & & & \\
\hline ₹ & & 8 & & & $\overline{8}_{\infty}^{8}$ & & & & & & \\
\hline $\begin{array}{l}0 \\
\vdots \\
0\end{array}$ & & & & & 站 & & & $\frac{0}{0}$ & $\frac{8}{2}$ & & \\
\hline 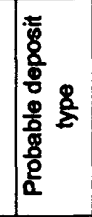 & 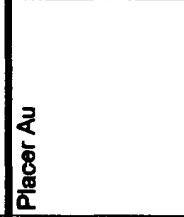 & 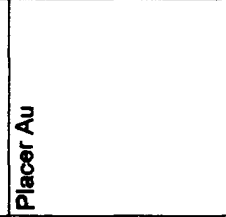 & \begin{tabular}{|l|}
$\frac{z}{3}$ \\
$\frac{8}{8}$ \\
$\frac{8}{a}$ \\
\end{tabular} & 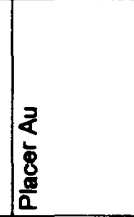 & 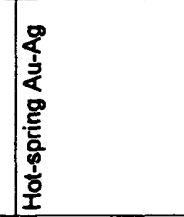 & 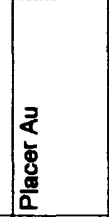 & 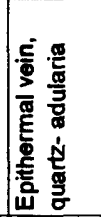 & 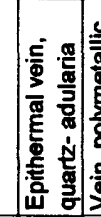 & 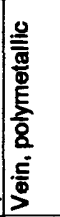 & 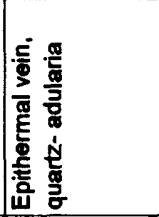 & 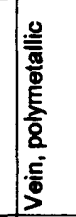 \\
\hline$\frac{8}{3}$ & 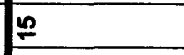 & is & a & 2 & 0 & + & $F$ & $\%$ & 8 & $\hat{\Lambda}$ & 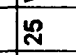 \\
\hline & 8 & F & $F$ & 8 & $\tilde{m}$ & ले & $\infty$ & 8 & $\infty$ & के & $\bar{m}$ \\
\hline ఏ5 & $\underline{N}$ & $\cong$ & $\stackrel{N}{\underline{N}}$ & $\cong$ & $\underline{\Sigma}$ & $\cong$ & $\cong$ & \begin{tabular}{l|l} 
& $\cong$
\end{tabular} & $\stackrel{7}{\mp}$ & $\cong$ & $\cong$ \\
\hline 8 & $B$ & 8 & $\tilde{N}$ & o & 8 & 7 & 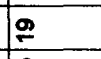 & 8 & $\tilde{N}$ & $\tilde{n}$ & 9 \\
\hline 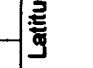 & $\frac{5}{5}$ & \begin{tabular}{|l|} 
年 \\
$g$
\end{tabular} & \begin{tabular}{|l|}
8 \\
9
\end{tabular} & \begin{tabular}{|l}
8 \\
9
\end{tabular} & $\frac{8}{6}$ & $\frac{\pi}{6}$ & \begin{tabular}{|l|} 
\\
6 \\
9
\end{tabular} & S & $\begin{array}{l}5 \\
0\end{array}$ & t5 & \begin{tabular}{|l|l}
5 \\
8
\end{tabular} \\
\hline 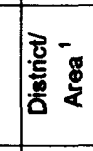 & 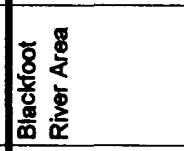 & 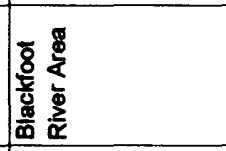 & 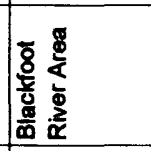 & 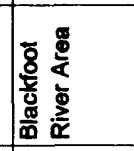 & 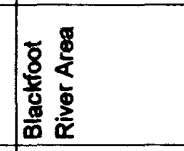 & 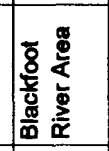 & 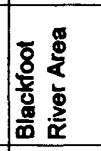 & 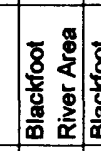 & $\sqrt[\bar{\alpha} \mathbf{\alpha}]{\bar{\alpha}}$ & 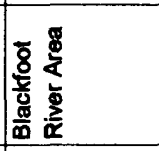 & 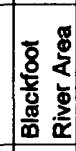 \\
\hline 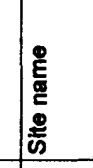 & 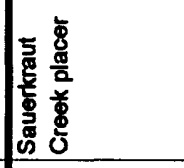 & 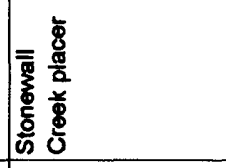 & 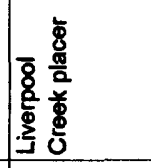 & 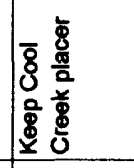 & 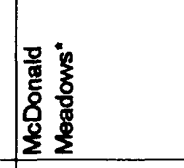 & 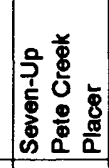 & 语 & 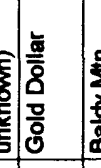 & & 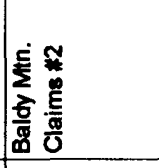 & 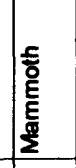 \\
\hline$\dot{z}$ & 9 & 迨 & In & in & 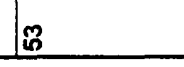 & I & 适 & 18 & 两 & $\infty$ & I \\
\hline
\end{tabular}

败 


\begin{tabular}{|c|c|c|c|c|c|c|c|c|c|c|c|}
\hline 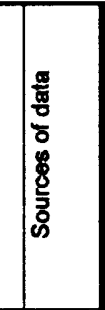 & 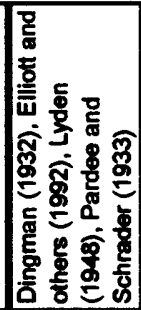 & 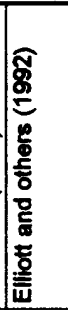 & 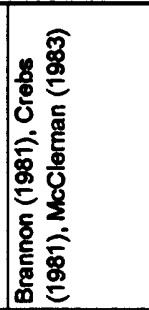 & 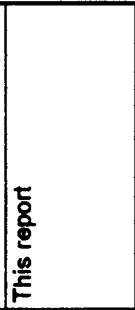 & 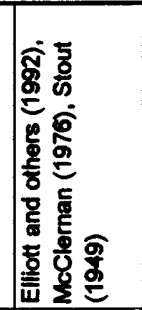 & 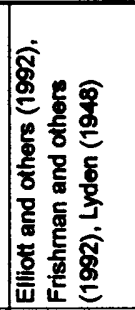 & 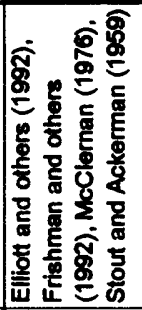 & 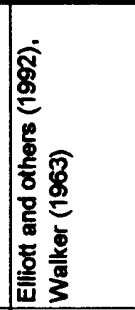 & 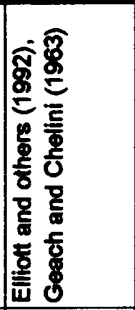 & 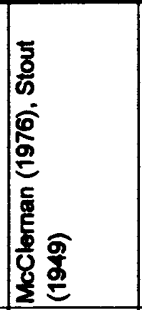 & 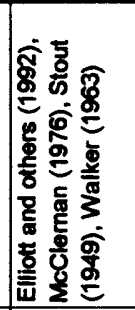 \\
\hline 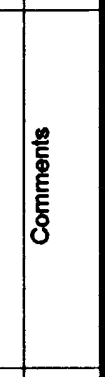 & & 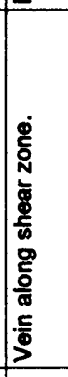 & 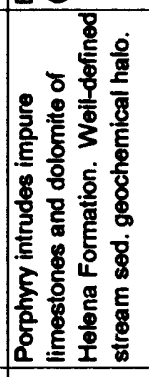 & 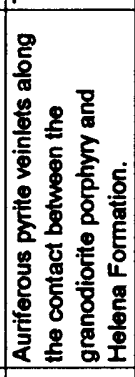 & 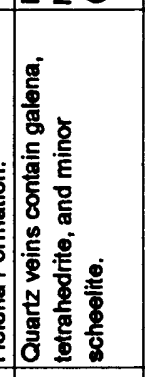 & & 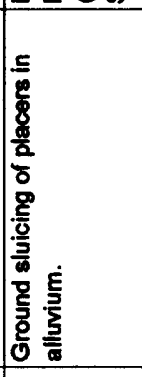 & 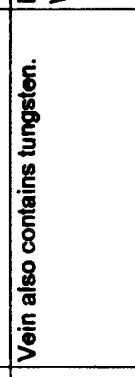 & & 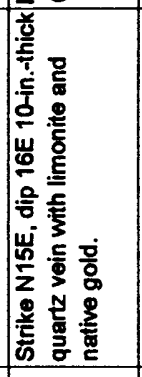 & 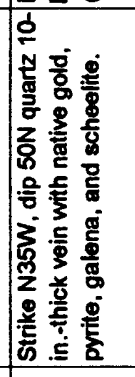 \\
\hline 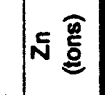 & & & & & & & & & & & \\
\hline$\frac{0}{2}$ & & & & & & & & & & & \\
\hline ठे & & & & & & & & & & & \\
\hline च्लेख & & & & & & & & & & & 18 \\
\hline ₹ $\bar{g}$ & 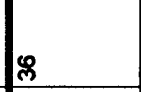 & & & & & & & & & & 우 \\
\hline | & 产 & & $\frac{2}{2}$ & & & & & 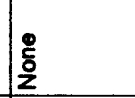 & & & $\mathscr{0}$ \\
\hline 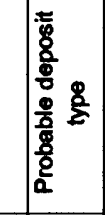 & $\mid \frac{z}{\frac{z}{8}}$ & 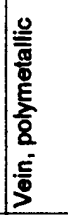 & 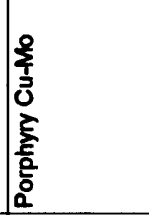 & 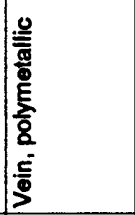 & 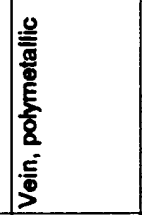 & $\begin{array}{l}\frac{z}{\alpha} \\
\frac{8}{\alpha} \\
\frac{\pi}{a}\end{array}$ & $\begin{array}{l}\frac{z}{4} \\
\frac{8}{8} \\
\frac{\pi}{a}\end{array}$ & 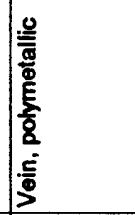 & $\begin{array}{l}\frac{z}{8} \\
\frac{8}{\alpha} \\
\frac{\Phi}{a}\end{array}$ & 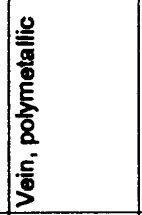 & 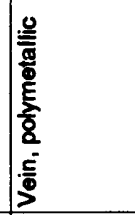 \\
\hline 8 & 10 & 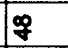 & $\stackrel{\infty}{\infty}$ & $\hat{m}$ & 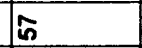 & 8 & N & n & N & 8 & 0 \\
\hline 童 & $\mathscr{R}$ & $\bar{m}$ & इ & gি & 8 & กิ & 8 & 8 & के & สิ & กิ \\
\hline$\overline{3}$ & $\cong$ & $\stackrel{2}{\rightleftharpoons}$ & $\cong$ & 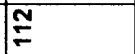 & $\stackrel{\mathfrak{Y}}{\rightleftharpoons}$ & $\cong$ & $\stackrel{2}{=}$ & $\stackrel{N}{=}$ & $\stackrel{N}{\mp}$ & $\stackrel{F}{=}$ & 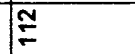 \\
\hline 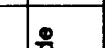 & N & 9 & $\checkmark$ & $\infty_{\infty}$ & m & 0 & ig & - & 18 & ส & $E$ \\
\hline 胥 & ๙ิ & ๙ & 8 & $\tilde{\kappa}$ & W & ज़ & กิ & สิ & $\bar{n}$ & กิ & ๙ \\
\hline & 9 & 9 & 9 & 9 & 9 & 9 & 9 & 9 & 9 & 9 & 9 \\
\hline 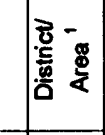 & 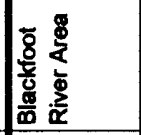 & 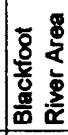 & 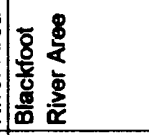 & 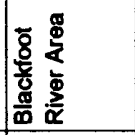 & 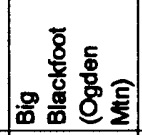 & 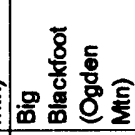 & 章总 & 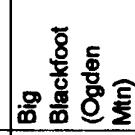 & 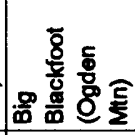 & 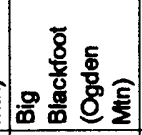 & 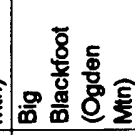 \\
\hline 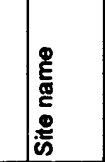 & 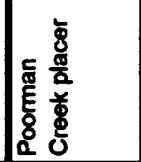 & 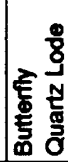 & $\frac{5}{\frac{5}{8}}$ & 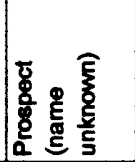 & 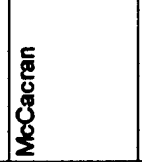 & 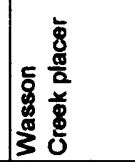 & 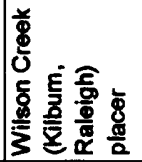 & 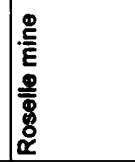 & 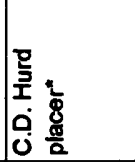 & 雨 & 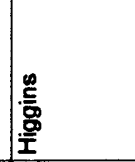 \\
\hline is & 8 & $\overline{0}$ & $\varepsilon$ & 18 & $\$$ & 8 & 18 & $\hat{0}$ & 18 & 8 & $R$ \\
\hline
\end{tabular}

$\hat{\omega}^{\infty}=$ 


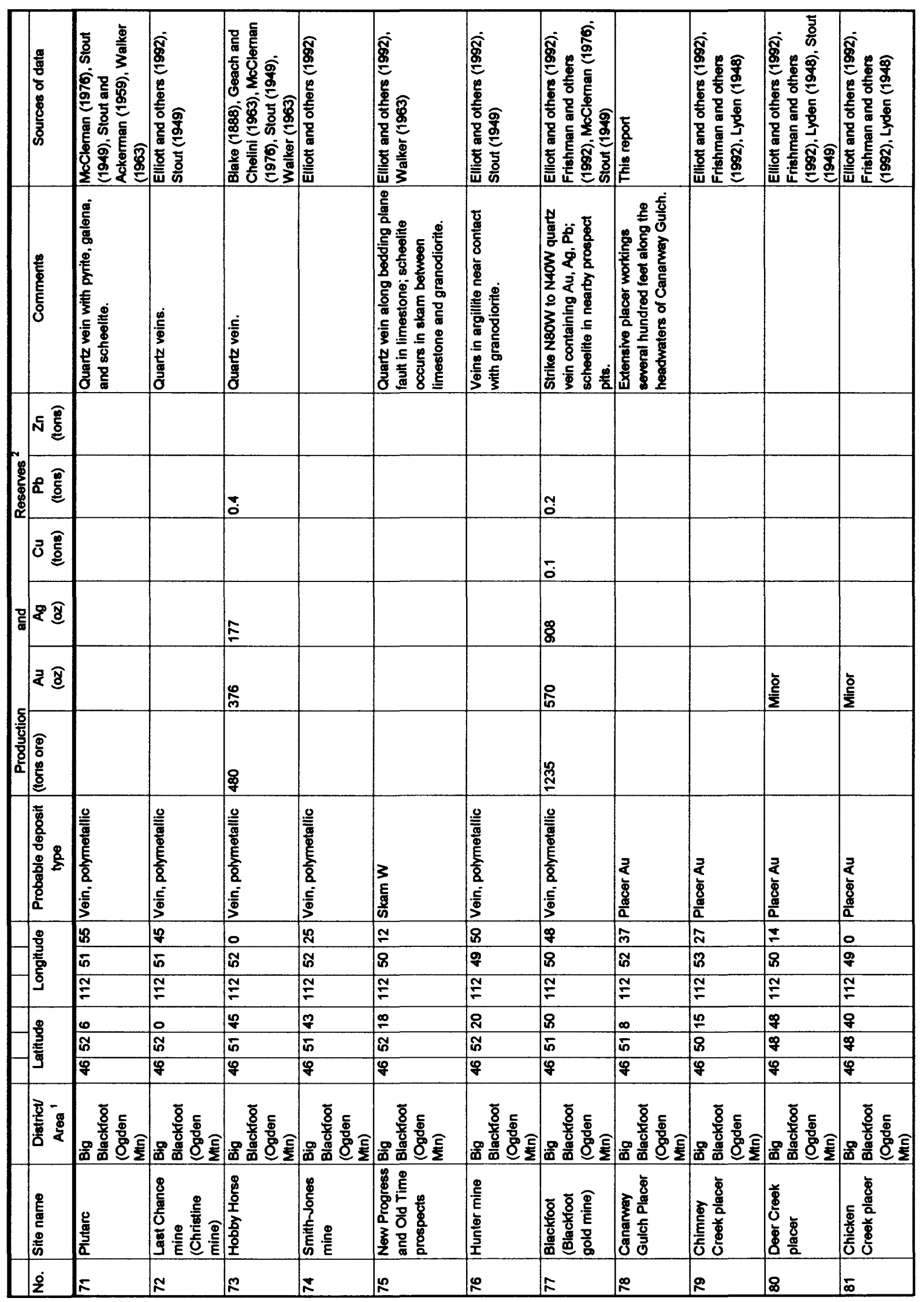

$\stackrel{\infty}{4}=$ 


\begin{tabular}{|c|c|c|c|c|c|c|c|c|c|c|c|}
\hline 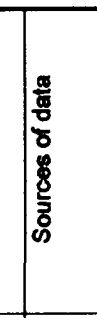 & 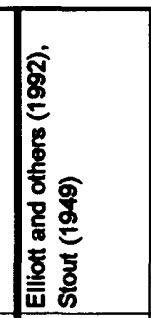 & 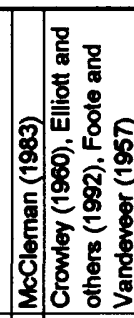 & & 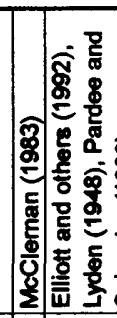 & 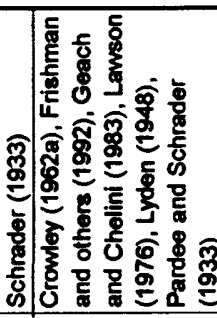 & 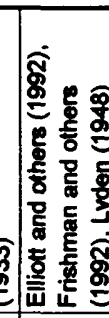 & 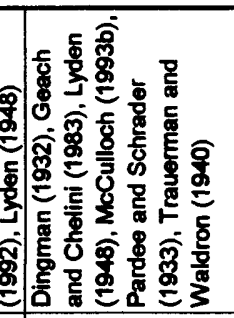 & 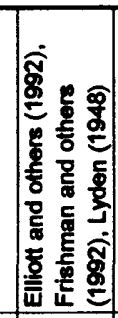 & 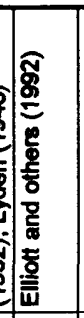 & 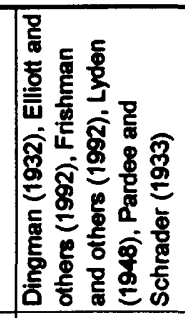 & 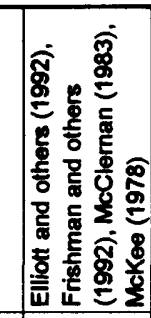 \\
\hline | & 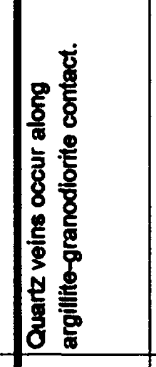 & 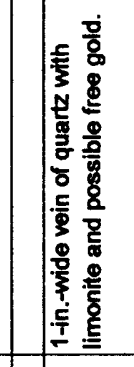 & & & & & & & 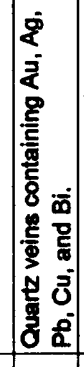 & 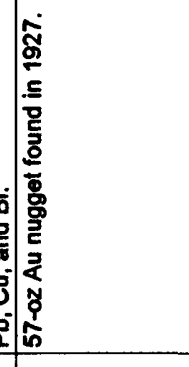 & 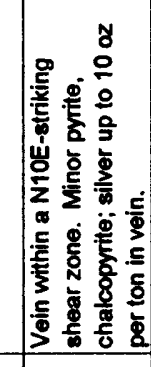 \\
\hline $\bar{N} \frac{\widehat{\underline{g}}}{\underline{\underline{g}}}$ & & & & & & & & & & & \\
\hline$\frac{8}{2}$ & & & & & & & & & & & $\rightleftarrows$ \\
\hline उे & & & & & & & & & & & $\cong$ \\
\hline 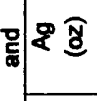 & & & & & & & & & & & 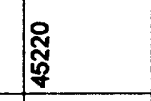 \\
\hline ₹ $\widehat{\alpha}$ & & & & 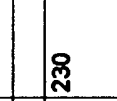 & 10 & $\kappa$ & 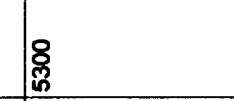 & $\frac{\bar{c}}{\frac{1}{2}}$ & & 8 & \% \\
\hline 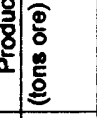 & & $\mid \frac{8}{2}$ & & & & & & & & & 㞔 \\
\hline 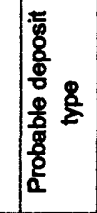 & 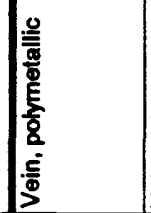 & 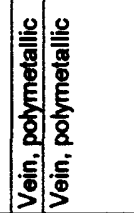 & 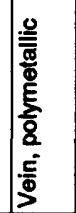 & 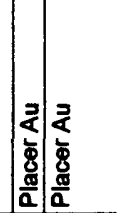 & 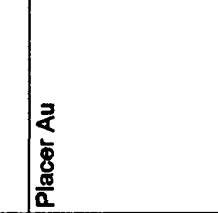 & 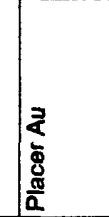 & 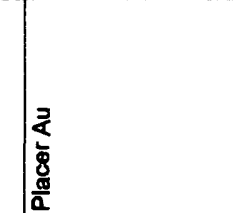 & \begin{tabular}{|l}
$\frac{z}{z}$ \\
$\bar{z}$ \\
$\frac{\pi}{a}$ \\
$\frac{\pi}{a}$
\end{tabular} & 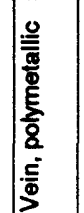 & 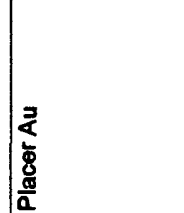 & 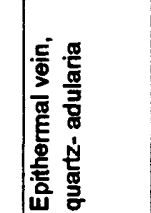 \\
\hline$-\frac{8}{3}$ & 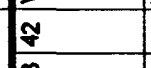 & 78 & 0 & $=8$ & 8 & 8 & $E$ & $\tilde{n}$ & 8 & 8 & T \\
\hline $7 \bar{z}$ & 98 & $|7|=$ & 8 & 97 & ₹ & 7 & $F$ & $\infty$ & $\infty$ & $\hat{m}$ & $\tilde{ల}$ \\
\hline 9 & $\stackrel{F}{F}$ & $\cong \cong$ & $\cong$ & $\cong \cong$ & $\cong$ & $\cong$ & $\cong$ & $\cong$ & $\cong$ & $\cong$ & $\cong$ \\
\hline$-\frac{8}{3}$ & $\frac{9}{9}$ & $-F$ & 0 & $\tilde{N}$ & 8 & $\infty$ & זั & $\bar{\pi}$ & $\frac{\infty}{0}$ & 落 & $\frac{\tilde{N}}{M}$ \\
\hline 接 & \begin{tabular}{|l}
8 \\
89 \\
9
\end{tabular} & \begin{tabular}{|l|l|}
$\bar{n}$ \\
$\log$
\end{tabular} & 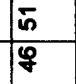 & \begin{tabular}{|l|l|}
8 \\
19
\end{tabular} & $\mid \vec{F}$ & $\mid \begin{array}{l}7 \\
9\end{array}$ & $\mid \frac{9}{9}$ & $\mid \frac{1}{9}$ & \begin{tabular}{|l|l}
8 \\
9
\end{tabular} & 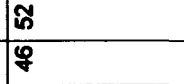 & $\frac{8}{98}$ \\
\hline 藁 & 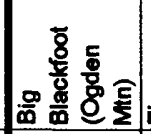 & $\sqrt{\frac{E}{L}}$ & 站 & 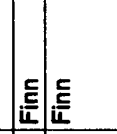 & 旋 & 站 & $\mid \frac{5}{\underline{\underline{L}}}$ & $\mid \begin{array}{l}\frac{\varepsilon}{L} \\
\underline{L}\end{array}$ & & 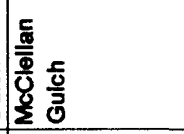 & 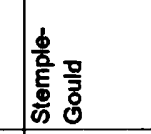 \\
\hline 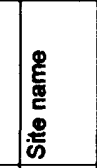 & 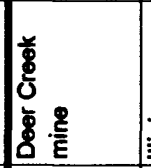 & 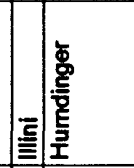 & & 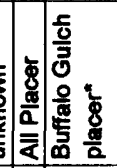 & 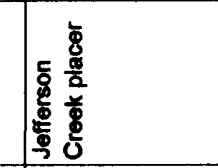 & 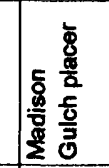 & 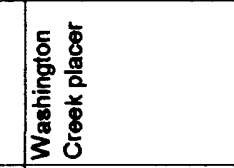 & 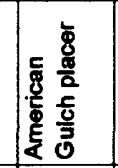 & 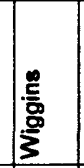 & 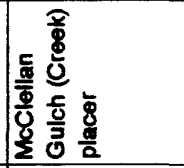 & 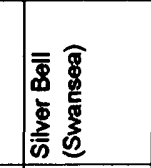 \\
\hline is & ఐ & வ & $\infty$ & 8 (क) & $\nsubseteq$ & $\mathbf{\infty}$ & 8 & $\overline{\mathbf{s}}$ & $\pi$ & $\cong$ & $\Phi$ \\
\hline
\end{tabular}




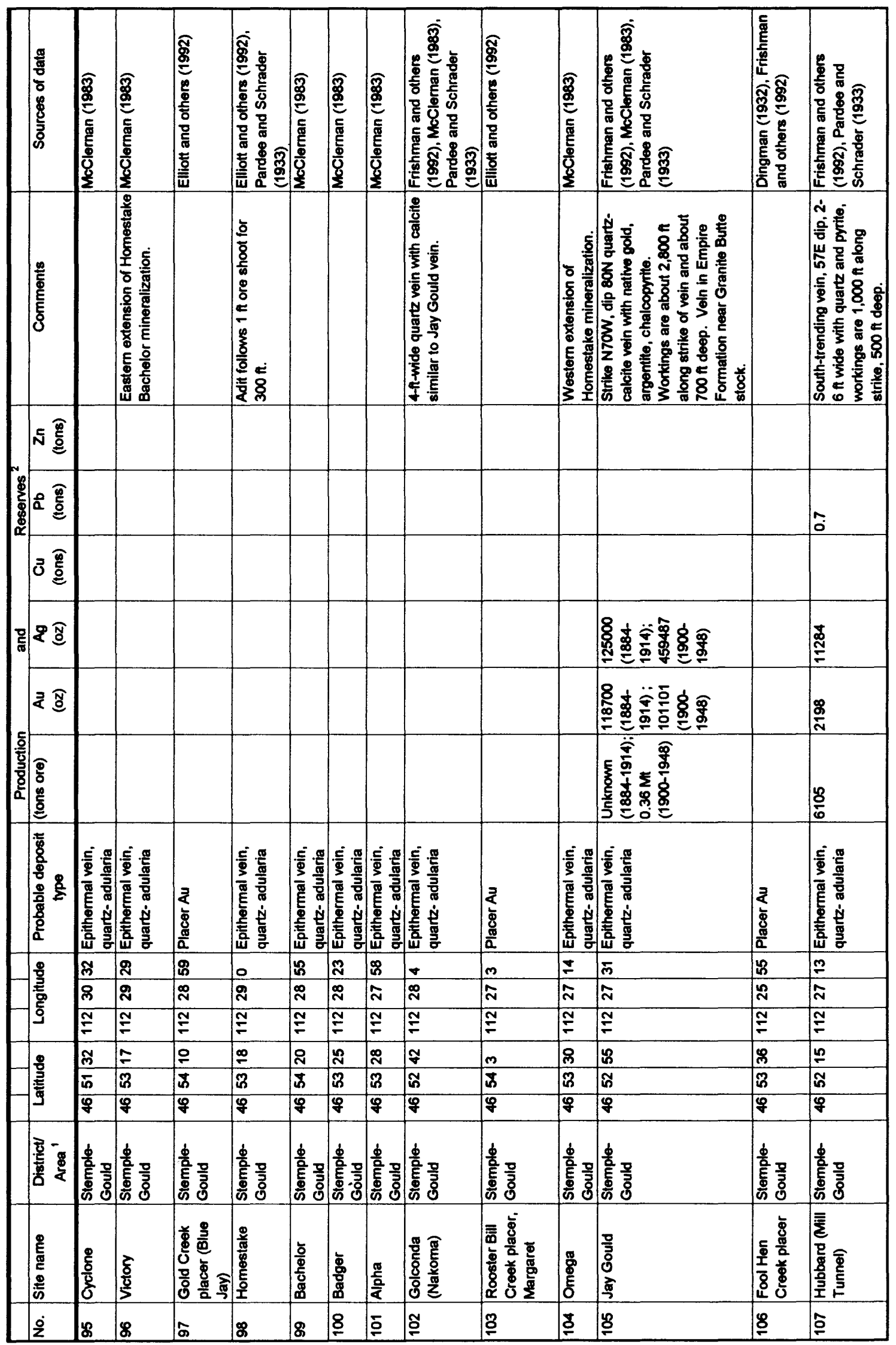

$\stackrel{ }{w} \underline{v}$ 


\begin{tabular}{|c|c|c|c|c|c|c|c|c|c|}
\hline 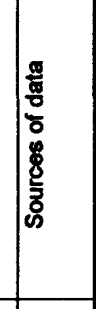 & 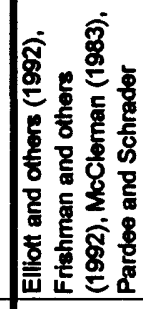 & 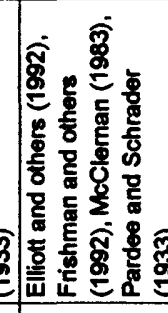 & 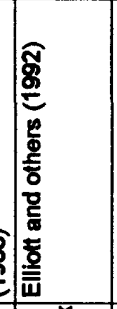 & 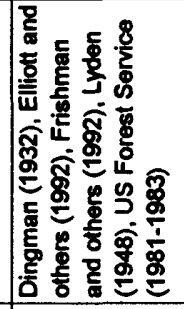 & 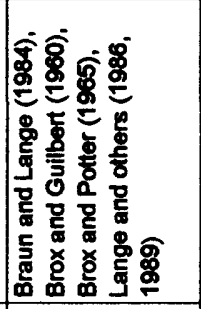 & 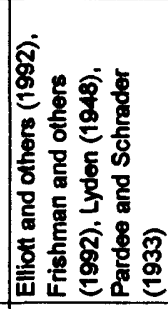 & 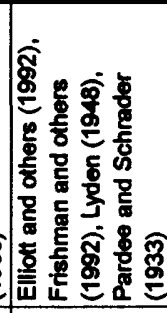 & 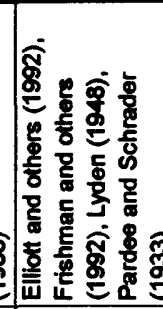 & 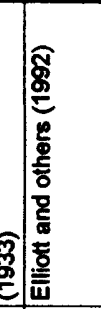 \\
\hline 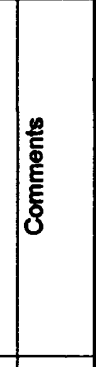 & 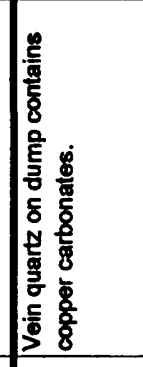 & 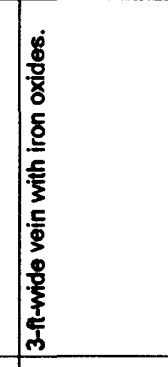 & 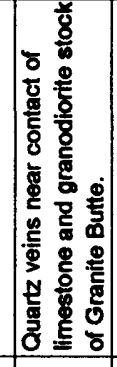 & & 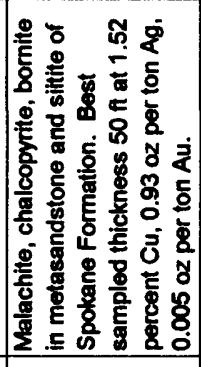 & & & & 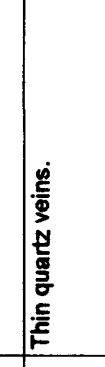 \\
\hline 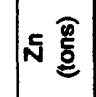 & & & & & & & & & \\
\hline 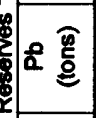 & & & & & & & & & \\
\hline$\overline{0}$ 兽 & & & & & 总 & & & & \\
\hline $2 \widehat{\gamma}$ & & & & & 8 & & & & \\
\hline ₹ $\bar{y}$ & & is & & & & & & & \\
\hline 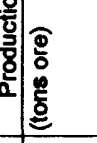 & & 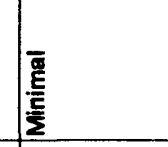 & & & 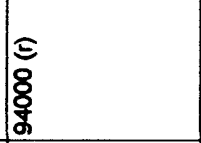 & & & & 总 \\
\hline 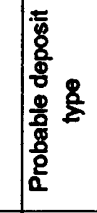 & 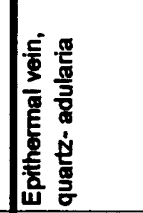 & 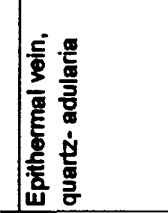 & 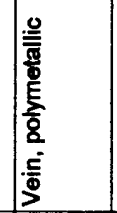 & 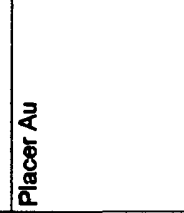 & 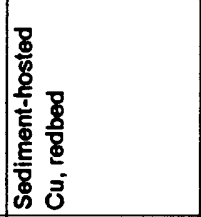 & 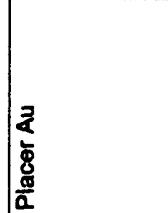 & 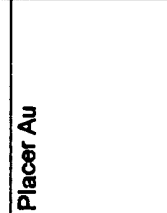 & \begin{tabular}{|l|}
$\frac{z}{2}$ \\
$\overline{8}$ \\
$\frac{d}{a}$ \\
$a$
\end{tabular} & 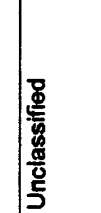 \\
\hline$-8 \frac{8}{2}$ & $\bar{d}$ & $\underline{m}$ & \pm & 9 & N & 7 & ? & 9 & $\tilde{\omega}$ \\
\hline $7 \frac{\bar{z}}{\Phi}$ & $\Phi$ & 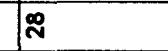 & $\hat{\Lambda}$ & $\mathbb{N}$ & $\%$ & i & $\bar{N}$ & \& & 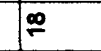 \\
\hline$\Xi$ & $\stackrel{乛}{\mp}$ & $\cong$ & $\cong$ & $\cong$ & $\cong$ & $\stackrel{N}{=}$ & $\stackrel{N}{\equiv}$ & $\stackrel{N}{=}$ & $\cong$ \\
\hline-18 & $\cong$ & 0 & 88 & m & q & $\stackrel{0}{\circ}$ & $\%$ & 8 & $\stackrel{2}{2}$ \\
\hline 盖 & 5 & $\bar{n}$ & 8 & 88 & 8 & 8 & (5) & $\tilde{n}$ & i \\
\hline & 9 & 9 & 9 & 98 & 9 & 9 & 98 & 98 & 19 \\
\hline 童- & 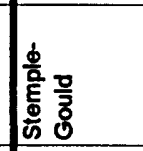 & 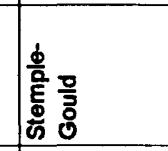 & 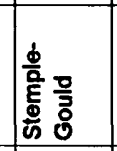 & 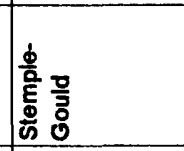 & 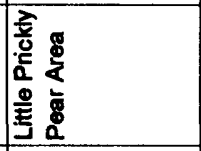 & 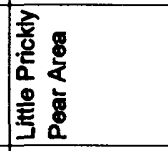 & 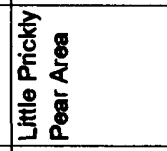 & 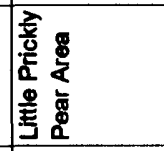 & 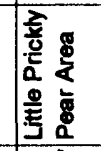 \\
\hline 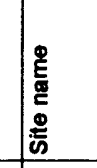 & 送 & $\begin{array}{l} \\
\\
\\
\end{array}$ & 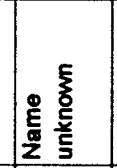 & 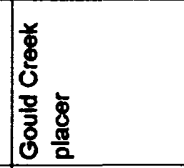 & 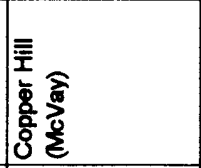 & 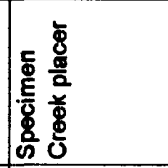 & 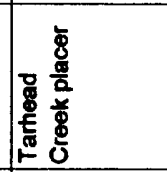 & 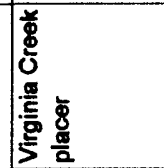 & 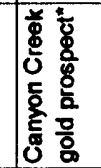 \\
\hline is & 8 & 8 & $\stackrel{9}{\div}$ & $\equiv$ & $\cong$ & $\stackrel{\rho}{\stackrel{\rho}{2}}$ & $\Xi$ & $\stackrel{m}{\leftrightarrows}$ & 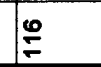 \\
\hline
\end{tabular}

ㄷำ 


\begin{tabular}{|c|c|c|c|c|c|c|c|c|c|c|}
\hline 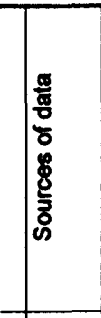 & 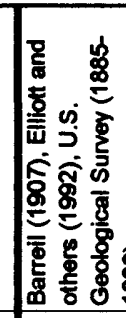 & 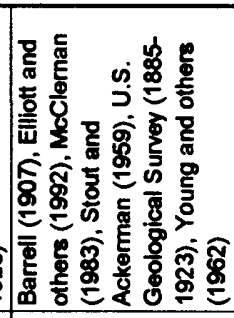 & 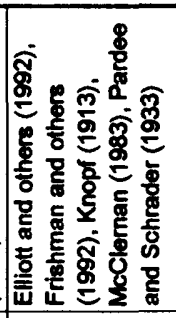 & 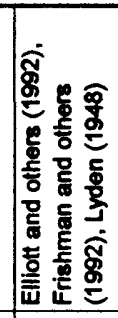 & 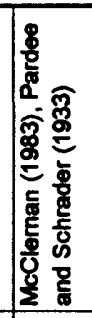 & 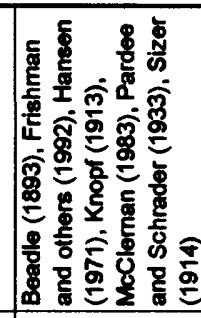 & 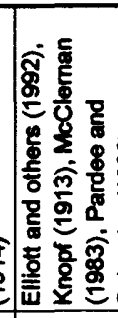 & 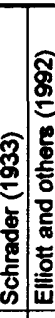 & & 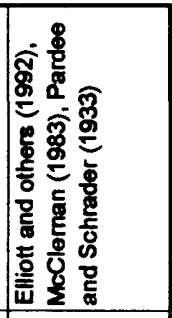 \\
\hline 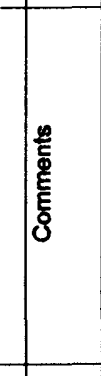 & 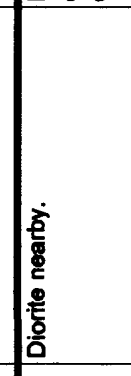 & 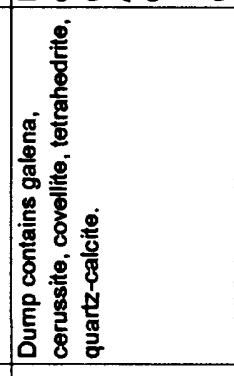 & 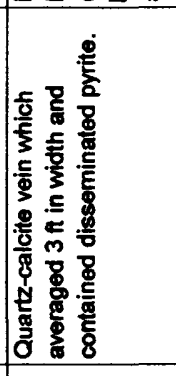 & & 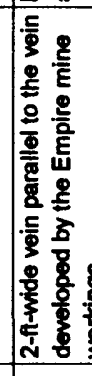 & 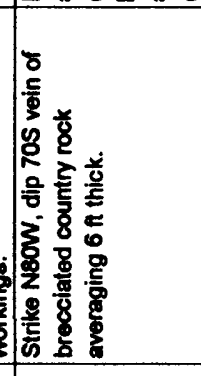 & 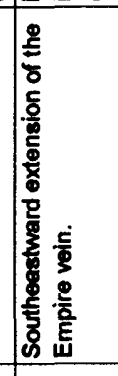 & & & 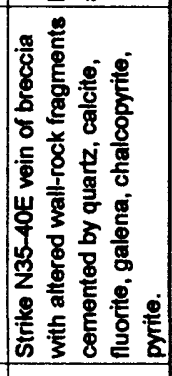 \\
\hline 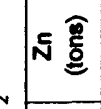 & & & & & & $\underline{\infty}$ & & & & \\
\hline 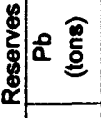 & & & & & & ฮ్ర & & & & \\
\hline 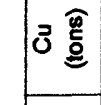 & & & $:$ & & & \ู & & & & \\
\hline 嗐 & & & 墕 & & & $\$$ & & & & \\
\hline ₹ $\widehat{\alpha}$ & & & 虽 & & & 居 & & & & \\
\hline 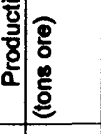 & & & \begin{tabular}{|l}
$\Sigma$ \\
\multirow{2}{*}{} \\
0 \\
0
\end{tabular} & 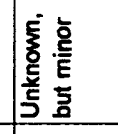 & & 产 & & $\frac{g_{0}^{2}}{2}$ & & \\
\hline 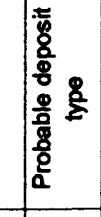 & 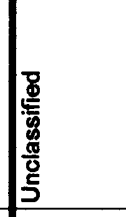 & 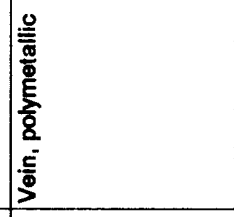 & 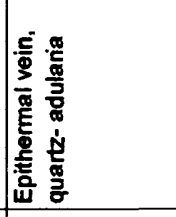 & 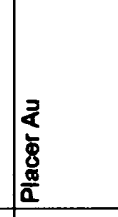 & 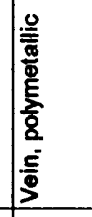 & 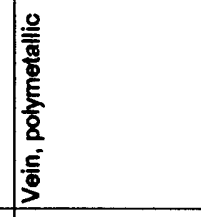 & 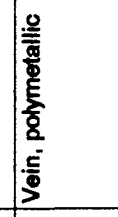 & 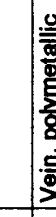 & & 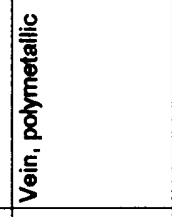 \\
\hline $1 \%$ & $g$ & $\underline{-1}$ & $\hat{N}$ & $\tilde{\tilde{n}}$ & $\%$ & $\tilde{\tilde{n}}$ & q & $\$$ & & a \\
\hline 」言 & $E$ & 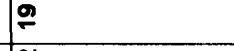 & i & $\bar{n}$ & I & $\approx$ & 2 & $\tilde{\tilde{\tau}}$ & & $\tilde{N}$ \\
\hline 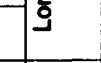 & $\underline{F}$ & $\cong$ & $\cong$ & $\cong$ & $\cong$ & $\cong$ & $\cong$ & 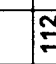 & & $\cong$ \\
\hline 电 & $\frac{\rho}{8}$ & 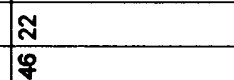 & $\begin{array}{l}8 \\
9\end{array}$ & $\frac{7}{9}$ & $\frac{m}{y}$ & $\frac{\bar{y}}{\bar{g}}$ & $\frac{9}{9}$ & $\frac{\pi}{\pi}$ & & $\frac{F}{I}$ \\
\hline & 8 & 8 & $\mid \frac{8}{8}$ & 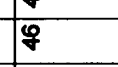 & 8 & 9 & 8 & $\$$ & & q \\
\hline 章- & 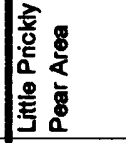 & 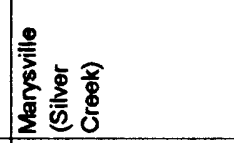 & 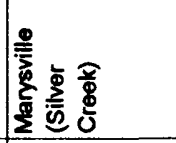 & 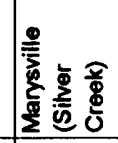 & 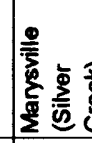 & 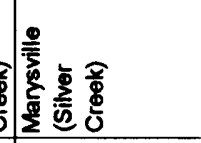 & 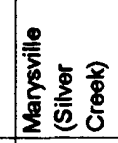 & & & 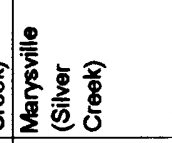 \\
\hline 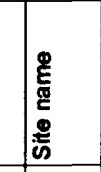 & 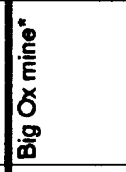 & 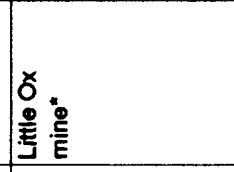 & 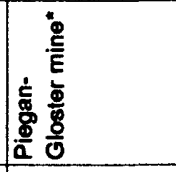 & 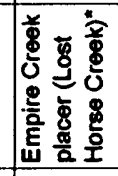 & 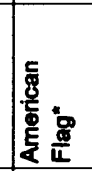 & $\mid$ & $\frac{\dot{y}}{\frac{1}{2}}$ & 8 & & 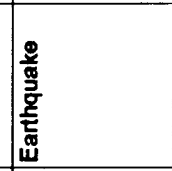 \\
\hline$\underline{\dot{2}}$ & $E$ & $\stackrel{\infty}{\frac{1}{2}}$ & $\stackrel{\rho}{\rightleftharpoons}$ & פְ & $\overline{\underline{\underline{j}}}$ & ָָ & $\mathbb{ָ}$ & 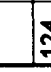 & & 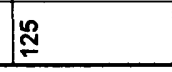 \\
\hline
\end{tabular}

ํํำ 


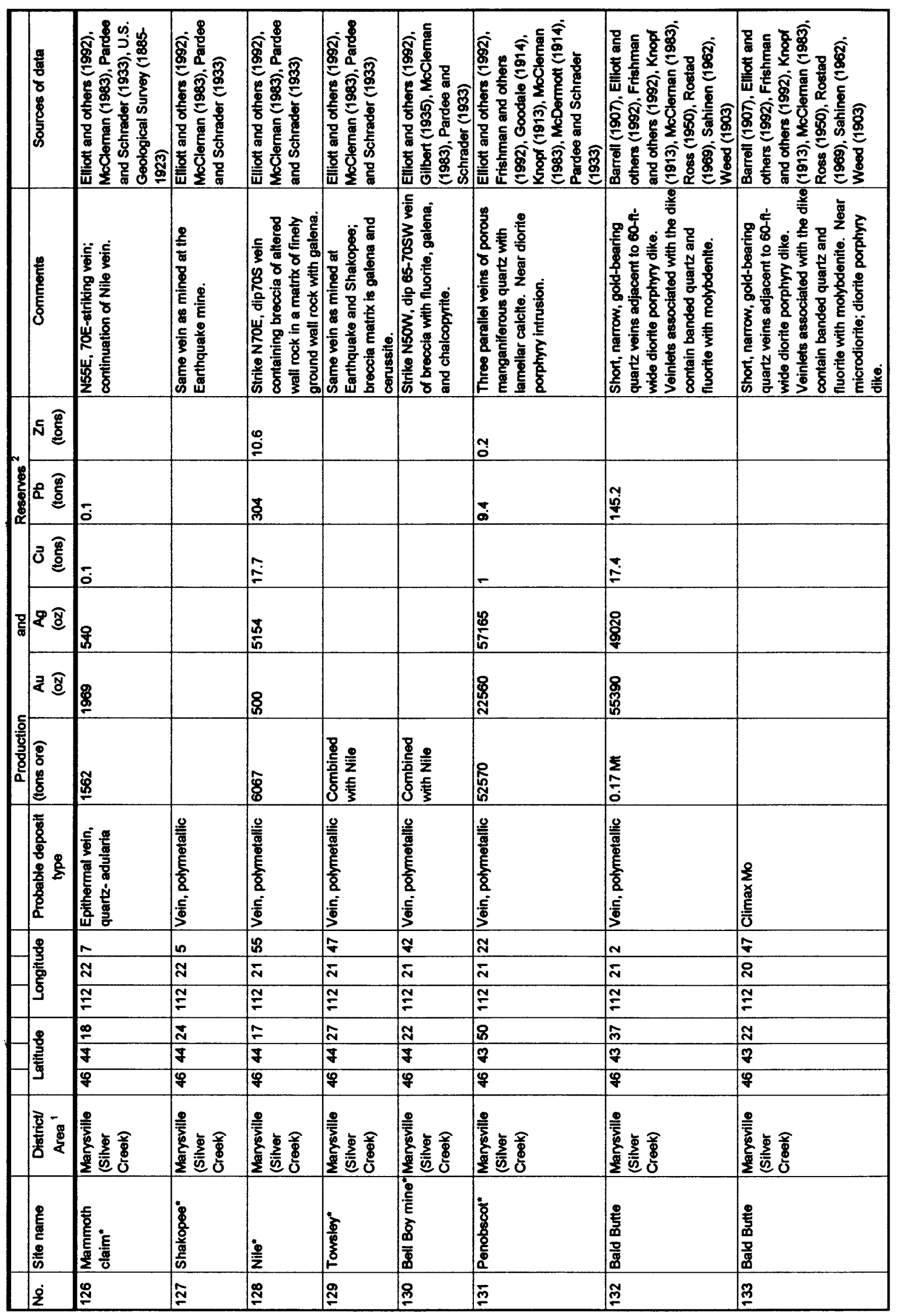

$\stackrel{m}{w} \stackrel{?}{?}$ 


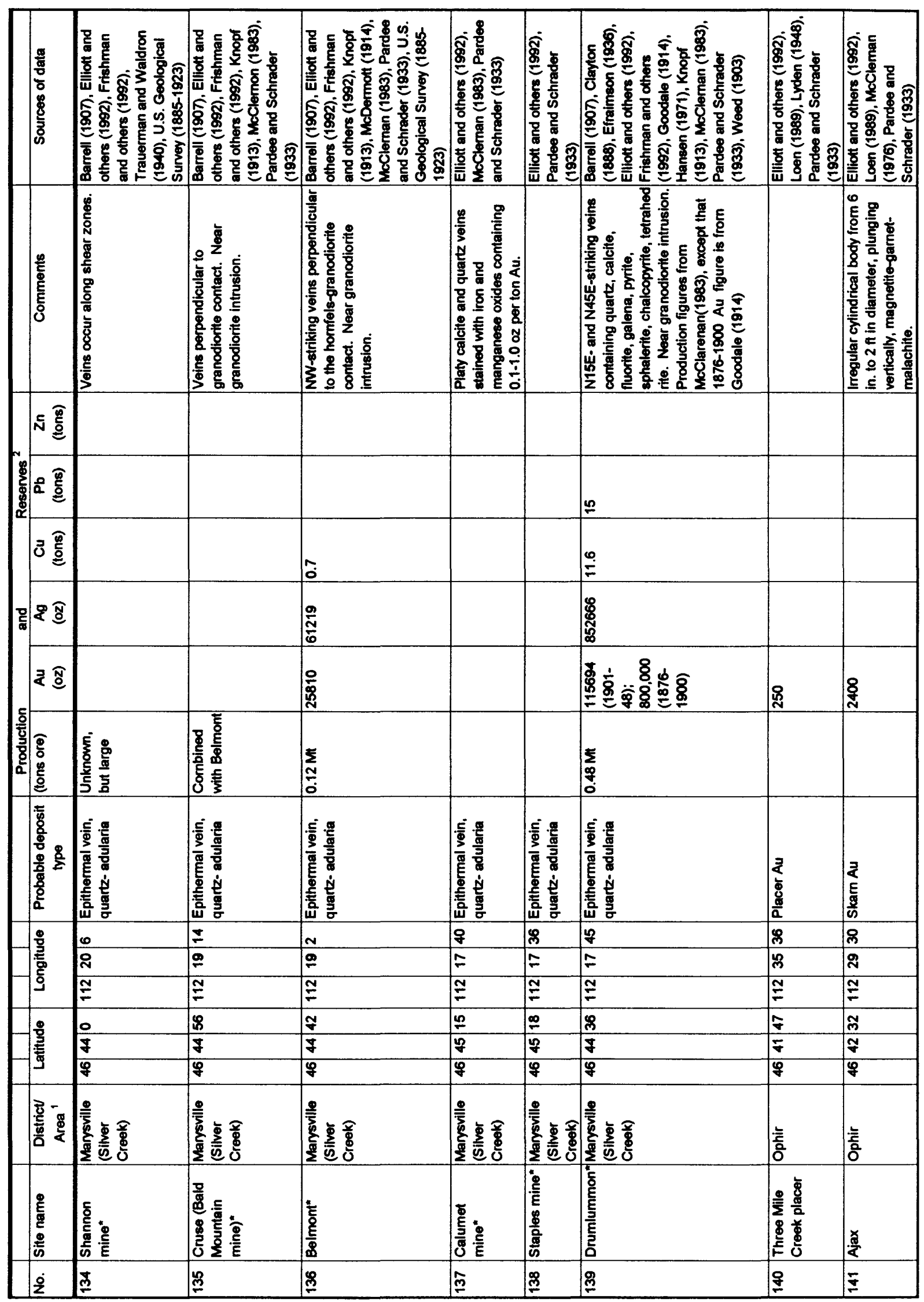

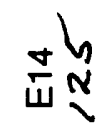




\begin{tabular}{|c|c|c|c|c|c|c|c|c|c|c|c|c|}
\hline 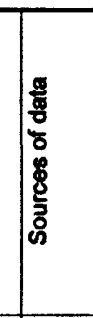 & 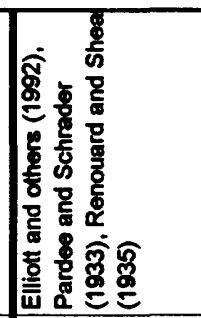 & 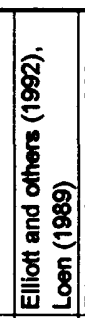 & 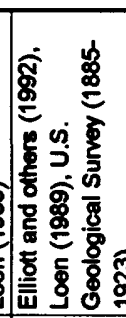 & 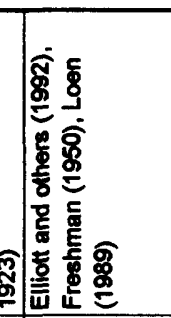 & 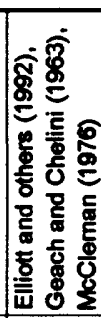 & 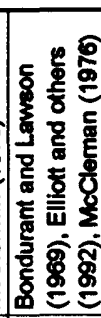 & 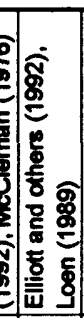 & 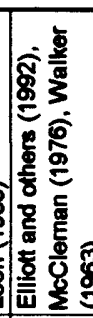 & 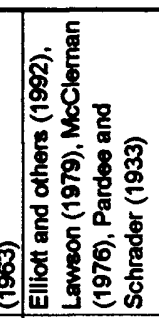 & 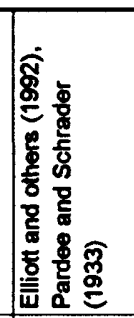 & 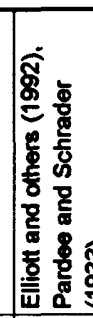 & 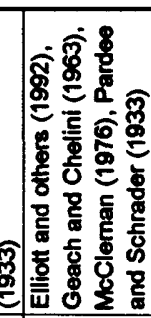 \\
\hline 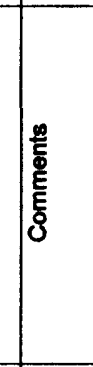 & 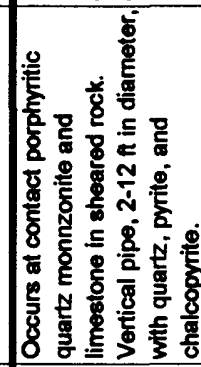 & & & 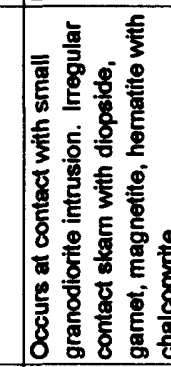 & 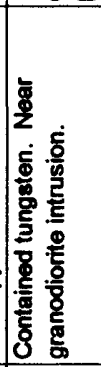 & 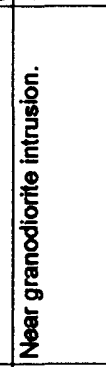 & 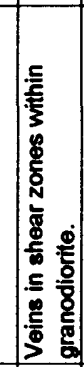 & 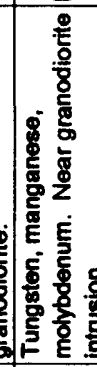 & 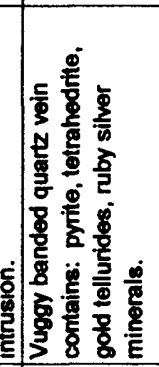 & 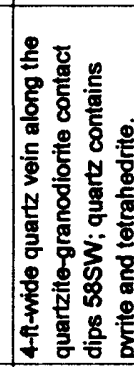 & 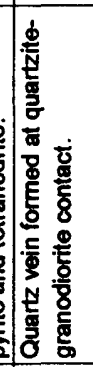 & 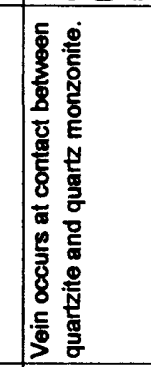 \\
\hline$\overline{\mathrm{N}} \frac{\overline{\mathrm{g}}}{\mathrm{g}}$ & & & & & & & & & & & & \\
\hline 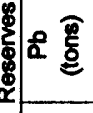 & & & & & & & & & m & & & \\
\hline 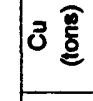 & & & & $\mathscr{0}$ & $\underset{\sim}{\infty}$ & & & & $\overline{0}_{0}$ & & & \\
\hline $8 \pi$ & & & & స్ & 8 & & & & హ్ & & & \\
\hline ₹ 증 & 8 & 道 & & $\Phi$ & $\infty$ & & & & $\hat{\mathbf{n}}$ & & & \\
\hline 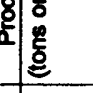 & & & & $\stackrel{8}{2}$ & $\bar{m}$ & & & & 7 & & $\div$ & \\
\hline 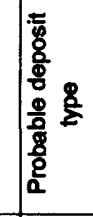 & 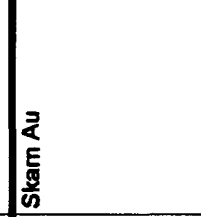 & $\mid \begin{array}{l}\frac{z}{2} \\
\frac{8}{8} \\
\frac{\mathrm{m}}{a}\end{array}$ & 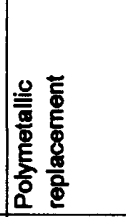 & 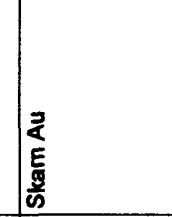 & 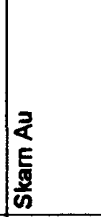 & $\mid \begin{array}{l}\frac{z}{8} \\
\frac{5}{5} \\
\frac{5}{n} \\
\end{array}$ & 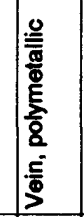 & \begin{tabular}{|l}
3 \\
E⿱ \\
$\frac{5}{n}$ \\
\end{tabular} & 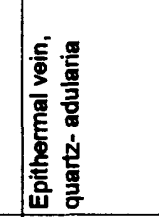 & 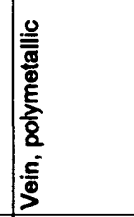 & 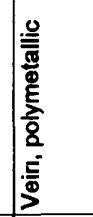 & 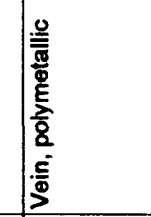 \\
\hline$-\frac{8}{2}$ & I & 8 & $\frac{6}{10}$ & $\frac{m}{9}$ & 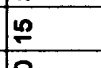 & $\frac{8}{8}$ & $\infty$ & 8 & $F$ & $\widetilde{m}$ & $\%$ & 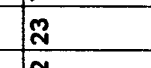 \\
\hline ] & $\varnothing$ & कి & \% & 8 & 8 & 8 & $\bar{m}$ & $\bar{m}$ & ल & $\widetilde{\kappa}$ & $\tilde{\pi}$ & $\widetilde{m}$ \\
\hline 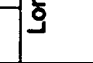 & $\cong$ & $\cong$ & $\cong$ & $\cong$ & $\cong$ & $\cong$ & $\cong$ & $\cong$ & $\cong$ & $\cong$ & $\cong$ & $\cong$ \\
\hline$f_{8}$ & 2 & \pm & $\infty$ & $t_{0}$ & 0 & 8 & $\mathbb{2}$ & $\underline{\infty}$ & 48 & Is & 8 & $\Lambda$ \\
\hline - & F & F & I & ₹ & F & $F$ & F & $F$ & 9 & q & 8 & 9 \\
\hline & 9 & 9 & 9 & 9 & 9 & 9 & 9 & 9 & $\%$ & 9 & 9 & 9 \\
\hline 豆要 & 言 & $\mid \begin{array}{c}\text { 产 } \\
\text { 音 }\end{array}$ & 圁 & 訚 & 言 & 豆 & 言 & 言 & 言 & 㢇 & 言 & 言 \\
\hline 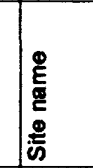 & 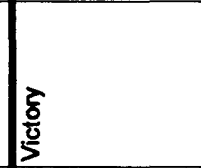 & 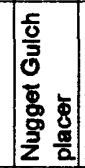 & 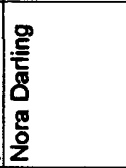 & 递 & 竞 & 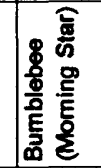 & 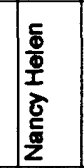 & 离 & 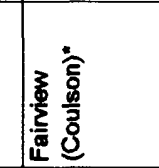 & 离 & 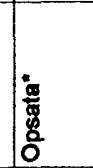 & 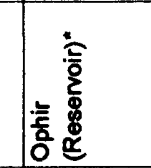 \\
\hline 12 & $\underline{\underline{z}}$ & 9 & $\$$ & 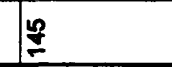 & $\$$ & 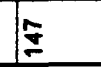 & $\frac{9}{9}$ & 9 & 8 & $\underline{\underline{\Phi}}$ & జِ & $\underline{R}$ \\
\hline
\end{tabular}

$\frac{n}{4} \mathcal{V}$ 


\begin{tabular}{|c|c|c|c|c|c|c|c|c|c|c|c|c|}
\hline 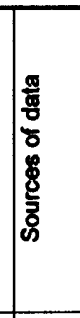 & 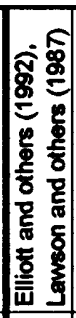 & 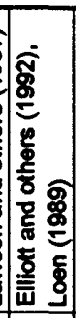 & 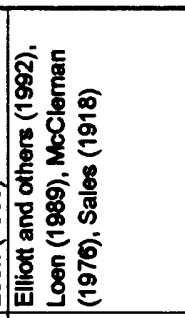 & 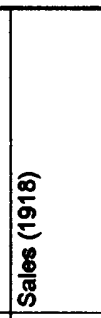 & & 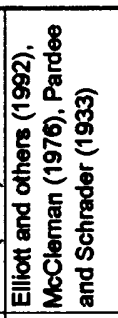 & 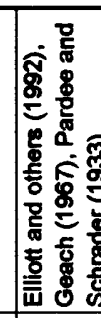 & 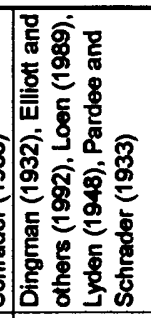 & 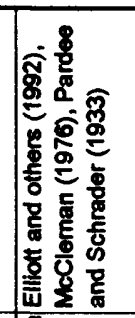 & 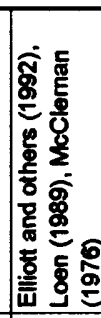 & 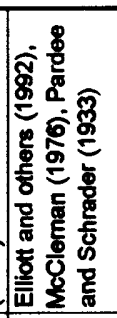 & 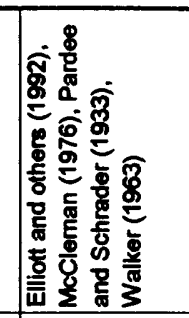 \\
\hline 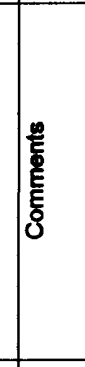 & 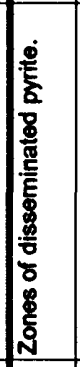 & & 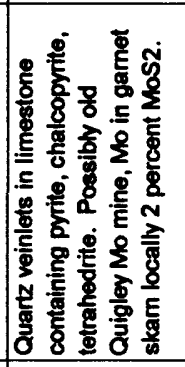 & 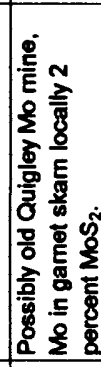 & & 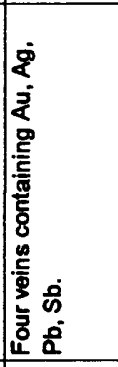 & 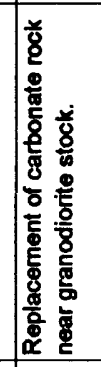 & 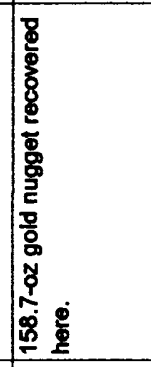 & 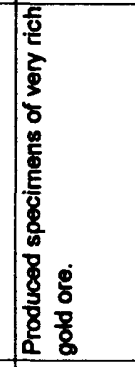 & & 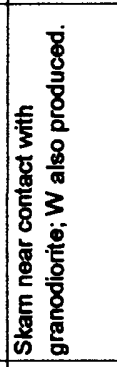 & 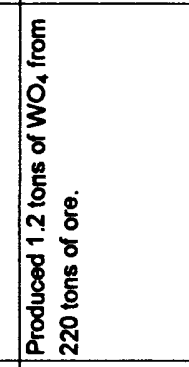 \\
\hline $5 \overline{\underline{g}}$ & & & & & & & & & & & & \\
\hline a $\frac{\widehat{d}}{\mathrm{~g}}$ & & & & & & & & & & & & \\
\hline उठ & & & & & & & & & & & & 苛 \\
\hline 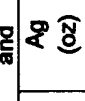 & & & & & & & & & & n & & $\Phi$ \\
\hline ₹ & & 8 & & & 8 & $\not 8$ & & 8 & & 0 & & ص \\
\hline 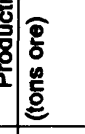 & & & & & & & & & & 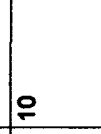 & & $\bar{\Phi}$ \\
\hline 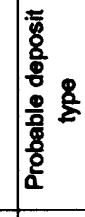 & 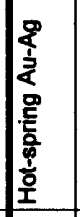 & 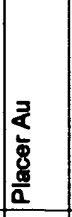 & 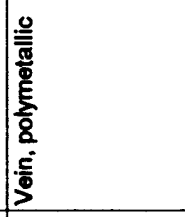 & \begin{tabular}{|l}
3 \\
E. \\
$\frac{9}{2}$
\end{tabular} & 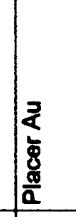 & 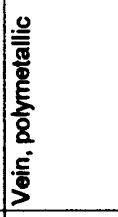 & 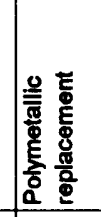 & 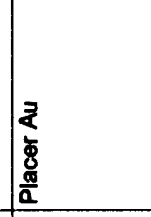 & 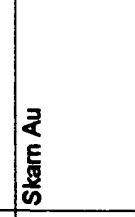 & 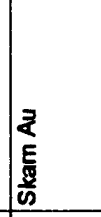 & \begin{tabular}{|l}
3 \\
E⿱ \\
$\frac{5}{\omega}$ \\
\end{tabular} & 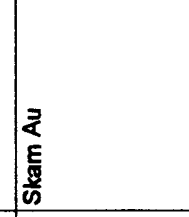 \\
\hline ) & $\frac{8}{\frac{8}{n}}$ & \begin{tabular}{|l|}
$\infty$ \\
8
\end{tabular} & $\mid \overrightarrow{\tilde{m}}$ & $\dot{\vec{m}}$ & $\frac{9}{8}$ & $\frac{\mid 2}{8}$ & $\frac{0}{2}$ & $\frac{\delta}{\hat{N}}$ & $\frac{10}{20}$ & 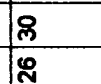 & $\frac{m}{\infty}$ & $\frac{\sim}{\infty}$ \\
\hline-1 & $\stackrel{1}{\stackrel{N}{*}}$ & $\underline{\underline{N}}$ & $\underline{\underline{N}}$ & $\stackrel{10}{\approx}$ & 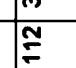 & 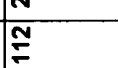 & $\cong$ & $\stackrel{N}{\simeq}$ & $\stackrel{N}{\cong}$ & $\stackrel{N}{\Sigma}$ & 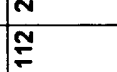 & $\mid$\begin{tabular}{|l}
$\tilde{N}$ \\
$\underline{\underline{N}}$
\end{tabular} \\
\hline - & \begin{tabular}{|l|}
8 \\
9 \\
9 \\
9
\end{tabular} & \begin{tabular}{|l|}
$\frac{1}{2}$ \\
$\frac{1}{2}$ \\
\end{tabular} & \begin{tabular}{|l|}
$\frac{9}{9}$ \\
$\frac{9}{8}$ \\
\end{tabular} & \begin{tabular}{|l|}
9 \\
9 \\
8 \\
\end{tabular} & \begin{tabular}{|l|}
8 \\
8 \\
8
\end{tabular} & \begin{tabular}{|l|}
$\frac{1}{5}$ \\
8 \\
8
\end{tabular} & \begin{tabular}{|l}
$\bar{N}$ \\
$\bar{q}$ \\
$\mathscr{q}$
\end{tabular} & \begin{tabular}{|l|}
$\frac{1}{2}$ \\
$\frac{1}{4}$ \\
\end{tabular} & \begin{tabular}{|l|}
$\tilde{N}$ \\
$\mathbf{F}$ \\
$\mathscr{q}$ \\
\end{tabular} & \begin{tabular}{|l|}
$\frac{1}{8}$ \\
$\frac{8}{9}$
\end{tabular} & \begin{tabular}{|l|}
$f$ \\
$q$ \\
$q$
\end{tabular} & \begin{tabular}{|l|}
8 \\
$q$ \\
8
\end{tabular} \\
\hline 童 & 圁 & & 㢇 & 棓 & 裘 & 衰 & 虔 & 圁 & 言 & 狺 & 訔 & 言 \\
\hline 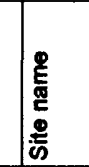 & 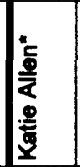 & 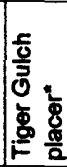 & & \begin{tabular}{|l} 
裹 \\
襄
\end{tabular} & 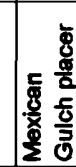 & 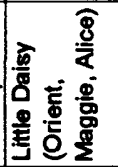 & $\frac{8}{a}$ & 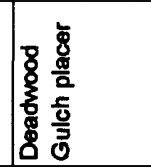 & 离 & 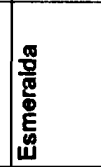 & 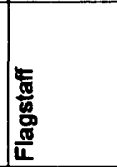 & 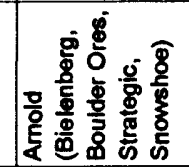 \\
\hline$\dot{2}$ & 苜 & 䓦 & $\$ 8$ & $\underline{\underline{\omega}}$ & 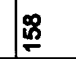 & $\underline{\underline{\rho}}$ & $\stackrel{8}{8}$ & $\Phi$ & ๕๕ & 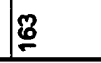 & $\Phi$ & $\dddot{8}$ \\
\hline
\end{tabular}

พำ 


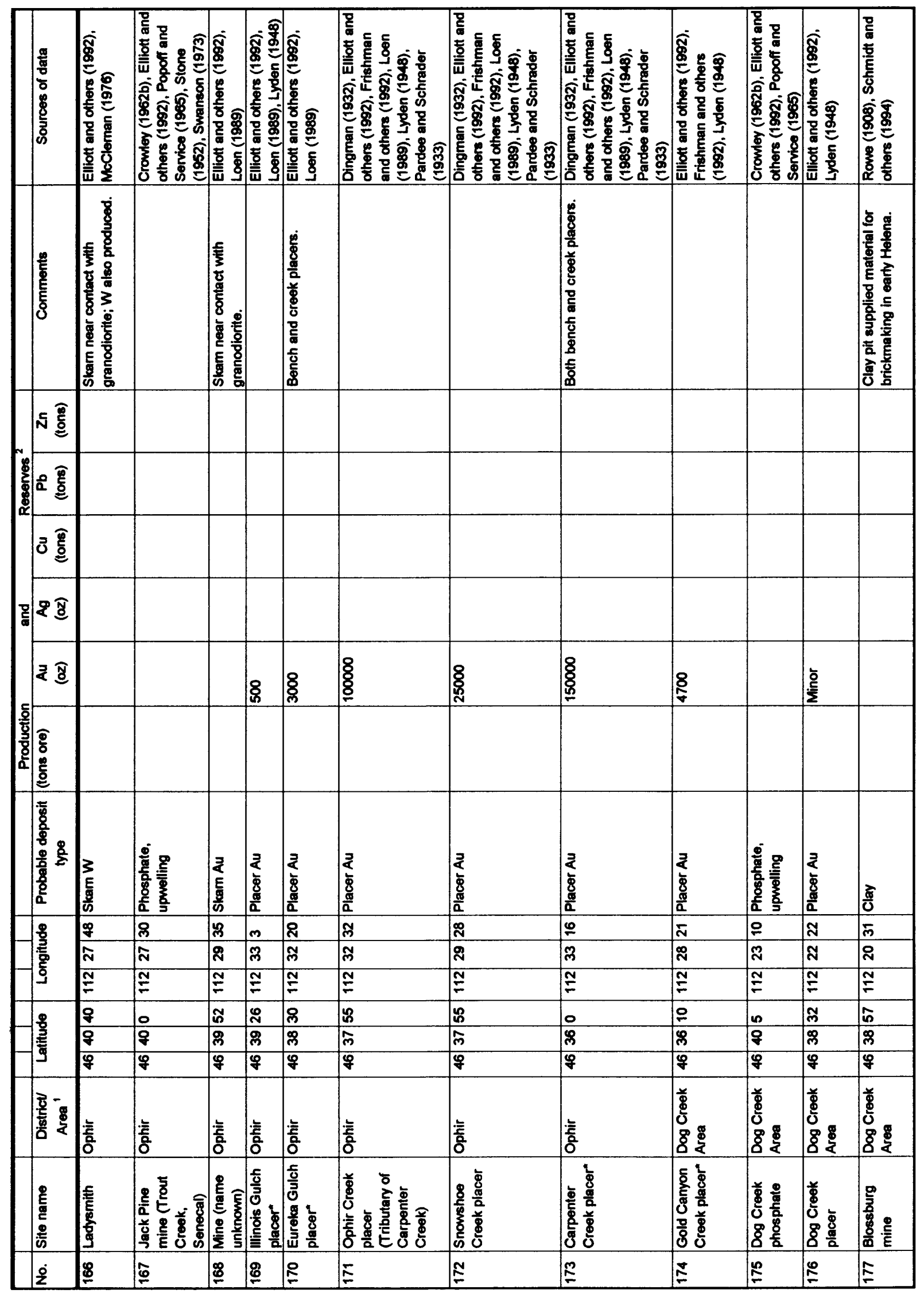

พิ 


\begin{tabular}{|c|c|c|c|c|c|c|c|c|c|c|c|c|}
\hline 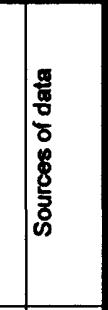 & 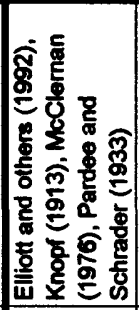 & 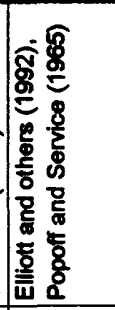 & 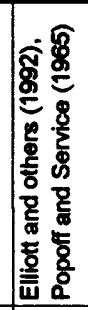 & 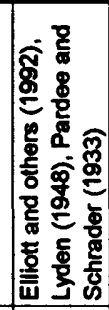 & 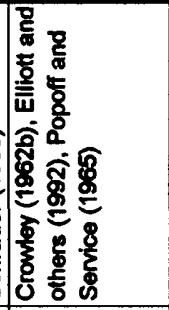 & 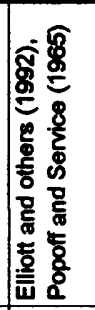 & 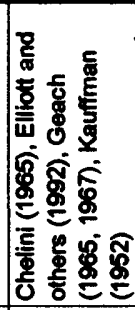 & 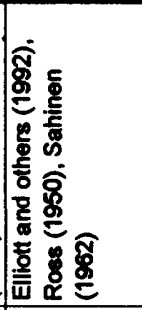 & 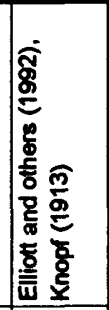 & 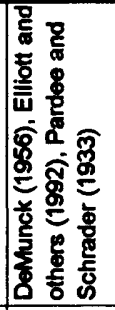 & 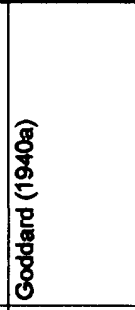 & 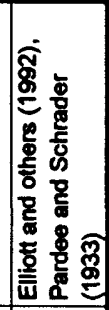 \\
\hline 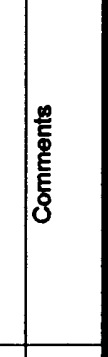 & 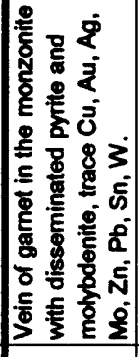 & & & & & 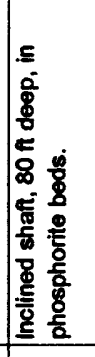 & & 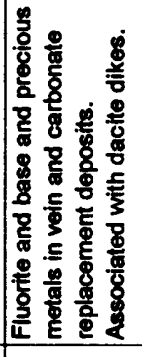 & 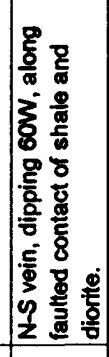 & 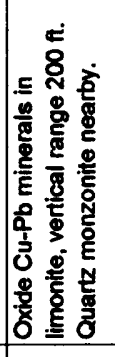 & 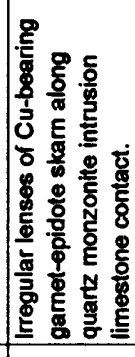 & 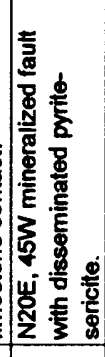 \\
\hline$\overline{\text { N }}$ & & & & & & & & & & & & \\
\hline 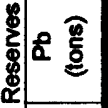 & & & & & & & & & & & & \\
\hline उं & & & & & & & & & & & & \\
\hline : & & & & & & & & & & & & \\
\hline इ त्र & & & & & & & & & & & & \\
\hline 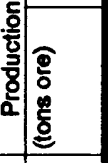 & & & & & & & & & & 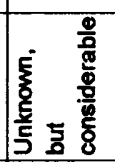 & & \\
\hline $\mid \begin{array}{l}\frac{1}{8} \\
\frac{8}{8} \\
\frac{8}{8} \\
\frac{8}{8} \\
\frac{8}{8} \\
\frac{8}{2} \\
\frac{8}{2} \\
0\end{array}$ & $\begin{array}{l}3 \\
E \\
\frac{E}{50} \\
5\end{array}$ & 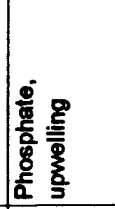 & 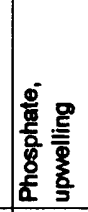 & 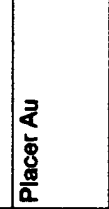 & 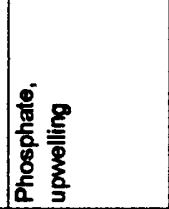 & 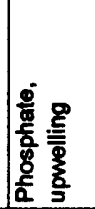 & 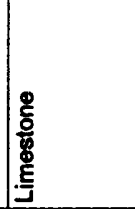 & 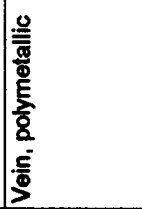 & 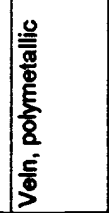 & 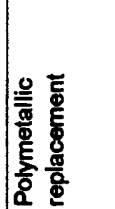 & $\mid \begin{array}{l}\frac{z}{\alpha} \\
E \\
\frac{w}{\omega}\end{array}$ & 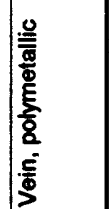 \\
\hline \$ & $\stackrel{2}{2}$ & 9 & $\infty$ & న్ల & 오 & 으 & 으 & sp & 18 & $\%$ & ম & 옹 \\
\hline 吾 & $\infty$ & $\boldsymbol{N}$ & $\dddot{\pi}$ & $\mathscr{2}$ & $\stackrel{n}{2}$ & $\bar{a}$ & 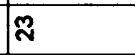 & $\infty$ & 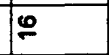 & \pm & $\%$ & $m$ \\
\hline 9 & $\stackrel{\mathbf{N}}{\rightleftharpoons}$ & $\cong$ & 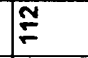 & $\cong$ & $\stackrel{N}{\rightleftharpoons}$ & $\cong$ & $\stackrel{N}{=}$ & $\stackrel{\Re}{\rightleftharpoons}$ & $\stackrel{N}{\rightleftharpoons}$ & $\stackrel{\cong}{\rightleftharpoons}$ & $\stackrel{ }{\mp}$ & $\stackrel{N}{F}$ \\
\hline : & $\infty$ & 0 & $m$ & 4 & 7 & 18 & if & 0 & $\overline{4}$ & 18 & 8 & 우 \\
\hline$\underline{3}$ & m & 18 & I & 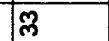 & $\mathscr{m}$ & $m$ & 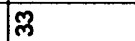 & gి & $\boldsymbol{F}$ & I & $\%$ & 9 \\
\hline & 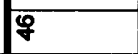 & 9 & 9 & 9 & 9 & 6 & 8 & io & 9 & 9 & 9 & 9 \\
\hline 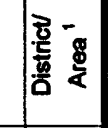 & 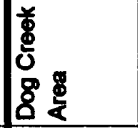 & \begin{tabular}{|l} 
\\
0 \\
0 \\
8 \\
8 \\
8
\end{tabular} & 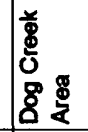 & 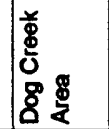 & 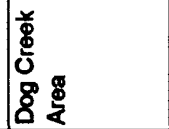 & 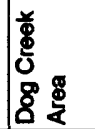 & 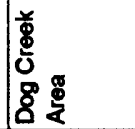 & 霓 & 憘 & 喜 & 喜 & 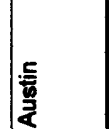 \\
\hline 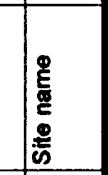 & 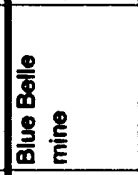 & 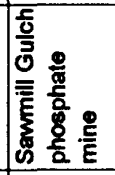 & 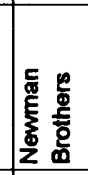 & 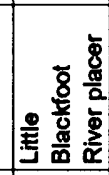 & 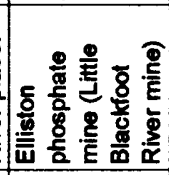 & 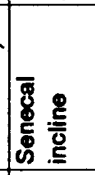 & 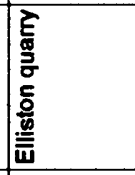 & 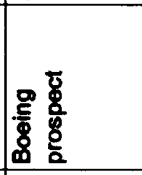 & 点 & 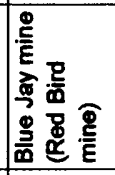 & 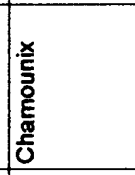 & 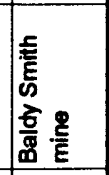 \\
\hline i & $\stackrel{\infty}{\mathscr{2}}$ & g & ळి & Ф્ळ & $\underset{\varpi}{\varpi}$ & $\dddot{\infty}$ & $\underset{\varpi}{\Phi}$ & $\dddot{\square}$ & $\not 2$ & 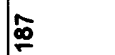 & $\dddot{\infty}$ & $\underset{\mathbf{m}}{\mathbf{\Phi}}$ \\
\hline
\end{tabular}

$\stackrel{\infty}{\omega} \underset{\tau}{\tau}$ 


\begin{tabular}{|c|c|c|c|c|c|c|c|c|c|c|}
\hline 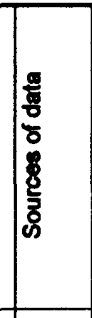 & 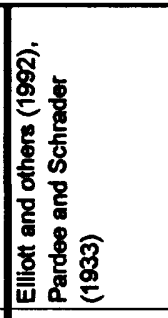 & 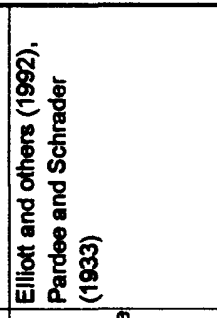 & 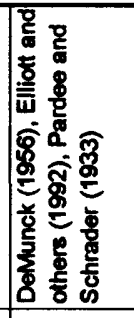 & 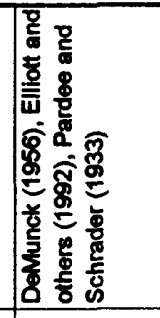 & 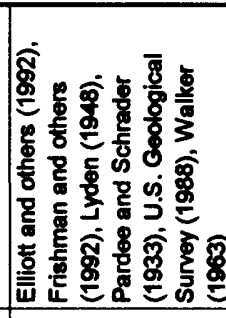 & 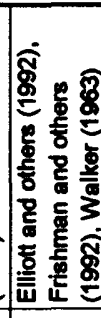 & 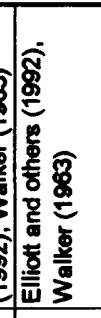 & 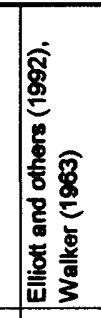 & 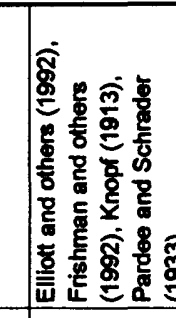 & 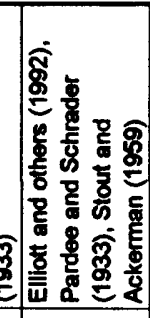 \\
\hline 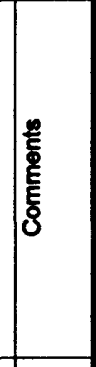 & 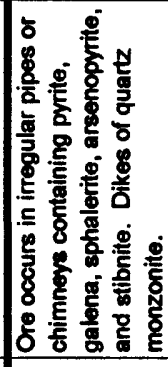 & 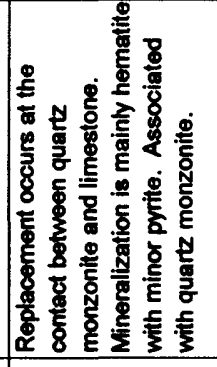 & 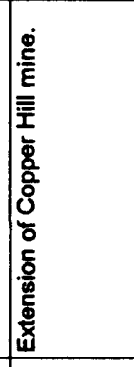 & 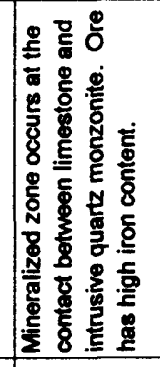 & 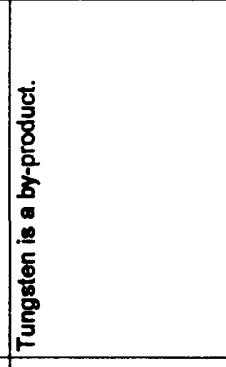 & 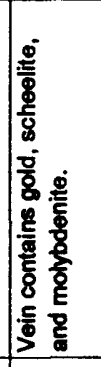 & 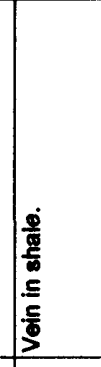 & 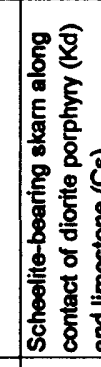 & 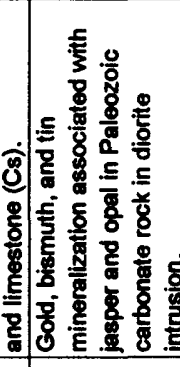 & 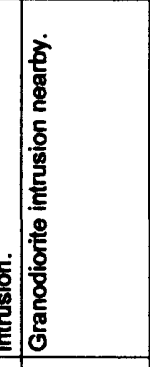 \\
\hline \multicolumn{11}{|l|}{$\tilde{N} \frac{\widehat{g}}{\tilde{g}}$} \\
\hline \multicolumn{11}{|l|}{ 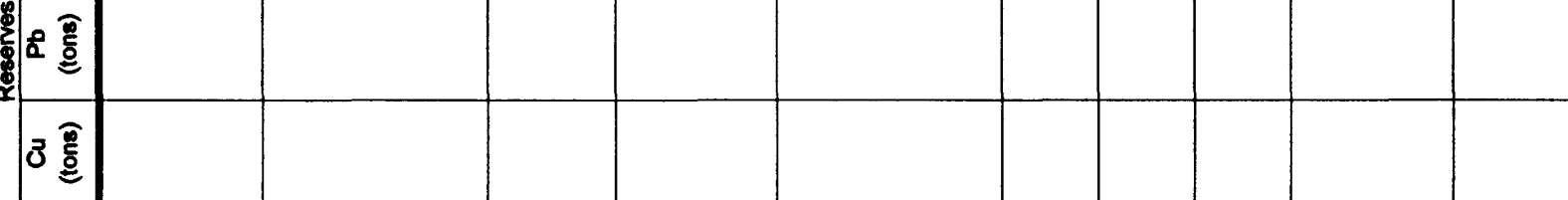 } \\
\hline \multicolumn{11}{|l|}{$2 \pi$} \\
\hline \multicolumn{11}{|l|}{ ₹ } \\
\hline 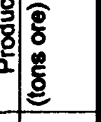 & \&్లి & & & 㫣 & & & $\mid \begin{array}{l}0 \\
\frac{0}{2} \\
2\end{array}$ & $\mid \begin{array}{l}\frac{\mathscr{g}}{2} \\
\frac{2}{2}\end{array}$ & & \\
\hline 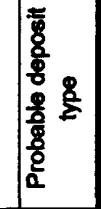 & 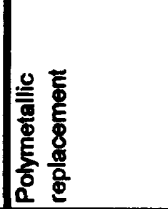 & 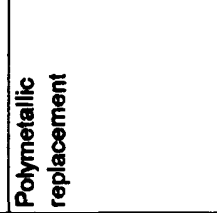 & 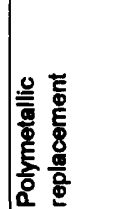 & 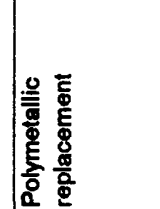 & 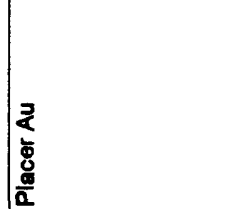 & 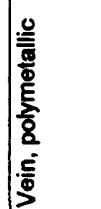 & 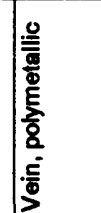 & $\mid \begin{array}{l}3 \\
E \\
\frac{E}{\omega} \\
\omega\end{array}$ & 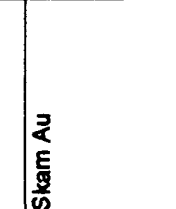 & $\mid \begin{array}{l}\frac{z}{z} \\
\frac{E}{\frac{g}{m}} \\
\frac{E}{m}\end{array}$ \\
\hline \multirow{3}{*}{ 蒡 } & $\frac{1}{9}$ & $\frac{\mathscr{N}}{\pi}$ & g & $m$ & $\%$ & $\frac{10}{-10}$ & $\frac{\pi}{0}$ & $\frac{\%}{0}$ & $\infty$ & $\mathscr{m}$ \\
\hline & E & \pm & \pm & $\cong$ & $=$ & $=$ & $?$ & 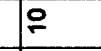 & $\infty$ & $\infty$ \\
\hline & $\stackrel{\Gamma}{\rightleftharpoons}$ & $\cong$ & $\cong$ & $\stackrel{\cong}{\cong}$ & $\stackrel{N}{=}$ & $\cong$ & $\cong$ & $\stackrel{N}{F}$ & $\stackrel{N}{\approx}$ & $\cong$ \\
\hline \multirow{3}{*}{ 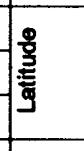 } & 7 & $\mathscr{m}$ & $N$ & 8 & $\%$ & $\mathscr{8}$ & $\infty$ & $\underbrace{\circ}_{0}$ & 8 & 9 \\
\hline & ge & I & 戹 & $\$$ & 屃 & $\$$ & $\$$ & $\infty$ & $\%$ & $\%$ \\
\hline & 8 & 8 & o & 9 & 9 & 8 & 19 & 9 & 8 & 8 \\
\hline 童- & 亮 & 毫 & $\mid \frac{\frac{5}{w_{3}}}{\frac{1}{4}}$ & 皆 & 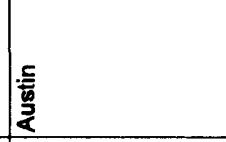 & 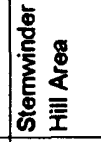 & 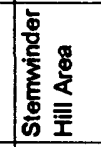 & 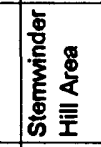 & 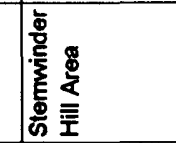 & 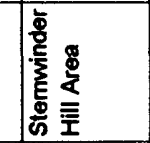 \\
\hline 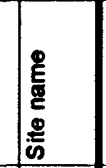 & 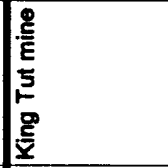 & 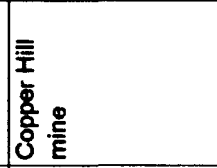 & 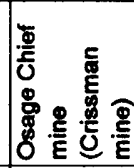 & 递 & 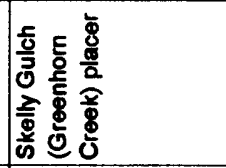 & 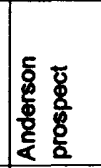 & 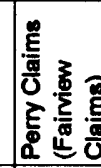 & 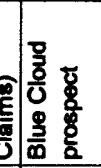 & 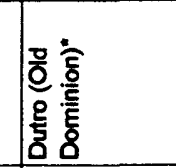 & 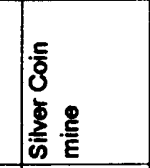 \\
\hline $\mid \dot{z}$ & $\underline{\underline{\alpha}}$ & $\Phi$ & $\Phi$ & $\stackrel{9}{9}$ & $\Phi$ & $g$ & 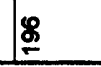 & $\underline{\mathbf{9}}$ & $\nsubseteq$ & פ \\
\hline
\end{tabular}




\begin{tabular}{|c|c|c|c|c|c|c|c|c|c|}
\hline 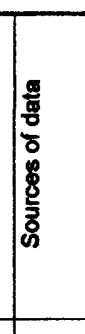 & 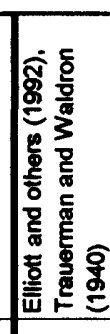 & 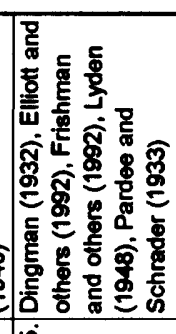 & 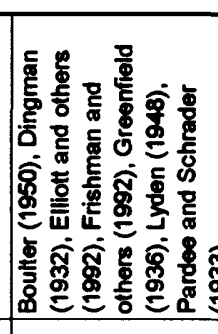 & 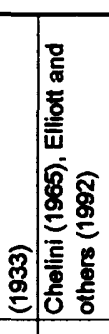 & 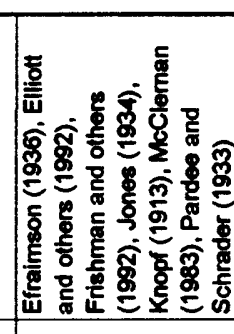 & 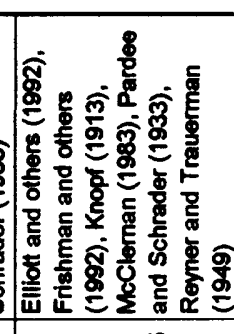 & 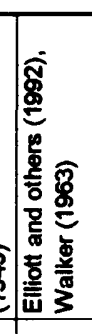 & 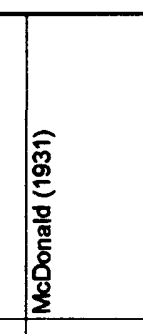 & 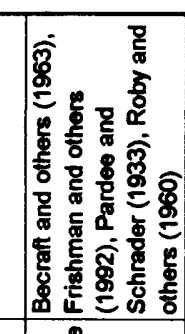 \\
\hline 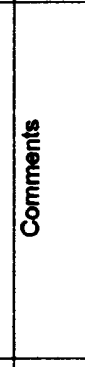 & & 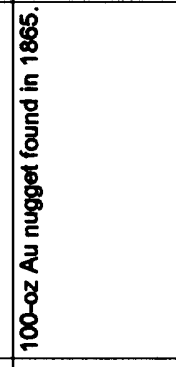 & & & 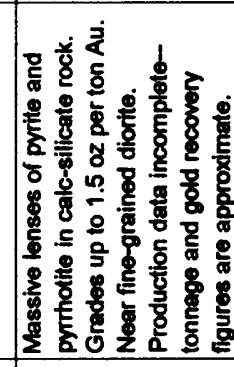 & 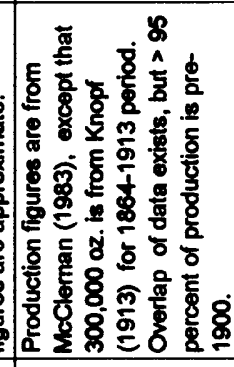 & 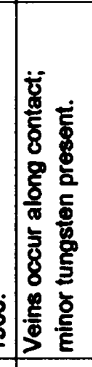 & 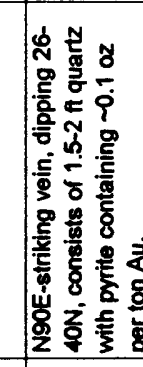 & 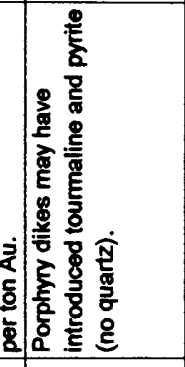 \\
\hline $\bar{N} \stackrel{\widehat{g}}{\underline{g}}$ & & & & & & มี & & & \\
\hline 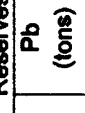 & & & & & & 岁 & & & \\
\hline वे & & & & & & $:$ & & & \\
\hline $2 \pi$ & & & & & & 居 & & & \\
\hline ₹ & & & 8 & & $\frac{8}{\frac{7}{1}}$ & 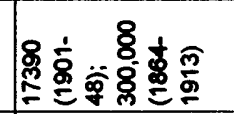 & & & 迶 \\
\hline $\mid$ & & & & 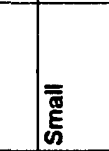 & 8 & 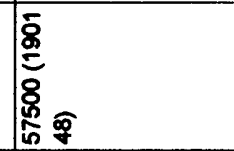 & & & \&్జి \\
\hline 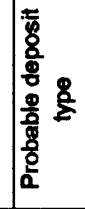 & 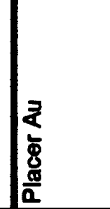 & 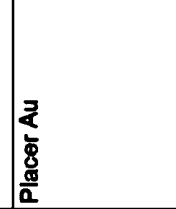 & \begin{tabular}{|l}
$\frac{z}{2}$ \\
$\frac{8}{a}$ \\
$\frac{m}{a}$
\end{tabular} & 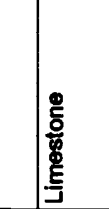 & 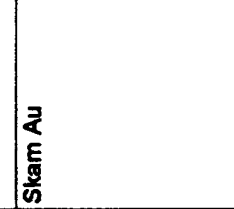 & 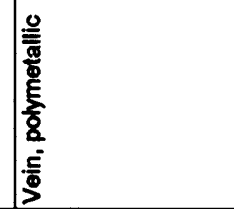 & 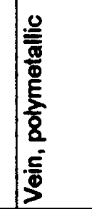 & 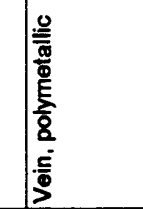 & 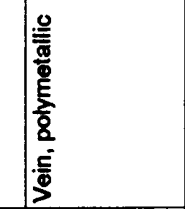 \\
\hline 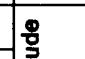 & 8 & 出 & $\tilde{m}$ & $\infty$ & 8 & 5 & $\infty$ & $\pi$ & 2 \\
\hline 柤 & r & $\infty$ & N & $\infty$ & $\infty$ & os & + & m & - \\
\hline$\Phi$ & 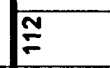 & $\cong$ & $\cong$ & $\cong$ & $\underline{N}$ & $\cong$ & $\cong$ & $\stackrel{N}{=}$ & $\cong$ \\
\hline 童 & $\frac{9}{18}$ & $\frac{1}{9}$ & 0 & $\frac{9}{9}$ & $\frac{\infty}{3}$ & $\frac{83}{\alpha}$ & $\frac{N}{2}$ & $=$ & $\frac{\hat{N}}{\mathrm{~N}}$ \\
\hline 拮 & & $\frac{m}{q}$ & $\mid \begin{array}{l}0 \\
\\
\end{array}$ & $\mid \begin{array}{l}\alpha \\
q\end{array}$ & $\frac{10}{9}$ & $\mid \frac{m}{q}$ & $\mid \begin{array}{l}m \\
\end{array}$ & $\mid \begin{array}{l}m \\
q\end{array}$ & $\mid \begin{array}{l}9 \\
\end{array}$ \\
\hline 屍- & & 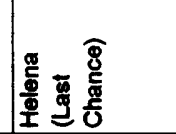 & 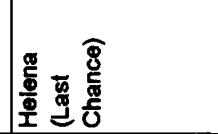 & 嘿 & 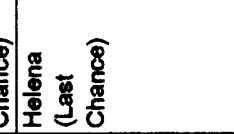 & 焉 & 离 & 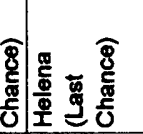 & 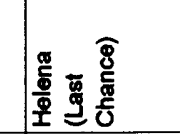 \\
\hline 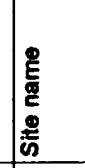 & 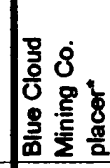 & 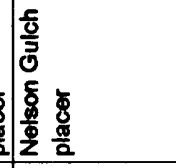 & 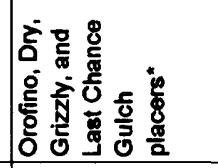 & 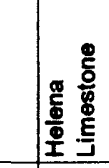 & 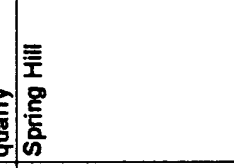 & 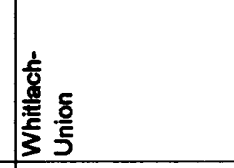 & 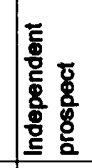 & 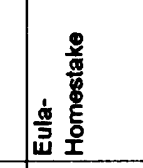 & 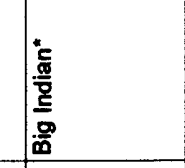 \\
\hline i & \&్ల & 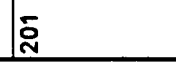 & జ్ & בֶ. & ర్లే & ళ्र & $\mathscr{e}_{-1}^{8}$ & సิ & 足 \\
\hline
\end{tabular}

พิญ 


\begin{tabular}{|c|c|c|c|c|c|c|c|c|c|c|c|c|}
\hline 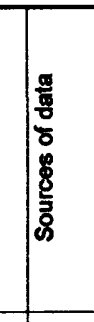 & 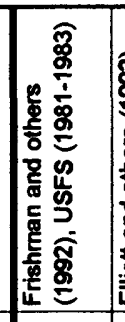 & 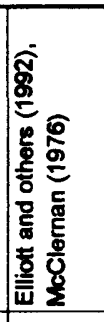 & 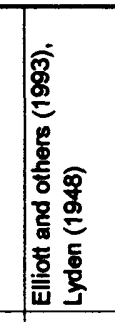 & 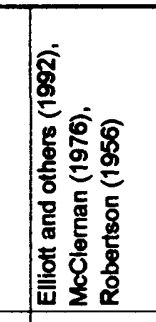 & 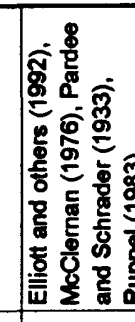 & 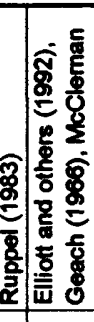 & 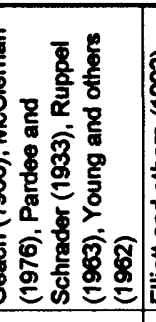 & 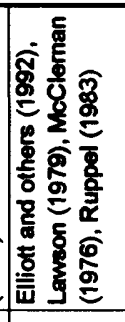 & 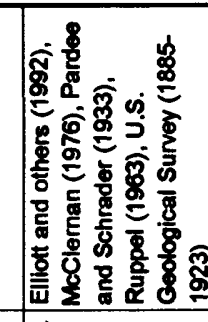 & 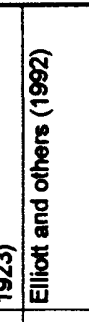 & 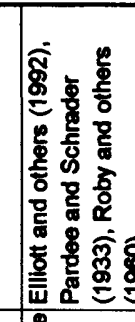 & 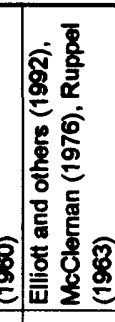 \\
\hline 墨 & & 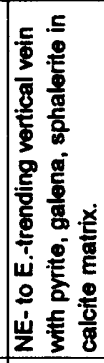 & & 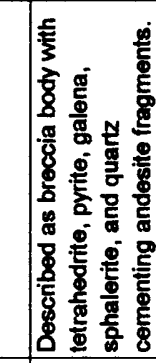 & 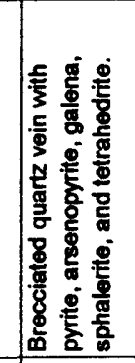 & 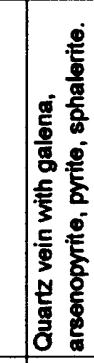 & & 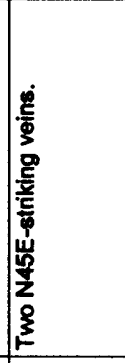 & 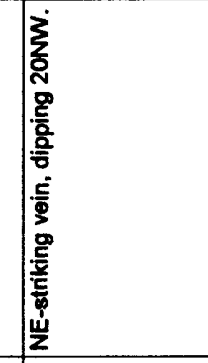 & & 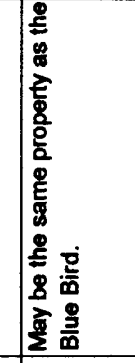 & 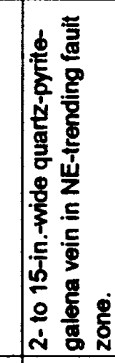 \\
\hline 西 & & & & & & 褢 & & $\infty$ & & & & \\
\hline 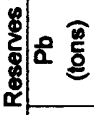 & & के & & & & 居 & & 8 & & & & \\
\hline $\bar{\partial}$ & & & & & & $\stackrel{?}{\circ}$ & & $:$ & & & & \\
\hline $9 \pi$ & & 总 & & & & 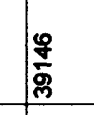 & & $\frac{\frac{\omega}{0}}{0}$ & & & & \\
\hline ₹ & & $\approx$ & $\Phi$ & & & $\tilde{\tilde{m}}$ & & 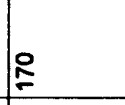 & & & & \\
\hline 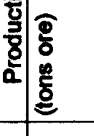 & & $\Phi$ & & & & $\tilde{\tilde{\tilde{\sigma}}}$ & & $\mathscr{\%}$ & & $\frac{2}{2}$ & & \\
\hline 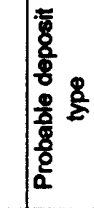 & & 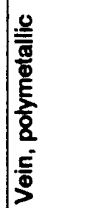 & 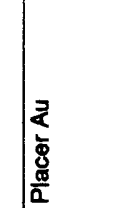 & 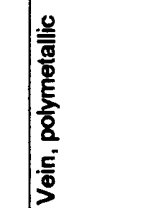 & 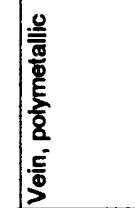 & 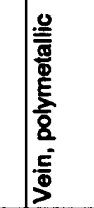 & & & 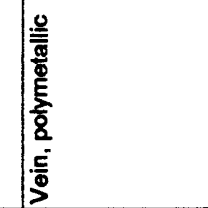 & 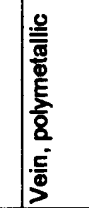 & 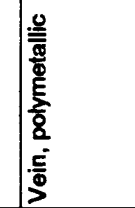 & 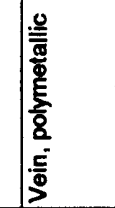 \\
\hline$-\frac{8}{3}$ & \begin{tabular}{|l|}
0 \\
0 \\
0
\end{tabular} & \begin{tabular}{|l}
5 \\
$\infty$
\end{tabular} & $\begin{array}{l}\overline{8} \\
2\end{array}$ & $\frac{F}{\pi}$ & 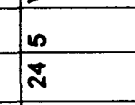 & 8 & & \begin{tabular}{|l|} 
\\
$\mathscr{D}$ \\
\end{tabular} & $\frac{7}{9}$ & $\bar{F}$ & $\frac{8}{8}$ & $\tilde{\Phi}$ \\
\hline$]^{5}$ & $\underline{E}$ & $\cong$ & $\cong$ & $\cong$ & $\cong$ & $\cong$ & & $\cong$ & $\cong$ & $\cong$ & $\cong$ & $\cong$ \\
\hline 题 & 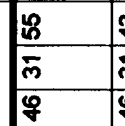 & \begin{tabular}{|l}
$\frac{7}{10}$ \\
$\frac{10}{9}$
\end{tabular} & \begin{tabular}{|l|}
9 \\
$\frac{1}{87}$ \\
8
\end{tabular} & \begin{tabular}{|l}
7 \\
8 \\
7
\end{tabular} & $\begin{array}{l}\frac{1}{6} \\
2 \\
0\end{array}$ & 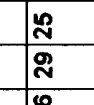 & & 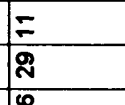 & $\begin{array}{l}\frac{\hbar}{5} \\
\frac{\pi}{2} \\
\end{array}$ & \begin{tabular}{|l}
$\tilde{\pi}$ \\
$\tilde{\pi}$ \\
0
\end{tabular} & $\begin{array}{l}\frac{0}{2} \\
\frac{10}{10} \\
\end{array}$ & 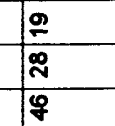 \\
\hline 憘要要 & 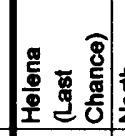 & 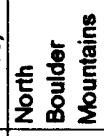 & 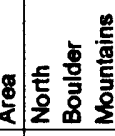 & 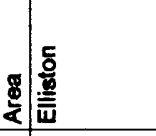 & $\mid \begin{array}{l}\frac{\mathrm{E}}{\underline{\underline{\underline{w}}}} \\
\end{array}$ & $\mid$ & & $\mid$ & 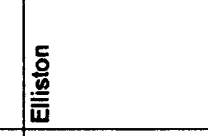 & $\mid \frac{\underline{\underline{\underline{\underline{w}}}}}{\underline{\underline{\underline{w}}}}$ & $\mid \frac{\delta}{\underline{\underline{\underline{w}}}}$ & 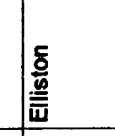 \\
\hline 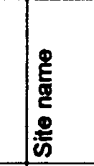 & 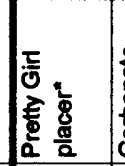 & 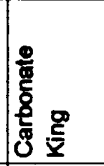 & 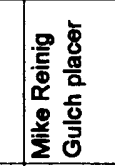 & 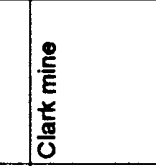 & 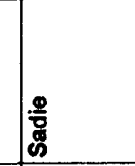 & 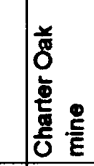 & & $\mid$ & 造 & 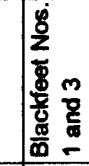 & 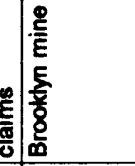 & 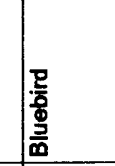 \\
\hline$\underline{i}$ & 禺 & 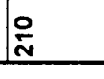 & $\equiv$ & $\frac{\tilde{N}}{\tilde{N}}$ & $\frac{m}{\pi}$ & $\frac{\pi}{\pi}$ & & $\frac{m}{N}$ & $\frac{0}{\mathbf{N}}$ & $\hat{\sim}$ & 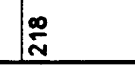 & $\frac{2}{2}$ \\
\hline
\end{tabular}




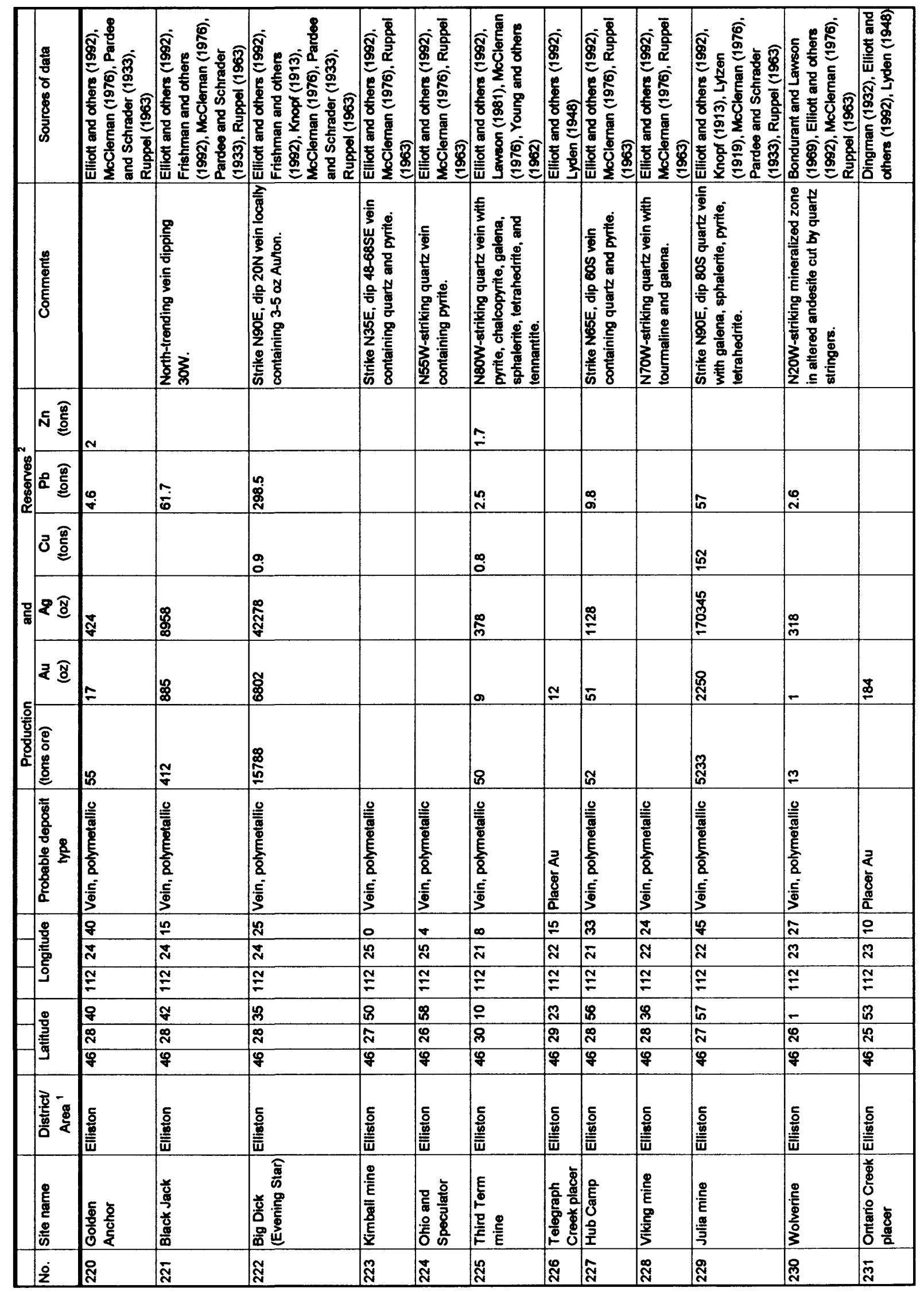

జิ ติ 


\begin{tabular}{|c|c|c|c|c|c|c|c|c|c|c|}
\hline 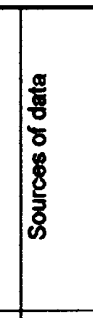 & 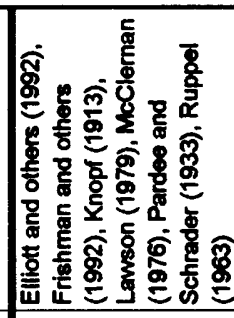 & 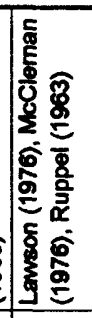 & 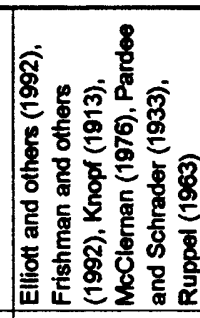 & 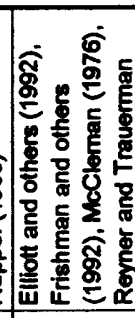 & 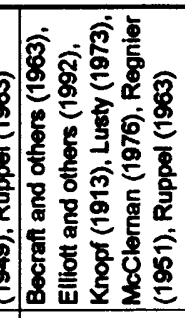 & 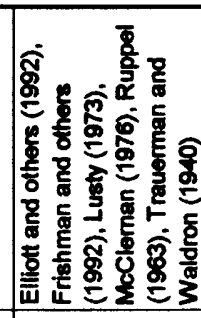 & 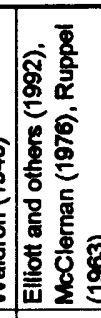 & 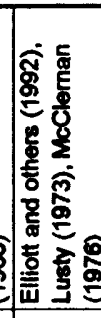 & 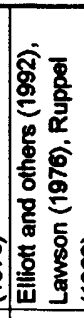 & 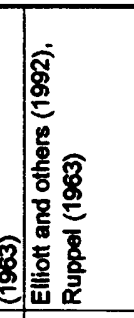 \\
\hline 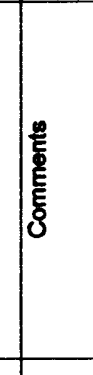 & 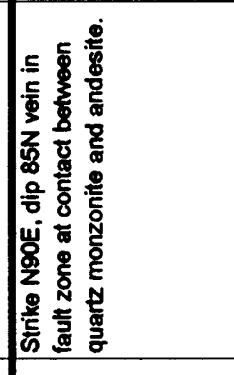 & 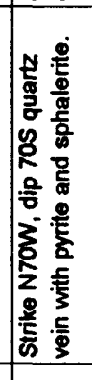 & 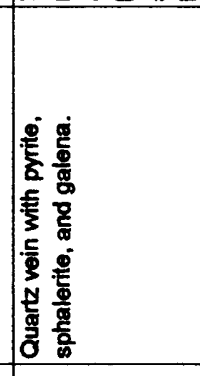 & 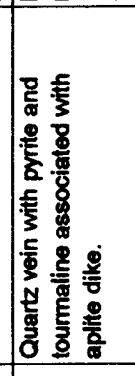 & 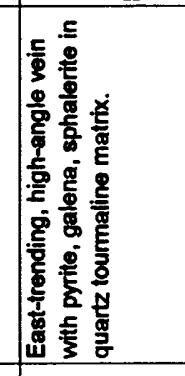 & 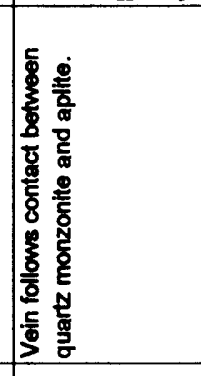 & 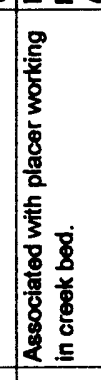 & 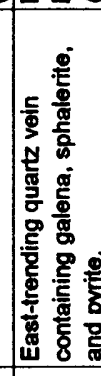 & & 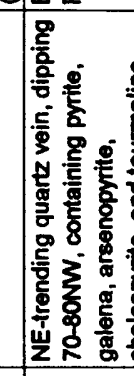 \\
\hline N & & & & & i & & & & & \\
\hline 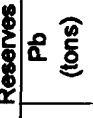 & $\tilde{q}$ & & $\mp$ & $\mathfrak{N}$ & $\hat{\mathrm{j}}$ & 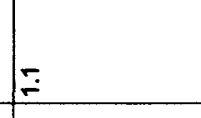 & $\dddot{8}$ & & & \\
\hline$\vec{\partial})$ & مْ & & & & $\stackrel{+}{\dot{*}}$ & I: & & & & \\
\hline $8 \widehat{\sigma}$ & $\underline{8}$ & & $\begin{array}{l}\hat{y} \\
\text { 柋 }\end{array}$ & 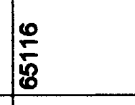 & 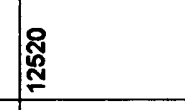 & 僮 & 8 & & & \\
\hline ₹ $\bar{\gamma}$ & 5 & & $\frac{0}{0}$ & . & 局 & $\approx$ & $E$ & & & 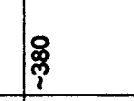 \\
\hline 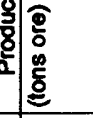 & 渠 & & జే & 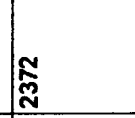 & స్ & 鮎 & ৪ & & & 을 \\
\hline \begin{tabular}{|l} 
\\
$\frac{5}{8}$ \\
$\frac{8}{8}$ \\
$\frac{8}{8}$ \\
$\frac{8}{2}$ \\
$\frac{8}{2}$ \\
$\frac{8}{2}$ \\
\end{tabular} & 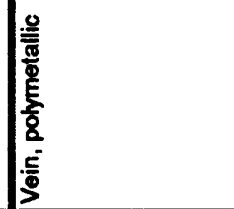 & 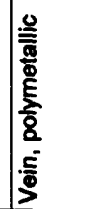 & 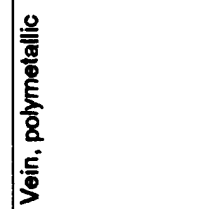 & 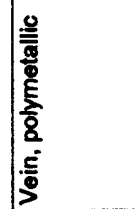 & 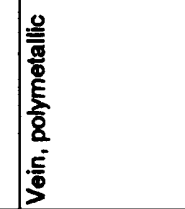 & 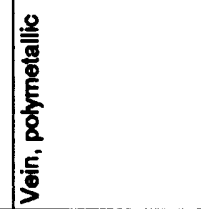 & 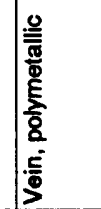 & 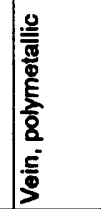 & 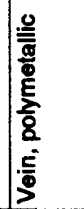 & 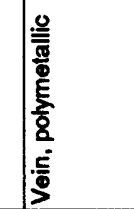 \\
\hline$-\frac{8}{3}$ & $\frac{1}{4}$ & $\simeq$ & $\frac{2}{0}$ & $\frac{5}{9}$ & $\hat{I}$ & $\infty$ & 8 & $\frac{R}{m}$ & $\underline{m}$ & to \\
\hline - & $\mid \frac{\tilde{N}}{\stackrel{\Sigma}{\Sigma}}$ & \begin{tabular}{|l}
$\tilde{N}$ \\
\\
\end{tabular} & \begin{tabular}{|l}
$\mathbb{R}$ \\
\\
\end{tabular} & \begin{tabular}{|l}
$\mathscr{2}$ \\
$\underline{\underline{N}}$
\end{tabular} & 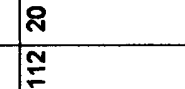 & $\frac{\mathbb{R}}{\tilde{N}}$ & \begin{tabular}{|l}
$\infty$ \\
$\underline{\infty}$ \\
$\underline{\Xi}$
\end{tabular} & \begin{tabular}{|l}
$\underline{\infty}$ \\
$\underline{\underline{N}}$
\end{tabular} & $\begin{array}{l}\tilde{F} \\
\underline{N}\end{array}$ & 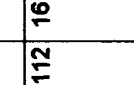 \\
\hline 18 & in & 7 & 8 & $\infty$ & 㐌 & $F$ & ก & if & a & $\bar{N}$ \\
\hline & $\bar{\alpha}$ & W & $\infty$ & $\%$ & $\mathscr{N}$ & $\hat{N}$ & $\hat{N}$ & $\hat{\mathbf{N}}$ & | & 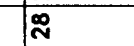 \\
\hline $7^{9}$ & 8 & 9 & 9 & $\$$ & 9 & 97 & 9 & 9 & 9 & 19 \\
\hline 萨- & 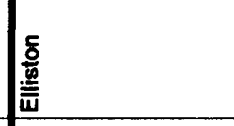 & 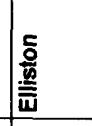 & 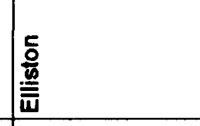 & 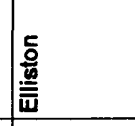 & 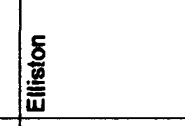 & 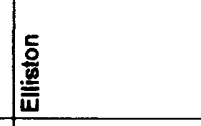 & 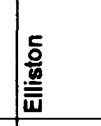 & $\mid$\begin{tabular}{|l}
$\frac{5}{\underline{\underline{\underline{m}}}}$ \\
$\underline{\underline{w}}$
\end{tabular} & 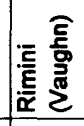 & 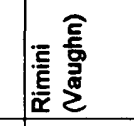 \\
\hline 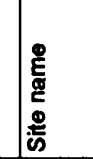 & $\mid \begin{array}{l}\frac{5}{5} \\
\frac{6}{6} \\
\frac{5}{2}\end{array}$ & 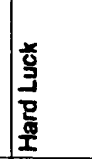 & 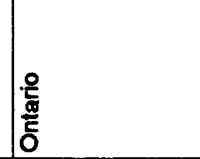 & 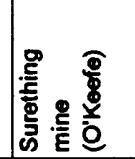 & 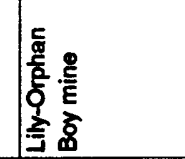 & 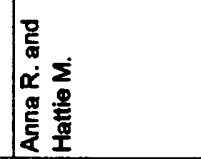 & 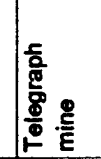 & 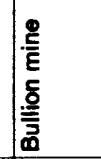 & 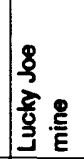 & 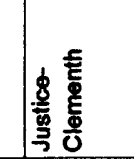 \\
\hline$\dot{2}$ & $\tilde{\widetilde{N}}$ & 芯 & 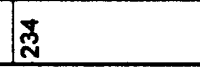 & 敢 & $\dddot{\pi}$ & $\hat{\mathbf{n}}$ & జ & 疍 & 旅 & $\bar{\Sigma}$ \\
\hline
\end{tabular}

พึำ 


\begin{tabular}{|c|c|c|c|c|c|c|c|c|c|}
\hline 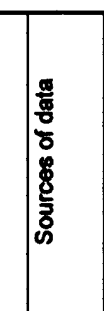 & 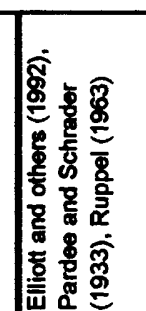 & 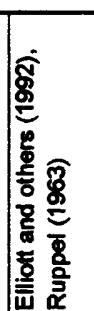 & 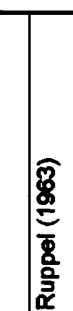 & 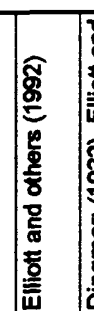 & 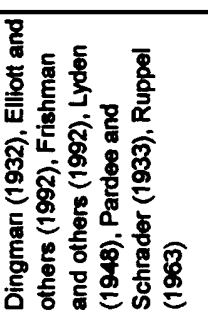 & 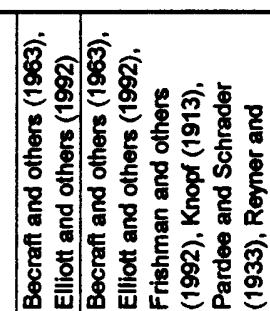 & 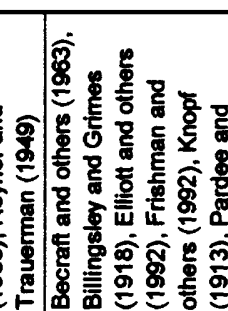 & 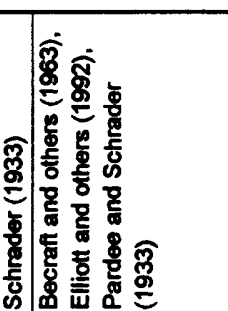 & 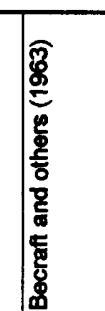 \\
\hline 量 & 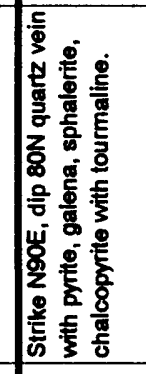 & 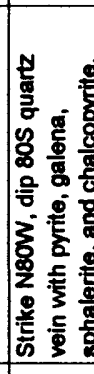 & & & & 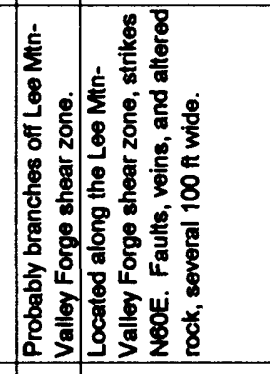 & 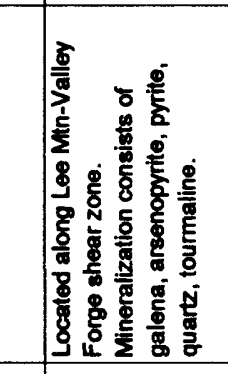 & 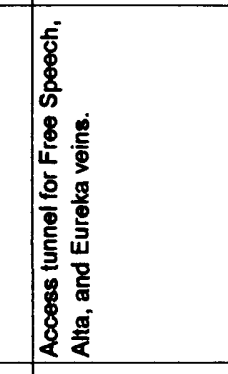 & 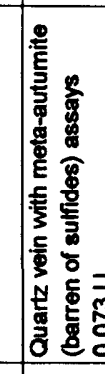 \\
\hline N $\frac{\bar{g}}{\underline{g}}$ & & & & & & $\mathscr{8}$ & & & \\
\hline 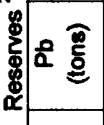 & & & & & & $\approx$ & & & \\
\hline$\overline{0} \frac{\bar{g}}{\underline{g}}$ & & & & & & & & & \\
\hline 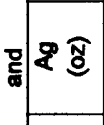 & & & & & & \% & & & \\
\hline$\geqslant \widehat{\widetilde{\theta}}$ & & & & & & 8 & 8 & & \\
\hline 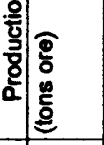 & 8 & & & 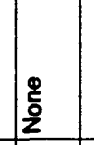 & & 夅 & & & \\
\hline 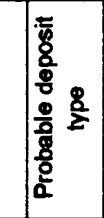 & 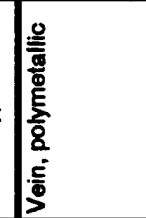 & 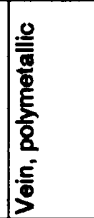 & $\mid \begin{array}{l}\frac{1}{8} \\
\frac{8}{6} \\
\frac{6}{5}\end{array}$ & 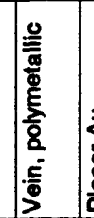 & & 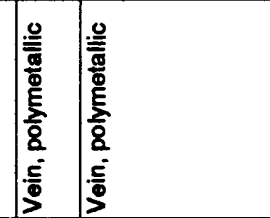 & 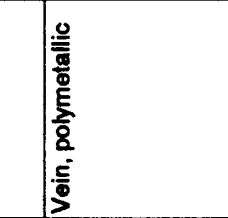 & 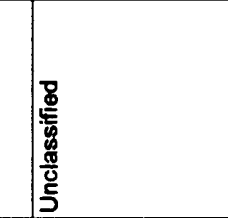 & 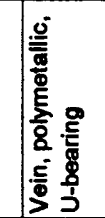 \\
\hline - & $E_{n}$ & 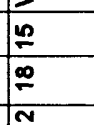 & $\frac{B}{\mp}$ & 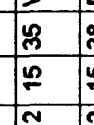 & & 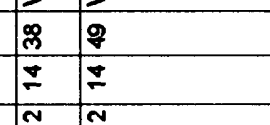 & 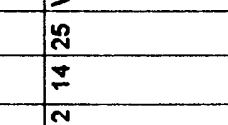 & $\begin{array}{l}\frac{1}{1} \\
\vdots \\
\\
\end{array}$ & $\frac{2}{\frac{\pi}{2}}$ \\
\hline$f^{3}$ & 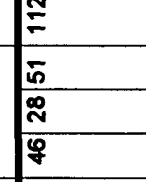 & 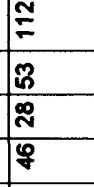 & 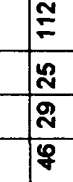 & 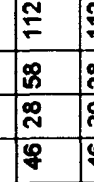 & & 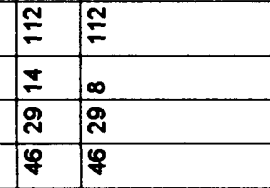 & 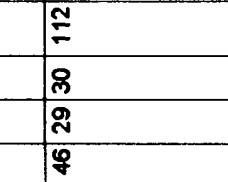 & 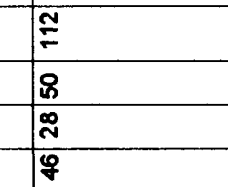 & 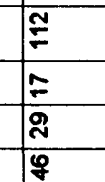 \\
\hline 謩 & 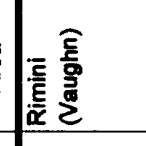 & 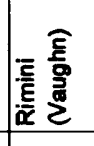 & & & & 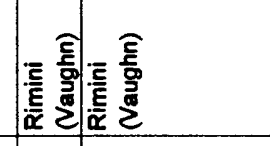 & 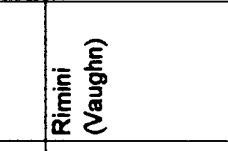 & 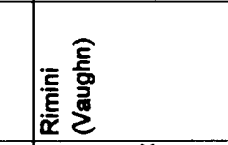 & 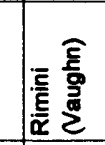 \\
\hline 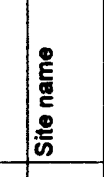 & 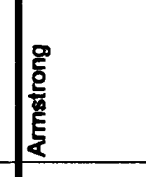 & $\frac{8}{8}$ & & & 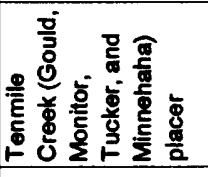 & 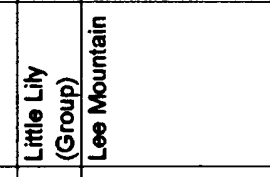 & 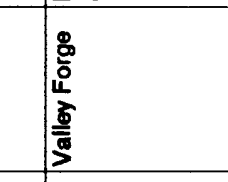 & 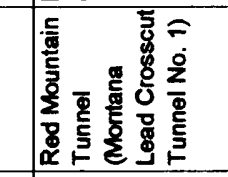 & 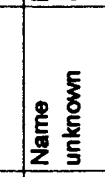 \\
\hline$\frac{1}{2}$ & $\tilde{\approx}$ & $\frac{\pi}{2}$ & \pm & 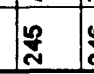 & & $\xi \frac{q}{\mathcal{N}}$ & 2 & 8 & $\overline{2}$ \\
\hline
\end{tabular}

พับำ 


\begin{tabular}{|c|c|c|c|c|c|c|c|c|c|c|c|c|c|}
\hline 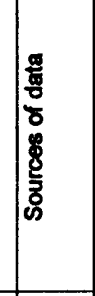 & 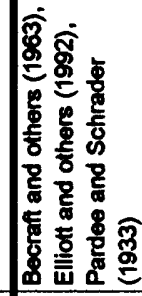 & 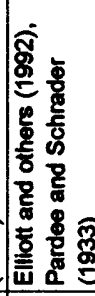 & 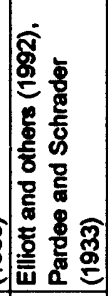 & 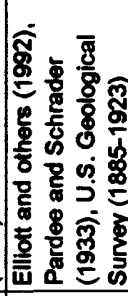 & 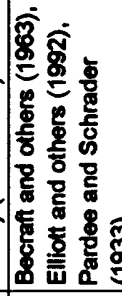 & 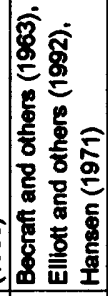 & 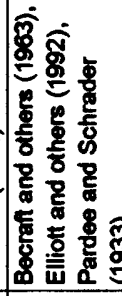 & 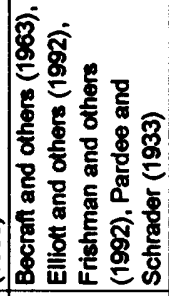 & 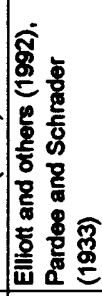 & 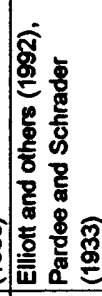 & 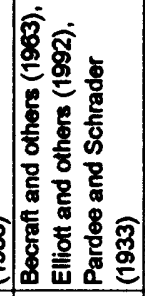 & 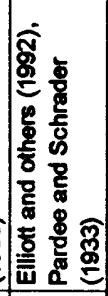 & 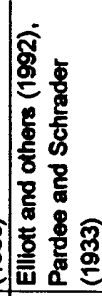 \\
\hline 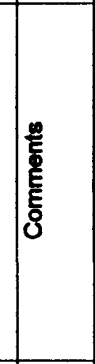 & 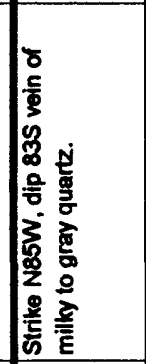 & 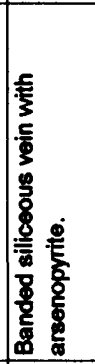 & 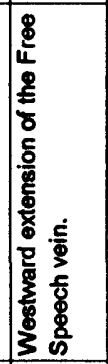 & 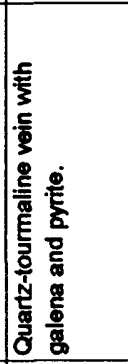 & 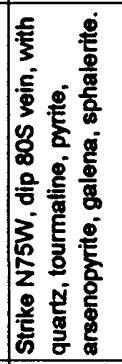 & 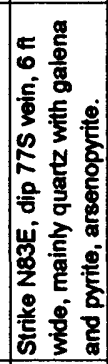 & 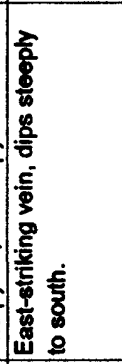 & 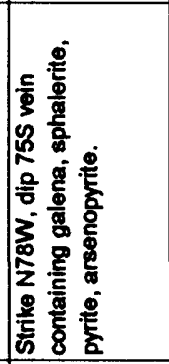 & 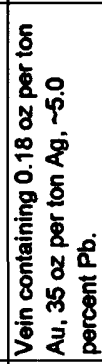 & 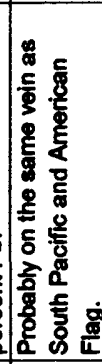 & 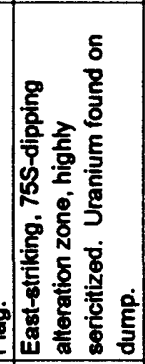 & 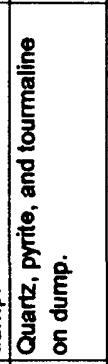 & \\
\hline 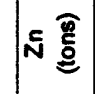 & & & & & & & & & & & & & \\
\hline 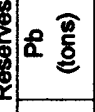 & & & & & & & & $\mathscr{\&}$ & & & & & \\
\hline 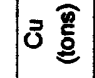 & & & & & & & & & & & & & \\
\hline$\& \overline{\underline{\sigma}}$ & & & & & & & & $\frac{8}{\frac{8}{5}}$ & & & & & \\
\hline ₹ $\bar{g}$ & & & & & & & & $\frac{0}{6}$ & & & & & \\
\hline 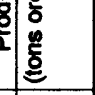 & & & & & & & & ఖ్స & & & & & \\
\hline $\mid \begin{array}{l}\frac{\bar{y}}{8} \\
\frac{8}{8} \\
\frac{8}{8} \\
\frac{8}{8} \\
\frac{8}{8} \\
\frac{2}{2} \\
\frac{2}{2}\end{array}$ & 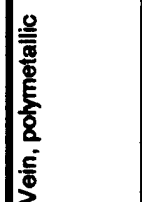 & 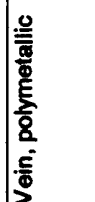 & 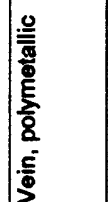 & 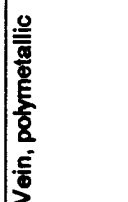 & 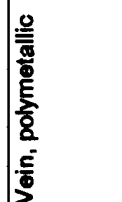 & 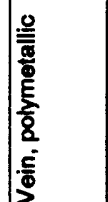 & 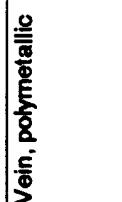 & 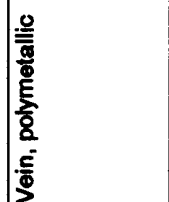 & 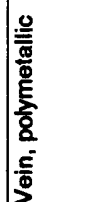 & 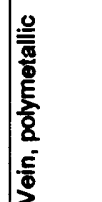 & 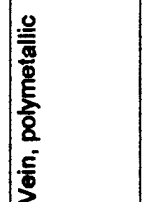 & 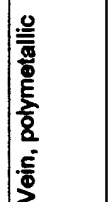 & 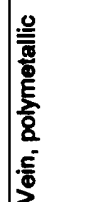 \\
\hline 8 & 0 & $\bar{m}$ & 19 & 88 & 8 & \% & 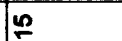 & $\bar{N}$ & J & $\%$ & \% & $\mathscr{N}$ & \pm \\
\hline 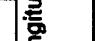 & \pm & \pm & \pm & I & \pm & \pm & I & \pm & \pm & \pm & \pm & \pm & \pm \\
\hline 9 & $\stackrel{2}{2}$ & $\stackrel{\underline{N}}{\rightleftharpoons}$ & $\cong$ & 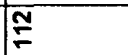 & $\stackrel{\mathbb{N}}{\rightleftharpoons}$ & $\stackrel{\mathbb{N}}{\mp}$ & $\cong$ & $\stackrel{\simeq}{\rightleftharpoons}$ & $\cong$ & $\stackrel{\underline{T}}{\rightleftharpoons}$ & $\stackrel{\mathbb{N}}{\cong}$ & $\stackrel{\underline{N}}{=}$ & $\stackrel{\cong}{\mp}$ \\
\hline 8 & ল్ల & 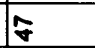 & న్ల & \pm & $\mathscr{2}$ & $\hat{N}$ & A & $\bar{N}$ & 2 & 오 & $\dddot{0}$ & 1 & 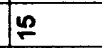 \\
\hline ב. & న & $\mathbf{I}$ & $\infty$ & ని & $\infty$ & $\infty$ & $\infty$ & $\infty$ & $\infty$ & $\infty$ & జ & 必 & क \\
\hline$\Phi$ & 9 & \% & 9 & $\%$ & iq & क & क & क़ & 9 & 9 & 9 & 9 & 9 \\
\hline 庵- & 施总 & 竞 & 站产 & 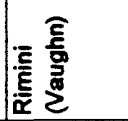 & 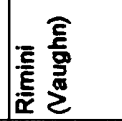 & 氞 & 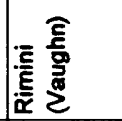 & 施 & 施 & 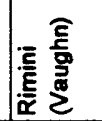 & 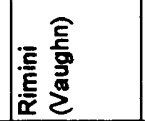 & 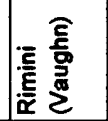 & 产总 \\
\hline 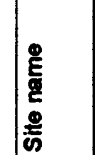 & | & 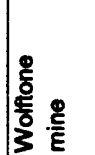 & 总 & 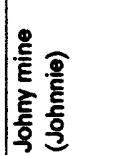 & 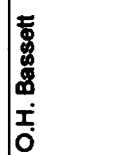 & $\begin{array}{l}\frac{5}{8} \\
\frac{8}{6} \\
0 \\
8 \\
8 \\
\frac{8}{4}\end{array}$ & 要 & 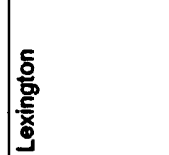 & 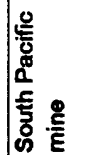 & 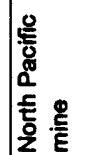 & 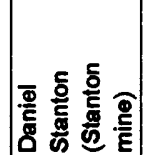 & 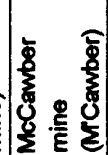 & 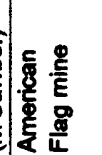 \\
\hline í & జ్ట్ & 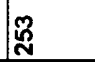 & 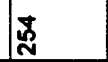 & $\stackrel{\mathscr{N}}{2}$ & $\$$ & న్న & 点 & 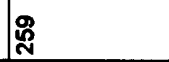 & $\underset{\text { \& }}{8}$ & $\overline{\mathscr{D}}$ & జ్ల & $\mathbb{\&}$ & 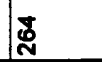 \\
\hline
\end{tabular}

พึ๊ 


\begin{tabular}{|c|c|c|c|c|c|c|c|c|c|c|c|c|c|}
\hline 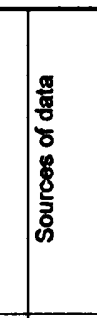 & 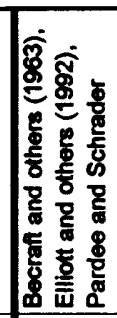 & 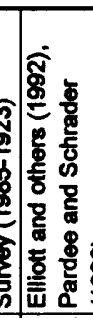 & 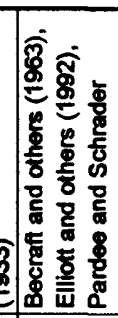 & 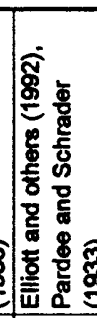 & 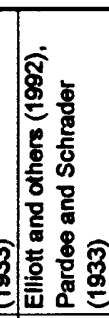 & 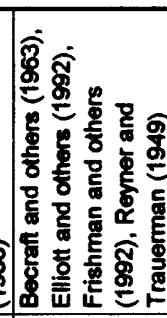 & 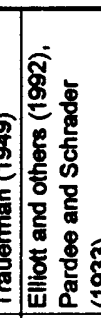 & 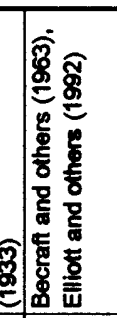 & 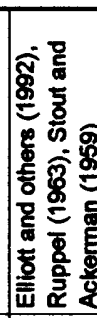 & 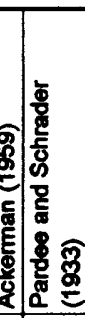 & 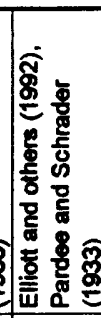 & 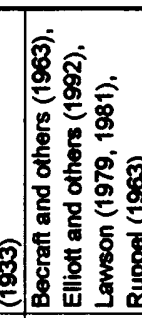 & 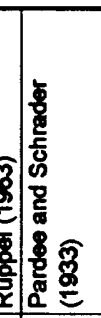 \\
\hline 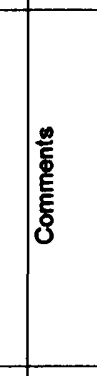 & 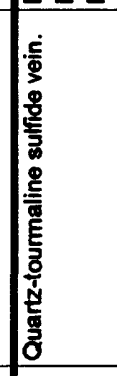 & 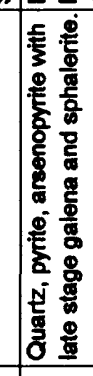 & 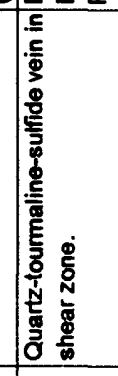 & 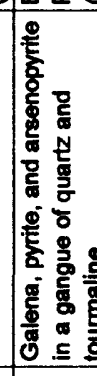 & 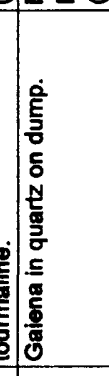 & 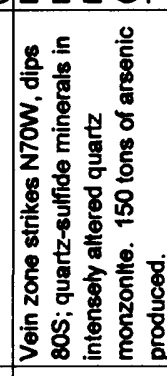 & 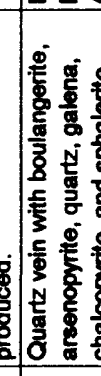 & 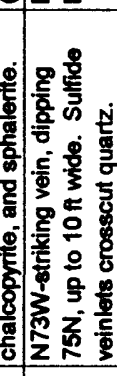 & & 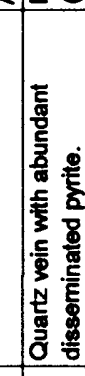 & 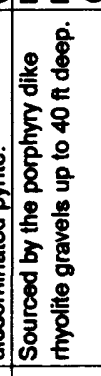 & 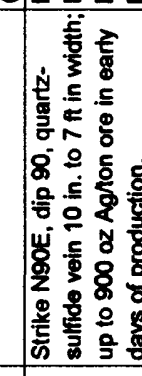 & 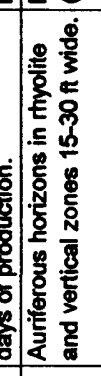 \\
\hline$\tilde{N} \overline{\underline{\alpha}}$ & to & & $\because$ & & & $\nsubseteq$ & & & & & & & \\
\hline 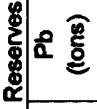 & 象 & & N & & & 8 & & & & & & & \\
\hline उठ & $F$ & & & & & $\stackrel{I}{E}$ & & & & & & & \\
\hline 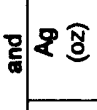 & 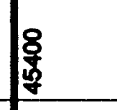 & & 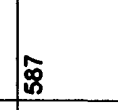 & & & $\frac{\mathscr{L}}{2}$ & & & & & & & \\
\hline ₹ & $\frac{1}{0}$ & & m & & & 8 & & & & & & & \\
\hline 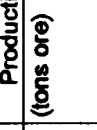 & 鹿 & & \pm & & & $\frac{m}{3}$ & & & & & & & \\
\hline 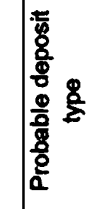 & 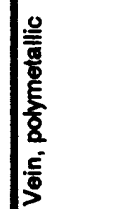 & 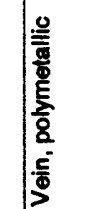 & 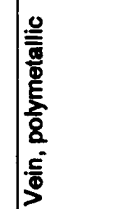 & 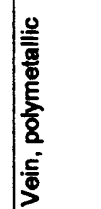 & 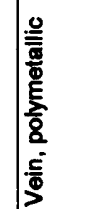 & 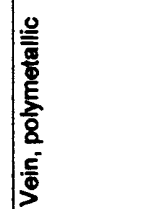 & 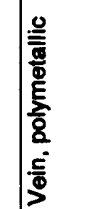 & 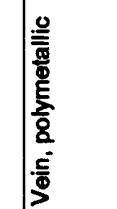 & 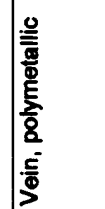 & 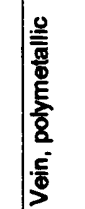 & 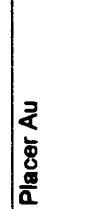 & 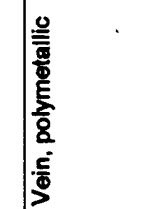 & 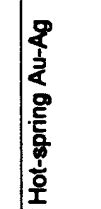 \\
\hline$-\frac{8}{2}$ & $\frac{\pi}{\sigma}$ & $\frac{8}{8}$ & $\frac{7}{7}$ & $\tilde{\tilde{m}}$ & $\frac{1}{7}$ & $\tilde{n}$ & $\frac{7}{7}$ & $\frac{8}{17}$ & $\frac{a}{10}$ & \begin{tabular}{|l|l}
$\infty$ \\
0
\end{tabular} & $\frac{\rho}{\infty}$ & 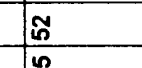 & $\frac{a}{a}$ \\
\hline 誩 & $\frac{\mathbb{Z}}{\stackrel{\Sigma}{\underline{N}}}$ & 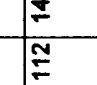 & $\mid$\begin{tabular}{|l}
$\bar{\Sigma}$ \\
\end{tabular} & \begin{tabular}{|l}
$\underline{\Xi}$ \\
$\underline{\Sigma}$
\end{tabular} & \begin{tabular}{|l}
$\mathbf{I}$ \\
$\stackrel{\underline{N}}{\mathbf{N}}$
\end{tabular} & \begin{tabular}{|l}
$\mid \underline{\Sigma}$ \\
$\underline{\Sigma}$
\end{tabular} & 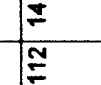 & \begin{tabular}{|l}
$\underline{\Sigma}$ \\
$\stackrel{\Sigma}{\Sigma}$
\end{tabular} & \begin{tabular}{|l}
$\underline{\mu}$ \\
$\underline{N}$
\end{tabular} & $\begin{array}{l}\stackrel{6}{\simeq} \\
\cong\end{array}$ & 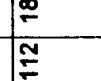 & 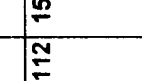 & $\stackrel{F}{\bar{N}}$ \\
\hline - & 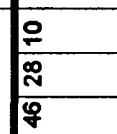 & \begin{tabular}{|l|}
$\infty$ \\
$\infty$ \\
\multirow{2}{*}{} \\
$q$
\end{tabular} & 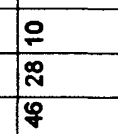 & 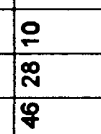 & 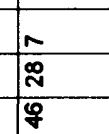 & 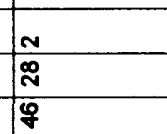 & \begin{tabular}{|l|}
0 \\
$\infty$ \\
$\infty$ \\
$q$
\end{tabular} & \begin{tabular}{|l|}
7 \\
$\mathbf{N}$ \\
$q$ \\
\end{tabular} & 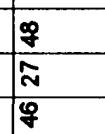 & \begin{tabular}{|l|}
$\bar{n}$ \\
$\bar{n}$ \\
\\
\end{tabular} & 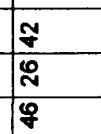 & \begin{tabular}{|l|}
0 \\
$\tilde{a}$ \\
$\mathscr{q}$
\end{tabular} & 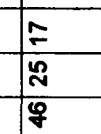 \\
\hline 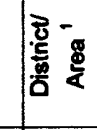 & 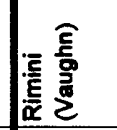 & 施离 & 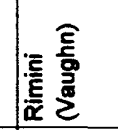 & 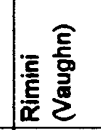 & 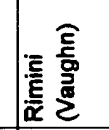 & 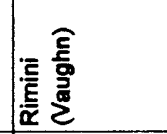 & 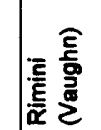 & 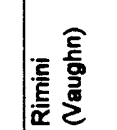 & 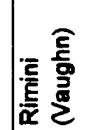 & & 施高 & 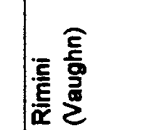 & 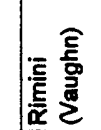 \\
\hline 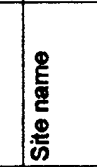 & $\mid$ & 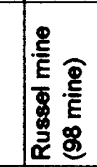 & 递 & 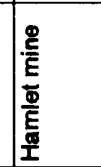 & 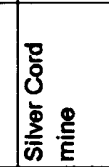 & 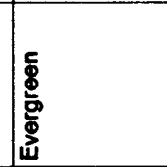 & 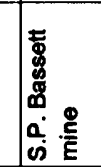 & $\frac{8}{2}$ & 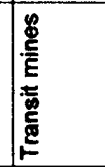 & & 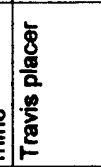 & 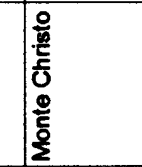 & 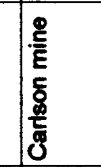 \\
\hline$\dot{i}$ & 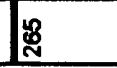 & $\$$ & $\widehat{\mathscr{D}}$ & 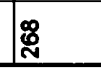 & : & R & $\overline{\mathbf{N}}$ & $\tilde{N}$ & 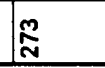 & $\frac{\pi}{n}$ & 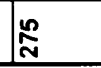 & $\stackrel{2}{N}$ & స \\
\hline
\end{tabular}

ํํำ 


\begin{tabular}{|c|c|c|c|c|c|c|c|c|c|c|}
\hline 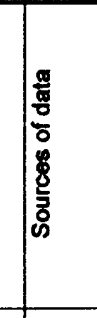 & 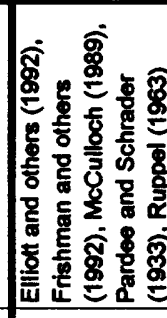 & 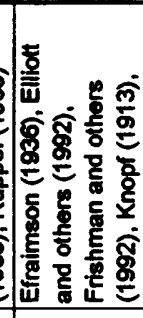 & 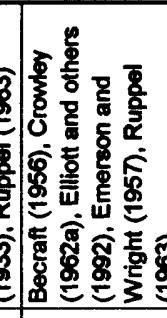 & 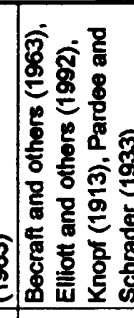 & 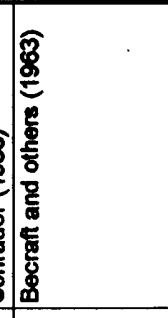 & 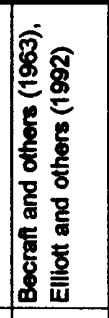 & 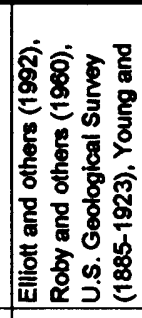 & 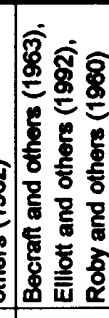 & 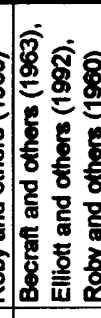 & 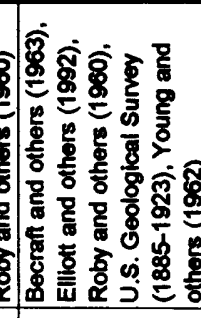 \\
\hline 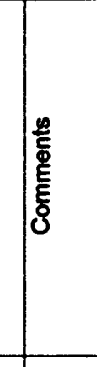 & 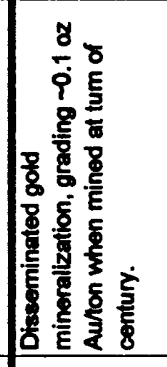 & 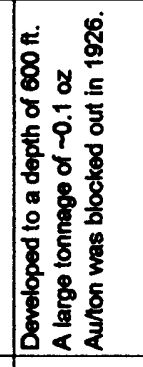 & 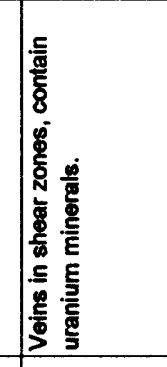 & 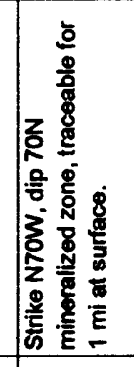 & 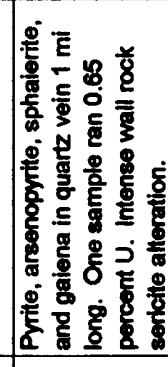 & 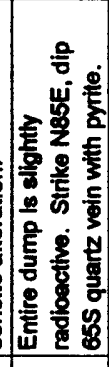 & 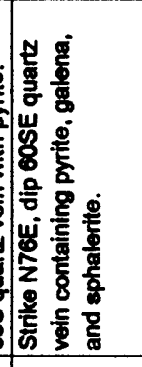 & 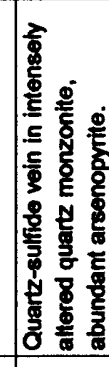 & 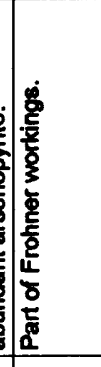 & 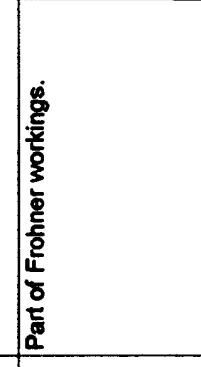 \\
\hline 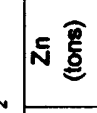 & & & & & & & : & & & \\
\hline 远 & & & & & & & 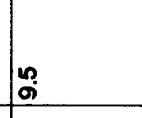 & & & \\
\hline 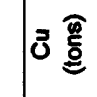 & & & & & & & & & & \\
\hline 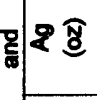 & & & & & & & 崫 & & & \\
\hline ₹ $\widehat{\underline{\sigma}}$ & $\frac{8}{8}$ & 串 & & & & & 은 & & & \\
\hline 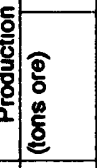 & 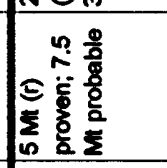 & 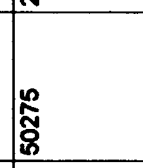 & & & & & 8 & 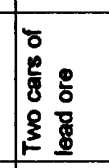 & & \\
\hline 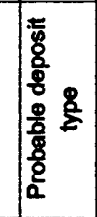 & 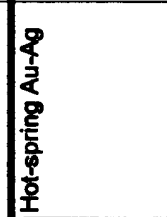 & 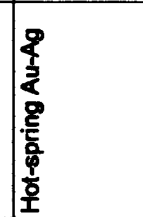 & 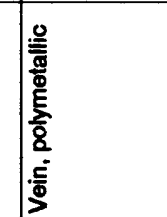 & 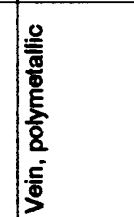 & 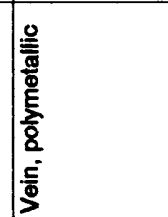 & 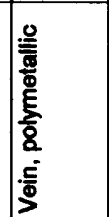 & 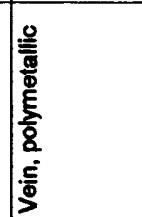 & 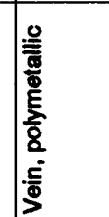 & 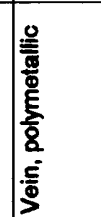 & 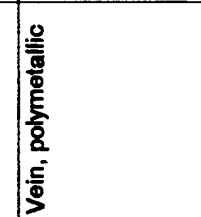 \\
\hline \% & 8 & to & 8 & $\pi$ & 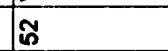 & $\infty$ & 8 & $\infty$ & 2 & in \\
\hline & $F$ & $\neq$ & $\cong$ & \pm & 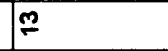 & $\simeq$ & $\simeq$ & $\simeq$ & $\simeq$ & $\simeq$ \\
\hline 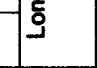 & $\cong$ & $\cong$ & $\stackrel{N}{=}$ & $\cong$ & $\cong$ & $\cong$ & $\cong$ & $\cong$ & $\cong$ & $\cong$ \\
\hline 1\% & 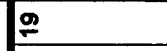 & q & $\stackrel{\infty}{\circ}$ & $\tilde{n}$ & 8 & ल & 8 & 8 & జ & $\approx$ \\
\hline 谞 & 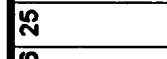 & $\infty$ & 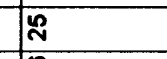 & 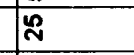 & $\infty$ & $\hat{n}$ & $\%$ & $\%$ & $\%$ & $\%$ \\
\hline & 9 & 9 & 9 & 9 & 9 & 9 & 9 & 9 & 9 & 9 \\
\hline 唔- & 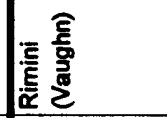 & 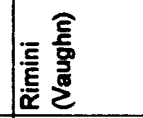 & 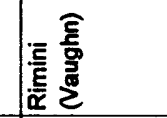 & 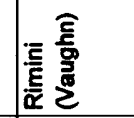 & 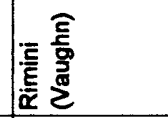 & 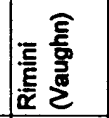 & 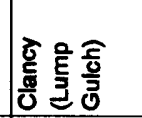 & 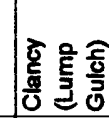 & 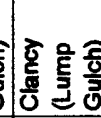 & 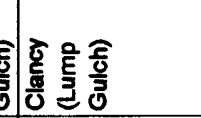 \\
\hline 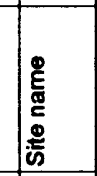 & 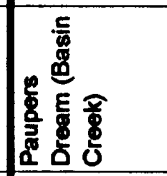 & 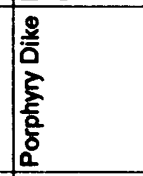 & 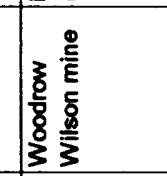 & 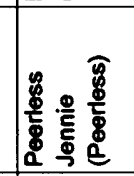 & 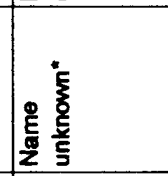 & 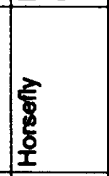 & 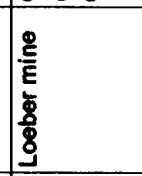 & 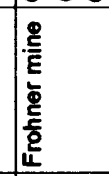 & 量 & 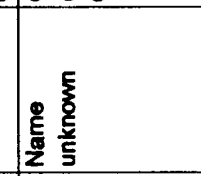 \\
\hline i & 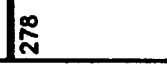 & 里 & 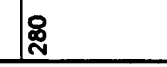 & $\overline{\mathbf{m}}$ & 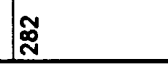 & $\mathbb{Z}$ & ఖ్ల & 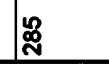 & 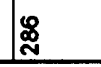 & $\widehat{\hat{\tilde{d}}}$ \\
\hline
\end{tabular}

สิบ 


\begin{tabular}{|c|c|c|c|c|c|c|c|c|c|c|}
\hline 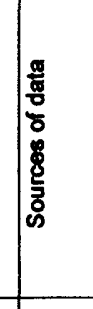 & 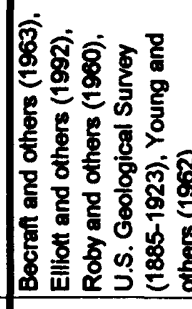 & 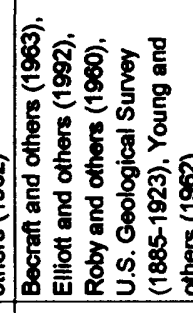 & 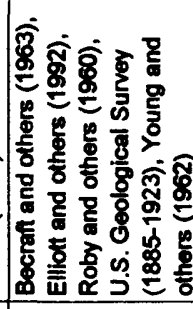 & 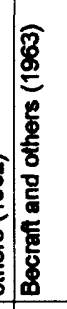 & 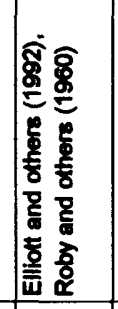 & 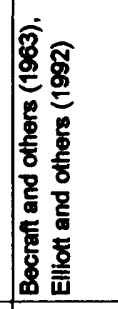 & 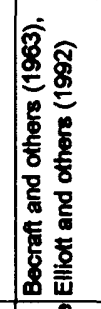 & 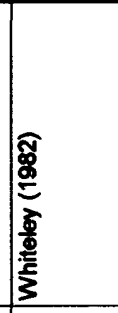 & 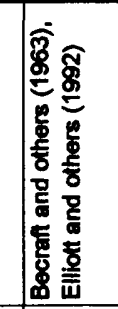 & 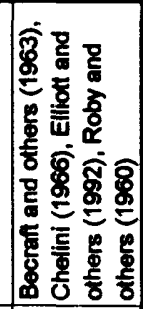 \\
\hline 嘉 & 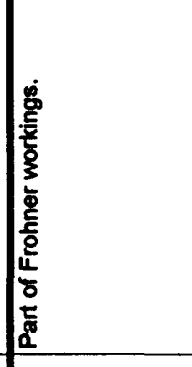 & 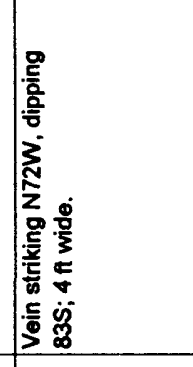 & 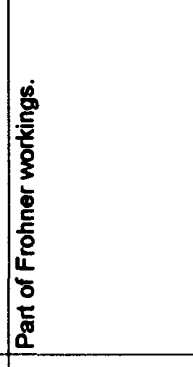 & & 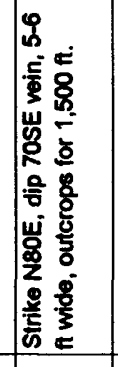 & 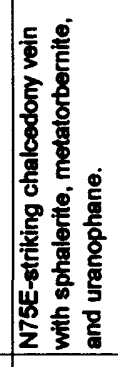 & 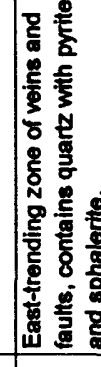 & 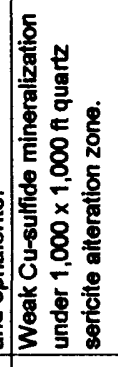 & 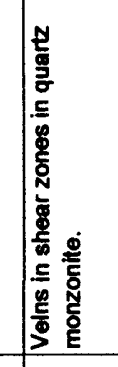 & 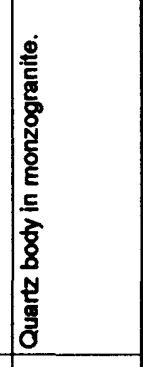 \\
\hline $\bar{N} \frac{\widehat{d}}{\underline{g}}$ & & & & & & & & & & \\
\hline$\stackrel{0}{0}$ & & & & & & & & & & \\
\hline $\overrightarrow{0} \frac{\widehat{\underline{d}}}{\mathrm{~g}}$ & & & & & & & & & & \\
\hline $2 \sqrt{0}$ & & & & & & & & & & \\
\hline ₹ $\widehat{\widetilde{\delta}}$ & & & & & & & & & & \\
\hline 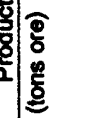 & & & & & $\infty_{\infty}$ & & & $\mid \frac{0}{5}$ & & $\mid \frac{9}{\frac{9}{2}}$ \\
\hline 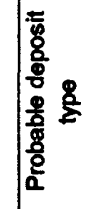 & 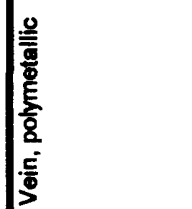 & 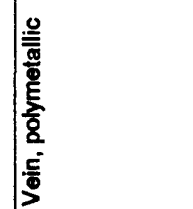 & 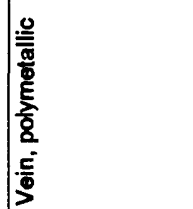 & 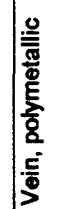 & 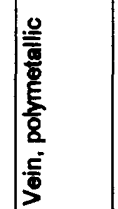 & 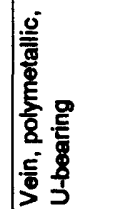 & 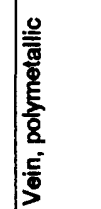 & 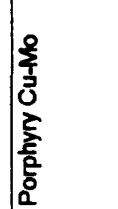 & 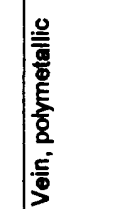 & 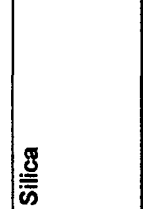 \\
\hline $8 \frac{8}{3}$ & $\frac{8}{N}$ & $\frac{m}{n}$ & N & $a$ & $\frac{2}{2}$ & $\frac{18}{10}$ & in & $\frac{8}{10}$ & $\tilde{\tilde{n}}$ & $F$ \\
\hline 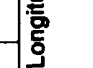 & $\cong$ & $\simeq$ & $\underline{\simeq}$ & $\cong$ & $\mid$ & $\frac{\mathscr{2}}{\underline{N}}$ & $\mid \frac{\sigma}{\underline{N}}$ & $\frac{P}{N}$ & $\frac{0}{N}$ & $\hat{N}$ \\
\hline & 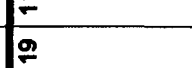 & $\overline{\tilde{a}}$ & $F$ & $\frac{F}{\mathscr{2}}$ & it & $\frac{\mid F}{9}$ & $F_{\bar{\Sigma}}$ & $F$ & $F$ & $F$ \\
\hline - & 2 & $\stackrel{2}{2}$ & $\mathscr{2}$ & $\mathscr{N}$ & $\overline{2}$ & \% & 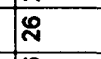 & 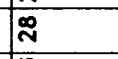 & $\bar{\lambda}$ & 必 \\
\hline & 9 & 9 & 9 & 9 & 9 & 98 & 9 & 9 & 19 & 9 \\
\hline 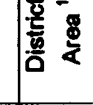 & | & 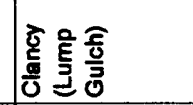 & 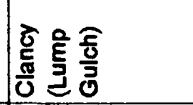 & 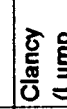 & 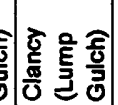 & 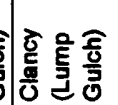 & 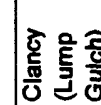 & 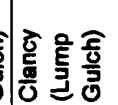 & 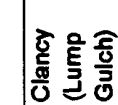 & 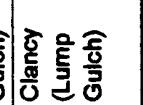 \\
\hline 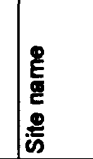 & 愿 & 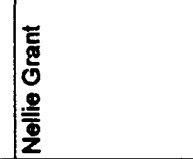 & 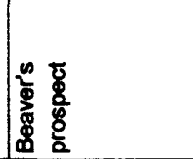 & 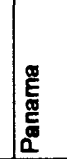 & 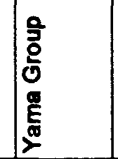 & 总 & 高 & | & 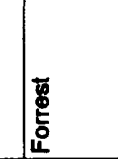 & 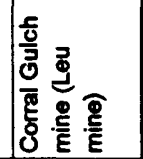 \\
\hline$\frac{8}{2}$ & 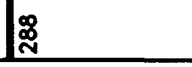 & 㘠 & \&్ల & $\overline{\mathbf{s}}$ & జ్ & $\cong$ & 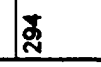 & ఝ్ & $\$$ & $\hat{\mathbf{s}}$ \\
\hline
\end{tabular}

$\stackrel{\text { พิ }}{\mathfrak{m}}$ 


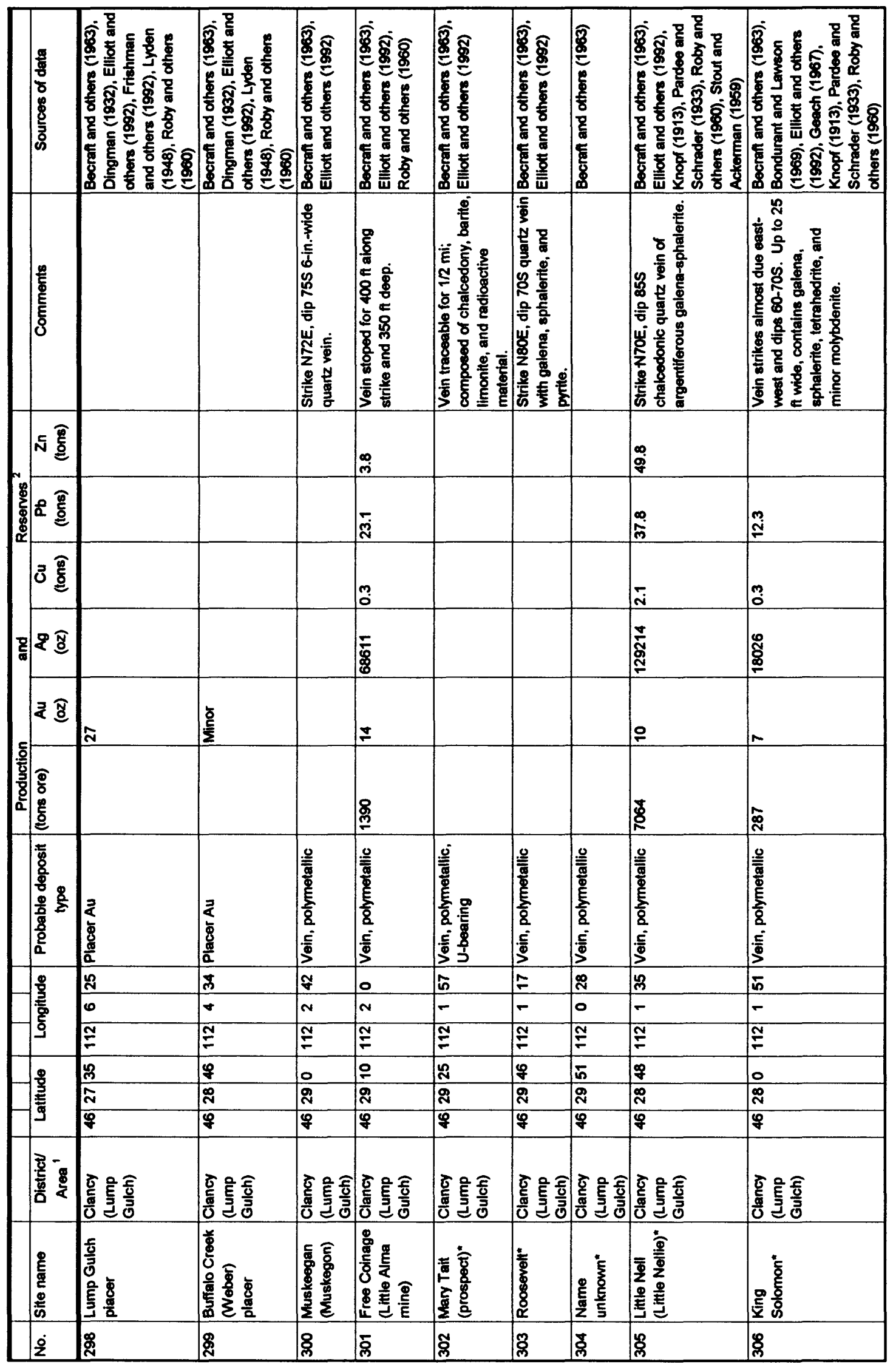

జ̊ํํ 


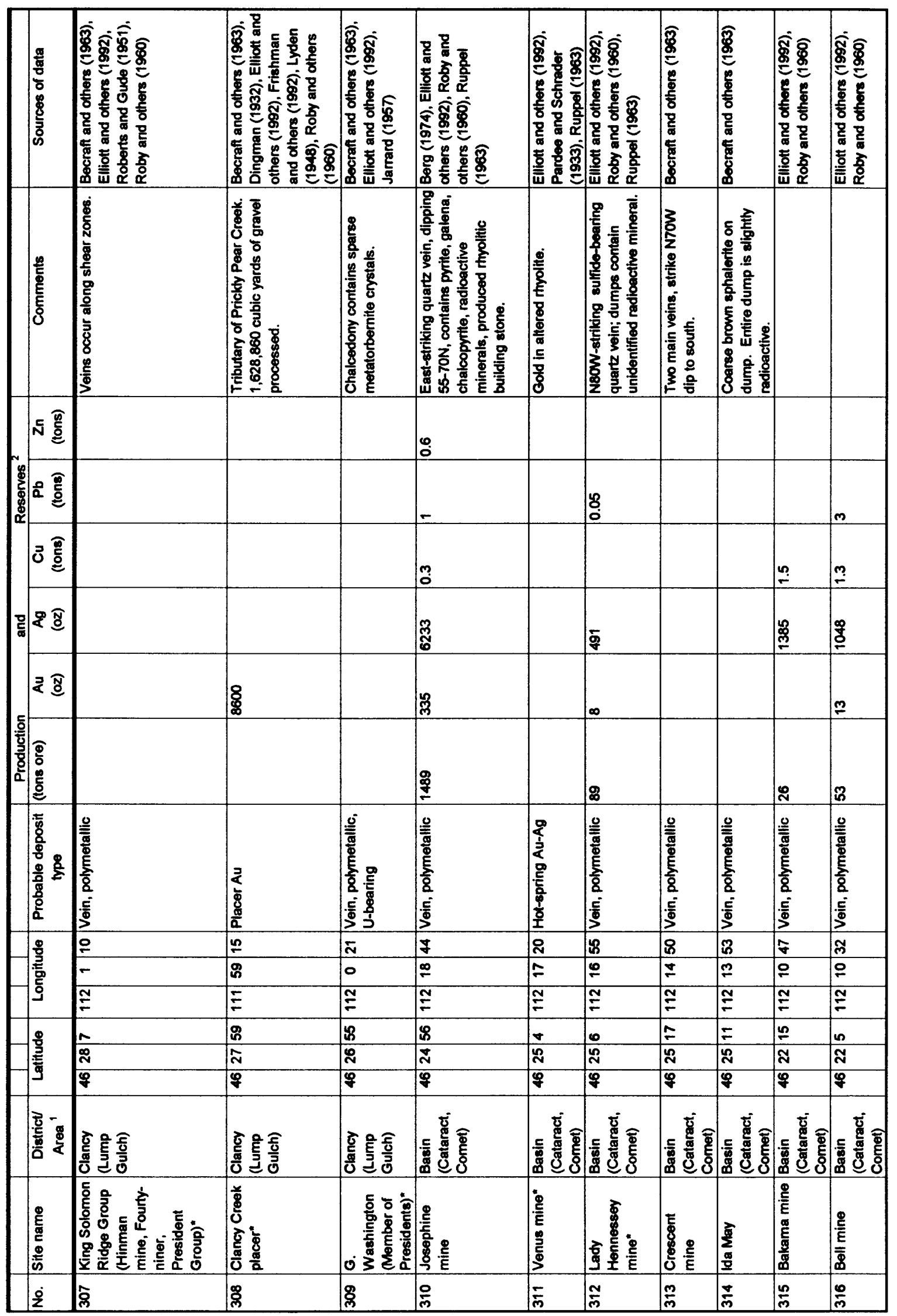




\begin{tabular}{|c|c|c|c|c|c|c|c|c|c|}
\hline 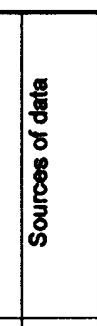 & 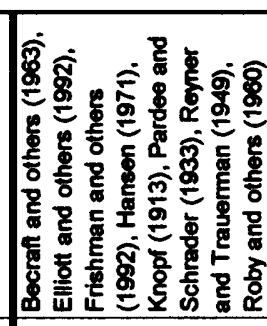 & 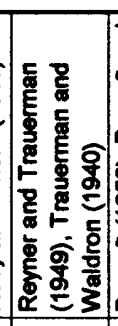 & 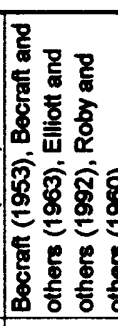 & 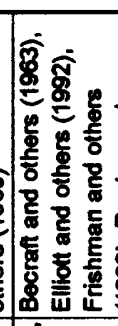 & 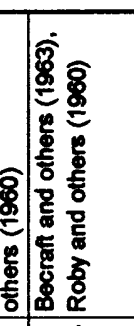 & 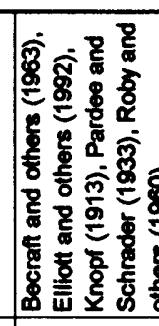 & 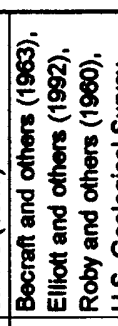 & 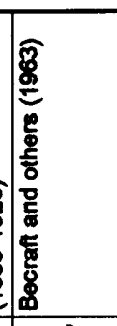 & 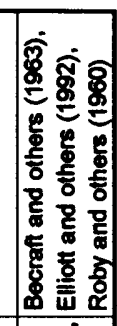 \\
\hline | & 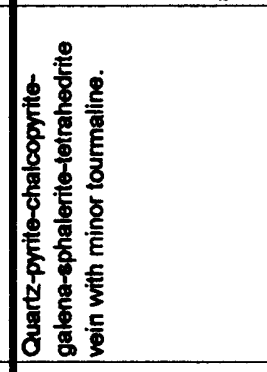 & & 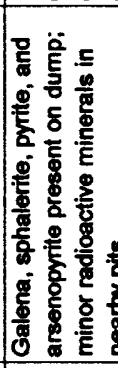 & 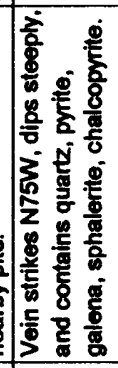 & 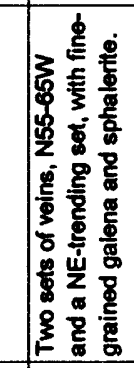 & 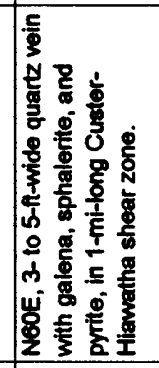 & 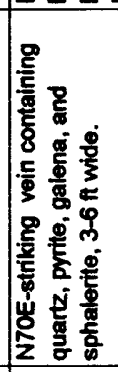 & 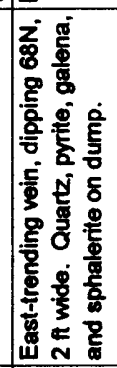 & 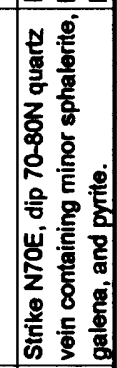 \\
\hline 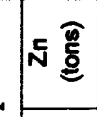 & & & & $\stackrel{\circ}{1}$ & $\infty_{\infty}^{\infty}$ & & & & \\
\hline$\frac{6}{0}$ & & & & 家 & $\frac{n}{\dot{\sigma}}$ & & & & $:$ \\
\hline ठ己 & & & & $\bar{\alpha}$ & $\overline{\mathrm{N}}$ & & & & \\
\hline 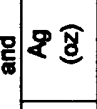 & & & & 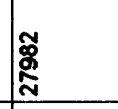 & 范 & 若 & \begin{tabular}{l}
0 \\
\multirow{2}{*}{} \\
\end{tabular} & & 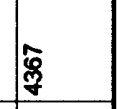 \\
\hline ₹ & & & & $\frac{m}{m}$ & 票 & 8 & E & & 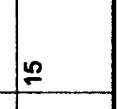 \\
\hline 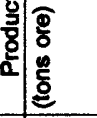 & & & 总 & $\frac{\mathscr{0}}{\underline{m}}$ & 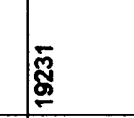 & $\tilde{\tilde{z}}$ & ฐู & & $\approx$ \\
\hline 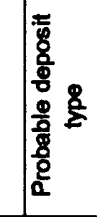 & 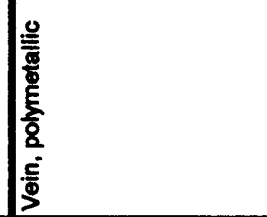 & 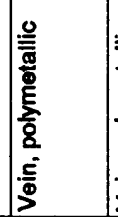 & 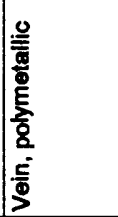 & 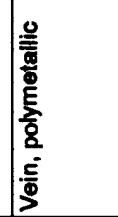 & 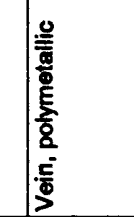 & 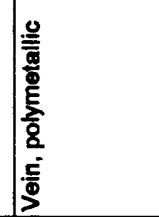 & 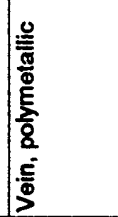 & 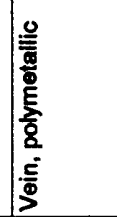 & 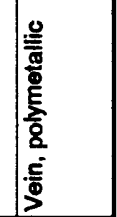 \\
\hline 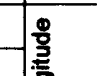 & $\frac{2}{m}$ & $\frac{\varphi}{=}$ & $\frac{\delta}{N}$ & $\frac{5}{4}$ & $\frac{\infty}{4}$ & $\frac{18}{10}$ & $\frac{m}{m}$ & $\infty$ & 0 \\
\hline-8 & $\underline{\Sigma}$ & $\cong$ & $\underline{\Sigma}$ & $\mid$ & 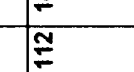 & \begin{tabular}{|l}
$\underline{N}$ \\
$\underline{N}$
\end{tabular} & $\cong$ & $\underline{\Sigma}$ & $\stackrel{\Sigma}{\approx}$ \\
\hline \% & 8 & 18 & 8 & $\infty$ & - & $\cong$ & $\tilde{N}$ & $\Phi$ & 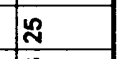 \\
\hline$-f^{\frac{2}{2}}$ & $\frac{8}{8}$ & $\frac{2}{9}$ & $\frac{9}{9}$ & $\frac{9}{9}$ & $\frac{9}{9}$ & $\frac{\infty}{9}$ & $\begin{array}{l}\infty \\
- \\
\end{array}$ & \begin{tabular}{|l}
$\underline{0}$ \\
$\underline{9}$
\end{tabular} & $\frac{\infty}{19}$ \\
\hline 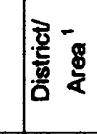 & 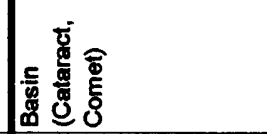 & 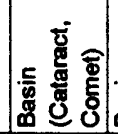 & 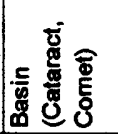 & 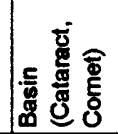 & 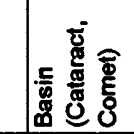 & 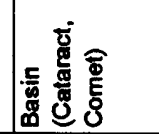 & 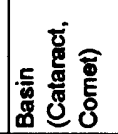 & 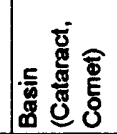 & 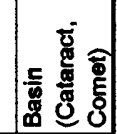 \\
\hline 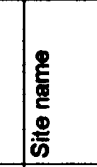 & 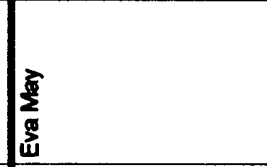 & 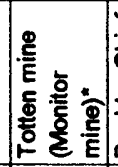 & 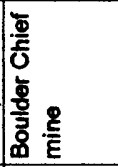 & 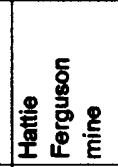 & 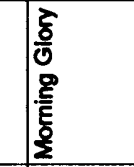 & 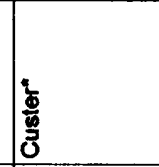 & 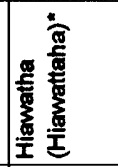 & \begin{tabular}{|l}
$\frac{3}{5}$ \\
$\frac{5}{3}$ \\
3
\end{tabular} & 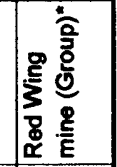 \\
\hline 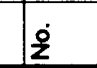 & 而 & $\frac{\infty}{m}$ & $\frac{O}{m}$ & \% & $\overline{\tilde{m}}$ & $\approx$ & 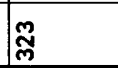 & 洰 & 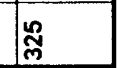 \\
\hline
\end{tabular}




\begin{tabular}{|c|c|c|c|c|c|c|c|c|c|}
\hline 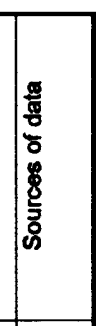 & 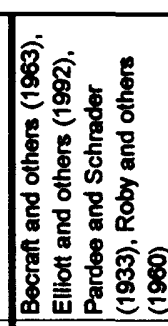 & 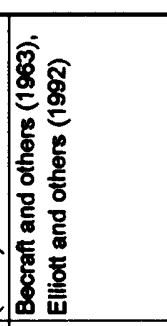 & 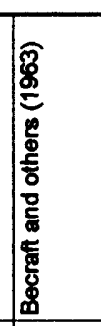 & 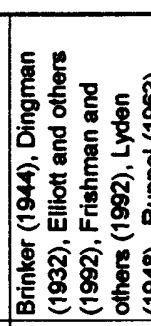 & 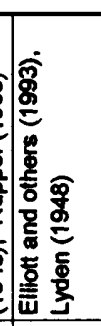 & 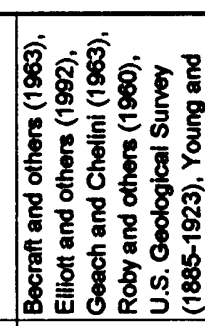 & 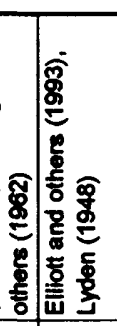 & 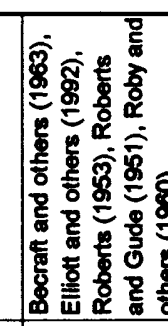 & 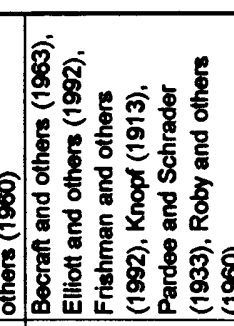 \\
\hline 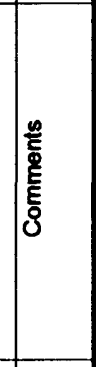 & 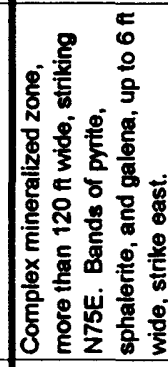 & 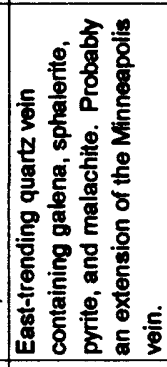 & & 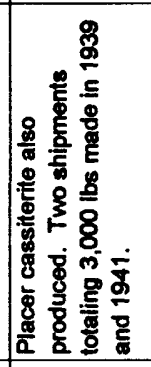 & & 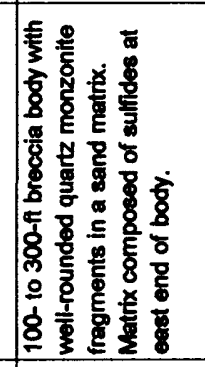 & & 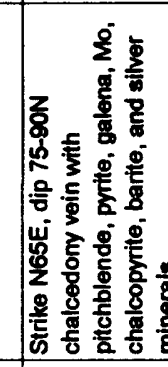 & 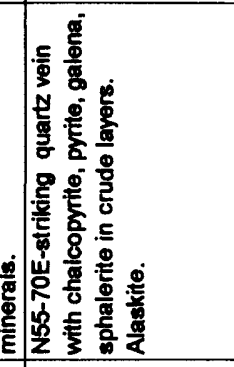 \\
\hline 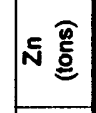 & ஜ̊ & & & & & : & & & i \\
\hline$\stackrel{0}{a}$ & 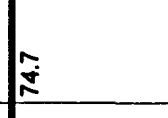 & & & & & I & & & \begin{tabular}{|l}
$\overline{8}$ \\
8 \\
\end{tabular} \\
\hline उँ & $\infty$ & & & & & & & & $\underline{\mathscr{\rho}}$ \\
\hline$q \bar{g}$ & 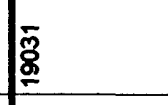 & & & & & $\frac{\tilde{z}}{\alpha}$ & & & 蒡 \\
\hline ₹ $\bar{Q}$ & to & & & 8 & & N & & & స్ \\
\hline 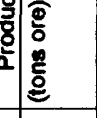 & $\stackrel{m}{=}$ & & & & & స్ & & & 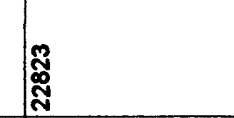 \\
\hline 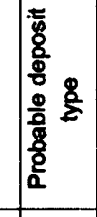 & 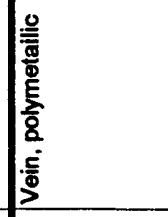 & 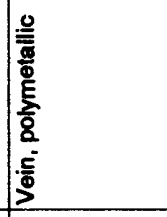 & 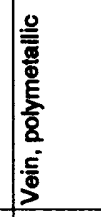 & 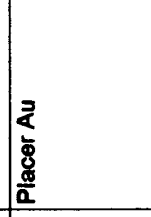 & 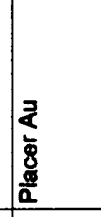 & 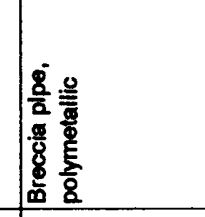 & \begin{tabular}{|l}
$\frac{z}{2}$ \\
$\bar{d}$ \\
$\frac{\mathbf{m}}{a}$ \\
\end{tabular} & 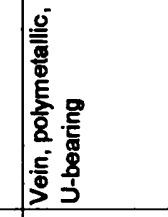 & 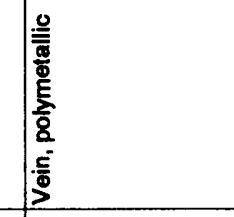 \\
\hline \% & $N$ & 18 & is & $\infty$ & 8 & $\mp$ & 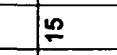 & $\tilde{n}$ & $\%$ \\
\hline 7 言 & E & 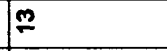 & $\cong$ & 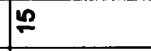 & \pm & $\cong$ & $\simeq$ & $\infty$ & $\infty$ \\
\hline 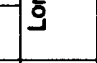 & 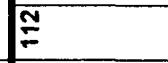 & $\stackrel{N}{=}$ & $\cong$ & $\cong$ & $\cong$ & 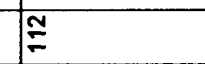 & 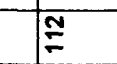 & $\stackrel{N}{\mp}$ & $\stackrel{1}{=}$ \\
\hline 里 & $\frac{8}{8}$ & $\frac{8}{12}$ & $\frac{\infty}{2}$ & 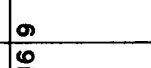 & $\frac{0}{\pi}$ & ${ }_{0}$ & $\frac{2}{0}$ & \begin{tabular}{|l}
$\bar{N}$ \\
$\infty$
\end{tabular} & $\frac{m}{\Omega}$ \\
\hline$f^{j \overline{\underline{\omega}}}$ & F & i & 5 & 8 & 9 & 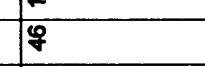 & 8 & 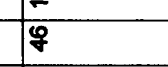 & $\mid$ \\
\hline 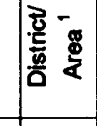 & 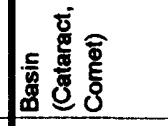 & 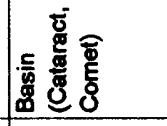 & 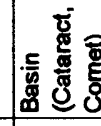 & 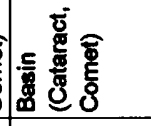 & 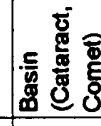 & 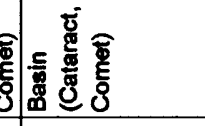 & 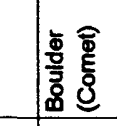 & 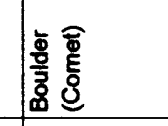 & 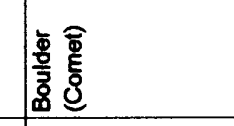 \\
\hline 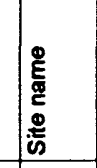 & 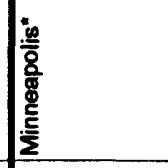 & 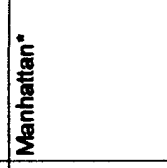 & 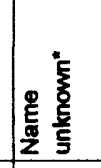 & 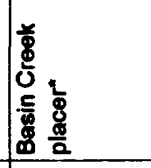 & 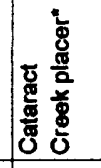 & \begin{tabular}{|l}
$\frac{5}{8}$ \\
$\frac{8}{8}$ \\
\end{tabular} & 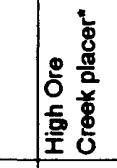 & 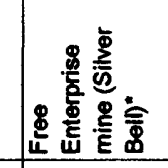 & 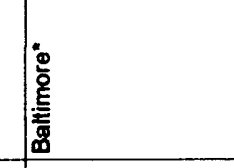 \\
\hline is & 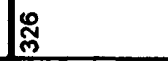 & 命 & 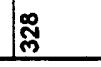 & \& & 㧀 & $\overline{8}$ & $\tilde{\tilde{n}}$ & 急 & 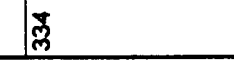 \\
\hline
\end{tabular}

政 


\begin{tabular}{|c|c|c|c|c|c|c|c|c|c|}
\hline 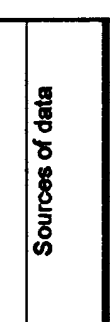 & 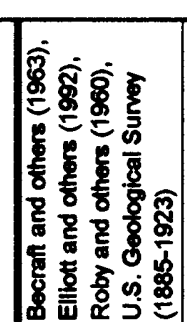 & 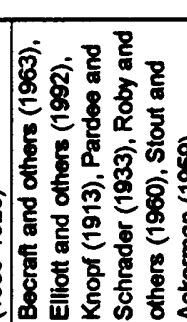 & & 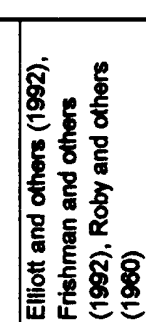 & 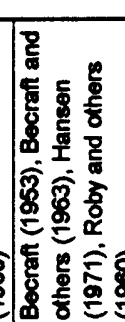 & 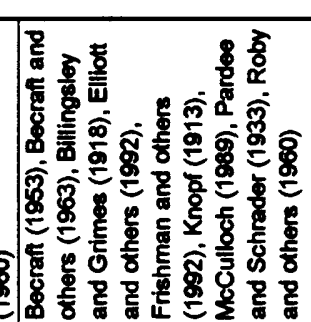 & 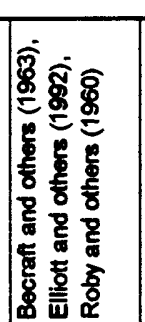 & 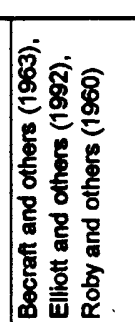 & 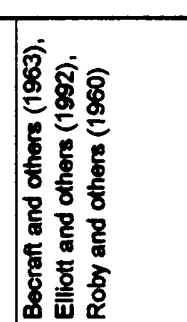 \\
\hline & 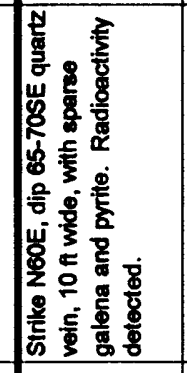 & 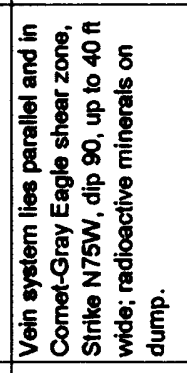 & & & 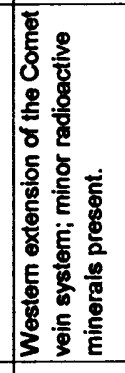 & 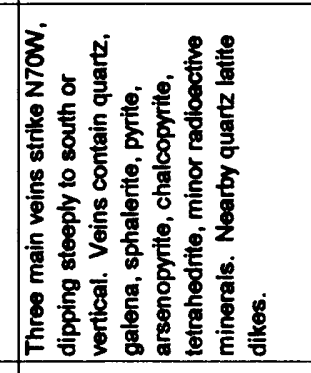 & 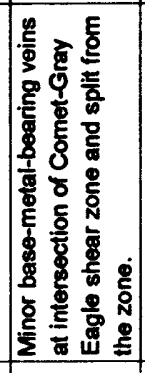 & 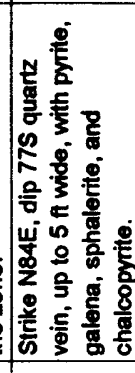 & 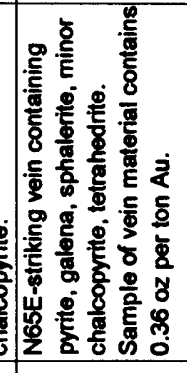 \\
\hline 象 & & 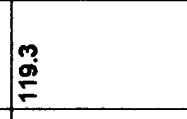 & $\tilde{\underline{N}}$ & & $\stackrel{8}{\circ}$ & 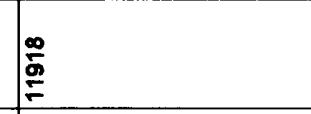 & & & \\
\hline 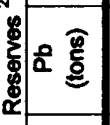 & & $\frac{8}{\pi}$ & $\oiiint_{\square}$ & $\circ$ & 吊 & 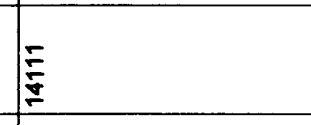 & & & \\
\hline$\overline{0} \frac{\vec{q}}{\underline{\underline{g}}}$ & & $\stackrel{\circ}{\circ}$ & $:$ & $m$ & $\hat{m}$ & $E$ & & & \\
\hline : & & 滂 & 赵 & $\frac{8}{8}$ & 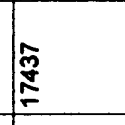 & 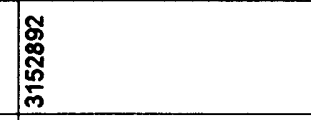 & & & \\
\hline ₹ $\widehat{\widehat{g}}$ & & 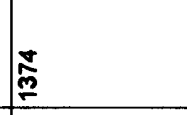 & $\$$ & 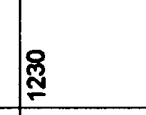 & $\hat{\tilde{a}}$ & 帝 & & & \\
\hline 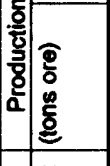 & & 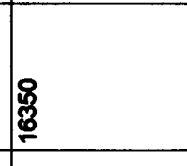 & $\$$ & $\frac{\bar{d}}{\bar{m}}$ & 理 & 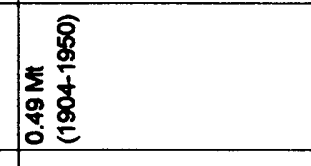 & $\frac{0}{\frac{0}{2}}$ & & \\
\hline 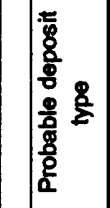 & 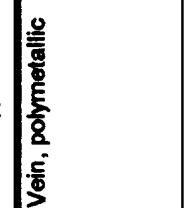 & 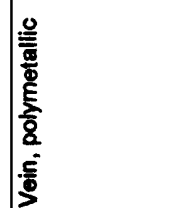 & 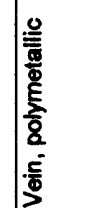 & 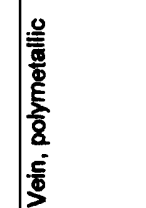 & 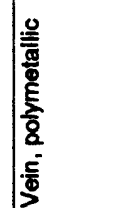 & 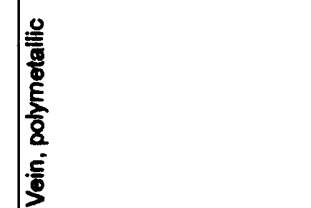 & 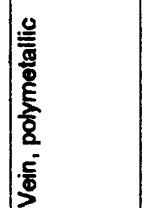 & 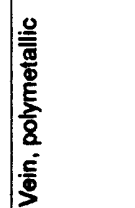 & 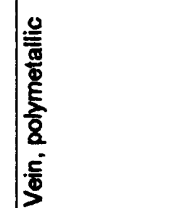 \\
\hline 意 & $\frac{8}{8}$ & \begin{tabular}{|l}
8 \\
5
\end{tabular} & $\frac{1}{\frac{1}{9}}$ & \begin{tabular}{|l}
3 \\
\\
\\
\end{tabular} & $\frac{p}{\frac{p}{2}}$ & $\div$ & $\frac{8}{8}$ & 足 & $\frac{1}{2}$ \\
\hline$\xi$ & $\cong$ & $\cong$ & $\cong$ & $\cong$ & $\cong$ & $\cong$ & $\cong$ & $\cong$ & $\cong$ \\
\hline$H^{\circ}$ & 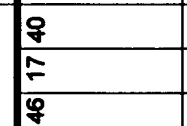 & \begin{tabular}{|l}
$\frac{9}{2}$ \\
$\frac{\infty}{-1}$ \\
10
\end{tabular} & $\begin{array}{l}\ddagger \\
-\infty \\
-\end{array}$ & \begin{tabular}{l}
$\infty$ \\
$\infty$ \\
\hdashline
\end{tabular} & 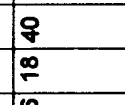 & $\begin{array}{l}\infty \\
\infty \\
\\
\end{array}$ & $\begin{array}{l}8 \\
8 \\
0 \\
\end{array}$ & $\begin{array}{l}\overline{5} \\
\overline{5} \\
0\end{array}$ & \begin{tabular}{|l}
8 \\
5 \\
0 \\
0
\end{tabular} \\
\hline 揞 & 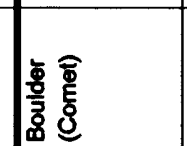 & 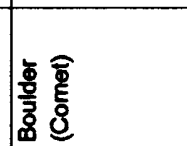 & 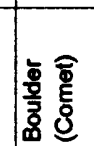 & 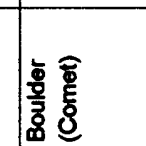 & 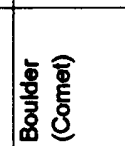 & 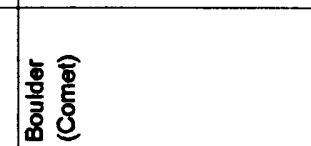 & 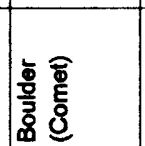 & 18 & | \\
\hline 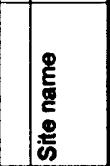 & 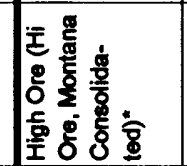 & 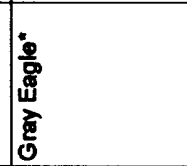 & 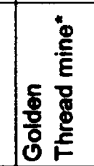 & 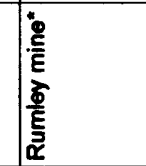 & 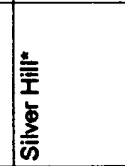 & 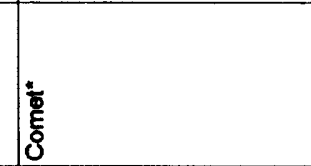 & 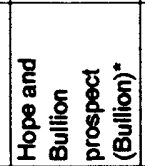 & & 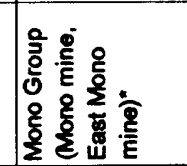 \\
\hline is & 嚍 & $\mathscr{8}$ & $\hat{\mathrm{m}}$ & 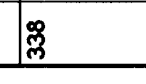 & 曾 & 里 & & 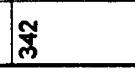 & $g$ \\
\hline
\end{tabular}

岧 


\begin{tabular}{|c|c|c|c|c|c|c|c|c|c|c|c|}
\hline 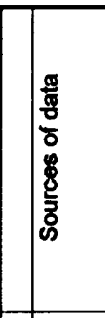 & 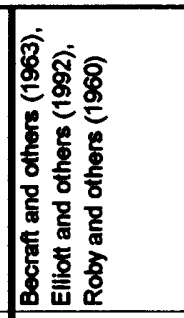 & & 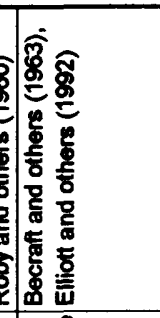 & 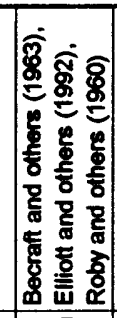 & 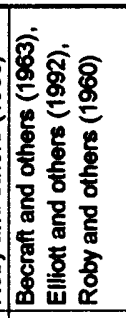 & 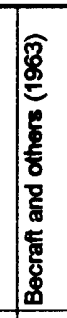 & 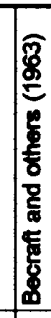 & 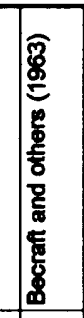 & 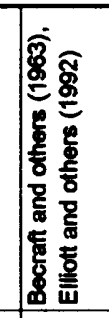 & 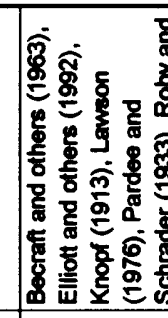 & 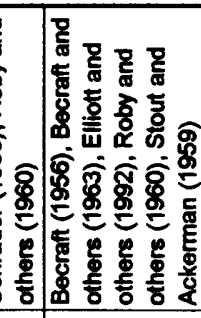 \\
\hline 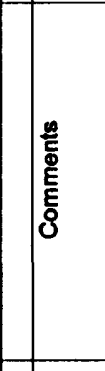 & 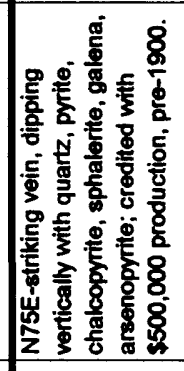 & & 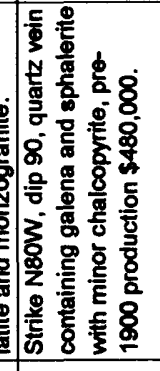 & 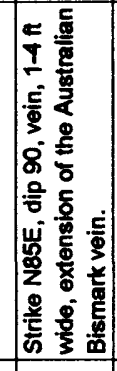 & 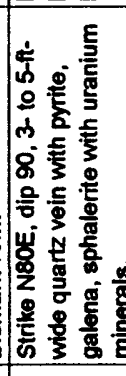 & & & & 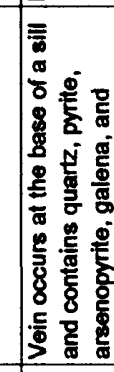 & 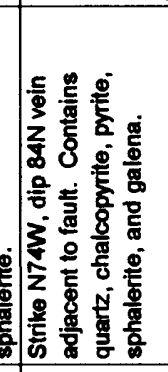 & 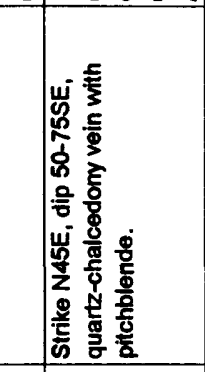 \\
\hline N & & & & & & & & & & 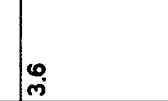 & \\
\hline 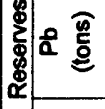 & 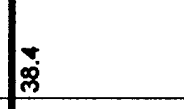 & $\$$ & & 8 & $\%$ & & & & & I & \\
\hline उ 0 产 & $\underset{⿱}{\infty}$ & בְּ & & & 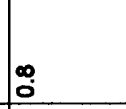 & & & & & $\dddot{m}$ & \\
\hline ष्ष & 扂 & 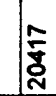 & & 8 & 诡 & & & & & $\frac{5}{\underline{5}}$ & \\
\hline द & న్ & 是 & & 龟 & I & & & & & - & \\
\hline 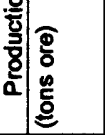 & శ్ & 路 & & 8 & $\frac{\sim}{n}$ & & & & & ฐ̃ & \\
\hline 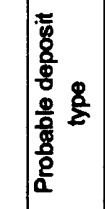 & 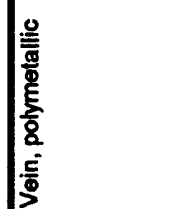 & 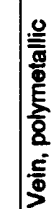 & 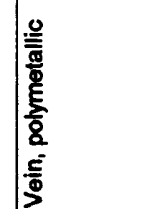 & 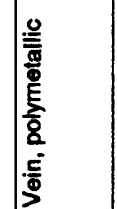 & 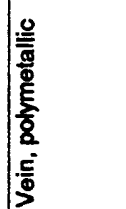 & 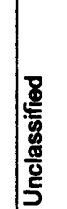 & 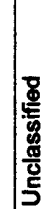 & 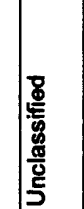 & 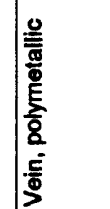 & 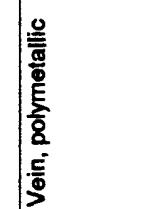 & 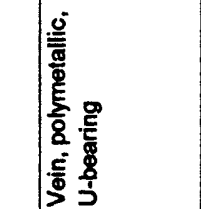 \\
\hline 8 & $\%$ & 18 & 78 & 8 & $n$ & $\infty$ & $\simeq$ & in & 2 & 18 & $\mathscr{n}$ \\
\hline 4 & $\infty$ & r & I & n & 0 & in & n & o & 10 & is & क \\
\hline - & $\cong$ & $\cong$ & $\cong$ & $\cong$ & $\cong$ & $\cong$ & $\cong$ & 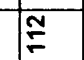 & $\tilde{F}$ & 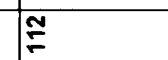 & $\cong$ \\
\hline 18 & 8 & 8 & $\%$ & $F$ & $\mathbb{N}$ & $\mathbf{\Sigma}$ & $F$ & $\mathscr{N}$ & ని & $\tilde{m}$ & $\infty$ \\
\hline 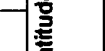 & 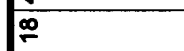 & 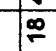 & $\stackrel{\infty}{\infty}$ & 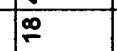 & $\underset{\infty}{\infty}$ & $\stackrel{\infty}{\infty}$ & 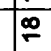 & $\infty$ & $\stackrel{9}{9}$ & $\%$ & $\sqrt[4]{2}$ \\
\hline $7^{9}$ & $\$$ & 9 & $\%$ & 9 & 9 & 9 & $\%$ & 9 & 9 & 19 & 19 \\
\hline 嚆 & 䇏 & 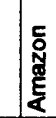 & 旅 & 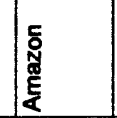 & 就 & 赔 & 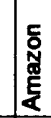 & 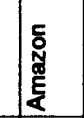 & \begin{tabular}{|l} 
\\
就 \\
\end{tabular} & 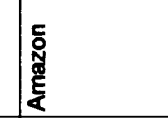 & 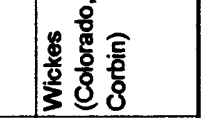 \\
\hline 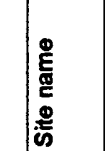 & $\mid$ & 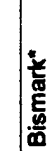 & 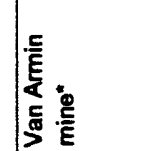 & 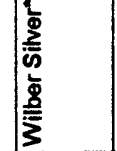 & 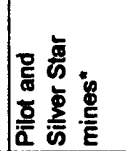 & 象 & & 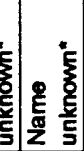 & 童 & 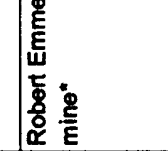 & 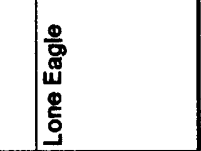 \\
\hline$\frac{j}{z}$ & I & I & 照 & 高 & 禺 & \% & 塥 & $\overline{\bar{n}}$ & $\tilde{\mathscr{m}}$ & 象 & 芯 \\
\hline
\end{tabular}

苟 


\begin{tabular}{|c|c|c|c|c|c|c|c|c|c|}
\hline 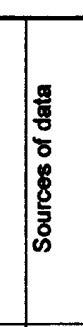 & 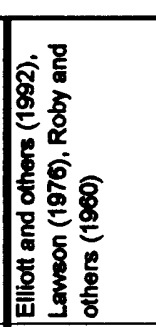 & 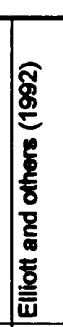 & 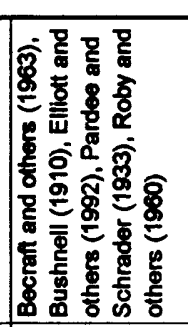 & 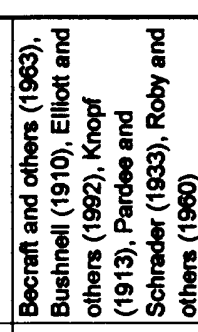 & 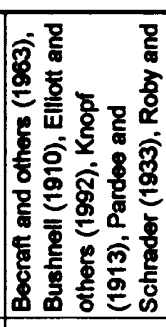 & 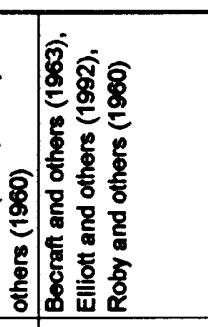 & 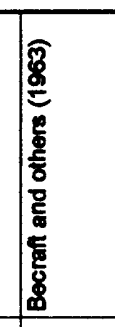 & 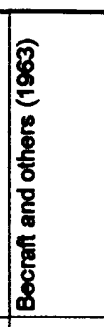 & 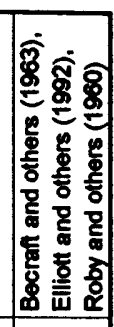 \\
\hline & 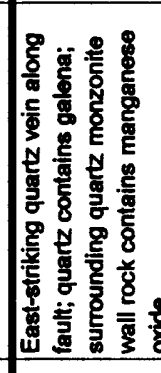 & 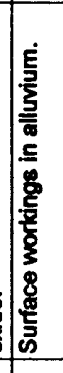 & 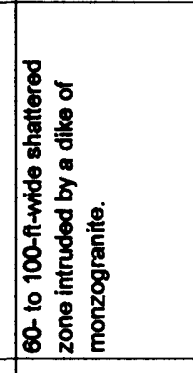 & 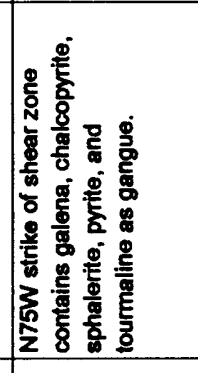 & 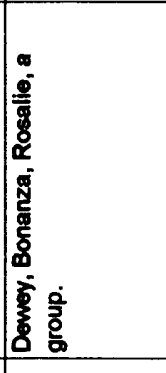 & 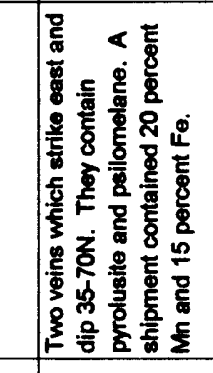 & 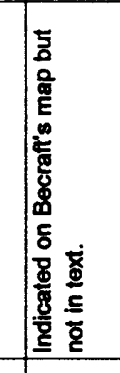 & 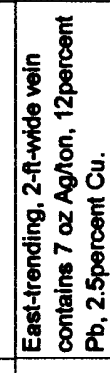 & \\
\hline$\tilde{N}$ & & & & & & & & & \\
\hline 醇 & & & & & & & & & $\%$ \\
\hline 0 & & & & & & & & & $\approx$ \\
\hline 98 & & & & & & & & & 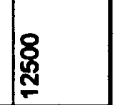 \\
\hline ₹ & & & & & & & & & \\
\hline$g$ & $\frac{8}{2}$ & & & & & & & & 茰 \\
\hline 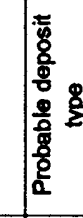 & 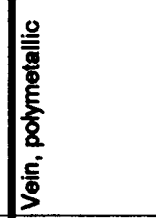 & 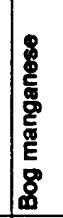 & 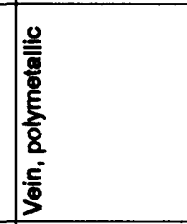 & 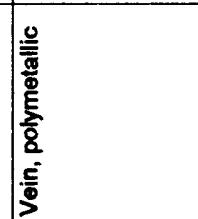 & 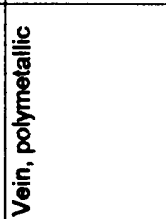 & 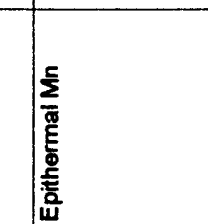 & 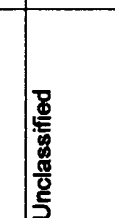 & | & $\mid$ \\
\hline 菨 & $\begin{array}{ll}\alpha \\
\alpha \\
\alpha\end{array}$ & $\frac{8}{9}$ & $\neq$ & $a$ & $\frac{8}{\infty}$ & $\frac{w}{\frac{w}{g}}$ & $\frac{m}{a}$ & $\begin{array}{l} \\
\end{array}$ & $\frac{9}{8}$ \\
\hline - & $\cong$ & $\cong$ & $\cong$ & $\cong$ & $\cong$ & $\cong$ & $\cong$ & $\cong$ & $\cong$ \\
\hline - & 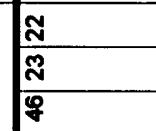 & \begin{tabular}{|l|}
$\infty$ \\
$\frac{\pi}{9}$ \\
9
\end{tabular} & \begin{tabular}{|l|}
$\frac{\pi}{\pi}$ \\
\multirow{2}{*}{}
\end{tabular} & \begin{tabular}{|l}
$\frac{\Phi}{2}$ \\
$\tilde{q}$ \\
$q$
\end{tabular} & $\frac{\overline{2}}{\bar{\pi}}$ & \begin{tabular}{|l|}
$n$ \\
$\pi$ \\
$\mathcal{Q}$
\end{tabular} & $\begin{array}{l}\bar{z} \\
\bar{\alpha} \\
g\end{array}$ & \begin{tabular}{|l|} 
\\
$\tilde{\pi}$ \\
\\
\end{tabular} & \begin{tabular}{|l|}
$\infty$ \\
$\tilde{\pi}$ \\
$\mathscr{q}$
\end{tabular} \\
\hline 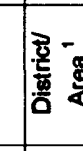 & 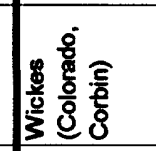 & & 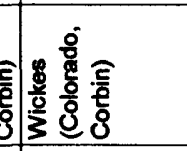 & 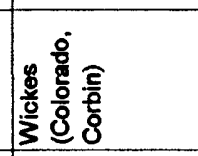 & 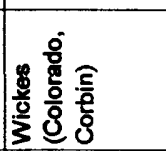 & 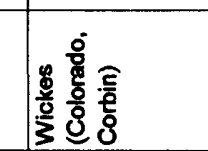 & 暑 & & \\
\hline 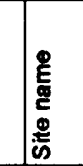 & 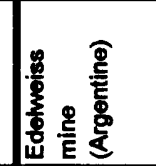 & 度 & 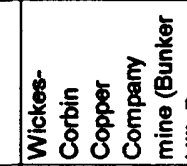 & 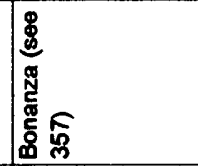 & 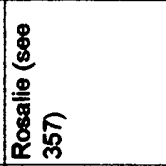 & 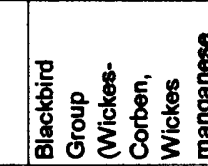 & $\frac{\sqrt{\frac{5}{5}}}{\underline{\underline{5}}}$ & 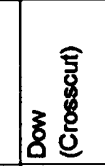 & 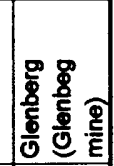 \\
\hline$\frac{\dot{i}}{2}$ & 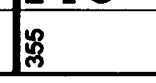 & 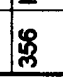 & 5 & 奠 & 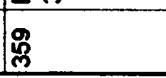 & 遇 & $\overline{8}$ & 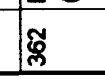 & $\mathscr{E}$ \\
\hline
\end{tabular}




\begin{tabular}{|c|c|c|c|c|c|c|c|}
\hline 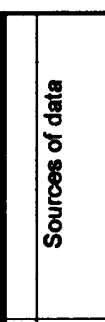 & 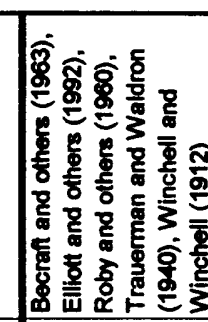 & 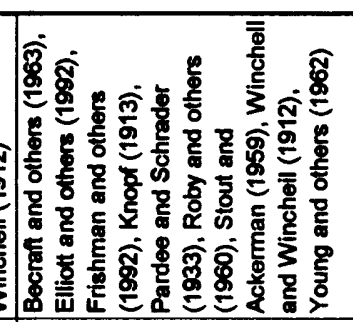 & 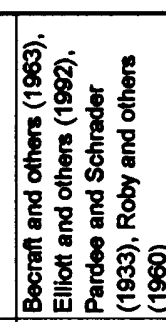 & 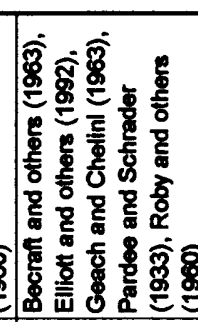 & 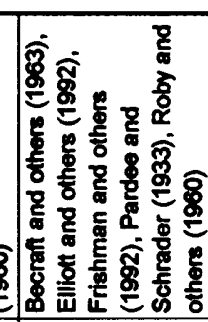 & 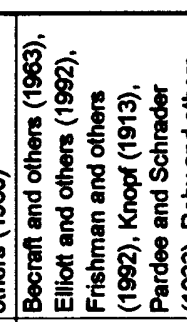 & 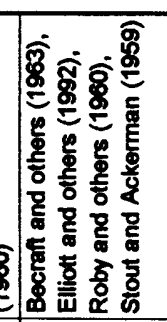 \\
\hline & 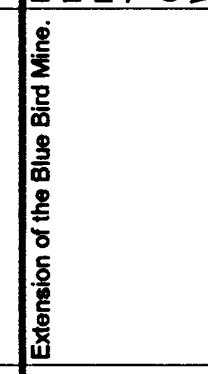 & 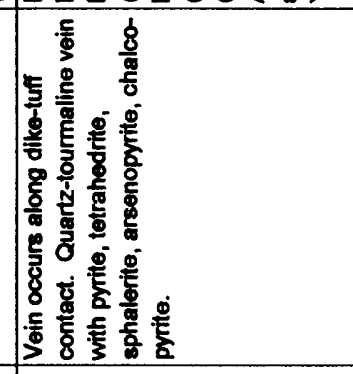 & 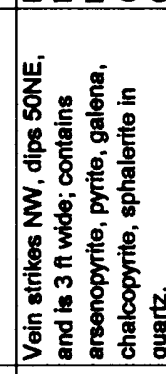 & 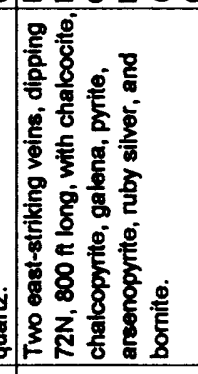 & 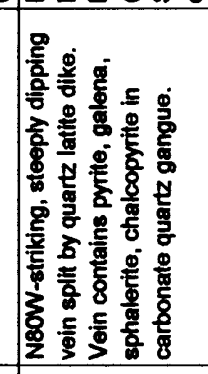 & 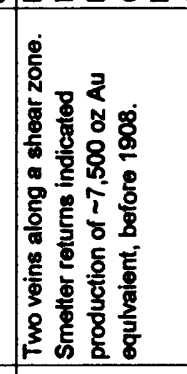 & 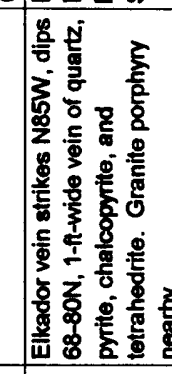 \\
\hline 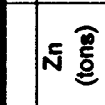 & & & & & జ్ & & \\
\hline$\frac{\mathrm{g}}{\mathrm{g}}$ & $:$ & $\underline{\underline{z}}$ & & & 总 & $\hat{\mathscr{b}}$ & \\
\hline$\overline{\mathrm{o}} \overline{\mathrm{g}}$ & $=$ & 多 & - & & $\tilde{\tilde{\Sigma}}$ & $\hat{a}$ & \\
\hline 용 & $\$$ & 嗻 & $\tilde{8}$ & & 蓶 & 愿 & \\
\hline$\geq \widehat{\approx}$ & 2 & 鴶 & m & & 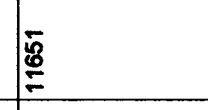 & 常 & \\
\hline 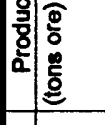 & ॄু & 叠 & $\mathscr{N}$ & & 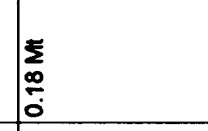 & $\frac{9}{\pi}$ & \\
\hline 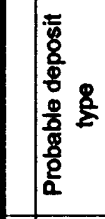 & 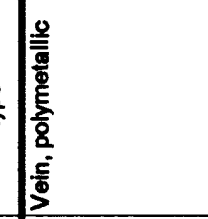 & 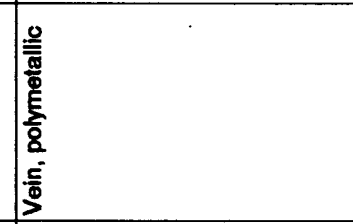 & 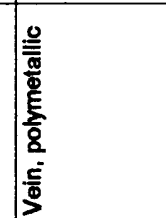 & 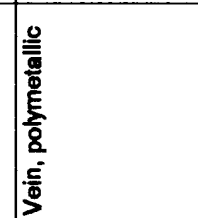 & 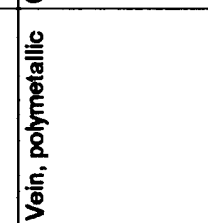 & 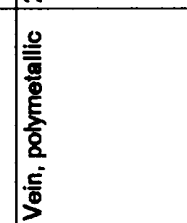 & 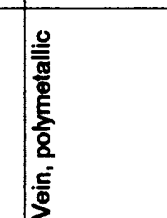 \\
\hline - & \begin{tabular}{l}
$\tilde{2}$ \\
\hdashline \\
0
\end{tabular} & $\frac{\infty}{\infty}$ & $\frac{5}{\infty}$ & $\infty$ & $\frac{n}{\infty}$ & $\frac{\infty}{\infty}$ & $\frac{\pi}{\infty}$ \\
\hline - 詰 & $\cong$ & $\cong$ & $\cong$ & $\cong$ & $\cong$ & $\cong$ & $\cong$ \\
\hline 薏 & 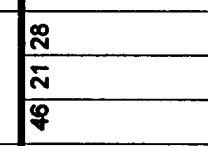 & \begin{tabular}{|l}
$\pi$ \\
$\frac{\pi}{q}$ \\
\end{tabular} & $\begin{array}{l}\frac{n}{2} \\
\frac{7}{9}\end{array}$ & \begin{tabular}{|l}
$\underline{n}$ \\
$\frac{a}{q}$ \\
\end{tabular} & \begin{tabular}{|l}
$\pi$ \\
$\frac{\pi}{9}$ \\
9
\end{tabular} & \begin{tabular}{|l}
$\frac{1}{n}$ \\
$\frac{1}{9}$
\end{tabular} & 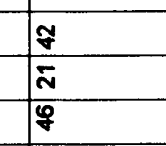 \\
\hline 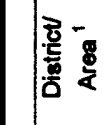 & 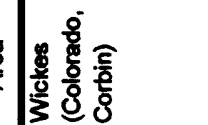 & 题高 & 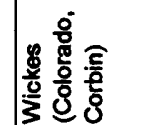 & 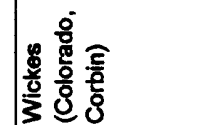 & 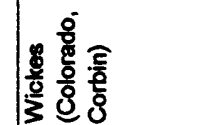 & 总 & 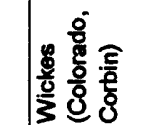 \\
\hline 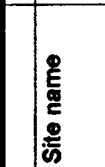 & | & 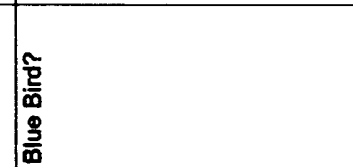 & | & 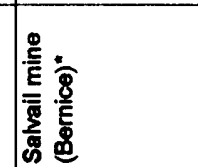 & 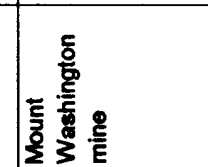 & $\frac{8}{\frac{1}{\omega}}$ & 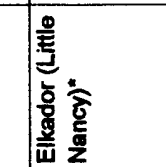 \\
\hline$\frac{1}{2}$ & 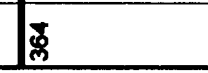 & 8 & $\$$ & 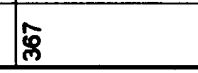 & $\$$ & \% & : \\
\hline
\end{tabular}

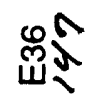




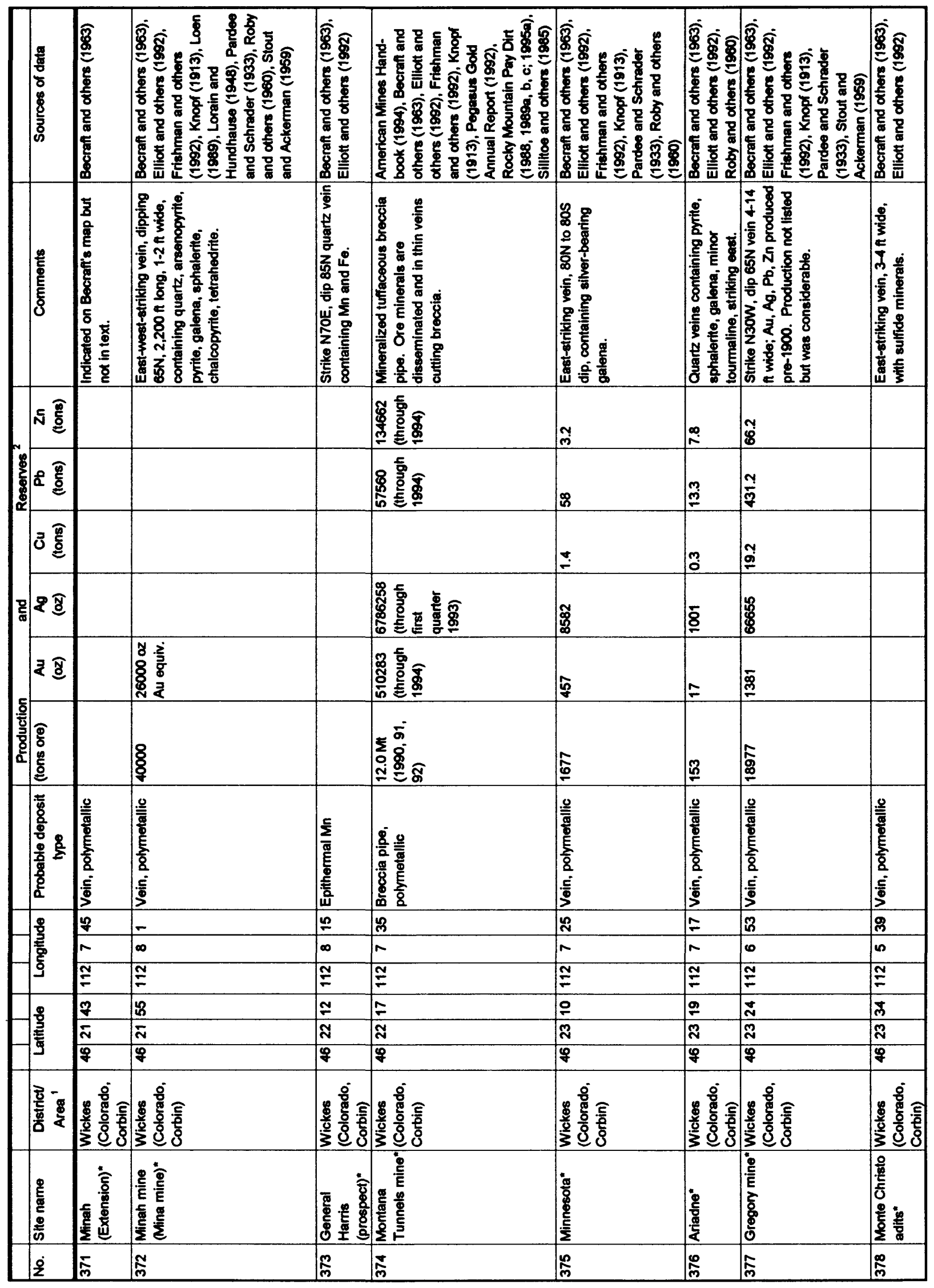

พิ山 


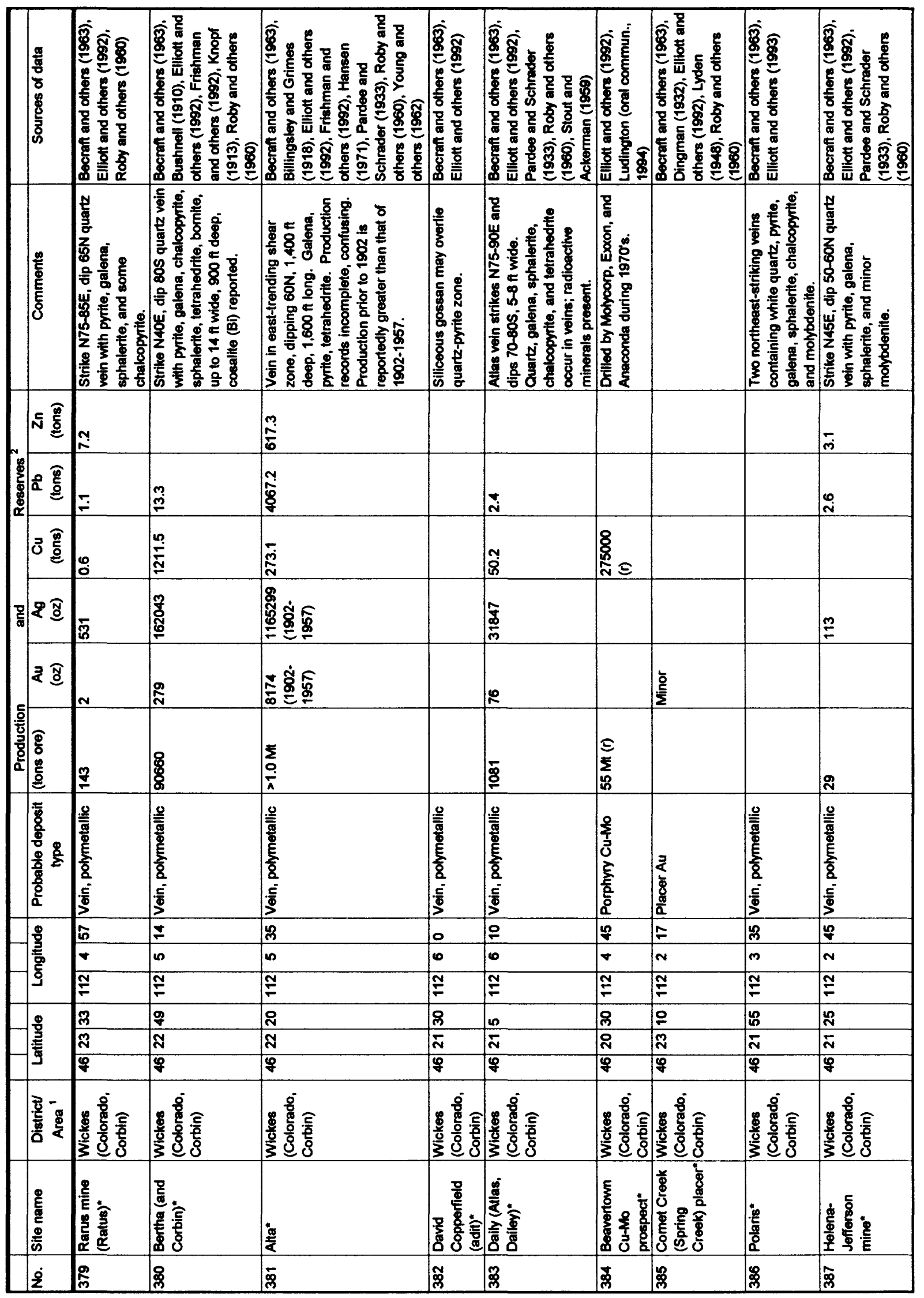

吕 


\begin{tabular}{|c|c|c|c|c|c|c|c|c|c|c|c|c|}
\hline 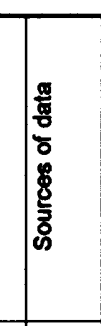 & 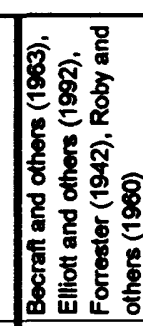 & 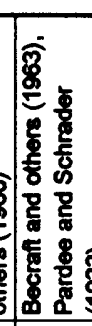 & 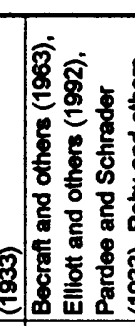 & 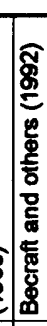 & & 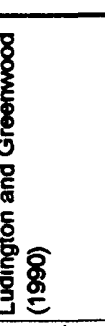 & 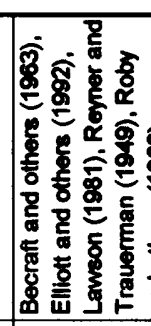 & 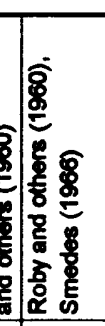 & 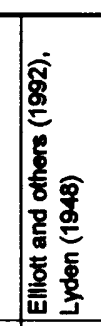 & 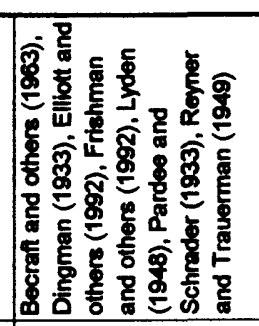 & 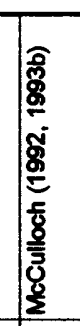 & 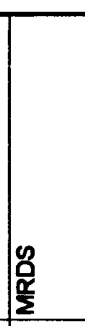 \\
\hline 施 & 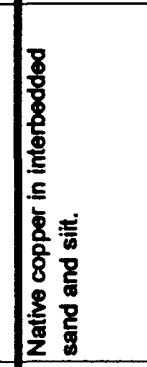 & 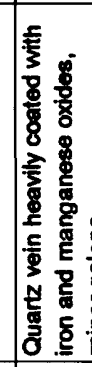 & 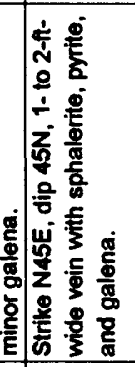 & & & 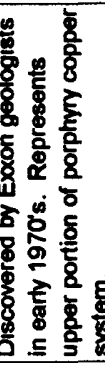 & 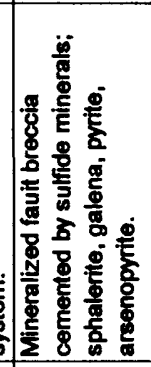 & 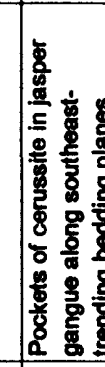 & & 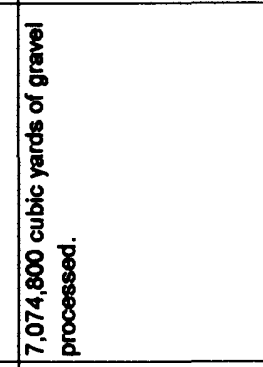 & & \\
\hline న & & & & & & & & & & & & \\
\hline 䍖 & & & $\stackrel{m}{\stackrel{m}{\circ}}$ & & & & & & & & & \\
\hline $\bar{\sigma} \frac{\widehat{\underline{g}}}{\underline{g}}$ & & & & & & & $q$ & & & & & \\
\hline : $2 \pi$ & & & 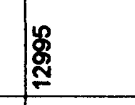 & & & & 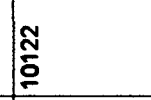 & & & & & \\
\hline ₹तु & & & 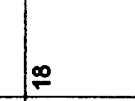 & & & & 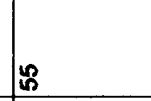 & & & 兽 & & \\
\hline 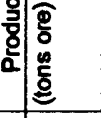 & & & ळ & & & 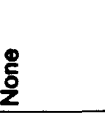 & $\dddot{8}$ & & & & & \\
\hline 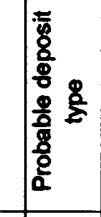 & $\begin{array}{l}\mathbf{8} \\
\frac{0}{8} \\
8 \\
8 \\
8\end{array}$ & 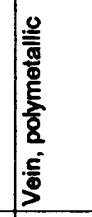 & 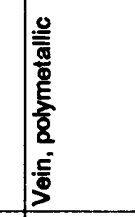 & 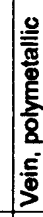 & & 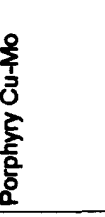 & 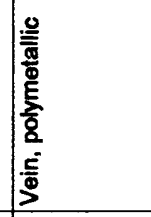 & 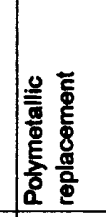 & 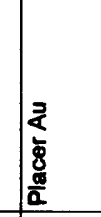 & \begin{tabular}{|l}
$\frac{z}{2}$ \\
$\frac{8}{2}$ \\
$\frac{m}{2}$
\end{tabular} & 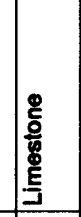 & 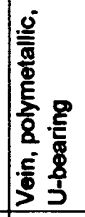 \\
\hline-8 & 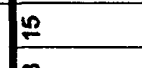 & $\infty$ & מ & क & & 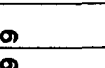 & $\frac{\pi}{0}$ & $\frac{0}{10}$ & $\frac{9}{10}$ & $\frac{m}{m}$ & 0 & $\frac{0}{\pi}$ \\
\hline ]言 & $\infty$ & 10 & 0 & 0 & & î & के & 品 & $\$$ & $B$ & \$ & $\$ 8$ \\
\hline 79 & $\stackrel{\Xi}{\equiv}$ & $\cong$ & $\underline{N}$ & $\cong$ & & $E$ & $\equiv$ & $\equiv$ & $\equiv$ & $\equiv$ & $\equiv$ & $E$ \\
\hline - & 兽 & 8 & $\frac{\pi}{\alpha}$ & $\frac{7}{-2}$ & & $\frac{8}{9}$ & $\frac{n}{-10}$ & $\frac{m}{m}$ & $\overline{\bar{n}}$ & $\frac{8}{18}$ & 8 & \begin{tabular}{|l|l|}
\multirow{2}{*}{} \\
0
\end{tabular} \\
\hline - & \begin{tabular}{|l|}
$\mathbf{R}$ \\
$\mathcal{P}$ \\
\end{tabular} & \begin{tabular}{|l|}
\multirow{2}{*}{} \\
$\mathscr{q}$
\end{tabular} & 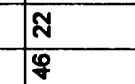 & \begin{tabular}{|l}
$\bar{\lambda}$ \\
$\mathscr{q}$
\end{tabular} & & $\frac{8}{8}$ & \begin{tabular}{|l|}
$\overline{1}$ \\
$\mathscr{q}$ \\
\end{tabular} & $\begin{array}{l}8 \\
8\end{array}$ & \begin{tabular}{|l|}
8 \\
89
\end{tabular} & \begin{tabular}{|l|} 
\\
\\
\end{tabular} & \begin{tabular}{|l|} 
N \\
$\mathscr{q}$
\end{tabular} & \begin{tabular}{|l|}
8 \\
8
\end{tabular} \\
\hline 嚆 & 造 & 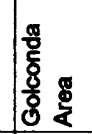 & 要 & 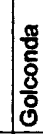 & & 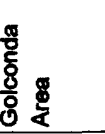 & 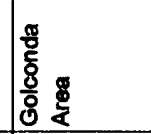 & 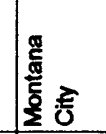 & 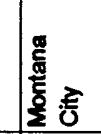 & $\mid$ & 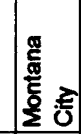 & 要 \\
\hline 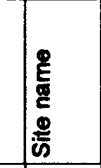 & 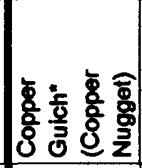 & 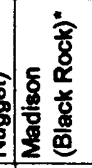 & 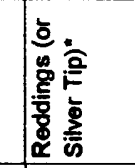 & $\frac{\$}{5}$ & & 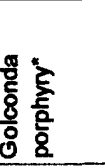 & 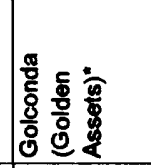 & |⿹ & 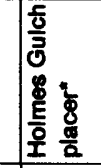 & 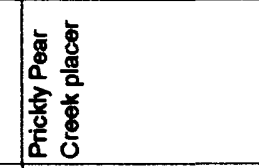 & & 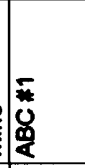 \\
\hline is & $\$$ & 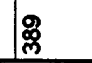 & 8 & 离 & & $\tilde{\tilde{g}}$ & 吕 & I & $\%$ & $\%$ & 盈 & 澹 \\
\hline
\end{tabular}




\begin{tabular}{|c|c|c|c|c|c|c|c|c|c|c|c|}
\hline 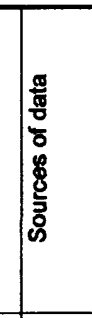 & 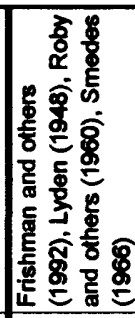 & 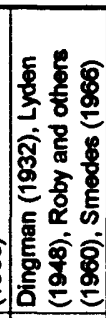 & 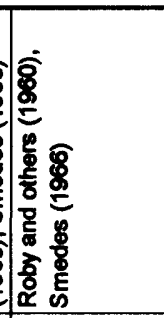 & 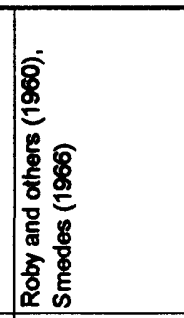 & 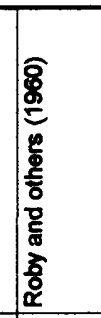 & 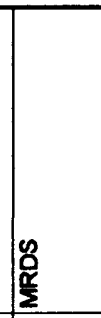 & 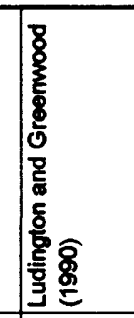 & 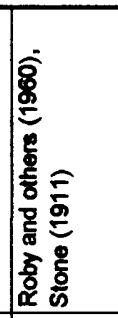 & 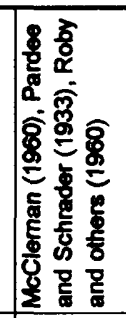 & 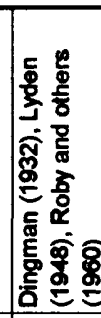 & 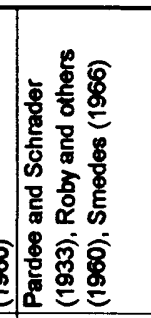 \\
\hline 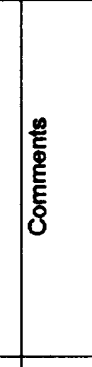 & & 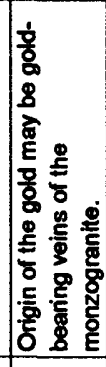 & 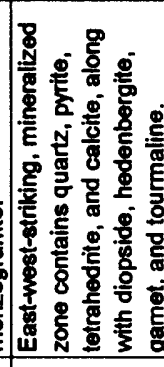 & 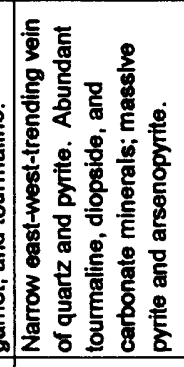 & 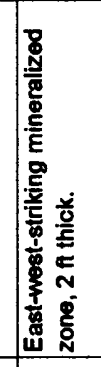 & 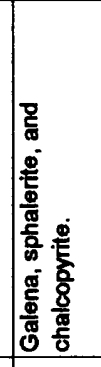 & 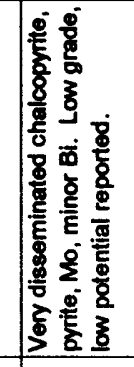 & 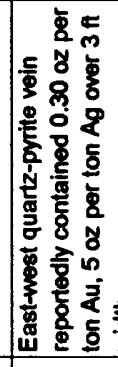 & 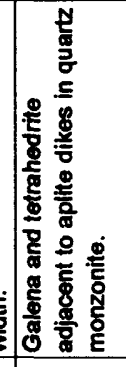 & 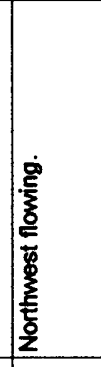 & 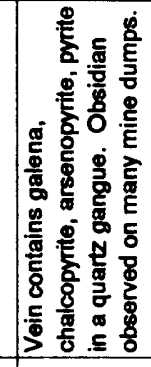 \\
\hline 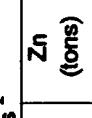 & & & & & & & & & i & & \\
\hline 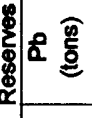 & & & & $\tilde{0}$ & & & & & $\hat{s}_{0}$ & & $\frac{8}{9}$ \\
\hline ठव⿱ & & & $\stackrel{9}{=}$ & $\overline{0}$ & & & & & & & $\begin{array}{l}\infty \\
8 \\
\end{array}$ \\
\hline च्ञ & & & 总 & $\underline{9}$ & & & & & 呤 & & 胥 \\
\hline ₹ $\bar{\sigma}$ & 8 & 高 & 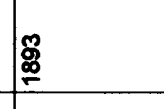 & $\infty$ & & & & & + & 高 & $\begin{array}{l}\mathscr{8} \\
\mathbf{D} \\
\underline{\infty}\end{array}$ \\
\hline 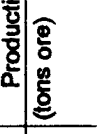 & & & 跑 & $\Phi$ & & & \begin{tabular}{|l}
$\frac{0}{50}$ \\
$\frac{5}{2}$
\end{tabular} & & 8 & & 敠 \\
\hline 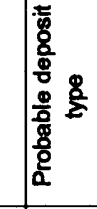 & 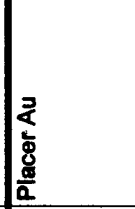 & $\mid \begin{array}{l}\frac{z}{2} \\
\frac{d}{6} \\
\frac{m}{a} \\
\end{array}$ & 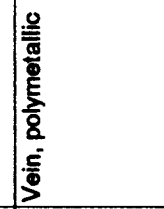 & 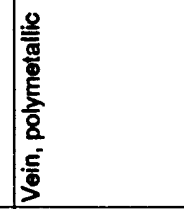 & 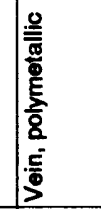 & 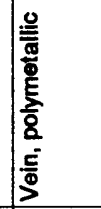 & 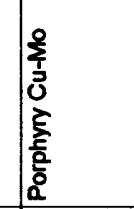 & 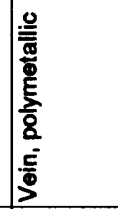 & 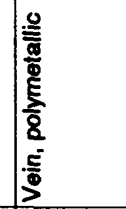 & 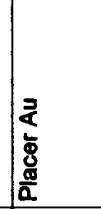 & 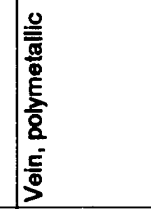 \\
\hline$-\frac{1}{3}$ & \begin{tabular}{|l} 
\\
8 \\
8
\end{tabular} & \begin{tabular}{|c|}
\multirow{2}{*}{} \\
กิ
\end{tabular} & $\frac{0}{15}$ & $\frac{0}{9}$ & $\mid \frac{2}{\bar{n}}$ & $\begin{array}{l}0 \\
8\end{array}$ & 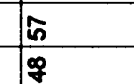 & $\frac{18}{18}$ & $\frac{8}{8}$ & $\begin{array}{l} \\
8 \\
8\end{array}$ & \begin{tabular}{|l|}
$\pi$ \\
8 \\
3
\end{tabular} \\
\hline 1 & $E$ & $\equiv$ & $E$ & $E$ & $\Xi$ & $E$ & 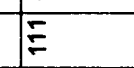 & $\equiv$ & $\Xi$ & $E$ & $E$ \\
\hline 撞 & $\frac{7}{17}$ & $E_{\alpha}$ & $\frac{8}{2}$ & $\frac{9}{8}$ & 㐌 & 6 & - & 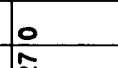 & $\infty$ & in & $\frac{7}{8}$ \\
\hline & 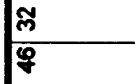 & \begin{tabular}{|l|} 
r \\
\multirow{q}{*}{}
\end{tabular} & $\frac{\bar{n}}{9}$ & $\frac{8}{9}$ & 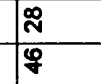 & $\frac{\mathscr{2}}{9}$ & $\mid \begin{array}{l}\infty \\
\end{array}$ & $\frac{\hat{N}}{q}$ & \begin{tabular}{|l}
$\tilde{N}$ \\
$q$
\end{tabular} & \begin{tabular}{|l}
$\hat{N}$ \\
\end{tabular} & $\frac{\mid \pi}{\pi}$ \\
\hline 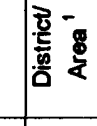 & 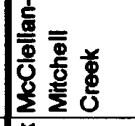 & 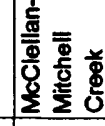 & 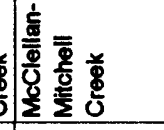 & 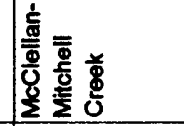 & 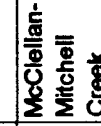 & 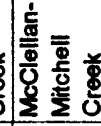 & 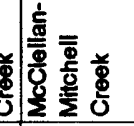 & 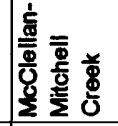 & 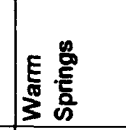 & 点 & 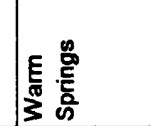 \\
\hline 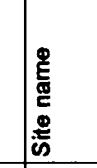 & 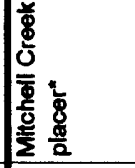 & 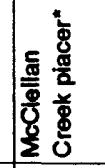 & 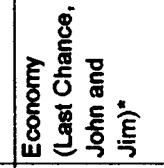 & 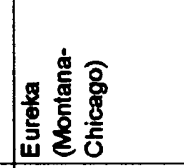 & 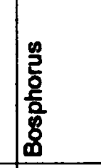 & 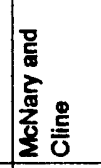 & 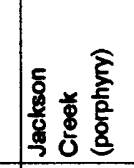 & 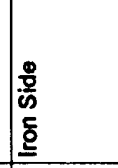 & 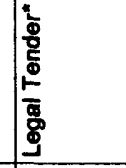 & 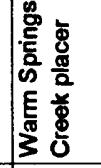 & 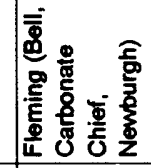 \\
\hline is & 居 & 8 & 京 & 文 & 骂 & 这 & 多 & 8 & 字 & 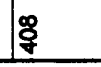 & 8 \\
\hline
\end{tabular}

吕 


\begin{tabular}{|c|c|c|c|c|c|c|c|c|c|c|c|}
\hline 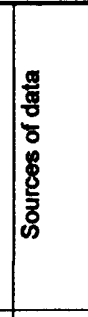 & 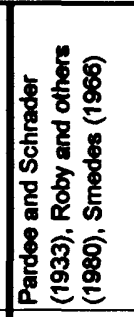 & 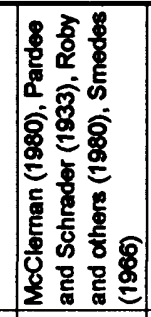 & 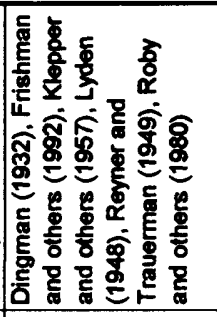 & 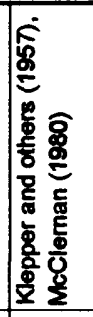 & 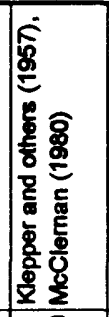 & 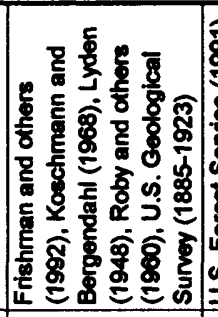 & & 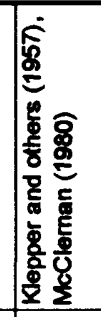 & 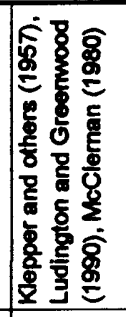 & 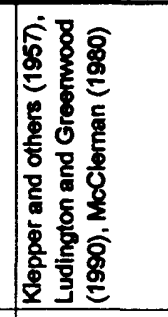 & 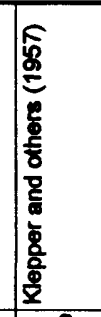 \\
\hline | & 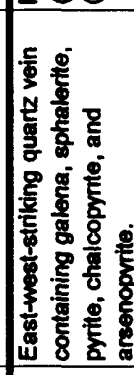 & 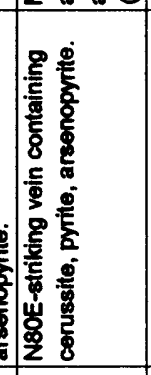 & 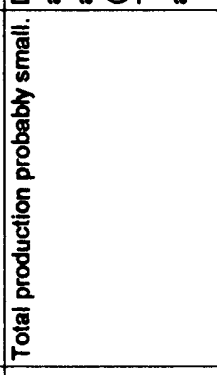 & 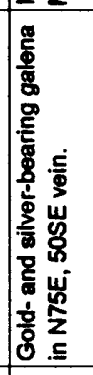 & 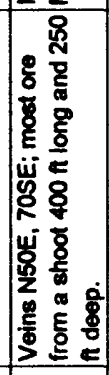 & & & 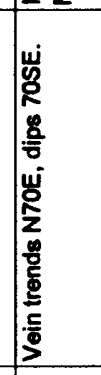 & 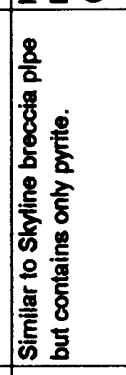 & 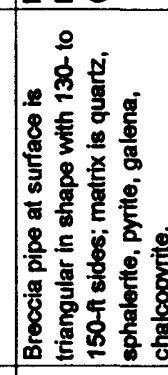 & 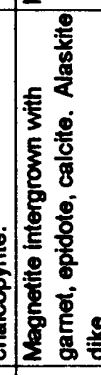 \\
\hline 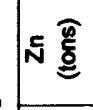 & i & & & & Io & & & & & & \\
\hline$\stackrel{0}{a} \frac{\underline{\underline{\underline{g}}}}{\underline{\underline{g}}}$ & 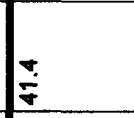 & 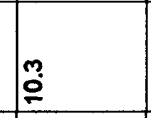 & & & $\approx$ & & & is & & & \\
\hline उ0 & 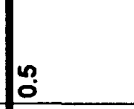 & - & & & I & & & & & & \\
\hline $2 \widetilde{g}$ & 疍 & 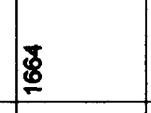 & & & 珺 & & & జ్ & & & \\
\hline ₹ $\widehat{\widehat{\delta}}$ & $\%$ & $\ddot{8}$ & & & $\frac{8}{\mathscr{8}}$ & & & ֶָ̃ & & & \\
\hline 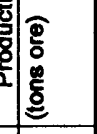 & 串 & $\cong$ & & & $\frac{8}{2}$ & & & : & & & \\
\hline 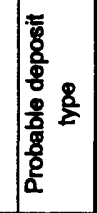 & 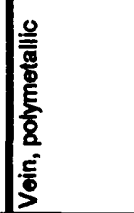 & 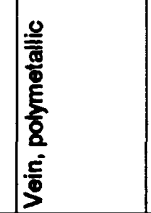 & $\mid$\begin{tabular}{|l}
$\frac{z}{z}$ \\
$\frac{\mathbf{d}}{2}$ \\
$\frac{m}{a}$ \\
\end{tabular} & 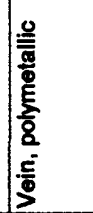 & 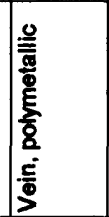 & 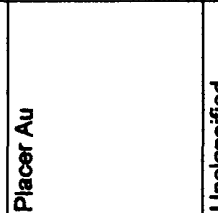 & & 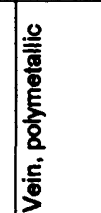 & 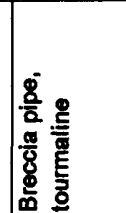 & 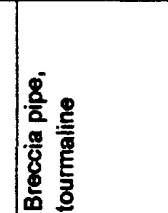 & 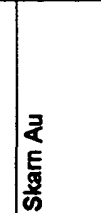 \\
\hline$-\frac{3}{3}$ & 8 & 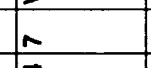 & 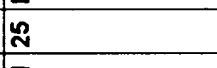 & 8 & $=$ & 8 & $\hat{n}$ & $\div$ & 8 & 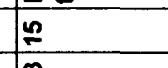 & $\%$ \\
\hline $7 \frac{z}{z^{2}}$ & 8 & के & $\overline{\bar{n}}$ & 88 & 18 & $\tilde{\kappa}$ & $\bar{n}$ & $\tilde{\tilde{\pi}}$ & $\tilde{\pi}$ & 8 & $\%$ \\
\hline$\Xi$ & $E$ & $\equiv$ & $\equiv$ & $\equiv$ & $\equiv$ & $\equiv$ & $\equiv$ & $E$ & $\equiv$ & $\equiv$ & $\equiv$ \\
\hline 19 & $\%$ & 8 & 8 & 9 & ? & $\tilde{\mathcal{N}}$ & $\cong$ & 88 & f & iి & \% \\
\hline 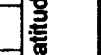 & $\sqrt{\alpha}$ & $\pi$ & $\bar{N}$ & $\bar{N}$ & $\overline{\boldsymbol{N}}$ & \% & \% & ๓ & $\underset{\mathbf{\infty}}{\mathbf{2}}$ & $F$ & 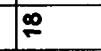 \\
\hline & क & 18 & 19 & 9 & 9 & 9 & 18 & 19 & 9 & 18 & 9 \\
\hline 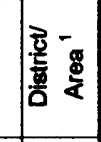 & 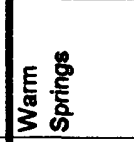 & 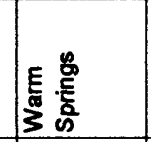 & 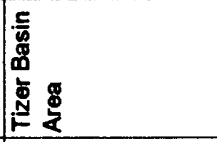 & 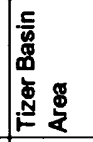 & 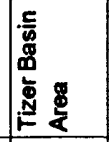 & 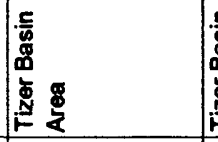 & 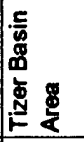 & 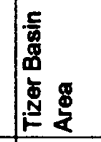 & 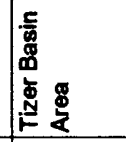 & 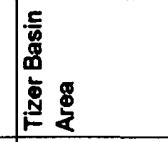 & 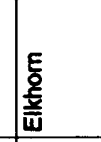 \\
\hline 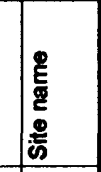 & 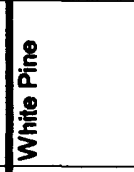 & 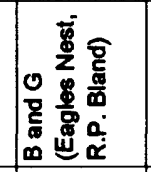 & 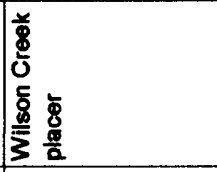 & 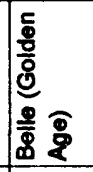 & 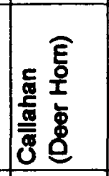 & 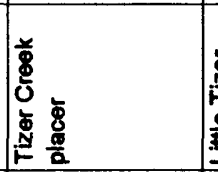 & 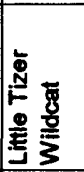 & 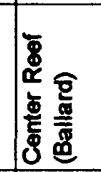 & 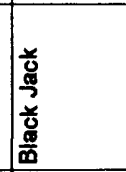 & $\mid \frac{\dot{c}}{\frac{2}{5}}$ & 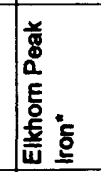 \\
\hline$\frac{\dot{2}}{2}$ & $\frac{9}{8}$ & $F$ & 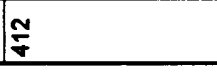 & $\frac{m}{5}$ & $\frac{7}{\ddagger}$ & $\frac{5}{7}$ & $\stackrel{0}{7}$ & 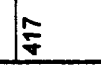 & $\frac{\infty}{F}$ & $\frac{9}{7}$ & \& \\
\hline
\end{tabular}




\begin{tabular}{|c|c|c|c|c|c|c|c|c|c|c|c|c|c|c|}
\hline 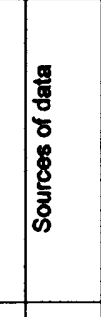 & 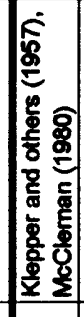 & & 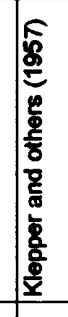 & 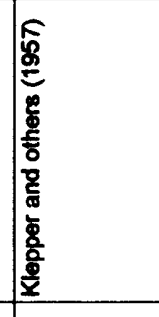 & 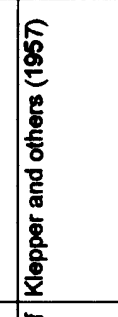 & 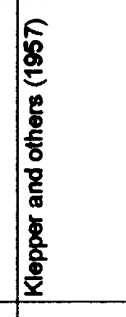 & 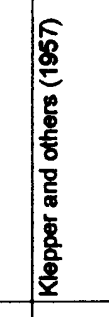 & 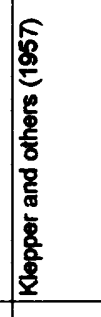 & 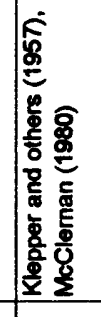 & 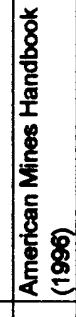 & 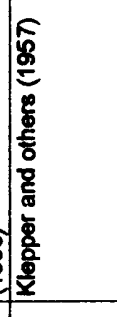 & 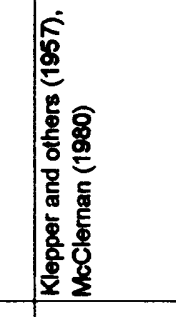 & 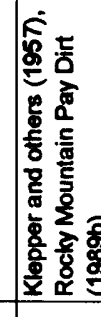 & 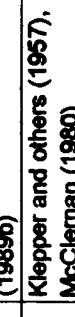 \\
\hline 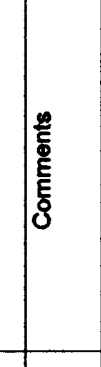 & & 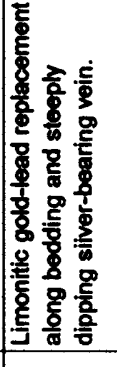 & 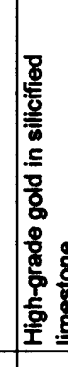 & 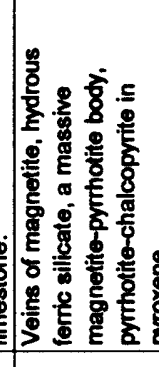 & 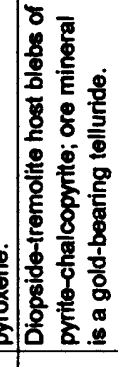 & 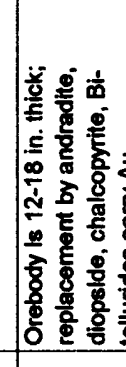 & 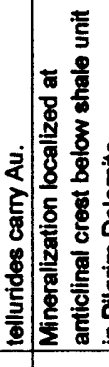 & 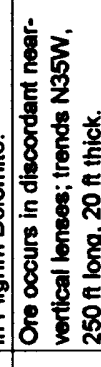 & 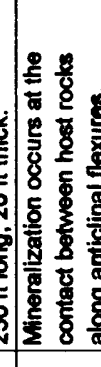 & 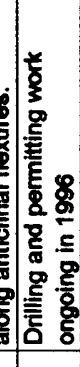 & 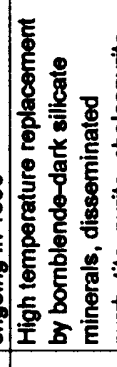 & 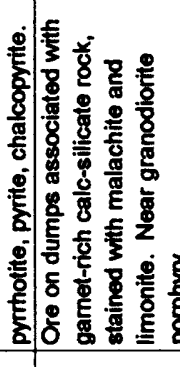 & 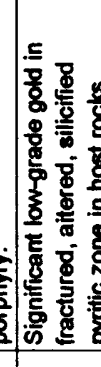 & 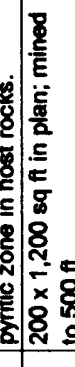 \\
\hline$\tilde{N} \frac{\widehat{\sigma}}{\underline{\underline{g}}}$ & & & & & & & & & \&్లి & & & & & q \\
\hline$\frac{\mathbb{\underline { V }}}{\mathrm{g}}$ & & & & & & & & & 8 & & & & & $\mathbb{R}$ \\
\hline ठठ & & & & 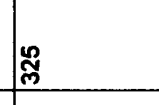 & & & & & 2 & & & In & & or \\
\hline : $2 \widehat{E}$ & & & & 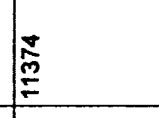 & & & & $\frac{8}{10}$ & 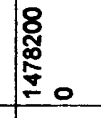 & & & & & \&్ల్లి \\
\hline ₹ $\bar{\sigma}$ & & & & \begin{tabular}{|l} 
磨 \\
\end{tabular} & & & & 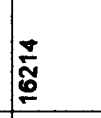 & 8 & 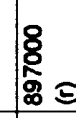 & 80 & & 站 & 薰 \\
\hline 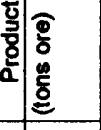 & & & & 辞 & & & 敢 & 遭 & & & 8 & $\hat{\phi}$ & 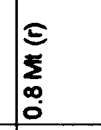 & 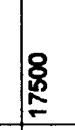 \\
\hline 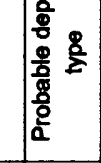 & & & 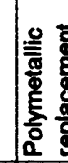 & & 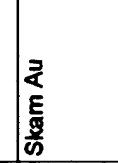 & 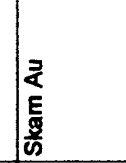 & 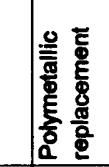 & 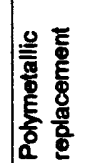 & 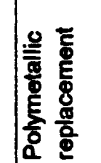 & 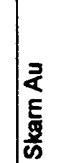 & 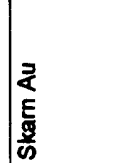 & 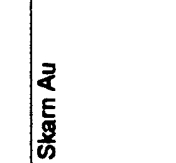 & 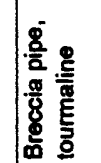 & 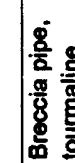 \\
\hline$\frac{9}{3}$ & 8 & & \pm & 9 & $\%$ & $\bar{s}$ & $m$ & $\%$ & 8 & 8 & $\%$ & 8 & 8 & 9 \\
\hline & $\$ 8$ & & 8 & is & 8 & 8 & 18 & 8 & 8 & $\%$ & 8 & 18 & 5 & 8 \\
\hline - & $E$ & $\equiv$ & $\equiv$ & $\equiv$ & $E$ & $\Xi$ & $\equiv$ & $\equiv$ & $\equiv$ & $\equiv$ & $\equiv$ & $\equiv$ & $\equiv$ & $\equiv$ \\
\hline 果 & $\bar{n}$ & 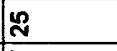 & $\mathfrak{n}$ & $m$ & 8 & $\tilde{\pi}$ & $\overline{5}$ & $\%$ & क & $\bar{\pi}$ & $\infty$ & $\hat{m}$ & \pm & $F$ \\
\hline$-f^{\frac{3}{2}}$ & $\frac{F}{8}$ & $\begin{array}{l}F \\
6\end{array}$ & \pm & $\neq$ & $\frac{\%}{6}$ & $\%$ & $\frac{\%}{0}$ & $\frac{2}{6}$ & $\div$ & \begin{tabular}{|l} 
\\
\end{tabular} & $\frac{10}{9}$ & $\frac{9}{6}$ & $\frac{5}{10}$ & \pm \\
\hline 案- & 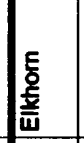 & 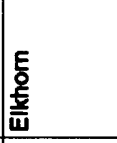 & $\mid$\begin{tabular}{|l} 
喜 \\
恙
\end{tabular} & 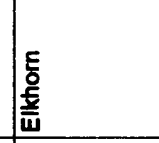 & 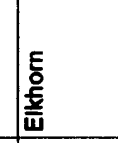 & | & 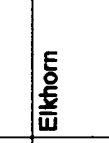 & 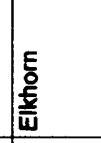 & 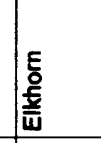 & $\mid \begin{array}{l}\text { 喜 } \\
\text { 厗 }\end{array}$ & 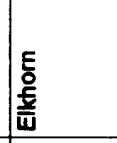 & 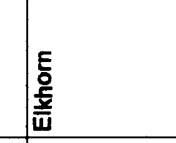 & 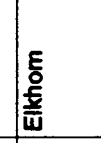 & 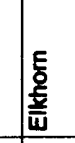 \\
\hline 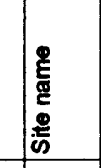 & & 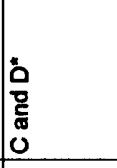 & 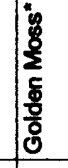 & 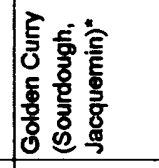 & 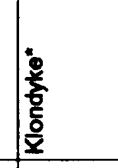 & $\mid \begin{array}{l}\frac{5}{8} \\
\frac{8}{8} \\
\end{array}$ & $\begin{array}{l}8 \\
8 \\
8 \\
\end{array}$ & 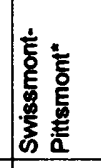 & 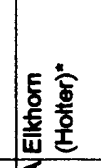 & |居 & 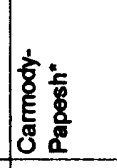 & 衰 & 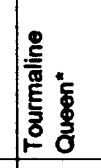 & 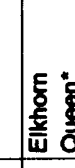 \\
\hline is & $\bar{a}$ & ฐุ & $\underset{\mathfrak{7}}{\mathfrak{3}}$ & $\frac{4}{4}$ & 尔 & 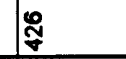 & $\widehat{\widehat{y}}$ & 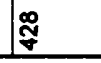 & ฐ్ళ & 倦 & 8 & $\overline{7}$ & 苯 & 8 \\
\hline
\end{tabular}




\begin{tabular}{|c|c|c|c|c|c|c|c|c|c|c|c|c|c|c|c|c|c|}
\hline 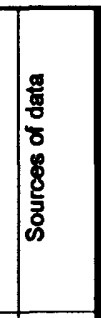 & 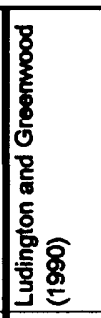 & 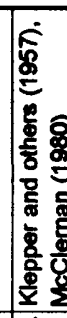 & 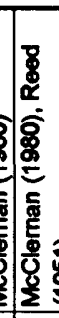 & & 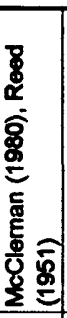 & 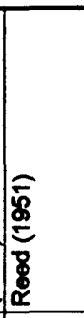 & & 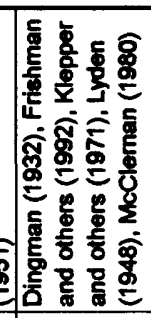 & 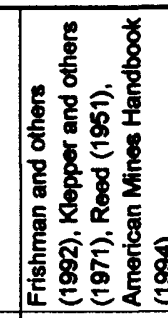 & 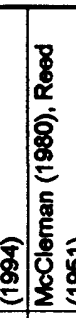 & 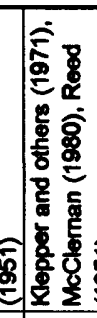 & & 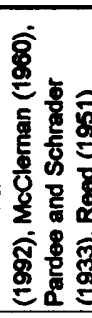 & 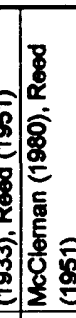 & $\sqrt{3}$ & 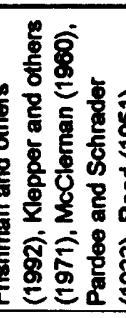 & 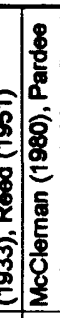 \\
\hline 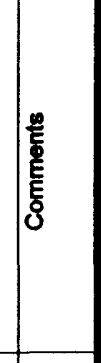 & 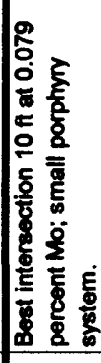 & 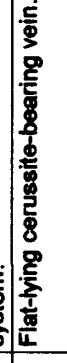 & & 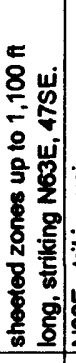 & & 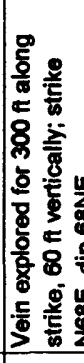 & 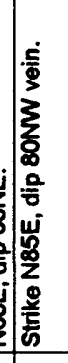 & 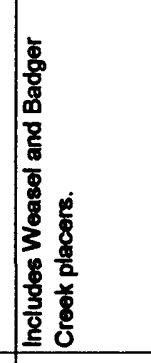 & 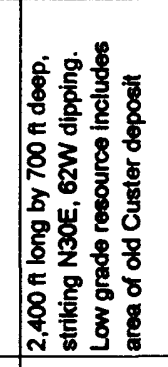 & 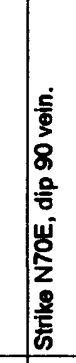 & 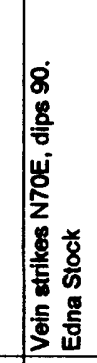 & 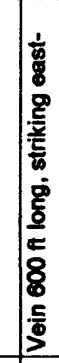 & 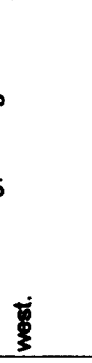 & & & & 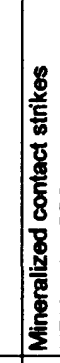 \\
\hline s & & & & & & & & & in & & & & & & $\frac{5}{y}$ & & \\
\hline 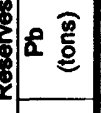 & & & & & & & & & $\bar{m}$ & & & 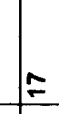 & & & స్ & & \\
\hline ठ己 & & & & & & & & & $\infty$ & & & & & & 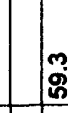 & & \\
\hline$q \bar{g}$ & & & & & & & & & 莩要 & & & 8 & & & 焉 & & \\
\hline z $\bar{\gamma}$ & & & & & & & & 8 & 议 & & & 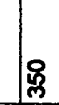 & & & 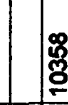 & & 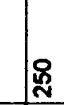 \\
\hline 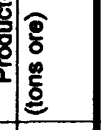 & 密 & & & & & & & & 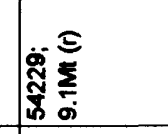 & & & 8 & & & 虎 & & \\
\hline 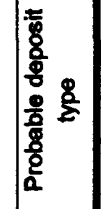 & 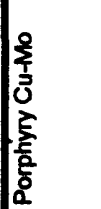 & 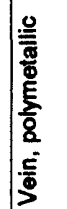 & 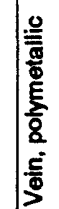 & & 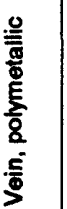 & 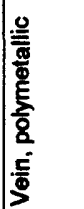 & 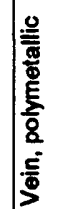 & \begin{tabular}{|l}
$\frac{z}{3}$ \\
$\frac{z}{8}$ \\
$\frac{8}{a}$ \\
$\frac{m}{a}$
\end{tabular} & 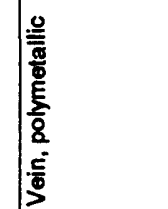 & 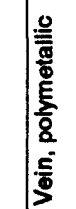 & 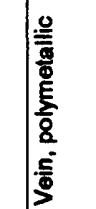 & 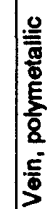 & & 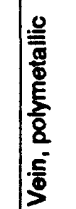 & 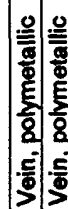 & & 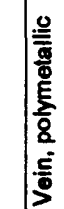 \\
\hline 犃 & $\hat{8}$ & $\begin{array}{l}\text { in } \\
\end{array}$ & $\frac{8}{7}$ & & $\frac{\pi}{7}$ & \begin{tabular}{|l|}
$\bar{\lambda}$ \\
$\bar{\lambda}$
\end{tabular} & $\begin{array}{l}7 \\
7\end{array}$ & $\frac{\pi}{2}$ & \begin{tabular}{|l|}
5 \\
8
\end{tabular} & \begin{tabular}{|l|}
$\tilde{\phi}$ \\
$\bar{\sigma}$
\end{tabular} & $\frac{d}{d}$ & $\frac{\hbar}{5}$ & & \begin{tabular}{|l|}
5 \\
\\
\end{tabular} & \begin{tabular}{|l|l}
5 & 48 \\
77 & \\
\end{tabular} & & \begin{tabular}{|l|} 
A \\
a
\end{tabular} \\
\hline - & $\equiv$ & $\equiv$ & $\risingdotseq$ & & $\bar{E}$ & $E$ & $\mp$ & $E$ & $\Xi$ & $\equiv$ & $\equiv$ & $\equiv$ & & $E$ & $\equiv \equiv$ & & $\equiv$ \\
\hline - & 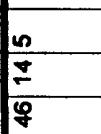 & \begin{tabular}{|l|}
- \\
5 \\
8
\end{tabular} & 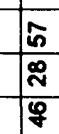 & & $\begin{array}{l}\frac{\infty}{\infty} \\
\stackrel{\infty}{\infty} \\
q\end{array}$ & \begin{tabular}{|l|} 
\\
$\infty$ \\
\multirow{2}{*}{} \\
\end{tabular} & \begin{tabular}{|l|}
8 \\
2 \\
9 \\
\end{tabular} & \begin{tabular}{|l|}
$\mathbf{A}$ \\
$\mathbf{N}$ \\
$\mathscr{q}$
\end{tabular} & 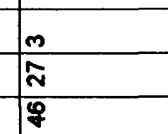 & \begin{tabular}{|l|}
$\frac{8}{2}$ \\
$\mathscr{2}$ \\
8
\end{tabular} & \begin{tabular}{|l}
8 \\
8 \\
8 \\
\end{tabular} & $\begin{array}{l}F \\
\frac{F}{2} \\
\end{array}$ & & 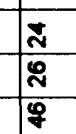 & 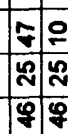 & & 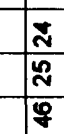 \\
\hline 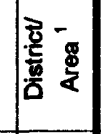 & 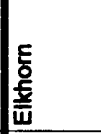 & 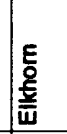 & 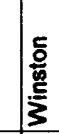 & & $\frac{5}{\frac{5}{20}}$ & 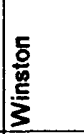 & 总 & 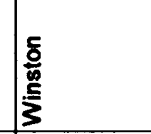 & 总 & 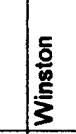 & 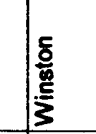 & 递 & & $\mid \begin{array}{l}\frac{5}{5} \\
\frac{\underline{\underline{n}}}{3} \\
\end{array}$ & 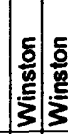 & & 亳 \\
\hline 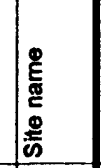 & 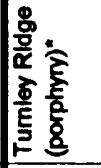 & 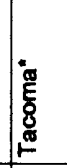 & 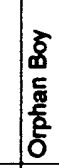 & & 훙 & 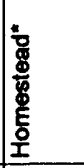 & 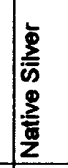 & 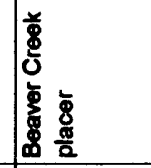 & 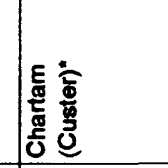 & $\sum 0$ & & 5 & & 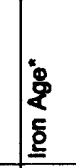 & 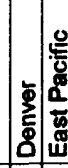 & & 产 \\
\hline io & 疍 & $\%$ & $\%$ & & $\bar{g}$ & 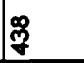 & 8 & o & 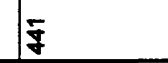 & $¥$ & $\frac{m}{q}$ & $\$$ & & $\varsubsetneqq$ & $\mid \frac{9}{9}$ & & $\frac{9}{7}$ \\
\hline
\end{tabular}

$\stackrel{9}{4}$ 


\begin{tabular}{|c|c|c|c|c|c|c|c|c|c|c|c|c|c|c|c|}
\hline 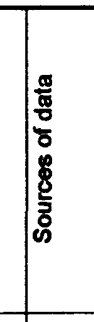 & 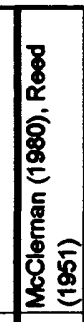 & 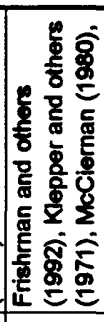 & 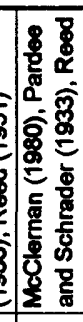 & & & 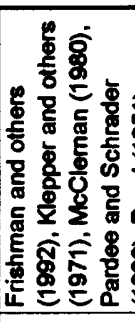 & 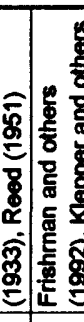 & 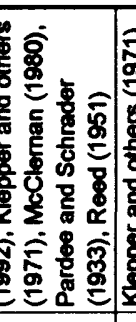 & 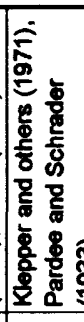 & 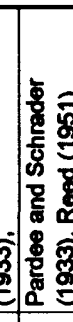 & 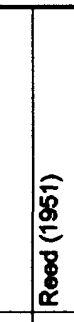 & & & 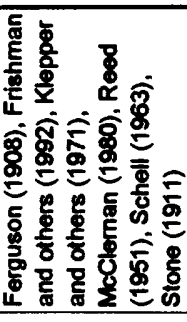 & 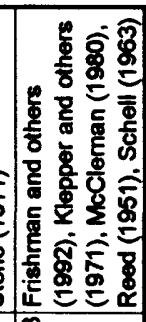 \\
\hline 量 & 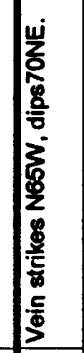 & 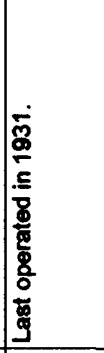 & 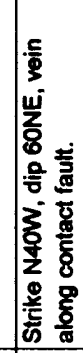 & 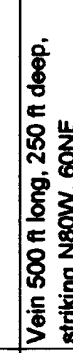 & & 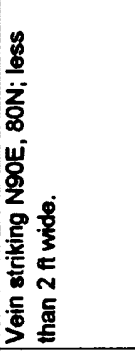 & 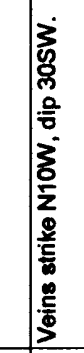 & & 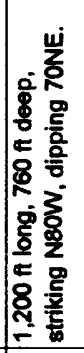 & 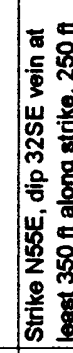 & 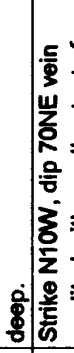 & & & & 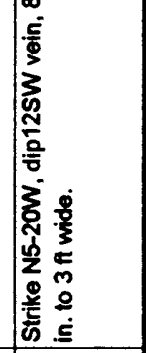 \\
\hline$\sqrt{\widehat{N}}$ & & $g$ & & $=$ & & & $\approx$ & & 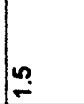 & & & & & & \\
\hline ¿ $\frac{\bar{g}}{\underline{\underline{g}}}$ & & $\stackrel{\leftrightarrow}{\stackrel{\leftrightarrow}{\circ}}$ & & $\ddot{8}$ & & 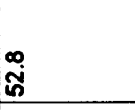 & 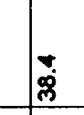 & & $\overline{\mathbf{A}}$ & & & & & 8 & 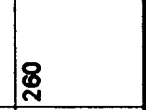 \\
\hline$\overline{0} \overline{\underline{g}}$ & & 乮 & & 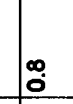 & & $E$ & กิ & & $\%$ & & & & & & $\bar{i}$ \\
\hline : $9 \overline{4}$ & & 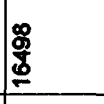 & & : & & 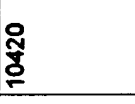 & 爱 & & 趼 & & & & & 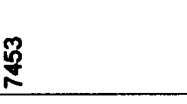 & 落 \\
\hline ₹专 & & $\ddot{8}$ & $\$$ & $=$ & & $\underline{\underline{\alpha}}$ & 8 & & $\hat{\text { a }}$ & דָ & & & & 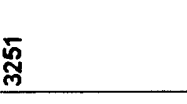 & $\hat{\hat{6}}$ \\
\hline | & & $\frac{8}{n}$ & & א్ల & & 要 & 斚 & & 商 & $\mid 8$ & & & 䓂 & 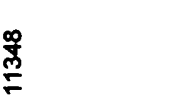 & $\frac{5}{5}$ \\
\hline 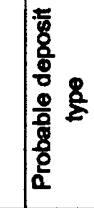 & 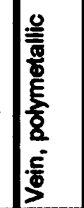 & 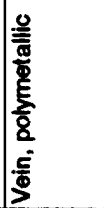 & 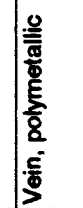 & 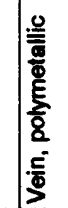 & & $\frac{8}{\frac{8}{6}}$ & \begin{tabular}{|l}
$\frac{1}{8}$ \\
$\frac{c}{5}$ \\
$\frac{5}{5}$
\end{tabular} & & 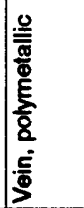 & 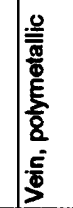 & 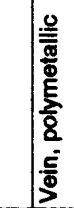 & & & & 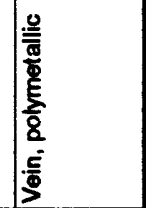 \\
\hline 搳 & \begin{tabular}{|l|} 
\\
$\frac{0}{8}$ \\
$=$ \\
\end{tabular} & $\begin{array}{l}9 \\
5 \\
=\end{array}$ & $\begin{array}{l}7 \\
5 \\
=\end{array}$ & $\begin{array}{l}\hat{b} \\
q \\
= \\
=\end{array}$ & & $\begin{array}{l}\overline{5} \\
\overline{5} \\
\overline{5}\end{array}$ & $\bar{\tau}$ & & 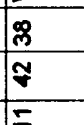 & $\begin{array}{l}\bar{q} \\
\bar{q} \\
=\end{array}$ & $\frac{\pi}{\pi}$ & & & $\frac{1}{7}$ & $\begin{array}{l} \\
\bar{n} \\
\end{array}$ \\
\hline 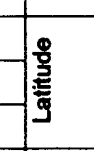 & \begin{tabular}{|l} 
\\
9 \\
0 \\
9 \\
9
\end{tabular} & \begin{tabular}{|l|} 
\\
\\
2 \\
8 \\
8
\end{tabular} & \begin{tabular}{|l|} 
\\
5 \\
\multirow{2}{*}{} \\
9
\end{tabular} & \begin{tabular}{|l}
- \\
0 \\
0 \\
1 \\
9 \\
\end{tabular} & & \begin{tabular}{l}
$\frac{F}{0}$ \\
$\frac{9}{\pi}$ \\
\multirow{q}{*}{}
\end{tabular} & $\begin{array}{l}F \\
F \\
\mathscr{B} \\
\mathscr{2} \\
\mathscr{q} \\
\end{array}$ & & 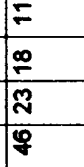 & 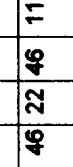 & 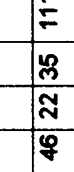 & & 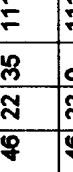 & 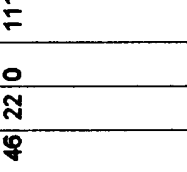 & \begin{tabular}{|l}
$\mid=$ \\
8 \\
$\frac{8}{5}$ \\
$\frac{9}{9}$
\end{tabular} \\
\hline 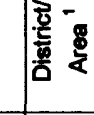 & & $\frac{5}{\frac{5}{3}}$ & 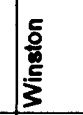 & 毫 & & $\frac{5}{\frac{5}{\underline{\underline{t e}}}}$ & 譥 & & $\frac{5}{\frac{2}{2}}$ & 产 & 咅 & & : & 产 & 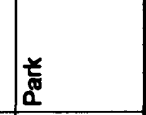 \\
\hline 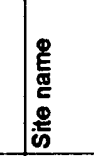 & 产 & |⿹ & | & 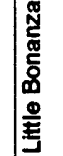 & & 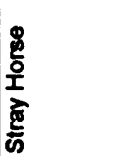 & 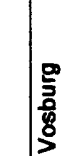 & & 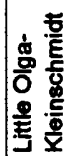 & 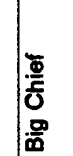 & 韵 & & & & | \\
\hline$\frac{j}{2}$ & 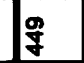 & 8 & $\overline{7}$ & $\tilde{\text { qิ }}$ & & 8 & 芓 & & $\underline{p}$ & $\%$ & $\sqrt{9}$ & & 䗆 & & 8 \\
\hline
\end{tabular}

吉乌 


\begin{tabular}{|c|c|c|c|c|c|c|c|c|c|c|c|c|c|c|c|}
\hline 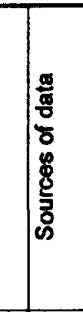 & 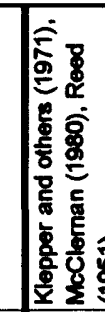 & 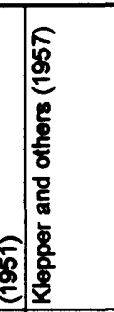 & 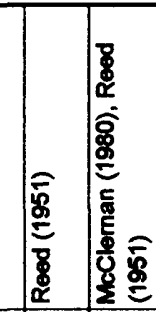 & 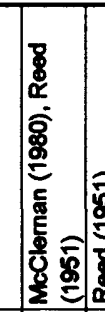 & & 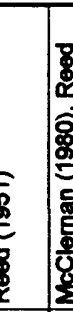 & & 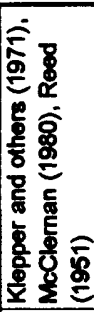 & 要 & 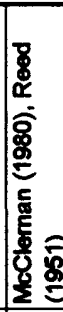 & & 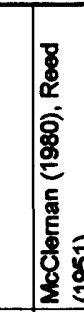 & 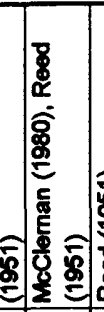 & 庶 & 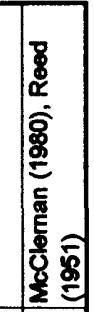 \\
\hline & 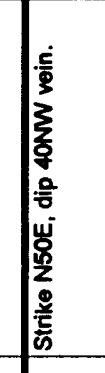 & 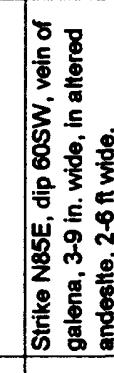 & 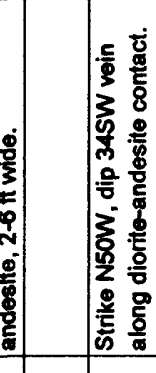 & 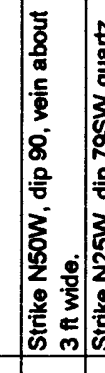 & & & & 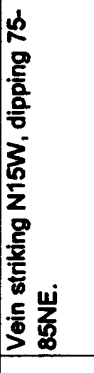 & & & 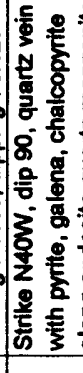 & 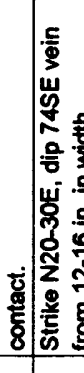 & 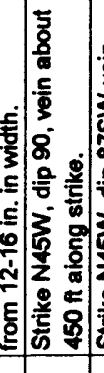 & 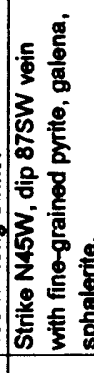 & 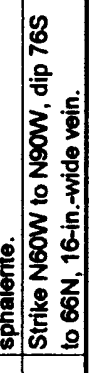 \\
\hline 5 可 & & & & & & & & 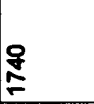 & & & & & & & \\
\hline 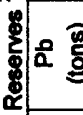 & : & & & & & & & ฐి & & 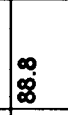 & $\hat{0}$ & 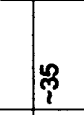 & & & \\
\hline उo & & & & $:$ & & & & 鄫 & & & $\hat{0}$ & & & & \\
\hline ? & $q$ & & & 串 & & & & 兽 & & 踞 & & & & & \\
\hline$z 8$ & N & & & $\overline{9}$ & & & & 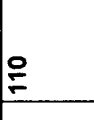 & & 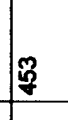 & $\dddot{q}$ & & & & \\
\hline$E$ & $\infty$ & & & 2 & & & & 滪 & & 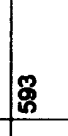 & $\tilde{๓}$ & 8 & & & \\
\hline 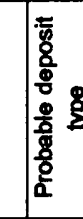 & 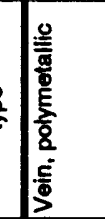 & 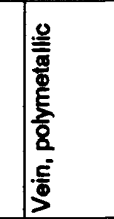 & 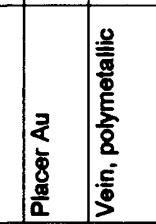 & 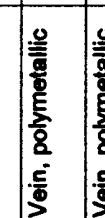 & & & & 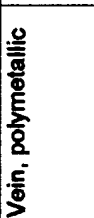 & & 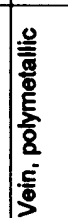 & & 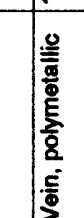 & $\mid$ & & 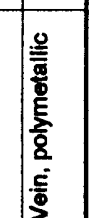 \\
\hline$-\stackrel{\circ}{2}$ & 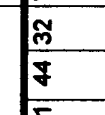 & $\frac{8}{7}$ & \begin{tabular}{|l|l}
$\underline{p}$ & 9 \\
7 & 7 \\
\end{tabular} & 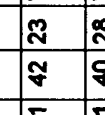 & \begin{tabular}{|l|}
$\infty$ \\
\multirow{2}{*}{} \\
- \\
\end{tabular} & 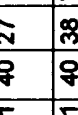 & & $\frac{5}{5}$ & & \begin{tabular}{|l} 
\\
\\
\end{tabular} & $\frac{\pi}{q}$ & \begin{tabular}{|l}
5 \\
8 \\
\end{tabular} & $\begin{array}{l}8 \\
\\
\end{array}$ & $\bar{F}$ & $\begin{array}{l}5 \\
5 \\
5\end{array}$ \\
\hline 15 & $E$ & $E$ & $\equiv \equiv$ & $\equiv$ & & $E E$ & & $E$ & & $E$ & $E$ & $E$ & $E$ & $E$ & $E$ \\
\hline - & 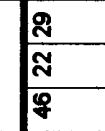 & \begin{tabular}{|l|}
$\frac{2}{12}$ \\
$\frac{1}{q}$
\end{tabular} & \begin{tabular}{|l|l}
8 & - \\
$\frac{2}{2}$ & $\overline{9}$ \\
9 & 9 \\
\end{tabular} & \begin{tabular}{|l|l}
0 & $\infty$ \\
$\pi$ & 5 \\
$q$ & 5 \\
$q$
\end{tabular} & 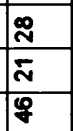 & \begin{tabular}{|l|l}
- & 8 \\
7 & 8 \\
9 & 9 \\
\end{tabular} & $f$ & $\begin{array}{l}\frac{8}{8} \\
\frac{8}{9} \\
\end{array}$ & & \begin{tabular}{|l|} 
\\
8 \\
9 \\
9
\end{tabular} & \begin{tabular}{|l|}
$\circ$ \\
8 \\
9
\end{tabular} & 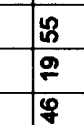 & \begin{tabular}{|l|l}
1 & $:$ \\
9 & 0 \\
& \\
\end{tabular} & $\begin{array}{l}8 \\
\frac{8}{9} \\
9 \\
9\end{array}$ & \begin{tabular}{|l}
$\frac{9}{9}$ \\
$\frac{9}{9}$ \\
\end{tabular} \\
\hline 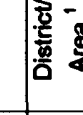 & 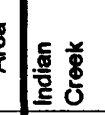 & 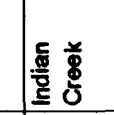 & 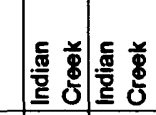 & 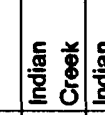 & & & 8 & 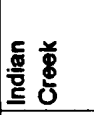 & & 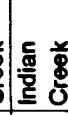 & & & 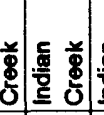 & 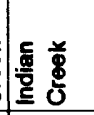 & 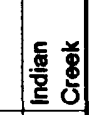 \\
\hline 鼬 & 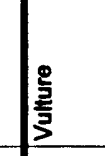 & 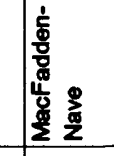 & 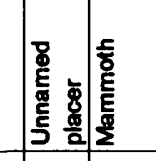 & 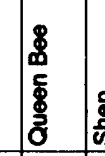 & & 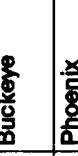 & & 曾 & & & 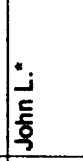 & 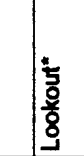 & , & 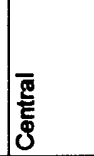 & 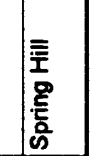 \\
\hline gi & $\sqrt{9}$ & 䒹 & $\mid \begin{array}{ll}8 \\
8\end{array}$ & 璟 & 8 & s & & 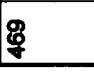 & & & $\tilde{z}$ & f & $\bar{F}$ & $\frac{9}{9}$ & $\%$ \\
\hline
\end{tabular}




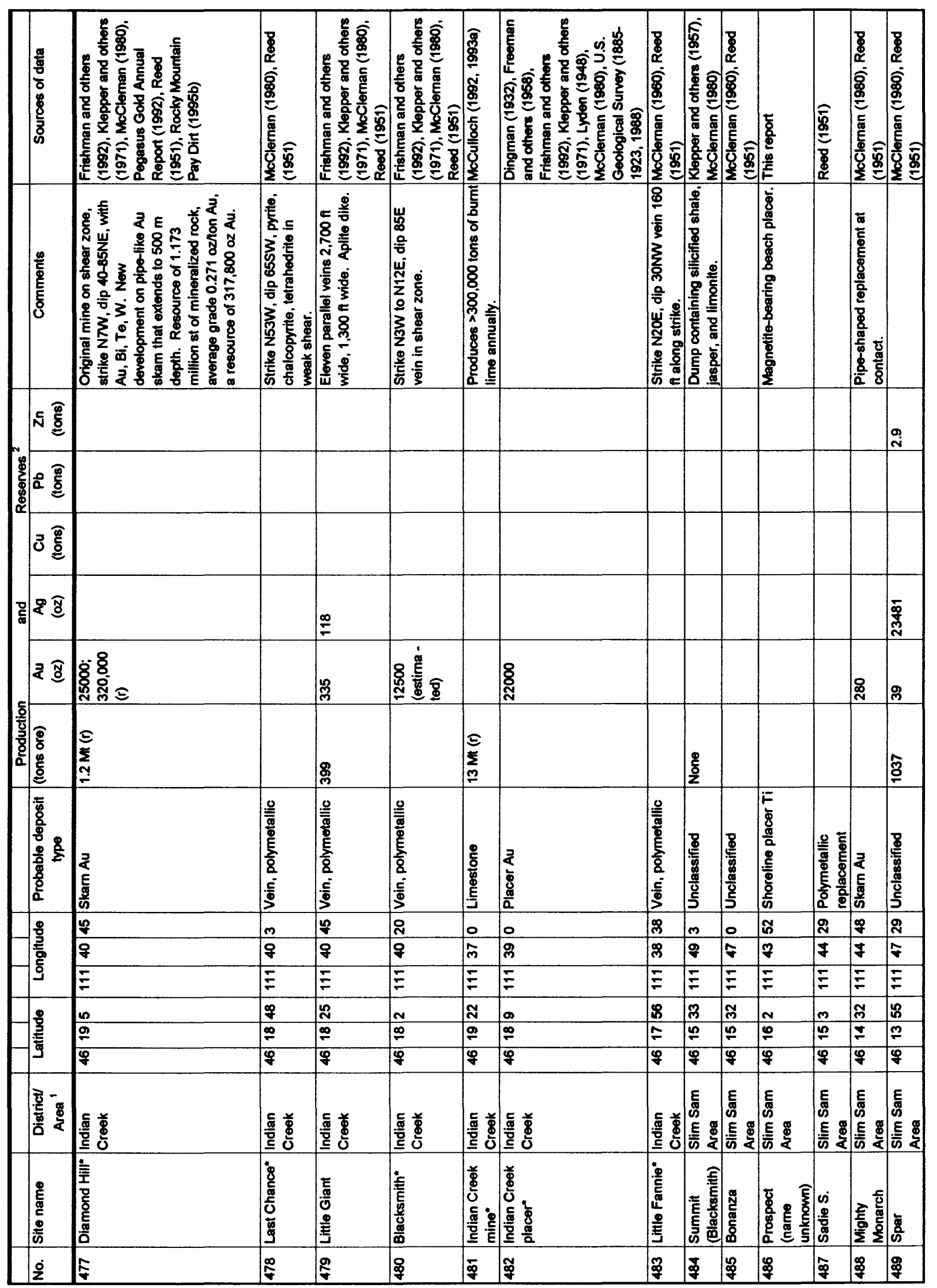

媱 


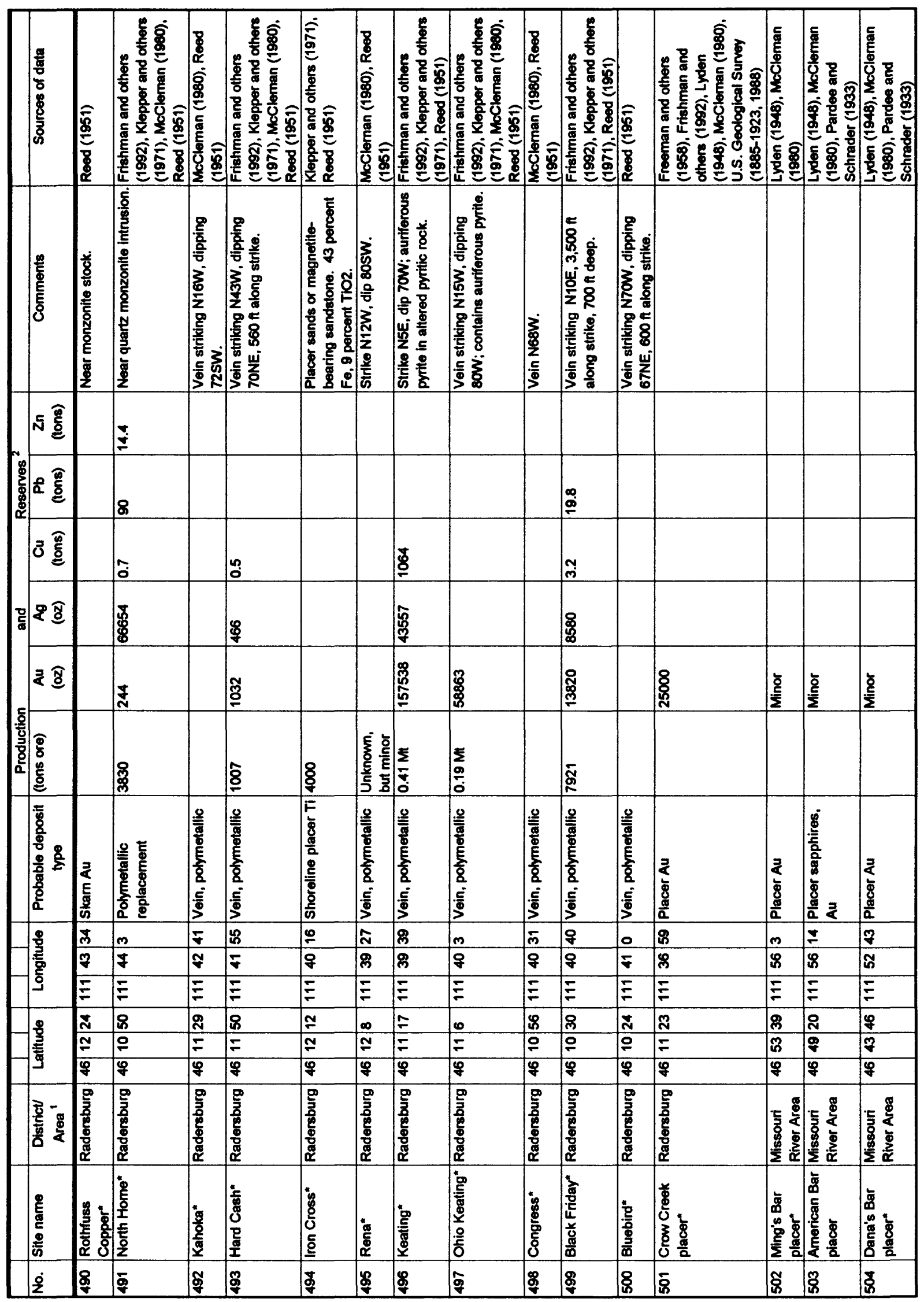

臵 


\begin{tabular}{|c|c|c|c|c|c|c|c|c|c|}
\hline | & 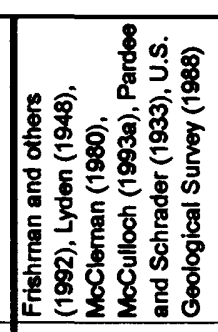 & 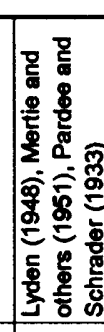 & 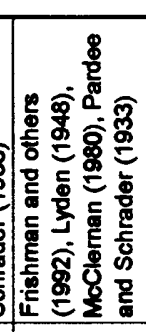 & 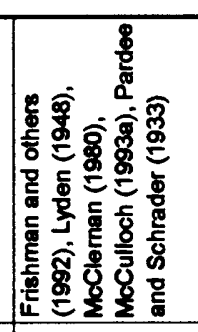 & 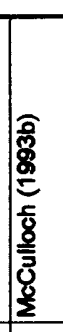 & 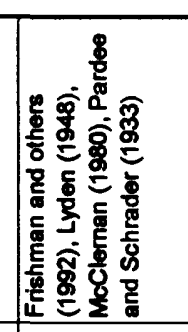 & 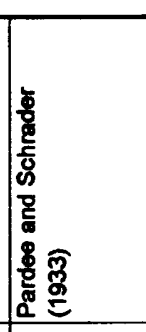 & 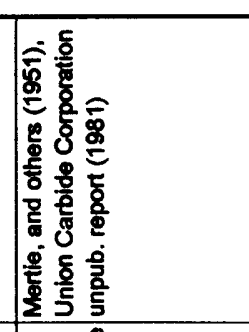 & 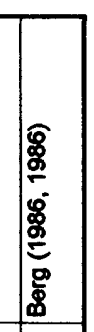 \\
\hline 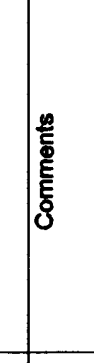 & 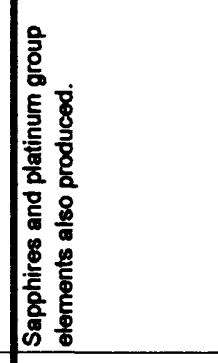 & & & & & 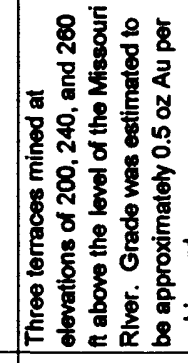 & 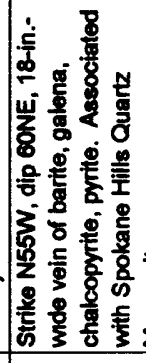 & 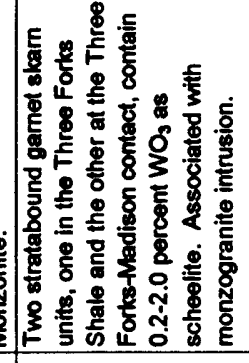 & 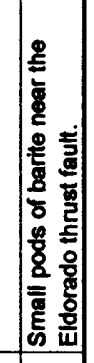 \\
\hline 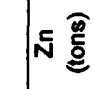 & & & & & & & & & \\
\hline Q & & & & & & & & & \\
\hline उ. & & & & & & & & & \\
\hline 92 & & & & & & & & & \\
\hline ₹ & $\frac{8}{8}$ & & 离 & 層 & & 店 & & & \\
\hline 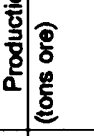 & & & & & & & & 8 & 产 \\
\hline 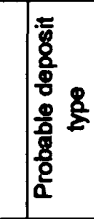 & 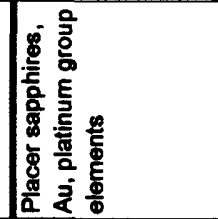 & & 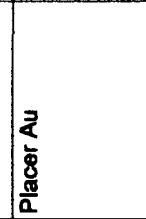 & 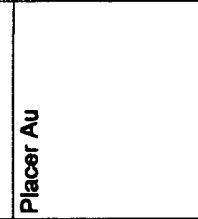 & 18 & & 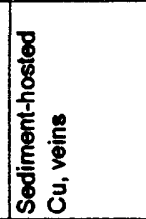 & 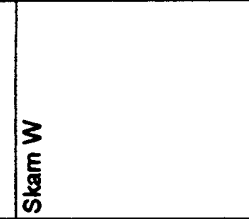 & 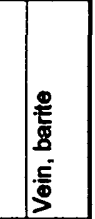 \\
\hline 害 & 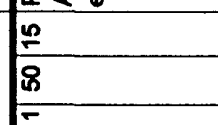 & $\begin{array}{l}\frac{\pi}{2} \\
9 \\
-10\end{array}$ & $\begin{array}{l}F \\
\end{array}$ & $\frac{8}{5}$ & 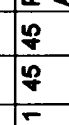 & $\begin{array}{l}7 \\
7 \\
\end{array}$ & $\frac{\pi}{y}$ & $\frac{0}{5}$ & 8 \\
\hline $\bar{\Xi}$ & $E$ & $E$ & $E$ & $E$ & $\equiv$ & $\equiv$ & $E$ & $E$ & $E$ \\
\hline 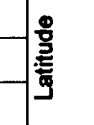 & \begin{tabular}{|l}
8 \\
8 \\
9 \\
9 \\
9
\end{tabular} & & $\begin{array}{l}\frac{\bullet}{9} \\
\vdots \\
\end{array}$ & $\begin{array}{l}\frac{\vdots}{q} \\
\vdots \\
q\end{array}$ & \begin{tabular}{|l}
$\begin{array}{l} \\
9 \\
9\end{array}$ \\
\end{tabular} & \begin{tabular}{|l|l} 
\\
\\
\\
\end{tabular} & $\begin{array}{l}\frac{m}{2} \\
\frac{8}{9} \\
9\end{array}$ & \begin{tabular}{|l}
$\frac{8}{8}$ \\
$\frac{8}{9}$
\end{tabular} & \begin{tabular}{|l}
$\frac{3}{7}$ \\
$\frac{7}{9}$
\end{tabular} \\
\hline 琶 & 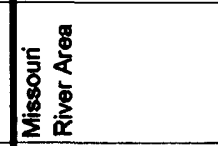 & 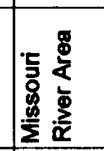 & 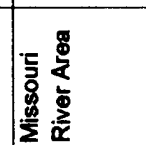 & 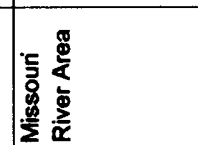 & & 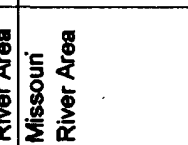 & $\mid$ & 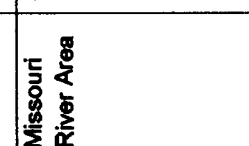 & 5 \\
\hline 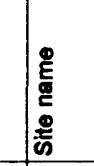 & 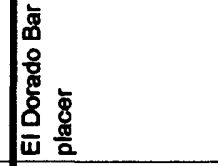 & 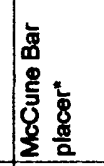 & 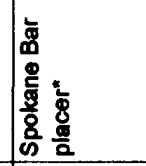 & 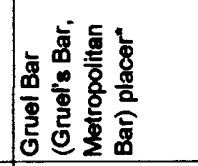 & & 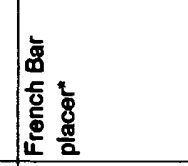 & 委 & $\mid$ & $\frac{2}{3}$ \\
\hline$\frac{\dot{2}}{2}$ & 迨 & 8 & ŝ. & 88 & 8 & $\frac{\circ}{10}$ & 涪 & $\frac{\pi}{n}$ & $\frac{2}{n}$ \\
\hline
\end{tabular}

通攴 


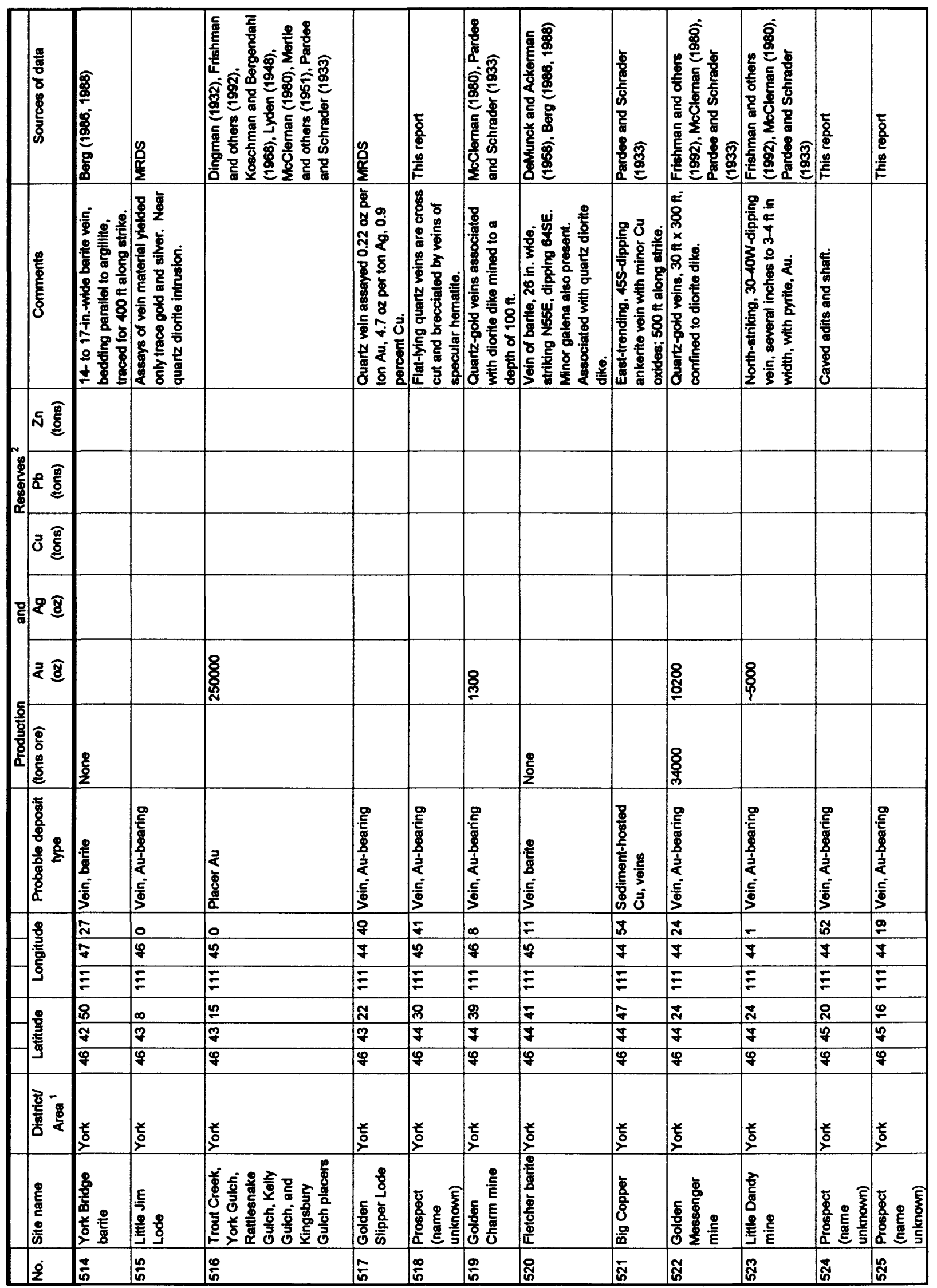

承是 


\begin{tabular}{|c|c|c|c|c|c|c|c|c|c|c|c|c|c|}
\hline 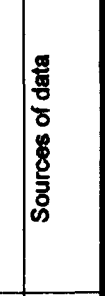 & 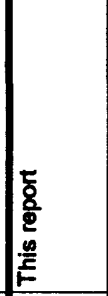 & 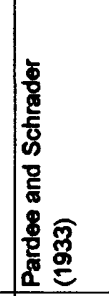 & 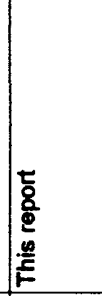 & 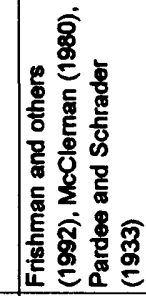 & 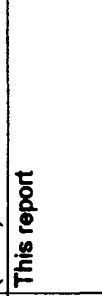 & $\begin{array}{l}5 \\
\frac{2}{2} \\
\frac{0}{2} \\
\frac{0}{F} \\
\end{array}$ & 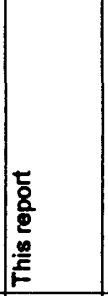 & 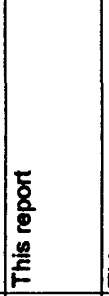 & 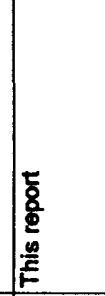 & 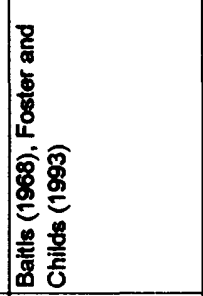 & 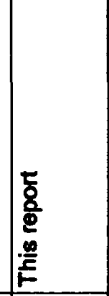 & 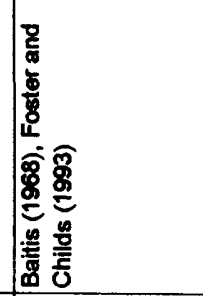 & 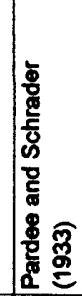 \\
\hline 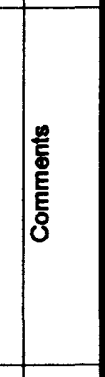 & 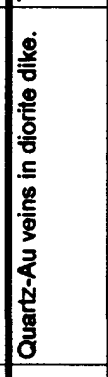 & 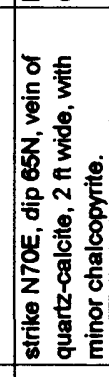 & 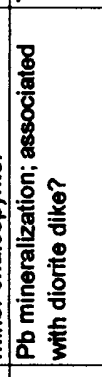 & 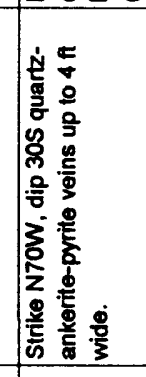 & 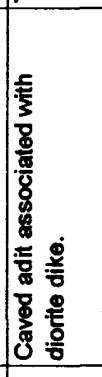 & 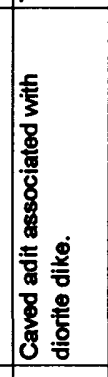 & 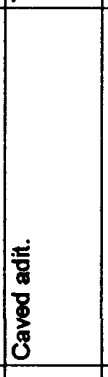 & & & 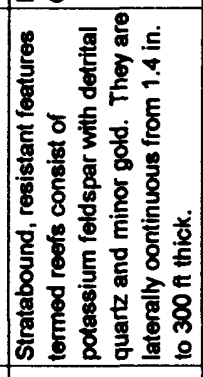 & 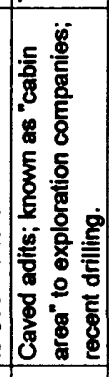 & 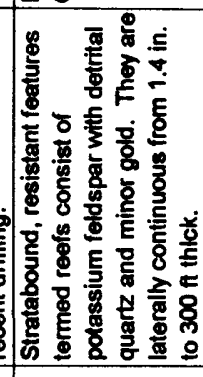 & 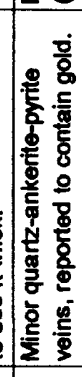 \\
\hline$\tilde{N} \frac{\widehat{Q ⿻}}{\underline{g}}$ & & & & & & & & & & & & & \\
\hline a & & & & & & & & & & & & & \\
\hline ỏ & & & & & & & & & & & & & \\
\hline श्ष & & & & & & & & & & & & & \\
\hline ₹त्रु & & & & 8 & & & & & & & & $\begin{array}{l}5 \\
8 \\
8 \\
0\end{array}$ & \\
\hline 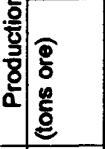 & & in & & & & & & & & & & 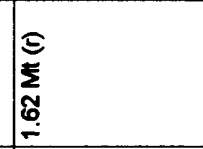 & \\
\hline 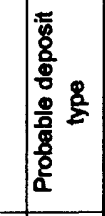 & 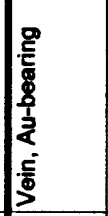 & 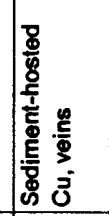 & 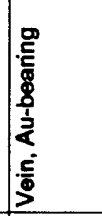 & 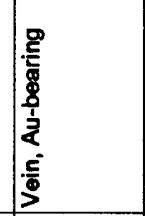 & 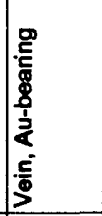 & 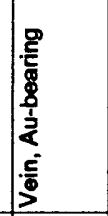 & 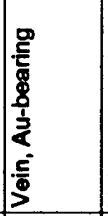 & 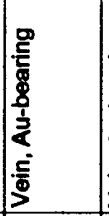 & 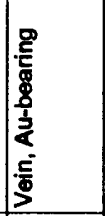 & 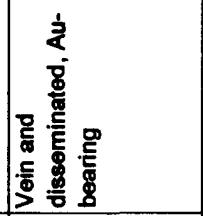 & 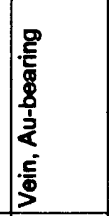 & 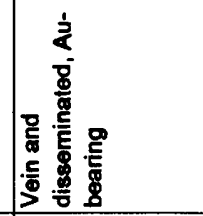 & 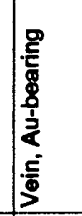 \\
\hline 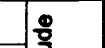 & $F$ & $\tilde{N}$ & 0 & 40 & 18 & 0 & in & $\infty$ & 0 & 7 & $\bar{N}$ & 48 & $=$ \\
\hline 憘 & 7 & $\mathcal{T}$ & $\checkmark$ & $F$ & $F$ & $F$ & q & q & q & g & I & 19 & $\infty$ \\
\hline 78 & 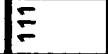 & $\Xi$ & $\equiv$ & 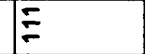 & $E$ & $\Xi$ & $\equiv$ & $\equiv$ & $\equiv$ & $\Xi$ & $\Xi$ & $\Xi$ & $\Xi$ \\
\hline 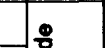 & กิ & $m$ & 9 & 2 & ig & $q$ & N & $\sigma$ & $\%$ & 9 & $\tilde{\kappa}$ & $=$ & 8 \\
\hline 苞 & 9 & 7 & 7 & 9 & \% & 7 & 7 & 7 & $\mathcal{F}$ & 7 & $\mathcal{F}$ & 9 & F \\
\hline & $\mathscr{q}$ & 9 & 9 & 9 & $\varphi$ & 9 & 9 & 9 & 9 & 9 & 9 & 9 & 9 \\
\hline 量 & 产 & ì & 它 & 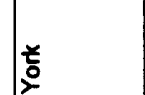 & 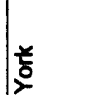 & $\frac{5}{2}$ & 旁 & 蒙 & $\frac{\check{z}}{\partial}$ & 宂 & 产 & 항 & 항 \\
\hline 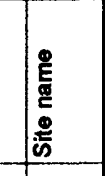 & 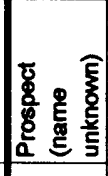 & 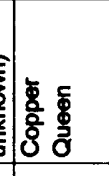 & 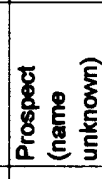 & 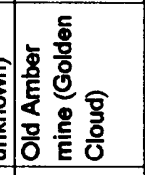 & 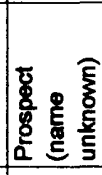 & 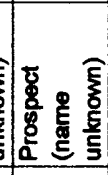 & 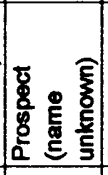 & 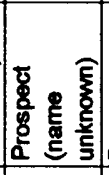 & 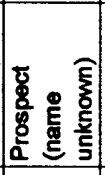 & 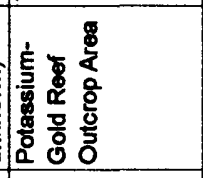 & 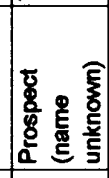 & 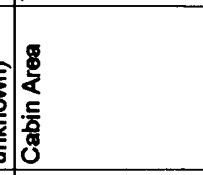 & $\frac{\frac{\delta}{0}}{\frac{0}{\omega x}}$ \\
\hline$\stackrel{0}{2}$ & 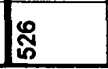 & 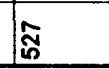 & జ్లో & స్ది & 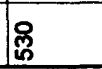 & $\overline{5}$ & 路 & 象 & 芯 & \begin{tabular}{l}
$n$ \\
\hdashline
\end{tabular} & $\begin{array}{l}2 \\
0\end{array}$ & 跑 & 总 \\
\hline
\end{tabular}

号 


\begin{tabular}{|c|c|c|c|c|c|c|c|c|c|c|c|}
\hline 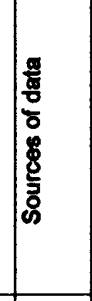 & 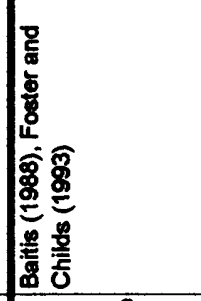 & 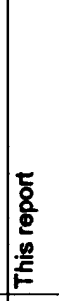 & 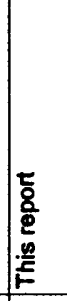 & 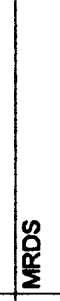 & 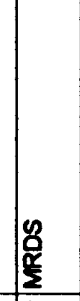 & 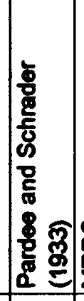 & & & 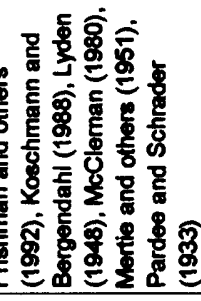 & 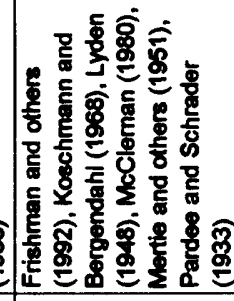 & 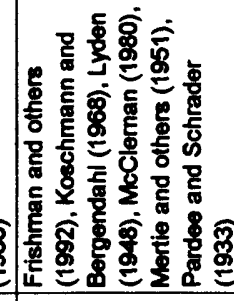 \\
\hline 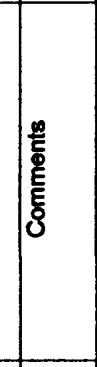 & 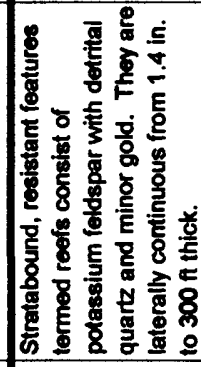 & 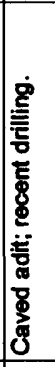 & 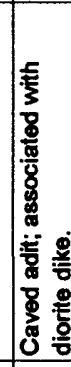 & & 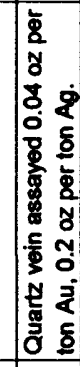 & & 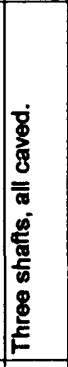 & & & & \\
\hline$\tilde{N} \frac{\widehat{g}}{\underline{g}}$ & & & & & & & & & & & \\
\hline$\stackrel{0}{0}$ & & & & & & & & & & & \\
\hline 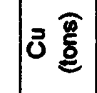 & & & & & & & & & & & \\
\hline \& & & & & & & & & & & & \\
\hline द $\widehat{\gamma}$ & \begin{tabular}{|l} 
E \\
8 \\
\\
\end{tabular} & & & & & & & 旡 & 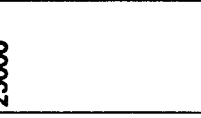 & 通 & 总 \\
\hline 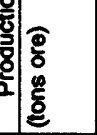 & 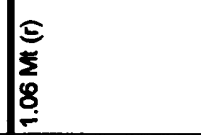 & & & & & & & & & & \\
\hline 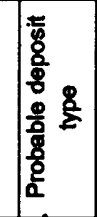 & 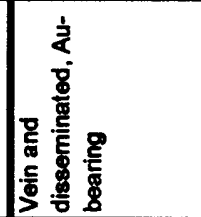 & 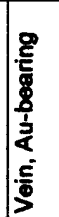 & 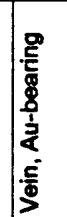 & $\begin{array}{l}\frac{z}{3} \\
\frac{8}{8} \\
\frac{8}{a}\end{array}$ & 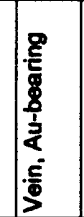 & \begin{tabular}{|l|}
$\frac{z}{8}$ \\
$\frac{8}{8}$ \\
$\frac{8}{2}$ \\
\end{tabular} & \begin{tabular}{|l}
$\frac{z}{3}$ \\
$\frac{8}{8}$ \\
$\frac{8}{a}$
\end{tabular} & 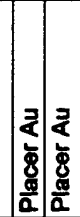 & & 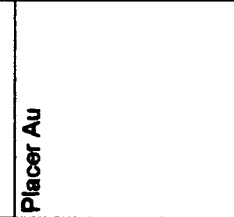 & 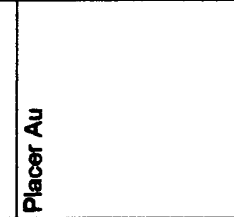 \\
\hline$\sqrt{3}$ & $\sim$ & 2 & 0 & is & 9 & $\tilde{N}$ & $\underline{m}$ & 60 & & 8 & 5 \\
\hline ] & $\%$ & $\infty$ & $\infty$ & $\hat{\mathbf{m}}$ & $\hat{m}$ & $\bar{m}$ & $\infty$ & 978 & & 7 & $F$ \\
\hline $\bar{\jmath}$ & $E$ & $E$ & $E$ & $\Xi$ & $E$ & $E$ & $\equiv$ & $E E$ & & $E$ & $E$ \\
\hline$\div$ & $\tilde{\omega}$ & $\bar{\Lambda}$ & $\%$ & $\approx$ & $\underline{m}$ & in & न & $\hat{N E}$ & & 9 & 9 \\
\hline$-{ }^{\underline{\underline{z}}}$ & \begin{tabular}{|l|l|}
\multirow{2}{*}{} \\
\end{tabular} & \begin{tabular}{|l|l}
1 \\
12
\end{tabular} & $\frac{9}{9}$ & $\begin{array}{l} \\
\end{array}$ & \begin{tabular}{|l|l}
\multirow{2}{*}{} \\
\end{tabular} & \begin{tabular}{|l|}
7 \\
$\frac{9}{8}$
\end{tabular} & $\begin{array}{l}\neq \\
g\end{array}$ & $\begin{array}{l}=5 \\
979\end{array}$ & & \begin{tabular}{|l|}
8 \\
$\%$
\end{tabular} & \begin{tabular}{|l|l}
8 \\
8
\end{tabular} \\
\hline 哣- & 类 & ț. & 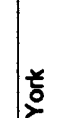 & to & $\underset{0}{2}$ & 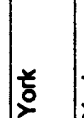 & 弟 & 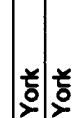 & & 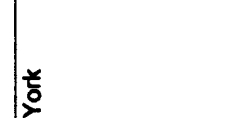 & 类 \\
\hline 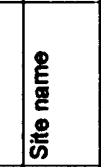 & 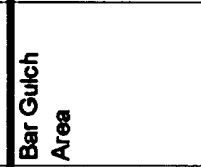 & & & & 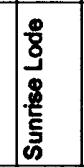 & 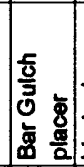 & 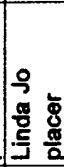 & 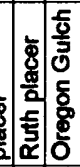 & 童 & 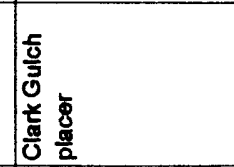 & 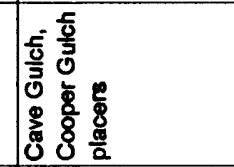 \\
\hline$\dot{s}$ & 遍 & 㷛 & 중 & 嘫 & 悉 & 通 & $\mid \begin{array}{l}5 \\
\end{array}$ & 影部 & & 造 & 量 \\
\hline
\end{tabular}




\begin{tabular}{|c|c|c|c|c|c|c|c|c|c|c|}
\hline 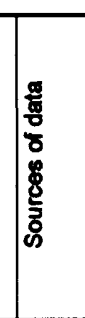 & 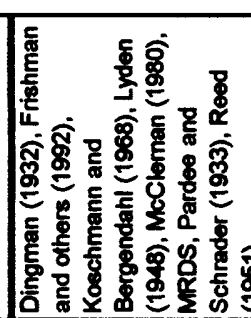 & 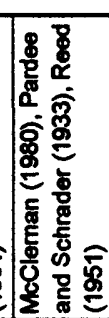 & 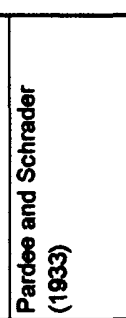 & 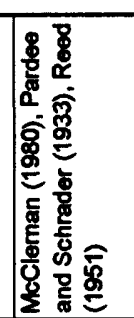 & 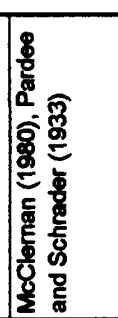 & 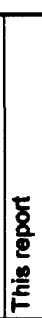 & 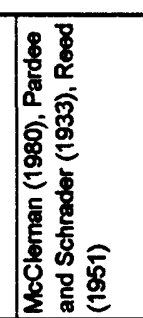 & 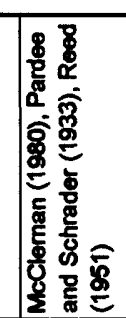 & 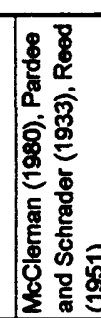 & 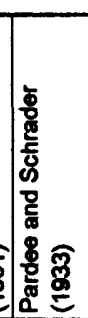 \\
\hline 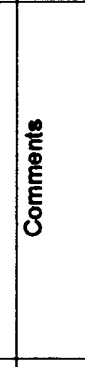 & & 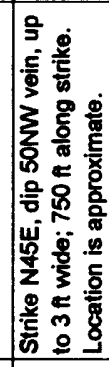 & 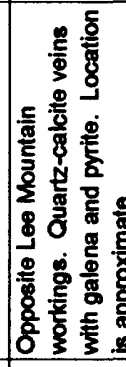 & 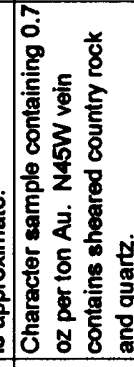 & 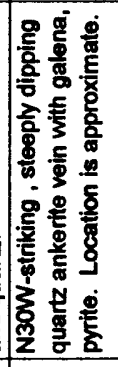 & & 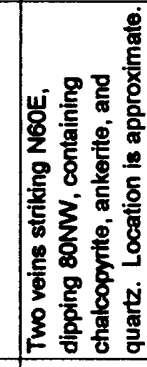 & 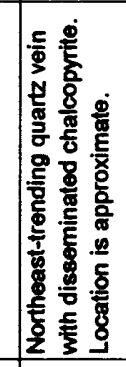 & 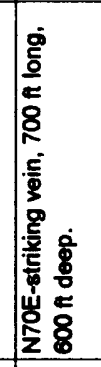 & 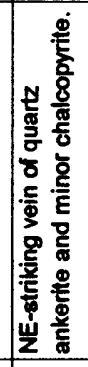 \\
\hline న工 & & & & & & & & & & \\
\hline 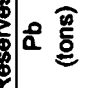 & & & & & & & & & & \\
\hline $\overrightarrow{0} \overline{\underline{\underline{g}}}$ & & $\hat{i}$ & & & & & & & 空 & \\
\hline 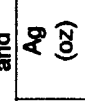 & & & & & & & & & $\hat{\mathfrak{N}}$ & \\
\hline द वर्ष & 8 & & & & & & & & $\$$ & \\
\hline 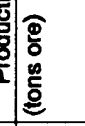 & & 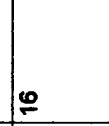 & \begin{tabular}{|l}
$\frac{9}{50}$ \\
$\frac{\mathbf{g}}{2}$
\end{tabular} & $\begin{array}{l}\frac{0}{0} \\
\frac{5}{2} \\
\underline{z}\end{array}$ & & & 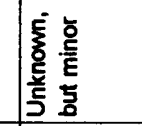 & 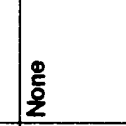 & 密 & \\
\hline 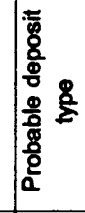 & 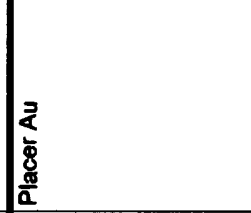 & 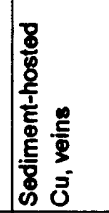 & 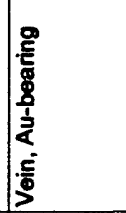 & 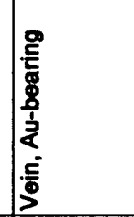 & 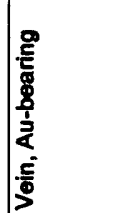 & 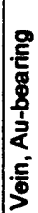 & 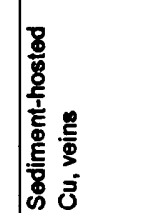 & 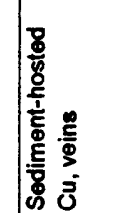 & 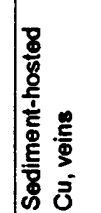 & 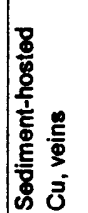 \\
\hline \% & 10 & $\infty$ & $\pi$ & $\infty$ & 9 & 8 & $\%$ & $F$ & N & 7 \\
\hline $7 \geq$ & $F$ & 曾 & $\%$ & స్ల & స్ & ळ & ले & m & $\infty$ & मे \\
\hline בَ & $E$ & $\equiv$ & $\equiv$ & $E$ & $\equiv$ & $E$ & $\equiv$ & $\equiv$ & $E$ & $\equiv$ \\
\hline $1 \%$ & en & 9 & $\cong$ & $\sigma$ & in & $\cong$ & $\hat{m}$ & 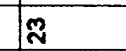 & \pm & $\%$ \\
\hline 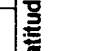 & $\infty$ & \& & 7 & 7 & F & F & $F$ & $\bar{F}$ & $F$ & 9 \\
\hline $7^{9}$ & 19 & 9 & iq & 18 & 98 & 9 & 9 & 19 & 18 & 9 \\
\hline 总要 & | & 易 & 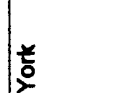 & 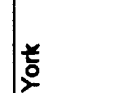 & 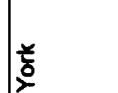 & | & 娄 & 弟 & 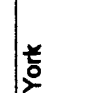 & 装 \\
\hline 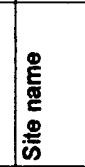 & 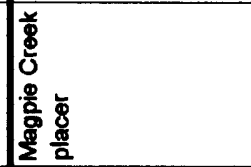 & 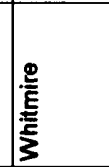 & 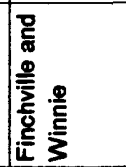 & $\mid \begin{array}{l}\frac{0}{2} \\
\frac{1}{3}\end{array}$ & 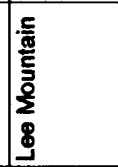 & & 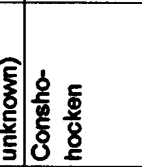 & 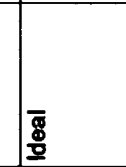 & 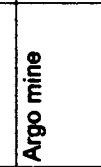 & 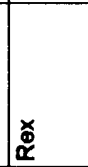 \\
\hline$\dot{i}$ & 遍 & $\overline{\bar{B}}$ & 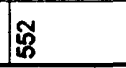 & 居 & 落 & 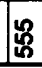 & 8 & 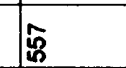 & 象 & 蛊 \\
\hline
\end{tabular}

政? 


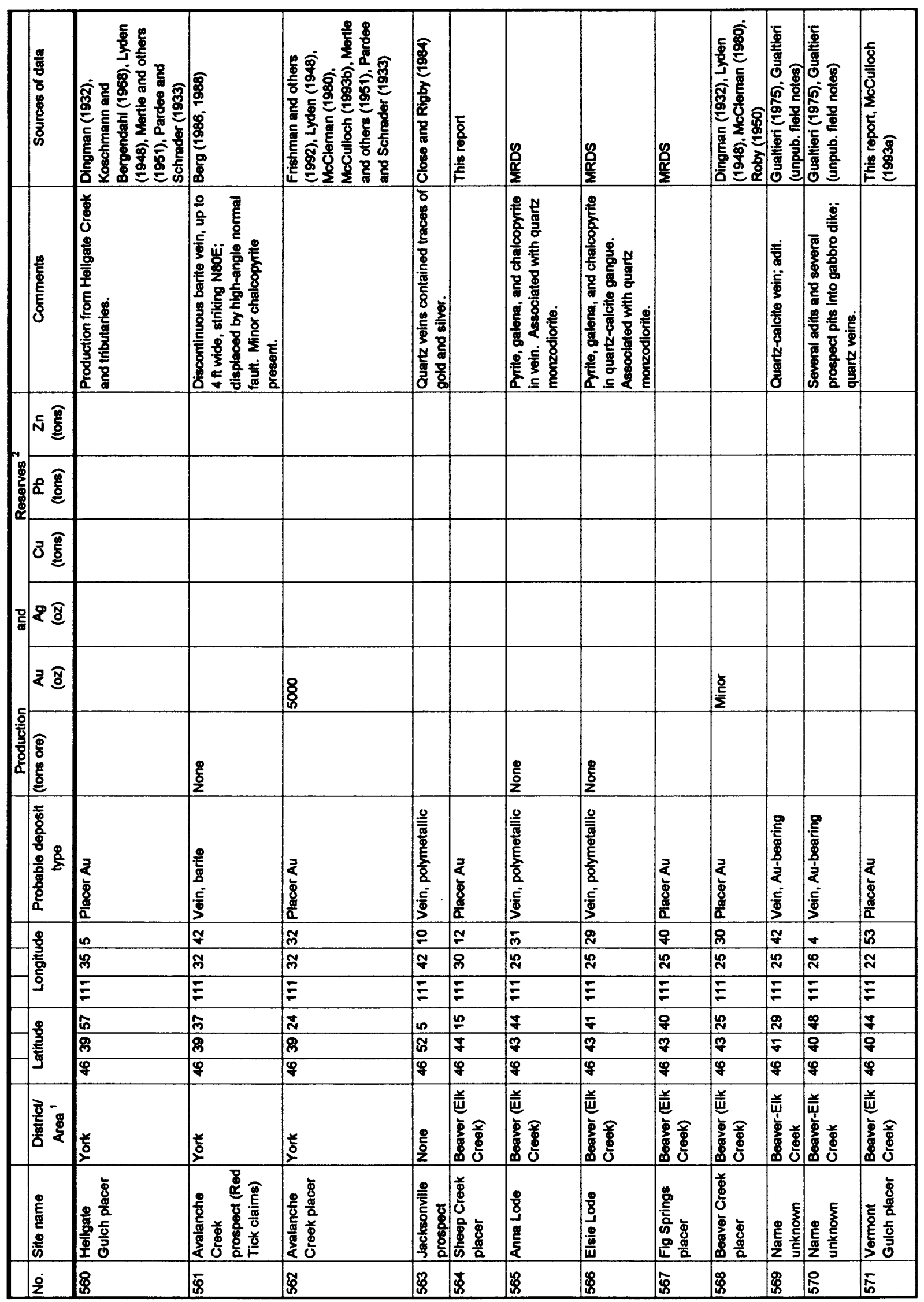

怘 


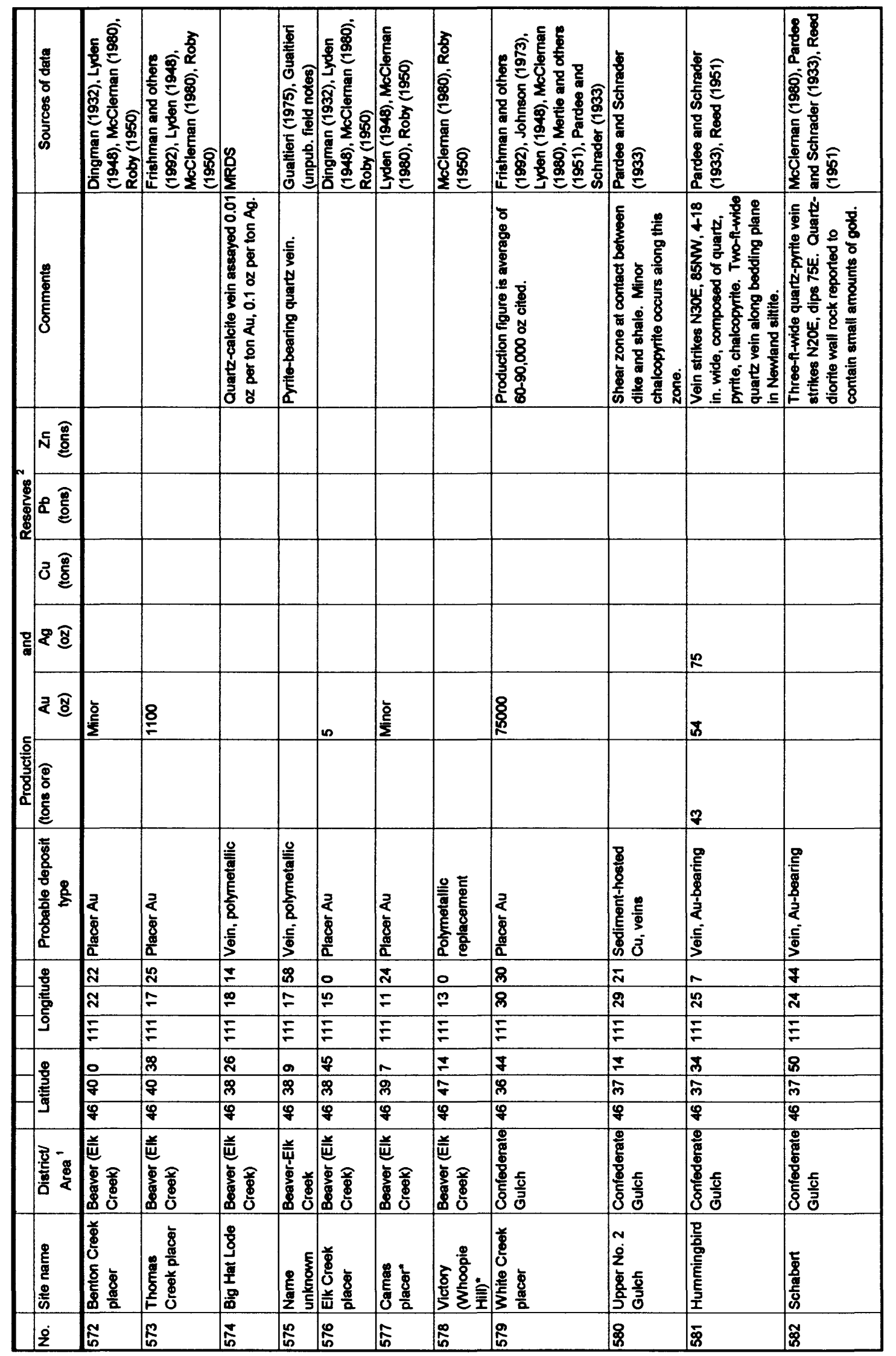

敬 


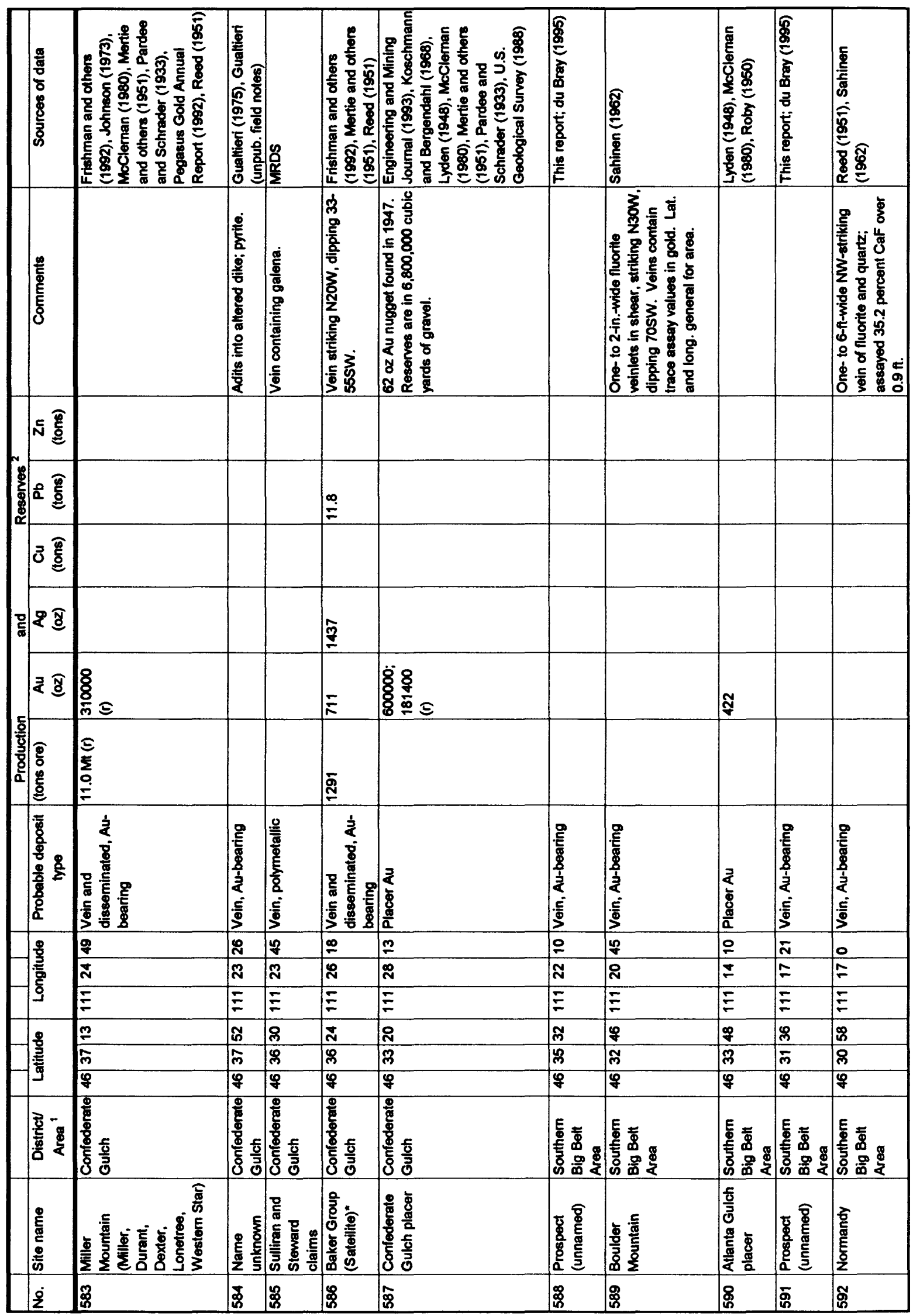




\begin{tabular}{|c|c|c|c|c|c|c|c|c|c|}
\hline 䢩 & | & 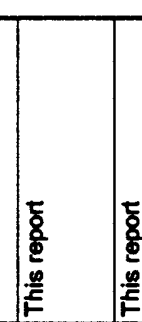 & 婄 & 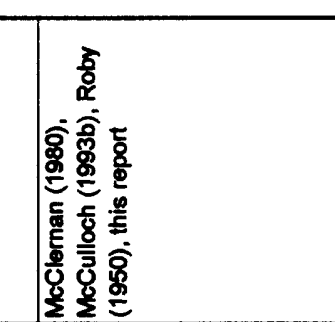 & 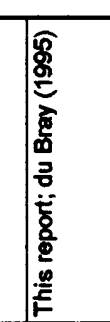 & 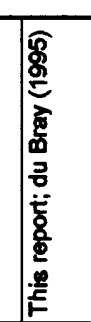 & 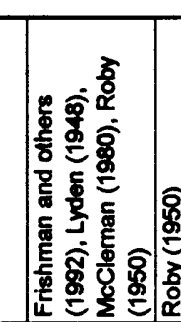 & & 毫 \\
\hline 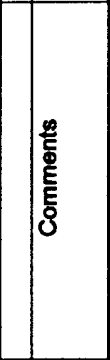 & & 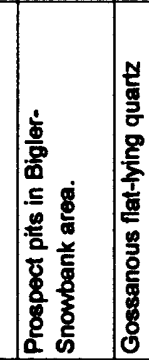 & 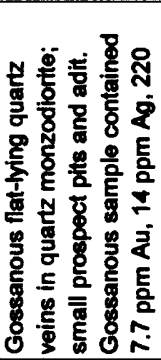 & 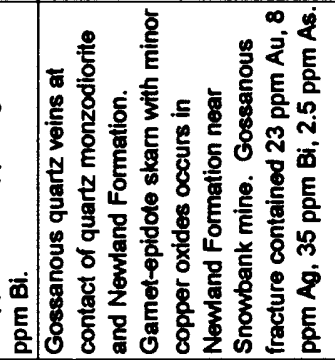 & 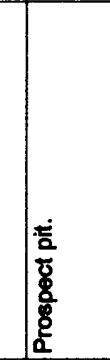 & 童 & & & 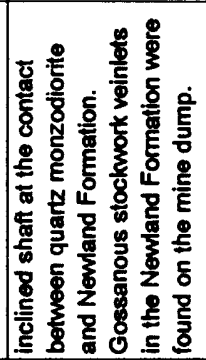 \\
\hline 5 醇 & & & & & & & & & \\
\hline 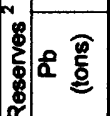 & & & & v & & & & & \\
\hline 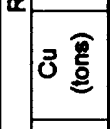 & & & & $:$ & & & & & \\
\hline $92 \pi$ & & & & $E$ & & & & & \\
\hline$\geq \mathbb{8}$ & & & & 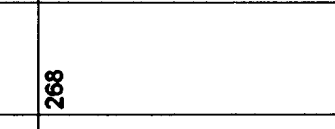 & & & 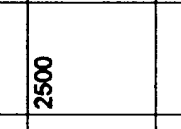 & & \\
\hline 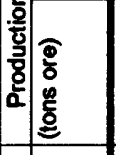 & & & & $\stackrel{\mathscr{\%}}{\mathscr{2}}$ & & & & & \\
\hline 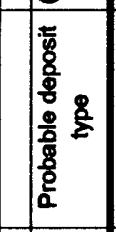 & & 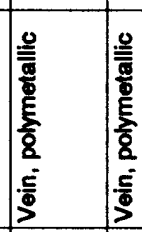 & 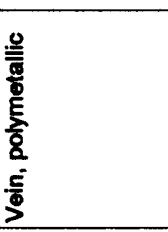 & 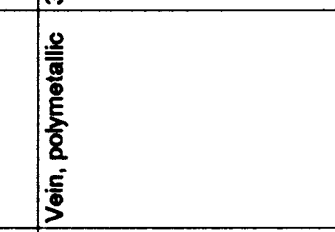 & 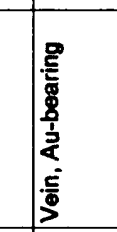 & 5 & & & \\
\hline 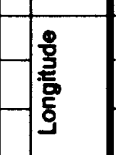 & & 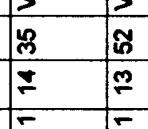 & $\begin{array}{l}\frac{\sigma}{0} \\
\frac{a}{2} \\
\frac{2}{4}\end{array}$ & $\stackrel{m}{ \pm}$ & $\stackrel{0}{\circ}$ & $\stackrel{2}{=}$ & $\approx$ & $\frac{7}{7}$ & t3 \\
\hline 謩 & 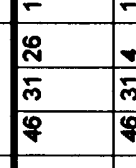 & \begin{tabular}{|l|l} 
& - \\
5 & 0 \\
5 & 5 \\
9 & 9 \\
\end{tabular} & $\begin{array}{l}\frac{5}{6} \\
\frac{0}{5} \\
9 \\
\end{array}$ & $\begin{array}{l}7 \\
8 \\
8 \\
9\end{array}$ & $\begin{array}{l}5 \\
\dot{8} \\
\\
8\end{array}$ & ff & 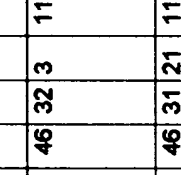 & $F$ & $\begin{array}{l}\frac{7}{\vdots} \\
\frac{8}{7}\end{array}$ \\
\hline 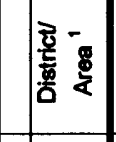 & 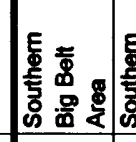 & 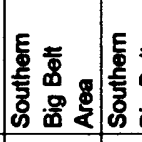 & 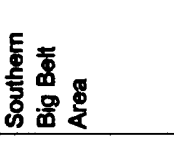 & 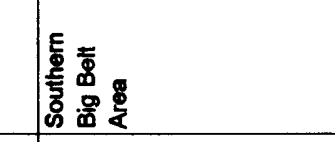 & & & 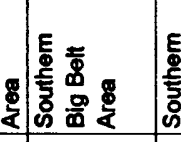 & & 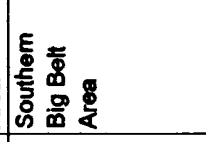 \\
\hline 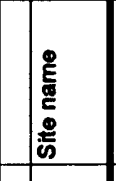 & 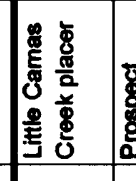 & 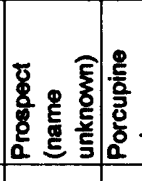 & 量裹 & 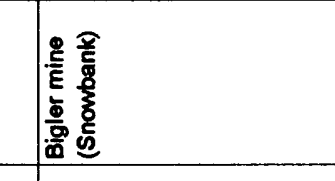 & 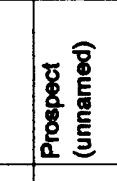 & & 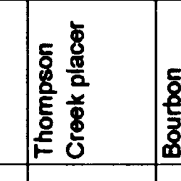 & & 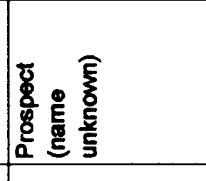 \\
\hline & 8 & 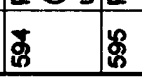 & & & & & & & \\
\hline
\end{tabular}

题 


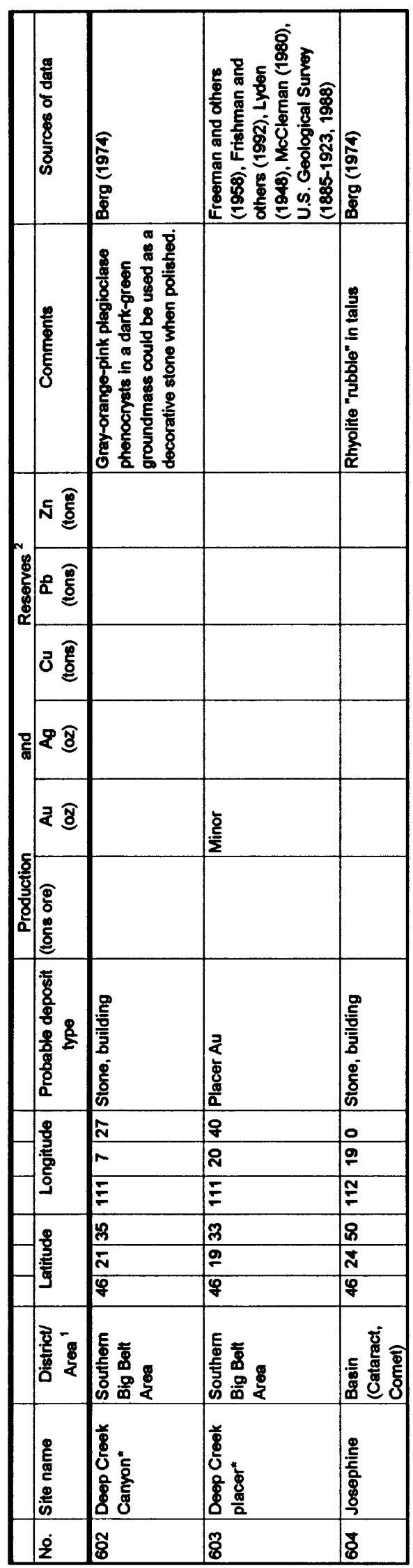

蛔 
American Mines Handbook, 1994, edited by Giancola, Diane, and Razvi, Nasreen: Don Mills, Ontario, Canada, Southam Magazine Group, Inc., 344 p. 1996, edited by Giancola, Diane, and Razvi, Nasreen: Don Mills, Ontario, Canada, Southam Magazine Group, Inc., 380 p.

Anonymous, 1972, Summary Report, Heddleston property, Lewis and Clark County, Montana: Anaconda Geological Documents Collection, International Archives of Economic Geology, American Heritage Center, Laramie, University of Wyoming, reference no. 703,7 p.

Baitis, H.W., 1988, York--A Proterozoic shale hosted gold system in Montana: Northwest Mining Association, 94th Annual Meeting, December 1988, 22 p.

Barrell, Joseph, 1907, Geology of the Marysville mining district, Montana--A study of igneous intrusion and contact metamorphism: U.S. Geological Survey Professional Paper 57, $178 \mathrm{p}$.

Beadle, H.M., 1893, The persistence of ores in lodes in depth--The Empire lode (Marysville, Montana): Engineering and Mining Journal, v. 55, no. 1, p. $154-155$.

Becraft, G.E., 1953, Preliminary report on the Comet area, Jefferson County, Montana: U.S. Geological Survey Circular 277, 8 p.

1956, Uranium deposits of the northern part of the Boulder batholith, Montana: Economic Geology, v. 51, p. 362-374.

Becraft, G.E., Pinckney, D.M., and Rosenblum, Sam, 1963, Geology and mineral deposits of the Jefferson City quadrangle, Jefferson and Lewis and Clark Counties, Montana: U.S. Geological Survey Professional Paper 428, 101 p.

Berg, R.B., 1974, Building stone in Montana: Montana Bureau of Mines and Geology Bulletin 94, 41 p.

1986. Characteristics and probable origin of barite veins in the Belt Supergroup, western Montana, in Roberts, S.M., ed., Belt Supergroup, A guide to Proterozoic rocks of western Montana and adjacent areas: Montana Bureau of Mines and Geology Special Publication 94, p. 258-267.

1988, Barite in Montana: Montana Bureau of Mines and Geology Memoir 61, $100 \mathrm{p}$.

Billingsley, P.R., and Grimes, J.A., 1918, Ore deposits of the Boulder batholith: American Institute of Mining and Metallurgical Engineers Transactions, v. 58, p. 284-361.

Blake, W.P., 1888, Rainbow Lode, Butte City, Montana: American Institute of Mining and Metallurgical Engineers Transactions, v. 16, p. 65-80.

Bondurant, K.T., and Lawson, D.C., 1969, Directory of mining enterprises, 1968: Montana Bureau of Mines and Geology Bulletin 72, 64 p.

Boulter, G.W., 1950, Placer deposits of Last Chance Gulch, Helena, Montana: Billings Geological Society, lst Annual Field Conference Guidebook, p. 7273.

Brannon, C.A., 1981, Rochester Gulch, land acquisition and target evaluation, Lewis and Clark County, Montana, 7 miles southeast of Lincoln, MT: Anaconda Geological Documents Collection, International Archives of Economic Geology, American Heritage Center, Laramie, University of Wyoming, reference no. 30552, $5 \mathrm{p}$.

Braun, E.R., and Lange, I.M., 1984, Organic control of sandstone-hosted copper-silver mineralization in the Spokane Formation near Rogers Pass, western Montana, in McBane, J.D., and Garrison, P.B., eds., Northwest Montana and adjacent Canada: Montana Geological Society 1984 Field 
Conference and Symposium Guidebook, p. 305-313.

Brinker, W.F., 1944, Placer tin deposits north of Basin, Montana: Butte, Montana School of Mines, M.S. thesis.

Brox, G.B., and Guilbert, J., 1960, Report on Copper Hill (McVay) Copper Prospect, Lewis and Clark County, Montana: Anaconda Geological Documents Collection, International Archives of Economic Geology, American Heritage Center, Laramie, University of Wyoming, reference no. 30601A, 5 p.

Brox, G.B., and Potter, C.W., 1965, Supplementary report on Copper Hill (McVay) Copper Prospect, Lewis and Clark County, Montana: Anaconda Geological Documents Collection, International Archives of Economic Geology, American Heritage Center, Laramie, University of Wyoming, reference no. 30601B, 5 p.

Bushne11, Floyd, 1910, The Corbin district, Jefferson County, Montana: Engineering and Mining Journal, v. 89, p. 1148-1154.

Canyon Resources Corporation, 1993, McDonald gold project in Montana moves forward with final feasibility study: News Release, 1 p.

Chelini, J.M., 1965, Limestone, dolomite, and travertine in Montana: Montana Bureau of Mines and Geology Bulletin 44, 53 p.

1966, Some high-purity quartz deposits in Montana: Montana Bureau of Mines and Geology Bulletin 54, 43 p.

Clayton, J.E., 1888, The Drumlummon group of veins and their mode of formation (Helena, Montana): Engineering and Mining Journal, v. 46, p. 85-86, 106108.

Close, T.J., and Rigby, J.G., 1984, Mineral investigation of the Gates of the Mountains Wilderness Study Area, Lewis and Clark County, Montana: U.S. Bureau of Mines Open-File Report MLA 3-84, 20 p.

Connor, J.J., and McNeal, J.M., 1988, Geochemistry of mineralized quartzite beds in the Spokane Formation (Belt Supergroup), Rogers Pass area, Lewis and Clark County, Montana: U.S. Geological Survey Bulletin 1762, 17 p.

Crebs, 1981, Lincoln geophysical programs: Anaconda Geological Documents Collection, International Archives of Economic Geology, American Heritage Center, Laramie, University of Wyoming, reference no. 30552, 6 p.

Crowley, F.A., 1960, Directory of known mining enterprises, 1959: Montana Bureau of Mines and Geology Bulletin 14, $83 \mathrm{p}$.

1962a, Directory of known mining enterprises, 1961: Montana Bureau of Mines and Geology Bulletin 25, $71 \mathrm{p}$.

1962b, Phosphate rock: Montana Bureau of Mines and Geology Special Publication 25, $8 \mathrm{p}$.

DeMunck, V.C., 1956, Iron deposits in Montana: Montana Bureau of Mines and Geology Information Circular 13, $49 \mathrm{p}$.

DeMunck, V.C., and Ackerman, W.C., 1958, Barite deposits in Montana: Montana Bureau of Mines and Geology Information Circular 22, 30 p.

Dingman, O.A., 1932, Placer-mining possibilities in Montana: Montana Bureau of Mines and Geology Memoir 5, p. 20-28.

Du Bray, E.A., 1995, Geologic map of Cretaceous intrusive rocks in the central Big Belt Mountains, Broadwater and Meagher Counties, Montana: U.S. Geological Survey Miscellaneous Field Studies Map MF-2291, scale 1:50,000, with text.

Earhart, R.L., Grimes, D.J., Leinz, R.W., Marks, L.Y., and Peterson, D.L., 1977. Mineral resources of the proposed additions to the Scapegoat Wilderness, Powe 11 and Lewis and Clark Counties, Montana: U.S. Geological Survey Bulletin 1430, 62 p. 
Earhart, R.L., Mudge, M.R., Whipple, J.W., and Connor, J.J., 1981, Mineral resources of the Choteau $1^{\circ} \times 2^{\circ}$ quadrangle, Montana: U.S. Geological Survey Miscellaneous Field Studies Map MF-858-A, scale 1:250,000.

Efraimson, L.E., 1936, Geology of some gold deposits of Montana: Butte, Montana School of Mines, B.S. thesis, $36 \mathrm{p}$.

Elliott, J.E., Loen, J.S., Wise, K.K., and Blaskowski, M.J., 1992, Maps showing locations of mines and prospects in the Butte $1^{\circ} \times 2^{\circ}$ quadrangle, western Montana: U.S. Geological Survey Miscellaneous Investigations Series Map I-2050-C, scale 1:250,000, with pamphlet.

Emerson, D.0., and Wright, H.D., 1957, Secondary uranium minerals at W. Wilson mine in the Boulder batholith, Montana: American Mineralogist, v. 42, p. 222-239.

Enders, M.S., Bartlett, M.W., Coffin, G.C., Volberding, J.E., and Young, D.P., 1995, The discovery of the McDonald gold deposit, Lewis and Clark County, Montana: Society for Mining, Metallurgy, and Exploration, Inc., preprint no, 95-225, 9 p.

Engineering and Mining Journal, 1993, This month in mining--Montana: v. 194, no. 3, p. 18 .

Ferguson, J.A., 1908, Eleventh report of the Bureau of Agriculture, Labor, and Industry of the State of Montana: Helena, State of Montana, $320 \mathrm{p}$.

Foote, C.S., and Vandeveer, P.L., 1957, Report on the Humdinger Mine in Lewis and Clark County, Montana: Anaconda Geological Documents Collection, International Archives of Economic Geology, American Heritage Center, Laramie, University of Wyoming, reference no. $32819,3 \mathrm{p}$.

Forrester, J.D., 1942, A native copper deposit near Jefferson City, Montana: Economic Geology, v. 37, p. 126-135.

Foster, Fess, and Childs, J.F., 1993, An overview of significant lode gold systems in Montana, and their regional geologic setting: Exploration and Mining Geology, v. 2, no. 3, p. 217-244.

Freeman, V.L., Ruppe1, E.T., and Klepper, M.R., 1958, Geology of part of the Townsend Valley, Broadwater and Jefferson Counties, Montana: U.S. Geological Survey Bulletin 1042-N, p. 481-556.

Freshman, (1950), Report on the Eldorado mine, near Avon, Powe11 County, Montana: Anaconda Geological Documents Collection, International Archives of Economic Geology, American Heritage Center, Laramie, University of Wyoming, reference no. 30537, 5 p.

Frishman, D., Elliott, J.E., Foord, E.E., Pearson, R.C., and Raymond, W.H., 1992, Map showing the location of productive lode and placer gold mines in Montana: U.S. Geological Survey Mineral Resource Map MR-96, scale 1:500,000, pamphlet.

Geach, R.D., 1965, Directory of mining enterprises, 1964: Montana Bureau of Mines and Geology Bulletin 46.

1966, Directory of mining enterprises, 1965: Montana Bureau of Mines and Geology Bulletin 49, $87 \mathrm{p}$.

1967, Directory of mining enterprises, 1966: Montana Bureau of Mines and Geology Bulletin 58, $95 \mathrm{p}$.

Geach, R.D., and Chelini, J.M., 1963, Directory of known mining enterprises, 1962: Montana Bureau of Mines and Geology Bulletin 33, 84 p.

Gilbert, F.C., 1935, Directory of Montana mining properties: Montana Bureau of Mines and Geology Memoir 15, $99 \mathrm{p}$.

Goddard, C.C., 1940a, Report on the Chamounix Group of mines, Greenhorn Mining District near Austin, Lewis and Clark Co., Montana: Anaconda Geological 
Documents Collection, International Archives of Economic Geology, American Heritage Center, Laramie, University of Wyoming, reference no. 30533, 3 p. 1940b, Report on the Mike Horse mine, Heddleston Mining District, 19 miles east of Lincoln, Lewis and Clark County, Montana: Anaconda Geological Documents Collection, International Archives of Economic Geology, American Heritage Center, Laramie, University of Wyoming, reference no. $31106,7 \mathrm{p}$.

Goodale, C.W., 1914, The Drumlummon mine, Marysville, Montana: American Institute of Mining and Metallurgical Engineers, Transactions, v. 49, p. $258-276$.

Greenfield, M., 1936, Gold dredging operations near Helena, Montana: Mining Journa1, v. 20 , no. 14, p. $5,29$.

Gualtieri, J.L., 1975, Geochemical data for the Confederate Gulch area, Broadwater and Meagher Counties, Montana: U.S. Geological Survey OpenFile Report 75-212.

Gualtieri, J.L., unpublished field notes .

Hansen, Miller, 1971, Directory of mining enterprises, 1970: Montana Bureau of Mines and Geology Bulletin 82, $59 \mathrm{p}$.

Jarrard, L.D., 1957, Some occurrences of uranium and thorium in Montana, with sections on prospecting for radioactive minerals: Montana Bureau of Mines and Geology Miscellaneous Contribution 15, $90 \mathrm{p}$.

Johnson, E.A., 1973, Geology and gold deposits of the Confederate Gulch-White Gulch area, Broadwater County, Montana: Butte, Montana College of Mineral Science and Technology, M.S. thesis, 53 p.

Jones, Verner, 1934, Spring Hill gold deposit near Helena, Montana: Economic Geology, v. 29, p. 544-559.

Kauffman, A.J., Jr., 1952, Industrial minerals of the Pacific Northwest: U.S. Bureau of Mines Information Circular 7641, $75 \mathrm{p}$.

Klepper, M.R., Ruppe1, E.T., Freeman, V.L., and Weeks, R.A., 1971, Geology and mineral deposits, east flank of the Elkhorn Mountains, Broadwater County, Montana: U.S. Geological Survey Professional Paper 665, 66 p.

Klepper, M.W., Weeks, R.A., and Ruppe1, E.T., 1957, Geology of the southern Elkhorn Mountains, Jefferson and Broadwater Counties, Montana: U.S. Geological Survey Professional Paper 292, 82 p.

Knopf, Adolph, 1913, Ore deposits of the Helena mining region, Montana: U.S. Geological Survey Bulletin 527, $143 \mathrm{p}$.

Koschmann, A.H., and Bergendahl, M.H., 1968, Principal gold producing districts of the United States: U.S. Geological Survey Professional Paper 610, p. $142-171$.

Lange, I.M., and Eby, D.E., 1981, Stratabound copper-silver-bearing oolitic deposits in the Belt Supergroup of Montana: Economic Geology, v. 76, p. 933-936.

Lange, I.M., and Sherry, R.A., 1986, Nonmassive sulfide deposits in the Late Precambrian Supergroup of western Montana, in Roberts, S.M., ed., Belt Supergroup, A guide to Proterozoic rocks in western Montana and adjacent areas: Montana Bureau of Mines and Geology Special Paper 94, p. 269- 278.

Lange, I.M., Moore, J.N., and Braun, E.R., 1986, Geology and diagenetic aspects of the Canyon Creek sandstone and Wolf Creek carbonate-hosted copper-silver deposits of the Belt Supergroup, northwestern Montana, U.S.A., in Craig, J.R., and others, eds., Mineral paragenesis: Theophrastus Publications, Athens, Greece, p. 411-442.

Lawson, D.C., 1976, Directory of mining enterprises, 1975: Montana Bureau of 
Mines and Geology Bulletin 100, 63 p.

1979, Directory of mining enterprises for 1978: Montana Bureau of Mines and Geology Bulletin 109, $55 \mathrm{p}$.

1981, Directory of mining enterprises for 1980: Montana Bureau of Mines and Geology Bulletin 115, $53 \mathrm{p}$.

Lawson, D.C., Berg, R.B., and Sholes, M.A., 1987, Mining and mineral developments in Montana--1987: Montana Bureau of Mines and Geology Open File Report MBMG 196, 16 p.

Loen, J.S., 1989, Lode and placer deposits in the Ophir district, Powel1 and Lewis and Clark Counties, Montana: Fort Collins, Colorado State University, $\mathrm{Ph}$. D. thesis, $268 \mathrm{p}$.

Lorain, S.H., and Hundhause, R.J., 1948, Investigation of the Minah leadsilver mine, Jefferson County, Montana: U.S. Bureau of Mines Report of Investigations $4359,9 \mathrm{p}$.

Ludington, Steve, and Greenwood, W.R., 1990, Geological and geochemical evaluation, in Greenwood, W.R., Ludington, Steve, Miller, W.R., Hanna, W.F., Wenrich, K.J., Suits, V.J., and McHugh, J.B., Mineral resources of the E1khorn Wilderness study area, Broadwater and Jefferson Counties, Montana: U.S. Geological Survey Bulletin 1805, 37 p.

Lusty, Q.C., 1973, Geology and mineral deposits of the eastern part of the E11iston mining district, Lewis and Clark and Powe1l Counties, Montana: Golden, Colorado School of Mines, M.S. thesis, 93 p.

Lyden, C.J., 1948, The gold placers of Montana: Montana Bureau of Mines and Geology Memoir 26, 151 p.

Lytzen, W.W., 1919, Shipments of ore from the Julia mine, Powell Co., Montana: Anaconda Geological Documents Collection, International Archives of Economic Geology, American Heritage Center, Laramie, University of Wyoming, reference no. 33207, 14 p.

McClernan, H.G., 1976, Metallic mineral deposits of Powe1l County, Montana: Montana Bureau of Mines and Geology Bulletin 98, 69 p. 1980, Metallogenic map of the White Sulphur Springs quadrangle, central Montana: Montana Bureau of Mines and Geology Geologic Map 7, scale $1: 250,000$.

1983, Meta1lic mineral deposits of Lewis and Clark County, Montana: Montana Bureau of Mines and Geology Memoir 52, 73 p.

McCulloch, Robin, 1989, Mining and mineral developments in Montana--1989:

Montana Bureau of Mines and Geology Open-File Report 223, $42 \mathrm{p}$.

1992, Montana mining directory 1991: Montana Bureau of Mines and Geology Bulletin 130, $135 \mathrm{p}$.

1993a, Mining and mineral developments in Montana--1992: Montana Bureau of Mines and Geology Open-File Report 262, $30 \mathrm{p}$.

1993b, Montana mining directory 1992: Montana Bureau of Mines and Geology Bulletin 131, 76 p.

McDermott, Walter, 1914, The Penobscot and Belmont mines: American Institute of Mining and Metallurgical Engineers Bulletin 92, p. 2113-2116.

McDonald, A.M., 1931, Report on Eula-Homestake mine, Lewis and Clark County, Montana: Anaconda Geological Documents Collection, International Archives of Economic Geology, American Heritage Center, Laramie, University of Wyoming, reference no. 30519, 5 p.

McKee, J., 1978, Petrology, alteration, and mineralization of the Poorman Creek-Silver Bell stock porphyry Cu-Mo deposit: Missoula, University of Montana, M.S. thesis, 97 p. 
Mertie, J.B., Fisher, R.P., and Hobbs, S.W., 1951, Geology of the Canyon Ferry quadrangle, Montana: U.S. Geological Survey Bulletin 972, 97 p.

Miller, R.N., Shea, E.P., Goddard, C.C., Jr., Potter, C.W., and Brox, G.W., 1973, Geology of the Heddleston copper-molybdenum deposit, Lewis and Clark County, Montana: American Institute of Mining, Metallurgical, and Petroleum Engineers, Pacific Northwest Metals and Minerals Conference, Coeur d'Alene, Idaho, $33 \mathrm{p}$.

Mudge, M.R., Earhart, R.L., Watts, K.C., Jr., Tuchek, E.T., and Rice, W.L., 1974, Mineral resources of the Scapegoat Wilderness, Powell and Lewis and Clark Counties, Montana, with a section on Geophysical surveys, by D.L. Peterson: U.S. Geological Survey Bulletin 1385-B, 82 p.

Pardee, J.T., and Schrader, F.C., 1933, Metalliferous deposits of the Greater Helena Mining Region, Montana: U.S. Geological Survey Bulletin 842, 318 p.

Parker, G.W., 1968, Progress Report, Heddleston Project, January: Anaconda Geological Documents Collection, International Archives of Economic Geology, American Heritage Center, Laramie, University of Wyoming, reference no. 26706,2 p.

Pegasus Gold Inc., 1992, Annual Report, 54 p.

Phelps Dodge Corporation, 1991, Annual Report, p. 14-15.

Popoff, C.C., and Service, A.L., 1965, An evaluation of the western phosphate industry and its resources (part 2--Montana): U.S. Bureau of Mines Report of Investigations $6611,146 \mathrm{p}$.

Potter, C.W., 1971, Summary of Heddleston District work by The Anaconda Company from May 1961 to date: Anaconda Geological Documents Collection, International Archives of Economic Geology, American Heritage Center, Laramie, University of Wyoming, reference no. 31028, 2 p.

Rankin, J.W., and Whitcomb, C.W., 1971, Report on Hayworth Claim Group, section 27, T. 16 N., R. 7 W., Lewis and Clark County, Montana: Anaconda Geological Documents Collection, International Archives of Economic Geology, American Heritage Center, Laramie, University of Wyoming, reference no. 31105,8 p.

Reed, G.C., 1951, Mines and mineral deposits (except fuels), Broadwater County, Montana: U.S. Bureau of Mines Information Circular 7592, 62 p.

Regnier, J., 1951, Mineralogy and paragenesis of the eastern part of the Elliston mining district, Montana: Butte, Montana College of Mineral Science and Technology, M.S. thesis.

Renouard, E., and Shea, E.P., 1935, Report on the New Victory Mining Company, Avon, Powe11 County, Montana: Anaconda Geological Documents Collection, International Archives of Economic Geology, American Heritage Center, Laramie, University of Wyoming, reference no. 33207, 5 p.

Reyner, M.L., and Trauerman, C.J., 1949, Directory of mining properties: Montana Bureau of Mines and Geology Memoir 31, $125 \mathrm{p}$.

Roberts, W.A., 1953, Geology of the area adjacent to the Free Enterprise Mine, Jefferson County, Montana: U.S. Geological Survey Bulletin 988-G, p. 143148.

Roberts, W.A., and Gude, A.J., 3d, 1951, Uranium-bearing deposits west of Clancy, Jefferson County, Montana: U.S. Geological Survey Bulletin 988-F, p. $69-87$.

Robertson, F., 1956, Geology and mineral deposits of the Elliston mining district, Powe11 County, Montana: Seattle, University of Washington, Ph. D. dissertation, $332 \mathrm{p}$. 
Roby, R.N., 1950, Mines and mineral deposits (except fuels), Meagher County, Montana: U.S. Bureau of Mines Information Circular 7540, 43 p.

Roby, R.N., Ackerman, W.C., Fulkerson, F.B., and Crowley, F.A., 1960, Mines and mineral deposits (except fuels), Jefferson County, Montana: Montana Bureau of Mines Bulletin 16, 122 p.

Rocky Mountain Pay Dirt, 1987, Sunshine to develop Big Blackfoot gold mine in Montana, November, p. 12A.

1988, Pegasus Gold posts big gains in precious metals output: April, p. 17A.

1989a, Pegasus Gold reports higher production, net in 1988: February, p. $17 \mathrm{~A}$.

1989b, of mines and men: March, p. 12A.

1989c Pegasus working to overcome Montana Tunnels problems: May, p. $16 \mathrm{~A}$.

1995a, Write-downs, exploration costs mean more red ink at Pegasus:

February, p. 17A.

1995b, Pegasus says reserves up 49 percent to 7.8 million ounces: August, P. $8 \mathrm{~A}$.

Rosenkranz, T.H., and Lyden, J.P., 1920, Report on Mineral Hill Mining District,

Powell County, Montana: Anaconda Geological Documents Collection, International Archives of Economic Geology, American Heritage Center, Laramie, University of Wyoming, reference no. 31023, $10 \mathrm{p}$.

Ross, C.P., 1950, Fluorspar prospects in Montana, 1950: U.S. Geological Survey Bulletin 955-E, p. 173-224.

Rostad, O.H., 1969, The use of geochemistry at the Bald Butte molybdenite prospect, Lewis and Clark County, Montana: Quarterly of the Colorado School of Mines, v. 64, no. 1, p. 437-449.

Rowe, J.P., 1908, Some economic geology of Montana: University of Montana, Bulletin no. 50, Geological Series No. 3, p. 54-56.

Ruppel, E.T., 1963, Geology of the Basin Quadrangle, Jefferson, Lewis and Clark, and Powel1 Counties, Montana: U.S. Geological Survey Bulletin $1151,121 \mathrm{p}$.

Sahinen, U.M., 1962, Fluorspar deposits in Montana: Montana Bureau of Mines and Geology Bulletin 28, $38 \mathrm{p}$.

Sales, R.H., 1918, Quigley molybdenum mine, unpublished field notes, Anaconda Company: Anaconda Geological Documents Collection, International Archives of Economic Geology, American Heritage Center, Laramie, University of Wyoming, reference no. $32620,1 \mathrm{p}$.

Schassberger, H.T., 1971, Heddleston K/Ar date and aeromag: AMAX Exploration, Inc., unpublished report, $2 \mathrm{p}$.

Schell, E.M., 1963, Ore deposits of the northern part of the Park (Indian Creek) district, Broadwater County, Montana: Montana Bureau of Mines and Geology Bulletin 35, $47 \mathrm{p}$.

Schmidt, R.G., Loen, J.S., Wallace, C.A., and Mehnert, H.H., 1994, Geology of the Elliston region, Powell and Lewis and Clark Counties, Montana: U.S. Geological Survey Bulletin 2045, 25 p.

Sillitoe, R.H., Grauberger, G.L., and Elliott, J.E., 1985, A diatreme-hosted gold deposit at Montana Tunnels, Montana: Economic Geology, v. 80, p. 1707-1721.

Sizer, F.L., 1914, The Empire mine: American Institute of Mining and Metallurgical Engineers Bulletin 92, p. 2116-2120.

Smedes, H.W., 1966, Geology and igneous petrology of the northern E1khorn 
Mountains, Jefferson and Broadwater Counties, Montana: U.S. Geological Survey Professional Paper 510, 116 p.

Stone, Jerome, 1952, The geology of the Elliston phosphate district, Powell County, Montana: Missoula, University of Montana, M.A. thesis.

Stone, R.W., 1911, Geologic relations of ore deposits in the E1khorn Mountains, Montana: U.S. Geological Survey Bulletin 470-B, p. 75-98.

Stout, K., 1949, Geology and mines of the Ogden Mountain Mining District, Powell County, Montana: Butte, Montana School of Mines, M.S. thesis, 56 p.

Stout, K.S., and Ackerman, W.C., 1959, Directory of known mining enterprises, 1958: Montana Bureau of Mines and Geology Bulletin 10, 80 p.

Swanson, R.W., 1973, Geology and phosphate deposits of the Permian rocks in central western Montana: U.S. Geological Survey Professional Paper 313-F, p. $779-833$.

Trammel, J.W., 1975, Strata-bound copper mineralization in the Empire Formation and Ravalli Group, Belt Supergroup, northwest Montana: Seattle, University of Washington, Ph. D. dissertation, $70 \mathrm{p}$.

Trauerman, C.J., and Waldron, C.R., 1940, Directory of mining properties: Montana Bureau of Mines and Geology Memoir 20, 135 p.

Union Carbide Corporation, 1981, unpublished company report and data.

U.S. Forest Service, Mineral Resource Files, Missoula, Montana, 1981-1983. 1991, Notice of Intent and Plan of Operation files obtained from Helena National Forest files.

U.S. Geological Survey, 1885-1923, Mineral resources of the United States [a series]: U.S. Bureau of Mines, 1924-1983, Mineral resources of the United States, and Minerals Yearbook [a series]: Washington, D.C., U.S. Government Printing office.

1988, Mineral resources of the United States, and Minerals Yearbook [a series]: Washington, D.C., U.S. Government Printing Office.

Walker, D.D., 1963, Tungsten resources of western Montana--Miscellaneous deposits: U.S. Bureau of Mines Report of Investigations 6334, $60 \mathrm{p}$.

Weed, W.H., 1903, Gold mines of the Marysville district, Montana: U.S. Geological Survey Bulletin 213, p. 88-89.

Whiteley, S.J., 1982, Chessman Reservoir Submittal--1982: Anaconda Geological Documents Collection, International Archives of Economic Geology, American Heritage Center, Laramie, University of Wyoming, reference no. 305.49, 4 p.

Winchel1, H.V., and Winchell, A.N., 1912, Notes on the Blue Bird mine [Wickes, Montana]: Economic Geology, v. 7, p. 287-294.

Young, F.M., Crowley, F.A., and Sahinen, U.M., 1962, Marketing problems of sma11 business enterprises engaged in lead and zinc mining: Montana Bureau of Mines and Geology Bulletin 30, 58 p. 


\section{C $\mathbf{H} \mathbf{A} \mathbf{T} \mathbf{R}$}

\section{MIMERAL REBOURCE GEOLOGY OF MIMING DI8TRICT8/AREAB}

\section{INTRODUCTION}

The mineral resource geology of the Helena National Forest (the Forest) is described by mining districts/areas that host, or potentially host, mineral deposits. The districts/areas are grouped by regions, which generally correspond with geographic divisions of the Forest. From northwest to southeast, the regions are Lincoln, Continental Divide, Elkhorn Mountains, and Big Belt Mountains (fig. F1). These regional divisions are used because they generally define contiguous geologic terranes and associated patterns of mineralization, facilitating the description of mining districts/areas and mineral deposits. Because of complex geology and diverse types of mineralization, the Continental Divide region is divided further, into three subregions: Granite Butte-Ogden Mountain, Black Mountain-Stemwinder Hil1, and North Boulder Mountains (fig. F1).

Most mineral deposits in the Continental Divide and Elkhorn regions are genetically and spatially related to granitic intrusions and extrusive to intrusive volcanic rocks. The Lincoln and Big Belt Mountains regions contain these same associations, but in addition contain stratabound mineral occurrences. The distribution of these stratabound occurrences, which are areally extensive tabular-shaped bodies, is determined partly by sedimentological properties of the host formations. The stratabound deposits do not fit neatly into the district/area classification scheme generally used in this chapter because the host formations, which are in the Middle Proterozoic Belt Supergroup, extend throughout the Lincoln and Big Belt Mountains regions, well beyond district/area boundaries. Hence, the stratabound occurrences are described by deposit type, and deposits within a district/area are discussed under the appropriate deposittype heading.

This chapter is arranged to reflect the genetic relationships of the deposits. The geology and mineral deposits of the Lincoln and Big Belt Mountains regions, which contain most of the genetically related stratabound deposits, are described first, followed by the Continental Divide and Elkhorn Mountains regions. 


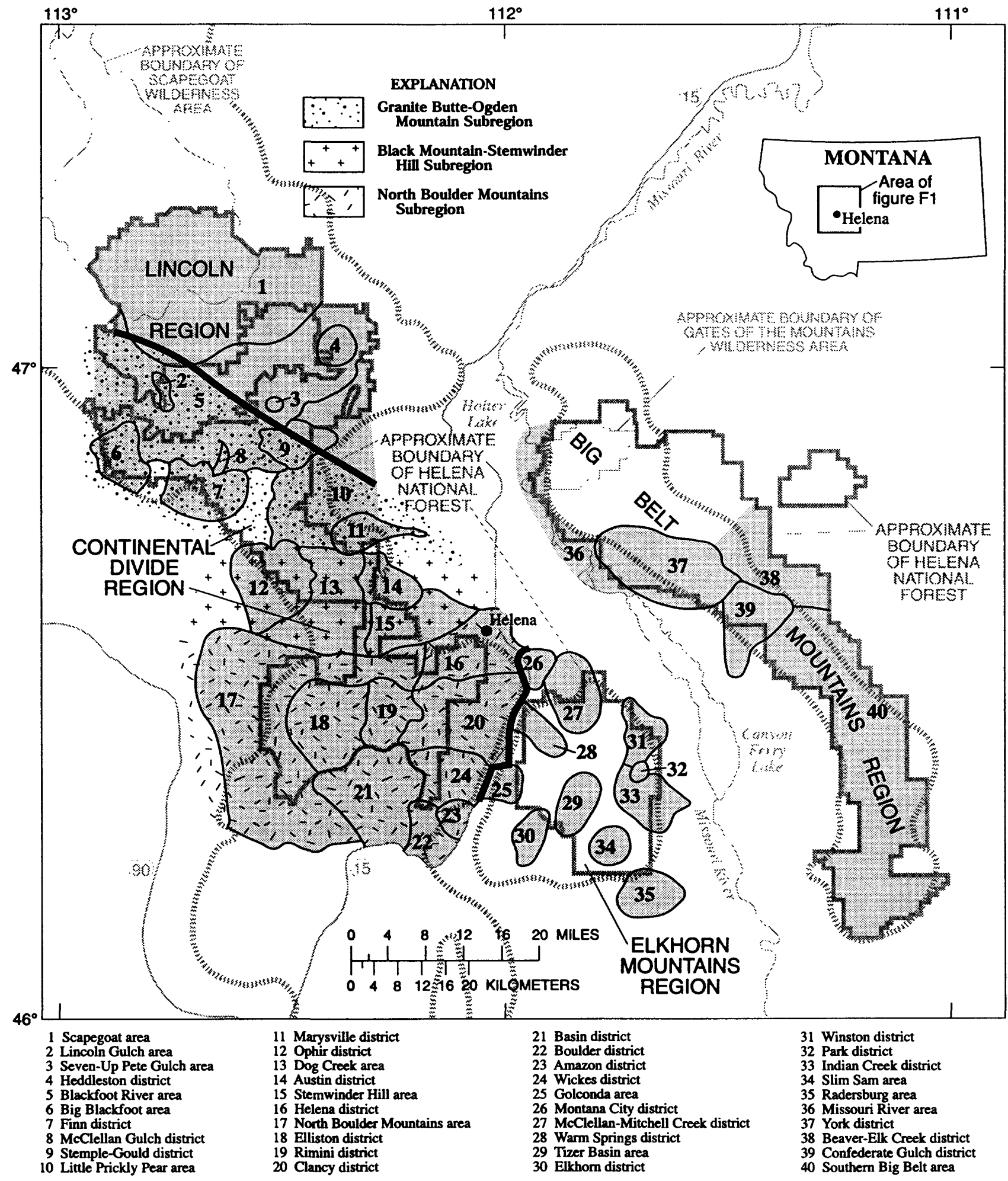

Figure F1. Map showing regions, subregions, and mining districts/areas of Helena National Forest. 


\section{LINCOLN REGION}

By R.G. Tysdal, J.E. Elliott, E.A. du Bray, and J.W. Whipple

The Lincoln Region lies north of the St. Mary-Helena Valley fault in the northwestern part of the Forest (fig. F1). It mainly includes rocks within the southeastern part of the Choteau $1^{\circ} \times 2^{\circ}$ quadrangle (fig. A2). The region hosts the Heddleston mining district and the recently discovered gold deposit at McDonald Meadows.

\section{Blackfoot River Area}

\section{Geologic setting}

The Blackfoot River area is adjacent to and includes parts of the Forest in the vicinity of Lincoln ( 1 1. 2, map B), and contains parts of both the Lincoln and Continental Divide regions (fig. F1). The principal rock types are sedimentary rocks of the Middle Proterozoic Belt Supergroup: the Spokane and Empire Formations of the Ravalli Group; the Helena Formation; and the Snowslip, Mount Shields, and Shepard Formations of the Missoula Group (pl. 1, map A). Broad, open folds and steeply dipping normal, reverse, and strike-slip faults are dominant structures. In the eastern part of the area the Proterozoic sedimentary rocks have been intruded and covered by Tertiary andesitic and rhyolitic volcanic rocks. Several granodiorite plutons (Tertiary and Cretaceous) are in the southern part of the area.

\section{Minera1 Deposits}

Mineral deposits of the Blackfoot River area historically produced metals from gold placers and polymetallic veins that yielded small quantities of gold, silver, copper, and lead. The area is a relatively small producer of metals, mainly gold, and the estimated total value of production for the area is about $\$ 27,000$ (Elliott and others, 1992). However, the area also contains the undeveloped McDonald Meadows gold deposit (described below), which contains several million ounces of gold.

Mineral deposits of the Blackfoot River area are spatially associated with igneous rocks. The associations are shown by geophysical studies of the Butte $1^{\circ} \times 2^{\circ}$ quadrangle (Hanna and others, 1994), which indicate several magnetic and gravity anomalies (figs. $\mathrm{Cl}, \mathrm{C} 4$ ). A magnetic high in the western part of the Blackfoot River area is associated with a small granodiorite stock near Mineral Hill. The terrace-magnetization pattern (fig. C4, anomaly 4) suggests a much larger subsurface extent than the small surface exposure indicates (Hanna and others, 1994; p1. 1, map A). Two magnetic highs along (figs. C1, C4, anomalies 3 and 6) the south and southeast borders of the Blackfoot River area are associated with the Dalton Mountain and Silver Bell (Stemple Pass) stocks, respectively. The Dalton Mountain stock (figs. $\mathrm{Cl}, \mathrm{C4}$, anomaly 3) continues into the adjacent Finn and McClellan Gulch districts. The magnetization pattern suggests that the stock extends to the south in the subsurface (Hanna and others, 1994), an interpretation confirmed by McCafferty (Chapter C of this report, fig. 
C4). The Silver Bell stock extends into the adjacent stemple-Gould district. The magnetic pattern for this stock suggests that its subsurface extent is two to three times larger than the surface extent (Hanna and others, 1994). A gravity low may be associated with the subsurface extent of the Silver Bell stock (Hanna and others, 1994). Another magnetic high along the eastern border of the Blackfoot River area may be related to part of the Lincoln volcanic field, composed of andesitic and rhyolitic rocks (Hanna and others, 1994); it may also be due to the presence of relatively thick low-density volcanic and volcaniclastic rocks of the Lincoln volcanic field.

Geochemical anomalies in arsenic, silver (fig. D13), gold (fig. D12), barium, bismuth (fig. D15), copper (fig. D16), iron (fig. D20), molybdenum (fig. D17), manganese (fig. D21), lead (fig. D14), antimony, tin, tungsten, and zinc (fig. D19) are present in the Blackfoot River area. The geochemicalanomalies in these figures (Alminas, Chapter D of this report) are for stream sediments and incorporate only the stream sediment dat of McDanal and others (1985).

\section{McDonald gold deposit}

The recently discovered McDonald deposit (p1. 2, no. 53) contains 8.2 million ounces of gold in 414 million tons of rock (Denver Post, 1994). It is within the Blackfoot River area, outside the Forest. It may be genetically related to epithermal veins in the Seven-Up Pete Gulch area to the south. The McDonald deposit, in rocks of the Tertiary Lincoln volcanic field, has many characteristics of a hot-spring gold-silver deposit. As described by Enders and others (1995), the deposit is within the upper part of the volcanic succession and is hosted by a 1,500-ft-thick sequence of rhyolite ash-flow tuffs that are underlain by a sequence of andesitic volcanic and volcaniclastic rocks. Approximately 90 percent of the mineralized rock is in the lower lithic-rich portion of the rhyolite tuffs. This lithic tuff is up to $1,200 \mathrm{ft}$ thick. An overlying crystal-rich tuff, up to $350 \mathrm{ft}$ thick, contains only about 7 percent of the deposit and an underlying sequence of andesitic flows and volcaniclastic rocks contains the remainder of the mineralized rock. Volcaniclastic rocks overlying the deposit contain depositional siliceous sinters with anomalous concentrations of trace elements that are primary indicators of the underlying mineral deposit.

The geometry of the mineralized zone is controlled by permeable zones in tuffaceous rocks and by steeply dipping, intersecting vein sets. The mineralized zone is nearly tabular and stratiform, dips $20^{\circ}$ to $25^{\circ}$ to the north, and has an average thickness of about $450 \mathrm{ft}$. Many of the vein sets may have been controlled by pre-mineral faults. The deposit is cut by two high-angle faults that have strikes of N. $60 \mathrm{E}$. and N. $15 \mathrm{~W}$. Many mineralized veins are parallel to the north-northwest (N. $15 \mathrm{~W}$.) fault and with west-northwest striking faults. The $\mathrm{N}$. $60 \mathrm{E}$. fault is probably a post-mineral structure.

Gold- and silver-bearing minerals and associated gangue minerals occur in veins and, to a lesser extent, are disseminated into the wall rock. Most of the gold occurs as electrum with a gold:silver ratio of about 56:44. Other ore and gangue minerals are native gold, acanthite, quartz, chalcedony, calcite, adularia, pyrite, marcasite, specular hematite, goethite, jarosite, and cryptomelane. Silicification and quartz-adularia are the main forms of alteration. Argillic alteration forms a halo around the mineralized area. 


\section{Seven-Up Pete Gulch Area}

\section{Geologic Setting and Mineral Deposits}

The Seven-Up Pete Gulch area (fig. F1; p1. 2, map B), 8 miles east of Lincoln, is a mineralized intrusive-extrusive volcanic center that was a probable source of much of the surrounding Tertiary andesitic and rhyolitic volcanic rocks (p1. 1, map A). Sulfide-bearing quartz veins are along shear and breccia zones in andesite. The area has produced moderate quantities of gold and silver. Recent exploration has indicated approximately 10 million short tons of mineralized rock with an average grade of 0.06 ounces of gold per ton, mainly in the vicinity of the Columbia mine ( $\mathrm{pl}$. 2, map B; Rocky Mountain Pay Dirt, 1991). The mineralization could be genetically related to that of the McDonald deposit, approximately $3 \mathrm{mi}$ to the north. The andesitic volcanic rocks that host the Seven-Up Pete mineralization are found to correlate with those that underlie the rhyolitic volcanic rocks of the McDonald deposit. Thus, the epithermal veins in the Seven-Up Pete Gulch area probably formed at a lower stratigraphic level than the McDonald deposit. The area is a small producer of metals with an estimated total value of production of about $\$ 25,000$ (E11iott and others, 1992). The results of a geochemical survey (McDanal and others, 1985) indicate geochemical anomalies for silver and gold in this relatively small area.

\section{Heddleston District}

\section{Geologic Setting}

The Heddleston district, 14 miles northeast of Lincoln (fig. A2, p1. 2), is largely underlain by quartzite (metasandstone) and siltite of the Middle Proterozoic Spokane and Empire Formations, and hornblende diorite and gabbro sil1-1ike intrusive masses of Late Proterozoic age (p1. 1). Multiple Tertiary felsic igneous bodies intrude the Proterozoic rocks. Miller and others (1973), from whose work the following descriptive material is derived, described the intrusive rocks as a series of feldspar porphyries, related breccia intrusions, and quartz porphyry. The oldest and largest is a steep-sided intrusion that is composed of quartz monzonite porphyry. Numerous dikes of the same rock type extend north and south of the main mass. Large phenocrysts of potassium feldspar, plagioclase, biotite, and rounded quartz in an aplitic groundmass are characteristic of the quartz monzonite porphyry. Miller and others (1973) reported that the intrusion is cut by zones of complex brecciation.

The granitic intrusions were believed by Miller and others (1973) to be younger than those of the Boulder Batholith (emplaced 78-70 Ma); Amax Exploration determined a middle Eocene $\mathrm{K}-\mathrm{Ar}$ age of $44.5 \pm 1.2 \mathrm{Ma}$ for sericite from altered rock.

Two prominent fault systems, an older N. 20-40 E.-striking set and a younger $\mathrm{N}$. 50-70 W. set, offset rocks in the district. Both fault systems contain mineralized rock, but the northeast-striking set seems to have influenced intrusion emplacement, whereas the vein deposits seem to have been controlled by the northwest-striking set (Miller and others, 1973). 


\section{Mineral Deposits}

The first ore deposits identified in the Heddleston district were large, supergene-enriched mineralized shear zones and veins. These were first exploited for gold and subsequently for their base metals. Known mines in the Heddleston district include the Anaconda (p1. 2, no. 40), Bobby Boy (no. 37), Calliope (no. 31), Carbonate (no. 32), Consolation (no. 35), Midnight (no. 33), Mike Horse (no. 43), Paymaster (no. 38), and Rex Beach (no. 34), all of which are inactive (Earhart and others, 1981). By 1930, lodes had produced about $\$ 2,000,000$ worth of gold, silver, lead, zinc, and copper. Additional ore was intermittently produced, principally from three major veins in the Mike Horse Mine, between 1930 and 1964 , by which time total production from the district was estimated at $\$ 25,000,000$.

During the period 1962-1970 the Anaconda Company conducted a full-scale exploration and development project that resulted in a fairly complete understanding of the geology and resource potential of the Heddleston district. Exploration and preliminary development work by Anaconda identified several significant porphyry copper-molybdenum prospects that are amenable to open-pit mining, but have not been developed. Early prospecting in the area led to smallscale mining of base and precious metals from vein systems that are probably related to the larger Heddleston copper-molybdenum deposit.

Mineralized rock in the Heddleston district is spatially and genetically associated with the numerous intrusions of quartz monzonite porphyry and quartz porphyry. As a group, the intrusions are responsible for the primary chalcopyrite-molybdenite deposits in the district and for extensive host rock hydrothermal alteration. Some of the most strongly mineralized rock is associated with the zones of complex autobrecciation, described above (Miller and others, 1973).

Several stages of quartz-chalcopyrite-molybdenite cut the main quartz monzonite porphyry of the Heddleston district. Important vein deposits containing galena, sphalerite, chalcopyrite, bornite, and tetrahedrite are spatially and genetically associated with copper-molybdenum deposits. Chalcopyrite and molybdenite are also broadly disseminated throughout the main quartz monzonite porphyry but their distributions are not well understood.

Much of the rock in the Heddleston district, including an area about 9000 ft long and $2000 \mathrm{ft}$ wide, has been affected by sericitic and pyritic alteration that broadly grades out to propylitic alteration. Weak alteration has resulted in zoning patterns that are not particularly well developed in this area. Alteration is widespread and affects the intrusive rocks as well as their host rocks. Supergene enrichment zones contain secondary chalcocite and covellite whose distribution appears to be controlled by steeply-dipping, premineralization structures. Miller and others (1973) stated that no extensive supergene copper enrichment blanket, such as those associated with many other copper-molybdenum deposits, has been identified. Much of the rock affected by supergene processes has been converted to montmorillonite.

The Kleinschmidt breccia pipe, in the southern part of the district, contains angular fragments of quartzite, siltite, quartz monzonite porphyry and highly altered quartz porphyry. The pipe is about $250 \mathrm{ft}$ wide and 400-500 ft long and contains abundant pyrite and minor chalcopyrite. A $600 \mathrm{ft}$ by $300 \mathrm{ft}$ intrusive breccia in the north part of the district, near the Midnight mine (no. 33 ), is one of the most strongly mineralized areas of the district. 


\section{Scapegoat Area}

\section{Geologic Setting}

The Scapegoat area, north and east of Lincoln (fig. F1, p1. 2), is mainly underlain by strata of the Middle Proterozoic Belt Supergroup. Except for the Newland Formation, all Belt formations present in the Forest are exposed in the Scapegoat area. The sequence is largely composed of clastic rocks that range in grain size from argilite to metasandstone. The Helena Formation, near the middle of the sequence, is comprised of dolomitic limestone, calcareous metasandstone, and siltite. These rocks locally are intruded by sills of Late Proterozoic diorite or gabbro. Paleozoic limestone and dolomite of Cambrian to Mississippian age overlie the Proterozoic strata locally in the northernmost part of the Scapegoat area.

\section{Mineral Deposits}

The Lincoln region (and the Big Belt Mountains region) contains mineral deposits that are stratabound. The mineralized rocks are described by deposit type, and deposits within a district/area are discussed under the appropriate deposit-type heading. Stratabound deposits of the Forest are present within some formations of the Middle Proterozoic Belt Supergroup.

\section{Sediment-hosted copper}

The main mineral deposits in the Scapegoat area are stratabound sedimenthosted copper-silver deposits within the Belt Supergroup. In Montana and northern Idaho, these deposits have been subdivided into greenbed, quartzite (metasandstone) or Revett, and carbonate deposits (Harrison, 1972, 1974; Lange and Sherry, 1986). Classification of sediment-hosted copper deposits is undergoing change and in this report are classified as follows, per written communication with D.A. Lindsey of the USGS:

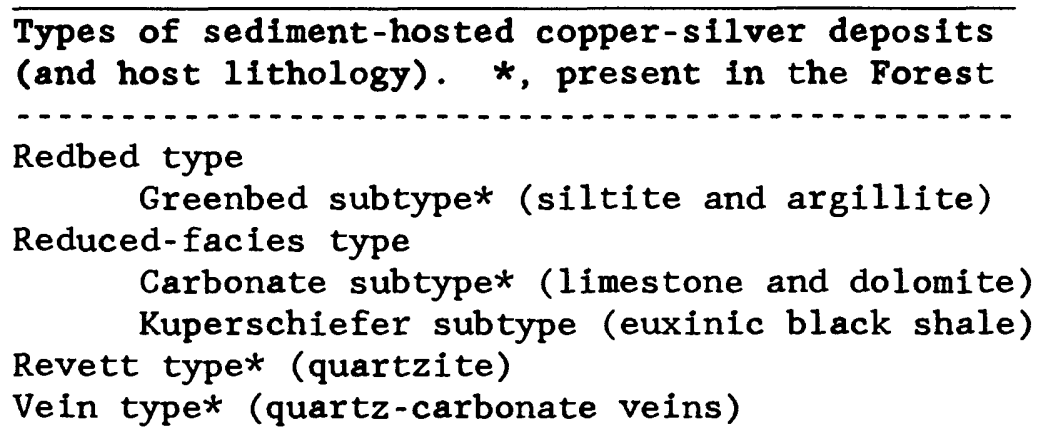

Greenbed subtype, Revett type, and carbonate subtype mineralized zones typically consist of sma11, disseminated mineral occurrences characterized by low 
tonnages and grades. The greenbed subtype and Revett type form mainly in localized, chemically reduced (green and gray) beds within sequences of oxidized (red) strata. The carbonate subtype also occurs within a chemically reduced environment but not necessarily within a redbed sequence. Vein type deposits consist of quartz-carbonate veins that are enriched in copper and silver during remobilization of these elements from nearby or contiguous stratabound greenbed, carbonate, and Revett occurrences. Geologic factors that control the distribution of the greenbed, Revett, and carbonate deposits also are directly related to distribution of the vein deposits. Mineralization processes that formed the stratabound copper-silver deposits of the Belt Supergroup are dependent on stratigraphic and sedimentologic properties of the host strata.

Redbed type, greenbed subtype. Green beds that contain copper - and silverbearing minerals are present in many of the Belt formations in the Forest--upper part of the Greyson, Spokane, Empire, Snowslip, Mount Shields, and McNamara Formations. The Spokane green beds contain most of the mineralized zones, according to Earhart and others (1981), who reported as many as 16 mineralized greenbed argillite and siltite sequences that are as thick as $60 \mathrm{ft}$ in the upper part of the Spokane. The middle part of the Spokane lacks green strata and the lower part contains some green beds that are sparsely mineralized. The copper and silver minerals commonly are near the middle of the greenbed units, within green to olive-gray argillite and siltite that are interbedded with red beds. In siltite, and locally very fine grained quartzite, concentration of minerals is in the coarser laminae at the base of beds. In argillite, the minerals are in discordant structures such as shrinkage cracks or along coarser grained laminae.

Where mineralized siltite or quartzite beds are more than 1-2 in. thick, they generally are assigned to the quartzite subtype of redbed deposit. Beds of quartzite 1-3 ft thick within greenbed sequences were classified as greenbed occurrences by Mudge and others (1974) and Earhart and others (1977, 1981), a time when classification of deposit types was still being determined. Such beds here are considered Revett type occurrences, discussed below.

The Empire Formation, above the Spokane, is mostly a greenbed unit and locally contains zones about $3 \mathrm{ft}$ thick of stratabound copper-silver-bearing zones, mainly in the middle and lower parts of the formation, but locally in the upper part within the Forest (Earhart and others, 1981). Some of these zones consist of mineralized quartzite beds and here are classified as the Revett type. Green beds of argillite and siltite are present throughout the Snowslip Formation, but mineralized zones typically are lenticular and difficult to trace for more than a few feet. Most mineralized zones in green beds of the McNamara Formation are discontinuous and are considered to have a low potential for mineral resources (Earhart and others, 1981).

Revett type. Quartzites of the Spokane Formation within the Scapegoat area are white, buff, gray, or pale-green fine-grained, noncalcareous, vitreous, feldspathic metasandstones (Whipple, 1980). The beds or lenses range up to about $7 \mathrm{ft}$ thick and occur within sequences of both red and green siltite and argillite. The mineralized quartzites of the Spokane have a higher potential for mineral resources than those in the younger formations of the Belt Supergroup because they are the thickest, most laterally extensive, and of the highest grade in copper and silver. Connor and McNeal (1988) reported that quartzites hosting copper- and silver-bearing minerals generally contain low but anonalous 
concentrations of mercury and barium as well.

On Green Mountain, northeast of Alice Creek (p1. 2, no. 19), quartzite beds occur through as much as $1,000 \mathrm{ft}$ of the middle part of the Spokane Formation (Tramme1, 1975; Earhart and others, 1981). Based on outcrop sampling, Connor and McNeal (1988) calculated that these quartzite lenses could contain more than $700,000 \mathrm{oz}$ of silver, mostly in two bodies near the crest of the mountain. Exploratory drilling of mineralized quartzite beds in this area was done in the 1970s by Bear Creek Mining Company (Whipple, 1980).

The Alice Creek mine ( $\mathrm{pl}$. 2, no. 19) is a small, high-grade, silver deposit that was mined from quartzite of the Spokane Formation in the late 1970's. Connor and McNeal (1988) stated that it has silver values that range up to 280 ounces per ton, but copper concentrations are no richer than those in other mineralized quartzite lenses. The exceptionally high silver content, along with high values of lead and mercury, led them to suggest that the deposit may reflect a hydrothermal source related to an inferred, concealed intrusive. The aeromagnetic data in this area of low resolution (fig. C2) and cannot be used to determine if hydrothermal alteration exists (A.E. McCafferty, oral commun., 1994). No other evidence of hydrothermal alteration exists, however. Neither the magnetic nor gravity data suggest a concealed intrusion in this area.

The Mount Shields Formation contains mineralized quartzites, in its middle and upper parts, in lenses within green beds of argillite and siltite. Quartzite beds similarly occur within the Empire and Snowslip Formations. Many of the quartzite occurrences were considered greenbed subtype deposits by Mudge and others (1974) and Earhart and others (1981), but here are assigned to the Revett type because the thickness of the quartzite beds exceeds 1-2 in., and commonly are in the $1 \mathrm{ft}$ range. Most of these quartzites are thinner than those at Green Mountain, although Earhart and others (1981) reported one 10-ft-thick mineralized quartzite in the Empire Formation, near the Copper Camp mine (p1. 2, no. 9). They stated that it averages 0.12 percent copper and about 0.25 ounces of silver per ton.

Reduced facies, carbonate subtype. Mineralization in this subtype is present in limestone and in calcareous metasandstone (quartzite) in the Scapegoat area. Limestone-hosted stratabound mineralization is known only from Red Mountain ( 1 1. 2, no. 8), in a clastic limestone of the basal part of the Helena Formation. The occurrence is a 2-7 ft thick mineralized zone that has a strike length of about $525 \mathrm{ft}$. The main copper-bearing mineral is chalcopyrite (Lange and Eby, 1981; Lange and Sherry, 1986).

Mineralized calcareous metasandstone is widely dispersed in the Scapegoat area, in basal strata of the Helena Formation. The mineralized rock contains disseminated minerals of lead and zinc, and less commonly copper and silver. Earhart and others (1977) reported that mineralized zones are planar to lenticular, as thick as $7 \mathrm{ft}$ and $200 \mathrm{ft}$ long. At Landers Fork (p1. 2, 1oc. 16), an $11.5 \mathrm{ft}$ wide zone of structurally thickened rock yielded values of 0.5 percent copper and 0.2 ounces per ton silver (Earhart and others, 1981).

Vein type.--Quartz-carbonate veins that are enriched in copper and silver are present at several places in the Scapegoat area of the Forest. The veins occur in the same Belt formations that host stratabound mineral occurrences of the greenbed subtype and Revett type. Such occurrences are believed to be metals of the stratabound Belt deposits that have been remobilized into veins. The veins and vein systems occupy small faults and shear zones and are most common 
in the Spokane, Empire, and Helena Formations. They also are present in the Late Proterozoic diorite to gabbro sills that intrude the Belt strata. Copper and silver minerals commonly are disseminated into strata adjacent to the veins and the mafic sills.

Prospecting and mining in the early 1900s in this area was focused mainly on the vein type copper-silver deposits. The Cotter Basin mine (p1. 2, no. 10) actively produced until the mid-1970s. The copper and silver deposits are in sheared dolomitic limestone of the Helena Formation. The principal shear zone is as wide as $5 \mathrm{ft}$ and more than $1,100 \mathrm{ft}$ long. The shear zone and adjacent mineralized wallrock form a zone that is at least $16 \mathrm{ft}$ thick and contains identified resources of $1,000,000$ tons of ore, which averages 1.2 percent copper and 0.2 ounces per ton silver (Earhart and others, 1977, p.54).

\section{Little Prickly Pear Area}

\section{Geologic Setting}

The Little Prickly Pear area of the Forest, southeast of Lincoln (fig. F1), is in the northeast part of the Butte $1^{\circ} \times 2^{\circ}$ quadrangle. The area is underlain by clastic strata of the Middle Proterozoic Spokane Formation, and locally the Empire Formation, of the Belt Supergroup. Late Proterozoic diorite to gabbro sills intrude the Spokane.

\section{Mineral Deposits}

\section{Sediment-hosted copper, Revett and redbed types}

The only reported mineral occurrence in this part of the Forest is of sediment-hosted copper and silver of the redbed type-greenbed subtype and Revett type deposits. (See Scapegoat area discussion of the deposit types, subtypes, and sedimentology pertinent to sediment-hosted copper.) Mineralized quartzite beds (Revett type deposit) of the lower Empire and the upper Spokane crop out in a $1 \mathrm{sq}$ mi area in the vicinity of the Copper Hill (McVay, Canyon Creek) deposit (p1. 2, no. 112). The deposit has been explored by drilling.

A drillhole penetrated basal beds of the Empire and extended into the middle of the Spokane; core from the hole permitted detailed study of ore-forming processes (Tramme1, 1975; Braun and Lange, 1984; Lange and others, 1986, 1989). Scattered copper sulfides were encountered in green beds of the Empire and upper part of the Spokane. Copper and silver minerals in the middle part of the Spokane occur through a $130 \mathrm{ft}$ interval comprised of light-gray quartzite beds and interstratified green siltite and argillite (greenbed subtype). Mineralized rock is most abundant and laterally persistent in the quartzites (Braun and Lange, 1984; Lange and others, 1989). Values of 1.52 percent copper, 0.93 ounces per ton silver, and 0.005 ounces per ton gold were reported from this deposit (Brox and Guilbert, 1960; Brox and Potter, 1965). 


\section{BIG BELT MOUNTAINS REGION}

By R.G. Tysdal, E.A. du Bray, and J.W. Whipple

York Distirct

\section{Geologic Setting}

The York district (fig. Fl; pl. 2) is on the southwest flank of the Big Belt Mountains, about $20 \mathrm{mi}$ northeast of Helena. Argillite, siltite, and metasandstone of the Middle Proterozoic Newland, Greyson, Spokane, and Empire Formations, and siltite and limestone of the Helena Formation underlie much of central and northern part of the district. The Helena Formation has been thinned by erosion. These formations are intruded by Late Proterozoic dikes and sills of gabbro, diorite, and andesite. On the southwest, adjacent to the Townsend Valley, the Proterozoic units are overlain by limestone and dolomite of Cambrian to Mississippian-age formations. An area of andesite to basalt flows of probable Cretaceous age overlies strata of the Greyson Formation in the eastern part of the district (Mertie and others, 1951; Gualtieri, 1975a).

The Proterozoic and Paleozoic rocks were folded and faulted during the Laramide orogeny, and a thrust fault trends southeast through the middle of the district (Pardee and Schrader, 1933; Mertie and others, 1951). The Helena Valley fault (fig. Bl; pl. 1) trends southeast through the southwestern part of the district. It is a Tertiary normal fault with right-lateral offset, separating the mountains from deposits of the Townsend Valley, and is the northern edge of the Lewis and Clark Line (Reynolds, 1979).

\section{Mineral Deposits}

The York district has experienced intermittent exploration or production from the late 1800 s to the $1990 \mathrm{~s}$. Mineralized zones are assigned to four types of mineral deposit models: sediment-hosted copper, redbed; sediment-hosted copper, veins; sedimentary exhalative, gold; and vein, gold-bearing. The first three models are for stratabound deposits; the vein, gold-bearing model is related to granitic intrusions.

\section{Sediment-hosted copper, redbed}

Sediment-hosted copper, redbed type, is present in scattered occurrences in the Spokane Formation of the York district. The mineralization is of the greenbed subtype. (See "Scapegoat (Mineral Hill) Area" for discussion of deposit types and subtypes.) Rocks of the Spokane Formation in the Big Belt Mountains contain fewer green interbeds than rocks in the Scapegoat area and are only parsely mineralized. Metasandstone (quartzite) beds in the Spokane of the Big Belt Mountains are very thin, discontinous, and generally not thick enough to host Revett deposits. 
Most copper in the York district is in veins. The deposits are characterized by chalcopyrite-rich, quartz-ankerite veins that are associated with the transition zone strata of the lower part of the Spokane Formation. The veins, and vein systems, are $1 \mathrm{ft}$ to several feet thick, occupy small faults and shear zones, and occur discontinuously along the length of the district. Although some of the copper deposits are of high grade, they usually are local and restricted. The copper mineralization of the veins generally is believed to have resulted primarily from remobilization, and concentration into veins, of metals from low grade, stratabound occurrences of copper-bearing minerals that are disseminated within green beds of the Belt strata (Baitis and others, 1988; Lange and others, 1992; Whipple and Morrison, in preparation).

The Argo mine (p1. 2, no. 558) was the main producer of copper in the York district. Copper ore was mined from a quartz-carbonate vein system in faulted strata near the contact of the Spokane and Greyson Formations. Pardee and Schrader (1933) described the main vein of the Argo as composed of 4-5 ft thick tabular bodies several hundred feet in length and depth consisting nearly entirely of chalcopyrite. Most of the ore was confined to green argillite--ore grades decreased markedly where red argillite was encountered. The Argo mine produced about 3,000,000 pounds of copper from 1902 to 1918 .

\section{Exhalative, gold}

A zone of gold-bearing sedimentary rocks is present in the Middle Proterozoic Greyson Formation of the York district. The zone is at least 12 mi long and consists of (1) mineralized orange-weathering siltite that contains abundant, intergranular potassium feldspar and locally iron carbonate; and (2) non-mineralized olive siltite that is thinly laminated (Whipple and Morrison, in preparation). The mineralized strata form resistant outcrops, termed "reefs," which are laterally discontinuous and range up to about $100 \mathrm{ft}$ thick and several thousand feet long (Baitis and others, 1988; Thorson and others, in preparation) (p1. 2, nos. 535, 537, 539).

Potassium feldspar of the reefs is orthoclase (Baitis and others, 1989) and appears to be authigenic; $\mathrm{K}_{2} \mathrm{O}$ is as much as 12 percent of the rock. At the base of some of the mineralized reefs is a distinct interval, a few tens of feet thick, of altered and bleached siltite that contains 2.4-4.7 percent $\mathrm{K}_{2} \mathrm{O}$ (Whipple and Morrison, in preparation). The map of potassium aeroradioactivity (fig. (6), which shows intensities of potassium detected in rocks, reveals that the potassium-rich rocks define a positive anomaly over Greyson strata in the York district (Pitkin, Chapter $C$ of this report).

The stratabound nature of gold mineralization in the district was first recognized by geologists of Noranda Exploration in the mid-1980s. Drilling by Noranda, and later other companies, showed that veins of quartz-carbonatepotassium feldspar cut all rocks at York but are most common in the reefs. Mineralization is disseminated within the reefs and in veins that are parallel and discordant to bedding within the reefs. The veins contain gold, pyrite, galena, and minor chalcopyrite (Baitis and others, 1988, 1989). Gold is absent from the bleached, altered siltite that occurs at the base of some reefs (Whipple and Morrison, in preparation). Reefs with greater than 0.01 ounces per ton gold were estimated to contain resources of more than 7,000,000 ounces of gold 
(Thorson and others, in preparation). Gold values in the reefs are 3-4 times those of background gold values for the Greyson (Baitis and others, 1988). Anomalous concentrations of gold in stream sediments from the York district are shown in figure D12 (Alminas, Chapter D of this report).

The Greyson Formation was examined by Whipple and Morrison (in preparation) to determine the stratigraphy and sedimentological features of the strata that host the gold. They recognized four informal map units. Unit 1, the basal unit, is 500-1,700 ft thick and mainly is carbonate-cemented siltite and argillite. Unit 2, which contains the mineralized gold-bearing reefs, is 3,500-4,000 ft thick. It is mostly very thinly laminated olive siltite that contains discontinuous interlaminae of blackish green siltite and argillite. Several tuffaceous, volcaniclastic beds, a few feet thick, occur in the Avalanche Gulch area of the district ( $p 1.2$ ) and are stratigraphically above and below the potassium-bearing rocks. Unit 3, about 1,200 ft thick, consists of blocky beds of gray to olive siltite and interbedded, very thinly laminated siltite. Some beds display hummocky crosslamination. Soft-sediment deformation structures occur in basal strata of this unit. Unit 4, about 1,000 ft thick, consists of mixed siliciclastic rocks and limestones that are lenticular and locally fragmental and stromatolitic. Mud-chip breccia, and rarely salt casts, are present in the uppermost beds. The succession of facies in the units 1-4 indicates a depositional environment that shallows upward from oshore basin plain to subtidal.

Sedimentary structures and lamination styles of some beds in unit 2 suggested deposition from turbidity currents, in deep parts of a restricted basin (Whipple and Morrison, in preparation; Thorson and others, in preparation). The volcaniclastic rocks and interbedded siltite of unit 2 suggest deposition in a deep-water environment that experienced periodic submarine volcanism (Whipple and Morrison, in preparation). The turbidites may have been generated by synsedimentary movement along intrabasin faults (Thorson and others, in preparation).

Several hypotheses have been proposed for genesis of the deposits, including granitic-intrusion related, epigenetic, syngenetic, and exhalative (Baitis and others, 1988, 1989; Thorson and others, in preparation; Whipple and Morrison, in preparation). The origin of these deposits is obscure. For this report we classified the gold deposits as exhalative, in the broadest sense. We include deposits that may have formed from metal-rich plumes issuing into the water column above a basin floor, as well as deposits that may have formed within the sedimentary pile beneath the basin floor, thus formed within the entire plumbing (feeder) system.

\section{Vein, gold-bearing}

The York mining district produced gold from veins in the early part of the 1900s. The deposits are typified by veins of the Golden Messenger mine (p1. 2, no. 522). The mine is within a Late Proterozoic mafic dike that is $200-400 \mathrm{ft}$ thick, several miles long, and cuts the Greyson and Spokane Formations. The dike contains replacement systems of gold-bearing quartz-carbonate veins, some as much as $30 \mathrm{ft}$ thick and 200-300 ft in other dimensions. Veins are not confined to the dike but extend into the adjacent sedimentary rock. Minerals are native gold, pyrite, and minor galena, sphalerite, and chalcocite; a small amount of silver was recovered from ore. Ore rich in gold also was rich in galena (Pardee and 
Schrader, 1933). Lode production from the York district was about 70,000 ounces of gold, mostly from the Golden Messenger mine (Koschmann and Bergendah1, 1968).

Woodward (1992) interpreted the lode-gold deposits of the Greyson and Newland Formations to be localized along basement faults that were active during Proterozoic sedimentation, but he was not certain of the timing of remobilization of elements and concentration into veins. Whipple and Morrison (in preparation) observed that the dike hosting the Golden Messenger mine, and nearby mines and prospects, intruded the northern end of the gold-enriched, potassium feldsparbearing zone of the Greyson Formation. They suggested that the gold in the dike could have been remobilized from the low-grade stratabound gold occurrences and concentrated into veins of the dike. In contrast, lead-isotope data suggests a Laramide age of mineralization, according to Lange and others (1993).

\section{Confederate Gulch District and Southern Big Belt Area}

\section{Geologic Setting}

The Confederate Gulch district is on the southwest flank of the Big Belt Mountains, about $30 \mathrm{mi}$ east of Helena, and the southern Big Belt area adjoins it on the southeast (fig. F1; p1. 2). The Confederate Gulch district and the Southern Big Belt area are discussed together because much of the mineralized rock of the two areas is related to one system of plutonic rocks, or is hosted by Proterozoic strata that extend through both areas.

Most of the sedimentary rocks of the Confederate Gulch district and the Southern Big Belt area are argillite, siltite, and metasandstone of the Middle Proterozoic Newland and Greyson Formations. The Spokane and Empire Formations are exposed in a small area in the westernmost part of the Confederate Gulch district. Late Proterozoic dikes and sills of gabbro and diorite are present locally. Limestone and dolomite of Paleozoic and Mesozoic ages constitute most of the strata in the southernmost part of the Big Belt Mountains (p1. 1).

Cretaceous(?) andesitic volcanic rocks are exposed on the west side of the Confederate Gulch district (Mertie and others, 1951; Gualtieri, 1975a) and similar Cretaceous extrusive rocks are present in the southernmost part of the Southern Big Belt area (Skipp and Peterson, 1965; Skipp and McGrew, 1977). Rocks of both areas are probably erosional remnants of the much larger Elkhorn Mountains Volcanics field.

Granitic intrusions crop out extensively in the northern part of the Southern Big Belt area. The Boulder pluton, about six miles in diameter, is centered between Boulder Baldy and Boulder Mountain, and a small satellitic stock lies about $1 \mathrm{mi}$ to the east (pl. 1) (Gualtieri, 1975a). To the south, another pluton crops out in the area of Mount Edith, in the headwaters of Big Birch Creek (du Bray, 1995). West of the Mount Edith pluton, and outside the limit of geology shown on plate 1, plutons mapped by Nelson (1963) yielded radiometric dates of $64.4 \pm 2.4 \mathrm{Ma}$ (biotite) and $71.5 \pm 3.2 \mathrm{Ma}$ (hornblende) (Daniel and Berg, 1981; ages recalculated by du Bray, 1995). The Boulder and Mount Edith plutons probably are coeval with the plutons mapped by Nelson (1963).

The Boulder pluton was mapped by Gualtieri (1975a), who recognized a concentric zonation, and later by du Bray (1995). The small pluton at Miller Mountain ( $\mathrm{pl}$. 1; p1. 2, no. 583) in the Confederate Gulch district was examined by Johnson (1973), who described rocks of differing compositions that comprise 
at least two stages of intrusion. The petrographic and intrusive relationships he described are similar to those observed in the middle and core zones of the Boulder pluton. The Miller Mountain pluton probably is an apophysis of the Boulder pluton; similarly, the other, very small intrusions in the district also probably are apophyses or are related to the same magma chamber as the Boulder pluton.

The Mount Edith pluton is petrographically similar to the intermediate zone of the Boulder pluton, implying temporal and genetic association of the plutons. The majority of the Mount Edith pluton is covered by a mantle of glacial deposits in the headwaters of Big Birch Creek.

The close spatial association and the petrographic and mineralogic similarities among the plutonic rocks of the central part of the Big Belt Mountains suggests they are genetically related and part of a single intrusive episode. Gravity and aeromagnetic data (McCafferty, Chapter C of this report, figs. C1, C4, anomalies 13, 14) corroborate the interpreted subsurface continuity of the intrusive masses. Thermal metamorphism associated with these intrusions produced aureoles that do not extend more than several hundreds of feet into sedimentary rocks of the Belt Supergroup.

\section{Mineral Deposits}

Exhalative(?), gold

Stratabound, sediment-hosted gold-bearing reefs like those in the York district are not known in the Confederate Gulch district and Southern Big Belt area. However, this area contains altered rocks similar to those of the York mineralized reefs and the underlying non-gold-bearing altered rocks of the York district. The altered rocks transgress stratigraphy (Thorson and others, in preparation), cutting downsection southeastward within the district (J.W. Whipple, oral commun., 1994). Light-gray, finely laminated, potassium-rich, altered siltite beds occur within the lower part of the Greyson Formation and upper part of the Newland Formation in the Southern Big Belt area. They crop out along State highway 12 on the west and east flanks of the mountains and were observed locally within the mountains as we11. The potassium aeroradioactivity map (fig. (6), which shows a positive anomaly over the potassium-rich rocks of the Greyson Formation in the York district (see York district, "Exhalative, gold"), reveals that the anomaly also overlies lower Greyson and (or) upper Newland strata southward, in the Confederate Gulch district. In the Southern Big Belt area, the anomaly is along the west flank of the mountains; across the southern part of the mountains where the strata cross the range in an anticline; and on the east flank of the mountains (see Pitkin, Chapter C of this report).

The relationship of the altered rocks in the southern part of the range to gold-rich and gold-poor, potassium-rich, altered rocks of the York district is unknown. Analyses of several altered rocks collected in the Southern Big Belt area during this study failed to detect gold. The continuity of the aeroradioactivity over potassium-rich rocks in the York and Confederate districts and the Southern Big Belt area, however, suggests that all of the potassium-rich rocks may be genetically related. 


\section{Cretaceous gold-bearing veins}

The Confederate Gulch area was a major producer of placer gold during the latter half of the 1800 s and sporadically thereafter (Lyden, 1948). Total gold production from the Confederate Gulch district through 1959 was between 550,000 and 600,000 ounces, all but about 10,000 ounces from placers (Kochmann and Bergendahl, 1968). A close spatial association between mineral deposits and intrusive rocks in the district suggests a genetic relationship. The gold in the placer deposits is commonly attributed to lode sources at Miller Mountain on the north side of Confederate Gulch (Pardee and Schrader, 1933).

Lode mining from veins in the vicinity of the pluton at Miller Mountain, and a small pluton to the south at the Satellite claims (pl. 2, no. 586), was conducted intermittently from the late 1800s to the early 1940s (Pardee and Schrader, 1933; Johnson, 1973). The gold vein deposits occur in two settings. In one setting quartz veins are in the contact zone of the granitic rocks with the Newland strata and contain gold, abundant pyrite, and minor sphalerite, digenite, and covellite. In the other setting, quartz veins occur entirely within the granitic rocks and mineralization consists mainly of gold and pyrite. The veins, up to $3 \mathrm{ft}$ thick, have a general north-south trend according to Johnson (1973); subhorizontal veins, up to $6 \mathrm{ft}$ thick, were emphasized by $\mathrm{G}$. Wittman (Pegasus Gold, oral commun., 1993).

Recent gold exploration at Miller Mountain has delineated a resource of at least 11 million short tons of ore containing 310,000 ounces gold (grade, 0.028 ounces/ton) (Pegasus Gold, Inc., 1992). Gold grades are highest near major structural intersections, particularly along low-angle shear zones. Johnson (1973) identified two main phases of intrusion of granitic rock at Miller Mountain; mineralization appears to be related to the second phase (G. Wittman, Pegasus Gold, oral commun., 1993).

Granitoid rock along the outer margin of the Boulder pluton and its satellitic stock to the east is coincident with a prospect ( 1 . 2, no. 591), the Porcupine Mine (no. 595), and a small mine nearby (no. 594), all observed during reconnaissance fieldwork for this study. Prospects (nos. 597, 598, 588) were observed in Newland strata near the pluton, as were the Snowbank and Bigler mines (both no. 596). Samples from these mines and prospects all contain anomalous abundances of elements (table E1). Fluorite is common at many of these mines and prospects, forming small veins in a shear zone in the core zone of the Boulder pluton (p1. 2, no. 589) and in veinlet-filled fractures near the edge of the pluton at the Normandy prospect (no. 592) (Sahinen, 1962; Ross, 1950; Roby, 1950; du Bray, 1995). Mineralized rock apparently is less common peripheral to the Mount Edith pluton, perhaps partly due to extremely poor exposures in the area.

Geochemical analyses of rocks collected by Gualtieri (1975b) in the Confederate Gulch-Boulder Mountain region show anomalous values mainly in the general area of Miller Mountain. The rocks are anomalous in gold, silver, and molybdenum, some of the same elements found in anomalous abundance in the present study for rocks in and adjacent to the Boulder and Mount Edith plutons. However, the other elements found during this study do not appear to be anomalous in Gualtieri's data, probably because analytical techniques used at the time had considerably higher threshhold values of detection. Anomalous values of gold and silver in stream sediments are shown in figures D12 and D13 (Alminas, Chapter D of this report). 


\section{Northern Part of Big Belt Mountains}

The area discussed here includes Snedaker basin in the northern part of the Big Belt Mountains and the separate, isolated part of the Forest in the Dry Range, directly east of the hamlet of Lingshire. The area is directly north of the York and Beaver-Elk Creek districts (fig. F1; p1. 2).

\section{Geologic Setting}

Paleozoic carbonate strata constitute most of the exposed rocks in the Dry Range area. The Forest area to the west is chiefly underlain by Paleozoic and Mesozoic carbonate strata, Middle Proterozoic Newland and locally Greyson Formations, and Tertiary volcanic rocks ( 1 1. 1). The pre-volcanic rocks were thrust into their present positions and consititute parts of several thrust sheets. Two magnetic anomalies (figs. C1, C4, anomalies 17 and 18) suggest that concealed intrusive bodies may lie beneath the Snedaker-Dry Range area (see Chapter $C$ of this report), as does the occurrence of an apparent polymetallic replacement deposit.

\section{Mineral Deposits}

The northern part of the Big Belt Mountains has very few prospects and no known significant mineralization. The two magnetic anomalies in the area, interpreted as indicating concealed plutons (McCafferty, Chapter $C$ of this report, figs. C1, C4, anomaly 18), could have associated mineralization even though 1ittle surface evidence of mineralization exists. Detailed examination of the Gates of the Mountains Wilderness, a small part of which lies in the westernmost part of the permissive terrane, yielded no significant evidence of mineralization (Close and Rigby, 1984; Reynolds, and Close, 1984). Mineralization, if present, likely is concealed beneath the surface rocks.

\section{Lower Belt Supergroup Strata in Big Belt Mountains}

Sediment-hosted massive sulfide deposits occur in lower strata of the Belt Supergroup in Montana, Idaho, Washington, and Canada. In the Helena structural salient (see Chapter B on geologic setting), copper-cobalt and lead-zinc mineral occurrences are present in the Newland Formation at Sheep Creek about 15 mi east of the Forest. South of Butte, near the southern margin of the Helena salient (fig. B1), Thorson (1984, in preparation) reported massive sulfide occurrences in the Greyson Formation. Newland and lower Greyson strata in the Forest were evaluated for this type of deposit.

\section{Geologic Setting}

The lower two-thirds of the Newland Formation of Big Be1t Mountains area is mainly noncalcareous to dolomitic and locally calcareous argillite and siltite, with sparse thin interbeds of sandstone. The upper third consists of 
interbedded dolomite, limestone, siltite, and argillite. The formation is at least 9,500 ft thick in the Big Belt Mountains (Nelson, 1963). Schieber (1985) measured only about 8,150 ft of the Newland along Deep Creek near highway U.S. 12 , but stated that a fault may cut out part of the sequence.

The lower member was deposited below wavebase in a basinal environment, punctuated by thin sandstone beds that are microturbidites; the upper member also was deposited below wavebase but in shoaling-upward environments in shallower water than the lower part, according to Zieg (1986). Schieber (1985, p. 160-163) concluded that most of the lower member was deposited towards the center of a basin in water "only a few meters deep." Later, (Schieber, 1989) stated that these strata represent sediments deposited below normal wavebase but above storm wavebase. Schieber (1992) concluded that limestone of the upper member in the Big Belt Mountains was primarily deposited in a distal, starved basin setting.

The Greyson Formation was described generally in the section on the York district. Turbidites are interpreted to make up a significant part of the lower three units of the Greyson, and were probably deposited in the deeper parts of a restricted basin (Whipple and Morrison, in preparation).

\section{Mineral Deposits}

\section{Massive sulfide deposits}

The massive sulfide deposits of the Belt sedimentary basin are commonly attributed to exhalative processes. Exhalative deposits are stratiform accumulations of sulfide and sulfate minerals that are interbedded with marine sediments. The sediments were deposited below wavebase, generally at depth within a marine basin. Deposits are sheet- or lens-like tabular bodies a few tens of feet to locally a few hundred feet thick. Mineralizing fluids moved upward along intrabasinal faults that were active during deposition of the sediments. During ore formation, the mineral-rich fluids permeated and altered rocks adjacent to the flow path, and emanated onto the sea floor and into the water column, where minerals precipitated to form deposits (Briskey, 1986).

The following description of the Sheep Creek mineralization, north of the Forest, is summarized, for comparative purposes, from Zieg and others (1991) and Zieg and Leitch (1996). The Sheep Creek area lies at the northern edge of the Helena embayment, along the Volcano Valley fault (fig. B1), on the south flank of the Little Belt Mountains. Metal-rich hydrothermal fluids moved upward along the ancestral Volcano Valley fault and were exhaled from vent centers. The fluids were rich in copper, cobalt, nickel, arsenic, and barium, as well as locally rich in lead, zinc, and silver. The minerals precipitated as sulfides that are bedded and interstratified within the Newland Formation. Exhalative activity must have been nearly continuous throughout deposition of the lower member of the Newland Formation and at least intermittent during deposition of the upper member. Drilling showed that the mineralized zones form several lenses along the fault and they extend for as much as 2,000 ft basinward (south) from the fault zone.

No massive sulfide mineral deposits of the Sheep Creek type were found during reconnaissance examination of areas of the Newland in the Big Belt Mountains, and we know of no reported occurrences of the deposit type in the range. However, the possibility of an occurrence cannot be eliminated because 
the region was active tectonically during deposition of the Newland, as demonstrated in the Sheep Creek and Highland Mountains areas. Syndepositional faults possibly could have been active within the central part of the Helena structural embayment, even though most of the Newland in the Forest is distant from either the basin-margin fault zones at Sheep Creek to the north or the Highland Mountains to the southwest.

Schieber (1991) stated that pyritic shale (argillite and siltite) horizons of the Newland Formation are similar to Proterozoic lead-zinc bearing pyritic shales in Australia (Mt. Isa, McArthur River). However, no lead-zinc orebodies have been found in the Newland of central Montana. Schieber's data from Montana samples show only minute quantities of these two metals and he stated that pyritic shales are not anomalous in their lead-zinc content.

Tourmalinites are commonly associated with a variety of stratabound mineral deposits, including lead-zinc-silver deposits in lower Belt strata in the western part of the Belt sedimentary basin. J.M Leask (personal commun. in Slack, 1993, p. 34) reported a tourmalinite locality in the Newland Formation of Cement Gulch, which is in the headwaters of Confederate Gulch. A thin section of rock collected from this area was examined and revealed no tourmaline; a chemical analysis showed only a few ppm boron. 


\section{CONTINENTAL DIVIDE REGION}

By J.E. Elliott

The Continental Divide region includes most of the Forest west of Interstate Highway 15 and southwest of the St. Marys-Helena Valley fault, which transects the Forest in the vicinity of Lincoln (fig. F1). Essentially all of this region was recently studied under a Conterminous United States Mineral Assessment Program (CUSMAP) evaluation of the Butte $1^{\circ} \times 2^{\circ}$ quadrangle. Reports of the many studies conducted in this quadrangle are described by Elliott and others (1993).

\section{Geologic Setting}

The Continental Divide region contains igneous, metamorphic, and sedimentary rocks and surficial deposits that range in age from Middle Proterozoic to Quaternary. The oldest rocks exposed are clastic and carbonate sedimentary rocks of the Middle Proterozoic Belt Supergroup, which are widespread and abundant in the northern and central parts of the region ( 1 . 1, map A). During the Late Proterozoic, sills and dikes of gabbro and diorite intruded the older sedimentary rocks. Paleozoic carbonate and carbonate-bearing clastic rocks, deposited in near-shore and shallow-water environments, are present in the central part of the region where they unconformably overlie the Belt rocks. Mesozoic sequences of marine and nonmarine clastic rocks, deposited in foreland basins, overlie Paleozoic rocks in the south-central part of the region.

Magmatic activity commenced in the region during the Late Cretaceous and continued intermittently until the middle Tertiary. This magmatism resulted in the formation of several volcanic sequences, a batholith, several stocks, and numerous dikes and sills. Most of the igneous rocks are in the southern part of the region and include part of the Boulder batholith, a large granitic pluton that extends south of the Forest (fig. B1), and three predominantly volcanic sequences, which include andesitic, dacitic, and rhyolitic rocks, of Late Cretaceous, middle Eocene, and late Eocene ages, respectively. Another relatively small volcanic field is in the northern part of the region, southeast and east of Lincoln, and consists of both andesitic and rhyolitic volcanic and volcaniclastic rocks. In the central part of the region, several stocks, mainly of granodioritic composition and Late Cretaceous age, are aligned along a northwesterly trend. Thermal aureoles adjacent to the Boulder batholith and the stocks resulted in contact metamorphism of the sedimentary rocks, converting them to hornfels, marble, and, locally, skarn. Most of the mineral deposits of the region are spatially and genetically associated with the igneous rocks. Hydrothermal activity during and following the waning stages of magmatism formed many of the mineral deposits that have been discovered in the region.

Surficial deposits include widespread lacustrine and fluvial deposits that accumulated in intermontane basins during mid- to late-Tertiary time and glacial, alluvial, colluvial, and landslide deposits formed during Quaternary time. The glacial deposits are most abundant in the northern part of the region where deposits of glacial till, moraine, and glaciofluvial deposits are common and widespread. 
The principal structural elements of the region are two major northwesttrending faults, the St. Marys-Helena Valley and Bald Butte faults (fig. B1), that are part of the Lewis and Clark line of western Montana and northern Idaho; a broad syncline including sedimentary rocks ranging from Middle Proterozoic to Late Cretaceous in age; and part of the Sapphire thrust terrane (p1. 1, map A). Although some of the movement along the Lewis and Clark line may have occurred during Proterozoic time (Wallace and others, 1990), most of the faulting and folding probably occurred during the Late Cretaceous when regional compression formed the laterally extensive Sapphire thrust terrane and caused major strikeslip movement along the Lewis and Clark 1ine (Wallace and others, 1990).

\section{Subregions}

To facilitate the description of mining districts and mineral deposits, the Continental Divide region is divided into subregions with boundaries that correspond closely to geologic terranes or structural features. From north to south these are the (1) Granite Butte-Ogden Mountain, (2) Black MountainStemwinder Hill, and (3) North Boulder Mountains subregions (fig. F1). The Granite Butte-Ogden Mountain subregion is south of the Lincoln region, described previously; their common boundary is the St. Marys-Helena Valley fault. The Black Mountain-Stemwinder Hill subregion is separated from the Granite ButteOgden Mountain subregion by the Bald Butte fault. The North Boulder Mountains subregion is separated from the Black Mountain-Stemwinder Hill subregion by U.S. Highway 12, which parallels and is near the northern boundary of the Boulder batholith.

\section{Granite Butte-Ogden Mountain Subregion}

\section{Geologic Setting}

Most of subregion is underlain by formations that are part of the Belt Supergroup. Sequences representative of the lower Belt (Spokane Formation) to the upper Belt (Bonner Formation) are present. Several stocks of Late Cretaceous age and one of possible Tertiary age occur along a northwest-trending belt. These include the Tertiary Silver Bell stock near Stemple Pass, and (from northwest to southeast) the Ogden Mountain, Dalton Mountain, Granite Butte, and Marysville stocks of Cretaceous age (pl. 1). For the Silver Bell stock, K-Ar ages from biotite are 50 and $52 \mathrm{Ma}$, but they may be reset ages due to the proximity of a Tertiary volcanic field (C.A. Wallace, U.S.G.S., written comm., 1989). Most of the stocks are granodiorite, but plutonic rocks range in composition to monzogranite, quartz monzonite, and quartz monzodiorite. The subregion includes most of a Tertiary volcanic field that extends mainly south and east of Lincoln. This volcanic field includes two compositionally distinct sequences: an older sequence of andesitic flows, breccia, air-fall tuff, and volcaniclastic rocks; and a younger sequence of rhyolitic flows, breccia, tuffs, and volcaniclastic sedimentary rocks. These volcanic rocks extend north into the Lincoln region and occur in isolated patches to the southeast and south of the main Lincoln field.

The Belt rocks are folded and cut by several normal faults, one thrust fault of probable small displacement, and two major regional faults, the St. Marys-Helena Valley and Bald Butte faults, both with strike-slip movement. The 
latter are two of several principal faults that form the Lewis-and-Clark line (Wallace and others, 1990). The St. Marys-Helena Valley fault cuts the northeastern part of the subregion and forms the northern boundary of the Lewis and Clark line. A right slip offset of about $8 \mathrm{mi}$ is indicated in the western segment of the fault (Wallace and others, 1990). The Bald Butte fault forms the southern border of subregion. Based on offset of stratigraphic units, about 17.4 mi of right-lateral separation is indicated for the Bald Butte fault (Wallace and others, 1990).

\section{Mineral Deposits}

The Granite Butte-Ogden Mountain subregion encloses all of the following mining districts and areas: Lincoln Gulch area and Big Blackfoot, Finn, McClellan Gulch, Stemple-Gould, and Marysville districts (fig. Fl; pl. 2, map B). It also includes parts of the Blackfoot River and Little Prickly Pear areas. The majority of lode deposits are either polymetallic veins or epithermal veins. Placer gold deposits are also numerous and widespread. Other types of known lode deposits include skarn tungsten, porphyry copper-molybdenum, and Climax molybdenum.

Lincoln Gulch Area--The Lincoln Gulch area is approximately four miles west of Lincoln and includes parts of the Forest. Calcareous siltite and argillite, and limestone of Middle Proterozoic Helena Formation have been intruded by thin Late Proterozoic dikes of intermediate to mafic composition. Quaternary till and alluvial and terrace gravels occupy the valley of Lincoln Creek. The area is one of the most famous districts of the region and was a very large producer of gold from placer deposits (approximately $\$ 7$ million, Pardee and Schrader, 1933; estimated 372,000 ounces, Elliott and others, 1992). An undetermined amount of gold has also been produced from the Blackfoot (Big Blackfoot) mine (p1. 2, no. 23), which is a vein deposit in a shear zone within a diorite dike. Over two million tons of gold ore containing approximately 100,000 ounces of gold are reported to be present at the Blackfoot mine (McCulloch, 1989; Rocky Mountain Pay Dirt, 1987).

The southern end of the Lincoln Gulch area is associated with a magnetic high that extends into the surrounding Blackfoot River area (fig. C1). This high is spatially associated with the Blackfoot mine. The source of this anomaly is probably an intrusive body similar in size and composition to the Ogden Mountain stock, to the southwest (Hanna and others, 1994).

Geochemical anomalies in arsenic, bismuth (fig. D15), copper (fig. D16), iron (fig. D20), molybdenum (fig. D17), lead (fig. D14), antimony, and tungsten are present in the Lincoln Gulch area, based on a geochemical survey of McDanal and others (1985). The geochemical anomalies in these figures (Alminas, Chapter $D$ of this report) are for stream sediments and incorporate only the stream sediment data of McDanal and others (1985).

Little Prickly Peak Area--The Little Prickly Pear area is north and northwest of Helena and east and southeast of Lincoln (fig. Fl). It includes parts of the Forest along the upper tributaries to Little Prickly Pear Creek. Most of the area is within the Lincoln Region and is described in the previous section of this chapter. 
Big Blackfoot (Ogden Mountain) Distirct--The Big Blackfoot district, approximately $10 \mathrm{mi}$ southwest of Lincoln, is within and adjacent to a granodiorite stock (fig. F1; p1. 2, map B). Much of the district is within the Forest. The Late Cretaceous Ogden Mountain stock, with an age of about 81-85 Ma (Schmidt and others, 1994), intruded sedimentary rocks of the Middle Proterozoic Helena, Snowslip, and Shepard Formations. The sedimentary rocks are offset by normal faults and, in the southwest part of the area, by right-lateral strike-slip faults of the Bald Butte fault zone. Tertiary volcanic and sedimentary rocks cover older rocks in places. Mineral deposits are polymetallic veins, gold placers, and a tungsten skarn. Two of the principal mines of the district are the Hobby Horse and Blackfoot mine (Elliott and others, 1992). The Hobby Horse ( $\mathrm{p} 1.2$, no. 73) produced gold, silver, and lead from quartz veins in granodiorite. At the Blackfoot mine (p1. 2, no. 77), weathered parts of quartz veins in granodiorite and limestone of the Helena Formation were mined adjacent to the contact of the Ogden Mountain stock. This was the largest mine in the district and produced gold, silver, copper, and lead (McClernan, 1976). The district has produced moderate quantities of gold (about 4,500 ounces), silver, copper, and lead and has a total value of recorded production of about $\$ 134,000$ (Elliott and others, 1992).

The aeromagnetic anomaly map (McCafferty, Chapter $C$ of this report, fig. $\mathrm{Cl}$, anomaly 2) shows a prominent magnetic high associated with the Ogden Mountain stock. Elements that are geochemically anomalous in the district are silver (fig. D13), gold (fig. D12), copper (fig. D16), iron (fig. D20), and tungsten (McDanal and others, 1985). The geochemical anomalies in these figures (Alminas, Chapter D of this report) are for stream sediments and incorporate only the stream sediment data of McDanal and others (1985).

McClellan Gulch District--The areally small McClellan Gulch district (fig. F1; p1. 2, map B) is approximately six miles south-southeast of Lincoln and within the Forest. The gulch is underlain by argillite, siltite, limestone, and quartzite of the Middle Proterozoic Helena, Snowslip, Shepard, and Mount Shields Formations. At the head of the gulch, granodiorite of Late Cretaceous Dalton Mountain stock, with an age of about $79 \mathrm{Ma}$ (Schmidt and others, 1994), is in contact with Mount Shields and Shepard Formations. The district was a very large producer of gold from placer deposits between 1864 and 1875 (about $\$ 7$ million, Pardee and Schrader, 1933; estimated 340,000 ounces, Elliott and others, 1992). The sources of the gold are probably vein deposits such as at the Wiggins mine (p1. 2, no. 92) where quartz veins occur within and near the contact of granodioirte of the Dalton Mountain stock. The south end of the district borders a magnetic high that is associated with the Dalton Mountain stock (McCafferty, Chapter $C$ of this report, fig. C1, anomaly 3 ).

Geochemical anomalies in barium, iron (fig. D20), lead (fig. D14), and tungsten are present in the district (McDanal and others, 1985). The geochemical anomalies in these figures (Alminas, Chapter $D$ of this report) are for stream sediments and incorporate only the stream sediment data of McDanal and others (1985).

Stemple-Gould District--The Stemple-Gould district is approximately $10 \mathrm{mi}$ southeast of Lincoln and is within or enclosed by Forest lands (fig. F1; p1. 2, map B). Sedimentary rocks of Middle Proterozoic Helena, Empire, and Spokane Formations have been intruded by Late Proterozoic diorite sills and dikes, the Cretaceous Granite Butte granodiorite stock, and the Tertiary Silver Bell quartz 
monzonite porphyry stock (p1. 1, map A). Proterozoic rocks are cut by northwesttrending, steeply dipping normal, reverse, and strike-slip faults. Ore deposits are epithermal gold-silver veins and gold placers. The district also includes a porphyry copper-molybdenum prospect in the western portion of the Silver Bell stock (Brannon, 1981; McKee, 1978). The district has produced gold, silver, copper, lead, and iron and the estimated total value of production is about $\$ 5.3$ million (Elliott and others, 1992). The largest producer in the district was the Jay Gould mine (p1. 2, no. 105; Pardee and Schrader, 1933), which is classified as an epithermal vein deposit based on its mineralogy and textures. It is largely composed of lamellar calcite and quartz with textures indicative of having filled an open fissure. The Jay Gould vein is west-trending and dips steeply north. The host rocks are argillites of the Empire Formation. Ore minerals are chalcopyrite, argentite, and native gold (Pardee and Schrader, 1933).

The magnetic and gravity anomaly maps (McCafferty, Chapter $C$ of this report, figs. C1, C3) show anomalies associated with intrusive rocks. A prominent magnetic high is associated with the Granite Butte stock (fig. Cl, anomaly 6) and a magnetic high combined with a gravity low is associated with the Silver Bell stock (fig. C1, anomaly 6).

Geochemical anomalies for arsenic, silver (fig. D13), gold (fig. D12), barium, bismuth (fig. D15), copper (fig. D16), iron (fig. D20), molybdenum (fig. D17), lead (fig. D14), antimony, tin ( $\mathrm{fig} . \mathrm{D18}$ ), and tungsten are present in the district (McDanal and others, 1985). The geochemical anomalies in these figures (Alminas, Chapter D of this report) are for stream sediments and incorporate only the stream sediment data of McDanal and others (1985).

Finn District--The Finn district is located about $10 \mathrm{mi}$ south of Lincoln and is adjacent to and includes parts of the Forest (fig. F1; p1. 2, map B). The district is known principally for gold placers that occur along several southwest-flowing tributaries to Nevada Creek. Thrust-faulted and folded sedimentary rocks of Middle Proterozoic Helena, Mount Shields, Shepard, and Snowslip Formations were intruded by the Late Cretaceous Dalton Mountain granodiorite stock in the mountainous northeastern part of the district (p1. 1, map A). A northwest-trending fault separates the mountainous terrain from the Nevada Creek Valley to the west, which is underlain by Tertiary and Quaternary deposits. The Finn district has produced gold and silver, mostly from placer deposits. The largest producer of placer gold was Washington Creek. The source of the gold was probably polymetallic veins that are near the contact of the stock. Some of these have been mined for gold, silver, and copper north of the Finn district, for example the Wiggins mine (table E1, no. 92). The district produced approximately 95,000 ounces of gold and minor silver with an estimated total value of about $\$ 1.8$ million (E1liott and others, 1992).

A magnetic anomaly high occurs along the north edge of the district, corresponding to the Dalton Mountain stock (McCafferty, Chapter $C$ of this report, fig. C1, anomaly 3) and a gravity low (fig. C3) in the western part of the district associated with low density sediments of the Avon Valley (Hanna and others, 1994).

Geochemical anomalies for silver (fig. D13), gold (fig. D12), barium, bismuth (fig. D15), iron (fig. D20), molybdenum (fig. D17), manganese (fig. D21), and tungsten are present in the district McDanal and others, 1985). Anomalies in these figures (Alminas, Chapter D of this report) are for stream sediments and incorporate only the stream-sediment data of McDanal and others (1985). 
Marysville (Silver Creek) District--Marysville, one of the oldest and most famous mining districts in this region, is located about $16 \mathrm{mi}$ northwest of Helena (fig. F1; p1. 2, map B). The southern and southwestern parts of the district include some Forest lands. Limestone and calcareous argilite and siltite of the Middle Proterozoic Helena and Empire Formations have been folded, cut by faults, and intruded by the Late Cretaceous Marysville granodiorite stock (p1. 1, map A). The age of this stock is $78.5 \pm 2.6 \mathrm{Ma}$, based on $\mathrm{K}-\mathrm{Ar}$ isotopic-age determination for biotite (Schmidt and others, 1994). The Bald Butte fault cuts the southwestern part of the district. A wide zone of contact metamorphosed rocks is present around the Marysville stock and above an unexposed granite stock (determined by drilling) in the area of Bald Butte. Mineral deposits include polymetallic veins, epithermal veins, a stockwork molybdenum zone, mineralized breccias, skarns, and gold placers. The district was active from about 1876 (Pardee and Schrader, 1933) until the late 1940's (McClernan, 1983) and was a large producer of gold, silver, lead, copper, and zinc with an estimated total value of production of over $\$ 40$ million (E1liott and others, 1992).

The most famous mine in the district, the Drumlummon (p1. 2, no. 139), produced ore valued at $\$ 15$ million, of which 60 per cent was in gold, before 1911 (Knopf, 1913). This value of gold ( $\$ 9$ million), at a price of $\$ 20.67$ per ounce, is equivalent to about 435,000 ounces. The recorded production for the Drumlummon mine during the period of 1901-1948 is 115,694 ounces (McClernan, 1983). The Drumlummon vein is an epithermal gold-silver vein along the contact of the Marysville stock with contact-metamorphosed Helena Formation. The vein has a general N. 15 E. trend and dips steeply to the east (Knopf, 1913). Most of the vein is oxidized; primary sulfide minerals, mainly tetrahedrite and chalcopyrite, are sparse. The gangue is composed of quartz, calcite, and dolomite that commonly has lamellar, vuggy, or drusy textures. The vein has been worked along a strike length of 3,000 ft and to a depth of 1,600 ft (Pardee and Schrader, 1933).

An interesting molybdenum prospect (p1. 2, no. 133) of the C1imax type occurs at Bald Butte in the southern part of the Marysville district. On the surface, contact metamorphosed Helena Formation is hydrothermally altered and cut by quartz veinlets that have molybdenite and fluorite. A geochemical survey of the area determined distinct molybdenum anomalies in soil (Rostad, 1969). Later drilling encountered an altered and mineralized porphyritic granite in the subsurface. A sample of this granite obtained from drill core (depth of 1,620 $f t$ ) on file at the Montana Bureau of Mines and Geology was analyzed by $x$-ray fluorescence and found to contain anomalously high concentrations of $t$ in, tungsten, rubidium, niobium, yttrium, thorium, and uranium. These anomalies are similar to those of other granitic rocks associated with Climax molybdenum deposits (Ludington, 1986). An age of $49 \mathrm{Ma}$ was determined by Blackwell and others (1975) on quartz porphyry, probably related to the porphyritic granite in the drill core, from the Bald Butte area.

Aeromagnetic and gravity maps (McCafferty, Chapter $C$ of this report, figs . $\mathrm{Cl}, \mathrm{C} 3$ ) show prominent anomalies associated with intrusive rocks in the district. A magnetic anomaly high is associated with the Marysville stock (fig. Cl, anomaly 11), which extends to the southwest in the subsurface. The subsurface extent of this body is approximately two times the area of the surface exposure. A gravity low (fig. C3) in the west part of the district may indicate subsurface bodies of granitic composition similar to the granite that underlies the Bald Butte molybdenum prospect (Hanna and others, 1994).

Geochemical anomalies for many elements are present in the district. These 
include arsenic, silver (fig. D13), gold (fig. D12), beryllium, bismuth (fig. D15), copper (fig. D16), molybdenum (fig. D17), manganese (fig. D21), lead (fig. D14), antimony, tin ( $f i g . D 18$ ), tungsten, and zinc (fig. D19) (McDanal and others, 1985). The geochemical anomalies in these figures (Alminas, Chapter D of this report) are for stream sediments and incorporate only the stream sediment data of McDanal and others (1985).

\section{Black Mountain-Stemwinder Hill Subregion}

\section{Geologic Setting}

Much of the subregion is underlain by a sequence of Middle Proterozoic, Paleozoic, and Mesozoic meta-sedimentary and sedimentary rocks that are folded into a broad, south-plunging syncline ( 1 1. 1, map A). The lowermost rocks exposed in this syncline are Middle Proterozoic Missoula Group (Be1t Supergroup) and the uppermost are Upper Cretaceous Blackleaf Formation. All of the Paleozoic and nearly all of the Mesozoic rocks, representative of those in the region, are exposed in the syncline.

The southeastern part of the subregion includes plutonic rocks, mainly monzogranite and granodiorite, that are northerly extensions of the Late Cretaceous Boulder batholith. Paleozoic and Mesozoic sedimentary rocks are in contact with these plutonic rocks. In the western part of the subregion, the Blackfoot City granodiorite stock, with an age of $76.6 \mathrm{Ma}$ (Schmidt and others, 1994), was emplaced into the axial portion of the syncline. There are several small exposures of plutonic rocks and moderate-sized areas where Tertiary rhyolitic rocks are exposed. The latter are probably late Eocene and related to the Helena volcanic field (Schmidt and others, 1994).

The major structural features of the subregion are the Bald Butte fault, a broad, south-plunging syncline, and the Sapphire thrust terrane. Imbricate thrust faults along the southwestern margin of this subregion mark the frontal edge of Sapphire thrust plate (Schmidt and others, 1994). This thrust terrane is present throughout much of the Butte $1^{\circ} \times 2^{\circ}$ quadrangle to the west of the Forest and has juxtaposed western lithofacies of Paleozoic and Mesozoic formations against eastern lithofacies of equivalent rock units. Therefore, the Sapphire thrust terrane represents a considerable, but undetermined, amount of crustal shortening. The syncline in the lower plate rocks probably formed concurrently with, and as a result of, the same regional compression that formed the Sapphire thrust. The subregion also has high-angle normal and reverse faults, including a range-front fault along the western side of the subregion that marks the eastern edge of Avon Valley.

\section{Mineral Deposits}

The Black Mountain-Stemwinder Hill subregion includes the Ophir and Austin mining districts and the Stemwinder Hill and Dog Creek areas (fig. F1). Metallic lode deposits include polymetallic vein, skarn gold, polymetallic replacement, skarn tungsten, epithermal vein, and hot spring gold-silver deposits. Nonmetallic deposits include phosphate, limestone, and clay deposits. Erosion of many pre-existing hydrothermal base- and precious-metal deposits and 
transportation and deposition of their gold by streams have resulted in the formation of numerous placer gold deposits. Many of the hydrothermal deposits, such as those in the Ophir and Austin districts, are located at or near contact zones between plutonic rocks and Paleozoic carbonate rocks.

Ophir (Snowshoe Creek-Carpenter Creek) District--The Ophir district is approximately $25 \mathrm{mi}$ west-northwest of Helena and $5 \mathrm{mi}$ northwest of Avon (fig. F1). The northeastern part of the district includes parts of the Forest. This part of the district is underlain by the Late Cretaceous Blackfoot City granodiorite stock, other small intrusives, and folded sedimentary rocks of Middle Proterozoic through Cretaceous ages that were intruded by the Blackfoot City stock. The Avon Valley, in the southwestern part of the district and outside of the Forest, occupies a basin formed by northwest-trending faults near the mountain front ( 1 l. 1). Lode deposits of the district, containing mainly gold, copper, silver, and tungsten, include skarns, polymetallic veins, breccia zones, and irregular replacement bodies. These are primarily in Paleozoic limestone near contacts with granodiorite stocks.

The Ajax and Victory mines were two of the most important mines in the district (Pardee and Schrader, 1933). The workings of the Ajax mine (pl. 2, no. 141) exploited a pipe-shaped body of skarn consisting of magnetite and hematite with minor garnet and quartz hosted by Paleozoic limestone. At the Victory mine (p1. 2, no. 142), ore minerals were found in a pipe-shaped replacement (skarn) zone of brecciated carbonate rock (Jefferson Formation) along the contact of the Blackfoot City stock.

Strata-bound phosphate deposits also occur in the district and are discussed in Chapter I on nonmetallic mineral deposits. Gold placers, discussed in Chapter $\mathrm{H}$ of this report, are in Quaternary alluvium and in Tertiary gravel. The largest and most productive placers were along Ophir and Carpenter Creeks.

The district has been a producer of gold, silver, copper, lead, tungsten, and phosphate. The district produced approximately 185,000 ounces of gold, mainly from placers, and has an estimated total value of production of about $\$ 4$ million (Elliott and others, 1992).

The aeromagnetic anomaly map (McCafferty, Chapter $C$ of this report, fig. $\mathrm{Cl}$, anomaly 8) shows a magnetic high associated with the Blackfoot City stock. In the subsurface, this intrusive body apparently extends to the southwest and is about two times the size (fig. C4) as indicated in mapped surface extent. A gravity low on the west side of the district is coincident with low-density sediments of the Avon Valley (Hanna and others, 1994).

A geochemical survey revealed anomalies for silver (fig. D13), gold (fig. D12), barium, bismuth (fig. D15), copper (fig. D16), iron (fig. D20), molybdenum (fig. D17), manganese (fig. D21), lead (fig. D14), tin (fig. D18), and tungsten (McDanal and others, 1985). The geochemical anomalies in these figures (Alminas, Chapter D of this report) are for stream sediments and incorporate only the stream sediment data of McDanal and others (1985).

Dog Creek Area--The Dog Creek area is approximately $15 \mathrm{mi}$ west of Helena, south of the Marysville district, and east of the Ophir district (fig. F1). The northern part of the area is within the Forest. Most of central and northern parts of the area are underlain by sedimentary rocks ranging in age from Middle Proterozoic to Cretaceous, which are folded into a southeast-plunging syncline. Several imbricated listric thrust faults of the frontal zone of Sapphire thrust 
terrane have been mapped in the southwestern part of area. Normal faults are in northern part of area. The southwestern corner of area contains Tertiary and Quaternary sedimentary rocks and Tertiary rhyolite flows. The area includes a few mineral deposits consisting mainly of gold placers and stratified phosphate deposits of the Permian Phosphoria Formation (discussed in Chapter I of this report). Some vein and skarn deposits are also in the area. The area has produced gold, phosphate, limestone, copper, and silver. Metallic production is mostly of gold (estimated 4,500 ounces) from placers (E1liott and others, 1992).

The aeromagnetic anomaly data (McCafferty, Chapter $\mathrm{C}$ of this report, fig Cl) indicates source rocks with high magnetization near the center of the Dog Creek area (figs. C1, C4). The high is over granitic rock that is mainly in the subsurface, indicated at the surface by small isolated exposures of intrusive rock (p1. 1, map A). The plutonic source rock of this anomaly may be similar in composition to, but somewhat smaller than, other stocks in the region such as the Blackfoot City and Marysville stocks (Hanna and others, 1994).

Although the number and size of metallic mineral deposits in the area is less than some neighboring districts, a large number of elements are geochemically anomalous in the area. A geochemical survey indicated anomalies in arsenic, silver (fig. D13), gold (fig. D12), boron, barium, beryllium, bismuth (fig. D15), copper (fig. D16), molybdenum (fig. D17), manganese (fig. D21), lead (fig. D14), antimony, tin (fig. D18), tungsten, and zinc (fig. D19) (McDanal and others, 1985). The geochemical anomalies in these figures (Alminas, Chapter D of this report) are for stream sediments and incorporate only the stream sediment data of McDanal and others (1985).

Austin District--The Austin district is approximately $10 \mathrm{mi}$ west-northwest of Helena and east of the Dog Creek area (fig. F1). The Forest adjoins the western part of the district, which includes part of the northern border of the Late Cretaceous Boulder batholith and a sequence of Middle Proterozoic, lower Paleozoic, and upper Paleozoic sedimentary rocks. This sedimentary sequence has been cut by numerous normal faults and intruded by several small bodies of Late Cretaceous granodiorite and Tertiary dacite. Mineral deposits include polymetallic vein, copper-gold skarn, and placer deposits. The district produced silver, copper, lead, gold, iron, zinc, and tungsten and has a total estimated value of production of about $\$ 340,000$ (E11iott and others, 1992).

The north edge of a large regional magnetic high (McCafferty, Chapter $C$ of this report, fig. C1) extends into the south part of the district, coincident with the northern end of the Boulder batholith (Hanna and others, 1994).

Geochemical survey results of McDanal and others (1985) indicate anomalies in arsenic, silver ( $f i g . D 13$ ), gold ( $f i g . D 12)$, bismuth (fig. D15), copper (fig. D16), iron (fig. D20), molybdenum (fig. D17), manganese (fig. D21), lead (fig. D14), antimony, tin ( $f i g . D 18$ ), and zinc (fig. D19). The geochemical anomalies in these figures (Alminas, Chapter D of this report) are for stream sediments and incorporate only the stream sediment data of McDanal and others (1985).

Stemwinder Hill Area- - The Stemwinder Hill area is west of Helena (fig. F1). Its western part is mostly in the Forest and is underlain by granodiorite of the Boulder batholith and by the Elkhorn Mountains Volcanics; the eastern part is underlain by a sequence of metamorphosed sedimentary rocks of Middle Proterozoic through late Paleozoic age. Mineral deposits include skarns and veins that contain silver, lead, gold, tungsten, and molybdenum as well as gold placers.

The Dutro mine (pl. 2, no. 198), although a small producer, is of geologic 
interest because of the occurrence of tin (as cassiterite) (Knopf, 1913). The mine produced gold ore from irregular masses of oxidized siliceous, iron-oxide rich zones in Paleozoic dolomite. The mine is situated near a diorite intrusive. The deposit has the geologic characteristics of a skarn gold deposit but with the unusual occurrences of tin minerals. The area produced small quantities of silver, lead, and gold and has an estimated total value of production of about $\$ 118,000$ (E11iott and others, 1992).

The aeromagnetic anomaly map (McCafferty, Chapter $C$ of this report, fig. C1) shows the western two-thirds of the area is coincident with a magnetic anomaly high (within heavy dashed line of fig. C1, C4) that marks the approximate northeastern edge of the Boulder batholith (Hanna and others, 1992).

A complex geochemical environment is indicated by anomalies in silver (fig. D13), gold (fig. D12), bismuth (fig. D15), copper (fig. D16), iron (fig. D20), molybdenum (fig. D17), manganese (fig. D21), lead (fig. D14), tin (fig. D18), tungsten, and zinc (fig. D19) (McDanal and others, 1985). The geochemical anomalies in these figures (Alminas, Chapter D of this report) are for stream sediments and incorporate only the stream sediment data of McDanal and others (1985).

\section{North Boulder Mountains Subregion}

\section{Geologic Setting}

Most of the rocks in the North Boulder Mountains subregion (fig. F1) are igneous in origin and include both plutonic and volcanic units. The dominant rock types are granodiorite and monzogranite that are major phases of the Boulder batholith, which extends into the Elkhorn Mountains region to the east and beyond the Forest to the southeast (Tilling, 1973). Four predominately volcanic map units of regional extent are the Late Cretaceous Elkhorn Mountains Volcanics, Paleocene or Late Cretaceous basaltic and andesitic volcanic rocks, Eocene Lowland Creek Volcanics, and Tertiary rhyolite (p1. 1). The Elkhorn Mountains Volcanics range in composition from rhyolite to basalt, but consist mostly of andesitic rocks, generally predate the Boulder batholith but plutonism associated with the emplacement of the batholith probably commenced before the volcanism ceased (Tilling, 1973). Basaltic and andesitic volcanic rocks in the northwest part of the subregion may be correlative with the Elkhorn Mountain Volcanics, but this correlation is uncertain due to obscure field relations and lack of geochronometric data. After the youngest plutonic rocks of the Boulder batholith were emplaced, about $68 \mathrm{Ma}$, and a period of quiescence, igneous activity was renewed during the Eocene when Lowland Creek Volcanics were formed about $50 \mathrm{Ma}$ (Smedes and Thomas, 1965). These rocks are predominantly quartz latite to dacite in composition. The youngest volcanic episode in the North Boulder Mountains subregion is represented by Tertiary rhyolitic rocks. These cover older rocks and are exposed at higher elevations along the Continental Divide. They are part of the Helena volcanic field with ages in the range of 37 to $39.8 \mathrm{Ma}$ (Schmidt and others, 1994).

Paleozoic and Mesozoic sedimentary rocks, older than the Late Cretaceous Elkhorn Mountain Volcanics, are present in the western part of the North Boulder Mountains subregion forming part of the leading edge of the Sapphire thrust terrane, as described in Chapter B of this report. 
Mineral Deposits

The Boulder batholith played a major role in the metallogeny of southwestern Montana, including a large part of the Forest. The batholith and older rocks within or adjacent to the batholith host a great number and variety of mineral deposits. Many of the largest and richest mines and districts in Montana, including the famous Butte district, are spatially and genetically related to the batholith. Many of the mineral deposits are probably just slightly younger than the batholith and formed from late-stage hydrothermal emanations of magmas that formed the batholith.

The North Boulder Mountains subregion includes all or parts of the Helena, Elliston, Rimini, Clancy, Basin, Wickes, Boulder, and Amazon districts and the North Boulder Mountains area (fig. F1). The predominant deposit type is this subregion is polymetallic veins. Of the approximately 180 known mines and prospects in the subregion, about 150 ( 83 percent) are of this type. The next most abundant deposit type is placer gold, which results from secondary processes of erosion of lode deposits and transportation and deposition of their gold by streams. Other secondary metallic deposits in the subregion include bog manganese and bog copper deposits. Other metallic deposits in the subregion include hot-spring gold-silver, breccia pipe polymetallic, porphyry coppermolybdenum, epithermal manganese, and skarn gold deposits. Nonmetallic deposits include limestone and silica.

Helena (Last Chance) District--Most of the Helena district is south and southwest of Helena, where it includes Forest lands, but the district also includes the gold placers along Last Chance Gulch in Helena (fig. F1). Rocks of the Helena district are mainly limestone, shale, and sandstone of Middle Proterozoic, Paleozoic, and Mesozoic ages that have been folded, faulted, and, along the south and west parts of district, intruded by Late Cretaceous monzogranite and granodiorite of the Boulder batholith.

Lode deposits are gold-bearing veins in granodiorite and polymetallic vein and skarn gold deposits in Paleozoic limestone at or near contacts with granodiorite. Last Chance Gulch was a major producer of gold from placers (Koschmann and Bergendah1, 1968). The sources of gold for placers were goldbearing skarn and polymetallic deposits along the contact of the Boulder batholith with sedimentary rocks. The most important deposits of these types are the Spring Hill mine (p1. 2, no. 204), a skarn deposit; Whitlach-Union mine (no. 205), a polymetallic vein; and Big Indian mine (no. 208), a polymetallic vein deposit. The district was a very large producer of gold (over one million ounces, as estimated by Elliott and others, 1992), mainly from placers. The total value of production is estimated at more than $\$ 28$ million (E1liott and others, 1992).

The aeromagnetic anomaly map (McCafferty, Chapter $C$ of this report, fig. C1) shows a prominent anomaly high across the southern part of the Helena district. The anomaly coincides with the mapped area of the strongly magnetic Unionville granodiorite ( $p 1.1$ ) that forms the northern rim of the Boulder batholith in this area (Hanna and others, 1994; within heavy dashed line on figs. $\mathrm{Cl}, \mathrm{C} 4)$.

A geochemical survey of McDanal and others (1985) showed that numerous elements are present in anomalous concentrations in the district. These elements include arsenic, silver (fig. D13), gold (fig. D12), boron, beryllium, copper 
(fig. D16), molybdenum (fig. D17), manganese (fig. D21), lead (fig. D14), antimony, tin (fig. D18), and zinc (fig. D19) (McDanal and others, 1985). The geochemical anomalies in these figures (Alminas, Chapter D of this report) are for stream sediments and incorporate only the stream sediment data of McDanal and others (1985).

E11iston District--The E1liston district is approximately $20 \mathrm{mi}$ southwest of Helena and mostly within the Forest (fig. F1). The district is underlain by andesitic tuff, breccia, and flows of the Elkhorn Mountains Volcanics and monzogranite of the Boulder batholith. Mineral deposits are polymetallic veins in volcanic rocks and monzogranite and small gold placers. The Big Dick mine was the largest producer; its workings follow two polymetallic veins in andesite breccia. Ore shoots up to $3.5 \mathrm{ft}$ thick were mined and some ore averaged 5 opt gold (Pardee and Schrader, 1933). The district produced gold, silver, lead, and copper with a total value of recorded production of about $\$ 3.1$ million.

Several connected magnetic highs in the eastern half of the district (MCafferty, Chapter $\mathrm{C}$ of this report, fig. C1) are due mainly to plutonic rocks of the Boulder batholith and, possibly, also in part to the Elkhorn Mountain Volcanics (Hanna and others, 1994). A steep north-trending magnetic gradient through the center of the district marks the approximate western margin of the batholith (heavy dashed line on figs. C1, C4).

Geochemical anomalies in arsenic, silver (fig. D13), gold (fig. D12), barium, beryllium, bismuth ( $f i g . D 15$ ), copper (fig. D16), manganese (fig. D21), lead (fig. D14), tin (fig. D18), tungsten, and zinc (fig. D19) are present in the district (McDanal and others, 1985). The geochemical anomalies in these figures (Alminas, Chapter D of this report) are for stream sediments and incorporate only the stream sediment data of McDanal and others (1985).

Rimini (Vaughn) District--The Rimini district is about $12 \mathrm{mi}$ southwest of Helena, mostly within or enclosed by the Forest (fig F1). The principal rocks in the district are Late Cretaceous monzogranite and aplite of the Boulder batholith. Along the northern margin of the district the batholith intruded andesitic rocks of the Elkhorn Mountains Volcanics. Tertiary rhyolitic volcanic and volcaniclastic rocks locally cover the older plutonic rocks of the batholith. The principal mineral deposits are polymetallic veins in granitic rocks that contain silver, lead, gold, and locally, copper and zinc. The district was a very large producer (estimated total value of production of more than $\$ 27$ million, Elliott and others, 1992) of gold, silver, lead, zinc, and copper.

The recently active Paupers Dream (Basin Creek) mine (p1. 2, map B, no. 278) has characteristics of a hot spring gold-silver deposit. The gold mineralization consists of fracture-controlled quartz veins, zones of silicified stockwork veins, argillized shear zones, and disseminated minerals in Tertiary volcanic and volcaniclastic rocks. In 1989 , the mine reportedly had proven reserves of 5 million tons and probable reserves of 7.5 million tons, both with an average grade of 0.042 opt gold (McCulloch, 1989).

Aeromagnetic and gravity maps (McCafferty $\mathrm{C}$ of this report, figs. C1, C3) show anomalies associated with mineralized areas. The district is entirely within the batholith and a magnetic anomaly low may coincide either with less magnetic plutonic rocks of the batholith or with areas of hydrothermal alteration, or a combination of both (Hanna and others, 1994). A gravity low in the southeast part of the district (fig. C3) is part of a large low that covers parts of the Clancy, Basin, Wickes, Boulder, and Amazon districts. The gravity 
low is mainly associated with the batholith (Hanna and others, 1994).

A geochemical survey showed anomalies for arsenic, silver (fig. D13), gold (fig. D12), boron, bismuth (fig. D15), copper (fig. D16), lead (fig. D14), antimony, tungsten, and zinc (fig. D19) (McDanal and others, 1985). The geochemical anomalies in these figures (Alminas, Chapter D of this report) are for stream sediments and incorporate only the stream sediment data of McDanal and others (1985).

Clancy (Lump Gulch) District--The Clancy district is south of the Helena district and its western part extends into the Helena National Forest (fig. F1). The district is entirely within the Boulder batholith and is underlain by Late Cretaceous granodiorite, monzogranite, alaskite, aplite, and pegmatite. Mineral deposits include polymetallic veins and gold placers. The principal lode deposits are veins consisting of chalcedonic quartz with sulfides, mainly galena, sphalerite, and tetrahedrite, in monzogranite of the batholith; the deposits were valuable mainly for silver (Knopf, 1913). The principal lode mines are the Little Nel1 (p1. 2, no. 305), Free Coinage (no. 301), and King Solomon (no. 306) mines. The largest placers were along Clancy and Prickly Pear Creeks (Lyden, 1948). The district produced gold, silver, and lead with a total value of recorded production of about $\$ 2.8$ million.

The magnetic anomaly map (McCafferty, Chapter $C$ of this report, fig. C1) shows a magnetic anomaly low on the west side of the district. The anomaly may be associated with less magnetic plutonic phases or with hydrothermal alteration, as in the Rimini district (Hanna and others, 1994).

Geochemical anomalies are present for the following elements: arsenic, silver (fig. D13), gold (fig. D12), bismuth (fig. D15), copper (fig. D16), molybdenum (fig. D17), manganese (fig. D21), lead (fig. D14), antimony, tungsten, and zinc (fig. D19) (McDanal and others, 1985). The geochemical anomalies in these figures (Alminas, Chapter $D$ of this report) are for stream sediments and incorporate only the stream sediment data of McDanal and others (1985).

Basin (Cataract) District--The Basin district is approximately $20 \mathrm{mi}$ southwest of Helena (fig. F1). The northern part of the district is adjacent to, but does not include Forest lands. The district is underlain by monzogranite of Boulder batholith and Tertiary and Cretaceous volcanic and volcaniclastic rocks. Most of the mineral deposits are in the eastern part of the area and hosted by granitic rocks of the batholith. These deposits include polymetallic veins and placer deposits.

Principal mines include the Custer, Josephine, and Crystal mines; these are polymetallic veins in monzogranite. The Crystal vein is along a fault zone that trends about $\mathrm{N} .75 \mathrm{~W}$. and dips about $70 \mathrm{~N}$. (Ruppel, 1963). The Crystal vein has been developed along a length of about $6 \mathrm{mi}$, explored to a depth of 1,500 ft, and has an average width of $30 \mathrm{ft}$ (Pardee and Schrader, 1933). Ore shoots are discontinuous and form separate fissures and overlapping lenses within the zone of altered country rock.

The district was a large producer with an estimated total value of production of about $\$ 15.6$ million (E11iott and others, 1992). Principal products were gold, silver, copper, lead, and zinc. A geochemical survey (McDanal and others, 1985) indicated anomalies in arsenic, silver (fig. D13), gold (fig. D12), boron, barium, beryllium, bismuth (fig. D15), copper (fig. D16), molybdenum (fig. D17), manganese (fig. D21), lead (fig. D14), antimony, tin (fig. D18), tungsten, and zinc (fig. D19). The geochemical anomalies in these figures 
(Alminas, Chapter D of this report) are for stream sediments and incorporate only the stream sediment data of McDanal and others (1985).

Wickes (Corbin) District--The Wickes district is approximately $15 \mathrm{mi}$ south of Helena and its western part includes Forest lands (fig. F1). It lies within the Boulder batholith and includes a large inlier of volcanic and volcaniclastic rocks, which consist of Elkhorn Mountains Volcanics and Lowland Creek Volcanics. Most mines and prospects in the district are in volcanic and plutonic rocks of the Elkhorn Mountains Volcanics and the Boulder batholith, which are of Late Cretaceous age. In contrast, the Montana Tunnels mine ( $\mathrm{pl}$. 2, no. 374) is a large-tonnage, low-grade gold-silver-lead-zinc deposit that is in Lowland Creek Volcanics, which is of Eocene age. Most mineral deposits are polymetallic veins in plutonic and volcanic and volcaniclastic rocks.

One of the most productive mines in the region, the Alta lead-silver mine (p1. 2, map B, no. 381), is located in the Wickes district. This deposit was discovered in 1869 and is thought to have produced more than 1.25 million tons of ore valued at $\$ 32$ million before 1893 (Pardee and Schrader, 1933). Between 1901 and 1948, about 10,000 tons of ore were produced and nearly 250,000 tons of tailings were reprocessed (Becraft and others, 1963). The ore bodies of the Alta mine apparently consisted of large overlapping lenses, veins, and replacement bodies along a well-defined east-trending shear zone in rocks of the E1khorn Mountains Volcanics. The ore minerals, consisting of galena, pyrite, tetrahedrite, and minor sphalerite, are in a gangue of mainly altered wallrock. The ore zone is about $1,600 \mathrm{ft}$ along strike and was mined to a depth of about $1,400 \mathrm{ft}$ (Becraft and others, 1963).

The Montana Tunnels deposit (p1. 2, no. 374), presently being mined, contains ore minerals of gold, silver, lead, and zinc as disseminations and veinlets in diatreme and hydrothermal breccias. The host rocks are dacitic lithic and crystal-rich welded ashflow tuffs and a dacite porphyry stock of the Eocene Lowland Creek Volcanics, which forms an inlier in the Boulder batholith (Sillitoe and others, 1985). The year-end 1990 reserves at Montana Tunnels were 32.1 million tons of proven and 2.2 million of probable reserves at a grade of 0.019 opt gold (Engineering and Mining Journal, 1991).

A stockwork copper-molybdenum prospect (p1. 2, no. 384) also occurs in monzogranite in the eastern part of the district. The district is very large producer (estimated total value of more than $\$ 125$ million, Elliott and others, 1992) and principal products are silver, gold, lead, copper, and zinc.

In the center of the district, a magnetic anomaly low (McCafferty, Chapter $C$ of this report, fig. C1) coincides with the area of exposure of the Lowland Creek and Elkhorn Mountain volcanic and volcaniclastic rocks (p1. 1). Much of the area is hydrothermally altered, and several large mineral deposits, including the Montana Tunnels and Alta mines, are within this magnetic low. The main cause of the low may be the hydrothermal alteration (Hanna and others, 1994).

Geochemical anomalies (McDanal and others, 1985) in arsenic, silver (fig. D13), gold (fig. D12), boron, bismuth (fig. D15), copper (fig. D16), molybdenum (fig. D17), manganese (fig. D21), lead (fig. D14), antimony, tungsten, and zinc (fig. D19) are present in the district (McDanal and others, 1985). The geochemical anomalies in these figures (Alminas, Chapter D of this report) are for stream sediments and incorporate only the stream sediment data of McDanal and others (1985). 
Boulder (Comet) District--The Boulder district is adjacent but outside of the Forest ( $f i g . F 1$ ). Most of the district is in granitic rocks of the Boulder batholith, but some of the north part of the district is underlain by Elkhorn Mountains Volcanics and Lowland Creek Volcanics. Nearly all of the deposits are polymetallic veins; one deposit is a gold placer. The district is a large producer of silver, gold, lead, copper, and zinc (Elliott and others, 1992).

The most important mine in the district is the Comet mine ( 1 . 2, no. 340). Discovered in 1874, this mine is reported to have produced silver, lead, zinc, gold, and copper with a total value that exceeded $\$ 20$ million. The mine is located along a strong and persistent regional structure called the Comet-Gray Eagle shear zone (Becraft and others, 1963). This complex shear zone, which trends N. $80 \mathrm{~W}$., cuts plutonic rocks of the Boulder batholith and volcanic rocks of the Elkhorn Mountain Volcanics. Other mines, including the Morning Glory and Gray Eagle, are located to the west on the same structure. The ore bodies at the Comet mine were mined from three main veins and many smaller veins along a 150 $\mathrm{ft}$ wide zone and from near the surface to a depth of about $800 \mathrm{ft}$. Most of the large ore bodies, however, bottomed out between $400 \mathrm{ft}$ and $600 \mathrm{ft}$ depth. The ore consisted of galena, sphalerite, pyrite, arsenopyrite, chalcopyrite, and tetrahedrite in a gangue of quartz, carbonate minerals, and altered wallrock (Becraft and others, 1963).

A magnetic low anomaly (McCafferty, Chapter $C$ of this report, fig. C1), probably associated with hydrothermal alteration, extends southwest from the adjacent Wickes district (Hanna and others, 1994; figs. C1, C4).

Geochemical anomalies (McDanal and others, 1985) are present for the following elements: silver (fig. D13), gold (fig. D12), boron, barium, bismuth (fig. D15), copper (fig. D16), molybdenum (fig. D17), manganese (fig. D21), lead (fig. D14), antimony, tungsten, and zinc (fig. D19). The geochemical anomalies in these figures (Alminas, Chapter D of this report) are for stream sediments and incorporate only the stream sediment data of McDanal and others (1985).

Amazon District--The Amazon district is located adjacent to the Wickes and Boulder districts and outside of the Forest (fig F1). The northwest part of the district is in Elkhorn Mountains Volcanics and the remainder of the district is in granitic rocks of the Boulder batholith. All of the known mines and prospects, for which information is available, are polymetallic veins. The Australian (p1. 2, no. 344) and Bismarck (no. 345), two of the most productive mines, are both located on the same vein. This vein strikes N. $75 \mathrm{E}$. and dips 65-89 N. and is about 8 to $15 \mathrm{ft}$ wide. Wall rock along the vein is monzogranite that is intensely altered to clay minerals and sericite (Becraft and others, 1963). The vein consists of sulfide minerals, mainly galena and sphalerite, in quartz and is valuable for gold and silver. The district produced silver, lead, gold, and copper with a total value of recorded production of about $\$ 120,000$.

A magnetic anomaly low trends southwest along the west side of the district (McCafferty, Chapter $\mathrm{C}$ of this report, within heavy dashed line of figs. Cl, C4) and is probably related to hydrothermal alteration (Hanna and others, 1994).

Geochemical anomalies in arsenic, boron, barium, lead (fig. D14), and zinc

(fig. D19) are present in the district (McDanal and others, 1985). The geochemical anomalies in these figures (Alminas, Chapter D of this report) are for stream sediments and incorporate only the stream sediment data of McDanal and others (1985). 


\section{ELKHORN MOUNTAINS REGION}

By Steve Ludington, Felix E. Mutschler, and Marta Franchini

\section{Introduction}

In this section, we describe geologic attributes of the mining districts and other mineralized areas in the Elkhorn Mountains. A mineralized area is an area whose characteristics are conveniently described together, and, ideally, correspond to regions affected by coherent mineralizing events and to historically defined mining districts. Because of our incomplete knowledge about the Forest and about the genesis of mineral deposits, strict adherence to this guideline is not always possible. In many cases, the groupings do correspond to mining districts, but the boundaries of mining districts are often poorly defined, and, even when known, may be accidents of history. The mineralized areas described here are shown in figure F2. These descriptions are prepared in the context of the geologic summary presented in Chapter B of this report, including the rock-unit descriptions on plate 2, and we assume the reader to be familiar with that summary.

\section{Geologic Setting}

The Elkhorn Mountains region of the Helena National Forest is underlain mostly by Elkhorn Mountains Volcanics and related intrusive rocks and by the Boulder batholith and satellitic stocks, all of Late Cretaceous age (fig. F2). A smaller part of the Forest is underlain by sedimentary strata of Paleozoic to Mesozolc age. In addition, sedimentary rocks of the Middle Proterozoic Belt Supergroup crop out directly south of the area, and presumably lie beneath the Paleozoic strata in most parts of the area. All of these rocks were intruded by igneous dikes and stocks and locally are overlain by related middle and late Tertiary volcanic rocks. The Elkhorn Mountains occupy a tectonically uplifted block, bounded on the east and north by major faults. This uplifted block has been eroded to its present form by glaciers and running water. Hydrothermal mineral deposits formed in the Elkhorn Mountains in concert with four distinct magmatic events, as shown in table F1. There are no hydrothermal deposits of unequivocal Tertiary age.

\section{Mineral Deposits}

\section{Montana City district}

The Montana City district lies directly outside the Forest. The most important metallic deposits are polymetallic veins and replacement deposits south of Helena and west of Interstate 15. The Montana City limestone quarry is in Paleozolc limestones. Similar limestones occur on the eastern margin of the Elkhorn Mountains, mainly outside the Forest, and in the core of the large antiform in the southern part of the region (plate 1). Limestone at Montana City is metamorphosed into marble by heat from the nearby Boulder batholith. 


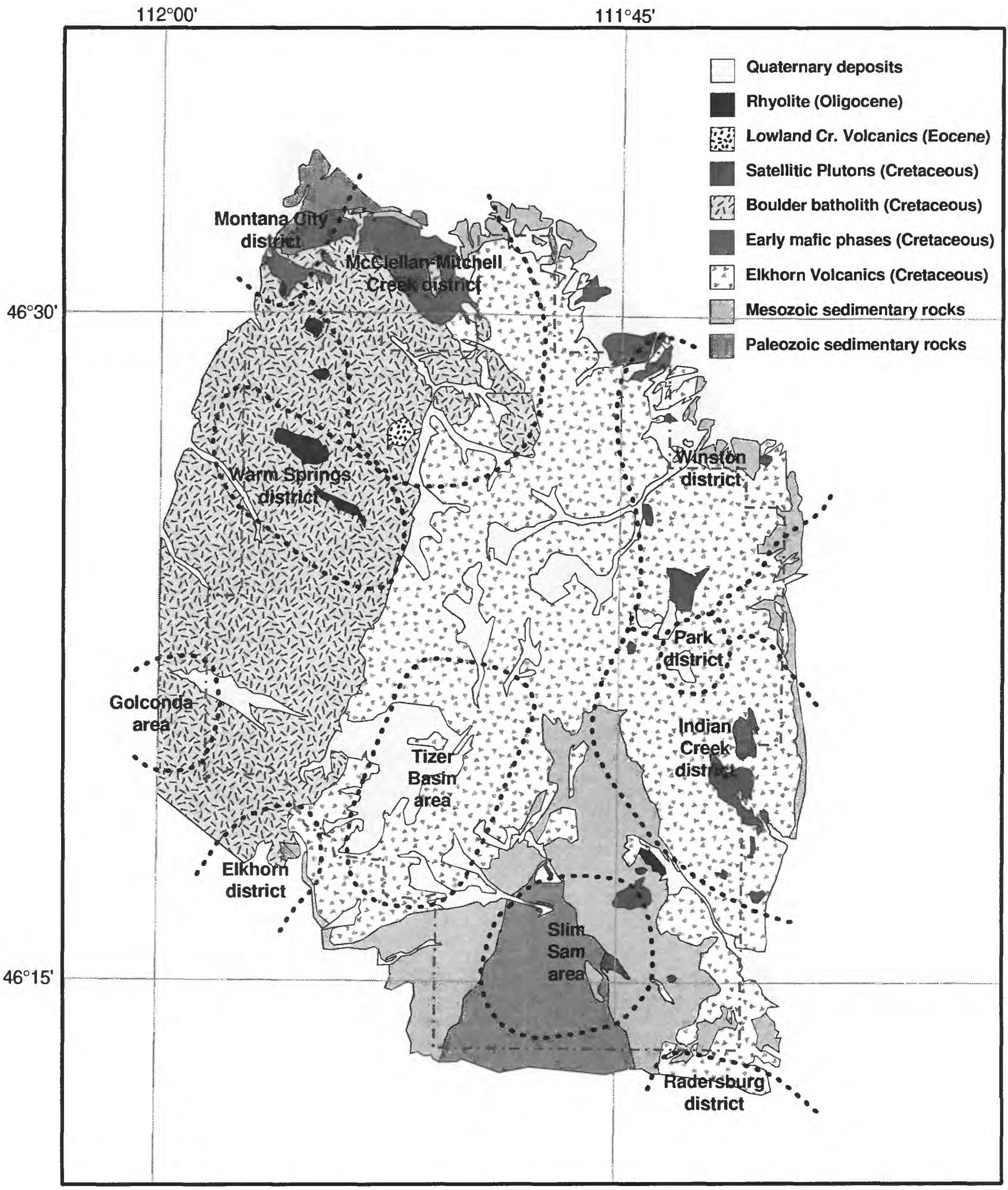

Figure F2. Map of the Elkhorn Mountains region, showing geology, Hele na National Forest boundary, and mining districts and mineralized areas discussed in the text. 
Table F1. Mineral deposits and correlative magmatic events in the Elkhorn Mountains region.

\begin{tabular}{|c|c|c|}
\hline $\begin{array}{l}\text { Magmatic } \\
\text { Event }\end{array}$ & $\begin{array}{l}\text { Age } \\
\mathrm{Ma}\end{array}$ & Type of Mineral Deposit (examples) \\
\hline $\begin{array}{l}\text { Elkhorn Mountains } \\
\text { Volcanics }\end{array}$ & $81-79$ & $\begin{array}{c}\text { Gold-bearing breccia pipes (Skyline, } \\
\text { Black Jack mines }\end{array}$ \\
\hline $\begin{array}{l}\text { Satellitic } \\
\text { stocks }\end{array}$ & $<80,>75 ?$ & $\begin{array}{l}\text { Skarn Au deposits (Diamond Hill, } \\
\text { Elkhorn) } \\
\text { Polymetallic veins (Radersburg, } \\
\text { East Pacific mines) }\end{array}$ \\
\hline $\begin{array}{l}\text { Boulder batholith, } \\
\text { main stage }\end{array}$ & $80-75$ & $\begin{array}{l}\text { Polymetallic veins (Warm Springs } \\
\text { district, McClellan-Mitchell } \\
\text { Creek district) }\end{array}$ \\
\hline $\begin{array}{l}\text { Boulder batholith, } \\
\text { aplites }\end{array}$ & $70-68$ & $\begin{array}{l}\text { Porphyry copper deposits (Golconda } \\
\text { deposit) }\end{array}$ \\
\hline
\end{tabular}

\section{McCle1lan-Mitche11 Creek district}

The remnants of an intriguing hydrothermal system, developed in intrusive rocks of the Boulder batholith, crop out along a tributary to Jackson Creek, in sections 2 and 11 of T.8N., R.2W. (Ludington and Greenwood, 1990) (fig. F3). Rock samples from this area contain up to $1,500 \mathrm{ppm}$ (parts per million) $\mathrm{Cu}, 50$ $\mathrm{ppm} \mathrm{Mo,} \mathrm{and} \mathrm{1,000} \mathrm{ppm} \mathrm{Bi.} \mathrm{Rock} \mathrm{types} \mathrm{vary} \mathrm{widely} \mathrm{in} \mathrm{this} \mathrm{small} \mathrm{area,} \mathrm{and} \mathrm{it} \mathrm{is}$ not clear how much of this apparent variation is due to hydrothermal alteration of the original rocks. Many of the rocks lack potassium feldspar, presumably because of hydrothermal alteration. However, secondary sericite (white mica) is only weakly and sporadically developed. Disseminated pyrite is common, and some samples contain as much as 10 percent sulfide minerals. The area was apparently explored by drilling in the $1960 \mathrm{~s}$, but the results are not known. The nature of this hydrothermal system remains enigmatic. Minor placer workings are present north of this area, downstream on Jackson Creek, but nothing is known of their history (Smedes, 1966).

Elsewhere in the McClellan-Mitchell Creek district, mineral deposits are widely scattered polymetallic vein deposits. Most are quite small, and the largest, the Economy mine, produced only about 2,000 oz (troy ounces) of $\mathrm{Au}$ and is about a mile outside the Forest. The Economy, Eureka, and Last Hope mines all contain skarn minerals (diopside, hedenbergite, garnet), as well as tourmaline, in the gangue and disseminated in the wall rocks; they appear to be similar to 
deposits in the Helena district (p1. 2), and suggest the possibility for undiscovered $\mathrm{Au}$ skarn deposits. The relationship of the polymetallic vein deposits to the Jackson Creek hydrothermal system is not known. Placer deposits have been worked on the lower reaches of both McClellan and Mitchell Creeks, with the deposit on Mitchell Creek, below the Economy mine, being much the larger.

\section{Warm Springs district}

The most important deposits in the Warm Springs district are a series of west-trending polymetallic veins found just south of the oligocene rhyolitic complex on Lava Mountain, in sections 29, 30, 31, and 32 of T8N, R2W. Some workers have speculated over the years that these veins are related to the 01igocene igneous activity (see Billingsley and Grimes, 1918, p. 337, as we11 as the discussion about radioactivity in Smedes, 1966, p. 110). However, the presence of unaltered obsidian in the same structures as the base-metal veins indicates that hydrothermal activity ceased before emplacement of the rhyolite. Perhaps uranium related to the rhyolite has subsequently been concentrated in some of the vein structures because of their permeability.

Placer deposits were discovered on Warm Springs Creek as early as 1865, but they were apparently not extensive, and little is known about the history of their exploitation (Smedes, 1966). All these deposits in the Warm Springs district are inside the Forest, and some of the ore produced in the past was very rich, but there has been little activity in recent years. There is little indication of potential in this district for the occurrence of any type of large, low-grade deposit that would be attractive today.

\section{$\underline{\text { Golconda }} \underline{\text { area }}$}

The Golconda porphyry copper-molybdenum deposit (possibly also known as Anderson Gulch) was apparently discovered by geologists of the Exxon Corporation in the early 1970s. The mineralization is primarily in, and closely related to, a large aplite body that intrudes the Boulder batholith. The deposit is in Anderson Gulch and Golconda Creek, directly south of Prickly Pear Creek, immediately adjacent to the Forest boundary, in sections 16, 17, 20, and 21 of T.7N., R.3W. (fig. F3). The area affected by mineralization and hydrothermal alteration is about 4-5 sq. mi.; perhaps 10 percent of that area lies within the Helena National Forest. Geologic details can be found in Ludington and Greenwood (1990). In 1977, exploration was in progress, and at least 4 diamond drill holes of unknown depth were completed at that time. Nothing is known of the subsequent exploration history. Though most of the potential ore lies outside the Forest, any development of this prospect would impact Forest lands.

Pripheral to the Golconda mineral system, but not clearly related to it, are a number of northeast-trending polymetallic veins, notably the Reddings, Big Chief, and Golconda or Golden Assets (fig. F2). These veins are typical of many polymetallic veins in the Boulder batholith, consisting of fault breccias, cemented by quartz and sulfide minerals, chiefly pyrite, galena, and sphalerite (Becraft and others, 1963; Stone, 1911). The Golden Assets property, a 1ittle more than a mile west of the Forest, was being developed in 1993 as a small openpit mine to exploit low-grade Au-mineralized rock that envelops the veins proper. 


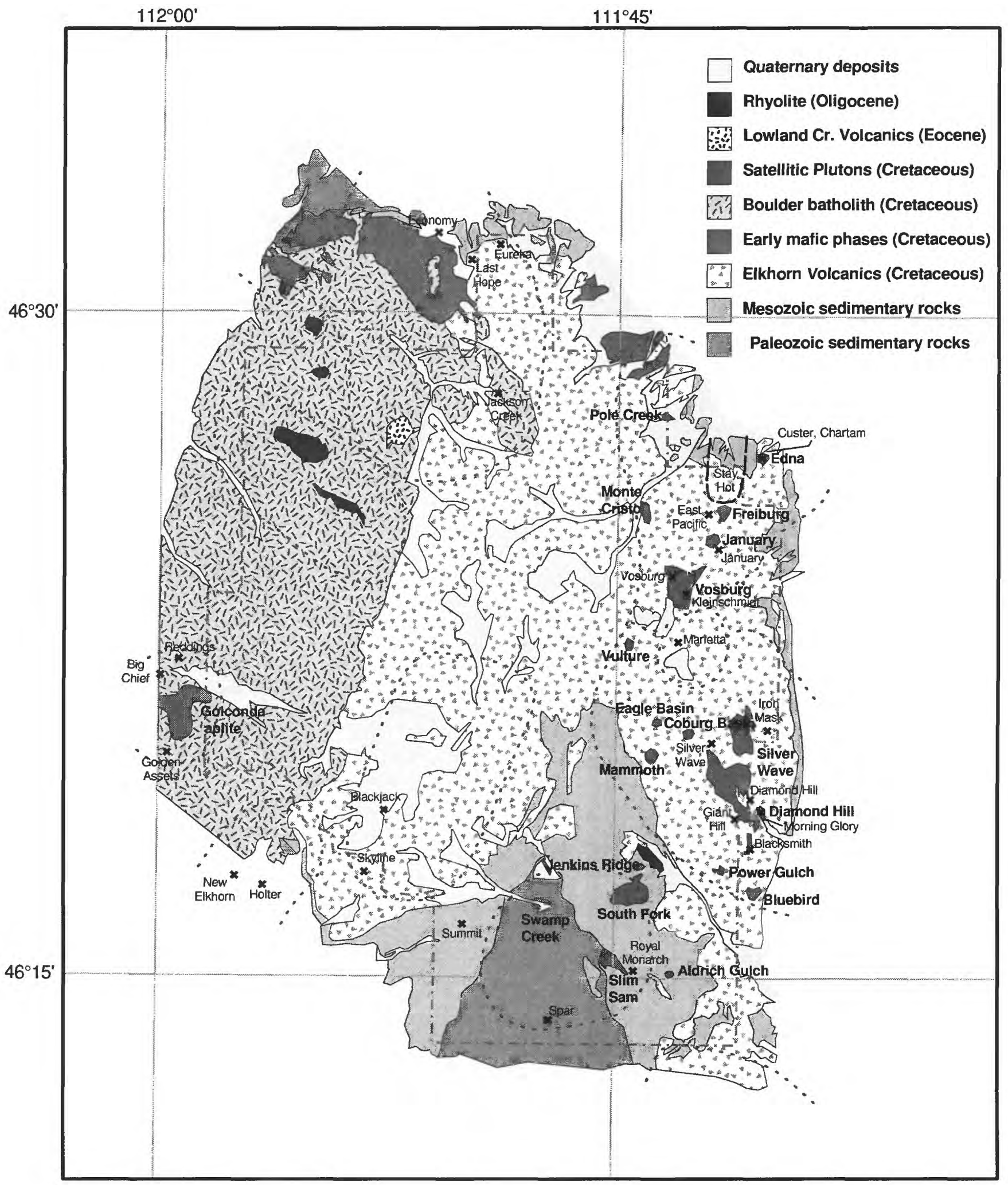

Figure F3. Map of the Elkhorn Mountains region, showing geology, Hele na National Forest boundary, and names of satellitic plutons (larger type) refe rred to in text. Mining districts and areas from fig. F2 are subdued and names are omitted. Sp ecific deposits and prospects referred to in the text are shown by $*$, with names in small type. 
Minor heap-leaching of ore from this deposit has been done in the last decade. Reserves were reported to be about 575,000 st (short tons) at a grade of 0.06 $\mathrm{oz} / \mathrm{st} \mathrm{Au}$ and $8.5 \mathrm{oz} / \mathrm{st} \mathrm{Ag} \mathrm{(Private} \mathrm{proprietary} \mathrm{report).}$

Minor placer deposits were exploited on Golconda and Prickly Pear Creeks (Stone, 1911).

\section{Elkhorn district}

We describe the Elkhorn district briefly, even though it is in the Deer Lodge National Forest, because it is the site of one of the most significant historic mines in the area, and is also the site of an important modern mineral discovery that is being actively explored. The new discovery also shows some similarities to the Diamond Hill deposit on the east flank of the Elkhorn Mountains.

The most important historic deposit in the Elkhorn district was exploited by the Holter (Elkhorn) mine, immediately adjacent to the historic townsite. In the years between, 1875 and 1952, this mine produced more than 500 st of $\mathrm{Ag}$ and about 7,000 st of $\mathrm{Pb}$ (Roby and others, 1960). This deposit, which at times was the leading Ag producer in the United States, would have a gross value of more than $\$ 100$ million at today's prices. It consists of replacement bodies along the contact between the dolomite of the Pilgrim Formation and the overlying Red Lion Formation, both Cambrian in age. The orebody had the form of two steep, stratabound shoots, localized along the crests of minor folds in the limestone, and was mined from the surface to depths of about $1,500 \mathrm{ft}$. Most of the ore consisted of argentiferous (silver-bearing) galena; it was not demonstrably related to any nearby igneous body.

In 1982, the Gold Fields Mining Corporation began exploration in the district, looking to develop new deposits of silver. Instead, they soon encountered gold-bearing skarn deposits to the north and west of the townsite. After considerable exploration and development during the 1980s, the property passed into the hands of the Santa Fe Pacific Minerals Corporation in 1993, and, as of the middle of 1995, drilling and permitting work was ongoing. The new deposit is reported to contain nearly 900,000 oz of Au (Giancola and Razvi, 1995).

In the southern part of the district is Turnley Ridge, a very low-grade low-fluorine molybdenite system that was drilled in the late 1970s, apparently with discouraging results (Ludington and Greenwood, 1990).

\section{$\underline{\text { Tizer }} \underline{\text { Basin }}$ area}

The Tizer Basin mineralized area, within the Forest, is characterized by two kinds of mineral deposit, polymetallic veins and tourmaline-bearing breccia pipes. The veins all strike northeast, similar to others in districts to the west and north, and produced $\mathrm{Cu}, \mathrm{Pb}, \mathrm{Zn}, \mathrm{Au}$, and $\mathrm{Ag}$ in small quantities. The total amount of ore produced is little more than $11,000 \mathrm{st}$, and none of the deposits were developed to a depth as great as $700 \mathrm{ft}$ (Klepper and others, 1957).

One of these deposits was examined during this study, an unnamed prospect in the northeast corner of section 3, T. $6 \mathrm{~N}$., R. $2 \mathrm{~W}$. Much of the exploration of this area appears to have taken place in the decades after World War II. 
Numerous trenches and prospect pits are developed in the Elkhorn Mountains Volcanics, near the base of the welded tuff units mapped by Klepper and others (1957). The material examined consisted largely of thin drusy quartz veins that cut the volcanic rocks along an $\mathrm{E}-\mathrm{W}$ trend that continues for at least $1,000 \mathrm{ft}$. Galena, sparse pyrite, and copper oxide were the only metallic minerals noted. Two geochemical samples were collected (HL44 and HL45, Table F2) that were anomalous in $\mathrm{Ag}, \mathrm{Au}, \mathrm{Cu}, \mathrm{Pb}$, and $\mathrm{Zn}$, and contained nearly $300 \mathrm{ppm} \mathrm{Ag}$ and as much as $1.6 \mathrm{ppm} \mathrm{Au}$.

Breccia pipes at the Blackjack and Skyline mines are both composed of fragments of Elkhorn Mountains Volcanics, cemented by quartz, tourmaline, and sulfide minerals. They were investigated in some detail by Ludington and Greenwood (1990). The Skyline is the larger of the two, shows more base-metal sulfide minerals in outcrop, and contains anomalous amounts of Mo, $\mathrm{Sn}$, and $\mathrm{W}$, whereas the Blackjack exhibits only pyrite at the surface, and contains only anomalous Mo. Both deposits could indicate the presence of a pluton at depth.

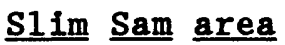

Production from scattered mines in this area within the southern part of the Forest (fig. F2) has been negligible. However, some exploration has taken place, and several long-abandoned mines yielded interesting geochemical results during the present study.

The most significant modern exploration effort in the area was known as the Royal Monarch. Hecla Mining Company geologists apparently recognized the potential for skarn Au deposits surrounding the S1im Sam stock in 1987, and substantial exploration efforts ensued. In 1989, Molycorp Inc. apparently completed 8 diamond drill holes in the area, of unknown depth, and with unknown results. Our studies added little information, although we can confirm that skarns exist in the area. Three mineralized samples were taken from the surface. One of them (HL36B, Table F2) contained about $300 \mathrm{ppm}$ Mo and nearly $20 \mathrm{ppm} \mathrm{Au} \mathrm{(>}$ $0.5 \mathrm{oz} / \mathrm{t}$ ).

At the Summit claim, Klepper and others (1957) reported silicification and limonite in argillaceous siltstone in the lower part of the Jurassic Morrison Formation. A brief visit revealed no more details, but a composite geochemical sample (HL71, Table F2) was surprisingly anomalous in $\mathrm{Ag}, \mathrm{As}, \mathrm{Au}, \mathrm{Cd}, \mathrm{Mo}, \mathrm{Pb}, \mathrm{Sb}$, $\mathrm{Sn}$, and $\mathrm{Zn}$, containing more than $60 \mathrm{ppm} \mathrm{Ag}$ and about $1 \mathrm{ppm} \mathrm{Au}$.

At the Spar mine, a small polymetallic replacement deposit in the Devonian Jefferson Dolomite produced a small amount of ore in the first half of this century (Klepper and others, 1957). The material that remains on the dumps consists primarily of brecciated chalcedony and barite that apparently has replaced limestone and dolomite. Two samples from the dumps (HL72, HL73, Table F2) were anomalous in $\mathrm{Ag}, \mathrm{As}, \mathrm{Au}, \mathrm{Cu}, \mathrm{Pb}, \mathrm{Sb}$, and $\mathrm{Zn}$, and contained as much as $200 \mathrm{ppm} \mathrm{Ag}, 500 \mathrm{ppm} \mathrm{Sb}$, and about $0.3 \mathrm{ppm} \mathrm{Au}$.

\section{Radersburg district}

The Radersburg district is almost entirely outside the Forest, with the richest mines about $4 \mathrm{~km}$ distant from the boundary (fig. F2). Historic 
production for the district is estimated to be about 11.1 st of $\mathrm{Au}$ and about 9.2 st of $\mathrm{Ag}$, along with modest amounts of $\mathrm{Cu}$ and $\mathrm{Pb}$, and small amounts of $\mathrm{Zn}$ (this study; Koschmann and Bergendah1, 1968). This production would have a gross value of more than $\$ 130$ million at today's prices. Most of this production came from two adjacent mines, the Keating and Ohio-Keating, which exploited veins that consist primarily of auriferous pyrite, along with minor gangue of quartz and calcite. Other sulfide minerals include chalcopyrite, bornite, chalcocite, galena, pyrite, pyrrhotite, and marcasite. At the Keating mine, these veins were developed to depths of $>1,300 \mathrm{ft}$.

A gold-rich porphyry copper system lies at depth beneath the district, apparently in the vicinity of the Keating and Ohio-Keating deposits. Indications of this deposit were known as early as the 1970s, when the prospect was first drilled to depths of several hundred feet. The system, related to a granodiorite with large potassium feldspar phenocrysts, is covered by $300-1,000 \mathrm{ft}$ of alluvium. Hydrothermal alteration is concentrically zoned around a potassium feldspar core zone that is nearly a mile in diameter. Fluid inclusions also display zonation, with filling temperatures of about $460^{\circ} \mathrm{C}$. for inclusions with 40-60 percent salinity in the center, indicating fluids typical of porphyry copper deposits. The best drill intercept was an interval of $>600 \mathrm{ft}$ with grades of about 0.25 percent $\mathrm{Cu}$ and $0.01 \mathrm{oz} / \mathrm{st} \mathrm{Au}$. The information presented in this paragraph was presented P.W. Mitchell at the July 1993 meeting of the Tobacco Root Geolocical Society, held near Whitehall, Montana.

Table F2 (facing page). Chemical analyses of mineralized rocks from the E1khorn Mountains, Helena National Forest, Montana.

[All values in parts per million, except $F e$, which is in weight percent. The samples were analyzed by 3 different methods: ICP-AES -40 element, ICP-AES-10 element, with organometallic halide extraction, and gold by Atomic Abosrbtion flame methods, with dissolution in $\mathrm{HF}$, aqua regia, and $\mathrm{HBr}-\mathrm{Br}_{2}$ (see Arbogast, 1996, for details). Detection limits among these methods vary. Where the same sample was analyzed for the same element by more than one method, an average is reported. The samples are listed in order of decreasing north latitude, i.e., from north to south.] 


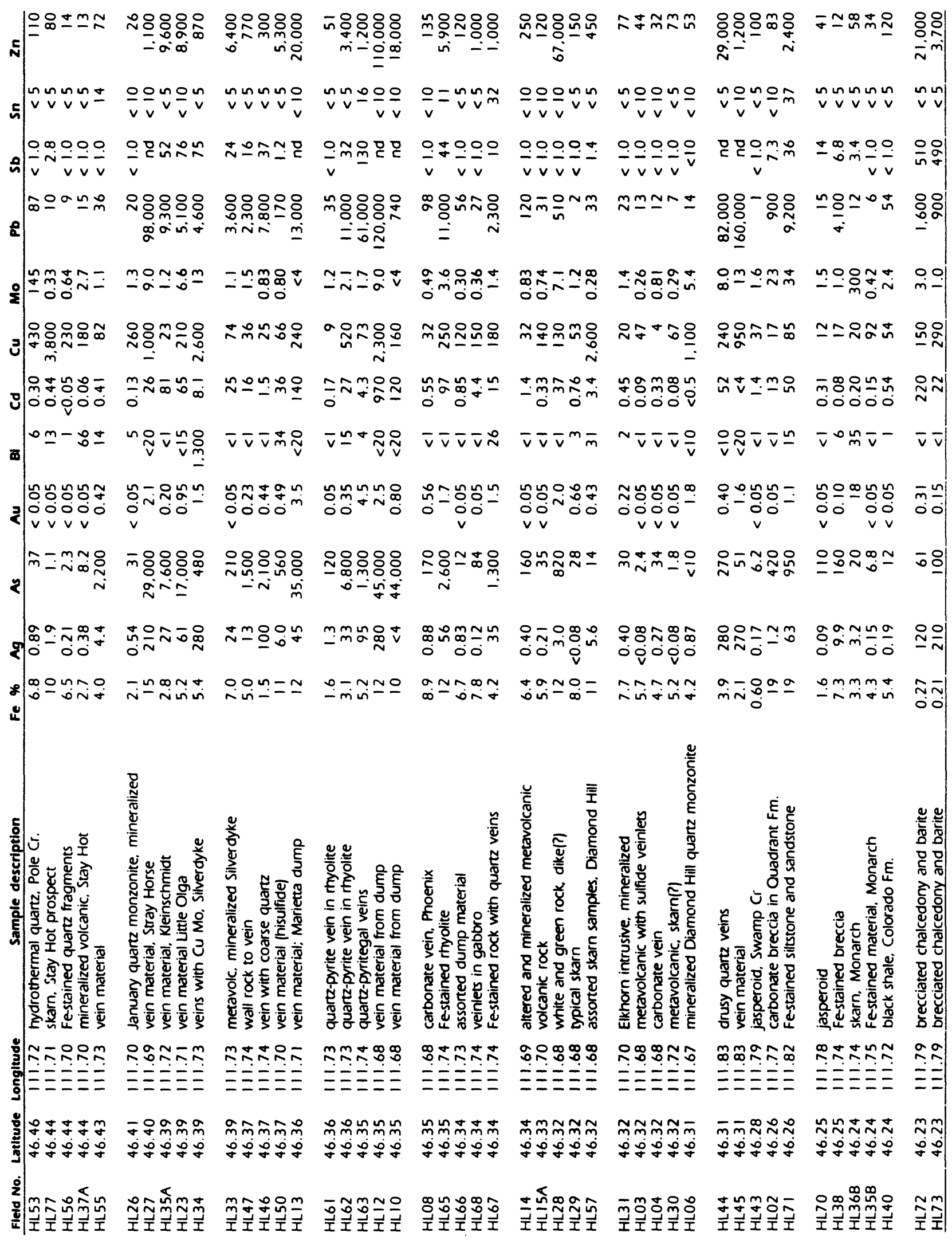




\section{Indian Greek district}

There are a number of important mineral deposits and prospects surrounding both bodies of the dioritic Silver Wave stock, which is one of the satellitic stocks that are marginal to the Boulder batholith along the eastern margin of the Elkhorn Mountains. There are numerous smaller deposits scattered throughout the district as well.

\section{Silver Wave vicinity}

Along the north margin of the Silver Wave stock, on the old Silver Wave and Silver Dollar claim groups, an exploration program was conducted within the Forest by Molycorp Incorporated in 1988 and Pathfinder Gold Corporation in 1989 (area marked Silver Wave on fig. F3). Surface rock samples indicated the possibility of good $\mathrm{Au}$ and $\mathrm{Ag}$ mineralization with strong base metals. Many samples contained more than 1 percent $\mathrm{Pb}, 0.05-0.1$ percent $\mathrm{Cu}$, and 0.1-0.5 percent $\mathrm{Zn}$, along with $\mathrm{Au}$ values $>0.01 \mathrm{oz} / \mathrm{st}$ and $\mathrm{Ag}>1 \mathrm{oz} / \mathrm{st}$. Drilling results (about 9 shallow holes) suggest that the material between the veins is not strongly mineralized, as composite assays are much lower, seldom exceeding 100 $\mathrm{ppb} \mathrm{Au}$. All precious-metal vein intercepts showed significant lead and zinc mineralization. The high base metals suggest that the mineralization in this area is related to the Marietta mine to the north, in the Park district, not to the Diamond Hill orebody to the south. Two rock samples collected from this area (HL14, HL15A, Table F2) yielded equivocal results on this question; they are weakly anomalous in $\mathrm{Zn}$, but do not contain large amounts of $\mathrm{Pb}, \mathrm{Cu}$, $\mathrm{As}$, or precious metals. The near-surface potential of this area has probably been adequately tested, but the nature of deeper portions of the hydrothermal system remains unknown.

\section{Giant $\underline{\text { Hi11 }}$}

At Giant Hill, more than 20 shallow (130-300 ft) holes were drilled by Pegasus Gold Incorporated in 1991. These holes were drilled at a $45^{\circ}$ angle to explore a series of gold-bearing veins that are detectable at the surface. We are indebted to Greg Wittman and Mike Maslowski of Pegasus for information about this project. The rocks exposed at the surface are andesites of the lower member of the Elkhorn Mountains Volcanics, intruded by diorite of the Silver Wave stock. Much of the rock is affected by argillic alteration and contains moderate amounts (as much as 3 percent) of disseminated pyrite. The rocks are cut by as many as 20 to 25 parallel faults that trend about N. 80W. Many of these faults host thin, high-grade precious-metal veins that were, in the past, mined to shallow depths. The drilling results were less favorable than expected. Typical 5 to $10 \mathrm{ft}$ intervals assayed 0.01-0.04 (rarely to 0.1 ) oz/st $\mathrm{Au}$, and generally even lower amounts of $\mathrm{Ag}$, with intervening intervals characterized by much lower values. Again, deeper portions of this system remain untested.

The adjacent Blacksmith prospect (not shown), has similar oxidized sulfide veins, probably related to a north-trending dike(?) of Silver Wave diorite, but has not been explored. Surface occurrences of skarn have also been reported from the Blacksmith prospect. No geochemical samples were collected from either area. 


\section{Diamond $\underline{\mathrm{Hil1}}$}

The major target in the Indian Creek district, presently being explored by Pegasus Gold Inc., is called Diamond Hill, after the old Diamond Hill mine. It is a skarn deposit that contains a minimum of about 1,173,000 short tons of mineralized material with an average grade of $0.271 \mathrm{oz} / \mathrm{st}$ Au (Giancola and Razvi, 1995). This is a resource of $318,000 \mathrm{oz}$ of $\mathrm{Au}$. The deposit is incompletely explored, and there is a good chance that more reserves will be found if exploration is continued. The deposit is located about 1,000 ft east of the Helena National Forest boundary. Much of the information in this section was provided by Mike Maslowski, Senior Geologist with Pegasus Gold.

Lode gold was first discovered at Diamond Hill in 1870. From then until about 1940, small- to medium-scale underground mining of high-grade gold ore, termed "quartz-poor pyrite veins" by Klepper and others (1971), yielded as much as 2.9 st of Au. From 1940 until 1971, there was only sporadic mining, principally in small open pits. First Amax Inc., in 1971-72, and then Kerr McGee Corporation, in 1973, drilled in the immediate area, searching for a porphyry copper deposit related to the adjacent Diamond Hill quartz monzonite stock. In 1974, Utah International explored the Diamond Hill skarns in search of tungsten, and, from 1978 to 1981, Diamond Hill was again explored for porphyry copper deposits, this time by Exxon Minerals Company. In 1982-83, gold became the focus of exploration again, as Utah International drilled nearly 30 holes on the property in search of gold in the skarns, followed by gold exploration conducted by Nord Resources Corporation in 1983-84. Finally, in 1988, Pegasus Gold signed a joint venture development agreement with Broadwater Developments Inc., who had acquired the property in 1984. Pegasus' initial exploration efforts were aimed at development of a low-grade open-pit mine, but, since 1992, exploration has been focused on delineation of underground mineable reserves. As of February 1995, a 4,000-footlong exploration decline had been completed that will permit detailed evaluation of grade distribution in the deposit; a decision regarding development of the property should follow soon.

The skarn is developed in the lower member of the Elkhorn Mountains Volcanics, along the southern margin of the dioritic Silver Wave stock (fig. F3). This skarn occurs in near-vertical pipe-like bodies, oval in cross-section, that are known from drilling to extend to at least 1,500 ft below the surface. The skarn bodies appear to be formed along north-south trending, nearly vertical fractures and faults (See Klepper and others (1971) for possible relation with "Diamond Hill Fault Zone"). The protolith of the skarn is not known with certainty. A distinctive feldspar porphyry, known to the project staff as diorite porphyry, appears to envelop the skarns; this rock may be either a finegrained facies of the Silver Wave diorite, or recrystallized, contactmetamorphosed andesite of the Elkhorn Mountains Volcanics. The skarn consists of medium-grained, inequigranular masses of quartz, calcite, actinolite, chlorite, epidote, and(or) garnet, along with minor pyrite, pyrrhotite, and chalcopyrite.

Four phases of high-temperature skarn formation and alteration are discernible at Diamond Hill. First is formation of a prograde skarn assemblage of garnet + diopside + calcite. This is followed by a retrograde event in which garnet and diopside are replaced by epidote and actinolite, respectively. The principal sulfide and gold mineralization accompanies this assemblage. This event appears to be followed by a potassic overprint that results in patchy 
development of orthoclase in the groundmass. In the fourth and final stage, many rocks show a late replacement of garnet by chlorite. These metamorphic assemblages are followed by development of two distinct episodes of quartz + calcite, which generally fill low-angle veins and other voids. Carbonate in the last phase is commonly iron-rich and accompanied by galena + sphalerite; this may be a distal manifestation of the silver and base-metal veins to the north (Marietta mine area).

Surrounding these skarns is an envelope of rock, termed endoskarn by the project staff, that is perhaps better referred to as skarnoid. It's igneous parentage is generally evident, and it may well be external to the Silver Wave stock. In this rock, an initial phase is represented by the development of finegrained granoblastic diopside in the groundmass. This is again followed by a potassic alteration event, represented either by orthoclase flooding or by the development of secondary biotite that replaces ferromagnesian minerals. Finally, many of these rocks show evidence of subsequent propylitization, signaled by the development of actinolite, chlorite, epidote, and calcite.

Opaque minerals found in the skarn include gold, pyrite, chalcopyrite, sphalerite, galena, rare pyrrhotite, minor silver, and microscopic tellurobismutite, which, along with gold, is spatially and temporally associated with pyrite. The gold occurs as free grains from 10 microns to $2 \mathrm{~mm}$ in size, and as thin films on pyrite.

Surface composite samples collected during the present study (HL29, 31, 57; table F2) of typical mineralized rock are anomalous only in $\mathrm{Au}, \mathrm{Cu}$, and $\mathrm{Zn}$, containing as much as $0.66 \mathrm{ppm} \mathrm{Au}, 2,600 \mathrm{ppm} \mathrm{Cu}$, and $450 \mathrm{ppm} \mathrm{Zn}$. These results contrast sharply with those from the polymetallic vein systems immediately to the north, in the Park and Winston districts. There, mineralized samples are commonly characterized by large amounts of $\mathrm{Ag}, \mathrm{As}, \mathrm{Cd}, \mathrm{Pb}$, and $\mathrm{Sb}$, in addition to $\mathrm{Au}, \mathrm{Cu}$, and $\mathrm{Zn}$. The contrast is perhaps best demonstrated by As. Arsenic contents in the samples from Diamond Hill are hundreds to thousands of times lower than in the mineralized rock to the north.

\section{Morning Glory}

On the Morning Glory claims, the Diamond Hill stock, just to the south of the Silver Wave stock, was drilled as a porphyry copper prospect in the 1970s by AMAX. The Diamond Hill stock is a quartz monzonite porphyry, apparently younger than the Silver Wave stock, that is pervaded by a quartz-pyrite stockwork. It lies just outside the Forest.

Five diamond drill holes were completed by AMAX in 1971 to explore this system, with results as follows (unpublished reports, Amax Incorporated and Pegasus Gold Incorporated):

AMAX-1: Total depth, $700 \mathrm{ft}$; begun in quartz monzonite, passed out of intrusive into metamorphosed Elkhorn Mountains Volcanics at about $260 \mathrm{ft}$. No assay results available.

AMAX-2; Total depth $495 \mathrm{ft}$; quartz monzonite from collar to $115 \mathrm{ft}$, then Elkhorn Mountains Volcanics; most $\mathrm{Cu}$ assays 0.03 to 0.1 percent. The entire hole showed $\mathrm{Au}>0.02 \mathrm{ppm}$, with values as high as 0.06 in $10 \mathrm{ft}$ composites.

AMAX-3; Total depth $1,090 \mathrm{ft}$; collared in quartz monzonite and remained 
in it; most $\mathrm{Cu}$ assays 0.03 to 0.16 percent. Six of eleven $10 \mathrm{ft}$ composites showed detectable $\mathrm{Au}$, with values to $0.18 \mathrm{ppm}$ (at top of hole).

AMAX-4; Total depth $865 \mathrm{ft}$; collared in Elkhorn Mountains Volcanics, and encountered only some dikes of quartz monzonite; most $\mathrm{Cu}$ assays 0.02 to 0.09 percent. Three of eight $10 \mathrm{ft}$ composites showed detectable $\mathrm{Au}$, with values to $0.1 \mathrm{ppm}$ at bottom of hole.

AMAX-5; Total depth $300 \mathrm{ft}$; entire hole in quartz monzonite.

AMAX-6; Total depth $985 \mathrm{ft}$; collared in quartz monzonite; Elkhorn Mountains Volcanics from 450-790 ft, then quartz monzonite to bottom of hole.

Surface rock geochemistry yielded values of about 0.05 to 0.18 percent $\mathrm{Cu}$, with $\mathrm{Pb}, \mathrm{Zn}$, and Mo almost all $<100 \mathrm{ppm}, \mathrm{Ag}$ mostly 0.1-1 ppm, with one sample of $18 \mathrm{ppm}$, and $\mathrm{Au}$ mostly less than $200 \mathrm{ppb}$, but one sample as high as $4.7 \mathrm{ppm}$. A single geochemical sample (HL06, table F2) collected during this study contained $1.8 \mathrm{ppm} \mathrm{Au}$ and $1,100 \mathrm{ppm} \mathrm{Cu}$. It is reported that drill core here shows the quartz monzonite to be younger than the Silver Wave stock, but older than the rhyolite porphyry. It is also reported that AMAX obtained a $\mathrm{K}$-Ar date on the Diamond Hill stock, but the results are not known to us. The prospect was later held by Heather Resources, but they relinquished in 1983. There has been no known activity since then.

\section{Indian Creek placers}

Beginning just downstream from the Diamond Hill target, at the townsite of Hasse1, Indian Creek has been worked repeatedly since as early as 1870 (Reed, 1951) for placer Au over a length of several miles. The source of this gold was probably outcropping portions of the Diamond Hill deposit, as well as the Aubearing polymetallic veins in the drainage area. Reed (1951) reports that there were significant amounts of scheelite (calcium tungstate) in the black sand residue from placer mining.

\section{Other deposits}

Other known deposits in the Indian Creek district are apparently quite small. Except for the Iron Mask, which produced more than 22,000 st of ore, production from them has been negligible. Study of their mineralogy and geochemistry, however, could prove valuable as exploration guides by allowing delineation of metallogenic zoning patterns in the district.

A series of geochemical samples were collected at unnamed prospects from the drainages of Eureka Creek (HL 61, 62, 63, 65; Table F2) and Eagle Creek (HL 66, 67, 68; Table F2) in the far western part of the district. All four samples from Eureka Creek contained anomalous values of $\mathrm{Ag}, \mathrm{As}, \mathrm{Au}, \mathrm{Pb}$, and $\mathrm{Zn}$, with $\mathrm{Au}$ values as high as $4.5 \mathrm{ppm}$. Most of these samples were also anomalous in $\mathrm{Sb}$, and two of them contained detectable $\mathrm{Sn}$ in amounts of 16 and $11 \mathrm{ppm}$. One of the three samples from Eagle Creek was anomalous in the polymetallic suite (Ag, As, $\mathrm{Au}, \mathrm{Pb}, \mathrm{Cu}, \mathrm{Sb}, \mathrm{Sn}$, and $\mathrm{Zn}$ ), with a $\mathrm{Au}$ content of $1.5 \mathrm{ppm}$. 


\section{Park district}

The Park district, entirely within the Forest, encompasses an important system of polymetallic veins that have been exploited by a number of mines, chief among them being the Marietta. Lode deposits in the district were discovered in the 1870s, with the highest level of activity occuring between 1880 and 1906 (Reed, 1951). Information about the Marietta mine comes primarily from Sanguinetti (1986).

The Marietta mine exploits three important vein systems, the Marietta, Blue, and Gold Dust. The Marietta vein has been mined along a strike length of $>1600 \mathrm{ft}$. The vein strikes nearly north-south, and dips west at a low angle $\left(25^{\circ}-40^{\circ}\right)$, so that, although it has been worked down dip for over $600 \mathrm{ft}$, it is only about $300 \mathrm{ft}$ below the surface at that point. The vein varies from about 6 in to nearly $10 \mathrm{ft}$ wide at depth. Mineralized material consists chiefly of pyrite and arsenopyrite, with minor amounts of galena, sphalerite, chalcopyrite, and native $\mathrm{Au}$, in a gangue of quartz and manganiferous carbonate. The wall rock, tuff of the Elkhorn Mountains Volcanics, is pyritized, silicified, and argillized for 3-6 ft on either side of the veins.

Drilling of 6 holes, designed to sample the Marietta vein down dip (west) from existing workings, found the vein to be 3-10 ft wide and to contain grades of $0.1-0.4 \mathrm{oz} / \mathrm{st} \mathrm{Au}$ and $0.1-0.8 \mathrm{oz} / \mathrm{st} \mathrm{Ag}$. Resources remaining in surface waste dumps were estimated to contain 0.2 st $\mathrm{Au}$ and 1.3 st $\mathrm{Ag}$. Activity subsequent to 1986 is unknown.

Two samples were collected from waste dumps in the Park district (HL 13, 50 ; table F2). Both contained anomalous amounts of $\mathrm{Ag}$, $\mathrm{As}$, and $\mathrm{Au}$. $\mathrm{HL} 50$, the more strongly mineralized of the two samples, contained $3.5 \mathrm{ppm} \mathrm{Au}$ and 3.5 percent As, along with anomalous amounts of $\mathrm{Pb}, \mathrm{Zn}, \mathrm{Cu}$, and $\mathrm{Cd}$.

It is difficult to assess ore controls in the Marietta vein system, because there are no intrusive rocks known to crop out nearby, and none are indicated by aeromagnetic or gravity data. Nevertheless, much of the system is unexplored, and it is likely that substantial reserves remain underground.

\section{Winston district}

Evidence of mineralization in the Winston district clusters around at least six quartz monzonite porphyry stocks, four of which appear to be aligned along the Weasel Creek fault. These are, from north to south, the Pole Creek, Edna, Monte Cristo, Freiburg, January, and Vosburg stocks (Klepper and others, 1971). Four of the stocks are associated with mines that had significant production from polymetallic vein deposits, Custer with the Edna stock, East Pacific with the Freiburg stock, January with the January Stock, and Vosburg and Little 0lgaKleinschmidt with the Vosburg stock. Earll (1964) estimated total production from these deposits to be about 3.57 st $\mathrm{Au}, 51$ st $\mathrm{Ag}$, and $>6,000$ st of $\mathrm{Cu}, \mathrm{Pb}$, and $\mathrm{Zn}$. There was additional production of about 0.3 st of placer gold.

A series of samples from waste dumps in the vicinity of these mines (HL 23, $26,27,33,34,35 \mathrm{~A}, 46,47$, and 55; table F2) are generally anomalous in the polymetallic suite of metals ( $\mathrm{Ag}, \mathrm{As}, \mathrm{Au}, \mathrm{Cu}, \mathrm{Pb}$, and $\mathrm{Zn}$ ), along with some instances of high values of $\mathrm{Bi}, \mathrm{Cd}$, or $\mathrm{Sb}$. Gold values are as high as $2.1 \mathrm{ppm}$, and the $\mathrm{Ag}$ : Au ratio is commonly about 100, distinctly higher than for the samples from the Diamond Hill area. 
$\underline{\text { Stay Hot }}$

The most promising area in the Winston district may be the stay Hot prospect, located in sections $14,15,22,23,26$, and 27 of T. 8 N., R. 1 W. This area includes the historic Sunshine and Maine-Sullivan deposits, which yielded only minor production. The area was explored by FMC Gold Company in 1988 and 1989. Most information comes from Hawksworth (1989).

A target called Scott's Ridge was developed, a zone 2,300 ft long that trends about N.70W., and contains both skarn $\mathrm{Au}$ and stockwork $\mathrm{Au}$ mineralization in the Slim Sam Formation. This area is mostly within the Forest, in the northwest quarter of section 23. Surface rock and soil sampling revealed a large area of 0.03 to $0.5 \mathrm{ppm} \mathrm{Au}$, with some samples as high as $3 \mathrm{ppm}$, along with sporadic anomalies in $\mathrm{Ag}, \mathrm{Bi}, \mathrm{Te}, \mathrm{Cu}$, and $\mathrm{As}$. Drilling in 1989 on $\mathrm{BLM}$ land to the north encountered zones of mineralized skarn and stockwork mineralization, but $\mathrm{Au}$ values were not as high as expected. The best intercept was $33 \mathrm{ft}$ at about $0.06 \mathrm{oz} / \mathrm{st} \mathrm{Au}$. The most promising ground, which is within the Forest, has not been tested.

Our field examination revealed two additional areas of interest. Directly west of the Scott's Ridge target, on the west bank of Weasel Creek (section 22), a large area of disseminated pyrite and pyrrhotite in Elkhorn Mountains Volcanics suggests a large hydrothermal system. North of there, on BLM land on the south bank of Beaver Creek (section 15), an area of unknown size contains strong skarn mineralization with disseminated sulfide minerals.

Three surface rock samples were collected from the Stay Hot area during this study (HL 37A, 56, 77; table F2). None of them contained important amounts of precious metals, but one contained as much as $3,800 \mathrm{ppm} \mathrm{Cu}$.

\section{Chartam}

A mineralized body called Chartam has been delineated at the Edna stock. Chartam is a group of low-angle quartz-pyrite veins in quartz monzonite porphyry that are closely enough spaced to be bulk-mined. The prospect is located in section 13, T. $8 \mathrm{~N}$., R. $1 \mathrm{~W}$, , on BLM land, less than a mile from the Forest boundary, and was not visited during this study. The resource is reported to be 9.1 million st at 0.033 opt $\mathrm{Au}$ and 0.065 opt Ag by Giancola and Razvi (1993), who report that Chartam was originally explored by Montana Power, but became a Canyon Resources Corporation property in 1992, and was explored by Phelps Dodge Corporation, who did more than 2,000 ft of new drilling in 1992. There has been about 15,000 ft of drilling by previous workers. The area was reportedly being restored in the summer of 1994. 


\section{REFERENCES CITED}

Arbogast, B.F., ed., 1996, Analytical methods manual of the Mineral Resource Surveys Program, U.S.G.S: U.S. Geological Survey Open-file Report 96-525, $248 \mathrm{p}$.

Baitis, Hart, Hall, Susan, and Howe1l, Roger, 1988, York--a Proterozoic shalehosted gold system in Montana: Northwest Mining Association, 94th annual convention, December 1988, Spokane, Wash., handout, 22 p.

Baitis, Hart, Hall, Susan, and Howell, Roger, 1989, York - A Proterozoic stratabound gold system in Montana [abs.]: Geological Society of America Abstracts with Programs, v. 21, no. 5, p. 52-53.

Becraft, G.E., Pinckney, D.M., and Rosenblum, Sam, 1963, Geology and mineral deposits of the Jefferson City quadrangle, Jefferson and Lewis and Clark Counties, Montana: U.S. Geological Survey Professional Paper 428, 101 p.

Billingsley, Paul, and Grimes, J.A., 1918, Ore deposits of the Boulder Batholith of Montana: Transactions of the American Institute of Mining Engineers, $v$. 63, P. 284-368.

Blackwell, D.D., Brott, C.A., Goforth, T.T., Holdaway, M.J., Morgan, Paul, Petefish, David, Rape, Thomas, Steele, J.L., Spafford, R.E., and Waibel, A.F., 1975, A brief description of the Marysville geothermal area, in Energy resources of Montana: Montana Geological Society Guidebook, 22nd Annual Field Conference, June 1975, p. 217-222.

Brannon, C.A., 1981, Report on proposed project for Rochester Gulch, Lewis and Clark County, Montana (November 24, 1981), [Geologic report], Document 30552.02, 4 p. [Available from Anaconda Geological Documents Collection, International Archive of Economic Geology, American Heritage Center, University of Wyoming, P.O. Box 3924, Laramie, WY 82071.]

Braun, E.R., and Lange, I.M., 1984, Organic control of sandstone-hosted copper-silver mineralization in the Spokane Formation near Rogers Pass, western Montana, in McBane, J.D., and Garrison, P.B., eds., Northwest Montana and adjacent Canada: Montana Geological Society 1984 Field Conference and Symposium Guidebook, p. 305-313.

Briskey, J.A., 1986, Descriptive model of sedimentary exhalative $\mathrm{Zn}-\mathrm{Pb}$, in Cox, D.P., and Singer, D.A., eds., Mineral deposit models: U.S. Geological Survey Bulletin 1963, p. 211-215.

Brox, G.B., and Guilbert, J., 1960, Report on Copper Hill (McVay) Copper Prospect, Lewis and Clark County, Montana: Anaconda Geological Documents Collection, International Archives of Economic Geology, American Heritage Center, Laramie, University of Wyoming, reference no. 30601A, 5 p.

Brox, G.B., and Potter, C.W., 1965, Supplementary report on Copper Hill (McVay) Copper Prospect, Lewis and Clark County, Montana: Anaconda Geological Documents Collection, International Archives of Economic Geology, American Heritage Center, Laramie, University of Wyoming, reference no. 30601B, 5 p.

Close, T.J., and Rigby, J.G., 1984, Mineral investigation of the Gates of the Mountains Wilderness Study Area, Lewis and Clark County, Montana: U.S. Bureau of Mines Open-File Report MLA 3-84, 20 p.

Connor, J.J., and McNeal, J.M., 1988, Geochemistry of mineralized quartzite beds in the Spokane Formation (Belt Supergroup), Rogers Pass area, Lewis and Clark County, Montana: U.S. Geological Survey Bulletin 1762, 17 p.

Daniel, Faith, and Berg, R.B., 1981, Radiometric dates of rocks in Montana: 
Montana Bureau of Mines and Geology Bulletin 114, $136 \mathrm{p}$.

Denver Post, 1994, Canyon Resources moving on gold mine in Montana: Denver Post, February 4, 1994, p. 10C.

du Bray, E.A., 1995, Geologic map showing distribution of Cretaceous intrusive rocks in the central Big Belt Mountains, Broadwater and Meagher

Counties, Montana: U.S. Geological Survey Miscellaneous Field Studies Map MF-2291, scale 1:50,000, with text.

Earhart, R.L., Grimes, D.J., Leinz, R.W., Marks, L.Y., and Peterson, D.L., 1977, Mineral resources of the proposed additions to the Scapegoat Wilderness, Powell and Lewis and Clark Counties, Montana: U.S. Geological Survey Bulletin 1430, 62 p.

Earhart, R.L., Mudge, M.R., Whipple, J.W., and Connor, J.J. , 1981, Mineral resources of the Choteau $1^{\circ} \times 2^{\circ}$ quadrangle, Montana: U.S. Geological Survey Miscellaneous Field Studies Map MF-858-A, text, scale 1:250,000.

Ear11, F.N., 1964, Economic geology and geochemical study of Winston mining district, Broadwater County, Montana: Montana Bureau of Mines and Geology, Bulletin 41, $56 \mathrm{p}$.

Elliott, J.E., Loen, J.S., Wise, K.K., and Blaskowski, M.J., 1992, Maps showing locations of mines and prospects in the Butte $1^{\circ} \times 2^{\circ}$ quadrangle, western Montana: U.S. Geological Survey Map I-2050-C, scale $1: 250,000$.

Enders, M.S., Bartlett, M.W., Coffin, G.C., Volberding, J.E., and Young, D.P., 1995, The discovery of the McDonald gold deposit, Lewis and Clark County, Montana: Society for Mining, Metallurgy, and Exploration, Inc., preprint no. 95-225, 9 p.

Engineering and Mining Journal, 1991, May 1991, p. 44.

Giancola, Diane, and Razvi, Nasreen, eds., 1993, American Mines Handbook 1994: Southam Magazine and Information Group, Toronto, Canada, $380 \mathrm{p}$. 1995, American mines handbook 1996: Southam Magazine and Information Group, Toronto, Canada, p. 188-189.

Gualtieri, J.L., 1975a, Preliminary unedited geologic map of the Confederate Gulch area, Broadwater and Meagher Counties, Montana: U.S. Geological Survey Open-File Report 75-211, scale 1:48,000.

Gualtieri, J.L., 1975b, Geochemical data for the Confederate Gulch area, Broadwater and Meagher Counties, Montana: U.S. Geological Survey OpenFile Report 75-212.

Hanna, W.F., Hassemer, J.H., Elliott, J.E., Wallace, C.A., and Snyder, S.L., 1994, Maps showing gravity and aeromagnetic anomalies in the Butte $1^{\circ} \mathrm{x}$ $2^{\circ}$ quadrangle, Montana: U.S. Geological Survey Map I-2050-I, scale $1: 250,000$.

Harrison, J.E., 1972, Precambrian Belt basin of northwestern United States.Its geometry, sedimentation, and copper occurrences: Geological Society of America Bulletin, v. 83, p. 1215-1240.

1974, Copper mineralization in miogeosynclinal clastics of the Belt Supergroup, northwestern United States, in Bartholomew, Paul, ed., Gisements Stratiformes et Provinces Cupriferes: Geological Sociaty of Belgium, Liege, Belgium, p. 353-366.

Hawksworth, M.A., 1989, 1989 summary report on the Stay Hot property: Unpublished private report, $33 \mathrm{p}$.

Hunt, J., Goddard, C., Potter, C., Brox, G., Trask, Jr., F., and Powell, L., 1970, Heddleston ore reserves: Anaconda Geological Documents Collection, International Archives of Economic Geology, American Heritage Center, Laramie, University of Wyoming, reference no. 26706.02, 2 p.

Johnson, E.A., 1973, Geology and gold deposits of the Confederate Gulch-White Gulch area, Broadwater County, Montana: Butte, Montana College of Mineral Science and Technology, M.S. thesis, $53 \mathrm{p}$.

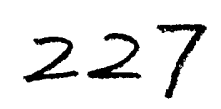


Klepper, M.T., Ruppe1, E.T., Freeman, V.L., and Weeks, R.A., 1971, Geology and mineral deposits, east flank of the Elkhorn Mountains, Broadwater County, Montana: U.S. Geological Survey Professional Paper 665, 66 p.

Klepper, M.T., Weeks, R.A., and Ruppe1, E.T., 1957, Geology of the southern Elkhorn Mountains, Jefferson and Broadwater Counties, Montana: U.S. Geological Survey Professional Paper 292, 82 p.

Knopf, Adolf, 1913, Ore deposits of the Helena mining region: U.S. Geological Survey Bulletin 527, $143 \mathrm{p}$.

Koschmann, A.H., and Bergendahl, M.H., 1968, Principal gold producing districts of the United States: U.S. Geological Survey Professional Paper 610, p. 142-171.

Lange, I.M., and Eby, D.E., 1981, Stratabound copper-silver-bearing oolitic deposits in the Belt Supergroup of Montana: Economic Geology, v. 76, p. 933-936.

Lange, I.M., and Sherry, R.A., 1986, Nonmassive sulfide deposits in the Late Precambrian Supergroup of western Montana, in Roberts, S.M., ed., Belt Supergroup, A guide to Proterozoic rocks in western Montana and adjacent areas: Montana Bureau of Mines and Geology Special Paper 94, p. 269 278 .

Lange, I.M., Moore, J.N., and Braun, E.R., 1986, Geology and diagenetic aspects of the Canyon Creek sandstone and Wolf Creek carbonate-hosted copper-silver deposits of the Belt Supergroup, northwestern Montana, U.S.A., in Craig, J.R., and others, eds., Mineral paragenesis: Theophrastus Publications, Athens, Greece, p. 411-442.

Lange, I.M., Herberger, D., Whipple, J.W., and Krouse, H.R., 1989, Stratabound $\mathrm{Cu}-\mathrm{Ag}$ mineralization in the Spokane and Helena Formations in the eastern part of the Belt basin, Montana--A common origin?, in Boyle, R.W., Brown, A.C., Jefferson, C.W., Jowett, E.C., and Kirkham, R.V., eds., Sediment-hosted stratiform copper deposits: Geological Association of Canada Special Paper 36, p. 287-304.

Lange, I.M., Woodward, L.A., and Krouse, H.R., 1992, Argo copper belt of the Helena embayment, Montana--Remobilized from Belt Supergroup (Middle Proterozoic) sulfide deposits?: Geological Society of America Abstracts with Programs, National Meeting, Cincinnati, v. 24, no. 7, p. A63.

Lange, I.M., Krouse, H.R., and Woodward, L.A., 1993, Implications of isotopically light sulfides in the Golden Messenger mine, York, Montana: Geological Society of America Abstracts with Programs, Cordilleran and Rocky Mountain Sections Meeting, Reno, v. 25, no. 5, p. 66.

Ludington, S.D., 1986, Descriptive model of Climax Mo deposits, in Cox, D.P., and Singer, D.A., eds., Mineral deposit models: U.S. Geological Survey Bulletin 1693, p. 73 .

Ludington, Steve, and Greenwood, W.R., 1990, Geological and geochemical evaluation, in Greenwood, W.R., Ludington, Steve, Miller, W.R., and Hanna, W.F., Mineral resources of the Elkhorn Wilderness Study Area, Broadwater and Jefferson Counties. Montana, with a section on Uranium and Thorium potential, by K.J. Wenrich, W.R. Miller, V.J. Suits, and J.B. McHugh: U.S. Geological Survey Bulletin 1805, p. 23-34.

Lyden, C.J., 1948, The gold placers of Montana: Montana Bureau of Mines and Geology Memoir 26, 151 p.

McClernan, H.G., 1976, Metallic mineral deposits of Powell County, Montana: Montana Bureau of Mines and Geology Bulletin 98, $69 \mathrm{p}$. 1983, Metallic mineral deposits of Lewis and Clark County, Montana: Montana Bureau of Mines and Geology Memoir 52, 73 p.

McCulloch, Robin, 1989, Mining and mineral develodments in Montana: Montana 
Bureau of Mines and Geology, Open-File Report MBMG 223, 42 p.

McDanal, S.K., Campbe11, W.L., Fox, J.P., and Lee, G.K., 1985, Magnetic tape containing analytical results form rocks, soils, stream sediments, and heavy-mineral concentrate samples: U.S. Geological Survey Report USGS-GD85-006. [Available only from U.S. Department of Commerce, National Technical Information Service, Springfield, Virginia 22151 as NTIS Report PB86-119484.]

McKee, J., 1978, Petrology, alteration, and mineralization of the Poorman Creek-Silver Bell stock porphyry $\mathrm{Cu}$-Mo deposit: Missoula, University of Montana, M.S. thesis, $97 \mathrm{p}$.

Mertie, J.B., Fisher, R.P., and Hobbs, S.W., 1951, Geology of the Canyon Ferry quadrangle, Montana: U.S. Geological Survey Bulletin 972, 97 p.

Miller, R.N., Shea, E.P., Goddard, C.C., Jr., Potter, C.W., and Brox, G.B., 1973, Geology of the Heddleston copper-molybdenum deposit, Lewis and Clark County, Montana: American Institute of Mining, Metallurgical and Petroleum Engineers, Inc., 1973 Pacific Northwest metals and minerals conference, p. $1-33$.

Mudge, M.R., Earhart, R.L., Watts, K.C., Jr., Tuchek, E.T., and Rice, W.L., 1974, Mineral resources of the Scapegoat Wilderness, Powell and Lewis and Clark Counties, Montana, with a section on Geophysical surveys, by D.L. Peterson: U.S. Geological Survey Bulletin 1385-B, 82 p.

Nelson, W.H., 1963, Geology of the Duck Creek Pass quadrangle: U.S. Geological Survey Bulletin $1121-\mathrm{J}, 56 \mathrm{p}$.

Pardee, J.T., and Schrader, F.C., 1933, Metalliferous deposits of the greater Helena mining region, Montana: U.S. Geological Survey Bulletin 842, 318 p.

Pegasus Gold Inc., 1992, Annual Report, 54 p.

Reed, G.C., 1951, Mines and mineral deposits (except fuels), Broadwater County, Montana: U.S. Bureau of Mines Information Circular 7592, 62 p.

Reynolds, M.W., 1979, Character and extent of basin-range faulting, western Montana and east-central Idaho, in Newman, G.W., and Goode, H.D., eds., Rocky Mountain Association of Geologists-Utah Geological Association 1979 basin and range symposium: Denver, Colorado, Rocky Mountain Association of Geologists, p. 185-193.

Reynolds, M.W., and Close, T.J., 1984, Gates of the Mountains Wilderness and additions, Montana, in Marsh, S.P., Kropschot, S.J., and Dickinson, R.G., Wilderness mineral potential, Assessment of mineral-resource potential in U.S. Forest Servcice lands studied, 1964-1984: U.S. Geological Survey Professional Paper 1300, p. 705-708.

Roby, R.N., 1950, Mines and mineral deposits (except fuels), Meagher County, Montana: U.S. Bureau of Mines Information Circular 7540, 43 p.

Roby, R.N., Ackerman, W.C., Fulkerson, F.B., and Crowley, F.A., 1960, Mines and mineral deposits (except fuels) Jefferson County, Montana: Montana Bureau of Mines and Geology Bulletin 16, 122 p.

Rocky Mountain Pay Dirt, 1987, Sunshine to develop Big Blackfoot gold mine in Montana: November, p. $12 \mathrm{~A}$.

1991, Phelps Dodge and Canyon Resources report Montana gold find: December, 1991 , p. 9A.

Ross, C.P., 1950, Fluorspar prospects in Montana, 1950: U.S. Geological Survey Bulletin 955-E, p. 173-224.

Rostad, 0.H., 1969, The use of geochemistry at the Bald Butte molybdenite prospect, Lewis and Clark County, Montana, in Canney, F.C., ed., 
International geochemical exploration symposium--Quarterly of the Colorado School of Mines, v. 64, no. 1, p. 437-449.

Ruppel, E.T., 1963, Geology of the Basin quadrangle, Jefferson, Lewis and Clark, and Powe11 Counties, Montana: U.S. Geological Survey Bulletin 1151, $121 \mathrm{p}$.

Sahinen, U.M., 1962, Fluorspar deposits in Montana: Montana Bureau of Mines and Geology Bulletin 28, 38 p.

Sanguinetti, M.H., 1986, Report on the Marietta mine property, Broadwater County, Montana: Unpublished private report, $28 \mathrm{p}$.

Schieber, Juergen, 1985, The relationship between basin evolution and genesis of stratiform sulfide horizons in mid-Proterozoic sediments of central Montana (Belt Supergroup): Eugene, University of Oregon Ph.D. dissertation, 811 p.

1989, Facies and origin of shales from the mid-Proterozoic Newland Formation, Belt Basin, Montana USA: Sedimentology, v. 36, p. 203-219.

1991, The origin and economic potential of sandstone-hosted disseminated $\mathrm{Pb}-\mathrm{Zn}$ mineralization in pyritic shale horizons of the Mid-Proterozoic Newland Formation, Montana, USA: Mineralium Deposita, v. 26, p. 290-297. 1992, Facies and deposition of a mixed terrigenous-carbonate suite in a Mid-Proterozoic epicratonic sea--The Newland Formation, Belt Supergroup, Montana, U.S.A.: Neues Jahrbuch for Geologie and Palaontologie Monatshefte, v. 184, p. 155-180.

Schmidt, R.G., Loen, J.S., Wallace, C.A., and Mehnert, H.H., 1994, Geology of the Elliston region, Powell and Lewis and Clark Counties, Montana: U.S. Geological Survey Bulletin 2045, 25 p.

Sillitoe, R.H, Grauberger, G.L., and Elliott, J.E., 1985, A diatreme-hosted gold deposit at Montana Tunne1s: Economic Geology, v. 80, p. 1707-1721.

Skipp, Betty, and McGrew, L.W., 1977, The Maudlow and Sedan Formations of the Upper Cretaceous Livingston Group on the west edge of the Crazy Mountains basin, Montana: U.S. Geological Survey Bulletin 1422-B, 68 p.

Slack, J.F., 1993, Models for tourmalinite formation in the Middle Proterozoic Belt and Purcell Supergroups (Rocky Mountains) and their exploration significance, in Current Research, part E: Geological Survey of Canada Paper 93-1E, p. 33-40.

Smedes, H.W., 1966, Geology and igneous petrology of the northern Elkhorn Mountains, Jefferson and Broadwater Counties, Montana: U.S. Geologica1 Survey Professional Paper 510, 116 p.

Smedes, H.W., and Thomas, H.H., 1965, Reassignment of the Lowland Creek Volcanics to Eocene age: Journal of Geology, v. 73, p. 508-510.

Stone, R.W., 1911, Geologic relation of ore deposits in the E1khorn Mountains, Montana, in Contributions to Economic Geology: U.S. Geological Survey Bulletin 470, p. 75-98.

Thorson, J.P., 1984, Suggested revisions of the lower Belt Supergroup stratigraphy of the Highland Mountains, southwestern Montana, in Hobbs, S.W., ed., The Belt, Abstracts with summaries, Belt symposium II: Montana Bureau of Mines and Geology Special Publication 90, p. 10-12.

in preparation, Controls of massive sulfide mineralization in the Belt Supergroup, in Berg, R.B., ed., Proceedings of Belt Symposium III: Montana Bureau of Mines and Geology Special Publication 111.

Thorson, J.P., White, B.G., and Baitis, H.W., in preparation, Gold resources in the Greyson Formation, Big Belt Mountains, Montana. Part II. Mineralization and genesis, in Berg, R.B., ed., Proceedings of Belt 
Symposium III: Montana Bureau of Mines and Geology Special Publication 111 .

Tilling, R.I., 1973, Boulder batholith, Montana--A product of two contemporaneous but chemically distinct magma series: Geological Society of America Bulletin, v. 84, p. 3879-3900.

Tramme1, J.W., 1975, Strata-bound copper mineralization in the Empire Formation and Raval1i Group, Belt Supergroup, northwest Montana: Seattle, University of Washington, $\mathrm{Ph}$. D. dissertation, $70 \mathrm{p}$.

Wallace, C.A., Lidke, D.J., and Schmidt, R.G., 1990, Faults of the central part of the Lewis and Clark line and fragmentation of the Late Cretaceous foreland basin in west-central Montana: Geological Society of America Bulletin, v. 102, p. 1021-1037.

Whipple, J.W., 1980, Depositional environment of the Middle Proterozoic Spokane Formation-Empire transition zone, west-central Montana: U.S. Geological Open-File Report 80-1232, 98 p.

Whipple, J.W., and Morrison, G.G., in preparation, Stratabound gold in the Greyson Formation, Big Belt Mountains, Montana. Part I. Geologic setting, in Berg, R.B., ed., Proceedings of Belt Symposium III: Montana Bureau of Mines and Geology Special Publication 111.

Woodward, L.A., 1992, Tectonic setting of Au-Ag deposits hosted by Proterozoic strata along the Lewis and Clark line, west-central Montana, in Bartholomew, M.J., Hyndman, W.W., Mogk, E.W., and Mason, R., eds., Basement tectonics 8--Characterization and comparison of ancient and Mesozoic continental margins; Proceedings of the 8 th International conference on basement tectonics: Dordrecht, the Netherlands, Kluwer Academic Publishers, p. 653-663.

Zieg, G.A., 1986, Stratigraphy and sedimentology of the Middle Proterozoic upper Newland Limestone, in Roberts, S.M., ed., Belt Supergroup--A guide to Proterozoic rocks of western Montana and adjacent areas: Montana Bureau of Mines and Geology Special Publication 94, p. 125-141

Zieg, G.A., and Leitch, C.H.B., 1996, The geology of the Sheep Creek copper deposits, Meagher County, Montana, in Berg, R.B., ed., Proceedings of Belt Symposium III: Montana Bureau of Mines and Geology Special Publication 111.

Zieg, G.A., Rankin, P.W., Ha11, S.M., and Tureck-Schwartz, K.R., 1991, The geology of the Sheep Creek Proterozoic copper deposits, central Montana: Society of Mining Engineers, AIME preprint, Rocky Mountain Section, Denver Meeting, February, 1991, 7 p. 


\section{MIMERAL RE8OURCE A88E88MENT FOR LOCATABLE MINERALS}

By Steve Ludington, J.E. Elliott, Christopher Osterman, and J.W. Whipple

\section{INTRODUCTION}

Resource assessments are of use to strategic planners, who plan for a nation's economic and military security; to economic planners, who estimate current and future mineral supplies and plan development; to mineral production companies, that use it to guide and help plan exploration; to governmental planning agencies, that help make decisions between competing land uses; and to public agencies that lease, trade, or sell publicly owned mineral resources. Our goal in the present study is to assess the undiscovered metallic and nonmetallic mineral resources in the Helena National Forest, Montana. An assessment of mineral resources (materials that are in such form that economic extraction of a commodity is currently or potentially feasible) can take many forms. The simplest might be a statement such as, "Yes, this is a good place to look for minerals." Another type of assessment might consist of an exhaustive inventory of the location, nature, and amount of known resources, which, in principle, is much like a shepherd counting sheep. An assessment of this sort would only be possible if the area were completely explored and no undiscovered deposits remained.

Qualitative estimates of the mineral content of specific areas have been with us for decades, if not centuries. All systematic mineral exploration requires decisions about the relative favorability of alternative tracts of ground. The assessment of undiscovered mineral resources in the Helena National Forest carries the evaluation process further, into the quantitative realm. A quantified assessment includes a numerical estimate of the amount and quality of mineralized rock and metal present within a tract (a specific area) to a specified depth. Because of the uncertainty inherent in assessment of the unknown, the results obtained are presented probabilistically. That is, the likelihood of the existence of undiscovered mineral deposits is expressed by a number. Because the resources being assessed are undiscovered, the assessment is necessarily subjective; it's quality is dependent on the collective knowledge and experience of the assessors. An assessment may be well done or poorly done, but because of the probabilistic nature of the result, it cannot be right or wrong. Likewise, it cannot be verified within the lifetimes of the assessors. Only with the passage of time, and with exhaustive exploration, will the accuracy of the assessment be known. Thus, resource assessment is clearly an estimate and not a precise measurement.

The quantitative assessment methods used in this study have been described by Singer (1993), Menzie and Singer (1990), Singer and Cox (1988), Drew and others (1986), and Singer and Ovenshine (1979). A thorough discussion of issues related to mineral resource assessment can be found in Barton and others (1995), along with an extensive bibliography on the subject. 


\section{Delineation of Permissive Tracts}

The first part of the assessment process defines areas where mineral deposits are, or could be, present. These areas are said to be permissive for the deposit type being evaluated. They are different from areas where deposits are most likely to occur, because, by definition, the permissive tract will contain all undiscovered deposits that are postulated in the assessment process. In other words, the permissive tract is the land that remains after excluding those areas where there is no reasonable chance that the deposit type could occur. The definition of a reasonable chance is critical; if it is defined to be too small, the maps of permissive tracts would commonly be all-inclusive and therefore, of limited value. Singer (1993) has stated that the boundaries should be defined such that the probability of deposits occurring outside the boundary is "negligible, that is, less than 1 in 100,000 to 1,000,000." Unfortunately, there are very few types of mineral deposits whose genesis we understand well enough that we can eliminate the last vestige of probability that they might occur in any particular place.

For this study, within some of the permissive tracts, we have outlined subareas that are particularly favorable for the occurrence of deposits. These favorable tracts were designated because the geologic environment contains a coincidence of a large number of important features that are characteristic of a particular deposit type. In these favorable tracts, we believe that the spatial density of undiscovered deposits is higher than throughout the permissive area in general, although we are, in general, unable to specify how much higher.

Boundaries of the permissive tracts may not correspond exactly to the outcrops of permissive rocks on the geologic map. This is because we considered undiscovered deposits at some depth below the surface, and permissive conditions may extend beneath the cover of younger rocks. This subsurface extension of permissive areas is largely subjective because we did not apply detailed geophysical information to estimate depths to basement rocks.

\section{GRADE AND TONNAGE MODELS}

The size of mineral deposits is commonly referred to as the tonnage, meaning the amount of ore that has been or might be mined in order to extract the valuable materials in the deposit. The grade of a mineral deposit refers to its quality. Deposits with higher grades contain more valuable material per unit mass of ore. Grade and tonnage models are used as part of the resource assessment to help classify the deposits, thus aiding in tract delineation, and to provide information about the potential value of undiscovered deposits. They are created using measured grades and tonnages of well explored or mined deposits. When estimates of undiscovered deposits are made, the grade and tonnage models define the deposits being estimated. Combined with estimates of the number of undiscovered deposits, they are instrumental in translating geologic expertise into statements that economists can use. Grade and tonnage are expressed in metric units because they are the worldwide standard for expressing size and mass.

Frequency distributions of tonnage and grade of wel1 explored or mined deposits are now available for many deposit types (Cox and Singer, 1986; Bliss, 1992; Orris and Bliss, 1991). For this study, we use some of those models, and have created some new ones. Table Gl provides a brief description of the deposit 


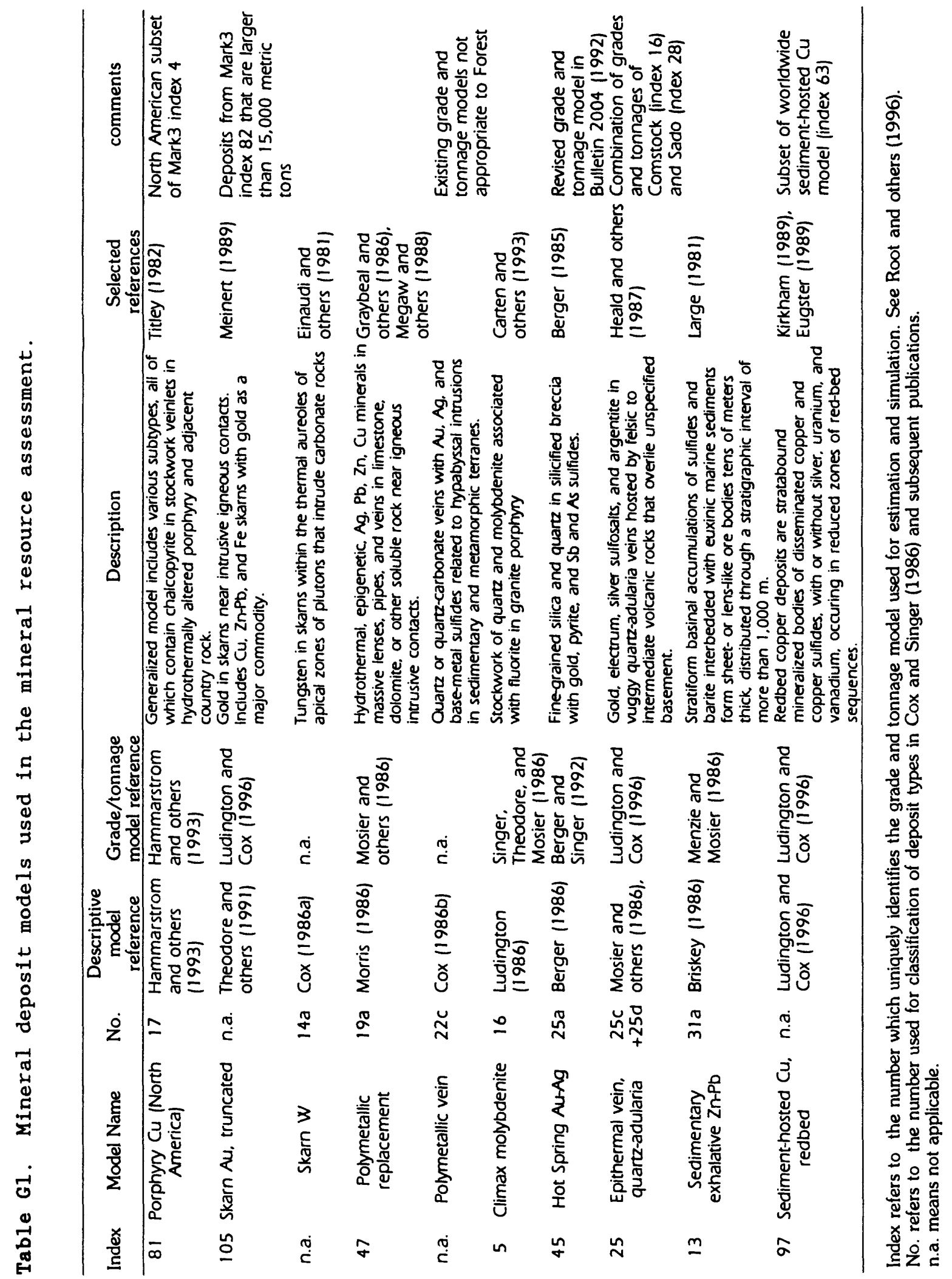


types used in this assessment, and provides a cross-reference to the original descriptions of the various descriptive and grade and tonnage models.

\section{Estimation of Number of Undiscovered Deposits}

The method used to estimate the number of undiscovered mineral deposits in this study was subjective and used the expert judgment of a number of geologists familiar with the geology and mineral deposits present in the Forest. The assessment team gathered available pertinent information about the various types of mineral deposits that might occur in the study area. After reviewing the geologic, geochemical, and geophysical information, and after delineating the boundaries of the permissive tracts, each member of the assessment team made estimates of the number of undiscovered deposits at five probability levels ( 0.9 , $0.5,0.1,0.05$, and 0.01 ). The estimates were shared among team members, and those with extreme estimates, both high and low, were asked to justify their responses. The estimates were discussed until consensus on a single set of five values was attained.

The deposits are estimated consistent with the appropriate grade and tonnage model. That is, if 10 deposits are estimated, 5 of them are visualized to be larger than the median tonnage, and 5 of them are visualized to have a higher grade than the median grade of the distributions that constitute the models. Most of them will fall within the range of values in the models, also.

Although a large number of mineral-deposit types were considered in this study, many were excluded from our final analysis because of limited importance or inadequate information on which to base an estimate. Table G2 summarizes information for those deposit types formally analyzed and provides a synopsis of the estimates of numbers of deposits. The team that made the estimates reported in this chapter consisted of Steve Ludington, J.E. Elliott, C. Osterman, and J.W. Whipple. Some members of the team participated in the estimation of only specific deposit types. Not all members of the team that studied the Forest participated in the quantitative estimation process, although they provided geologic, geochemical, or geophysical input and discussion that aided those who made the estimates. The team that made the estimates includes geologists who have spent an extensive period of time (years) working in and adjacent to the Forest and (or) with the types of deposits that were evaluated.

\section{Simulation}

A computer program, the Mark3 simulator, is used to combine information about grade, tonnage, and estimated number of deposits into information about amounts of metal that may be present and about the quantity of ore, or mineralized rock that would be mined in order to recover the metal. Probabilities for the existence of undiscovered deposits are stated as inequalities because mineral deposits occur only as discrete numbers of deposits. A simulator must be used because the probability distributions used to describe grade, tonnage, and number of deposits are empirical (i.e., not mathematical functions) and cannot be combined mathematically. The quantiles of the grade and tonnage distributions cannot be multiplied to generate the quantiles of contained metal. Multiplying quantiles could be successful only if the ordinal lists of 
grades and tonnages were identical, a very unlikely event. The result of the simulation is a probability distribution of contained metal and ore, or mineralized rock.

The results of the simulations can be presented in various ways. Classinterval histograms emphasize, and make it easy to highlight, those amounts of metal that are estimated to be most likely to exist. Cumulative histograms are especially useful because all the information generated by the simulation can be read from a single plot. Various quantiles and the means are best used for comparisons between and among estimates for different deposit types or different permissive tracts. No single number can adequately represent the magnitude of an estimate, because no single number can represent the uncertainty in judgment that is inherent in the estimation process or the range of values that make up the grade and tonnage models.

\section{UNDISCOVERED DEPOSITS IN THE FOREST}

Below, we describe the particular estimates for undiscovered deposits in the Helena National Forest, along with the most important information that affected the estimates. The maps that portray the permissive and favorable tracts to which the estimates pertain are on plates 5 and 6 , maps $K$ through $R$. table G2 summarizes the estimates.

\section{PORPHYRY COPPER DEPOSITS}

Porphyry copper deposits are the most valuable type of metallic mineral deposit commonly found in North America. They commonly consist of hundreds of millions of tons of mineralized rock, and are usually mined by open-pit methods. They are genetically associated with epizonal igneous porphyry intrusions, and contain chalcopyrite in stockwork veinlets in hydrothermally altered porphyry and adjacent country rock. They are commonly associated with polymetallic vein, skarn, and replacement deposits.

\section{Choice of Model}

In this assessment, we used a grade and tonnage model for porphyry copper deposits that is made up of 107 examples from North America (Mark3 index 81) extracted from the worldwide model (Mark3 index 4) of Singer, Mosier, and Cox (1986). Mark3 index is a numerical identifier that refers to which grade and tonnage model the Mark3 simulator uses in any particular case (see table G1). The North American model was created for, and first used, in the Custer-Gallatin National Forest assessment (Hammarstrom and others, 1993). The 107-deposit North American model contains an average of 6 percent less mineralized rock, 20 percent less copper, 45 percent less molybdenum, 31 percent less gold, and 8 percent more silver than the worldwide model. The team judged that the slightly smaller size and lower copper grades of the North American model are more representative of the population of undiscovered porphyry copper deposits that might exist in central Montana. 
Table G2. Summary of mineral deposit types analyzed, with numerical estimates of numbers of undiscovered mineral deposits (locatable minerals) in Helena National Forest. The columns labeled $0.9,0.5,0.1,0.05$, and 0.01 list the least number of deposits estimated to exist at the specified probability or more (Root and others, 1992). The Mark3 index identifies the specific grade and tonnage model used for the estimates (see Table G1).

\begin{tabular}{|c|c|c|c|c|c|c|c|}
\hline Deposit Type & 0.9 & $0.5^{f}$ & $\begin{array}{c}\text { robabill } \\
0.1\end{array}$ & 0.05 & 0.01 & $\begin{array}{l}\text { Mark3 } \\
\text { index }\end{array}$ & Comments \\
\hline Porphyry Cu (North America) & 0 & 0 & 1 & 2 & 3 & 81 & $\begin{array}{l}\text { There are } 5 \text { important prospects in or } \\
\text { near the Forest; both permissive and } \\
\text { favorable tracts were delineated. }\end{array}$ \\
\hline Skarn Au & 1 & 1 & 5 & 7 & 8 & 105 & $\begin{array}{l}\text { Au-poor, } \mathrm{Cu}-\mathrm{Fe} \text {, and } \mathrm{Zn} \text {-bearing } \\
\text { skarns were not analyzed separately, } \\
\text { but the area is also permissive for } \\
\text { them. }\end{array}$ \\
\hline Skarn Au in Elkhorn Mountains & 0 & 1 & 2 & 3 & 4 & 105 & $\begin{array}{l}\text { Separate estimate made because of } \\
\text { different geologic environment. }\end{array}$ \\
\hline Skarn W(Mo) & - & - & - & 一 & - & - & $\begin{array}{l}\text { No formal estimate made because } \\
\text { deposits in the Forest are very small. }\end{array}$ \\
\hline Polymetallic Replacement & 0 & 0 & 0 & 1 & 3 & 47 & none \\
\hline Polymetallic Vein & - & - & - & 一 & - & - & $\begin{array}{l}\text { No appropriate grade and tonnage } \\
\text { models; deposits may occur in entire } \\
\text { Forest. }\end{array}$ \\
\hline Polymetallic Vein (U-bearing) & - & - & - & 一 & - & - & $\begin{array}{l}\text { No appropriate grade and tonnage } \\
\text { models. }\end{array}$ \\
\hline Climax molybdenite & 0 & 0 & 1 & 1 & 2 & 5 & none \\
\hline York-type exhalative (?) Au & 一 & - & - & 一 & - & - & $\begin{array}{l}\text { No numerical estimate; permissive } \\
\text { tract only. }\end{array}$ \\
\hline Cretaceous Au-bearing vein & 一 & - & - & - & - & - & $\begin{array}{l}\text { No numerical estimate; permissive } \\
\text { tract only. }\end{array}$ \\
\hline Hot-spring Au-Ag & 1 & 1 & 2 & 2 & 2 & 45 & $\begin{array}{l}\text { Hot-spring and epithermal vein } \\
\text { deposits share the same permissive } \\
\text { tract. }\end{array}$ \\
\hline $\begin{array}{l}\text { Epithermal vein, quartz- } \\
\text { adularia }\end{array}$ & 0 & 1 & 2 & 3 & 4 & 25 & $\begin{array}{l}\text { Hot-spring and epithermal vein } \\
\text { deposits share the same permissive } \\
\text { tract. }\end{array}$ \\
\hline Sedimentary exhalative $\mathrm{Zn}-\mathrm{Pb}$ & 一 & - & - & - & - & - & $\begin{array}{l}\text { Area is permissive for deposits like } \\
\text { Sheep Creek; no estimate made. }\end{array}$ \\
\hline Sediment-hosted $\mathrm{Cu}$, redbed & - & - & - & - & - & - & $\begin{array}{l}\text { Deposits and prospects in the Forest } \\
\text { are generally small; no estimate } \\
\text { made. }\end{array}$ \\
\hline
\end{tabular}




\section{Known Examples}

There are several known examples of porphyry copper deposits and prospects within or near the Helena National Forest. Probably the best known deposit is at Heddleston (plate 2, map B; table E1, no. 42), which is related to a group of Eocene quartz monzonite plutons. This deposit was extensively explored by the Anaconda Company before 1973, but has never been mined.

In addition, a prospect known informally as Golconda (plate 2, map B; table E1, no. 392), is situated immediately adjacent to the Forest boundary, along the west flank of the Elkhorn Range, just east of Jefferson City. Some exploratory drilling was done here in the late 1970s, but its tonnage and grade are not known, nor is it known why the exploration effort was abandoned. The area is described in Ludington and others (1990).

Just outside the Forest, immediately west of Interstate 15, and to the south of Corbin, is another small porphyry copper deposit, known as Beaverton. It was explored extensively by several companies also in the late 1970s, although the present status of the prospect is not known. Much of the possible ore consisted of oxidized protore, and is probably enriched above the original tenor.

Directly outside the eastern boundary of the Elkhorn part of the Forest, the Morning Glory claims, on the Diamond Hill stock, were explored as a porphyry copper target from 1971 until 1983. Limited exploration yielded equivocal results. At Radersburg, about $3 \mathrm{mi}$ southeast of the Forest boundary, exploration was conducted in the $1970 \mathrm{~s}$ and two deep $(>1,000 \mathrm{ft})$ holes were reportedly drilled. Details results of this exploration are not known, although anomalous copper was reportedly encountered.

On Jackson Creek (plate 2, map B; table E1, no. 405), southeast of Helena, an area with anomalous copper, molybdenum, and bismuth in bedrock samples, shows some similarities to a porphyry copper system, although its nature is very poorly understood (Greenwood and others, 1990).

Rochester (plate 2, map B; table E1, no. 62), which is about $7 \mathrm{mi}$. southeast of Lincoln, was explored in the 1980s (Brannon, 1981), but little is known about the results. Near Chessman Reservoir (plate 2, map B; table E1, no. 295), mineralized and altered rock typical of porphyry copper deposits was encountered during exploration of the Montana Tunnels deposit (Whiteley, 1982).

\section{Tract Delineation}

The permissive tract for porphyry copper deposits was delineated using the procedure outlined below, and is depicted on plate 5, map $K$. First, areas of exposed plutonic rocks of the Boulder batholith were delineated, including an approximately $2 \mathrm{mi}$-wide buffer to capture areas underlain by concealed plutons and subsurface extensions. This area was extended to include areas where aeromagnetic data suggested the possibility for other buried plutonic rocks, and also to include known polymetallic vein and polymetallic replacement districts, which are often spatially associated with porphyry copper deposits. All the known porphyry copper prospects fall within the resulting permissive tract. The tract was found to be generally coincident with the areas where high values of $\mathrm{Cu}$ occur in stream-sediment samples (see Chapter D).

In addition, two areas north and south of Helena were delineated to constitute a favorable tract. These are areas where all the characteristics 
listed above are present. The northerly area contains the Heddleston deposit; the southern one is centered on the northern end of the Boulder batholith, and includes most of the Elkhorn Mountains.

\section{Estimate of Undiscovered Deposits}

For the $90 \mathrm{th}, 50 \mathrm{th}, 10 \mathrm{th}, 5 \mathrm{th}$, and 1st percentiles, the team estimated 0 , $0,1,2$, and 3 undiscovered porphyry copper deposits consistent with the North American subset (Mark3 index 81) of the porphyry copper grade and tonnage model of Singer, Mosier, and Cox (1986). The estimates were strongly influenced by the fact that there has been substantive exploration on at least five prospects within the permissive tract, and within, or very near to, the Forest boundaries. All of these prospects have been explored extensively, many by diamond drilling. The team considered that Golconda and Rochester were the prospects most likely, if fully evaluated, to qualify as deposits in terms of size and grade. In addition, there is already one known deposit (Heddleston) within the Forest, and another (Beaverton) very near the Forest boundary.

\section{GOLD SKARN DEPOSITS}

Skarn deposits are mineral deposits wherein the ore consists primarily of carbonate and calc-silicate minerals, formed by metasomatic replacement of the original rocks, adjacent to, and sometimes, in, plutons. Valuable metals are found in and in association with sulfide minerals distributed throughout the skarn. Gold skarns are often attractive targets for mineral exploration. They tend to have higher grades and are smaller than many other important types of gold-bearing deposits. Hence, they may be mined less-expensively and with smaller excavations and infrastructure investments.

\section{Choice of Model}

The abundance of various metals in skarn deposits may well form a continuum; their categorization is based on the materials present that have the greatest value. Thus, gold skarns are those skarn deposits in which gold is the most valuable metal in the deposit. Exploration for skarn deposits in Montana has focused closely on gold during the last decade. Thus, although there are a few examples of copper- and (or) zinc-lead-bearing skarns in the region that did not contain significant gold, we chose not to evaluate them separately, as they are unlikely to be a target for mineral development during the lifespan of this as ses sment.

Theodore and others (1991) presented a grade and tonnage model for gold skarns, and included data for 90 deposits. They restricted deposits in the model to those that had gold grades greater than $1 \mathrm{~g} /$ metric ton. This model has since been used for quantitative assessment in a number of instances. However, this team believes that many of the deposits that make up that model are inappropriate, in that they are too small to be significant attractions for development today. Therefore, we created a truncated model (Mark3 index 105), discarding the 27 deposits that contained less than 15,000 metric tons of 
mineralized rock. The remaining 63 deposits contain more than 99 percent of the metal present in the deposits that make up the original model.

\section{Known Examples}

The Spring Hill mine (plate 2, map B; table E1, no. 476) is one of several good examples of small gold skarn deposits; in addition, there are others in the Helena district. In the Ophir district, a number of mines have produced small amounts of gold from skarn deposits in the past.

More significantly, the Diamond Hill deposit in the Elkhorn Mountains (plate 2, map B; table El, no. 477), is presently undergoing active exploration by Pegasus Gold. Diamond $\mathrm{Hill}$ is located immediately adjacent to the eastern boundary of the Elkhorn region of the Forest, and contains a known resource of greater than 10 metric tons of gold, with opportunities to discover more. The deposit is found in the lower member of the Elkhorn Mountain Volcanics, along the southern margin of the Silver Wave stock, and occurs in near-vertical pipe-like bodies that extend to at least $500 \mathrm{~m}$ below the surface. The skarn bodies consist of medium-grained, inequigranular masses of quartz, calcite, actinolite, chlorite, epidote, and(or) garnet, along with minor pyrite, pyhrrotite, and chalcopyrite that replace the enclosing volcanic rocks. Gold is temporally associated with pyrite and occurs as free grains and as thin films on pyrite.

Nine other areas in the Elkhorn Mountains were judged to have a specific probability of occurrence for gold skarn deposits. They include areas near the Antelope, Vosburg-January-Freiburg, Monte Cristo, Vulture, Silver Wave, South Fork, Slim Sam, and Swamp Creek stocks, and the Marietta Mine area (plate 2, map B; table E1, no. 460).

Gold skarn deposits in the Elkhorn district (p1ate 2, map B; table E1) are about $3 \mathrm{mi}$. southwest of the Forest boundary, near the townsite of E1khorn, in the Deer Lodge National Forest. A silver-rich replacement deposit was exploited here in the 19th and early part of the 20th centuries. Exploration in the 1980s encountered gold skarn deposits nearby that are currently undergoing continued exploration and evaluation. Deposits here could contain 1 million troy oz. of gold.

\section{Tract Delineation}

In deciding which areas to include within the permissive tract for this deposit type, we used the basic criteria that a skarn deposit requires a reactive host rock and an intrusion to provide heat, fluids, and metals. Accordingly, we began by outlining those areas that are near plutons, both exposed and inferred, and that are underlain by carbonate-bearing strata. Formations included in the permissive tract are the Helena, Empire, Shepard, Red Lion, and Hasmark Formations; the Park Shale, Meagher Limestone, Wolsey Shale, and Flathead Sandstone; the Three Forks, Jefferson, and Maywood Formations, and members of the Madison Group; and the Amsden, Phosphoria, and Kootenai Formations. We excluded rocks of the Colorado Group, the Blackleaf, Mount Shields, and Bonner Formations, all intrusive rocks, and volcanic rocks outside of the Elkhorn Mountains. An area underlain by the Elkhorn Mountains Volcanics was included in the permissive tract because of the occurrence of the Diamond Hill deposit, which proves that 
subaerial andesites can be reactive in some cases. We then ensured that this area included all the known gold skarn deposits and prospects. The resulting permissive tract is depicted on plate 5 , map $L$.

We then delineated a smaller area, designated as favorable. To qualify as favorable, areas had to meet the above criteria, including being near important deposits or prospects, and, in addition, they had to exhibit anomalous gold geochemical values, as shown by the NURE stream-sediment data (Chapter D). The resulting favorable tract consists of three small areas west and south of Helena, and a large area composed of volcanic rocks that form the roof of the Boulder batholith along the east flank of the Elkhorn Mountains (plate 5, map L).

\section{Estimate of Undiscovered Deposits}

Two discrete estimates were made of numbers of undiscovered deposits, both consistent with the gold skarn grade and tonnage model of Theodore and others (1991), modified as described above (Mark3 index 105). For the 90th, 50th, 10th, 5 th, and 1st percentiles, the team estimated $1,1,5,7$, and 8 undiscovered gold skarn deposits for the part of the permissive tract exclusive of the Elkhorn Mountains, and $0,1,2,3$, and 4 or more undiscovered gold skarn deposits for the part of the permissive tract in the Elkhorn Mountains. The presence of important representatives of this deposit type in the immediate vicinity of the Forest weighed heavily in our estimate. In the Elkhorn Mountains, many of the mafic alkaline satellitic stocks on the east flank of the range are petrochemically similar to source plutons for gold skarns elsewhere.

\section{TUNGSTEN-(MOLYBDENUM) SKARN DEPOSITS}

Skarn deposits are mineral deposits wherein the ore consists primarily of carbonate and calc-silicate minerals, and that formed by metasomatic replacement of the original rocks, adjacent to, and sometimes, in, plutons. Very few tungsten skarn deposits are polymetallic, and most are exploited only for tungsten, although many contain trace amounts of molybdenum (including some of the prospects in the Forest) which is sometimes produced as a byproduct. Meinert and others (1990) showed that tungsten skarn deposits are associated with intrusive rocks that are somewhat richer in silica than the intrusive rocks that are characteristic of gold, copper, or zinc-lead skarns. The Butte Quartz Monzonite (plate 1) and many other rocks of the Boulder batholith are of the appropriate composition to generate tungsten skarn deposits.

\section{Choice of Model}

Tungsten skarn deposits are an important source of tungsten worldwide, but known deposits and occurrences in Montana, and those in and near the Forest are small, and the available grade and tonnage model was judged to be inappropriate to this area. Exploration for this deposit type in the United States is limited. Worldwide tungsten production is dominated by low-cost operations in Asia, and deposits like those that might be found in the Forest are unlikely to be exploited without a significant increase in the price of tungsten. 


\section{Known Examples}

Skarn deposits in the Ophir district (plate 2, map B; table E1), 7-10 mi northwest of Elliston, have produced a small amount of tungsten, certainly less than 100 tons. Recent exploration in the Ophir district, however, has been directed toward gold-bearing deposits. Other possible historic tungsten skarn deposits and prospects are in the Big Blackfoot and Stemwinder Hill districts, and in the Dog Creek area.

\section{Tract Delineation}

For the permissive tract for tungsten skarn deposits, we delineated the same area as for gold skarns, with one important exception; areas underlain by the Elkhorn Mountains Volcanics were also excluded from the permissive tract because tungsten skarns are not known to occur in volcanic rocks. We then ensured that this area included all the known tungsten skarn deposits and prospects; the result is depicted on plate 5, map $\mathrm{L}$.

Again, we designated three small areas to constitute a favorable tract (plate 5, map L). These three areas had to meet all of the above criteria, including being near important deposits or prospects; they are west and south of Helena and are apparently related to outlying granodiorite plutons at the north end of the Boulder batholith.

\section{Estimate of Undiscovered Deposits}

Because the team believed that Montana tungsten skarn deposits are likely to be small, compared to others found worldwide, the existing grade and tonnage model was judged to be inappropriate to this area, and no numerical estimates were made.

\section{POLYMETALLIC REPLACEMENT DEPOSITS}

Polymetallic replacement deposits typically form tabular, pod-, and pipelike ore bodies that may be localized by faults or favorable sedimentary strata. The deposits are found in sedimentary rocks, chiefly carbonates (limestone and dolomite), which are intruded by porphyritic plutons. Massive carbonate beds that may fracture readily during intrusion and deformation are the preferred host rock. Mineral zoning is common, with inner zones rich in chalcopyrite or enargite and outer zones containing only sphalerite and rhodochrosite. Jasperoid is commonly found near ore bodies.

\section{Cholce of Model}

Polymetallic replacement ores have been important sources of lead, $z$ inc, and silver throughout history. However, Helena Forest examples are smaller than 
the majority of the districts that make up the worldwide grade and tonnage model (Mosier and others, 1986); most important U.S. districts are in Nevada and Utah. The Elkhorn mine, nearby in the Deer Lodge Forest, was a significant deposit, however, and we determined that the worldwide model is appropriate to the Forest.

\section{Known Examples}

Polymetallic replacement-type mineralization is found in the Austin and Ophir districts within the Forest (plate 2, map B; table E1). In addition, the Elkhorn mine (plate 2, map B; table E1), nearby in the Deer Lodge National Forest is representative of this type of deposit.

\section{Tract Delineation}

In a particular mineral system, polymetallic replacement deposits are usually found farther from intrusive rocks than related skarn deposits. However, at the small scale of this assessment, this distinction becomes trivial, and we considered the criteria for delineation of the permissive tract to be the same for polymetallic replacement as for skarn deposits. We used the criteria that a polymetallic replacement deposit requires a reactive host rock and an intrusion to provide heat, fluids, and metals. Accordingly, we outlined areas near plutonic rocks, both exposed and inferred, that are underlain by carbonatebearing strata. Formations included in the permissive tract are the Helena, Empire, Shepard, Red Lion, and Hasmark Formations; the Park Shale, Meagher Limestone, Wolsey Shale, and Flathead Sandstone; the Three Forks, Jefferson, and Maywood Formations, and members of the Madison Group; and the Amsden, Phosphoria, and Kootenai Formations. We excluded rocks of the Colorado Group, the Blackleaf, Mount Shields, and Bonner Formations, all intrusive rocks, and all volcanic rocks. We then ensured that the resulting area included all the known polymetallic replacement deposits and prospects; the result is depicted on plate 5 , map M. No favorable area was delineated.

\section{Estimate of Undiscovered Deposits}

Polymetallic replacement deposits rarely form in isolation, and examples in the grade and tonnage model are districts, which commonly cover tens of square miles. Thus, the team reasoned that, because of their size, undiscovered districts are unlikely to be concealed beneath alluvial cover, and are unlikely to have been overlooked by past exploration. Thus, a relatively small estimated number of deposits seemed appropriate. For the 90th, 50th, 10th, 5th, and 1st percentiles, the team estimated $0,0,0,1$, and 3 or more polymetallic replacement deposits, consistent with the grade and tonnage model of Mosier and others (1986) (Mark3 index 47).

\section{POLYMETALLIC VEIN DEPOSITS}

Polymetallic vein deposits are the most common type of metallic mineral 
deposit in the Helena National Forest, and possibly, in the world. They appear to be a product of the emplacement of intrusive rocks into shallow to moderate levels of the crust. Thus, areas permissive for the occurrence of polymetallic vein deposits are the same as those for porphyry copper deposits. Indeed, polymetallic vein deposits are commonly used in prospecting, as an indicator of porphyry copper deposits. These vein deposits are associated with a wide spectrum of compositions of igneous rocks. Quartz is commonly the most important gangue mineral, whereas the most important metallic minerals are pyrite, sphalerite, galena, and various silver-bearing minerals, along with electrum.

Although polymetallic vein deposits have provided a large part of the past production of mineral wealth in Montana, and in the entire western United States, there is, at present, no suitable grade and tonnage model for these deposits. The existing model (B1iss and Cox, 1986) is not representative of deposits in the Rocky Mountains. The known deposits, like polymetallic replacement deposits, occur in districts, often consisting of dozens of individual veins and(or) mines. In contrast, many of the deposits used in the Bliss and Cox (1986) model are individual mining entities, and consist of only a very small part of the district in which they occur. The sizes of deposits in the model are too small to be used reliably in Montana, and no quantitative estimates were made, even though we believe the remaining resource in these known districts is substantial.

Districts within the scope of this study that incorporate many polymetallic vein deposits include Basin, Big Blackfoot, Clancy, Corbin-Wickes, Elliston, Heddleston, Indian Creek, Marysville, Park, Radersburg, Rimini, and Winston.

Because of the large size of polymetallic vein districts, the team believes that there are few undiscovered districts in exposed areas. Any that might exist would be in areas covered by surficial sediments. Perhaps of more significance is the resource remaining in known districts. Mining halted in nearly all these districts for economic or political reasons, not because mineralized rock was exhausted. The remaining resource in these areas is probably as large or larger than the resource that may exist in undiscovered districts. However, we presently lack a technology for estimating this resource.

\section{URANIUM-BEARING POLYMETALLIC VEIN DEPOSITS}

Some polymetallic vein deposits in the Forest contain trace amounts of uranium, and a few produced small amounts of uranium ore. Most of these veins are in a northeast-trending band that extends from southwest of Clancy to the vicinity of Lava Mountain. It is probable that the uranium in the veins is related to the intrusion of the rhyolites, because of the veins spatial distribution and coincidence with an area of northeast-trending, highly-evolved, rhyolitic dikes and minor intrusions of oligocene age. Wenrich and others (1990, their plate 5) demonstrated that aerial gamma ray spectroscopic data outline an area of anomalous radioactivity coincident with these veins. Some of these deposits have also been exploited as "health mines" for some years. The amount of uranium in these deposits is not known, but must be very small. We do not believe these veins are likely to be a significant source of uranium, nor will they be an attractive exploration target during the lifetime of this assessment. 


\section{CLIMAX MOLYBDENITE DEPOSITS}

Climax molybdenite deposits are invariably associated with high-silica granite or rhyolite. These high-silica rocks display a characteristic geochemical signature and are strongly enriched in $\mathrm{Rb}, \mathrm{Y}, \mathrm{Nb}, \mathrm{Th}, \mathrm{Sn}$, and $\mathrm{W}$ (Ludington, 1986). They exhibit higher molybdenite grade, and higher fluorine contents than other large, porphyry-type molybdenite deposits. Climax deposits included in the grade-tonnage model (Singer, Theodore, and Mosier, 1986) all have three or more ore bodies, formed by repeated porphyry intrusion, but included together as one deposit in each case. Considering this fact and the restricted permissive environment, the occurrence of a Climax deposit should be considered an uncommon event. Topaz-bearing rhyolites, like those at Lava Mountain in the Elkhorn Mountains, have been suggested (Burt and others, 1982) to mark the tops of deeply buried deposits.

\section{Known Examples}

There is one important C1imax deposit in Montana, at Big Ben (376 million metric tons at 0.098 percent Mo; Carten and others, 1993), in the Little Belt Mountains, about $50 \mathrm{mi}$ northeast of the Forest. The Bald Butte deposit (plate 2, map B; table El, no. 133), a few thousand feet outside the Forest southwest of Marysville, appears to have most of the characteristics of a Climax deposit. A sample of drill core from the deposit analyzed for this study showed elevated contents of $\mathrm{Rb}(>350 \mathrm{ppm}), \mathrm{Nb}(>70 \mathrm{ppm}), \mathrm{Y}(>150 \mathrm{ppm}), \mathrm{Sn}(>100 \mathrm{ppm})$, and W $(>100$ $\mathrm{ppm})$. These are all important components in the geochemical signature of Climax deposits. The presently known resource at Bald Butte is smaller (14 million metric tons at 0.1 percent Mo; Carten and others, 1993) than any of the deposits in the grade and tonnage model and is unlikely to be exploited within the lifespan of this assessment.

\section{Tract Delineation}

The following criteria were used to delineate the permissive tract for Climax deposits: 1) stream-sediment anomalies for Mo, sometimes accompanied by $\mathrm{Bi}, \mathrm{Sn}$, and $\mathrm{W} ; 2$ ) the presence or suspected presence of high-silica rhyolite flows, dikes, and small stocks; 3 ) trace-element geochemistry of rhyolites that exhibits elevated $\mathrm{Rb}, \mathrm{Y}, \mathrm{Nb}, \mathrm{W}, \mathrm{Sn}$, or $\mathrm{Th}$. To meet any one of these criteria was deemed sufficient evidence to make an area permissive.

We delineated a permissive tract that consists of three discrete areas in the western part of the Forest (plate 5, map $N$ ). In the north, an area that encompasses the McDonald Meadows area and the Stemple-Gould district was delineated. Some of the rhyolites at McDonald Meadows display elevated Y, Nb, and $\mathrm{Th}$, even though hydrothermal alteration may have altered their $\mathrm{Rb}, \mathrm{Sr}$, and Ba contents. The Stemple-Gould district is the site of a pronounced Mo anomaly in stream-sediment data, but no rhyolitic rocks are known in the area. Farther south, a nearly circular area was delineated north of MacDonald Pass on U.S. 12. The basis for delineation of the northern part of this area is the deposit at Bald Butte, which is apparently responsible for another large Mo anomaly in stream-sediment data. The southern part of this area contains a cluster of 
rhyolite intrusions west of Mullan Pass that can be inferred to be the source of the Avon volcanics, part of which are high-silica rhyolites. Farther south, a third permissive area stretches from near Basin, north-northeast to the vicinity of Lava Mountain in the Elkhorn Mountains. This area was delineated primarily because of the occurrence of a series of high-silica flows and intrusions, and it also displays a moderately-strong Mo anomaly in stream-sediment data, centered just northeast of Chessman Reservoir. The tract is delineated on plate 5, map N.

\section{Estimate of Undiscovered Deposits}

We envisioned a number of possible sites for undiscovered Climax deposits within the permissive tract: McDonald Meadows, the Stemple-Gould district, the Bald Butte area, west of Mullan Pass, the vicinity of Buffalo Gulch (near Chessman Reservoir), the Basin area, and the vicinity of Lava Mountain. We evaluated the possibility that each of these might be the site of an undiscovered deposit. The area west of Mullan Pass is probably the most highly favorable target, and we estimated that there is about one chance in 20 that it contains an undiscovered deposit. Stemple-Gould is difficult to evaluate because no rhyolites are known from the area. We doubt that there is room in the Bald Butte area for a new undiscovered deposit, though Mo resource there could likely be increased with further exploration. McDonald Meadows, Basin, Buffalo Gulch, and Lava Mountain were judged to be much less interesting, with estimated probabilities of occurrence between 1 in 10,000 and 1 in 1,000 . Our final estimate was, for the $90 \mathrm{th}, 50 \mathrm{th}, 10 \mathrm{th}, 5 \mathrm{th}$, and 1st percentiles, $0,0,1,1$, and 2 or more Climax deposits (Singer, Theodore, and Mosier, 1986) (Mark3 index 5).

\section{YORK-TYPE EXHALATIVE(?) GOLD DEPOSITS (AND AU-BEARING VEINS}

The York district, on the southwest flank of the Big Belt Mountains, contains a unique combination of geologic characteristics that suggest the possible presence of a heretofore unknown mineral deposit type (see Chapter $F$, York district). An extensive zone in the Middle Proterozoic Greyson Formation is characterized by uncommon amounts of potassium, with as much as 12 weight percent $\mathrm{K}_{2} \mathrm{O}$. These zones, termed reefs, are also characterized in part by elevated gold contents. Very little of this rock apparently contains enough gold to be classified as ore, but there is a large low-grade resource. Thorson and others (in preparation) quote a resource of more than 7 million oz., using a cutoff grade of 0.01 opt. Within this zone, some gold-bearing vein deposits are known, most notably the Golden Messenger mine (Pardee and Schrader, 1933). The Late Proterozoic mafic dike that hosts the Golden Messenger and associated prospects intrudes the northwest end of the gold-and potassium-enriched zone described above; thus these vein deposits may contain gold that has been remobilized from the potassic reefs.

\section{Tract Delineation}

The gold- and potassium-enriched reefs can be difficult to recognize in the 
field, especially in areas of poor outcrop. However, the map of aeroradioactivity due to potassium (fig. C6) delineates the known anomalous zones (reefs) quite well. For this reason, we relied primarily on this map to delineate a permissive tract where similar chemically anomalous zones and possible associated mineral deposits may be found. This area extends the length of the Big Belt Mountains (plate 6, map 0).

\section{Estimate of Undiscovered Deposits}

In the absence of a grade and tonnage models (even the descriptive model is very preliminary), no estimates of undiscovered resources could be made. However, we expect the permissive area to continue to be the site of prospecting for possible gold deposits.

\section{GRETACEOUS GOLD-BEARING VEIN DEPOSITS}

The gold-bearing vein deposit at Miller Mountain, in the Confederate Gulch district in the Big Belt Mountains (see Chapter F, Confederate Gulch district), is difficult to classify. Polymetallic vein deposits can be quite variable in their mineralogy, and deposits that are gold-rich and those that show an almost complete lack of gold can both be so classified. There are several gold-rich prospects and occurrences of this type around the margin of the Boulder pluton, south of Miller Mountain. But the deposits at Miller Mountain are notably poor in base metals and contain almost no sulfide minerals except pyrite and arsenopyrite. Veins with mineralogy similar to that at Miller Mountain are usually classified as low-sulfide gold-quartz veins, but the tectonic setting at Miller Mountain is not indicative of that deposit type, which is normally associated with deep-seated shear zones, and low- to medium-grade metamorphic terranes. The polymetallic veins around the Boulder pluton and the deposits at Miller Mountain are all apparently Late Cretaceous in age, however, and, largely for this reason, we have classified them together. In the Big Belt Mountains, we have delineated a permissive tract for these vein deposits, based largely on proximity to pluton margins, and to the location of known prospects (plate 6, map $0)$. In essence, this tract describes the area where we predict that exploration for deposits like Miller Mountain will continue. As with the York-type occurrences, there is no grade and tonnage model, and we made no estimate of undiscovered resources.

\section{QUARTZ-ADULARIA AND HOT-SPRING GOLD-SILVER DEPOSITS}

Quartz-adularia deposits consist of epithermal veins or groups of veins that typically have a sulfide assemblage that includes argentite, tetrahedrite, tennantite, and variable amounts of galena and sphalerite. Gold, as electrum, is usually present. Hydrothermal alteration associated with these deposits is characterized by sericitic and argillic alteration assemblages, along with adularia and carbonate; primary alunite is not associated with the main stage of mineralization. The mineralized area is often elongate, and some of the deposits and districts are very large, with vein systems many miles long. Structural 
controls for intrusion and mineralization are complex; caldera ring and radial fracture settings are not uncommon, yet some deposits occur remote from any caldera. Most deposits formed at paleodepths of less than a few thousand feet, with many forming within a few hundred feet of the paleosurface.

Hot-spring gold-silver deposits, defined by Berger $(1985,1986)$, represent the uppermost parts of epithermal systems, and formed within a few tens to hundreds of feet from the surface. They appear to form above quartz-adularia deposits, thus will be found in the same regions and geologic environments. We estimated numbers of undiscovered deposits for both types independently.

\section{Choice of Model}

Epithermal quartz-adularia gold deposits have been divided into three subtypes-Creede, Comstock, and Sado (Mosier and others, 1986). Because of difficulties in distinguishing among the environments likely to contain these subtypes, we evaluated the possible occurrence of epithermal quartz-adularia vein districts using a combination of the Comstock and Sado grade and tonnage models (Mark3 index 25). The grade and tonnage model of Berger and Singer (1992) (Mark3 index 45) was used to evaluate hot-spring gold-silver deposits.

\section{Known Examples}

Important deposits in the Forest that were classified as quartz-adularia deposits include Drumlummon, Jay Gould, and Piegan-Gloster (plate 2, map B; table E1, nos. 139, 105, and 119). The most significant hot-spring deposit, which lies outside the Forest, is clearly McDonald Meadows (plate 2, map B; table El, no. 53), one of the largest gold deposits in the United States (see Chapter F, Blackfoot River area).

\section{Tract Delineation}

The following criteria were used to delineate the permissive tract for quartz-adularia and hot-spring gold-silver deposits: 1) presence of Tertiary volcanic rocks and 2) presence of epithermal prospects or deposits in or near the Forest. The Elkhorn Mountains Volcanics were not included because there is minimal evidence that this type of mineralization is associated with the Cretaceous volcanic rocks. We also excluded areas that appear to be too deeply eroded to preserve epithermal deposits. The resulting permissive tract is depicted on plate 6 , map $P$.

\section{Estimate of Undiscovered Deposits}

Our resource estimates were based primarily on the number of known and suspected eruptive centers and very shallow plutons, combined with the occurrence of known deposits and prospects. For the $90 \mathrm{th}, 50 \mathrm{th}, 10 \mathrm{th}, 5 \mathrm{th}$, and 1st percentiles, the team estimated $1,1,2,2$, and 2 or more hot spring deposits and $0,1,2,3$, and 4 or more quartz-adularia districts. 


\section{SEDIMENTARY EXHALATIVE ZINC-LEAD DEPOSITS}

Sedimentary exhalative (sedex) deposits are large deposits of zinc, lead, and silver; their shape is generally similar to that of the host sedimentary strata. They are an important worldwide source of zinc and lead, and some of the largest deposits in the world are in North America (Menzie and Mosier, 1986). They are formed during, or shortly after, deposition of clastic rocks in the deep part of ocean basins, apparently due to expulsion of metal-bearing hydrothermal fluids from the basins along faults that are active during deposition. Individual deposits are commonly not directly associated with igneous rocks.

\section{Known Examples}

The Sullivan deposit, in British Columbia, Canada, is an important example of sedex deposits. It is one of the largest in the world, and is found in a tectonic and stratigraphic setting that is similar to parts of the Belt Supergroup in the Forest. The Sheep Creek deposit (see Chapter F, Big Belt Mountains region), east of the Forest on the south flank of the Little Belt Mountains, contains many similarities to sedex deposits, especially style of alteration and tectonic setting. Its metallogeny is, however, quite distinct; Sheep Creek is rich in copper, cobalt, and nickel. Nevertheless, we believe that Sheep Creek and any other deposits like it formed in a similar environment to that in which zinc-and lead-rich sedex deposits developed.

\section{Tract Delineation}

A permissive tract for sedex deposits, including those similar to the Sheep Creek deposit was defined by inclusion of Proterozoic rocks that were deposited in deep water environments (below wave base). This restriction indicates that most of the Newland Formation and the lower two-thirds of the Greyson Formation are permissive. The tract was delineated using the presence of these stratigraphic units, and their possible subsurface extensions within about 1,000 feet from the surface. Syndepositional faults may be present in other nearby parts of the Belt Basin, but this feature has not been clearly demonstrated in the Forest. The resulting permissive tract is depicted on plate 6 , map $Q$.

\section{Estimate of Undiscovered Deposits}

Although the tectonic and stratigraphic environment supports the delineation of a permissive tract for sedex deposits, the absence of demonstrable syndepositional faulting argues against their presence. Our assessment team estimated that there is a chance of approximately 1 in 1,000 that an undiscovered sedex deposit exists in the Helena National Forest.

\section{SEDIMENT -HOSTED COPPER DEPOSITS, REDBED TYPE}

Sediment-hosted copper deposits are layered, stratabound, and locally stratiform mineralized bodies of disseminated copper and copper sulfides, with 
or without silver, uranium and vanadium. Their formation is associated with acidic, reducing environments, and they often form at the boundary between oxidized and reduced sedimentary rocks.

\section{Choice of Model}

Sediment-hosted copper deposits and prospects in the Helena Forest are in Proterozoic shallow-water clastic and mixed clastic and carbonate rocks, and appear to be of the redbed type. Three subtypes were recognized locally (see Chapter F), deposits that occur in (1) quartzite, 2) greenbeds (siltite and argillite), and 3) carbonate (limestone and dolomite). In addition, some small copper-bearing vein deposits appear to have formed by remobilization of the sediment-hosted deposits. These vein occurrences are not assessed separately, as they are generally small and of little significance.

\section{Known Examples}

Known examples of prospects and occurrences in the Forest include the Hayworth claims (Alice) (quartzite subtype), Copper Hill (greenbed subtype), and Klondike (carbonate subtype) (plate 2, map B; table El, nos. 19, 112, and 2). All are small, and the total amount of production from deposits of this type in the Forest is negligible. A few deposits have small to moderate identified resources.

\section{Tract Delineation}

A permissive tract in the Forest was defined by inclusion of Proterozoic rocks that were deposited in shallow water. This restriction indicates that the Spokane and Empire Formations, the upper third of the Greyson Formation, the Missoula Group, the lower $2 \mathrm{~m}$ of the Helena Formation, and the Bonner and McNamara Formations are permissive. That is, of the Proterozoic section, only the Helena and Newland Formations and the lower two-thirds of the Greyson Formation are excluded. The tract was delineated using these stratigraphic units, and their possible subsurface extensions, to a depth of about 500 feet.

A favorable tract was delineated within the permissive tract. It was restricted to the Spokane and Empire Formations, and used the additional criteria that prospects must be present, and that stream-sediment samples contain anomalous amounts of copper. The resulting permissive tract is depicted on plate 6, map $R$.

\section{Estimate of Undiscovered Deposits}

Deposits and prospects in the Forest are small, and the team judged that there is less than a 1 in 100 chance that any deposits are present that are representative of any applicable grade and tonnage models. 


\section{SIMULATION RESULTS AND ESTIMATED CONTAINED METAL}

The results of the Mark3 simulations are reported in table G3, and figures G1 through G7.

A summary of the results of this assessment is presented in table G4. It shows the amount of undiscovered contained metal predicted to exist in the 6 deposit types that were evaluated. Although the table is a convenient summary, it does not convey a complete answer to the question, "What is the magnitude of undiscovered resources in the study area?" The mean and median values in the table are statistical characteristics of populations, which are products of simulation, using the computer program called MARK3, described in Root and others (1992). The amount of undiscovered resource, for any given metal, cannot be properly represented by any single number.

\section{Nature of Distributions}

The probability distributions that result from the computer simulations are different from those with which many users are familiar, due primarily to the marked asymmetry of most known tonnage distributions. In all the deposit types considered in the study area, a few large deposits in the grade and tonnage models account for a large proportion of the contained metal. Logarithmic standard deviations representing nearly an order of magnitude or more are common among the tonnage models compiled by Cox and Singer (1986). This is one reason why the mean amounts of metal are commonly significantly larger than the medians, and why amounts as large as the mean are unlikely to exist. For example, with reference to figure G3, the median (50th percentile) copper estimate is 780 metric tons, whereas the mean estimate is 42,000 metric tons, nearly two orders of magnitude larger. For this copper estimate, there is less than a 16 percent probability of occurrence of an amount as large as the mean.

In addition, the proportion of this undiscovered metal that can be recovered at a profit is not determined here. The estimated undiscovered metal is that present in deposits like those in the associated grade and tonnage models (see table Gl). Some deposits in the models are economic, and some are not. The economic viability of any individual mineral deposit is a function of current metal prices and mining and recovery costs. Also, of course, new resources may well be discovered in the form of deposit types not considered in this study, or not yet conceived of.

\section{Meaning of the Estimates}

Whatever form is used to communicate the results of these estimates, it is we11 to remember that they reflect a large uncertainty in the number and sizes of undiscovered deposits of the types evaluated. Undiscovered deposits are, by their very nature, not known with a high degree of certainty. Furthermore, not all types of deposits known to be present in the study area could be evaluated.

The most important conclusion that can be drawn from the probability distributions represented in tables G3 and G4, and figures G1 through G7, is the dominant role that copper and gold play in the Forest. These two commodities constitute approximately 90 percent of the potential value of the undiscovered 
Table G3. Summaries of simulated metal and mineralized rock in undiscovered deposits in the Helena National Forest at 5 probability levels, and at the mean (all amounts are in metric tons). Each subtable describes predicted resources in one type of mineral deposit, A) Porphyry Cu deposits, North American subset, B) Skarn Au deposits for all areas except Elkhorn Mountains, model truncated to those with $>15,000$ t of ore, C) Skarn Au deposits for Elkhorn Mountains, model truncated to those with $>15,000$ t of ore, D) Polymetallic replacement deposits, E) Climax Mo deposits, F) Hot-spring Au-Ag deposits, and G) Epithermal quartz-adularia vein deposits. Index refers to the number which uniquely identifies the grade and tonnage model used for estimation and simulation. See Root and others (1996). Estimated numbers of deposits are in Table G2.

A. Mark3 Index 81: Porphyry Cu (North America)

\begin{tabular}{crrrrrr} 
quantile & \multicolumn{1}{c}{ Cu } & Mo & Au & Ag & \multicolumn{1}{c}{ rock } \\
\hline 0.95 & 0 & 0 & 0 & 0 & 0 \\
0.90 & 0 & 0 & 0 & 0 & 0 \\
0.50 & 0 & 0 & 0 & 0 & 0 \\
0.10 & $2,000,000$ & 21,000 & 10 & $300420,000,000$ \\
0.05 & $4,300,000$ & 78,000 & 37 & 1,100 & $840,000,000$ \\
& mean & 920,000 & 20,000 & 9 & 260 & $170,000,000$ \\
Probability of mean & 0.15 & 0.10 & 0.10 & 0.10 & 0.16 \\
Probability of zero & 0.70 & 0.81 & 0.86 & 0.84 & 0.70 \\
\hline
\end{tabular}

B. Mark3 Index 105: Skarn Au, truncated (except Elkhorn Mts.)

\begin{tabular}{crrrrrrr} 
quantile & \multicolumn{1}{c}{ Cu } & Au & \multicolumn{1}{c}{ Fe } & Ag & PD & \multicolumn{1}{r}{ rock } \\
\hline 0.95 & 0 & 0 & 0 & 0 & 0 & 0 \\
0.90 & 0 & 0 & 0 & 0 & 0 & 53,000 \\
0.50 & 12,000 & 9 & 0 & 18 & 0 & $2,800,000$ \\
0.10 & 240,000 & 70 & 665,000 & 290 & 11,000 & $29,000,000$ \\
0.05 & 470,000 & 110 & $1,600,000$ & 570 & 33,000 & $44,000,000$ \\
& mean & 85,000 & 25 & 390,000 & 130 & 32,000 & $9,800,000$ \\
Probability of mean & 0.19 & 0.28 & 0.12 & 0.20 & 0.05 & 0.29 \\
Probability of zero & 0.20 & 0.07 & 0.86 & 0.19 & 0.80 & 0.07 \\
\hline
\end{tabular}

C. Mark3 Index 105: Skarn Au, truncated (Elkhorn Mts.)

\begin{tabular}{crrrrrrr} 
quantile & \multicolumn{1}{c}{ Cu } & Au & & Fe & Ag & Pb & \multicolumn{1}{r}{ rock } \\
\hline 0.95 & 0 & 0 & 0 & 0 & 0 & 0 \\
0.90 & 0 & 0 & 0 & 0 & 0 & 0 \\
0.50 & 780 & 2 & 0 & 2 & 0 & 510,000 \\
0.10 & 80,000 & 33 & 0 & 110 & 0 & $16,000,000$ \\
0.05 & 230,000 & 54 & 460,000 & 250 & 9,300 & $25,000,000$ \\
& mean & 42,000 & 12 & 150,000 & 59 & 11,000 & $4,600,000$ \\
Probability of mean & 0.16 & 0.25 & 0.06 & 0.16 & 0.05 & 0.22 \\
Probability of zero & 0.43 & 0.32 & 0.94 & 0.42 & 0.91 & 0.32 \\
\hline
\end{tabular}


Table G3. (continued)

D. Mark3 Index 47: Polymetallic replacement

\begin{tabular}{|c|c|c|c|c|c|c|}
\hline quantile & $\mathrm{Cu}$ & Au & $\mathrm{Zn}$ & Ag & PD & rock \\
\hline 0.95 & 0 & $\overline{0}$ & 0 & 0 & 0 & $\overline{0}$ \\
\hline 0.90 & 0 & 0 & 0 & 0 & 0 & 0 \\
\hline 0.50 & 0 & 0 & 0 & 0 & 0 & 0 \\
\hline 0.10 & 0 & 0 & 0 & 0 & 0 & 0 \\
\hline 0.05 & 1.700 & 1 & 99,000 & 290 & 110,000 & $2,800,000$ \\
\hline mean & 2,000 & 1 & 49,000 & 170 & 47,000 & 880,000 \\
\hline Probability of mean & 0.05 & 0.05 & 0.06 & 0.06 & 0.06 & 0.06 \\
\hline Probability of zero & 0.93 & 0.94 & 0.92 & 0.93 & 0.92 & 0.92 \\
\hline
\end{tabular}

E. Mark3 Index 5: Climax Mo

\begin{tabular}{|c|c|c|}
\hline quantile & Mo & rock \\
\hline 0.95 & $\overline{0}$ & $\overline{0}$ \\
\hline 0.90 & 0 & 0 \\
\hline 0.50 & 0 & 0 \\
\hline 0.10 & \multicolumn{2}{|c|}{$710,000420,000,000$} \\
\hline 0.05 & \multicolumn{2}{|c|}{$1,200,000 \quad 630,000,000$} \\
\hline mean & \multicolumn{2}{|c|}{$200,000 \quad 100,000,000$} \\
\hline Probability of mean & 0.23 & 0.24 \\
\hline Probability of zero & 0.70 & 0.70 \\
\hline
\end{tabular}

F. Mark3 Index 45: Hot spring Aur-Ag

\begin{tabular}{|c|c|c|c|}
\hline quantile & Au & $\mathrm{Ag}$ & rock \\
\hline 0.95 & 0 & 0 & 0 \\
\hline 0.90 & 1 & 0 & 510,000 \\
\hline 0.50 & 28 & 45 & $20,000,000$ \\
\hline 0.10 & 140 & 670 & $94,500,000$ \\
\hline 0.05 & 230 & 1.000 & $200,000,000$ \\
\hline mean & 58 & 240 & $41,000,000$ \\
\hline Probability of mean & 0.28 & 0.27 & 0.27 \\
\hline Probability of zero & 0.07 & 0.38 & 0.07 \\
\hline
\end{tabular}

G. Mark3 Index 25: Epithermal vein, quartz-adularia

\begin{tabular}{crrrrrrr} 
quantile & Cu & Au & Zn & Ag & Pb & \multicolumn{2}{r}{ rock } \\
\hline 0.95 & 0 & 0 & 0 & 0 & 0 & 0 \\
0.90 & 0 & 0 & 0 & 0 & 0 & 0 \\
0.50 & 0 & 2 & 0 & 22 & 0 & 400,000 \\
0.10 & 300 & 52 & 0 & 2.000 & & 0 & $8,700,000$ \\
0.05 & 1.100 & 100 & 0 & 4,600 & & 0 & $15,000,000$ \\
& mean & 380 & 21 & 18 & 1.400 & 2 & $3,700,000$ \\
Probability of mean & 0.09 & 0.20 & 0.02 & 0.12 & 0.03 & 0.18 \\
Probability of zero & 0.87 & 0.31 & 0.98 & 0.31 & 0.96 & 0.31 \\
\hline
\end{tabular}




\section{Porphyry copper deposits (North America)}

\section{Cumulative Distributions of Contained Metal and Mineralized Rock}

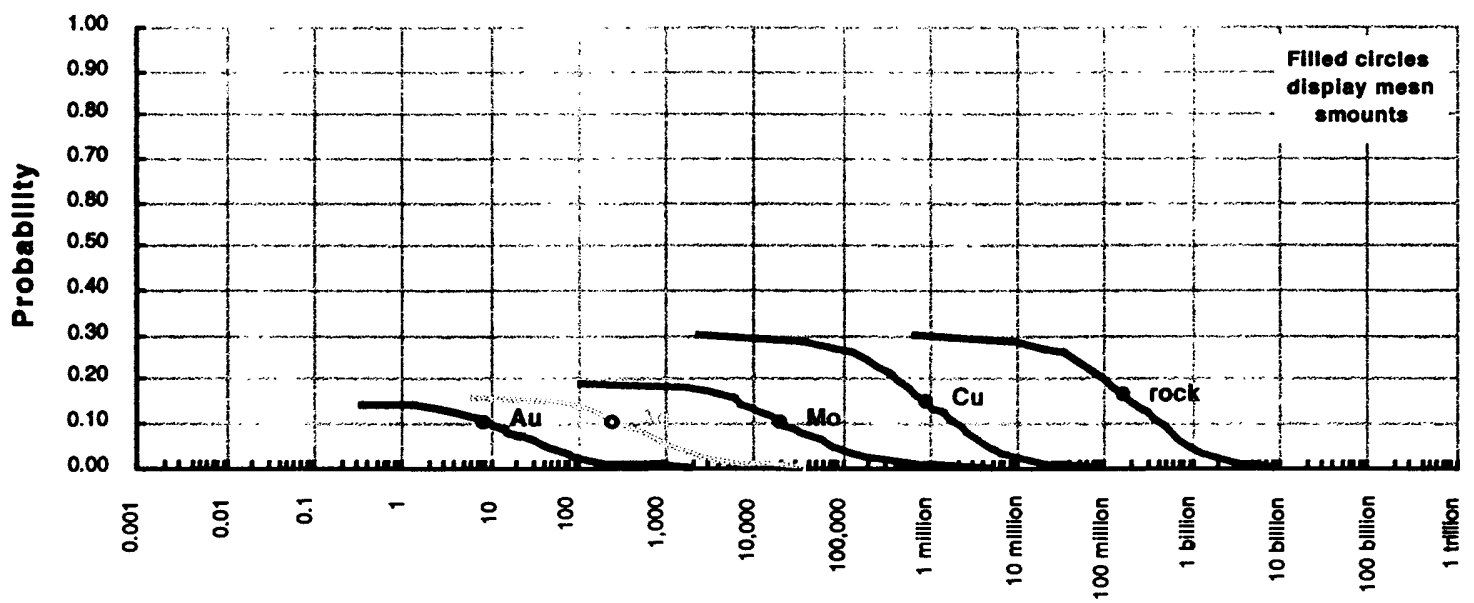

metric tons

Histograms of Contalned Metal and Mineralized Rock (metric tons)
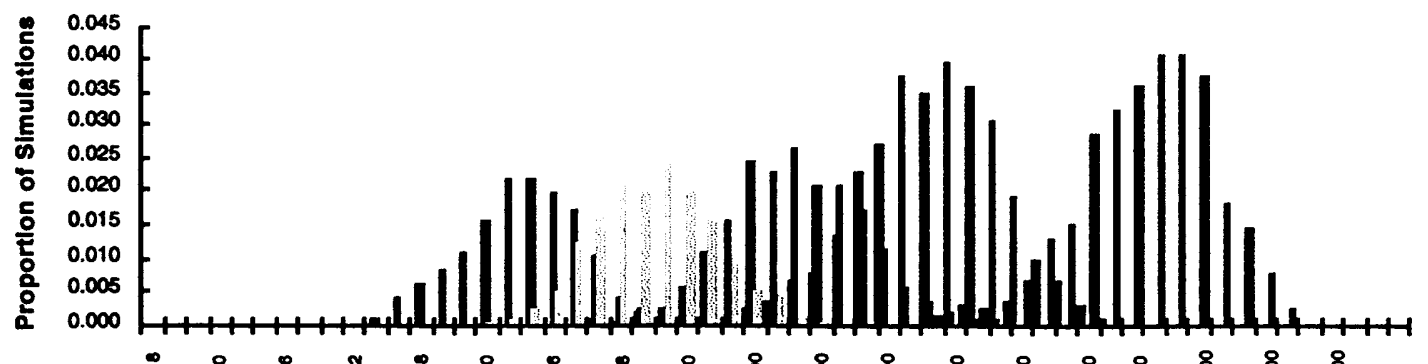

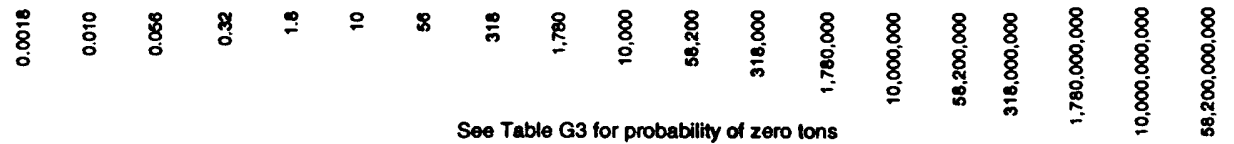

DCu Mo au Ma

Figure G1. Simulated metal content in undiscovered porphyry copper deposits in the Helena National Forest (all amounts in metric tons). 


\section{Skarn Au deposits (Elkhorn Mountains)}

\section{Cumulative Distributions of Contained Metai and Mineralized Rock}

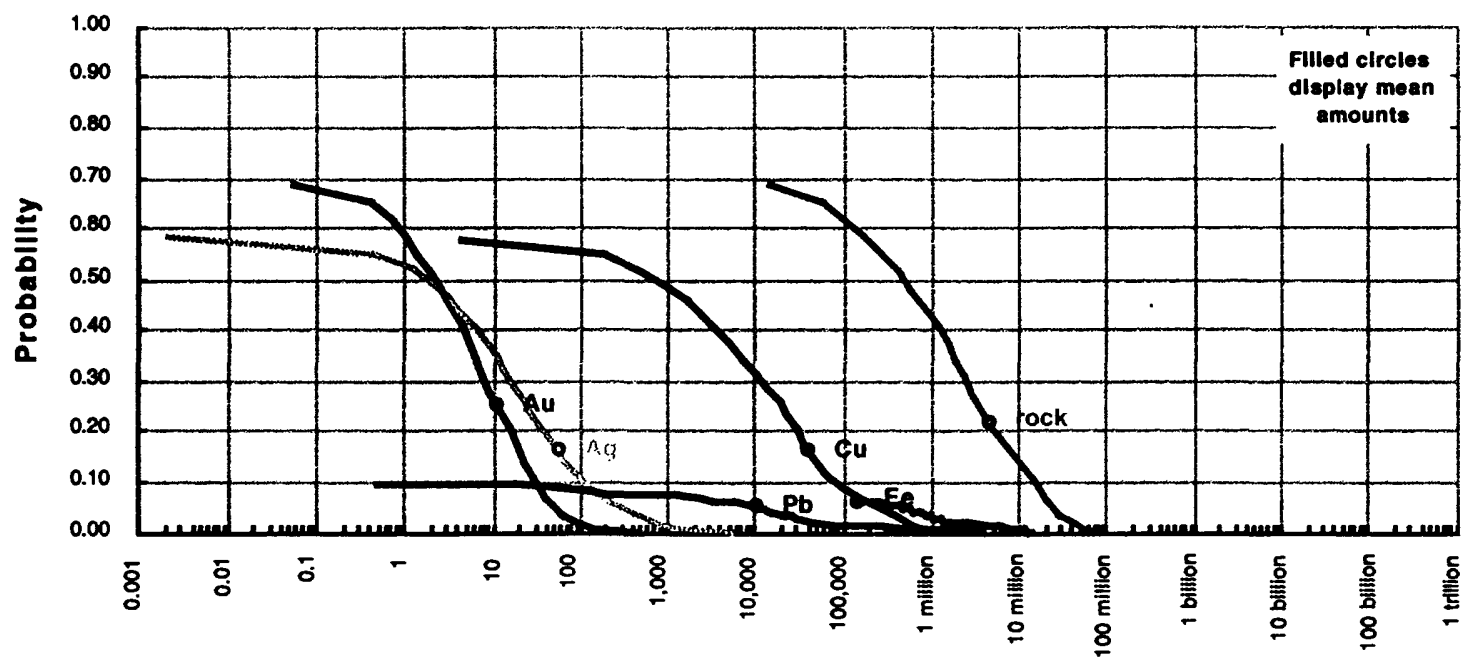

Histograms of Contalned Metal and Minerallzed Rock (metric tons)

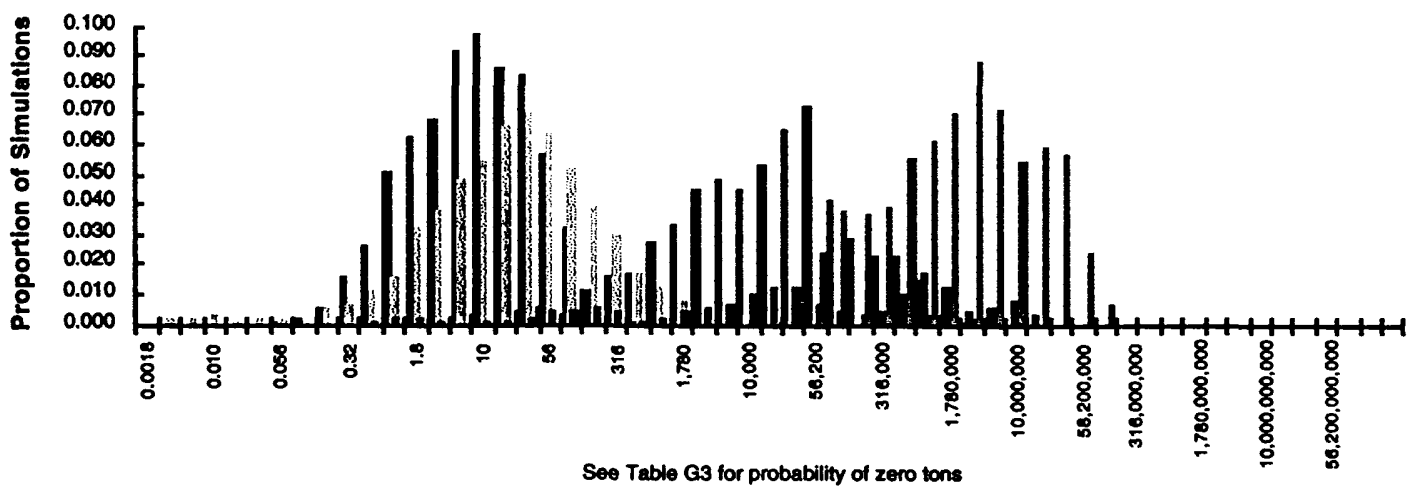

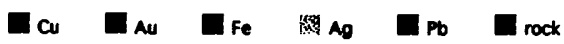

Figure G2. Simulated metal content in undiscovered skarn gold deposits in the Helena National Forest (exclusive of the Elkhorn Mountains). All amounts in metric tons. 


\section{Skarn Au deposits (exclusive of Elkhorn Mountains)}

Cumulative Distributions of Contained Metal and Mineralized Rock

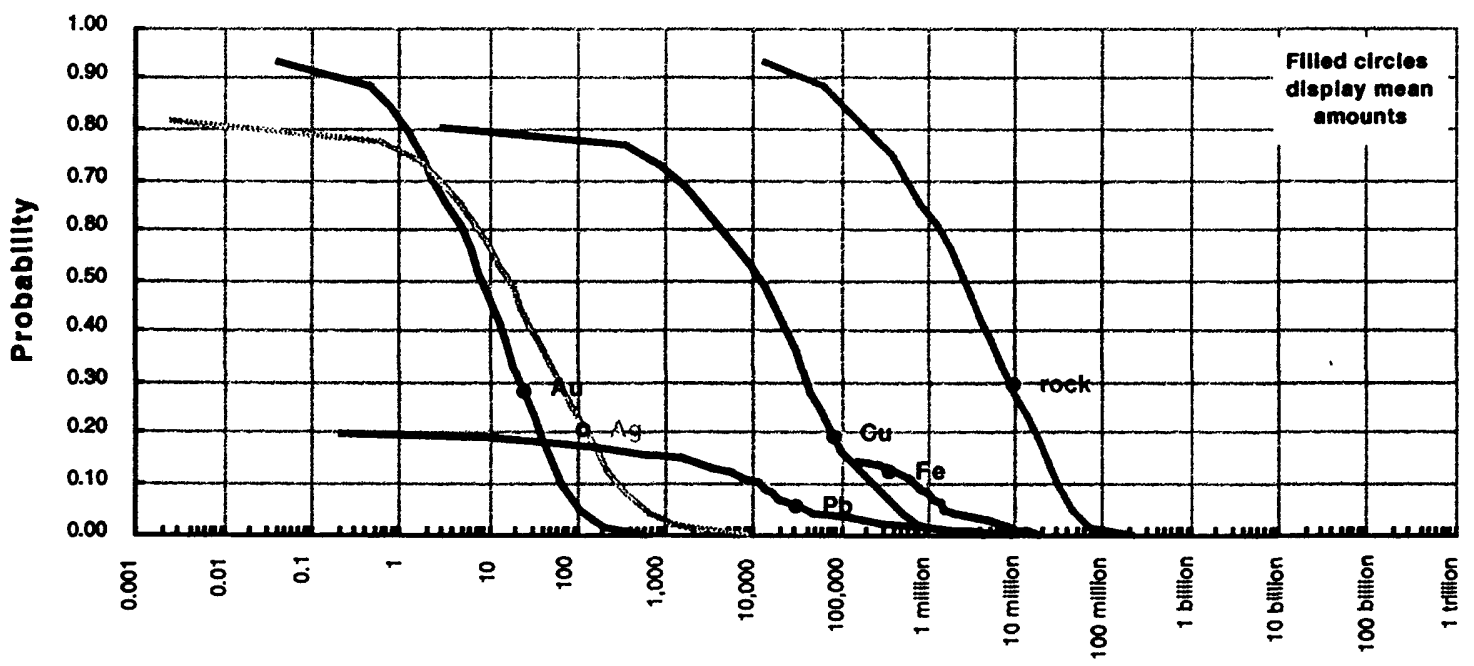

Histograms of Contalned Metal and MInerallzed Rock

(metric tons)

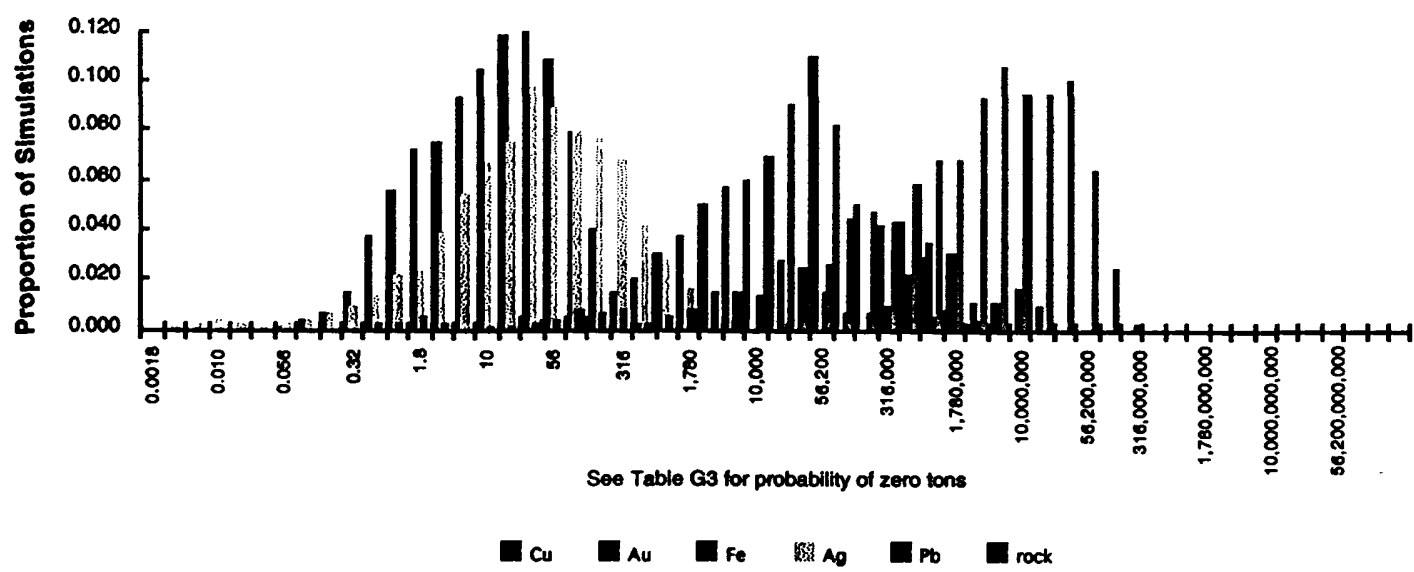

Figure G3. Simulated metal content in undiscovered skarn gold deposits in the Helena National Forest (Elkhorn Mountains only). All amounts in metric tons. 


\section{Polymetailic replacement deposits}

\section{Cumulative Distributions of Contained Metal and Mineralized Rock}

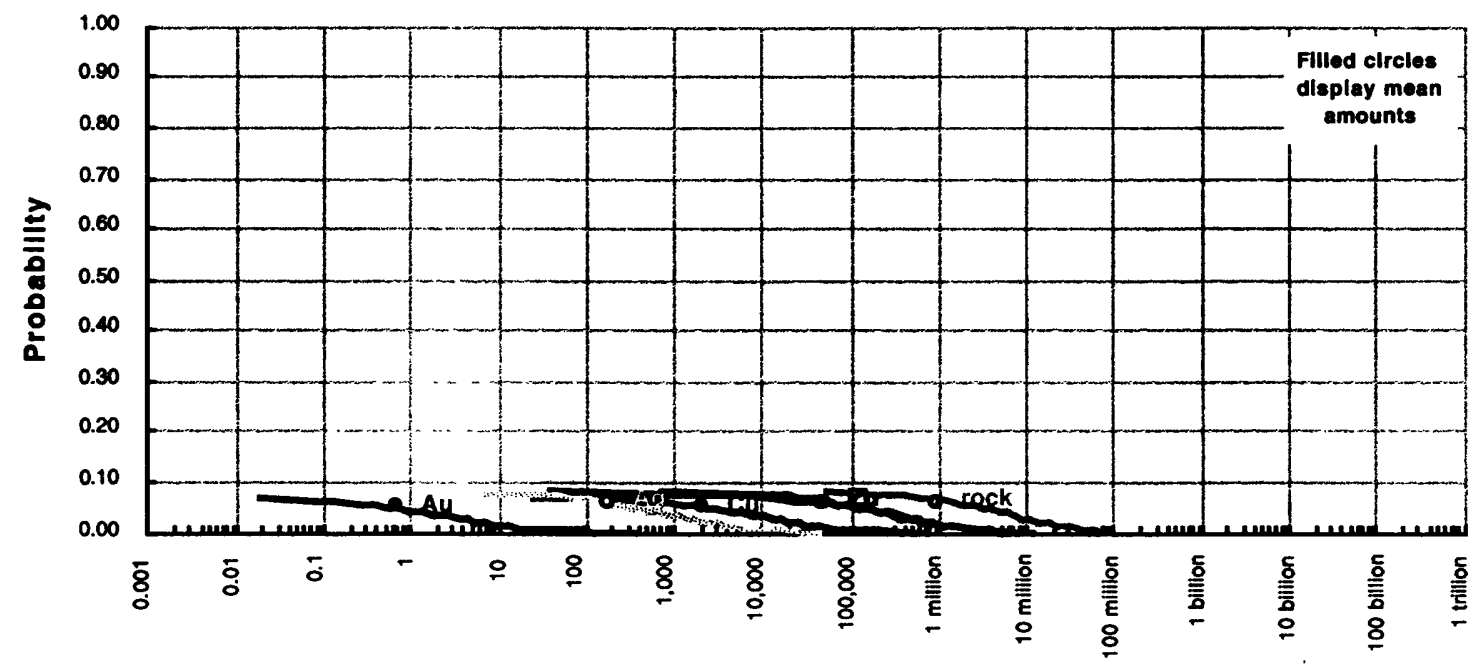

Histograms of Contalned Metal and Minerallzed Rock (metric tons)

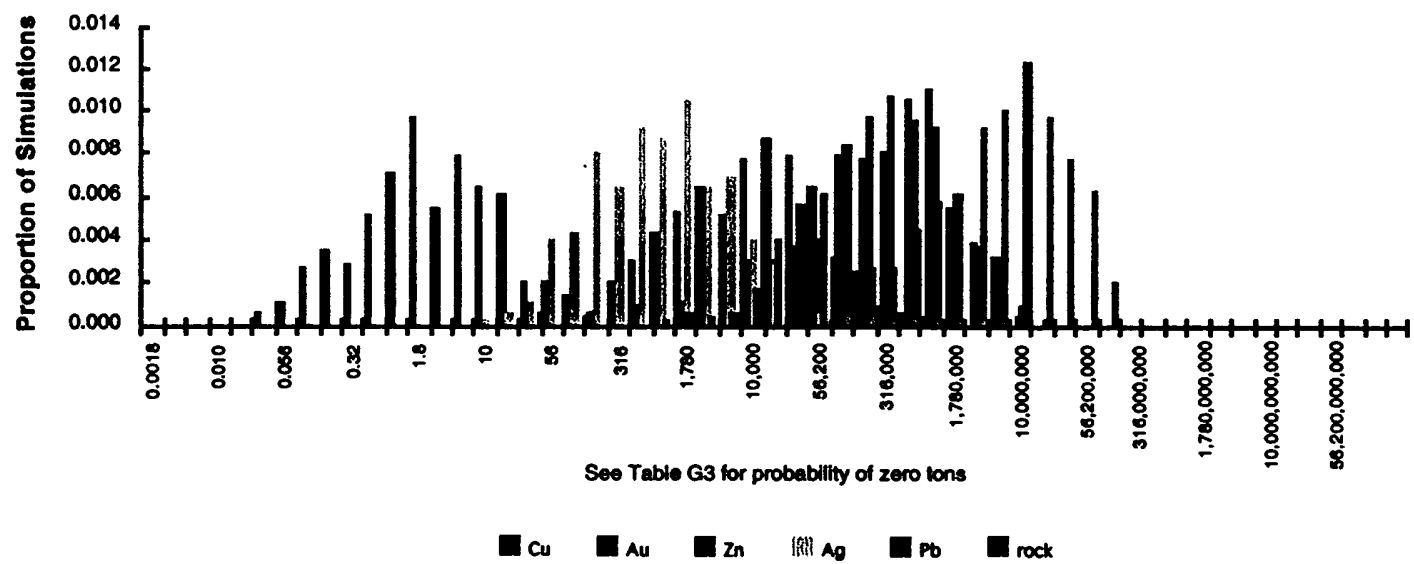

Figure G4. Simulated metal content in undiscovered polymetal contentlic replacement deposits in the Helena National Forest (all amounts in metric tons). 


\section{Ciimax molybdenite deposits}

\section{Cumulative Distributions of Contained Metal and Mineralized Rock}

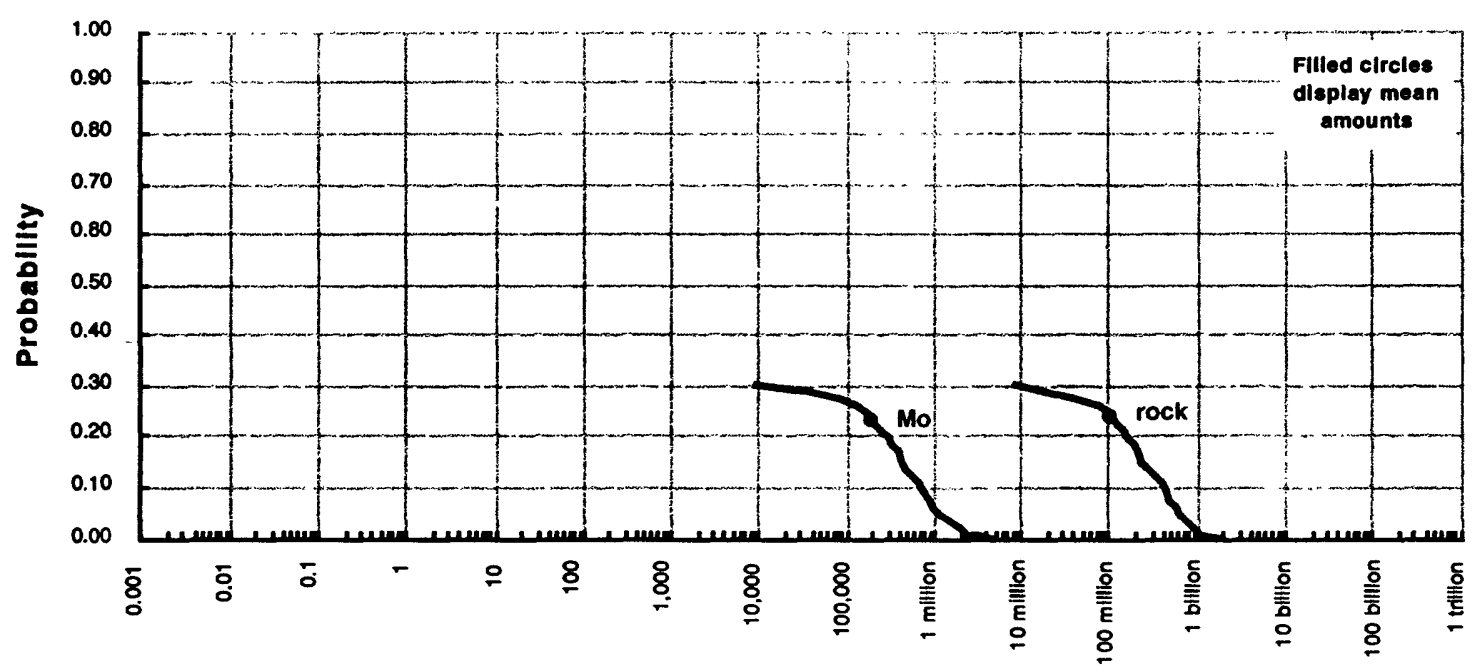

Histograms of Contained Metal and Mineralized Rock (metric tons)

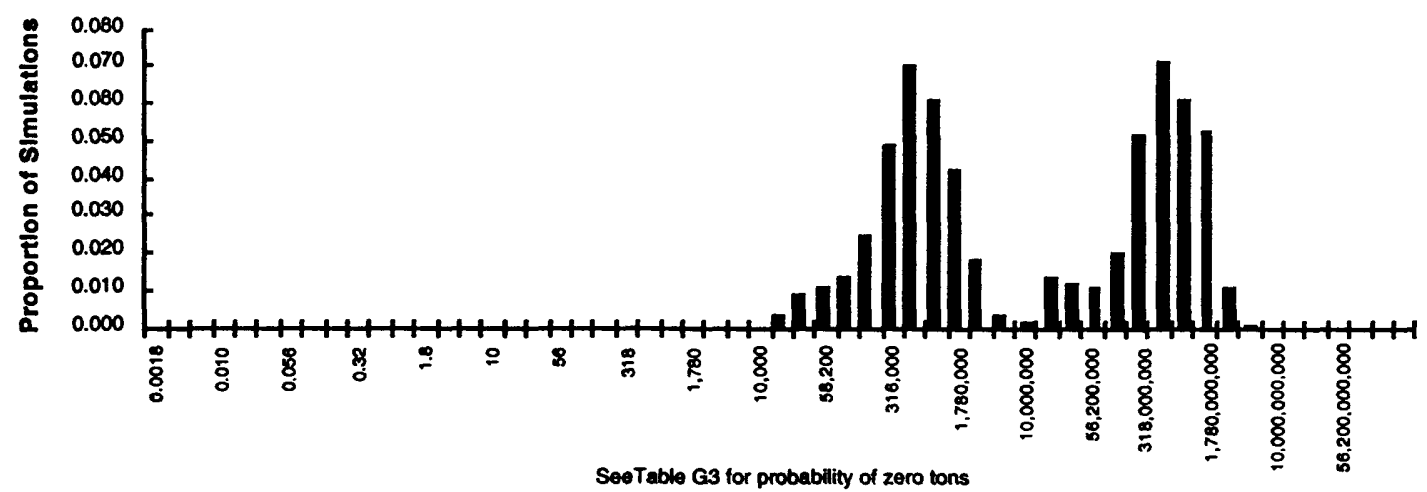

$\square_{\text {Mo }} \mathbf{D}_{\text {rock }}$

Figure G5. Simulated metal content in undiscovered Climax molybdenite deposits in the Helena National Forest (all amounts in metric tons). 
Hot-spring Au-Ag deposits

Cumulative Distributions of Contained Metal and Mineralized Rock

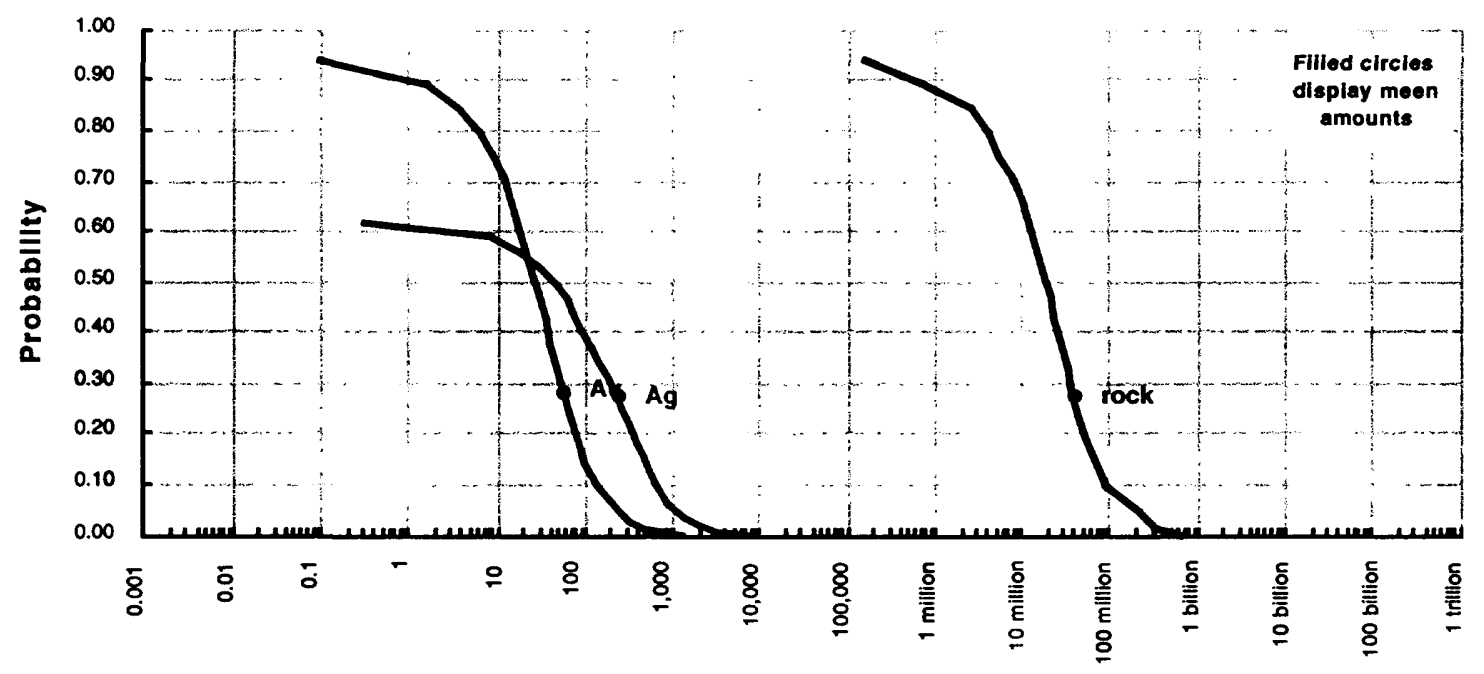

Histograms of Contained Metal and Mineralized Rock (metric tons)

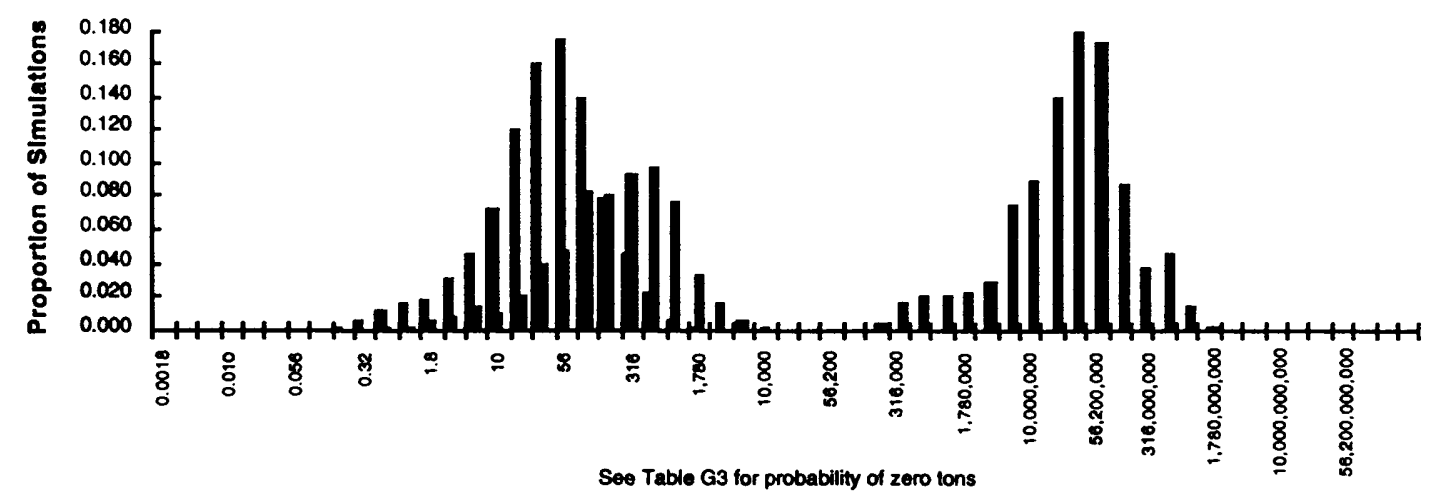

au a a nock

Figure G6. Simulated metal content in undiscovered hot-spring Au-Ag deposits in the Helena National Forest (all amounts in metric tons). 


\section{Epithermal quartz-adularia vein deposits}

\section{Cumulative Distributions of Contained Metal and Mineralized Rock}

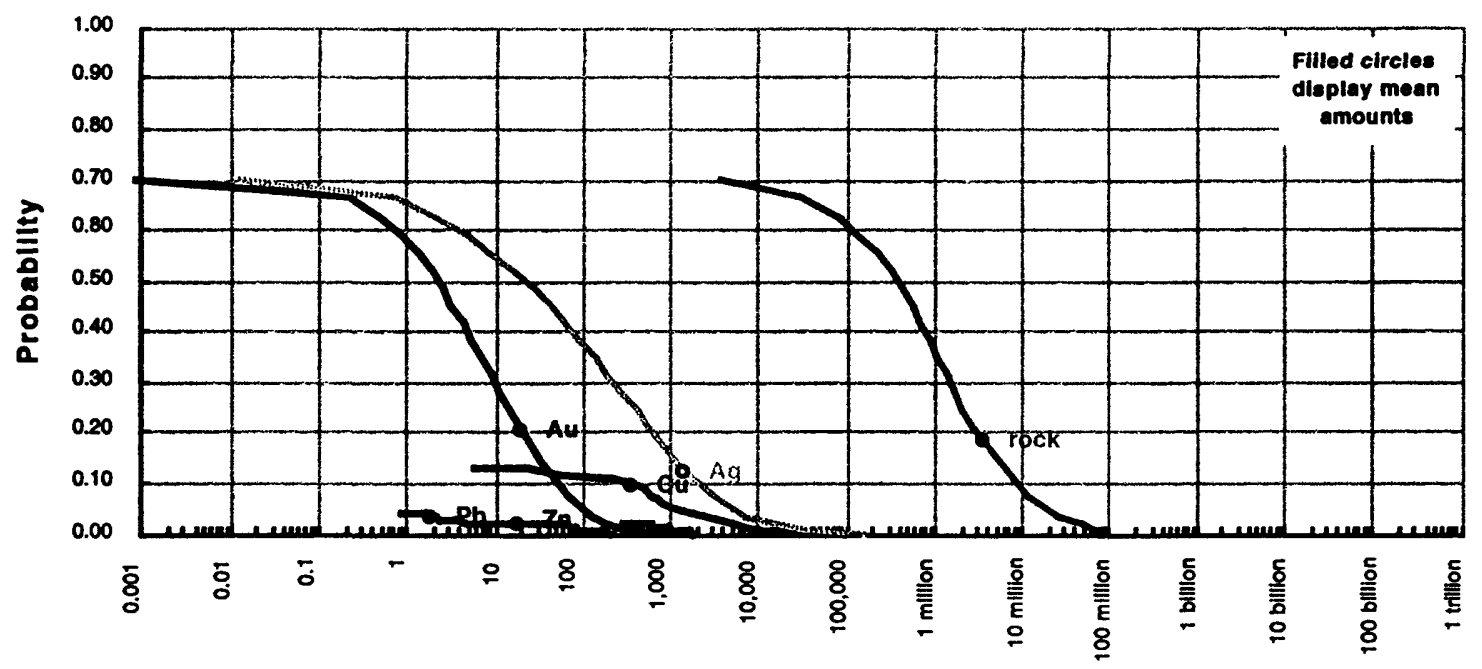

Histograms of Contained Metal and Mineralized Rock (metric tons)

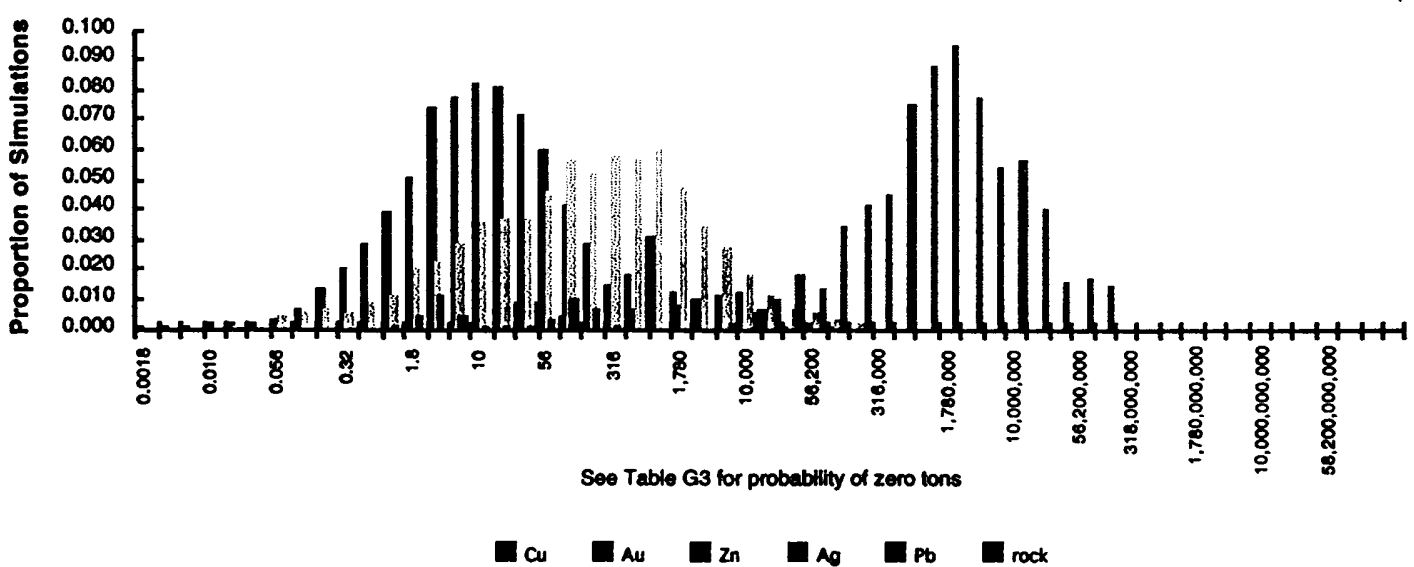

Figure G7. Simulated metal content in undiscovered epithermal quartz-adularia deposits in the Helena National Forest (all amounts in metric tons). 
Table G4. Mean and median estimated metal endowment for undiscovered metallic deposits evaluated in the Helena National Forest, Montana.

[A11 amounts are in metric tons. See Figures G1 through G7 for mean and median values for individual deposit types that were evaluated. Note that estimates are not made for all of the deposit types that might be found in the area; see text for explanation. Values for Butte, taken from Meyer and others (1968), are for production during the period 1880-1964, from the underground mines only, and are provided for perspective only.]

\begin{tabular}{lrrr}
\hline Commodity & Median & \multicolumn{1}{c}{ Mean } & Butte \\
\hline Gold & 85 & 130 & 78 \\
Silver & 590 & 2,200 & 20,000 \\
Copper & 83,000 & $1,000,000$ & $7,300,000$ \\
Lead & 0 & 90,000 & 380,000 \\
Zinc & 0 & 49,000 & $2,200,000$ \\
Molybdenum & 0 & 220,000 & - \\
Iron & 0 & 540,000 & - \\
mineralized rock & 100,000 & $330,000,000$ & - \\
\hline
\end{tabular}

resources predicted in this study. Most of the copper would come from porphyry copper deposits; most of the gold from hot-spring, quartz-adularia, and skarn gold deposits. Lead and zinc are minor constituents of the deposits predicted to exist. Silver and molybdenum are byproducts in deposits that are primarily exploited for gold and copper at present. Iron is generally recovered from sources other than the skarn deposits predicted to occur here.

It is also important to remember that not all of the possible resources known to be present, or that might be present, in the Forest were quantified in this study. We did not quantitatively evaluate the non-metallic deposits, as well as a few metallic deposit types, such as polymetallic veins. Until sufficient information is available regarding these other types of deposits, the resource estimates presented herein should be considered incomplete.

Finally, the spatial distribution of the predicted deposits is not addressed by the quantitative part of this assessment. That information is presented on the maps on plate 5. The maps of permissive and favorable tracts depict where exploration activity will take place in the future.

\section{SOME ENVIRONMENTAL CONSIDERATIONS}

The abundance of metal sulfide minerals is a major factor that affects the ability of a body of rock to generate acid when in contact with natural waters. Acidic water generated in this way is an important environmental concern in land management. Unfortunately, we have virtually no direct information about the sulfide mineral content of various mineralized areas in the Forest, because much of the sulfur in metallic mineral deposits is in 


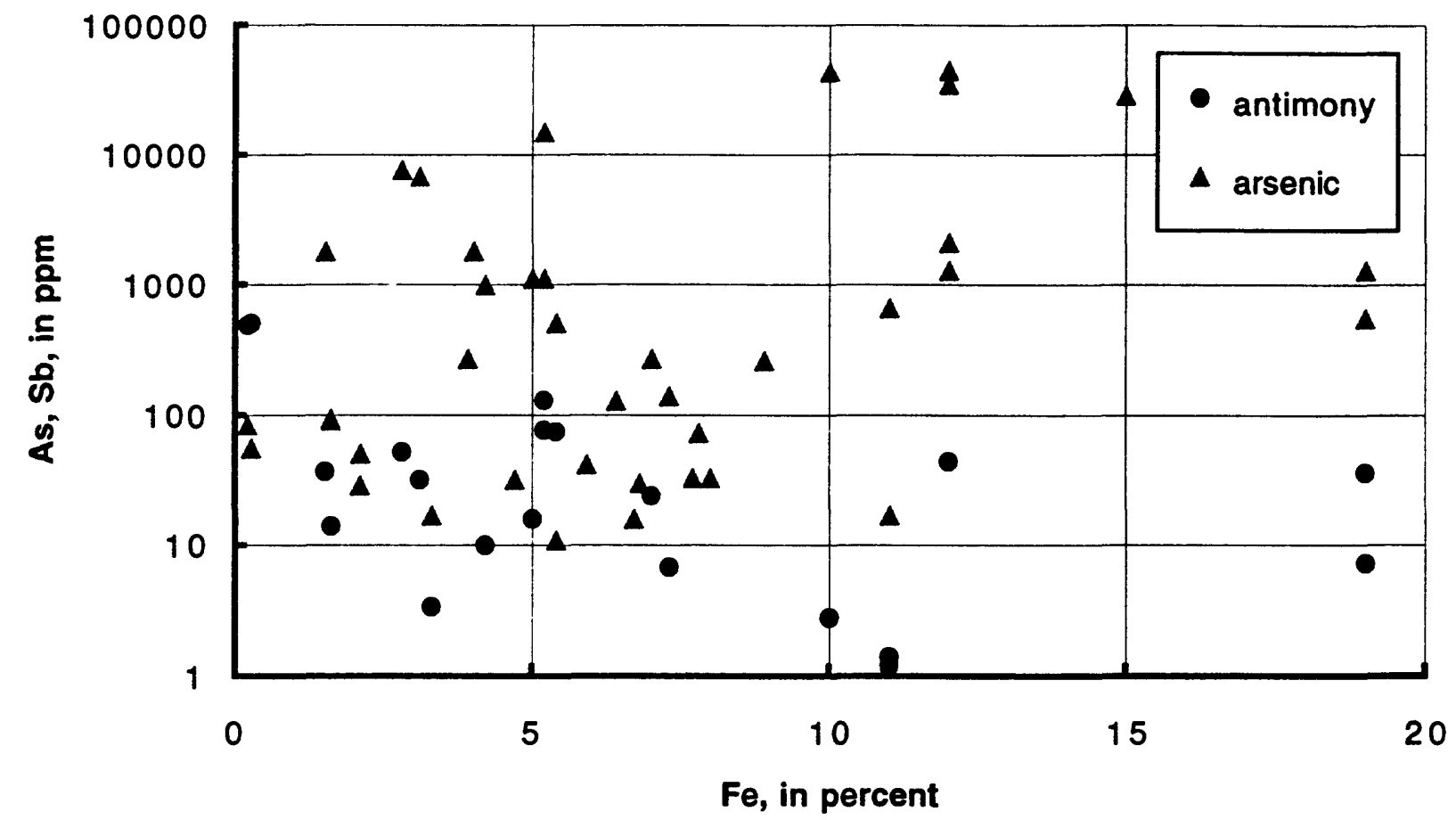

Figure G8. Arsenic and antimony content of mineralized samples from the Elkhorn Mountains. Arsenic and antimony units are parts per million, iron is in weight percent.

samples from the Elkhorn Mountains do not directly imply high toxicity to plant and animal life. Studies to determine levels of bio-available toxic metals would be necessary to establish toxicity. 
iron-bearing sulfides that contribute nothing to the value of the deposit, and are not commonly measured. However, it is possible that geochemical maps can serve as a proxy for the abundance of sulfides. In an earlier chapter (Alminas, this volume, Chapter D), two important geochemical associations (lead and gold) were developed and mapped. Both the lead and gold associations are associated with the common sulfide-bearing polymetallic vein and skarn gold deposits found over wide areas of the Forest.

Those districts that have especially high values for the lead- and goldassociation models are: Heddleston ( $\mathrm{Pb}, \mathrm{Au}$ ), Finn ( $\mathrm{Au}$ ), Stemple-Gould ( $\mathrm{Pb}$, $\mathrm{Au})$, Marysville $(\mathrm{Pb}, \mathrm{Au})$, north part of Dog Creek ( $\mathrm{Au}$ ), Austin ( $\mathrm{Au}$ ), E1liston $(\mathrm{Pb}, \mathrm{Au})$, Rimini ( $\mathrm{Au}), \mathrm{McCl}$ ellan-Mitche11 ( $\mathrm{Au}$ ), Warm Springs ( $\mathrm{Pb}, \mathrm{Au})$, Elkhorn $(\mathrm{Au})$, Winston $(\mathrm{Pb}, \mathrm{Au})$, Park $(\mathrm{Pb}, \mathrm{Au})$, and Indian Creek ( $\mathrm{Pb}, \mathrm{Au})$. If high values of these element-association models are good proxies for sulfide content, those areas are more likely than average to be the sites of acid drainage, associated with both natural outcrops and historic mining operations.

Hot-spring gold, porphyry copper, and Climax molybdenite deposits are the largest deposits expected to occur in the Forest. Any development of these would create the most significant potential source of surface disturbance and generation of large amounts of tailings and waste rock.

A survey of data recently compiled on the geoenvironmental character of a number of mineral deposit types (du Bray, 1995) reveals a paucity of information about toxic metal concentrations in materials from mineralized systems like those in the Helena National Forest. Perhaps the most interesting and relevant data are some that relate to gold skarn deposits. Hammarstrom and others (1995) reported on the Fortitude skarn gold deposit in Nevada. In the border zone, lower ore zone, and upper ore zone, respectively, mean values are as follows: As-96, 1,732, 633; Sb-11, 7, 7. Figure G8 shows arsenic and antimony values for a suite of mineralized rock and ore samples collected during this study from the Winston, Park, and Indian Creek mineralized areas. Mineralized areas in the Elkhorn Mountains appear to be generally comparable to the data from Nevada. It should be emphasized that the high arsenic and antimony contents noted in mineralized samples from the Elkhorn Mountains do not directly imply high toxicity to plant and animal 1ife. Studies to determine levels of bio-available toxic metals would be necessary to establish toxicity.

\section{SUMMARY}

The general location and number of selected undiscovered metallic mineral deposits, and the quantity of metal that might be contained in them, are predicted in this section.

To assess location, we defined permissive tracts to be those areas where mineral deposits are, or could be, present. These permissive tracts are defined by important geologic, geochemical, and geophysical features that are characteristic of a particular deposit type. Within some of the permissive tracts, we outlined subareas that are particularly favorable for the occurrence of deposits. These favorable tracts were designated because the geologic environment contains a coincidence of a large number of important features that are characteristic of a particular deposit type. In these 
favorable tracts, we believe that the areal density of undiscovered deposits is higher than throughout the permissive tract in general, although we have made no attempt to specify how much higher. The permissive and favorable tracts are shown on a series of maps on plates 5 and 6 .

The quantity of contained metal in predicted undiscovered deposits was subjectively estimated by a team of earth scientists, using the Mark3 computer program to simulate amounts by extrapolation from the grade and tonnage models, and from probabilistic estimates of the number of undiscovered deposits present. Those amounts are shown in Figures G1 through G7 and in tables G2, G3, and G4.

The broad range of values in the estimates reflects an inherent large uncertainty in the number and sizes of undiscovered deposits of the types evaluated. Copper and gold together constitute approximately 90 percent of the potential value of the undiscovered resources predicted in this study. Most of the copper would come from porphyry copper deposits; most of the gold from hot-spring, quartz-adularia, and skarn gold deposits.

\section{REFERENCES CITED}

Barton, P.B., Ludington, Steve, Brew, D.A., Lindsey, D.A., Ayuso, R.A., Force, E.R., Gamble, B.M., Goldfarb, R.J., John, D.A., and Johnson, K.M. , Recommendations for assessments of undiscovered mineral resources, U.S. Geological Survey Open-File report 95-82, 90 p.

Berger, B.R., 1985, Geologic-geochemical features of hot-spring precious metal deposits, in Tooker, E.W., ed., Geologic characteristics of sediment-and volcanic-hosted disseminated gold deposits: U.S. Geological Survey Bulletin 1646 , p. 47-48.

1986, Descriptive model of hot-spring Au-Ag deposits, in Cox, D.P., and Singer, D.A., eds., Mineral deposit models: U.S. Geological Survey Bulletin 1693 , p. 143 .

Berger, B.R., and Singer, D.A., 1992, Grade and tonnage model of hot-spring $\mathrm{Au}-\mathrm{Ag}$, in Bliss, J.D., ed., Developments in mineral deposit modeling: U.S. Geological Survey Bulletin 2004, p. 23-25.

Bliss, J.D., ed, 1992, Developments in mineral deposit modeling: U.S. Geological Survey Bulletin 2004, $168 \mathrm{p}$.

Bliss, J.D., and Cox, D.P., 1986, Grade and tonnage model for polymetallic veins, in Cox, D.P., and Singer, D.A., eds., Mineral deposit models: U.S. Geological Survey Bulletin 1693, p. 125-129.

Brannon, C.A., 1981, Rochester Gulch land acquisition and target evaluation, Lewis and Clark County, Montana, 7 miles southeast of Lincoln, Montana: Anaconda Geological Documents Collection, International Archives of Economic Geology, American Heritage Center, laramie, University of Wyoming, reference no. 30552,5 p.

Briskey, J.A., 1986, Descriptive model of Appalachian Zn, in Cox, D.P., and Singer, D.A., Mineral deposit models: U.S. Geological Survey Bulletin 1693, p. 222 .

Burt, D.M., Sheridan, M.F., Bikun, J.V., and Christiansen, E.H., 1982, Topaz rhyolites-distribution, origin, and significance for exploration: Economic Geology, v. 77 , no. 8, p. 1818-1836.

Carten, R.B., White, W.H., and Stein, H.J., 1993, High-grade granite-related 
molybdenum systems-classification and origin, in Kirkham, R.V., Sinclair, W.D., Thorpe, R.I., and Duke, J.M., eds., Mineral deposit modeling:

Geological Association of Canada Special Paper 40, p. 521-554.

Cox, D.P., 1986a, Descriptive mode1 of $\mathrm{W}$ skarn deposits, in Cox, D.P., and Singer, D.A., eds., Mineral deposit models: U.S. Geological Survey Bulletin 1693, p. 55 .

1986b, Descriptive model of polymetallic veins, in Cox, D.P., and

Singer, D.A., eds., Mineral deposit models: U.S. Geological Survey Bulletin 1693 , p. 125.

Cox, D.P., and Singer, D.A., eds., 1986, Minera1 deposit models: U.S. Geological Survey Bulletin 1693, 379 p.

Drew, L.J., Bliss, J.D., Bowen, R.W., and Bridges, N.J., Cox, D.P., DeYoung, J.H. , Jr., Houghton, J.C., Ludington, Steve, Menzie, W.D., Page, N.J, Root, D.H., and Singer, D.A., 1986, Quantification of undiscovered minera1resource assessment-The case study of U.S. Forest Service wilderness tracts in the Pacific Mountain System: Econ. Geo1., 81, 1, p. 80-88.

du Bray, E.A., ed., 1995, Preliminary coimpilation of descriptive geoenvironmental mineral deposit models: U.S. Geological Survey Open-File Report 95-831, $272 \mathrm{p}$.

Einaudi, M.T., Meinert, L.D., and Newberry, R.J., 1981, Skarn deposits, in Skinner, B.J., ed., Economic Geology 75th Anniversary Volume, 1905-1980: Economic Geology, p. 317-391.

Eugster, H.P., 1989, Geochemical environments of sediment-hosted $\mathrm{Cu}-\mathrm{Pb}-\mathrm{Zn}$ deposits, in Boyle, R.W., Brown, A.C., Jefferson, C.W., Jowett, E.C., and Kirkham, R.V., eds., Sediment-hosted stratiform copper deposits: Geological Association of Canada Special Paper 36, p. 111-126.

Graybea1, F.T., Smith, D.M., Jr., and Vickre, P.G., 1986, The geology of silver deposits, in Wolf, K.H., ed., Handbook of strata-bound and stratiform ore deposits: Amsterdam, Elsevier, v. 14, p. 1-184.

Greenwood, W.R., Ludington, Steve, Miller, W.R., and Hanna, W.F., 1990, Mineral resources of the Elkhorn Wilderness Study Area, Broadwater and Jefferson Counties, Montana: U.S. Geological Survey, Bulletin 1805, 37 p., 5 plates.

Hammarstrom, J.M., Zientek, M.L., and E11iott, J.E., eds., 1993, Minera1 resource assessment of the Absaroka-Beartooth study area, Custer and Gallatin National Forests, Montana: U.S. Geological Survey Open-File Report 93-207, 296 p., 19 plates.

Hammarstrom, J.M., Kotlyar, B.B., Theodore, T.G., Elliott, J.E., John, D.A., Doebrich, J.L., Nash, J.T., Carlson, R.R., Lee, G.K., Livo, K.E., and Klein, D.P., 1995, Cu, $\mathrm{Au}$, and $\mathrm{Pb}-\mathrm{Zn}$ skarn deposits, in du Bray, E.A., ed., Preliminary compilation of descriptive geoenvironmental mineral deposit models: U.S. Geological Survey Open-File Report 95-831, p. 90-111.

Hayes, T.S., and Einaudi, M.T., 1986, Genesis of the Spar Lake strata-bound copper-silver deposit, Montana. Part I-Controls inherited from sedimentation and pre-ore diagenesis: Economic Geology, v. 81, no. 8, p. $1899-1931$.

Heald, Pamela, Foley, N.K., and Hayba, D.0., 1987, Comparative anatomy of volcanic-hosted epithermal deposits-Acid-sulfate and adularia-sericite types: Economic Geology, v. 82, no. 1, p. 1-26.

Kirkham, R.V., 1989, Distribution, settings, and genesis of sediment-hosted stratiform copper deposits, in Boyle, R.W., Brown, A.C., Jefferson, C.W., Jowett, E.C., and Kirkham, R.V., eds., Sediment-hosted stratiform copper 
deposits: Geological Association of Canada Special Paper 36, p. 3-38.

Large, D.E., 1981, Sediment-hosted submarine exhalative lead-zinc deposits-A review of their geological characteristics and genesis, in Wolf, K.H., ed., Handbook of strata-bound and stratiform ore deposits: Amsterdam, Elsevier, v. 9, p. 469-508.

Ludington, Stephen D., 1986, Descriptive mode1 of Climax Mo deposits, in Cox, D.P., and Singer, D.A., eds., Mineral deposit models: U.S. Geological Survey Bulletin 1693, p. 73.

Ludington, Steve, and Greenwood, W.R., 1990, Geological and geochemical evaluation, in Greenwood, W.R., Ludington, Steve, Miller, W.R., and Hanna, W.F., Mineral resources of the Elkhorn Wilderness Study Area, Broadwater and Jefferson Counties, Montana, with a section on Uranium and Thorium potential, by K.J. Wenrich, W.R. Miller, V.J. Suits, and J.B. McHugh: U.S. Geological Survey Bulletin 1805, p. 23-34.

Ludington, Steve, and Cox, Dennis, eds., 1996, Data base for a National mineral-resource assessment of undiscovered deposits of gold, silver, copper, lead, and zinc in the conterminous United States: U.S. Geological Survey Open-File Report 96-96, CD-ROM.

Menzie, W.D., and Singer, D.A., 1990, A course on mineral resource assessment: Proceedings of the International Symposium on Mineral Exploration-the case of artificial intelligence: Tokyo and Tsukuba, Japan, Mining and Materials Processing Institute of Japan, p. 177-188.

Menzie, W.D., and Mosier, D.L., 1986, Grade and tonnage model of sedimentary exhalative $\mathrm{Zn}-\mathrm{Pb}$, in Cox, D.P., and Singer, D.A., eds., Mineral deposit models: U.S. Geological Survey Bulletin 1693, p. 212-215.

Megaw, P.K.M., Ruiz, Joaquin, and Titley, S.R., 1988, High-temperature, carbonate-hosted $\mathrm{Ag}-\mathrm{Pb}-\mathrm{Zn}(\mathrm{Cu})$ deposits of northern Mexico: Economic Geology, v. 83, no. 8, p. 1856-1885.

Meinert, L.D., 1989, Gold skarn deposits-Geology and exploration criteria, in Keays, R.R., Ramsay, W.R.H., and Groves, eds., Geology of gold deposits: Economic Geology Monograph 6, p. 537-552.

Meinert, L.D., Myers, G.W., and Brooks, J.W., 1990, Skarn deposits in Nevada-geology, geochemistry, mineralogy, and petrology of $\mathrm{Au}, \mathrm{Cu}$, W, and Zn skarns, in Buffa, R.H, and Coyner, A.R., eds., Geology and ore deposits of the Great Basin-Field trip guidebook compendium, v. 1, p. 37-80.

Meyer, Charles, Shea, E.P., Goddard, C.C., and staff, 1968, Ore deposits at Butte, Montana: in Ridge, J.D., ed., Ore deposits of the United States, 1933-1967-The Graton-Sales Volume, v. II, American Institute of Mining, Metallurgical, and Petroleum Engineers, Inc., New York, p. 1373-1416.

Morris, H.T., 1986, Descriptive model of polymetallic replacement deposits, in Cox, D.P., and Singer, D.A., eds., Mineral deposit models: U.S. Geological Survey Bulletin 1693, p. 99-100.

Mosier, D.L., Morris, H.T., and Singer, D.A., 1986, Grade and tonnage model of polymetallic replacement deposits, in Cox, D.P., and Singer, D.A., eds., Mineral deposit models: U.S. Geological Survey Bulletin 1693, p. 101-104.

Mosier, D.W., Singer, D.A., and Berger, B.R., 1986, Descriptive model of Comstock epithermal veins, in Cox, D.P., and Singer, D.A., eds., Minera1 deposit models: U.S. Geological Survey Bulletin 1693, p. 150.

Mosier, D.W., Berger, B.R., and Singer, D.A., 1986, Descriptive model of Sado epithermal veins, in Cox, D.P., and Singer, D.A., eds., Mineral deposit models: U.S. Geological Survey Bulletin 1693, p. 154.

Orris, G.J., and Bliss, J.D., eds., 1991, Some industrial mineral deposit 
models; descriptive deposit models: U.S. Geological Survey Open-file report $91-11 \mathrm{~A}, 73 \mathrm{p}$.

Pardee, J.T. and Schrader, F.C., 1933, Metalliferous deposits of the greater Helena mining region, Montana: U.S. Geological Survey Bulleting 842, 318 p.

Root, D.H., Menzie, W.D., and Scott, W.A., 1992, Computer Monte Carlo simulation in quantitative resource assessment: Nonrenewable Resources, $v$. 1, p. $125-138$.

Root, D.H., Scott, W.A., Jr., Selner, Gary I., 1996, Computer program for aggregation of probabilistic assessments of mineral resources: U.S. Geological Survey Open-File Report 96-94,

Singer, D.A., 1993, Basic concepts in three-part quantitative assessments of undiscovered mineral resources: Nonrenewable resources, v. 2, p. 69-81.

Singer, D.A., and Cox, D.P., 1988, Application of mineral deposit models to resource assessments: U.S. Geological Survey Yearbook, Fiscal Year 1987, p. 55-57.

Singer, D.A., and Ovenshine, A.T., 1979, Assessing mineral resources in Alaska: American Scientist, v. 67, p. 582-589.

Singer, D.A., Mosier, D.L., and Cox, D.P., 1986, Grade-tonnage model of porphyry copper, in Cox, D.P., and Singer, D.A., eds., Mineral deposit models: U.S. Geological Survey Bulletin 1693, p. 77-81.

Singer, D.A., Theodore, T.G., and Mosier, D.L., 1986, Grade and tonnage mode1 of Climax Mo deposits, in Cox, D.P., and Singer, D.A., eds., Mineral deposit models: U.S. Geological Survey Bulletin 1693, p. 73-75.

Spanski, G.T., 1992, Quantitative assessment of future development of copper/silver resources in the Kootenai National Forest, Idaho/Montana:

Part 1-Estimation of the copper and silver endowments: Nonrenewable Resources, v. 1, no. 2, p. 163-183.

Theodore, T.G., Orris, G.J., Hammarstrom, J.M., and Bliss, J.D., 1991, Goldbearing skarns: U.S. Geological Survey Bulletin 1930, 61 p.

Thorson, J.P., White, B.G., and Baitis, H.W., in preparation, Gold resources in the Greyson Formation, Big Belt Mountains, Montana. Part II-Mineralization and genesis, in Berg, R.B., ed., Proceedings of Belt Symposium III: Montana Bureau of Mines and Geology Special Publication 111.

Titley, S.R., 1982, The style and progress of mineralization and alteration and alteration in porphyry copper systems, in Titley, S.R., ed., Advances in geology of the porphyry copper deposits-Southwestern North America: Tucson, University of Arizona Press, p. 93-116.

Wenrich, K.J., Miller, W.R., Suits, V.J., and McHugh, J.b., 1990, Uranium and thorium potential, in Greenwood, W.R., Ludington, Steve, Miller, W.R., and Hanna, W.F., Mineral resources of the Elkhorn Wilderness Study Area, Broadwater and Jefferson Counties, Montana, with a section on Uranium and Thorium potential, by K.J. Wenrich, W.R. Miller, V.J. Suits, and J.B. McHugh: U.S. Geological Survey Bulletin 1805, p. 34-35.

Whiteley, S.J., 1982, Chessman Reservoir submitta1-1982: Anaconda Geologica1 Documents Collection, International Archives of Economic Geology, American Heritage Center, laramie, University of Wyoming, reference no. 305.49, 4 p. 


\title{
C $\begin{array}{llllllllllllll}\mathbf{A} & \mathbf{P} & \mathbf{T} & \mathbf{E} & \mathbf{R} & \mathbf{H}\end{array}$
}

\section{PLACER GOLD OF THE HELENA NATIONAL FORE8T}

\author{
GEOLOGY OF PLACER GOLD DEPOSITS
}

By R.G. Tysdal

\section{Introduction}

Placer gold deposits consist of elemental gold in grains and rarely nuggets in gravel, sand, silt, and clay, and their consolidated equivalents, in alluvial, beach, eolian, and rarely glacial deposits. Descriptive models for gold placer deposits are given by Yeend (1986) and Yeend and Shawe (1989), which is the basis for the following discussion.

Placers form by weathering of gold from bedrock sources and mechanical concentration of the particles by running water in streams, rivers, and locally slopewash. The richness (concentration) of gold in placer deposits depends on the supply of gold in source rock and on conditions favorable for its concentration and preservation. Gold has a high specific gravity and works its way into joints, fractures, or irregular rock surfaces in stream beds. In some deposits, however, gold is dispersed throughout a gravel mass and lacks significant concentration. Most alluvial placers are of Cenozoic age, but "fossil" placers may be preserved within sediments protected beneath younger rocks. Placer deposits are subject to destruction and the gold may be recycled by the same processes that led to their formation. The most prospective areas for placer deposits are in, or near, known lode gold districts.

\section{Placers in and adjacent to the Forest}

Placer deposits of the Forest include those in unconsolidated sediments of streams, terraces adjacent to streams, eluvium (residum of weathered rock), glacial debris, and Quaternary-Tertiary deposits in consolidated materials of alluvial fans and benches, chiefly along mountain flanks. Placer locations are shown on plate 2, along with other mines, prospects, and mineral occurrences in and adjacent to the Forest. Placers also are shown on map D ( 1 . 3), which depicts streams and stream-segments where publications indicate placer operations yielded gold in the past. The placer-bearing streams primarily were compiled from Lyden (1948), but also include data from publications cited in the "Sources of Data" column of table El, including field observations for this report. Coordinates for each placer deposit represent only one point along a stream, generally at the lower end, whether inside or outside the Forest. However, in some cases tributary streams also are represented by coordinates (table H1), which corresponds to a data entry in table $\mathrm{H} 1$.

Production records of gold recovery from the various streams within and adjacent to the Forest are incomplete. Nevertheless, data show that most of the gold was produced from only a few streams, primarily in the $1880^{\prime}$ s to first third 
of the 1900's. Recovery of gold included the use of dredges on some drainages. Koschmann and Bergendah1 (1968) reported the following cumulative production of placer gold, through 1959: Helena (Last Chance) district--at least 940,000 ounces ; Confederate Gulch- -550,000-600,000 ounces; Lincoln Gulch- $-342,000$ ounces ; McClellan Gulch district (near Lincoln)--340,000 ounces; Ophir district--minimum of 180,000 ounces (Loen [1989] estimated 300,000 ounces for this district); western part of the York district (York, Oregon, Clark, Cave, and Magpie Creeks) -minimum of about 160,000 ounces; Missouri River terraces--minimum of about 105,000 ounces; and Finn district- $-81,000$ ounces.

Almost certainly every stream within and adjacent to the Forest has been prospected for placer gold. Some streams never contained gold and others contained placers that have been exhausted of their gold. The following streams within the Forest had placering operations that were producing or developing in 1992 (McCulloch, 1993a). In the Big Belt Mountains: Avalanche Creek (York district) and Vermont Gulch (Beaver-Elk Creek district). In the western part of the Forest: Lincoln Gulch area, and Washington Gulch (Finn district); and outside but directly adjacent to the Forest--Sauerkraut Creek (Finn district).

Exploration (testing) of permissive placer ground was ongoing at the following locations in or adjacent to the Forest in 1992 (McCulloch, 1993b). In the Big Belt Mountains: Confederate and Cement Gulches (Confederate Gulch district); Benton, Thompson, and Vermont Gulches, and Beaver and Elk Creeks (Beaver-Elk Creek district). In the western part of the Forest: Mike Reinig Creek (E11iston district), Greenhorn Creek (Austin district), and Hope Creek (Dog Creek area).

Fossil stream-1aid deposits ("bars") are present loca11y along the Missouri River, on terraces as much as 200-250 ft above water leve1. These terrace deposits yielded gold, and locally sapphires, in past and some present placer operations. Gold in the bars probably came from lodes in the York district; the amount of gold decreased rapidly downstream from the district (Pardee and Schrader, 1933). The richest deposits were in French Bar (p1. 3, map D, no. 510), close to the district. Eldorado Bar (no. 505) is partly within the Forest and lies below the mouth of Trout Creek, which is the probable source of its gold (Pardee and Schrader, 1933); dredging ground was reportedly exhausted by 1942 (Lyden, 1948). American Bar (no. 503) also is partly within the Forest. It is composed of bedrock that is overlain by about $3 \mathrm{ft}$ of river gravel and cobbles, which in turn is overlain by as much as $11 \mathrm{ft}$ of alluvium. About 13 acres of the bar was hydraulically mined for placer gold in the late 1800's and is believed to have yielded only a small amount of gold (Close and Rigby, 1984).

The only modern study of placer deposits within or adjacent to the Forest is that of Loen (1989) for the Ophir district (p1. 2). Loen reported that placering has taken place in terrace gravel, alluvium, lag deposits (old stream deposits that have undergone prolonged in-place weathering), and eluvium (gold concentrated in-place from prolonged weathering of bedrock). Gold was freed from the Blackfoot City stock (plate 1) and the surrounding mineralized rocks that lie at the head of streams of the district.

Alluvium of the present-day streams was extensively placered for their gold, but the richest deposits of the Ophir district were found in consolidated (cemented by calcium carbonate) stream gravels of Pleistocene(?) and Pliocene age (Loen, 1989). These strata deposits the present streams and underlie terraces a few feet above stream level. The terrace gravels lie almost wholly outside the Forest, contained within map unit QTg (p1. 1).

Three placer deposits of the Ophir district lie within the Forest: at the head of Nugget Gulch (p1. 3, map D, no. 143), Mexican Gulch (no. 158), and 
Table H1. Placer Au deposits and production, grouped by district, Helena National Forest, Montana

[", mine or prospect on or just outside Forest boundary; ( $r$ ), reserves; oz, ounces; data corresponds to that of table E1, plate 2, and map D of plate 3. 'Alternate name(s) in parentheses; ${ }^{2}$ Value given only when recorded in data source, as cited in table E1.]

\begin{tabular}{|c|c|c|c|}
\hline No. & Site name & District/ Area ${ }^{1}$ & $\begin{array}{c}\text { Au } \\
\text { Production' } \\
\text { Reserves } \\
(0 z)^{2}\end{array}$ \\
\hline 24 & Lincoln Gulch (Lincoln Creek) placer & Lincoln Gulch Area & 350000 \\
\hline 48 & Moose Creek placer & Blackfoot River Area & \\
\hline 49 & Sauerkraut Creek placer & Blackfoot River Area & \\
\hline 50 & Stonewall Creek placer & Blackfoot River Area & 703 \\
\hline 51 & Liverpool Creek placer & Blackfoot River Area & \\
\hline 52 & Keep Cool Creek placer & Blackfoot River Area & \\
\hline 54 & Seven-Up Pete Creek Placer & Blackfoot River Area & \\
\hline 60 & Poorman Creek placer & Blackfoot River Area & 36 \\
\hline 65 & Wasson Creek placer & Big Blackfoot (Ogden Mtn) & \\
\hline 66 & Wilson Creek (Kilburn, Raleigh) placer & Big Blackfoot (Ogden Mitn) & \\
\hline 68 & C.D. Hurd placer" & Big Blackfoot (Ogden Mitn) & \\
\hline 78 & Canarway Gulch Placer & Big Blackfoot (Ogden Mitn) & \\
\hline 79 & Chimney Creek placer & Big Blackfoot (Ogden Mtn) & \\
\hline 80 & Deer Creek placer & Big Blackfoot (Ogden Mitn) & Minor \\
\hline 81 & Chicken Creek placer & Big Blackfoot (Ogden Mitn) & Minor \\
\hline 86 & All Placer & Finn & \\
\hline 87 & Buffalo Gulch placer & Finn & 230 \\
\hline 88 & Jefferson Creek placer & Finn & 15 \\
\hline 89 & Madison Gulch placer & Finn & 75 \\
\hline 90 & Washington Creek placer & Finn & 5300 \\
\hline 91 & American Gulch placer & Finn & Minor \\
\hline 93 & McClellan Gulch (Creek) placer & McClellan Gulch & 350000 \\
\hline 97 & Gold Creek placer (Blue Jay) & Stemple-Gould & \\
\hline 103 & Rooster Bill Creek placer, Margaret & Stemple-Gould & \\
\hline 106 & Fool Hen Creek placer & Stemple-Gould & \\
\hline 111 & Gould Creek placer & Stemple-Gould & \\
\hline 113 & Specimen Creek placer & Little Prickly Pear Area & \\
\hline 114 & Tarhead Creek placer & Little Prickly Pear Area & \\
\hline 115 & Virginia Creek placer & Little Prickly Pear Area & \\
\hline 120 & Empire Creek placer (Lost Horse Creek) & Marysville (Silver Creek) & \\
\hline 140 & Three Mile Creek placer & Ophir & 250 \\
\hline 143 & Nugget Gulch placer & Ophir & 250 \\
\hline 155 & Tiger Gulch placer & Ophir & 200 \\
\hline 158 & Mexican Gulch placer & Ophir & 500 \\
\hline 161 & Deadwood Gulch placer & Ophir & 500 \\
\hline 169 & Illinois Gulch placer & Ophir & 500 \\
\hline 170 & Eureka Gulch placer & Ophir & 3000 \\
\hline 171 & Ophir Creek placer (Tributary of Carpenter Creek) & Ophir & 100000 \\
\hline 172 & Snowshoe Creek placer & Ophir & 25000 \\
\hline 173 & Carpenter Creek placer & Ophir & 150000 \\
\hline 174 & Gold Canyon Creek placer & Dog Creek Area & 4700 \\
\hline 176 & Dog Creek placer & Dog Creek Area & Minor \\
\hline 181 & Little Blackfoot River placer & Dog Creek Area & \\
\hline 194 & Skelly Gulch (Greenhorn Creek) placer & Austin & \\
\hline 200 & Blue Cloud Mining Co. placer & Stermwinder Hill Area & \\
\hline 201 & Nelson Guich placer & Helena (Last Chance) & \\
\hline 202 & Orofino, Dry, Grizzly, and Last Chance Gulch placers* & Helena (Last Chance) & 800000 \\
\hline
\end{tabular}




\begin{tabular}{|c|c|c|c|}
\hline 209 & Pretty Girl placer & Helena (Last Chance) & \\
\hline 211 & Mike Relnig Gulch placer & North Boulder Mountains Area & 180 \\
\hline 226 & Telegraph Creek placer & Elliston & 12 \\
\hline 231 & Ontario Creek placer & Elliston & 184 \\
\hline 246 & $\begin{array}{l}\text { Tenmile Creek (Gould, Monitor, Tucker, and Minnehaha) } \\
\text { placer }\end{array}$ & Rimini (Vaughn) & \\
\hline 275 & Travis placer & Rimini (Vaughn) & \\
\hline 298 & Lump Gulch placer & Clancy (Lump Gulch) & 27 \\
\hline 299 & Buffab Creek (Weber) placer & Clancy (Lump Gulch) & Minor \\
\hline 308 & Clancy Creek placer & Clancy (Lump Gulch) & 8600 \\
\hline$\overline{329}$ & Basin Creek placer" & Basin (Cataract, Comet) & 1300 \\
\hline 330 & Cataract Creek placer & Basin (Cataract, Comet) & \\
\hline 332 & High Ore Creek placer & Boulder (Comet) & \\
\hline 385 & Comet Creek (Spring Creek) placer" & Wickes (Colorado, Corbin) & Minor \\
\hline 395 & Holmes Gulch placer" & Montana City & \\
\hline 396 & Prickly Pear Creek placer & Montana City & 59000 \\
\hline 399 & Mitchell Creek placer & McClellan-Mitchell Creek & 14000 \\
\hline 400 & McClellan Creek placer & McClellan-Mitchell Creek & Minor \\
\hline 408 & Warm Springs Creek placer & Warm Springs & Minor \\
\hline 412 & Wilson Creek placer & Tizer Basin Area & \\
\hline 415 & Tizer Creek placer & Tizer Basin Area & \\
\hline 440 & Beaver Creek placer & Winston & 10000 \\
\hline 463 & Unnamed placer & Indian Creek & \\
\hline 482 & Indian Creek placer* & Indian Creek & 22000 \\
\hline 501 & Crow Creek placer" & Radersburg & 25000 \\
\hline 502 & Ming's Bar placer" & Missouri River Area & Minor \\
\hline 503 & American Bar placer & Missouri River Area & Minor \\
\hline 504 & Dana's Bar placer" & Missouri River Area & Minor \\
\hline 505 & El Dorado Bar placer & Missouri River Area & 41000 \\
\hline 506 & McCune Bar placer & Missouri River Area & \\
\hline 507 & Spokane Bar placer & Missouri River Area & 27500 \\
\hline 506 & Gruel Bar (Gruel's Bar, Metropolitan Bar) placer* & Missouri River Area & 2050 \\
\hline 509 & Lovestone* & Missouri River Area & \\
\hline 510 & French Bar placer & Missouri River Area & 75000 \\
\hline$\overline{516}$ & Trout Creek, York Gulch, Rattlesnake Gulch, Kelly Gulch, & York & 250000 \\
\hline 542 & Garnet placer & York & \\
\hline 544 & Bar Gulch placer & York & \\
\hline 545 & Linda Jo placer & York & \\
\hline 546 & Ruth placer & York & \\
\hline 547 & Oregon Gulch placer & York & 25000 \\
\hline 546 & Clark Gulch placer & York & 15000 \\
\hline 549 & Cave Gulch, Cooper Gulch placers & York & 20000 \\
\hline 550 & Magpie Creek placer & York & 14000 \\
\hline 550 & Hellgate Gulch placer & York & \\
\hline 562 & Avalanche Creek placer & York & 5000 \\
\hline 564 & Sheep Creek placer & Beaver (Elk Creek) & \\
\hline 567 & Fig Springs placer & Beaver (Elk Creek) & \\
\hline 568 & Beaver Creek placer & Beaver (Elk Creek) & Minor \\
\hline 571 & Vermont Gulch placer & Beaver (Elk Creek) & \\
\hline 572 & Benton Creek placer & Beaver (EIk Creek) & Minor \\
\hline 573 & Thomas Creek placer & Beaver (Elk Creek) & 1100 \\
\hline
\end{tabular}




\begin{tabular}{llll}
\hline 576 & Elk Creek placer & Beaver (Elk Creek) & 5 \\
577 & Camas placer* & Beaver (Elk Creek) & Minor \\
\hline 579 & White Creek placer & Confederate Gulch & 75000 \\
587 & Confederate Gulch placer & Confederate Gulch & $600000 ;$ \\
& & & $181400(r)$ \\
\hline 590 & Attanta Gulch placer & Southern Big Belt Area & 422 \\
593 & Little Camas Creek placer & Southern Big Belt Area & 2500 \\
599 & Thompson Creek placer & Southem Big Belt Area & Minor \\
603 & Deep Creek placer & Southern Big Belt Area & \\
\hline
\end{tabular}


Deadwood Gulch (no. 161). All are eluvial deposits that had an estimated total production of only 1,250 ounces. These deposits lie above the level of modern streams, along hill slopes that have been exposed to weathering, possibly for as much as several million years (Loen, 1989).

\section{Permissive Tracts}

Map D (pl. 3) shows extensive shaded areas that are a composite of all geologic terranes containing, or are permissive for, gold-bearing rocks, as explained in Chapter $G$ of this report. Streams on map $D$ are shown in a "drainage net" of differing line weights. The fine-line weight represents the following: (1) streams within the Forest, and within the areas of geologic mapping directly adjacent to the Forest, for which we found no record of placer gold recovery, and for which the streams do not flow across geologic terrane considered permissive for gold-bearing mineralization; and (2) all streams that lie outside the Forest and outside the limit of geologic mapping, whether or not the streams yielded placer gold, flow across geologic terrane permissive for gold, or flow across terrane considered permissive for placer gold.

The bold and intermediate weight lines of the drainage net are shown only within the Forest and within the areas of geologic mapping directly adjacent to the Forest. Bold lines represent streams or stream-segments that yielded gold from placer operations, as determined from a cited geologic publication (table E1). An asterisk is placed after the name of each placer deposit for which the publication(s) indicates the gold-bearing stream or stream-segment lies entirely outside the Forest. In many cases, a bold line clearly represents a stream within (or partly within) the Forest. In other cases, however, the actual occurrence of placer mining within the Forest is uncertain (whether or not a bold line lies entirely outside the Forest), due to the unclarified nature of statements in some publications.

Intermediate weight lines show streams for which cited publication(s) did not definitively indicate placer production. The streams flow across geologic terrane that is considered permissive for gold-bearing mineral deposits, thus these streams could yield gold to form placers. Such streams (or streamsegments) are considered prospective for placer deposits. However, because it is almost certain that every stream within and adjacent to the Forest has been prospected for placer gold, streams most likely to yield gold probably will be those from which gold has been recovered in the past.

Occurrences of placer gold have led to discoveries of many lode gold deposits by tracing the placer anomalies to bedrocks sources upstream. In the Confederate Gulch district, the gold in the streams of Confederate and White Gulches has long been considered to come from quartz veins in the vicinity of Miller Mountain (Pardee and Schrader, 1933). Pegasus Gold (1992) reported reserves of 310,000 ounces of lode gold in the Miller Mountain area.

A placer prospect at Sheep Creek (Scapegoat area), north of Lincoln, was reported by Earhart and others (1977), but not included in a later compilation of mines, prospect, and mineral occurrences of Earhart and others (1981). They found no record of gold recovery and their sampling yielded no gold. Similarly, Willow Creek (southern part of Blackfoot River area) was reported by Lyden (1948) as having placer activity, but Elliott and others (1992) found no evidence or record of placering and did not include the stream as productive.

In the northwestern part of the Forest, Stonewall, Liverpool, and Keep Cool Creeks flow south from the Scapegoat area of the Forest into the Blackfoot River 
area (p1. 2; map D of p1. 3). Placering operations were reported along each of these streams within the Forest (Earhart and others, 1977, 1981), and along the southern parts of the streams, which are outside the Forest. These reports led us to classify the headwaters of these streams as prospective for placer gold (p1. 3, map D). However, Earhart and others $(1977,1981)$ cited no record of production and Lyden (1948) stated that the lower parts of the Liverpool and Keep Cool Creeks yielded only minor gold from irregular and low-grade concentrations. Lyden (1948) reported that the lower end of Stonewall Creek was dredged but yielded less than 1,000 ounces of gold. The source of this gold may not be from rocks in the headwaters of the three creeks, within the Forest, but may be from gold-bearing rocks in the vicinity of the McDonald Meadows gold property (p1. 2, no. 53) about $7 \mathrm{mi}$ east of Lincoln.

Three Mile Creek ( 1 l. 3, map D, no. 140) heads in terrane not considered prospective for gold. Gold in the placers may have come from the QuaternaryTertiary conglomeratic terrace gravels of rock unit (QTg) that directly underlies the southern placered stream segment or from glacial till that underlies the northern placered segment. Such gold would be considered recycled from a previously formed sedimentary placer concentration.

\section{COMPOSITION OF GOLD GRAINS FROM PLACERS}

by W.H. Raymond and G.A. Desborough

\section{Introduction}

Gold in stream sediments reported here is from two separate studies. Data collected in 1970 (table H2, nos. M001-M027) are from a study of p1acer deposits of Montana by Raymond. The study was designed to sample coarse-grained gold of placer deposits and use gold composition as an aide in determining the sources of the gold. Data collected in the 1990-94 period (table H2, nos. M050-M148) are from an extensive, ongoing stream-sediment sampling program of Raymond and Desborough. The study focused on Idaho and Montana and is designed to determine the feasibility of mechanical panning techniques for recovery of, and tracing sources of, very fine grained gold and other metals.

Data from the two studies are not directly comparable because the sampled gold is from two different size fractions, collected by different techniques, and likely represents different histories of transport and origin. The two sets of data are distinguished by different symbols on map $E$ ( 1 1. 3), which shows only general, qualitative abundance: no gold recovered, some gold recovered, and a significant amount of gold recovered. Only data for samples collected within and adjacent to the Forest during the two studies are presented here. None of the data have been published previously.

Placer Gold Collected in 1970

Each sample of the 1970 data set (table H2, nos. M001-M027) was collected 
and processed by a standard procedure. A set of portable riffles was placed in the stream and about 30 shovelfuls (about $300 \mathrm{lbs}$ ) of gravel were worked and reduced to a concentrate. The goal was to recover at least 20 usable colors ("The specks of gold seen after successful operation of a gold pan ..." , Thrush, 1968 ) for a statistical analysis of the composition of gold grains. Field panning of the concentrates indicated when sufficient colors had been obtained. In a few cases, additional gravel was processed to obtain sufficient colors. In the laboratory, some loss of gold grains occurred during mounting and polishing, reducing the number of gold grains to insufficient levels for statistically significant analysis. The existing data for these samples are included in the table, however, along with notes on other minerals observed in the concentrates.

Gold grains were mounted in epoxy, ground to expose the cores of grains, and polished in preparation for analysis by electron microprobe. Readings on the microprobe were taken at a minimum of four sites in the core of each grain, avoiding measurement of leached rims. Grains suspected of having been ground to the leached rims on the underside were discarded. Analyses were made for gold, silver, and copper. The mounts later were repolished and examined by energy dispersive analysis for platinum group elements (PGE), but none were detected.

The resulting data set shows that the gold is remarkably consistent in composition, almost without regard to the number of grains analyzed. The samples from the Ophir district (table H2, nos. M001, M002, M004) and the one sample from Grizzly Gulch (no. M008) are the only grains that contain gold of significantly higher-than-average fineness. Copper was detected in small amounts only, near the southwesternmost part of the Elkhorn Mountains (p1. 3, map E, no. M020), and in Tenmile Creek (no. M007) west of Helena. PGE's were not found in these fairly coarse-grained gold samples, although copper and palladium were detected in the much finer grained gold of the 1990-94 data set from some of the same areas. These samples are thought to have been derived directly from the Middle Proterozoic host rocks of the Big Belt Mountains and nearby localities, as described below with the 1990-94 data set.

Repeat collection of samples at sites on the west slope of the Big Belt Mountains yielded a startling decrease in gold population between 1970 and 1993. Local inquiry indicated that a large, catastrophic flood in the late 1970's removed much of the sediment in the lower reaches of many of the streams. Some sites along Confederate Gulch that yielded abundant gold in 1970 yielded little or none in 1993 (table H2), suggesting that gold was flushed downstream and redeposited in alluvium outside the Forest.

\section{Placer Gold Collected in 1990-94}

The 1990-94 placer samples were collected in a manner to recover very fine grained gold. At each sample site, two one-kilogram (2.2 1bs) bags of sediment were collected from the stream bed near the thread of maximum water velocity. In the laboratory, each sample was weighed and screened to five size fractions, and each fraction was panned mechanically according to the procedure described in English and others (1987). The concentrate was examined under a microscope, the colors inventoried and reported in colors per kilogram of sample. Compositional variations in the gold grains were determined for selected sites by using energy dispresive techniques on a scanning electron microscope (SEM).

The very fine-grained gold $(<0.2 \mathrm{~mm})$ has less silver and more detectable copper and palldium than the coarse-grained gold of the 1970 data set, which is of the type known and recovered by miners throughout the region. The very fine- 
grained gold generally is not recovered in commonly used panning techniques. Gold grains in particles large enough to be recovered in most commercial operations are from faults, veins, and dikes that have consolidated and enriched the very fine-grained gold into larger, localized particles. These larger and younger grains generally have lost copper, palladium, and some other elements.

Native arsenic is associated with the fine-grained gold in Sulphur Bar Creek and tributaries in the southern part of the Big Belt Mountains (p1. 3, map $E$, nos. M127, M128, and M129). Its presence is anomalous and is not understood.

\section{REFERENCES CITED}

Close, T.J., and Rigby, J.G., 1984, Mineral investigation of the Gates of the Mountains Wilderness Study Area, Lewis and Clark County, Montana: U.S. Bureau of Mines Open-File Report MLA 3-84, 20 p.

Earhart, R.L., Grimes, D.J., Leinz, R.W., Marks, L.Y., and Peterson, D.L., 1977, Mineral resources of the proposed additions to the Scapegoat Wilderness, Powe 11 and Lewis and Clark Counties, Montana: U.S. Geological Survey Bulletin 1430, $62 \mathrm{p}$.

Earhart, R.L., Mudge, M.R., Whipple, J.W., and Connor, J.J., 1981, Mineral resources of the Choteau $1^{\circ} \times 2^{\circ}$ quadrangle, Montana: U.S. Geological Survey Miscellaneous Field Studies Map MF-858-A, scale 1:250,000.

Elliott, J.E., Loen, J.S., Wise, K.K., and Blaskowski, M.J., 1992, Maps showing locations of mines and prospects in the Butte $1^{\circ} \times 2^{\circ}$ quadrangle, western Montana: U.S. Geological Survey Map I-2050-C, scale 1:250,000.

English, B.L., Desborough, G.A., and Raymond, W.H., 1987, A mechanical panning technique for separation of fine-grained gold and other heavy minerals: U.S. Geological Survey Open-File Report 87-364, 9 p.

Koschmann, A.H., and Bergendah1, M.H., 1968, Principal gold producing districts of the United States: U.S. Geological Survey Professional Paper 610, p. $142-171$.

Loen, J.S., 1989, Lode and placer deposits in the Ophir district, Powell and Lewis and Clark Counties, Montana: Fort Collins, Colorado State University, Ph.D. dissertation, $268 \mathrm{p}$.

Lyden, C.J., 1948, The gold placers of Montana: Montana Bureau of Mines and Geology Memoir 26, 151 p.

McCulloch, Robin, 1993a, Mining and mineral developments in Montana--1992:

Montana Bureau of Mines and Geology Open-File Report 262, 30 p.

1993b, Montana mining directory 1992: Montana Bureau of Mines and Geology Bulletin $131,76 \mathrm{p}$.

Pardee, J.T., and Schrader, F.C., 1933, Metalliferous deposits of the Greater Helena Mining Region, Montana: U.S. Geological Survey Bulletin 842, 318 p.

Pegasus Gold, Inc., 1992, Annual report, 54 p.

Thrush, P.W., compiler, 1968, A dictionary of mining, mineral, and related terms, compiled and edited by P.W. Thrush and the staff of the Bureau of Mines, U.S. Department of Interior: Special Publication of the U.S. Bureau of Mines, 1269 p.

Yeend, Warren, 1986, Descriptive model of placer Au-PGE, in Cox, D.P., and Singer, D.A., eds., Mineral deposit models: U.S. Geological Survey Bulletin 1693, p. 261-264.

Yeend, Warren, and Shawe, D.R., 1989, Gold in placer deposits: U.S. Geological Survey Bulletin 1857-G, p. G1-G13. 


\begin{tabular}{|c|c|c|c|c|c|c|c|c|c|c|c|c|c|c|c|c|c|c|c|c|c|c|}
\hline \\
\hline & 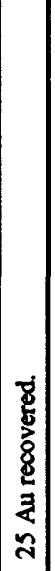 & 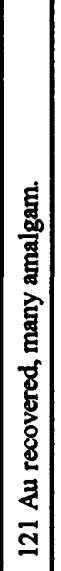 & 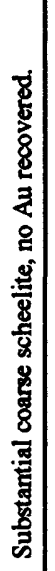 & 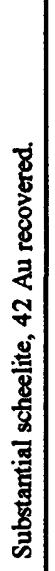 & 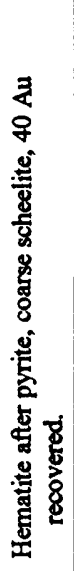 & 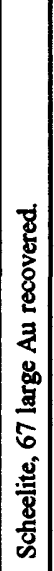 & 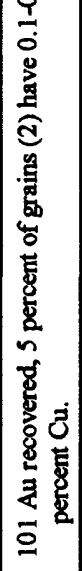 & 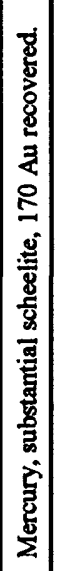 & 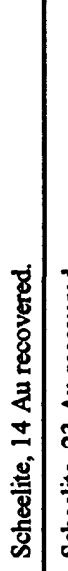 & 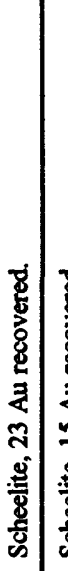 & 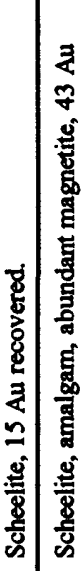 & 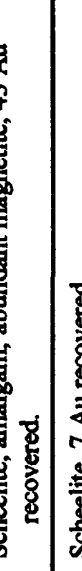 & 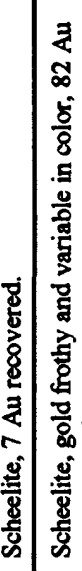 & 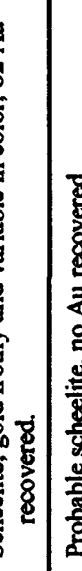 & 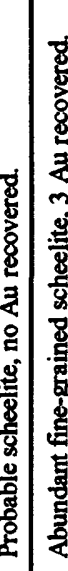 & 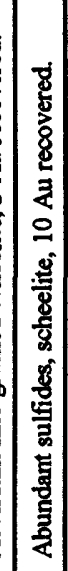 & 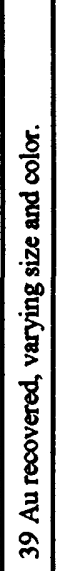 & 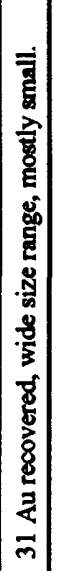 & 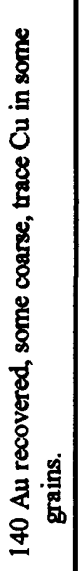 & 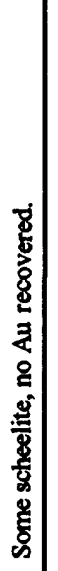 & 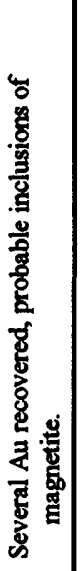 & \\
\hline \multirow{2}{*}{ 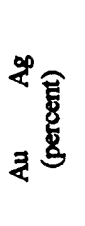 } & \begin{tabular}{|c|}
8 \\
6
\end{tabular} & $\begin{array}{l}8 \\
9\end{array}$ & 1 & $\begin{array}{l}\text { q } \\
6\end{array}$ & 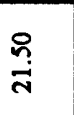 & $\begin{array}{l}0 \\
9 \\
9\end{array}$ & $\begin{array}{l}8 \\
\end{array}$ & a & 1 & 11 & 18 & 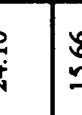 & 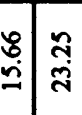 & & 11 & $\mid \begin{array}{l}R \\
\dot{X}\end{array}$ & $\mid \begin{array}{c}0 \\
\stackrel{n}{n} \\
\end{array}$ & $\begin{array}{l}8 \\
5 \\
5\end{array}$ & 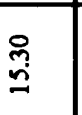 & 1 & 1 & \\
\hline & 임 & \begin{tabular}{|c|} 
\\
$\dot{1}$ \\
\end{tabular} & 1 & $\stackrel{8}{\circ}$ & 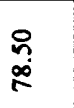 & \& & ¿ & 8 & 1 & 11 & 12 & & 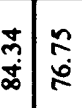 & & 111 & 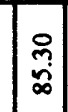 & $\begin{array}{l}? \\
\dot{\infty} \\
\dot{\infty}\end{array}$ & $\mid$ & $\infty$ & 11 & 1 & \\
\hline
\end{tabular}

过 语

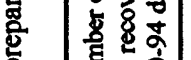

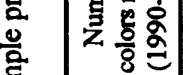

है

氬 论

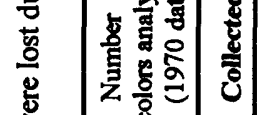

i

焉

.

이

总

吾

8

这

8 음

䨔

을

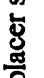

通

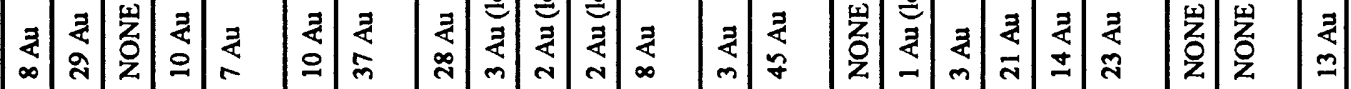

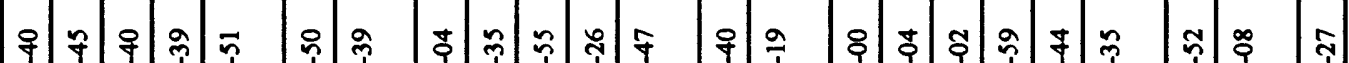

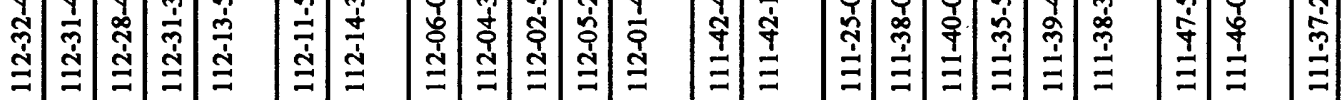

9

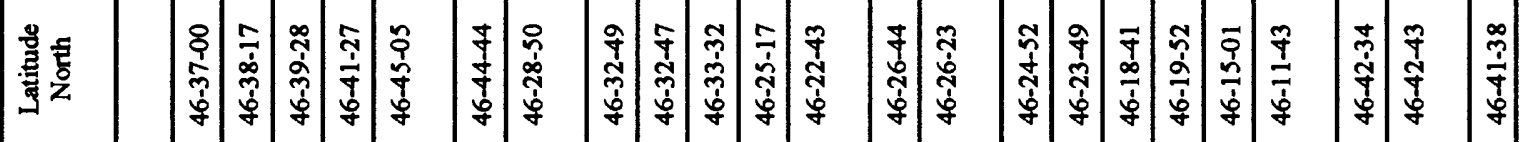

通

焉

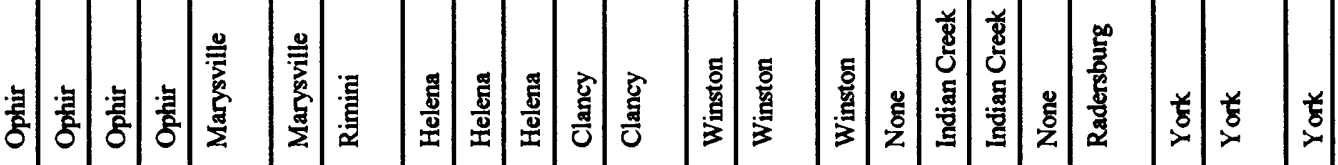

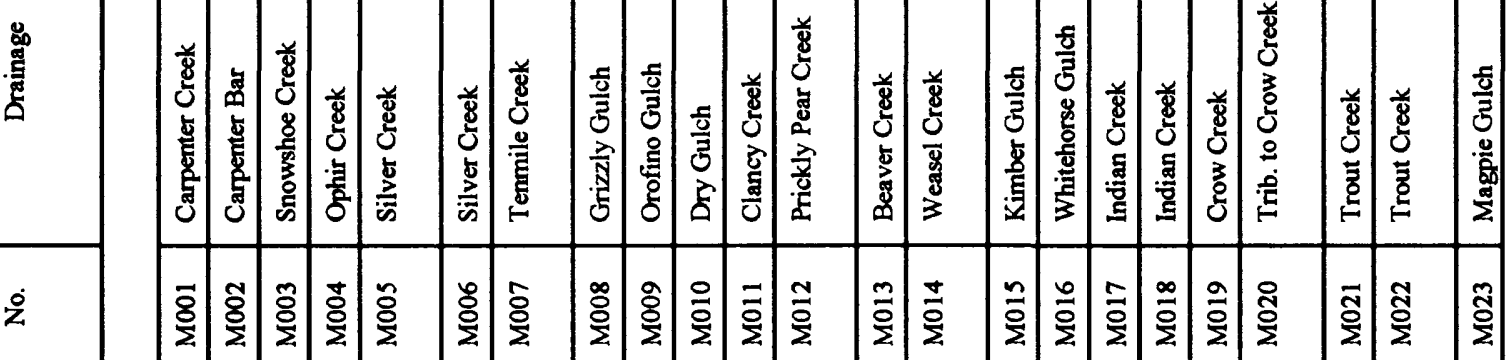




\begin{tabular}{|c|c|c|c|c|c|c|c|c|c|c|c|c|c|c|c|c|c|c|c|c|c|c|c|c|c|c|c|}
\hline 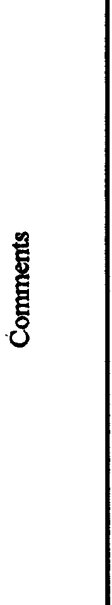 & 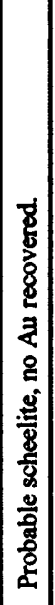 & 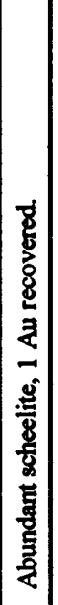 & 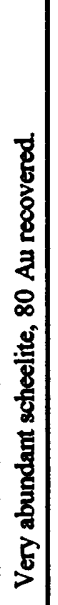 & 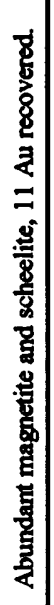 & & 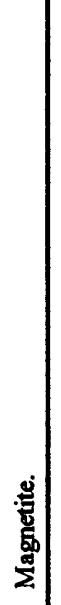 & & 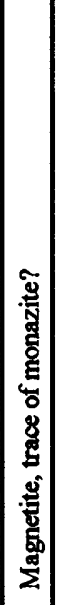 & 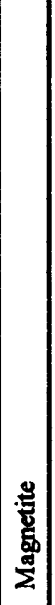 & 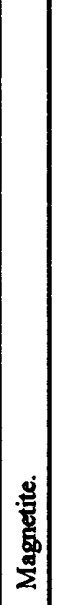 & 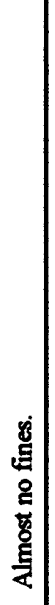 & 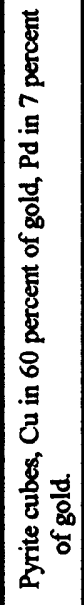 & & & 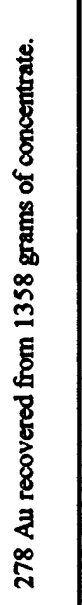 & & & & & & 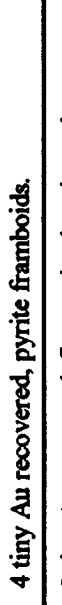 & 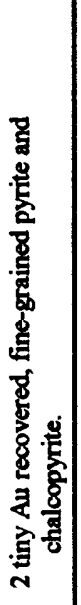 & & & & & \\
\hline \multirow{2}{*}{ 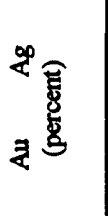 } & 1 & 1 & $\begin{array}{l}R \\
2 \\
-1\end{array}$ & 1 & & 1 & 1 & 1 & 1 & 1 & 1 & 웅 & 1 & 1 & 1 & 1 & 1 & 1 & 1 & 1 & 1 & 1 & 1 & 1 & 1 & 1 & 1 \\
\hline & 1 & 1 & 윯 & 1 & & 11 & 1 & 11 & 1 & 1 & 1 & $\begin{array}{l}\text { ஃ̊ } \\
\text { ஃ }\end{array}$ & 1 & 1 & 1 & 1 & 1 & 11 & 1 & 1 & 1 & 1 & 1 & 1 & 1 & 1 & 1 \\
\hline 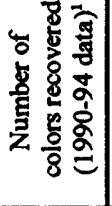 & 1 & 1 & 1 & 1 & ప్ & 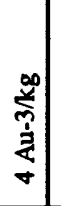 & 竞 & 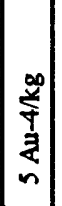 & 呈 & 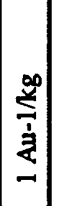 & 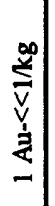 & 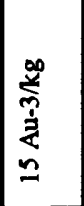 & $\stackrel{z}{z}$ & 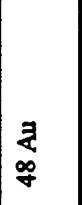 & 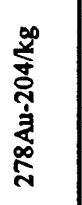 & 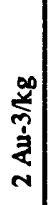 & 崩 & 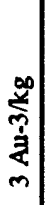 & 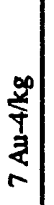 & 帘 & 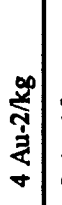 & 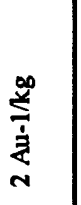 & 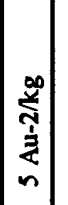 & \begin{tabular}{l|l}
$\stackrel{\infty}{\infty}$ \\
$\stackrel{p}{3}$ \\
$m$ \\
$m$
\end{tabular} & 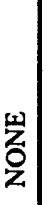 & 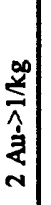 & 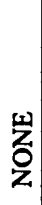 \\
\hline 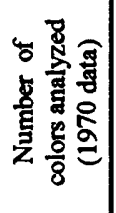 & 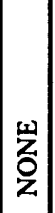 & $\mid \begin{array}{l}\text { w } \\
\vdots \\
\text { z }\end{array}$ & 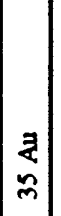 & 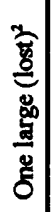 & 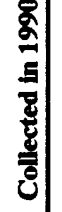 & 11 & 1 & 1 & 1 & 1 & 1 & 1 & 1 & 1 & 1 & 1 & 1 & 1 & 1 & 1 & 1 & 1 & 1 & 1 & 1 & 1 & 1 \\
\hline 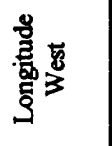 & 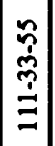 & 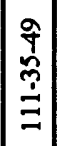 & $\begin{array}{l}\infty \\
\vdots \\
\vdots \\
\vdots\end{array}$ & 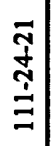 & & 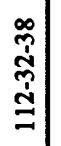 & 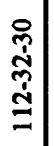 & $\mid \begin{array}{c}\stackrel{ \pm}{\grave{n}} \\
\stackrel{\overbrace{}}{\Xi} \\
\Xi\end{array}$ & 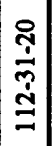 & 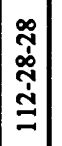 & $\begin{array}{l}\tilde{\vdots} \\
\frac{\bar{\Xi}}{\Xi} \\
\vdots\end{array}$ & $\begin{array}{l}\overrightarrow{0} \\
\infty \\
⿱ 亠 䒑 \\
\vdots \\
\equiv\end{array}$ & $\frac{9}{\frac{1}{3}}$ & $\begin{array}{l}\$ \\
\vdots \\
\text { İ } \\
\vdots\end{array}$ & $\frac{n}{\grave{b}}$ & $\begin{array}{l}\infty \\
⿱ 亠 䒑 \\
\pm \\
\vdots\end{array}$ & $\begin{array}{l}\bar{s} \\
\tilde{y} \\
\Xi\end{array}$ & 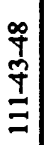 & 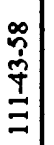 & $\begin{array}{c}\hat{s} \\
\tilde{s} \\
\Xi \\
\Xi\end{array}$ & $\begin{array}{l}\bar{I} \\
\bar{I} \\
\Xi\end{array}$ & $\begin{array}{l}\bar{n} \\
\dot{y} \\
\vdots \\
\Xi\end{array}$ & $\mid \begin{array}{l}\stackrel{0}{0} \\
\dot{0} \\
\stackrel{\Xi}{\Xi} \\
\Xi\end{array}$ & 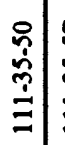 & 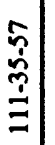 & 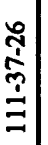 & ơ \\
\hline 㺃 & $\begin{array}{c}a \\
\hat{a} \\
\hat{o} \\
\dot{b}\end{array}$ & 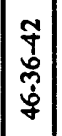 & $\begin{array}{l}\frac{1}{d} \\
\frac{d}{b}\end{array}$ & 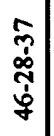 & & 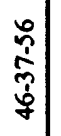 & 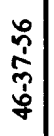 & 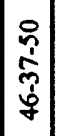 & $\begin{array}{c}\infty \\
\stackrel{0}{7} \\
\dot{7} \\
\dot{f}\end{array}$ & 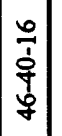 & $\frac{\hat{9}}{\overline{7}}$ & 官 & 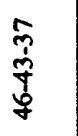 & 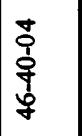 & 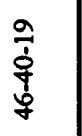 & 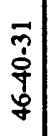 & 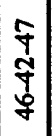 & 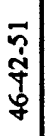 & 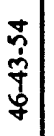 & $\begin{array}{l}n \\
\vdots \\
⿱ 亠 乂 \\
\vdots \\
\vdots\end{array}$ & $\begin{array}{l}\infty \\
\frac{1}{5} \\
\vdots \\
\vdots\end{array}$ & 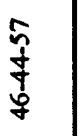 & 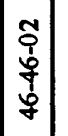 & 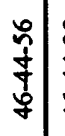 & 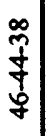 & 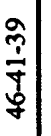 & 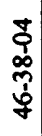 \\
\hline 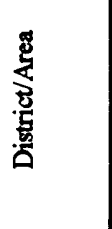 & 芦 & 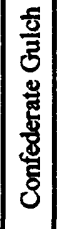 & 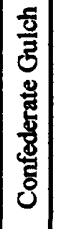 & 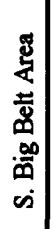 & & 氡 & :향 & 咅 & 紊 & 常 & 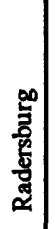 & z & 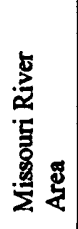 & 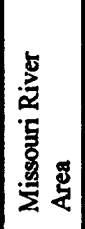 & 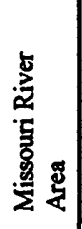 & 兑 & 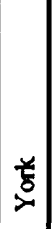 & 芦 & $\stackrel{\text { 라 }}{2}$ & 芦 & 总 & 兑 & $\mid \begin{array}{l}0 \\
\text { g } \\
z\end{array}$ & 总 & 营 & 曾 & 항 \\
\hline $\begin{array}{l}\text { 品 } \\
\text { 苞 } \\
\text { हू }\end{array}$ & 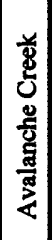 & 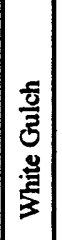 & 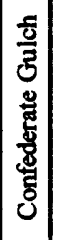 & 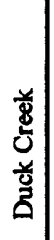 & & 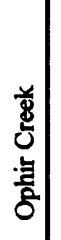 & 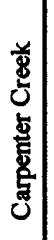 & 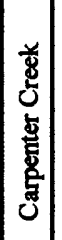 & 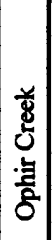 & 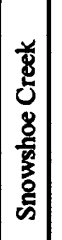 & $\begin{array}{l}\text { 惫 } \\
0 \\
3 \\
0 \\
0 \\
0\end{array}$ & 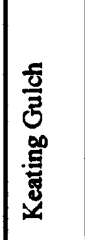 & 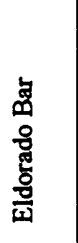 & 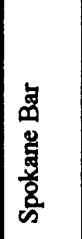 & 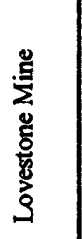 & 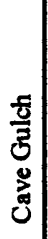 & 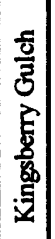 & 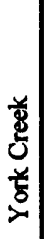 & 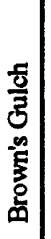 & 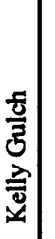 & 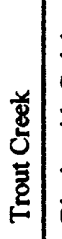 & 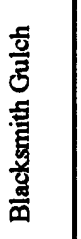 & 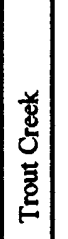 & 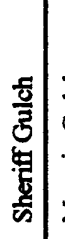 & 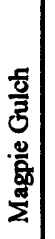 & 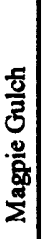 & 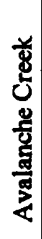 \\
\hline$\dot{z}$ & 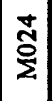 & ָ⿳亠े冖ౖ & 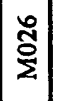 & స్ & & $\begin{array}{l}0 \\
\grave{2} \\
\Sigma\end{array}$ & 产 & 䓂 & $\stackrel{\tilde{n}}{\Sigma}$ & 浐 & 言 & ֻัڤ & 全 & $\aleph_{\tilde{\Sigma}}^{\infty}$ & 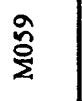 & $\Sigma$ & 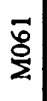 & స్ & ஜे & 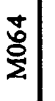 & 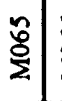 & $\frac{8}{\sum}$ & 容 & $\begin{array}{l}\infty \\
0 \\
\vdots \\
\Sigma\end{array}$ & $\begin{array}{l}\text { oे } \\
\text { ¿े }\end{array}$ & 尽 & $\overline{5}$ \\
\hline
\end{tabular}




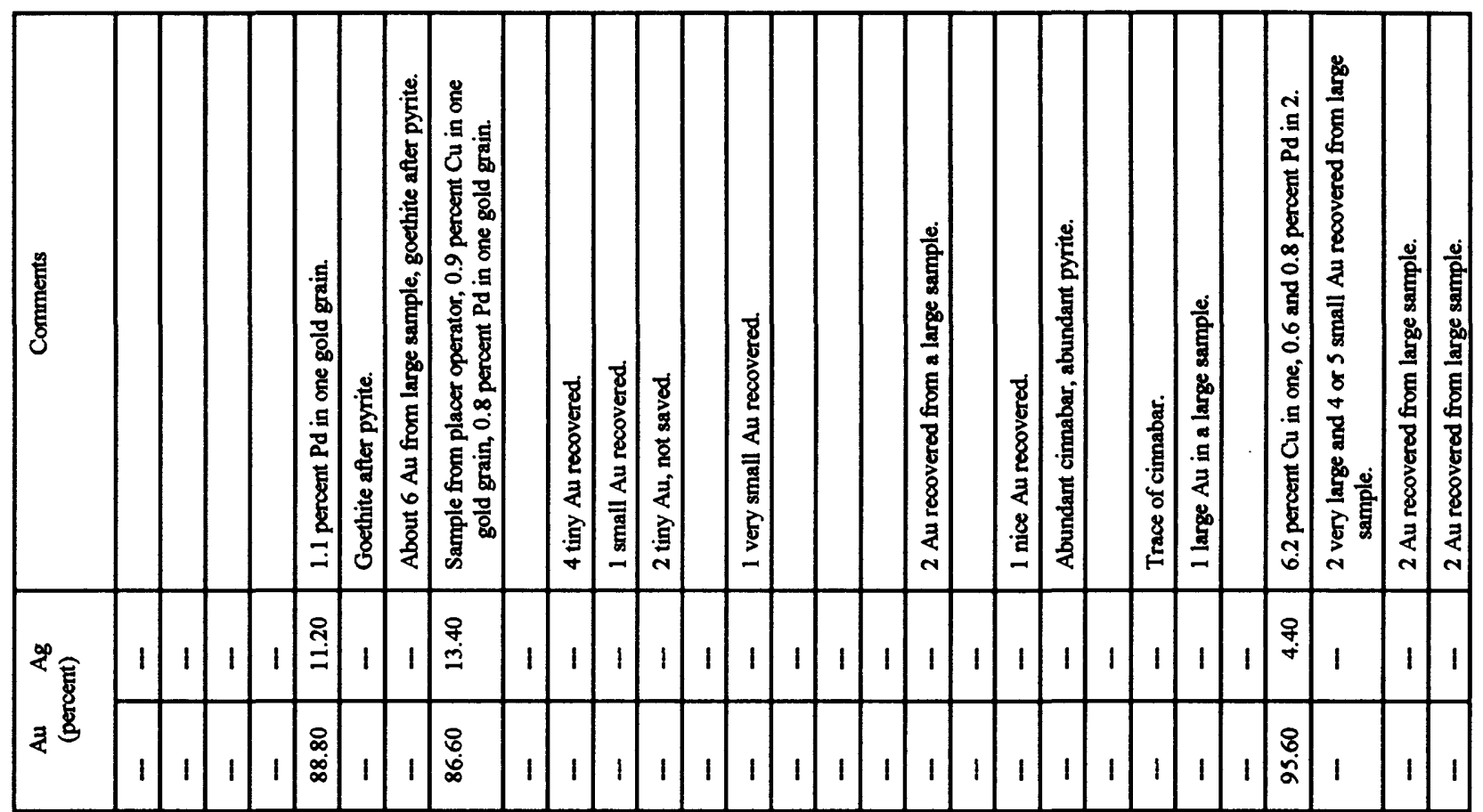

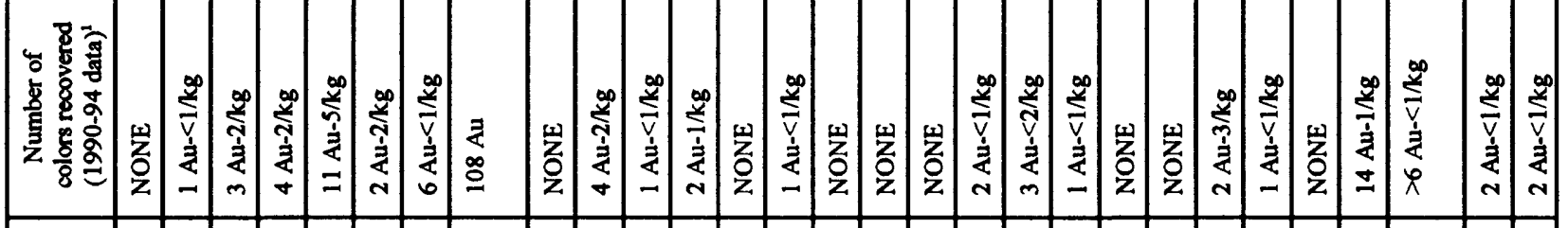

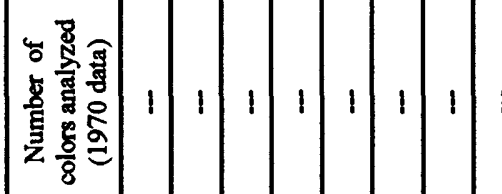

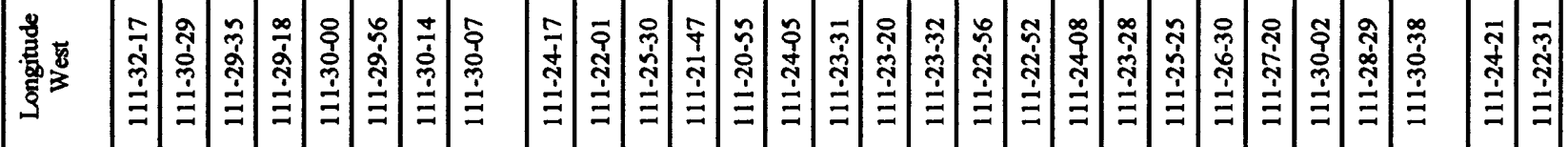

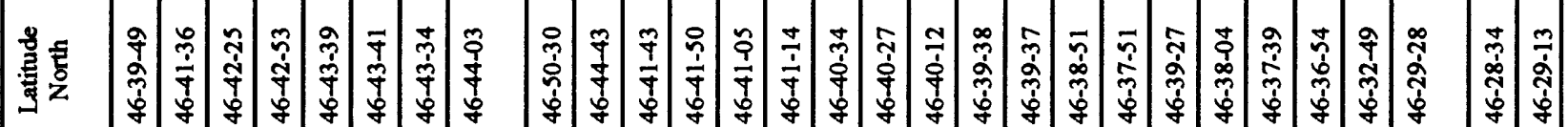

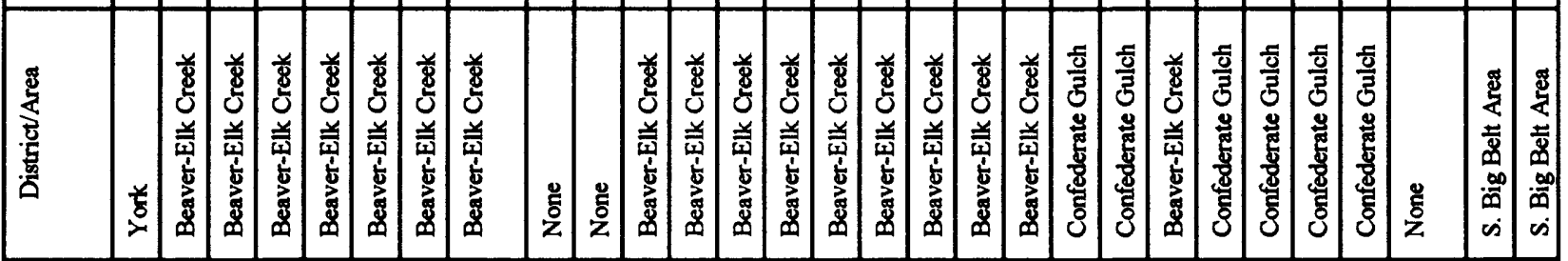

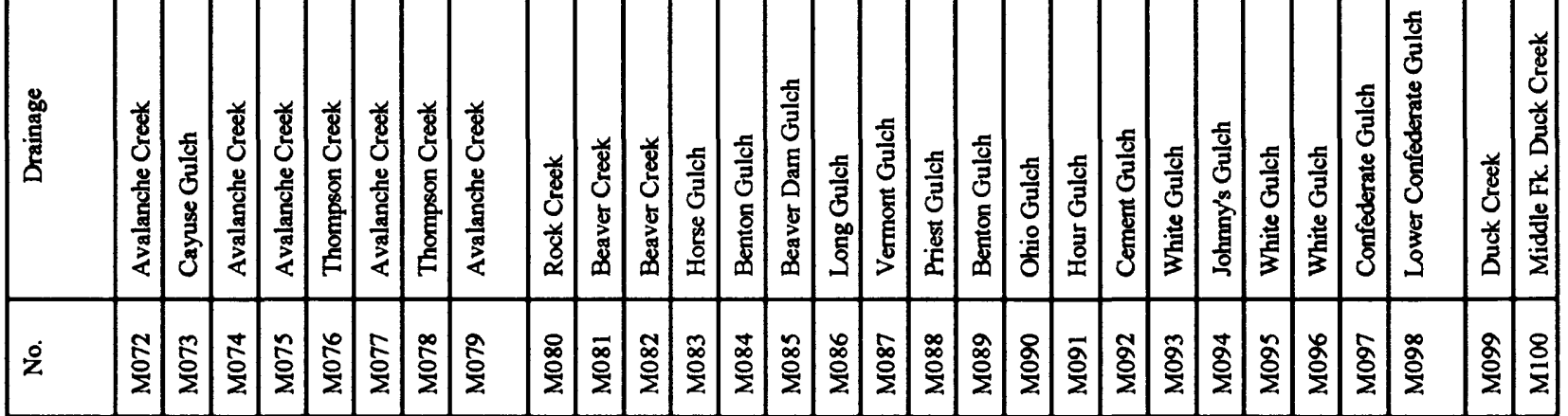




\begin{tabular}{|c|c|c|c|c|c|c|c|c|c|c|c|c|c|c|c|c|c|c|c|c|c|c|c|c|c|c|c|c|}
\hline 卷 & & & & 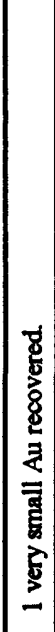 & & & & & & & & & & & & & 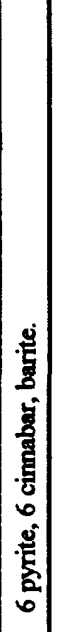 & & है & & & & 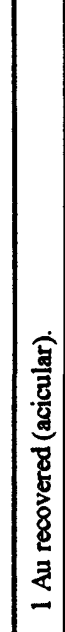 & & 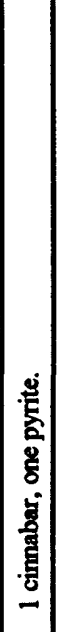 & 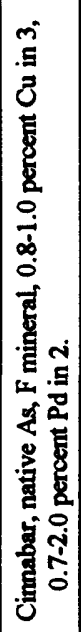 & 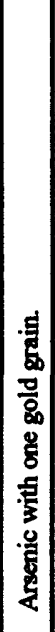 & 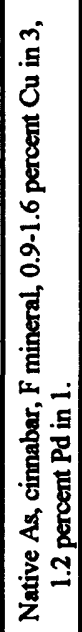 \\
\hline \multirow{2}{*}{ 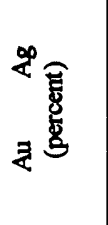 } & 1 & 1 & 1 & 1 & 1 & 1 & 1 & 1 & 1 & 1 & 1 & 1 & 1 & 1 & 1 & 1 & 1 & 1 & 1 & 1 & 1 & 11 & 1 & 1 & 1 & $\stackrel{0}{\circ}$ & 1 & $\underset{6}{8}$ \\
\hline & 1 & 1 & 1 & 1 & 1 & 1 & 1 & 1 & 1 & 1 & 1 & 1 & 1 & 1 & 1 & 1 & 1 & 1 & 1 & 1 & 1 & 111 & 1 & 1 & 11 & \& & 1 & $\begin{array}{l}8 \\
+ \\
1\end{array}$ \\
\hline 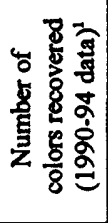 & 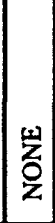 & 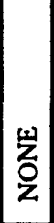 & 包 & 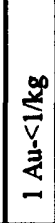 & 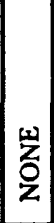 & \begin{tabular}{|l|} 
\\
$\vdots$ \\
2 \\
$z$
\end{tabular} & 离 & 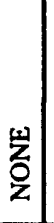 & $\begin{array}{l}\text { 坚 } \\
0 \\
\text { z }\end{array}$ & 岂 & $\begin{array}{l}\text { 品 } \\
\text { 乙 } \\
\text { 乙 }\end{array}$ & ㄹ & 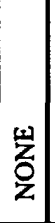 & 号 & 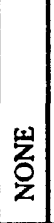 & 岂 & 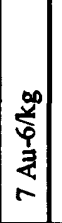 & 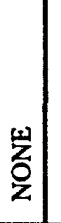 & 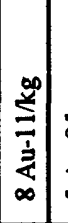 & 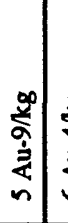 & 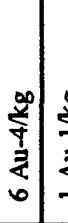 & 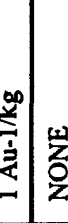 & 竞 & 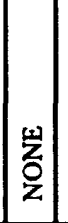 & 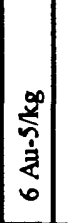 & 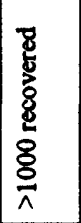 & 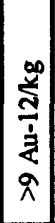 & $\begin{array}{l}\overline{8} \\
8 \\
8 \\
8 \\
8 \\
8 \\
8 \\
\frac{8}{\Lambda}\end{array}$ \\
\hline 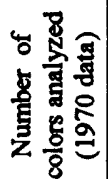 & 1 & 1 & 1 & 1 & 1 & 11 & 1 & 1 & 1 & 1 & 1 & 1 & 1 & 1 & 1 & 1 & 1 & 1 & 1 & 1 & 1 & 11 & 1 & 1 & 1 & 1 & 1 & 1 \\
\hline 总范 & 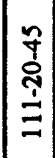 & $\begin{array}{l}\swarrow \\
\vdots \\
\vdots \\
\vdots \\
\vdots\end{array}$ & $\begin{array}{l}0 \\
\hat{\gamma} \\
\vdots \\
\vdots \\
\vdots\end{array}$ & $\begin{array}{l}\tilde{5} \\
\dot{\Xi} \\
\vdots \\
\vdots\end{array}$ & 号 & 站 & 울 & 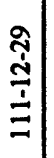 & $\stackrel{\sim}{\stackrel{0}{ٍ}}$ & $\begin{array}{l}\stackrel{\Xi}{\grave{d}} \\
\vdots \\
\vdots\end{array}$ & $\begin{array}{l}\stackrel{9}{9} \\
\\
\end{array}$ & $\begin{array}{l}\hat{n} \\
0 \\
\vdots \\
\vdots\end{array}$ & $\begin{array}{l}\text { oे } \\
0 \\
0 \\
\end{array}$ & 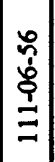 & $\begin{array}{l}\frac{0}{5} \\
\frac{1}{9} \\
ٍ\end{array}$ & 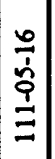 & 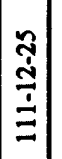 & $\begin{array}{l}\stackrel{\Im}{ড} \\
\stackrel{\vdots}{\Xi}\end{array}$ & $\begin{array}{l}\frac{9}{0} \\
\vdots \\
\vdots\end{array}$ & 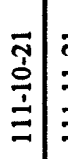 & 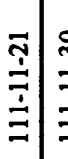 & 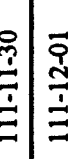 & $\begin{array}{l}a \\
\grave{\Delta} \\
\vdots \\
\vdots\end{array}$ & $\mid \begin{array}{l}\hat{n} \\
\stackrel{亏}{\Xi} \\
\vdots\end{array}$ & $\mid \begin{array}{l}\frac{9}{a} \\
\vdots \\
\vdots \\
\vdots\end{array}$ & 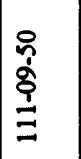 & \begin{tabular}{|c|}
0 \\
$\vdots$ \\
$\vdots$ \\
$\vdots$ \\
$\vdots$
\end{tabular} & 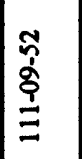 \\
\hline 胥 & 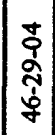 & 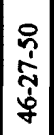 & 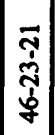 & 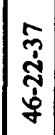 & 客 & $\left|\begin{array}{c}\tilde{\hat{\alpha}} \\
\frac{\hat{\sigma}}{\dot{b}}\end{array}\right|$ & 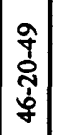 & $\begin{array}{l}\bar{n} \\
\frac{a}{b}\end{array}$ & $\frac{\tilde{c}}{\stackrel{0}{b}}$ & $\frac{\infty}{\grave{\alpha}}$ & $\frac{m}{\frac{1}{4}}$ & $\begin{array}{l}\vec{t} \\
\frac{\vec{t}}{\dot{b}}\end{array}$ & $\mid$ & 蒿 & 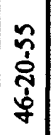 & $\frac{n}{\alpha}$ & $\mid \begin{array}{c}\frac{\infty}{1} \\
\frac{a}{b} \\
\frac{b}{b}\end{array}$ & $\frac{a}{\frac{a}{b}}$ & $\frac{m}{\frac{3}{b}}$ & $\frac{\tilde{n}}{\dot{0}}$ & 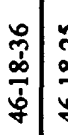 & 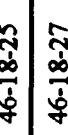 & $\frac{\frac{0}{\alpha}}{\frac{1}{b}}$ & $\mid \begin{array}{l}n \\
\frac{n}{5} \\
\dot{5}\end{array}$ & $\mid \begin{array}{l}\overrightarrow{5} \\
\vdots \\
\dot{b}\end{array}$ & $\frac{9}{\frac{9}{5}}$ & $\frac{\frac{q}{2}}{\frac{3}{b}}$ & $\frac{5}{\frac{5}{6}}$ \\
\hline 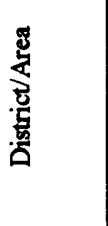 & 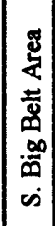 & 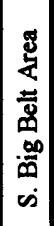 & 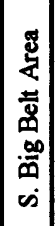 & 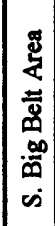 & 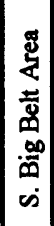 & 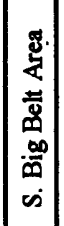 & 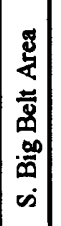 & 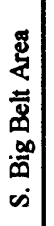 & 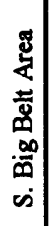 & 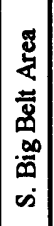 & 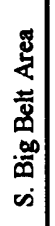 & 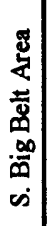 & 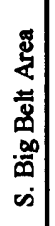 & 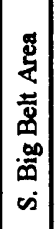 & 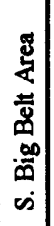 & 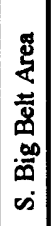 & 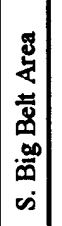 & 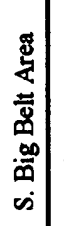 & 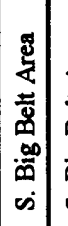 & 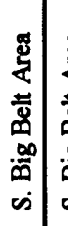 & 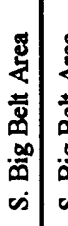 & 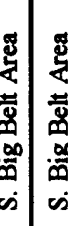 & 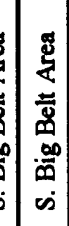 & 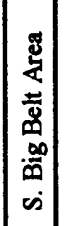 & 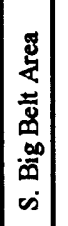 & 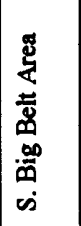 & 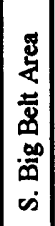 & 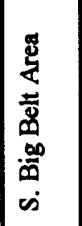 \\
\hline 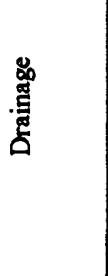 & 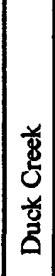 & 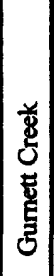 & 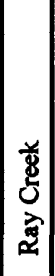 & 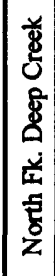 & 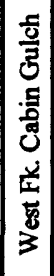 & 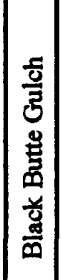 & 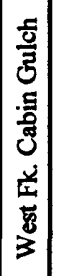 & 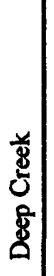 & 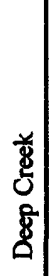 & 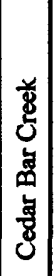 & 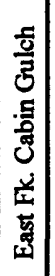 & 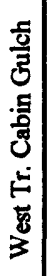 & 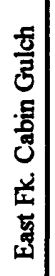 & 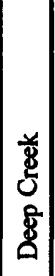 & 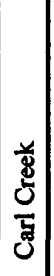 & 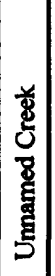 & 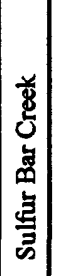 & 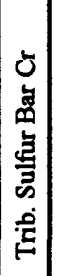 & 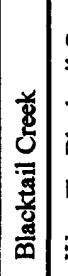 & 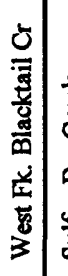 & 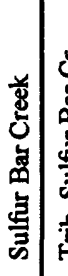 & 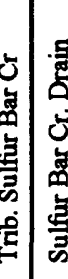 & 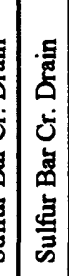 & 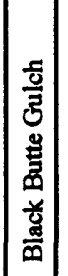 & 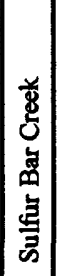 & 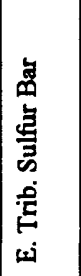 & 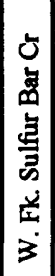 & 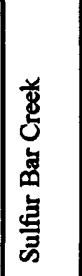 \\
\hline ż & $\frac{\overrightarrow{0}}{\Sigma}$ & $\stackrel{\Sigma}{\Sigma}$ & $\stackrel{\varrho}{0}$ & $\frac{ \pm}{\Sigma}$ & $\frac{n}{\Sigma}$ & $\frac{\xi}{\Sigma}$ & 官 & $\stackrel{\infty}{\stackrel{\infty}{\Sigma}}$ & ఏे & 올 & $\bar{\Sigma}$ & $\underset{\Sigma}{\Sigma}$ & $\stackrel{m}{\bar{\Sigma}}$ & 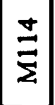 & $\cong$ & $\stackrel{\circ}{\bar{z}}$ & 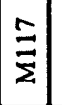 & $\stackrel{\infty}{\Xi}$ & $\stackrel{a}{\Sigma}$ & $\underset{\Sigma}{\stackrel{\Xi}{\Sigma}}$ & $\underset{\Sigma}{\vec{\Sigma}}$ & $\underset{\Sigma}{\Sigma} \stackrel{\Xi}{\Sigma}$ & $\stackrel{\vec{J}}{\Sigma}$ & $\underset{\Sigma}{\Sigma}$ & $\underset{z}{\Sigma}$ & $\stackrel{\grave{\Sigma}}{\grave{\Sigma}}$ & $\stackrel{\infty}{\stackrel{\Sigma}{\Sigma}}$ & 产 \\
\hline
\end{tabular}




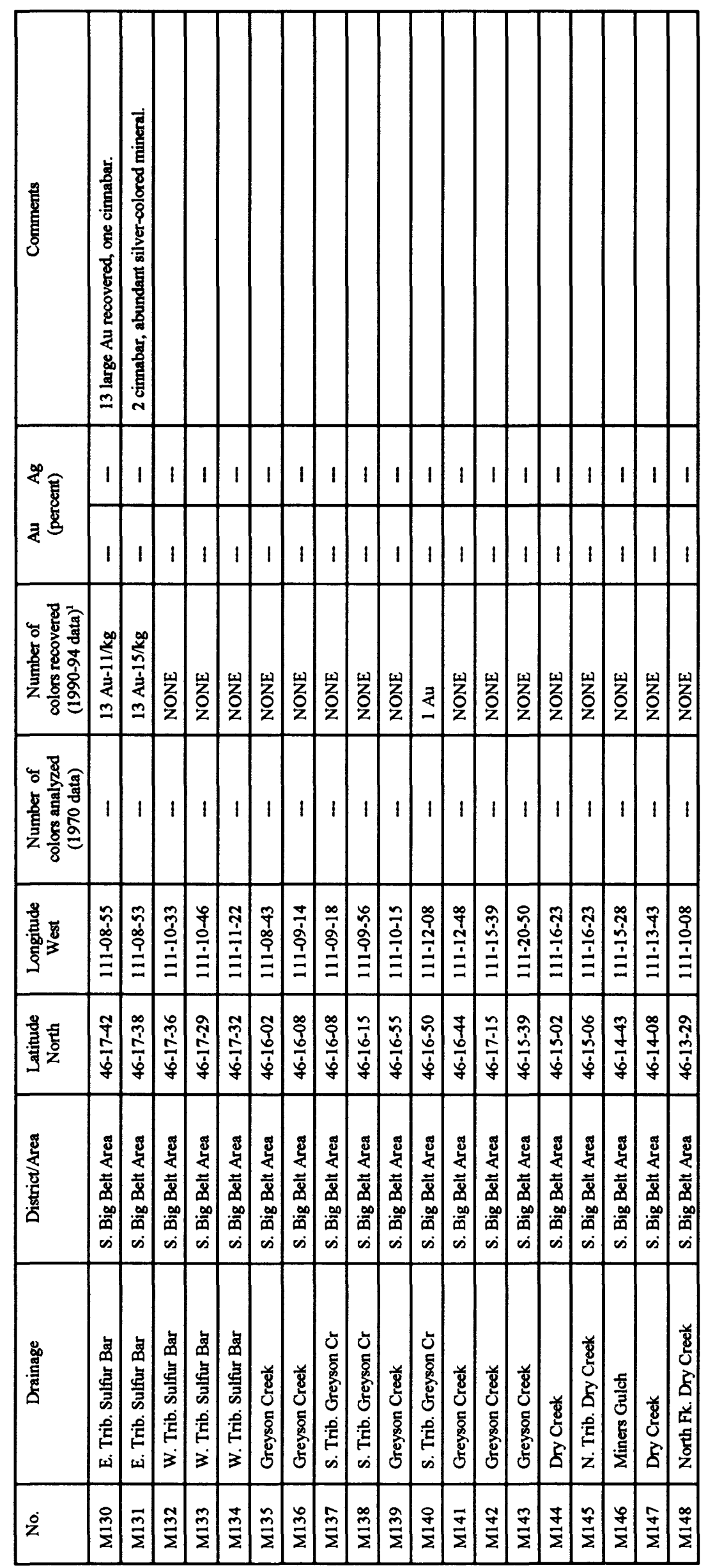




\title{
MONMETALLIC MINERAL DEPOBITS OF THE HELENA MATIONAL FORE8T
}

\author{
By R.G. Tysdal
}

\section{BARITE}

Barite forms veins and pods in the Middle Proterozoic Spokane Formation at a few widely separated localities along the southwest flank of the Big Belt Mountains (Berg, 1988) in the York mining district (fig. A2, p1. 2). The veins and pods belong to a class of barite vein deposits that occur within metasedimentary rocks of the Middle Proterozoic Belt Supergroup and generally occur along faults (Berg, 1986). Barium in the veins probably was derived from the host Belt strata: (1) the veins are most abundant in the Belt rather than younger strata; (2) the veins generally are not associated with igneous intrusives; and (3) the Belt rocks are known to contain fairly high values of disseminated barium that locally could be concentrated into veins and pods (Berg, 1986).

The barite veins and pods in the Big Belt Mountains are too small and localized to be considered of economic significance (p1. 2 and table E1, nos. $513,514,520,561)$. Further, some of the veins contain quartz that would need to be separated before the barite could be used for industrial purposes.

\section{BUILDING STONE}

Building (dimension) stone is naturally occurring rock that is selected, shaped, or cut into blocks, slabs, or other specified shape for use in structures such as pavements, walkways, fireplaces, or walls of buildings. Building stone is not known to have been quarried from within the Forest. Near State highway 12 in Deep Creek Canyon, a thick granitic dike has been quarried for road metal (p1. 2, no. 602). This appears to be the same locality that $\operatorname{Berg}(1974$, p. 16) applied the term andesite, stating that when polished, the rock would produce a decorative stone. Rhyolite has been obtained from a large accumulation of talus west of the Josephine mine in an area north of Basin (p1. 2, no. 604). This stone has been termed "rubble" by Berg (1974, p. 11 and 25), which he defined as a variety of stone that has at least one relatively flat surface produced by splitting or rough sawing.

\section{CLAY}

Clay used in bricks and paving stones was produced from the Blossburg mine (p1. 2, no. 177), in the Mullan Pass area west of Helena, from the late 1800's (Rowe, 1908) to about 1950. Clay was mined from a Tertiary unit (Ts, p1. 1), possibly 01igocene in age, comprised of nonmarine claystone, siltstone, sandstone, and volcanic ash (Schmidt and others, 1994). The Blossburg mine is on private land that lies inside the Forest boundary, but the clay-bearing 
stratigraphic unit extends westward onto Forest land.

\section{LIMESTONE}

The distribution of limestone, dolomite, and calcareous and dolomitc strata in the Forest is shown on map C (p1.3). Limestone of quality that is adequate for use in cement or as a smelting flux is present locally in the Gates of the Mountains Wilderness (Reynolds and Close, 1984) and nearby strata in the northern part of the Big Belt Mountains. Analyses by the U.S. Bureau of Mines indicate that most of this limestone is suitable for cement and for other construction uses (Close and Rigby, 1984).

On the east flank of the Elkhorn Mountains, outside the Forest, limestone is produced from the Indian Creek mine (p1. 2, no. 481) in the Mississippian Mission Canyon Formation. The operation produces about 1 million tons of raw limestone per year (McCulloch, 1992, 1993). The host formation also is present in the southeastern part of the Forest in the Elkhorn Mountains, but we have conducted no analyses of composition to determine suitability for industrial use. Limestone for cement is produced from the Montana City quarry (p1. 2, no. 397) south of Helena, from altered limestone (McCulloch, 1992). A small amount of limestone is quarried from Cambrian limestone at the Helena limestone quarry ( $p 1$. 2, no. 203) (Chelini, 1965). The adjacent Forest contains an extensive area of limestone of Cambrian to Mississipian age. Similarly, limestone has been quarried near the Forest about $3 \mathrm{mi}$ east of Elliston, and an extensive area of Cambrian to Mississippian limestone is present in the Forest north of Elliston, in the Ophir district--Dog Creek area.

The Forest contains Cambrian to Mississippian-age limestones that could be utilized for industrial purposes, but large areas of limestone also lie outside the Forest and generally are closer to major transportation routes.

\section{PHOSPHATE}

In western Montana, the Lower Permian Phosphoria Formation is the host for minable phosphate. The phosphate is of the upwelling type. Deep, cold ocean water rich in phosphorous welled upward to replace near surface water, and phosphate precipitated due chiefly to a decrease in pressure and an increase in temperature (Sheldon, 1964; Mosier, 1986).

The Phosphoria Formation is present only locally in the northern part of the Big Belt Mountains, where it was generally removed by erosion prior to deposition of younger strata. No phosphatic rocks were reported to occur within the Gates of the Mountains Wilderness, or proposed additions thereto, in the northern part of the Forest (Reynolds and Close, 1984; Close and Rigby, 1984). Robinson and others (1969) stated that as much as $40 \mathrm{ft}$ of the formation is present locally near American Bar (p1. 2, vicinity of no. 503) (along the Missouri River directly east of Holter Lake); at the northwesternmost edge of the Forest, west of the Missouri River; and near Hauser Dam. They reported none of the phosphatic strata that produces phosphate in western Montana, noting only phosphatic sandstone in the isolated remnants of the formation. The Big Belt Mountains area lies east of the phosphate producing region, where the formation is thin and sandy and does not have minable grade, thickness, or extent. 
In the southeastern part of the Elkhorn Mountains, the Phosphoria Formation has limited exposures. It consists mainly of chert and quartzitic sandstone, but in places includes one or two thin beds of phosphate-bearing rock. A lower phosphatic bed, only 9 in. thick, and chips of phosphatic material in a quartzite bed at the top of the Phosphoria, occur east of the Forest; none of the phosphate rock in the Forest is of minable grade or thickness (Klepper and others, 1971, p. 6).

In the westernmost part of the Forest, in the Ophir district-Dog Creek area north of E11iston (pl. 2), most of the phosphate occurs in a 2.5-7.5 ft thick single zone (the Retort Phosphatic Shale Member) of the Phosphoria Formation. Swanson (1973, p. 827) stated that the phosphate varies widely in quality, with some beds containing too little phosphate to mine whereas other beds are thick enough to mine by themselves. The irregular distribution was attributed to the strata representing landward deposits of the Phosphoria sea. A small tonnage has been produced from each of several small underground exposures, mostly before World War II. In the area where the Phosphoria is contiguous in and adjacent to the Forest, Swanson (1973, p. 829) calculated resources of the Phosphatic Shale Member to be about 200 million short tons of rock containing greater than 18 percent $\mathrm{P}_{2} \mathrm{O}_{5}$. About half of the area of distribution of the member lies within the Forest.

\section{SAPPHIRES IN PLACER DEPOSITS}

Sapphires have been recovered from terrace deposits of gravel ("bars") near the Missouri River along the west side of the Big Belt Mountains (Pardee and Schrader, 1933). The sapphires originally were recovered as a byproduct of placer gold operations, and later for gems, but their pale colorations failed to find a significant market. Most seem to have been used for industrial purposes (Clabaugh, 1952). The bars presently produce small-scale concentrates for commercial use and fee digging for recreational recovery (Ziehen, 1987; Voynick, 1988; McCulloch, 1993). Parts of only two of the bars, American and Eldorado (p1. 2, nos. 503 and 505), lie partly inside the Forest. Washed commercial concentrates of sapphires were being recovered from the Eldorado and some of the other bars as recently as 1992 (McCulloch, 1993).

The source of the sapphires has not been satisfactorally determined. A few sapphires have been found in an igneous dike about 1 mi down stream from Canyon Ferry Dam, on the west bank of the Missouri River. Kunz (1893) and Pardee and Schrader (1933) believed this dike and perhaps others to be the source of the placer sapphires. Baker (1992) attributed a sapphire-bearing dike of the Little Belt Mountains (east of the Forest study area) to emplacement from a deep-seated crustal magma during Eocene regional magmatic activity, which is in accord with modern concepts of the origin of sapphires. Several decades earlier, Mertie and

others (1951) hypothesized that the sapphires were derived from contact-metamorphic rocks formed in the roof zone of a granitic intrusive that crops out directly south of Canyon Ferry Dam, along the west side of the reservoir; they speculated that the lode deposits were entirely removed by erosion.

No sapphire-bearing dikes, or sapphire-bearing contact-metamorphic rocks, are known to be present within the Forest in this vicinity. 


\section{SILICA}

Deposits of high silica content are of both igneous and sedimentary origin. The only sedimentary unit of very high silica content is the Pennsylvanian Quadrant Formation, which is known to be of high purity locally in western Montana (Chelini, 1966). No evaluations were made of the purity of the Quadrant in the Forest. Only one deposit type, of igneous origin, is considered here.

A $150 \mathrm{ft}$ wide dike that is nearly pure quartz, the Corral Gulch deposit (p1. 2, no. 297), cuts granitic rocks of the Boulder batholith west of Clancy (West, 1959; Chelini, 1966). The quartz is of hydrothermal origin, likely formed from fluids that crystallized during waning stages of formation of the Boulder batholith. The deposit has a measured reserve of 275,000 tons of quartz (Chelini, 1966, p. 20). No production is known to have occurred from the deposit (Elliott and others, 1992).

\section{SHORELINE PLACER DEPOSITS}

Shoreline placer deposits are composed of heavy minerals that have been concentrated by wave processes. The concentrations form beds and lenses which, over a large area, constitute elongate "shoestring" deposits indicatve of a strandline. Most such placer deposits formed in a marine beach environment and, to a lesser extent, associated dune and inlet environments. The heavy minerals typically are dark gray to black, well sorted sands of fine to medium grain size. Some minerals that may be present in the placers, and of economic value, include ilmenite (titanium), rutile (titanium), zircon (zirconium), and monazite (rare earths and thorium). Magnetite also commonly is present, although by itself generally is not likely to be of economic value. Quartz, resistant to weathering, also generally is abundant. Present-day production from shoreline placer deposits generally is from late Tertiary-Quaternary deposits, most of which have not been lithified (Force, 1986; E.R. Force, oral commun., 1994).

In the vicinity of the Forest, heavy minerals in fossil (ancient) shoreline placer deposits form black sandstones that were deposited along the margin of the Late Cretaceous Western Interior seaway (Houston and Murphy, 1977). A black sand-bearing zone yielded magnetite and ilmenite from a 7-15 ft thick sandstone at the Iron Cross mine (p1. 2, no. 494), about 1 mi southeast of the southeast corner of the Forest in the Radersburg district of the Elkhorn Mountains (Reed, 1951, p. 60; Klepper and others, 1957, 1971; Freeman and others, 1958). Heavy mineral-bearing sandstone also is present in the southeasternnmost part of the Forest (p1. 2, no. 486). These deposits are within the basal part of the Cretaceous Eagle Sandstone (plate 1), which Tysdal (unpub. data) separated from the Slim Sam Formation as originally defined by Klepper and others $(1957,1971)$. The host Eagle Sandstone ( 1 1. 1) is discontinuous within the Forest of the Elkhorn Mountains and adjacent area, due to erosion during the Cretaceous, and the black sand-bearing zone is thin and discontinuous within the preserved strata of the formation (Klepper and others, 1971, p. 12; Tysdal, unpub. data). 
Baker, D.W., 1992, Central Montana alkalic province--Critical review of Laramide plate tectonic models that extract alkalic magmas from abnormally thick Precambrian lithospheric mantle, in Elliott, J.E., ed., Guidebook for the Red Lodge-Beartooth Mountains-Stillwater area: Northwest Geology, v. 20/21, p 71-95.

Berg, R.B., 1974, Building stone in Montana: Montana Bureau of Mines and Geology Bulletin 94, 41 p.

1986, Characteristics and probable origin of barite veins in the Belt Supergroup, western Montana, in Roberts, S.M., ed., Belt Supergroup--A guide to Proterozoic rocks of western Montana and adjacent areas: Montana Bureau of Mines and Geology Special Publication 94, p. 258-267.

1988, Barite in Montana: Montana Bureau of Mines and Geology Bulletin $94,41 \mathrm{p}$.

Chelini, J.M., 1965, Limestone, dolomite, and travertine in Montana: Montana Bureau of Mines and Geology Bulletin 44, $53 \mathrm{p}$.

1966, Some high-purity quartz deposits in Montana: Montana Bureau of Mines and Geology Bulletin 44, $54 \mathrm{p}$.

Clabaugh, S.E., 1952, Corundum deposits of Montana: U.S. Geological Survey Bulletin 983, $100 \mathrm{p}$.

Close, T.J., and Rigby, J.G., 1984, Mineral investigation of the Gates of the Mountains Wilderness Study Area, Lewis and Clark County, Montana: U.S. Bureau of Mines Open-File Report MLA 3-84, 20 p.

Elliott, J.E., Loen, J.S., Wise, K.K., and Blaskowski, M.J., 1992, Maps showing locations of mines and prospects in the Butte $1^{\circ} \times 2^{\circ}$ quadrangle, western Montana: U.S. Geological Survey Miscellaneous Field Investigations Map I-2050-C, scale 1:250,000, with pamphlet.

Freeman, V.L., Ruppel, E.T., and Klepper, 1958, Geology of part of the Townsend Valley, Broadwater and Jefferson Counties, Montana: U.S. Geological Survey Bulletin 1042-N, p. 481-556.

Force, E.R., 1986, Descriptive model of shoreline placer Ti, in Cox, D.P., and Singer, D.A., eds., Mineral deposit models: U.S. Geological Survey Bulletin 1693, p. 270.

Houston, R.S., and Murphy, J.F., 1977, Depositional environment of Upper Cretaceous black sandstones of the Western Interior: U.S. Geological Survey Professional Paper 994-A, 29 p.

Klepper, M.R., Weeks, R.A., and Ruppel, E.T., 1957, Geology of the southern Elkhorn Mountains, Jefferson and Broadwater Counties, Montana: U.S . Geological Survey Professional Paper 292, 82 p.

Klepper, M.R., Ruppe1, E.T., Freeman, V.L., and Weeks, R.A., 1971, Geology and mineral deposits, east flank of the Elkhorn Mountains, Broadwater County, Montana: U.S. Geological Survey Professional Paper 665, 66 p.

Kunz, G.F., 1893, Precious stones, in Mineral resources of the United States, 1891: Mineral resources of the United States: U.S. Geological Survey, p. $542-544$.

McCulloch, Robin, 1992, Montana mining directory 1991: Montana Bureau of Mines and Geology Bulletin 130, 135 p.

1993, Mining and mineral developments in Montana--1992: Montana Bureau of Mines and Geology Open-File Report 262, 30 p.

Mertie, J.B., Jr., Fischer, R.P., and Hobbs, S.W., 1951, Geology of the Canyon 
Ferry quadrangle, Montana: U.S. Geological Survey Bulletin 972, 97 p.

Mosier, D.L., 1986, Descriptive model of upwelling type phosphate deposits, in Cox, D.P., and Singer, D.A., eds., Mineral deposit models: U.S. Geological Survey Bulletin 1693, p. 234.

Pardee, J.T., and Schrader, F.C., 1933, Metalliferous deposits of the greater Helena mining region, Montana: U.S. Geological Survey Bulletin 842, 318 p.

Reed, G.C., 1951, Mines and mineral deposits (except fuels), Broadwater County, Montana: U.S. Bureau of Mines Information Circular 7592, 58 p.

Reynolds, M.W., and Close, T.J., 1984, Gates of the Mountains Wilderness and additions, Montana, in Marsh, S.P., Kropschot, S.J., and Dickinson, R.G., Wilderness Mineral Potential, Assessment of Mineral-Resource Potential in U.S. Forest Service Lands Studied, 1964-1984: U.S. Geological Survey Professional Paper 1300, p. 705-708.

Robinson, G.D., McCallum, M.E., and Hays, W.H., 1969, Geologic map of the Upper Holter Lake quadrangle, Lewis and Clark County, Montana: U.S. Geological Survy Geologic Quadrangle Map GQ-840, scale 1:24,000.

Rowe, J.P., 1908, Some economic geology of Montana: University of Montana, Bulletin no. 50, Geological Series No. 3, p. 54-56.

Schmidt, R.G., Loen, J.S., Wallace, C.A., and Mehnert, H.H., 1994, Geology of the Elliston region, Powe 11 and Lewis and Clark Counties, Montana: U.S . Geological Survey Bulletin 2045, 25 p.

Sheldon, R.P., 1964, Paleolatidunal and paleogeographic distribution of phosphorite: U.S. Geological Survey Professional Paper 501-C, p. 106-113.

Swanson, R.W., 1973, Geology and phosphate deposits of the Permian rocks in central western Montana: U.S. Geological Survey Professional Paper 313-F, p. $779-833$.

West, J.M., 1959, Mineral resources (except oil and gas) of Helena-Great Falls division, Montana: U.S. Bureau of Mines Preliminary Report 127, 139 p.

Voynick, Steve, 1988, French bar sapphires: Rock and Gem, v. 18, no. 12, p. $16-27$.

Zeihen, L.G., 1987, The sapphire deposits of Montana, in Lawson, D.C., compiler, Directory of Montana mining enterprises for 1986: Montana Bureau of Mines and Geology Bulletin 126, p. 28-40. 


\section{C $\begin{array}{llllllllll}\mathbf{A} & \mathbf{P} & \mathbf{T} & \mathbf{E} & \mathbf{R} & \mathbf{J}\end{array}$ \\ PETROLEUM POTENTIAL OF THE HELENA NATIONAL FOREST}

By William J. Perry, Jr., Mark J. Pawlewicz, Ted A. Daws, Thaddeus S. Dyman and George Desborough

\section{INTRODUCTION}

The Helena National Forest (the Forest) lies primarily within southern Lewis and Clark, northeastern Powe11, northern Jefferson, northeastern and western Broadwater and northwestern Meagher Counties, and extends into the southern edge of Cascade and the northern edge of Gallatin Counties, Montana. It spans four geologic provinces (fig. B1), from north to south - (1) the Montana disturbed belt (Mudge, 1983; Perry, 1989) or simply the disturbed belt (fig. B1); (2) the Montana transverse zone (Lewis and Clark line of Reynolds, 1979, and Sears, 1995); (3) the Helena salient of the Cordilleran thrust belt; and (4) the Boulder batholith, its satellite bodies and fringing volcanic rocks along the western edge of the Helena salient. To date, only one of these provinces has produced hydrocarbons in commercial quantities, the shut-in two-we11 Knowlton gas field and adjacent three-we11 Blackleaf Canyon gas field in T. 26 N., R. 8 W. of the disturbed belt, more than 50 miles north of the northern edge of the Forest. These two small gas accumulations have produced a total of 7 BCF (billion cubic feet) of natural gas and 0.03 million barrels of condensate ( $\mathrm{J} . \mathrm{W}$. Halvorson, Montana 011 and Gas Conservation Division, 1993, written communication).

The purpose of this chapter is to qualitatively evaluate the petroleum potential of the Forest. First, the oil and gas plays recognized in the vicinity of the Forest are discussed. Second, we summarize the available drillhole data, and third, the available hydrocarbon source-rock data. Structural geology is a recurring theme throughout the chapter. The districts mentioned in this chapter are the Forest Service districts named on the Helena National Forest, Forest Visitor Map (Anonymous, 1991). This chapter discusses the geology of a region greater than the Helena National Forest because regional geologic features, hydrocarbon-bearing rocks, and structures cross National Forest boundaries.

\section{OIL AND GAS PLAYS}

Oil and gas plays are sets of known or postulated oil and gas "accumulations sharing similar geologic, geographic, and temporal properties such as source rock, migration pathway, timing, trapping mechanism, and hydrocarbon type" (Gautier, 1996). Play areas are regions over which specific play concepts are considered to be valid.

The Lincoln District of the Forest, north of T. $13 \mathrm{~N} .\left(-46^{\circ} 55^{\prime} \mathrm{N}\right)$ lies within the disturbed belt and the confirmed Imbricate Thrust Gas Play (2701) of Perry (1996, fig. J1). This gas play is based on the likelihood of undiscovered gas-bearing reservoir rocks, primarily carbonate rocks of the Mississippian Madison Group, in structural traps for hydrocarbons in antiformal stacks of thrust-bounded imbricate zones and related anticlines in the footwall of the 
Eldorado-Lewis thrust system (Perry, 1996). Such likely structural traps are inferred to be sourced by gas generated from the Upper Devonian to Lower Mississippian marine rocks and (or) Cretaceous marine shales. The northeastern margin of the Lincoln District lies within the southwestern part of the hypothetical Cone Member, Marias River Shale Oil Play (2703) of Perry (1996, fig. J1). This unconventiona1, continuous-type play is based on the oil-prone character and above average organic carbon content of the Cone Member and its proven ability to yield oil from fractures, apparently irrespective of structural relief. Source and reservoir are the same; the oil has not been expelled from its source, and conventional oil-water contacts are not anticipated (Perry, 1996).

The northeastern part of the Helena District (Hogback Mountain and Gates of the Mountains Wilderness areas) lies along the southeastern edge of the disturbed belt, abutting the Helena salient and disrupted by Tertiary dextral, oblique-slip and normal faults of the Montana transverse zone (Lorenz, 1984, and Sears, 1995). This area also lies within the southeastern margin of Play 2701 as well as Play 2707, the hypothetical Imbricate Thrust 0il Play of Perry (1996, play areas shown in fig. J1). Play 2707 is defined on the basis of both anticipated source rocks (chiefly Upper Devonian and Lower Mississippian) and reservoirs (in carbonate rocks of the Madison Group), in inferred structural traps similar to those of Play 2701 but within the oil window. Examination of potential reservoir rocks in the southern part of the area of Play 2707 revealed no residual oil in pores or fractures (Reynolds and Close, 1984). Analyses of potential hydrocarbon source rocks they collected suggest that, regardless of the structural position of the samples, these "rocks have passed through temperatures of oil and wet gas generation and have been in the temperature range of dry gas generation" (Reynolds and Close, 1984).

To the west, the main part of the Helena District lies within the batholithic province of McMannis (1965), specifically the northeastern part of the Boulder batholith and related rocks ( $f i g . B 1$ ). This area is considered unfavorable for hydrocarbons because of the intense thermal activity associated with emplacement of these igneous rocks and because of expected, related, vertical fractures that would permit the escape of hydrocarbons. To the east, the Big Belt Mountains in the eastern Helena and Townsend Districts expose primarily Middle Proterozoic formations of the lower Belt Supergroup. Based on results from the American Petrofina \#1 Manger-Skyline drillhole (no. 32 of table $\mathrm{J} 1$ and fig. J1), as well as unpublished seismic data obtained from farther south across the Big Belt Mountains, we interpret these mountains to overlie a major thrust-imbricate-cored structural culmination of Proterozoic Belt strata that have been thrust over other Belt rocks. Shepard and Precht (1989) reported values as high as 3 to 5 percent organic carbon in exposures of Proterozoic Newland Formation in Swimming Woman Canyon in the Big Snowy Mountains about 85 miles east of the northeastern boundary of the Forest. Aram (1993) suspected a Proterozoic source for some oils farther to the northeast (Cat Creek field oils of Aram, 1993). For these reasons and because of the organic-rich appearance of some of these rocks, a source-rock evaluation of these rocks was undertaken by Pawlewicz (1994, see table J3).

All but the westernmost part of the Townsend District of the Forest lies in the area of the Helena Salient Gas Play (2704) of Perry (1996). Anticlinal and thrust imbricate closures within the salient and presence of Mississippian Heath-equivalent and Lower Cretaceous source rocks, plus favorable migration pathways and timing, define the play. The central part of the play area contains 


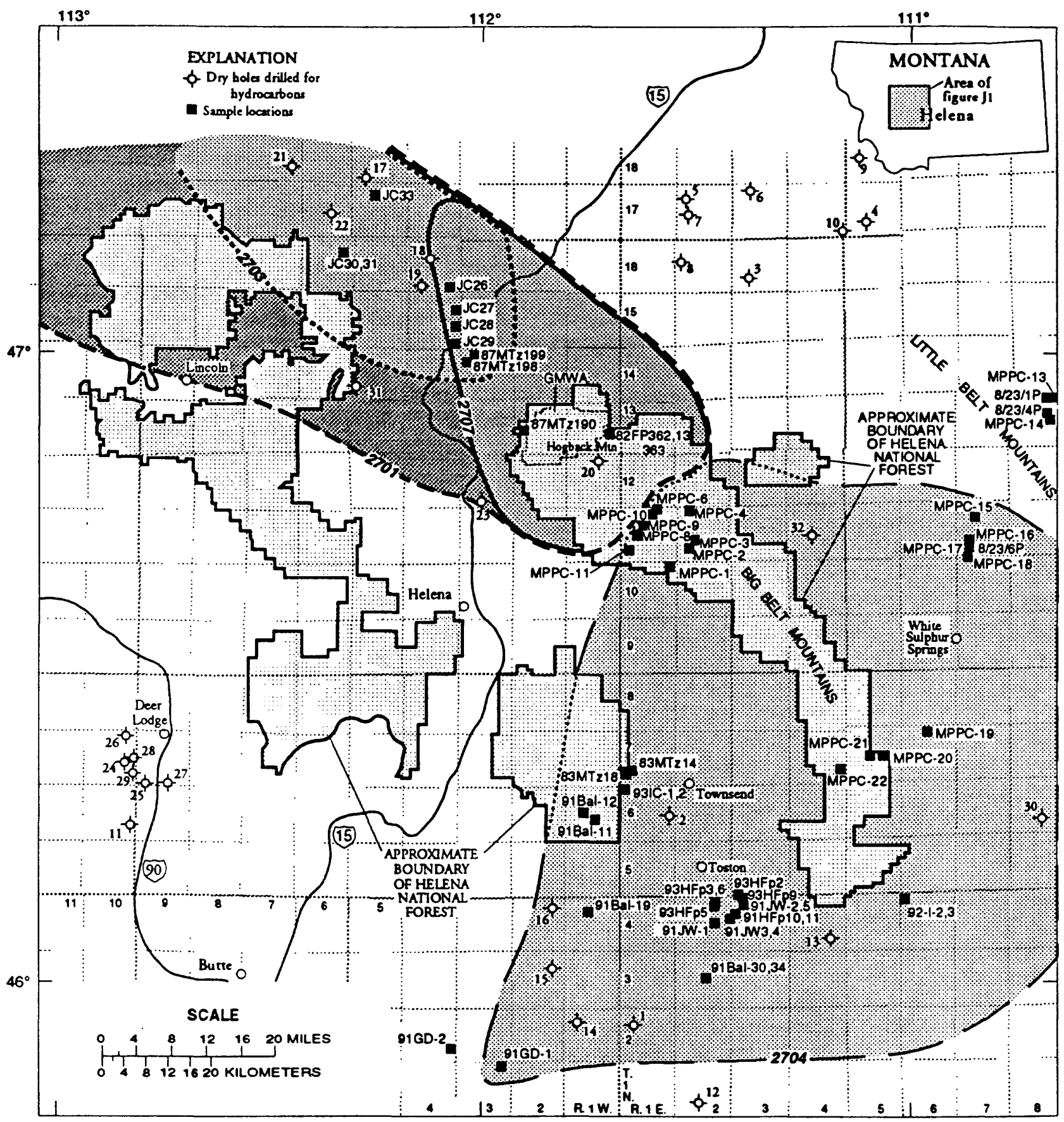

Figure J1. Index map showing Helena National Forest (light shaded areas), outlines of hydrocarbon plays (darker shaded areas) assessed by Perry (1996), dry holes drilled for hydrocarbons in the vicinity of the Forest (Table J1), sample localities (Tables J2 - J6), township-range grid, and geographic features mentioned in the text. GMWA - Gates of the Mountains Wilderness Area. Numbers (i. e., 2704) associated with each hydrocarbon play are the play numbers from Perry (1996). 
12,000 to $25,000 \mathrm{ft}$ of Middle Proterozoic sedimentary rocks unconformably overlain by about 9,000 ft of Phanerozoic sedimentary rocks, with nearly half of this sequence composed of Cretaceous strata. No exploratory holes for hydrocarbons (table J1) drilled in this play have proved productive nor have significant shows of oil or gas been reported. A deep exploratory hole (no. 16, table $\mathrm{J} 1$ and fig. J1) drilled through the Lombard thrust plate (fig. B1). in Jefferson County about 7 miles south of the western area of the Townsend District (Elkhorn Mountains region of fig. F1) was interpreted by Ballard and others (1993) to have significant hydrocarbon potential. Their interpretation of formations encountered below the Lombard thrust in this drillhole is not supported by our data, summarized in tables J3, J4, J6, figure J2 and discussed below.

\section{DRILLHOLE DATA}

Data from 32 exploratory holes drilled in the search for hydrocarbon accumulations in or near the Forest, for which encountered formation tops have been reported, are summarized in table J1. The tops given are those reported by the operators, except for the upper and lower lithic equivalents of the Lower Mississippian and Upper Devonian Bakken Formation (Macqueen and Sandberg, 1970), which are, respectively, the Cottonwood Canyon Member of the Lodgepole Formation and the basal black shale (unit 1) of the underlying Sappington Member of the Upper Devonian (Poole and others, 1992, fig. 2) Three Forks Formation. The depths and apparent thickness of these two units were picked by Perry from geophysical borehole logs. Of the 32 exploratory holes listed in table $\mathrm{Jl}$, seven were drilled west of the Forest in the Tertiary basin south and west of Deer Lodge (fig. Jl, T. 6-7 N., R. 9-10 W.); these holes targeted Eocene to Oligocene lake deposits with inferred adjacent source and reservoir rocks. Prospective parts of this basin do not underlie the Forest, nor was this exploration effort successful.

Eight of the holes were drilled in the southern part of the Helena salient (T. 1-6 N., R. 2 W. to R. 8 E.) during exploration for hydrocarbons in structural traps in Paleozoic and Mesozoic rocks. No shows of oil were reported from these eight holes in information at hand. Gas shows were reported in the Phillips Petroleum 1 Brainard-B and Amoco Production 1-R Kiff Ranch holes (respectively, nos. 13 and 30 , table J1). The former is located 3.4 miles south, and the latter is 16 miles east-northeast of the southeast margin of the Townsend District (fig. $\mathrm{J} 1$ ). Lithologies shown on the hydrocarbon well $\mathrm{log}$ (includes drilling time and description of well cuttings) of the former hole indicate a very different drilled sequence of units from that predicted by the operator (see columns 2 and 3, no. 13, table J1, for Perry's preliminary interpretation of formations encountered in this hole). Perhaps the most controversial of these eight holes drilled in the southern part of the Helena salient, the Norcen Explorer 1-11 Kimpton Ranch (no. 16, table J1 and fig. J1), was completed most recently $(4 / 01 / 91)$, controversial because the Cretaceous Colorado Group, reported to have been encountered below 7,824 ft in this hole, requires major revisions in the interpretation of Cordilleran structure and Cretaceous stratigraphy of the area. Nine large samples from this hole, kindly provided by Norcen Energy Resources Ltd., spaced roughly 1,000 ft apart, were analyzed in detail (tables J3, J4 and $\mathrm{J} 6$ ), as discussed below.

Three of the 32 holes were drilled along the northern margin of the Helena 
salient (T. 11-12 N., R. 3 W. to R. 4 E.). One of these, the Getty 0il 3-10 Federal (no. 20, table J1 and fig. J1) reveals great structural complexity and minor gas shows as well as traces of oil staining. The many lost circulation zones reported are consistent with intense fracturing inferred from the various logs available from this hole.

Six of the 32 holes were drilled in the southern part of the disturbed belt (T. 14-18 N., R. 4-7 W.), of which the Unocal 1-B30 Federal (no. 31, table J1), completed $9 / 26 / 89$ to a total depth (TD) of $17,818 \mathrm{ft}$, through the Eldorado thrust, revealed an extensive Mesozoic and Paleozoic footwall section within the oil window (table J1, Peterson and Nims, 1992). The report of low-salinity formation water in the Mississippian Madison Group in the Unocal drillhole by Peterson and Nims (1992; data on file at Montana Oil and Gas Conservation Division) is summarized in table J1. Eight additional holes were drilled within the map area outside of the Forest in the foreland northwest of the Little Belt Mountains (T. 16-18 N., R. 2-5 E., fig. J1).

\section{POTENTIAL SOURCE ROCKS FOR HYDROCARBONS}

Potential source rocks for hydrocarbons in the vicinity of the Helena National Forest include marine shales and (or) organic mudstones of Devonian, Mississippian, and Cretaceous age. Organic-rich mudstones of the Permian Phosphoria Formation are not present in the disturbed belt or in the Helena salient east of the Boulder batholith (Peterson, 1986, fig. 13). Tertiary oil shales have been identified and analyzed for lithium by Brenner-Tourtelot and others (1978) in T. $15 \mathrm{~N}$., R. 9 W., 6-8 miles northwest of Lincoln, along the northwestern edge of the Forest, but their organic matter was not analyzed. Based on analogy with a study (Curiale and others, 1988) of similar rocks in the Kishenehn basin along the western margin of Glacier Park, 40-50 miles northwest of the Forest, these 0ligocene oil shales in the Lincoln area are inferred to be thermally immature with respect to oil generation. Post-0ligocene burial has apparently been regionally insufficient for thermogenic hydrocarbon generation. Proterozoic lower Belt rocks of the Helena salient and Belt embayment farther east have also been considered potential hydrocarbon source rocks (Shepard and Precht, 1989; Aram, 1993). A preliminary evaluation of these Proterozoic rocks by Pawlewicz (1994) did not support this contention. A more extensive discussion of the potential source rocks mentioned in this paragraph follows, but first we provide a brief explanation of methods and terminology.

\section{Methods and terminology}

We use the term kerogen to designate insoluble organic material in sedimentary rocks; in contrast, bitumen is soluble in organic solvents (Tissot and Welte, 1978, p. 123). Kerogen is composed of many different types of organic matter. We use Types I, II, and III as defined by Tissott and Welte (1978, p. 147). Type I kerogen has a high $\mathrm{H} / \mathrm{C}$ (hydrogen/carbon) ratio, is primarily "derived from algal lipids or from organic matter enriched in lipids [fats], and has a high potential for oil and gas generation". Type II kerogen has a lower $\mathrm{H} / \mathrm{C}$ ratio and oil and gas potential than Type $\mathrm{I}$, "is usually related to marine organic matter deposited in a reducing environment, with medium to high sulfur 
content." Type III kerogen is mainly derived from terrestrial higher plants; H/C ratio is low, and oil-generating capacity is moderate to low; it is primarily capable of generating gas. Rock-Eval pyrolysis is the controlled heating of a rock sample under dry conditions. It is presently the most common laboratory screening technique in hydrocarbon source rock evaluation (Bordenave and others, 1993) and was conducted on 56 samples in conjunction with the present study (results summarized in Tables $\mathrm{J} 2$ and $\mathrm{J} 4$ ). Tmax is the temperature in ${ }^{\circ} \mathrm{C}$ (degrees Celsius) recorded for the maximum generation of hydrocarbons from kerogen during the artificial maturation caused by dry pyrolysis. It is a measure of thermal maturity. The high-temperature end of the oil window in source rocks occurs at a Tmax of about $460^{\circ} \mathrm{C}$ (Tissot and Welte, 1978, p. 454, fig. V.1.16). More recently, Bordenave and others (1993, fig. 2-17) showed that the oil and gas window varies slightly for different types of organic matter. Total organic carbon (TOC) is measured by combustion of the organic material of a sample at $600^{\circ} \mathrm{C}$ after Rock-Eval pyrolysis is complete. Highly mature Type III organic matter can give Rock-Eval TOC values as much as 48 percent lower than actual (Bordenave and others, 1993, table 2-4), because $600^{\circ}$ combustion does not completely oxidize such material. The $S_{1}$ peak is a measure of hydrocarbons that exist in a rock sample and "can be used as a tool to detect migrated hydrocarbons" (Bordenave and others, 1993, p. 241). The $\mathrm{S}_{2}$ peak represents hydrocarbons produced by heating of kerogen (Types I, II III). It "gives a reasonable evaluation of the current [hydrocarbon generation] potential of a rock sample"; 70-80 percent of Type I, 45-50 percent of Type II, and only 10-25 percent of Type III kerogen are transformed into hydrocarbons during pyrolysis (Bordenave and others, 1993, p. 242). Rocks with $S_{2}<4 \mathrm{mg} / \mathrm{g}$ of sample have low or no potential; $S_{2}$ between 4 and 8 indicates fair potential (Bordenave and others, 1993, p. 260). None of our samples had $S_{2}$ values as high as 2 (tables $\mathrm{J} 2$, J4). The $\mathrm{S}_{3}$ peak is a measure of carbon dioxide generated in the sample during pyrolysis of oxygen-bearing organic compounds. It is recorded below $400^{\circ} \mathrm{C}$ because of thermal decomposition of some carbonates (especially siderite, see table J6) and other poorly crystallized inorganic crystalline materials. The accuracy of this peak is low for rocks with low organic content (TOC $<0.5$ percent, especially those containing a large amount of siderite), affecting the oxygen index [OI], computed from $S_{3} / T O C$. The hydrogen index [HI], computed from $\mathrm{S}_{2} / \mathrm{TOC}$, correlates well with $\mathrm{H} / \mathrm{C}$ [atomic] ratios of kerogen. HI vs OI diagrams are used in classifying Types I, II, and III kerogen (Bordenave and others, 1993, p. 251). Obviously, false values of OI, primarily caused by low TOC, will affect such analyses. Vitrinite reflectance (table $\mathrm{J} 3$ ), is the standard reflected light technique for measurement of the thermal maturity of organic material (Tissot and Welte, 1978). It is a better measure of thermal maturity than Tmax: oil generation begins at $R_{o}$ (mean random reflectance in oil) of about 0.7 percent for Type I kerogen and about 0.6 percent for Types II and III. Cracking is sudden for type $I$ and primary hydrocarbon generation is ended by $R_{0}$ about 1 percent; most type II kerogen is also transformed by the time $R_{o}=1.0$ percent (Bordenave and others, 1993, p. 247). For Type III organic matter, transition to gas condensate generation corresponds to an $R_{\circ}$ of 1.3 percent, and dry gas generation continues above an $R_{0}$ of 1.6 percent.

Mineralogy of the samples (table J6 and figure J2) was determined using nickel-filtered $\mathrm{Cu}-\mathrm{K}_{\alpha}$ radiation at an $\mathrm{X}$-ray diffractometer scale of $2^{\circ} /$ minute. The same equipment and operating conditions were used for all analyses. Powdered samples were packed into 2 -mm-thick mounts. X-ray peak intensities reported 
were corrected for background. For each mineral, the $x$-ray diffraction maximum was used (table J6 and figure J2).

\section{Precambrian}

The Middle Proterozoic Newland Formation is 7,000 to $10,600 \mathrm{ft}$ thick in the Big Belt Mountains (Mitchell W. Reynolds, U.S. Geological Survey, 1987, written communication), at the south end of which Robinson (1967) described the upper part as a gray to yellow-brown [weathered] limy siltite, limestone, and fine quartzite. The lower member consists of medium gray siltite and limy siltite (Tysdal, Chapter B and plate 1, this report, description of map units). Outcrops sampled by Pawlewicz and Perry in the Little Belt and Big Belt Mountains consisted of dark gray to black, thin to very thin-bedded, often laminated siltite and limestone, yielding 0.06-0.63 percent TOC (table J2). Reliable Tmax values of these Newland samples ranged from 473 to $545^{\circ} \mathrm{C}$. The fine-grained facies of the possibly equivalent LaHood Formation was sampled by Desborough (samples 91GD-1 and 91GD-2, table J2) near the southern edge of the Helena salient, about 25 miles south of the Forest. These samples yielded 0.75 and 1.09 percent TOC respectively, with a reliable Tmax only on the latter of $485^{\circ} \mathrm{C}$. A11 Proterozoic samples obtained (table J2), except one from the LaHood, were remarkably low in TOC, in spite of their general dark gray to black color and our care in collecting only unweathered surface samples. XRD (X-Ray diffraction) analyses by Desborough (table J6) showed a relatively high chlorite content in many of the Newland samples. The chlorite peaks were characteristic of clinochlore IIB, indicating that these rocks had been exposed to temperatures of more than $200^{\circ} \mathrm{C}$. Dolomite to calcite peak height ratios indicated that dolomite generally predominated over calcite in the 10 Newland samples analyzed (three from the southwestern edge of the Little Belt Mountains and seven from the Big Belt Mountains).

The dolomite/calcite and chlorite/illite ratios for the Newland Formation and Cretaceous(?) samples from the Kimpton Ranch well (no. 16, table Jl, fig. J1) are nearly identical (table $\mathrm{J} 6$ ) and very different from well-documented Cretaceous samples obtained from both hanging wall and footwall rocks of the

Lombard thrust. D.J. Nichols (U.S. Geological Survey, 1993, written communication) examined unoxidized palynologic preparations derived from carefully washed drill cuttings from four depths $(8,000,10,000,11,000$, and $13,000 \mathrm{ft}$ ) from the Kimpton Ranch drillhole. He found amorphous organic matter, but no palynomorphs. A sample of the bentonite used in the drilling mud, kindly furnished by Jack Warne (Consultant, Billings, Montana), yielded a "sparse but diverse assemblage of fossil and modern palynomorphs including (among fossils) marine forms; none of these were observed in the drill cuttings samples" (D.J. Nichols, U.S. Geological Survey, 1993, written communication). E.I. Robbins (1993, U.S. Geological Survey, written communication) examined the same four preparations and found only very dark brown pellet-shaped microfossils, algal balls, and algal filaments (Robbins and others, 1985) that she considered indigenous. Modern and/or Cretaceous organic matter, probably derived from drilling mud or subsequent contamination, is much lighter in color than the organic matter deemed indigenous. Neither specialist found any trace of vascular plant remains (of the appropriate color to be indigenous), common in Cretaceous samples from this region. The samples from the Kimpton Ranch well are, therefore, all tentatively considered Middle Proterozoic. Reliable Tmax data 
(table J4) of these samples ranges from $510-592^{\circ} \mathrm{C}$, and TOC from $0.26-1.31$ ? percent, both ranges slightly higher than the surface Newland samples to which they bear a remarkable resemblance (color, texture, dolomite/calcite and chlorite/illite peak height ratios - table J6, fig. J2). The abundance of chlorite and pyrite is similar to samples collected by Desborough from the finegrained facies of the LaHood near the southern edge of the Helena salient; the chlorite (clinochlore IIB) is another link to the Middle Proterozoic suite of samples. We conclude that Proterozoic rocks in the Forest area are generally low in TOC and overmature with respect to liquid hydrocarbons and marginal with respect to gas. Organic material appears to be primarily algal, and some solid pyrobitumen is present, such that these rocks may have generated liquid hydrocarbons long ago, probably prior to Cambrian time.

\section{Upper Devonian - Lower Mississippian}

The source of most 1iquid hydrocarbons on the Sweetgrass arch to the north of the Helena salient is considered to be the Upper Devonian to Lower Mississippian Bakken Formation of southern Alberta (Dolson and others, 1993), termed Exshaw Formation by Macqueen and Sandberg (1970). Typically, the Bakken Formation consists of an upper organic-rich shale member, a medial siltstone member, and a lower organic-rich shale member. The upper Bakken-equivalent unit in the area of the Forest is the Cottonwood Canyon Member of the basal Lodgepole Formation (Sandberg and Klapper, 1967). The lower Bakken-equivalent is the basal black shale (unit 1) of the underlying Sappington Member of the Three Forks Formation (Macqueen and Sandberg (1970, fig. 4). The upper unit is 2 to $3 \mathrm{ft}$ thick where encountered (drillholes 20 and 30 , table J1) in the Helena salient but pinches out against a basal Mississippian unconformity to the north (in $T$. 27-28 N., Dolson and others, 1993, fig. 10). The black shale of the basal Sappington Member is not present in drillhole 30 (Table J1; fig. J1) in the southern Helena salient and is only locally preserved beneath an intraformational unconformity within the Sappington Member farther south (Gutschick and others, 1976). In the Getty 0il 3-10 Federal drillhole (no. 20, table J1) a major thrust encountered at a depth of 7,166 ft separates two Devonian source rock sequences: (1) The upper sequence contains a drilled interval of $15 \mathrm{ft}$ of the lower black shale (carbonaceous mudstone), $58 \mathrm{ft}$ of upper Sappington Member, and $3 \mathrm{ft}$ of Cottonwood Canyon Member. (2) The lower sequence contains a drilled interval of $6 \mathrm{ft}$ of the lower black shale, $44 \mathrm{ft}$ of upper Sappington Member, and $2 \mathrm{ft}$ of Cottonwood Canyon Member. Because the upper sequence (4,351-4,354 ft) probably dips more steeply than the parautochthonous sequence at 12,416-12,468 ft, only the relative proportion of black shale (20 percent in the upper section of Sappington Member, 12 percent in the lower) is deemed significant. Because the upper sequence is displaced eastward with respect to the lower, the carbonaceous mudstone of the basal Sappington Member appears to have originally thickened westward with respect to the entire Sappington interval. Mitchell W. Reynolds (1995, written communication) reports 5.97-15.01 percent TOC from carbonaceous mudstone of the basal Sappington Member and 5.0 percent TOC from the Cottonwood Canyon Member in surface samples collected nearby. To the north, Dolson and others (1993) report 12.9 percent TOC from the Bakken(?) Formation in the Pan American G-1 State drillhole ( $\mathrm{sec} .36$, T. $20 \mathrm{~N} ., \mathrm{R} .3 \mathrm{~W}$.$) . The upper and lower$ shales/mudstones of the Bakken-equivalent Cottonwood Canyon-Sappington sequence are considered excellent petroleum source rocks and are oil-prone. 
The Heath Formation of the Upper Mississippian Big Snowy Group is a major source for oil on the Central Montana platform northeast of the Forest (Aram, 1993). Samples of the Heath-equivalent Lombard Formation, primarily shale, provided by Jack Warne (samples 91JW-2 to 91-JW5, table J2) and others collected by Perry (93HFp10 and 93HFpl1, table J2) ranged from 0.16 to 2.89 percent TOC with reliable Tmax values generally within the oil window. These Heathequivalent carbonaceous shale/mudstone samples were all collected at or near the type section of the Lombard Formation of the Big Snowy Group (Blake, 1959, SW 1/4 section $7, T .4 \mathrm{~N} ., \mathrm{R} .4 \mathrm{E}$.) in the footwall of the Lombard thrust plate, about 5 miles south of the Forest area of the Big Belt Mountains. These rocks are gasprone (Perry, 1996, discussion of play 2704) and are absent farther west, on the hanging wall of the Lombard thrust as well as northwest of the Gates of the Mountains Wilderness area. Source-rock evaluation of the Heath Formation in the northeastern part of the Helena salient is discussed by Longden and others (1988), who concluded that only dry gas would be present in the subsurface near their sampling locality, consistent with their reported $R_{0}$ values of 3.7-4.7. Forrest G. Poole (U.S. Geological Survey, 1986, written communication) collected two Heath samples in T. $13 \mathrm{~N}$., R. $01 \mathrm{~W}$. (table J6) which had the highest (4.804.86 percent) TOC reported from the Heath in the vicinity of the Forest. These samples yielded $T$ max values of 504 to $519^{\circ} \mathrm{C}$ (approximately equivalent to an $R_{0}$ of 1.7 to 2.0 percent) in the gas generating range and were not exposed to the intense heating illustrated by the very high $R_{0}$ of the samples of Longden and others (1988) collected farther east.

If the Lombard thrust was encountered in the Kimpton Ranch drillhole (no. 16, table J1) discussed above, then the footwall cutoff (western limit in the footwall) of these Mississippian rocks is east of this drillhole. The "black shales" encountered in this drillhole (Kimpton Ranch samples discussed above, generally highly pyritic and dolomitic) are clearly not Heath equivalents (see table J6 and fig. J2). Therefore, the likelihood of the Heath-equivalent rocks sourcing liquid hydrocarbons in the close vicinity of the central and southern Forest is considered unlikely; although gas generation is likely to have occurred. The Heath Formation may have also yielded dry gas in the subsurface in the vicinity of $T .13 \mathrm{~N} ., \mathrm{R} .01 \mathrm{~W}$. in the northeastern segment of the Helena District.

\section{Cretaceous}

The Cretaceous sequence is the thickest and most complex body of rocks in the vicinity of the Forest. The nature of the primarily siliciclastic Cretaceous sequence has been recently summarized by Dyman and others (1994). Marine tongues, principally represented by the Thermopolis Shale (Flood Member of the Blackleaf Formation), Mowry Shale, Belle Fourche Shale, Carlile Shale, Cody Shale, Marias River Shale, and Bearpaw Shale extend westward and interfinger with the Cretaceous nonmarine sequence. The total thickness of the Blackleaf Formation through Cody Shale in the hanging wall block west of Townsend (fig. J1) is about 2,000 $\mathrm{ft}$ (Dyman and others, 1996). This interval contains about 600-700 cumulative ft of black shale intervals (R.G. Tysdal and T.S. Dyman, U.S. Geological Survey, unpub. data, 1995). The Thermopolis Shale is the most organic 
rich of these shales sampled to date in the footwall of the Lombard thrust (0.791.25 percent TOC, samples $92-\mathrm{I}-2$ and $92-\mathrm{I}-3$, table J2), where it is about $160 \mathrm{ft}$ thick. These rocks are lean in organic carbon and the organic matter is considered gas-prone. The correlative Flood Member of the Blackleaf Formation on the hanging wall of the Lombard thrust (0.11-0.71 percent TOC, samples 93ICl and 93IC2, table J2) is very lean where sampled on the eastern flank of the Boulder batholith complex. The $R_{0}$ values obtained from these samples, ranging from 4.2-4.4 (table J3), indicate exposure to high paleotemperatures.

The source rock potential of Cretaceous rocks in the sleeping Giant Wilderness area (directly north of the Gates of the Mountains Wilderness) has recently been discussed by Tysdal and others (1991). This area appears to be close to the southern limit of the Upper Cretaceous Marias River Shale of the disturbed belt. Sample 87MTz198 (table J5) yielded 1.38 percent TOC with a Tmax within the oil window. The other two Cretaceous samples from the Sleeping Giant area (87MTz190 and $87 \mathrm{MTz} 199$, table J5) were from the Flood Member of the Blackleaf and yielded 1.01 and 1.44 percent TOC respectively; the latter yielded a reliable Tmax well within the oil window.

North of the Forest, Clayton and others (1983) provided organic geochemical analyses of a number of Cretaceous samples; those relevant to the present study are summarized in table J5. The Cone Member of the Marias River Formation, the only oil-prone Cretaceous source rock discovered to date in western Montana, apparently is not found south of T. $16 \mathrm{~N}$. (Clayton and others, 1983; also W.A. Cobban, U.S. Geological Survey, 1988, oral communication). Both the Blackleaf and Marias River Shales are considered source rocks for natural gas in play 2701 (Perry, 1996), which includes the northern part of the Forest as shown on figure J1. Cretaceous shales are also considered a source for biogenic gas and for oil in Cretaceous reservoirs in the adjacent central Montana Province of Dyman (1996).

\section{CONCLUSIONS}

In conclusion, only the Lincoln District, northeastern (Gates of the Mountains Wilderness) part of the Helena District, and southern edge of the Townsend District of the Forest are considered to have economically recoverable hydrocarbon potential. The first area lies within the southwestern part of the Imbricate Thrust Gas Play (2701) of Perry (1996). The second area lies within both Play 2701 and the hypothetical Imbricate Thrust 0il Play (2707) of Perry (1996). The presence in or near both areas of normal faults, oblique slip faults, and extensive vertical fracturing associated with the Montana transverse zone reduce the probability of hydrocarbon retention in thrust-related reservoirs. The report of low-salinity formation water in the Mississippian Madison Group in the Unocal 1-B30 Federal drillhole (no. 31, table J1) by Peterson and Nims (1992) also appears to reduce the likelihood of undiscovered economically recoverable hydrocarbons beneath the northern part of the Forest, as the Madison Group is the principal potential reservoir rock for hydrocarbons in this part of the Montana thrust belt.

The third area, the southern edge of the Townsend District, east of Toston (Fig. J1), lies within the Helena Salient Gas Play (2704) of Perry (1996). Drillholes to the south and east have abundant gas shows. However, virtually the entire rock sequence plunges south along the southern edge of Townsend District, 
and all prospective reservoir rocks are exposed just to the north, along the southern margin of the Big Belt Mountains. Therefore, this must be considered an area of low gas potential within the play. Remaining portions of the Forest within the Helena Salient Gas Play are considered to have no to very low hydrocarbon potential based on our interpretation of the available source rock, paleogeothermal regime, drillhole, and structural data.

\section{REFERENCES CITED}

Anonymous, 1991, Forest Visitor Map, Helena National Forest, Montana: U.S Department of Agriculture, Forest Service, scale 1:126,720.

Aram, R.B., 1993, Source rock study of central Montana, in Hunter, L.D.V., ed., Energy and mineral resources of central Montana: Montana Geological Society 1993 Field Conference Guidebook, p. 179-193.

Ballard, D.W., Burton, B.R., Lageson, D.R., Warne, J.R., 1993, Drilling experiences at Devils Fence Anticline, Montana: Baseline data for future activity in a promising overthrust province, in Hunter, L.D.V., ed., Energy and mineral resources of central Montana: Montana Geological Society 1993 Field Conference Guidebook, p. 159-168.

Blake, O.D., 1959, Big Snowy stratigraphy in the area adjacent to the Rocky Mountain front [Montana]: Billings Geological Society 10th Annual Field Conference Guidebook, 1959, p. 64-68.

Bordenave, M.L., Espitalié, J., Leplat, P., Oudin, J.L., and Vandenbroucke, M., 1993, Screening techniques for source rock, evaluation, in Bordenave, M.L., ed., Applied petroleum geochemistry: Editions Technip., 27 rue Ginoux 75015, Paris, p. 217-278.

Brenner-Tourtelot, E.F., Meier, A.L., and Curtis, C.A., 1978, Lithium in rocks from the Lincoln, Helena and Townsend areas, Montana: U.S. Geological Survey Open-File Report 78-430, 27 p.

Clayton, J., Mudge, M.R., Lubeck, Sr.C., and Daws, T.A., 1983, Hydrocarbon source rock evaluation of the disturbed belt, northwestern Montana, in Powers, R. B., ed., Geologic studies of the Cordilleran thrust belt 1982: Rocky Mountain Association of Geologists, v. 2, p. 817-830.

Curiale, J.A., Sperry, S.W., and Senftle, J.T., 1988, Regional source rock potential of lacustrine oligocene Kishenehn Formation, northwestern Montana: American Association of Petroleum Geologists Bulletin, v. 72, p. 1437-1449.

Dolson, J., Piombino, J., Franklin, M., and Harwood, R., 1993, Devonian oil in Mississippian and Mesozoic reservoirs--Unconformity controls on migration and accumulation, Sweetgrass arch, Montana: The Mountain Geologist, v. 30, no. 4, p. $125-146$.

Dyman, T.S., 1996, North-Central Montana Province (028), in Gautier, D.L., Dolton, G.L., Takahashi, K.I., and Varnes, K.L., eds., 1995 National Assessment of United States oil and gas resources - Results, methodology, and supporting data: U.S. Geological Survey Digital Data Series 30, release 2 [CD-ROM].

Dyman, T.S., Cobban, W.A., Fox, J.E., Hammond, R.H., Nichols, D.J., Perry, W.J., Jr., Porter, K.W., Rice, D.D., Setterholm, D.R., Shurr, G.W., Tysdal, R.G., Haley, J.C., and Campen, E.B., 1994, Cretaceous rocks from southwestern Montana to southwestern Minnesota, northern Rocky Mountains, and Great Plains region, in Shurr, G.W., Ludvigson, G.A., and Hammond, 
R.H., eds., Perspectives on the eastern margin of the Cretaceous Western Interior Basin: Geological Society of America Special Paper 287, p. 5-28. Dyman, T.S., Tysdal, R.G., Porter, K.W., and Thompson, G.A., 1995, Measured sections and correlations of mid-Cretaceous rocks near the Lombard thrust of the Helena salient in southwestern Montana: U.S. Geological Survey Open-File Report OF-95-841, 11 p.

Gautier, D.L., 1996, Introduction, in Gautier, D.L., Dolton, G.L., Takahashi, K.I., and Varnes, K.L., eds., 1995 National Assessment of United States Oil and Gas resources -Results, methodology, and supporting data: U.S. Geological Survey Digital Data Series 30, release 2 [CD-ROM].

Gutschick, R.C., McLane, M., and Rodrigues, J., 1976, Summary of Late DevonianEarly Mississippian biostratigraphic framework in western Montana, in Guidebook, The Tobacco Root Geological Society 1976 Field Conference: Montana Bureau of Mines and Geology Special Publication 73, p. 91-124.

Longden, M.R., Banowsky, B.R., and Woodward, L.A., 1988, Source rock evaluation of the Heath Formation (Mississippian) in Montana thrust belt: 011 and Gas Journal [May 9, 1988 Issue], p. 60-63.

Lorenz, J.C., 1984, The function of the Lewis and Clark fault system during the Laramide orogeny, in McBane, J.D., and Garrison, P.B., eds., Northwest Montana and adjacent Canada: Montana Geological Society 1984 Field Conference and Symposium Guidebook, p. 221-230.

Macqueen, R.W., and Sandberg, C.A., 1970, Stratigraphy, age, and interregional correlation of the Exshaw Formation, Alberta Rocky Mountains: Bulletin of Canadian Petroleum Geology, v. 18, p. 32-66.

McMannis, W.J., 1965, Resume of depositional and structural history of western Montana: American Association of Petroleum Geologists Bulletin, v. 49, p. $1801-1823$.

Mudge, M.R., 1983, A resume of the structural geology of the northern disturbed belt, northwestern Montana, in Powers, R.B., editor-in-chief, Geologic studies of the Cordilleran thrust belt - 1982: Rocky Mountain Association of Geologists, v. 1, p. 91-122.

Pawlewicz, Mark, 1994, Organic petrographic and Rock-Eval pyrolysis analyses of Proterozoic Belt Supergroup rocks, west-centra1 Montana: U.S. Geological Survey Open-File Report 94-155, 11 p.

Perry, W.J., Jr., 1989, A review of the geology and petroleum potential of the Montana thrust belt: U.S. Geological Survey Open-File Report 88-450C, 28 p.

Perry, W.J., Jr., 1996, Montana Thrust Be1t Province (027), in Gautier, D.L., Dolton, G.L., Takahashi, K.I., and Varnes, K.L., eds., 1995 National Assessment of United States oil and gas resources - Results, methodology, and supporting data: U.S. Geological Survey Digital Data Series 30, release 2 [CD-ROM].

Peterson, J.A., 1986, General stratigraphy and regional paleotectonics of the western Montana overthrust belt, in Peterson, J.A., 1986, Paleotectonics and sedimentation in the Rocky Mountain Region, United States: American Association of Petroleum Geologists Memoir 41, p. 57-86.

Peterson, C.H., and Nims, R.C., 1992, How Montana's deepest hole cut Mississippian carbonates underneath Precambrian Belt: $0 i 1$ and Gas Journal, v. 90 , no. 31 , p. $67-70$.

Poole, F.G., Stewart, J.H., Palmer, A.R., Sandberg, C.A., Madrid, R.J., Ross, R.J., Jr., Hintze, L.F., Miller, M.M., and Wrucke, C.T., 1992, Latest Precambrian to latest Devonian time; Development of a continental margin, 
in Burchfiel, B.C., Lipman, P.W., and Zoback, M.L., eds., The Cordilleran orogen: Geological Society of America, The Geology of North America, v. G-3, p. $9-56$.

Reynolds, M.W., 1979, Character and extent of basin-range faulting, western Montana and east-central Idaho: Rocky Mountain Association of Geologists-Utah Geological Society 1979 Basin and Range Symposium, p. 185-193.

Reynolds, M.W., and Close, T.J., 1984, Gates of the Mountains Wilderness and additions, Montana, in Marsh, S.P., Kropschot, S.J., and Dickinson, R.G., eds., Wilderness mineral potential - Assessment of mineral-resource potential in U.S. Forest Service lands studied 1964-1984: U.S. Geological Survey Professional Paper 1300, p. 705-708.

Robbins, E.I., Porter, K.G., and Haberyan, K.A., 1985, Pellet microfossils Possible evidence for metazoan life in early Proterozoic time: Proceedings, National Academy of Sciences, U.S.A., v. 82, p. 5809-5813.

Robinson, G. D., 1967, Geologic map of the Toston quadrangle, southwestern Montana: U.S. Geological Survey Miscellaneous Geologic Investigations Map I-486, scale $1: 24,000$.

Sandberg, C.A., and Klapper, G., 1967, Stratigraphy, age, and paleotectonic significance of the Cottonwood Canyon Member of the Madison Limestone in Wyoming and Montana: U.S. Geological Survey Bulletin 1271-B, 70 p.

Sears, J.W., 1995, Tectonic significance of the Lewis and Clark line in the Sevier orogenic belt, Idaho and Montana [abs]: Geological Society of America Abstracts with Programs, v. 27, no. 4, p. 54.

Shepard, W., and Precht, W., 1989, Trip 3 Road Log - Waulsortian mounds (Mississippian Lodgepole $\mathrm{Fm}$. ) and their relation to rifting, central Montana, in French, D.E., and Grabb, R.F., Geologic resources of Montana: Montana Geological Society 1989 Field Conference Guidebook, Montana Centennial Edition, v. 2, road logs, p. 450-460.

Tissot, B.P., and Welte, D.H., 1978, Petroleum formation and occurrence, A new approach to oil and gas exploration: Berlin, Springer-Verlag, 538 p.

Tysdal, R.G., Reynolds, M.W., Carlson, R.R., Kleinkopf, M.D., Rowan, L.C., and Peters, T.J., 1991, Mineral Resources of the Sleeping Giant Wilderness Study Area, Lewis and Clark County, Montana: U.S. Geological Survey Bulletin 1724, $31 \mathrm{p}$. 


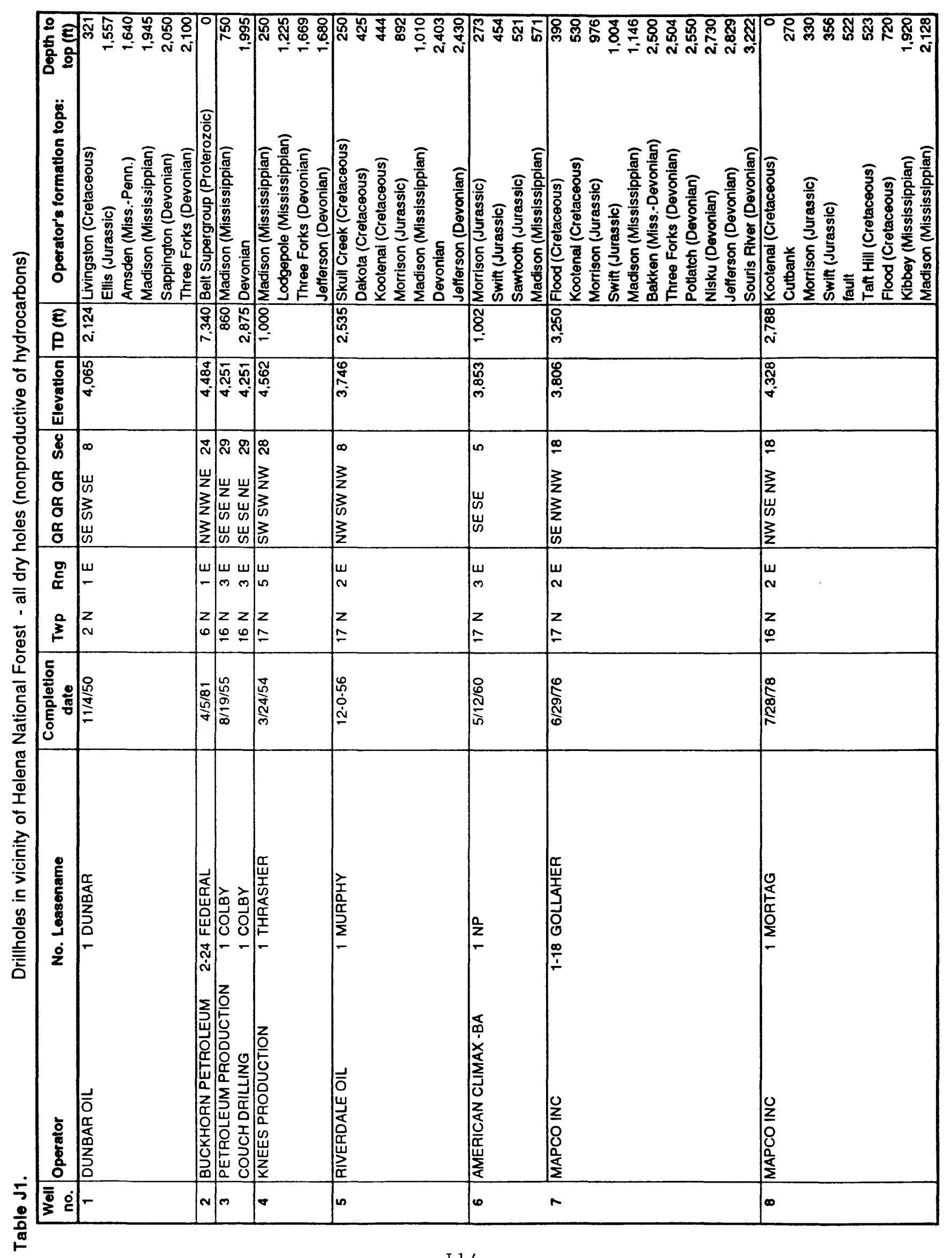




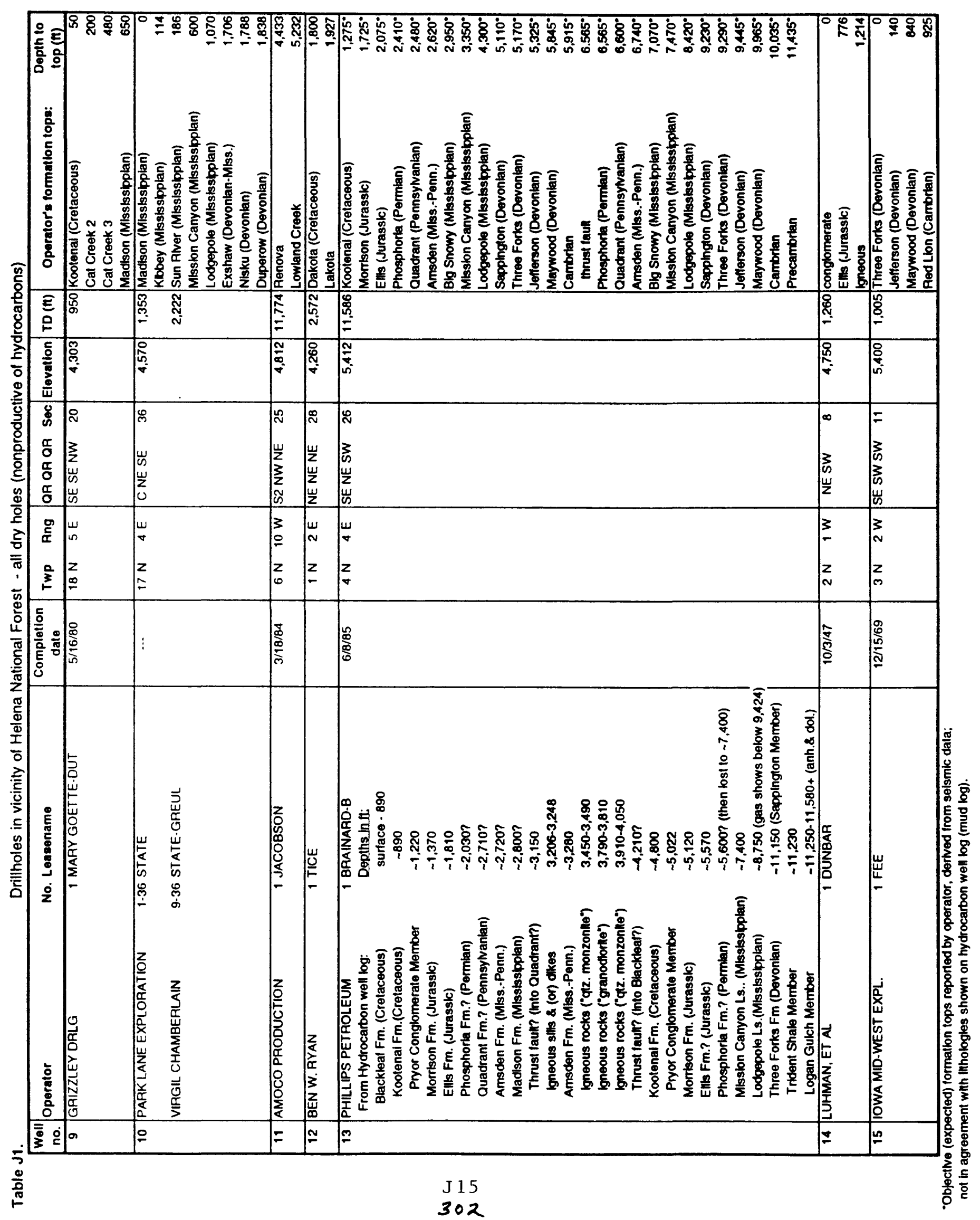




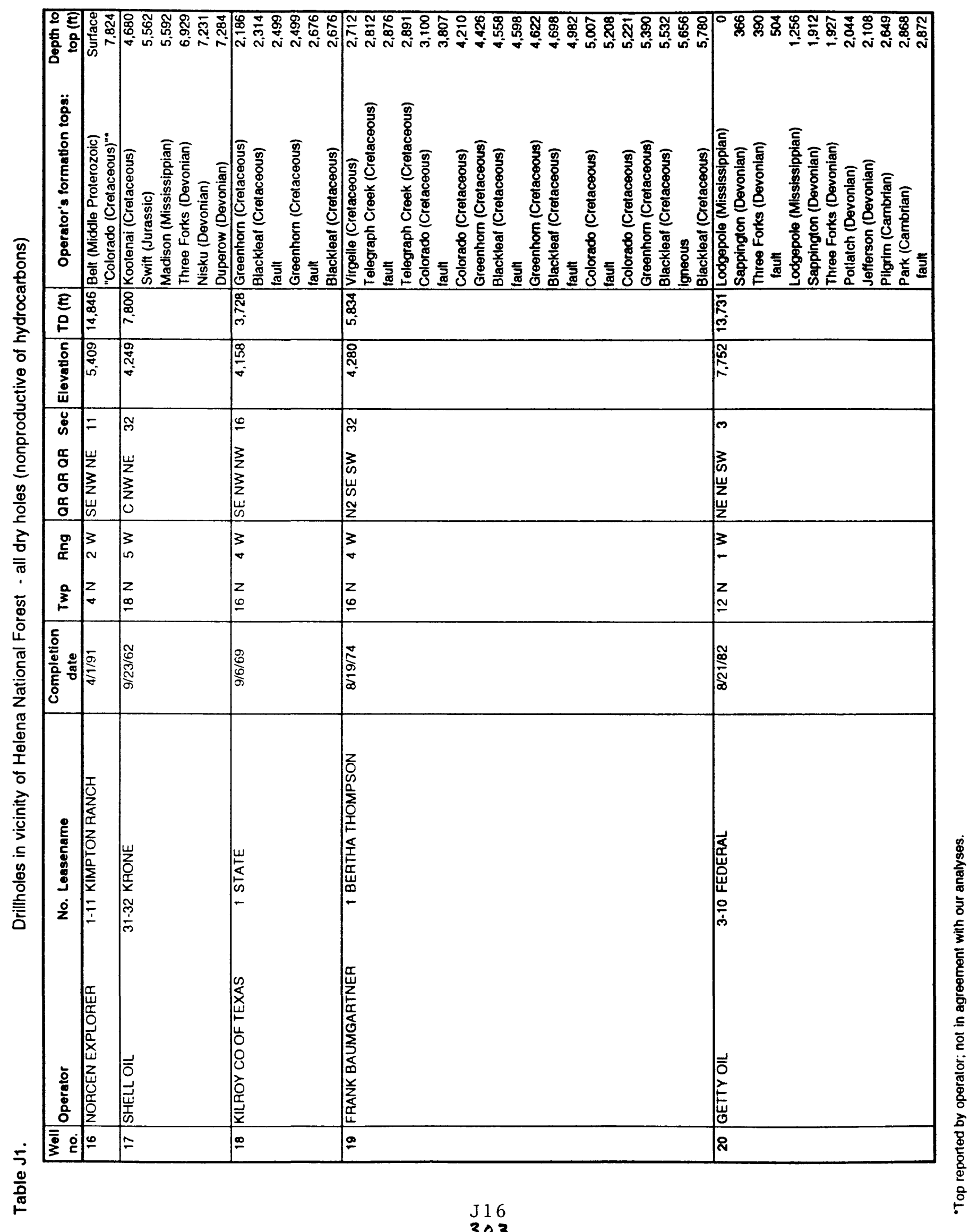




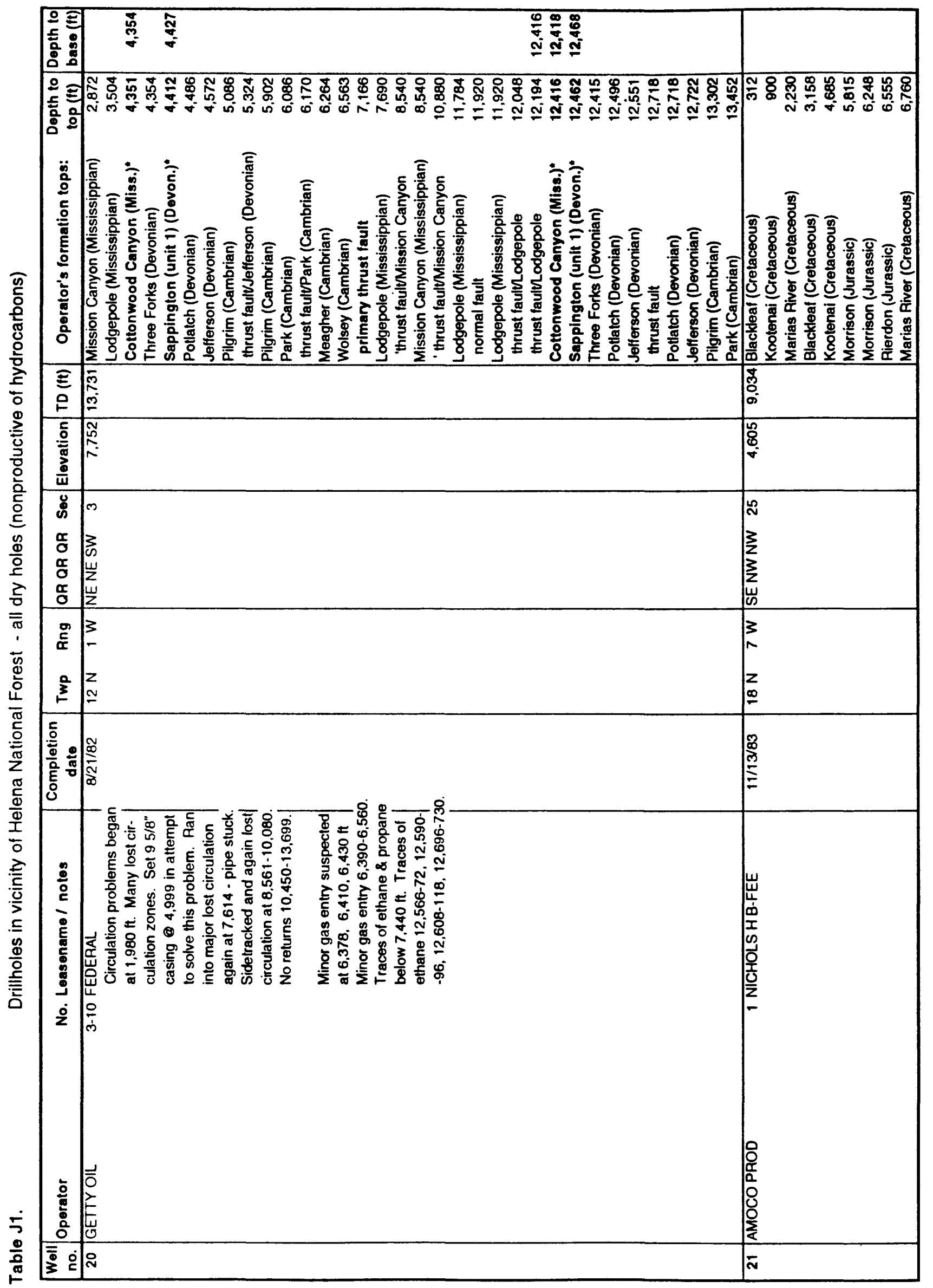

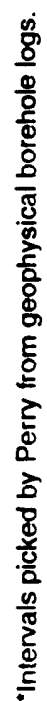




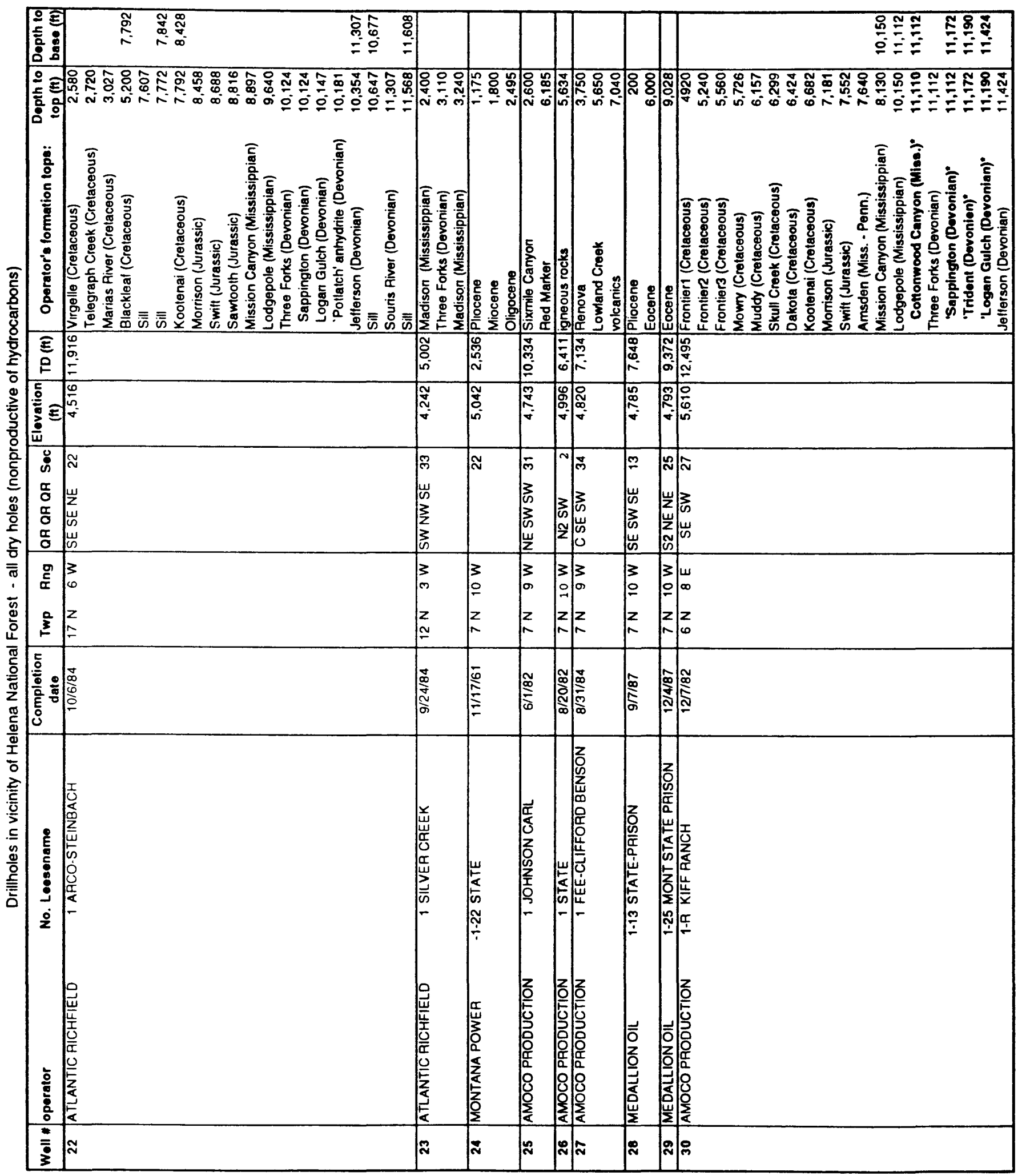

$\frac{5}{5}$ 


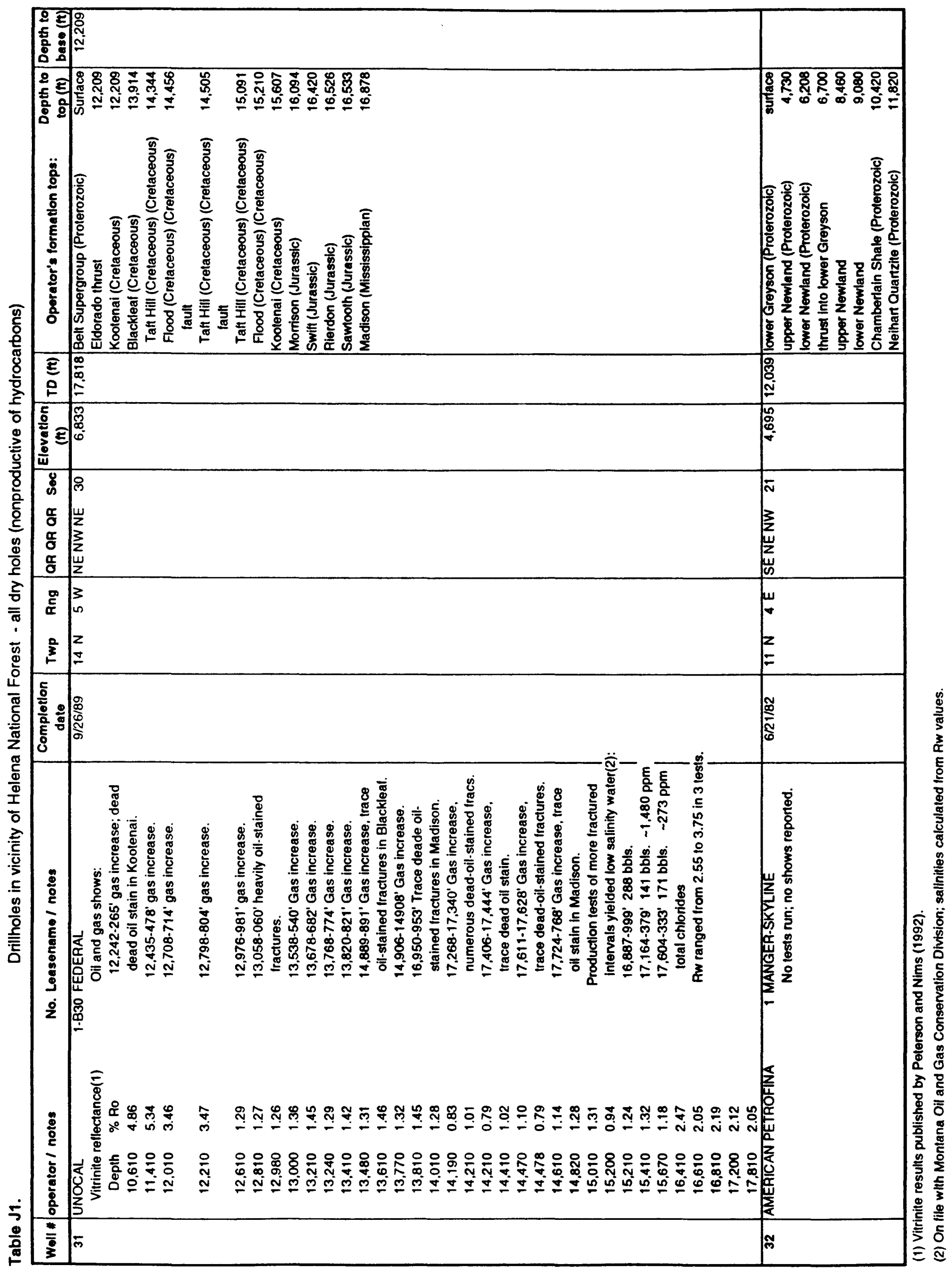




\begin{tabular}{|c|c|c|c|c|c|}
\hline $\bar{\sigma}$ & 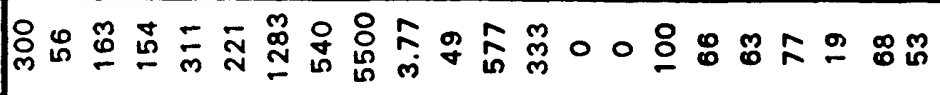 & F号 & $\stackrel{\infty}{\sim}$ & \multicolumn{2}{|c|}{$\overline{0}$ ถิธ } \\
\hline$*$ & 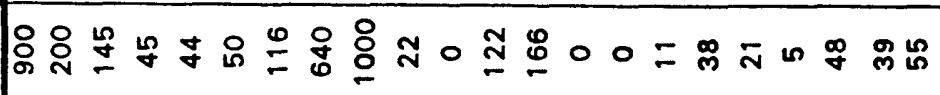 & $\stackrel{\sim}{\sim}$ & 芯吕 & \multicolumn{2}{|c|}{ 임요 } \\
\hline ๙ู & 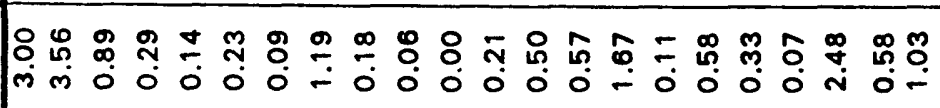 & 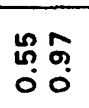 & & \multicolumn{2}{|c|}{ 융 $=\frac{m}{0}$} \\
\hline$\frac{n}{\alpha}$ & 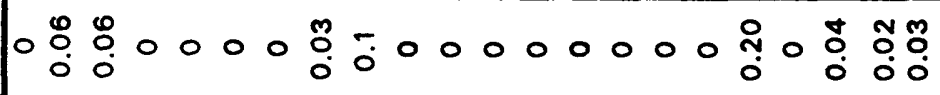 & $\circ \frac{1}{0}$ & 菓 & \multicolumn{2}{|c|}{ 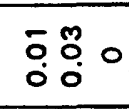 } \\
\hline$\tilde{\tilde{B}}$ & 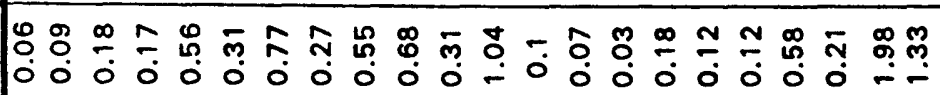 & $\stackrel{\leftrightarrow}{\circ} \stackrel{\mathscr{m}}{0}$ & $\begin{array}{ll}0 \\
\stackrel{0}{0} \\
0 \\
0\end{array}$ & \multicolumn{2}{|c|}{ 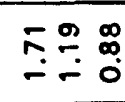 } \\
\hline స్ & 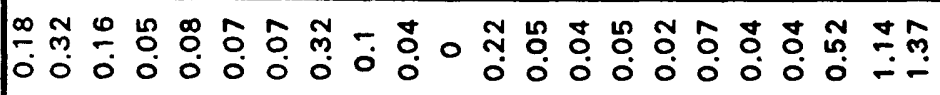 & సׁุ̆ & 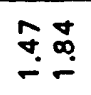 & \multicolumn{2}{|c|}{ 品 } \\
\hline$\overline{\text { is }}$ & 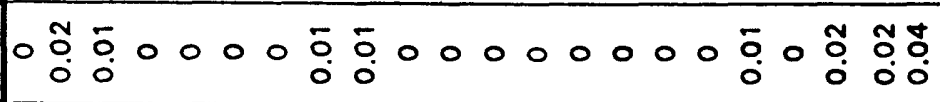 & $\circ \stackrel{0}{0}$ & 萬 & \multicolumn{2}{|c|}{ 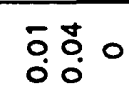 } \\
\hline $\begin{array}{lll}0 & 0 \\
0 & \xi \\
1 & \xi\end{array}$ & 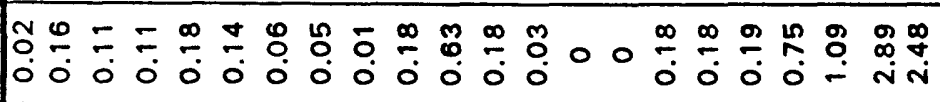 & 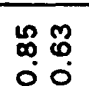 & $\hat{\sim} \mathfrak{\sim}$ & \multicolumn{2}{|c|}{$\stackrel{\infty}{\sim} \underset{\sim}{\sim} \underset{\sim}{\infty}$} \\
\hline 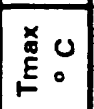 & 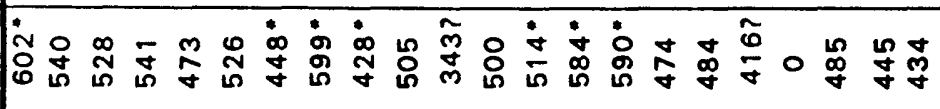 & 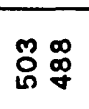 & $\stackrel{2}{\circ}$ & \multicolumn{2}{|c|}{$\int \frac{a}{d}$} \\
\hline $\begin{array}{l}0 \\
0 \\
0 \\
0\end{array}$ & 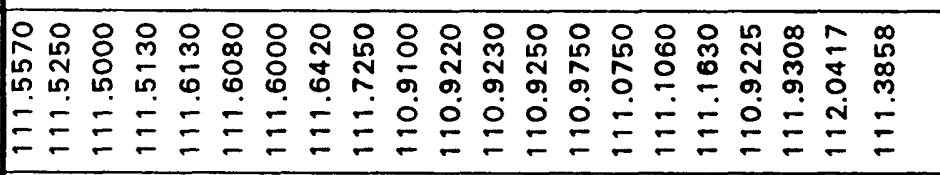 & $\begin{array}{l}\Xi \\
\vdots \\
\end{array}$ & $\begin{array}{l}\equiv \\
\vdots \\
\vdots\end{array}$ & 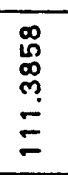 & \\
\hline $\mid$ & 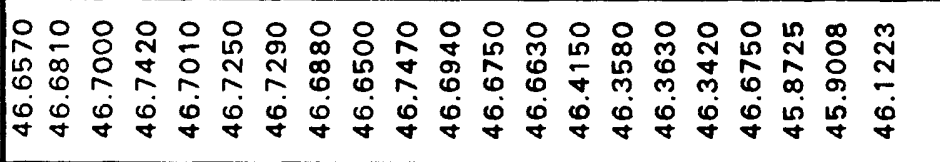 & 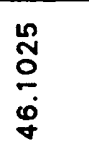 & 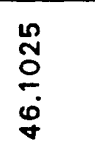 & $\underset{\stackrel{m}{N}}{\stackrel{m}{\dot{q}}}$ & \\
\hline $\begin{array}{l}\stackrel{a}{\sigma} \\
\stackrel{\alpha}{\sigma}\end{array}$ & 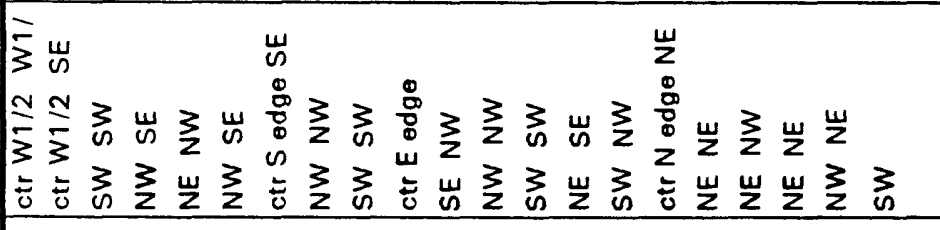 & $\begin{array}{l}\mathrm{u} \\
\mathrm{w} \\
\end{array}$ & $\begin{array}{l}\mathrm{u} \\
\mathrm{u} \\
\mathrm{Z}\end{array}$ & 3 & $\begin{array}{l}3 \\
w \\
w \\
\end{array}$ \\
\hline 岃 & 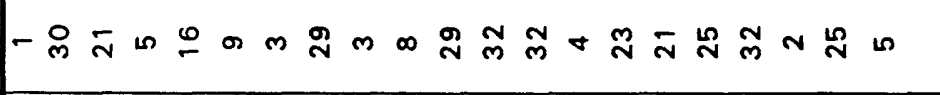 & $m$ & $m$ & n & N \\
\hline$\underset{凶}{\ddot{J}}$ & ن & $\stackrel{\text { บे }}{\text { ù }}$ & $\underset{\sim}{w}$ & $\underset{m}{\dot{m}}$ & \\
\hline$\sum_{k}^{a}$ & 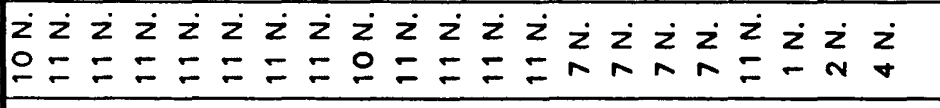 & i & $\dot{ }$ & $\dot{z}$ & \\
\hline 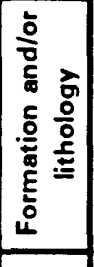 & 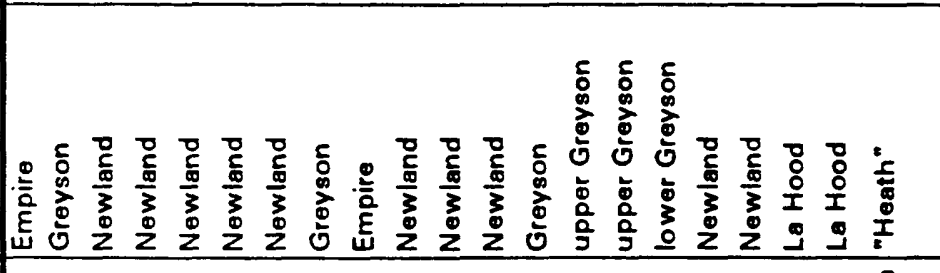 & $\frac{1}{\underbrace{\prime}_{0}}$ & $\frac{1}{2}$ & 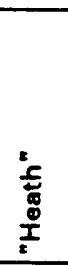 & $\begin{array}{l}\dot{0} \\
\vdots \\
0 \\
0 \\
0 \\
0 \\
0 \\
\end{array}$ \\
\hline 这 & 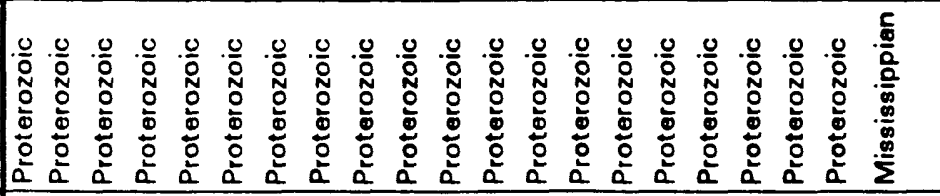 & 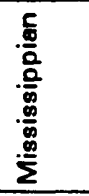 & 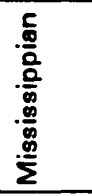 & 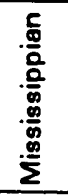 & 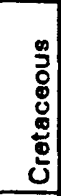 \\
\hline $\begin{array}{l}0 \\
\frac{0}{0} \\
\frac{0}{5} \\
\infty\end{array}$ & 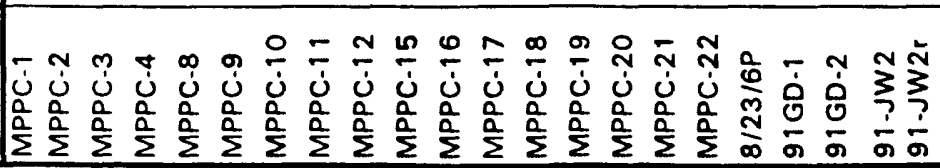 & 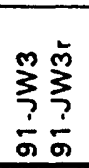 & $\frac{1}{3} \frac{1}{3} \frac{1}{9}$ & & \\
\hline
\end{tabular}




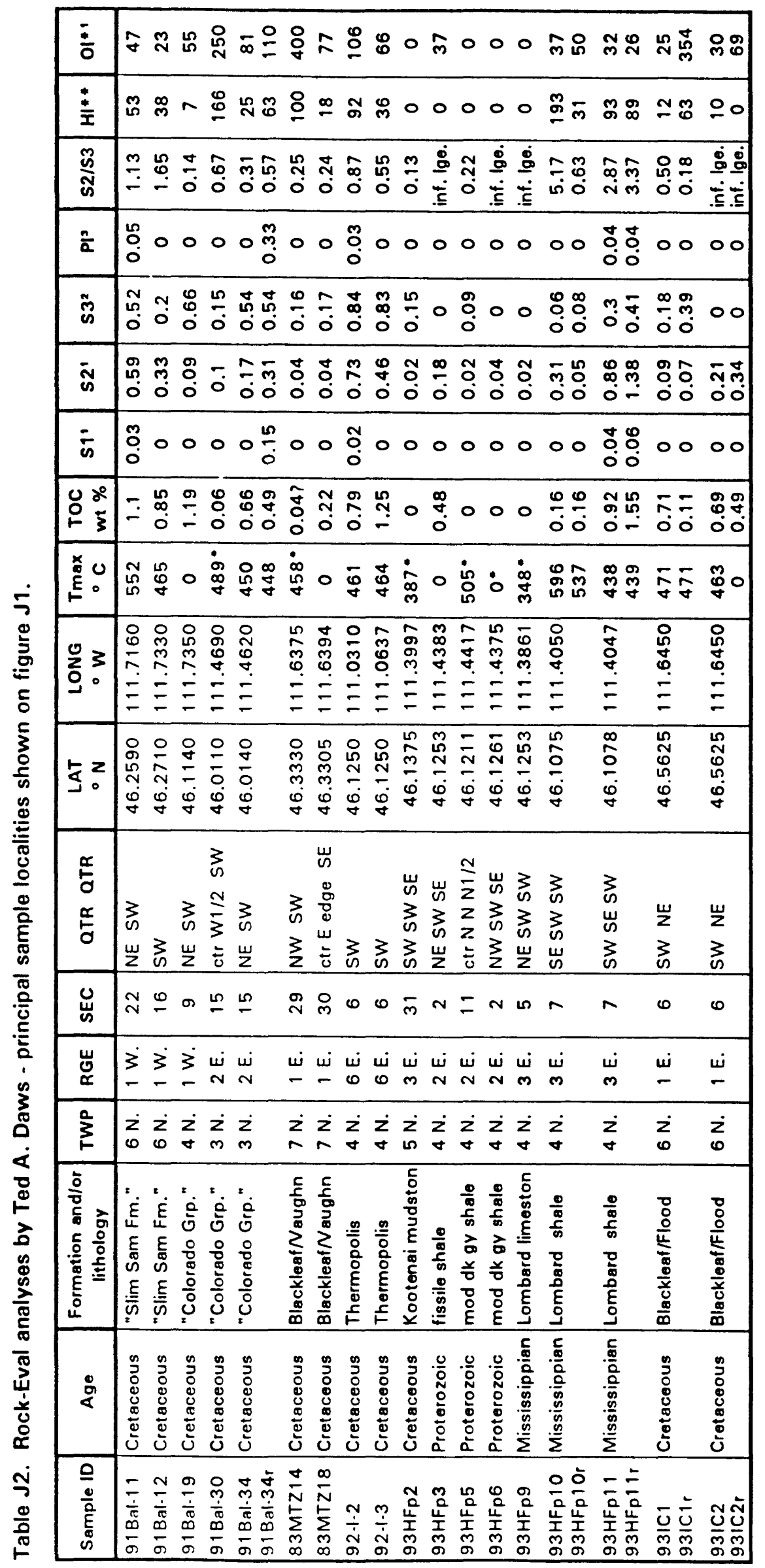




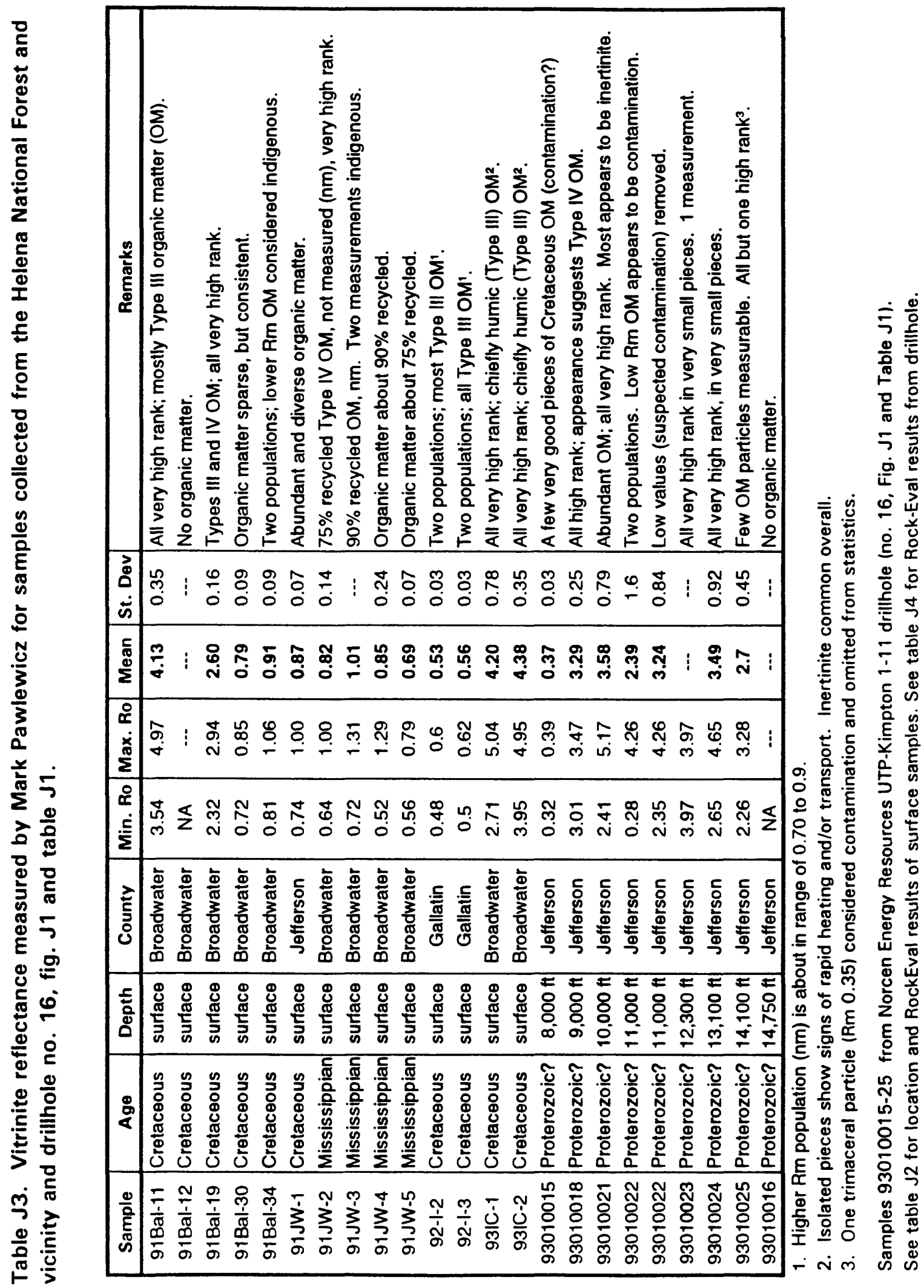




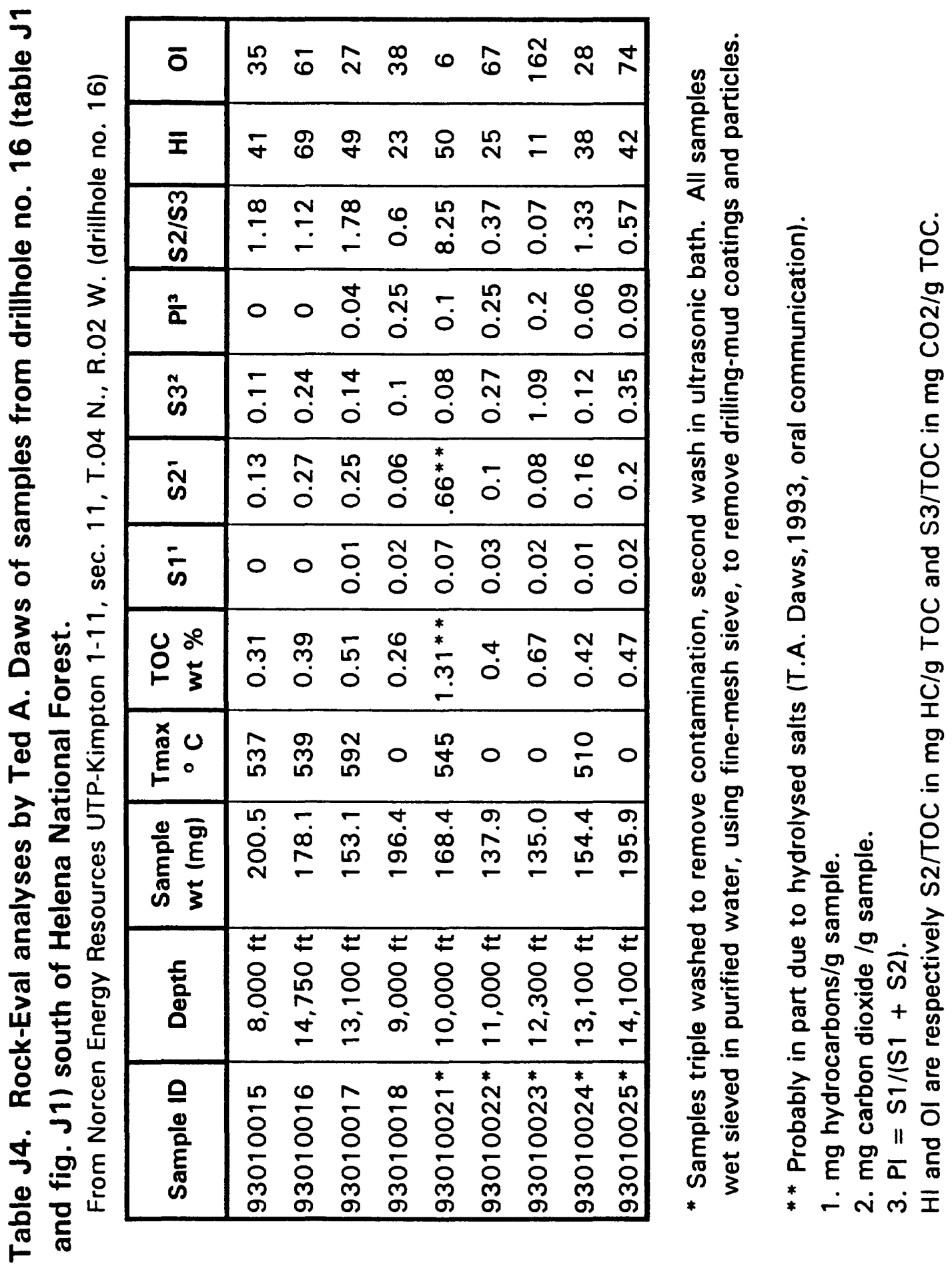




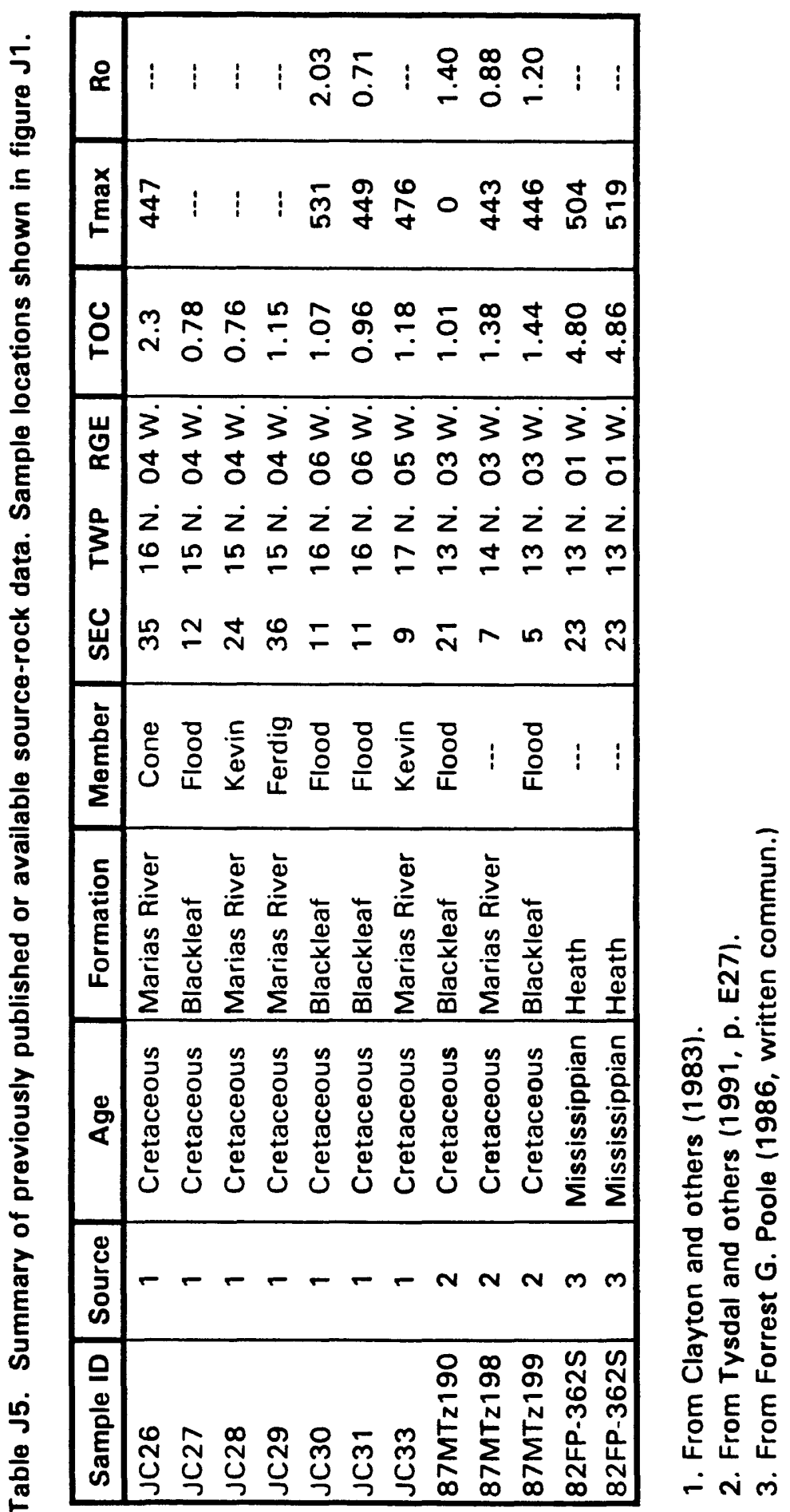


Table J6. XRD relative peak heights (analyses by George Desborough) from samples collected in the Helena National Forest and vicinity. Sample bations shown on figure $\mathrm{J1}$.

Cretaceous samples from Helena Salient

\begin{tabular}{|c|c|c|c|c|c|c|c|c|c|c|}
\hline Sampla & chlorite & nite & pyitte & elderite & calcite & dolomite & plag-eper & K-spar & Dol/Cal & chlorfilite \\
\hline 83MTZ14 & 9 & 4 & 0 & $\overline{0}$ & 27 & 0 & 30 & 0 & 0 & 2.25 \\
\hline 83MTZ18 & 6 & 3 & 0 & 0 & 0 & 0 & 60 & 0 & 0 & 2.00 \\
\hline $92-1-3$ & 0 & 0 & 0 & 0 & 0 & 0 & 0 & 12 & 0 & 0 \\
\hline $92-1-2$ & 0 & 0 & 0 & 0 & 0 & 0 & 0 & 8 & 0 & 0 \\
\hline 91Bat-11 & 5 & 7 & 0 & 0 & 0 & 0 & 4 & 0 & 0 & 0.71 \\
\hline $91 \mathrm{Bat}-12$ & 9 & 51 & 0 & 10 & 0 & 0 & 14 & 7 & 0 & 0.18 \\
\hline 91Bat-19 & 0 & 0 & 0 & 0 & 0 & 0 & 0 & 0 & 0 & 0 \\
\hline 91Bar-30 & $\operatorname{tr}$ & tr & 0 & 0 & 0 & 0 & 9 & 0 & 0 & 0 \\
\hline 91Bal-34 & 0 & 0 & 0 & 0 & 0 & 32 & 7 & 0 & inf large & 0 \\
\hline $93 I C-1$ & 3 & 4 & 0 & 3 & 0 & 0 & 5 & 0 & 0 & 0.75 \\
\hline $93 \mid C-2$ & 0 & 4 & 0 & 2 & 4 & 0 & 9 & 0 & 0 & 0 \\
\hline Average & 2.89 & 6.64 & 0 & 1.36 & 2.82 & 2.81 & 12.65 & 2.45 & 1.03 & 0.44 \\
\hline
\end{tabular}

Cretaceous samples from Lombard thrust plate (vitrinite where obtained yielded $>1.5$ mean Ro)

\begin{tabular}{|c|c|c|c|c|c|c|c|c|c|c|}
\hline Ssmple & chlonte & Ulite & pyrite & siderite & calcite & dolomite & plag-spar & K-epar & Dol/Cal & chlornillte \\
\hline $83 M T 214$ & 9 & 4 & 0 & 0 & 27 & 0 & $\overline{30}$ & 0 & 0 & 2.25 \\
\hline 83MTZ18 & 6 & 3 & 0 & 0 & o & 0 & 60 & 0 & 0 & 2.00 \\
\hline 91Bal-11 & 5 & 7 & 0 & 0 & 0 & 0 & 4 & 0 & 0 & 0.71 \\
\hline 91Bat-12 & 9 & 51 & 0 & 10 & o & 0 & 14 & 7 & 0 & 0.18 \\
\hline 91Bat-19 & 0 & 0 & 0 & 0 & 0 & 0 & 0 & 0 & 0 & 0 \\
\hline $931 \mathrm{C}-1$ & 3 & 4 & 0 & 3 & 0 & 0 & 5 & 0 & 0 & 0.75 \\
\hline $931 \mathrm{C}-2$ & 0 & 4 & 0 & 2 & 4 & 0 & 9 & 0 & 0 & 0 \\
\hline Average & 4.57 & 10.43 & 0 & 2.14 & 4.43 & 0 & 17.43 & 1.00 & 0 & 0.44 \\
\hline
\end{tabular}

Mississippian samples from Helena Salient

\begin{tabular}{|c|c|c|c|c|c|c|c|c|c|c|}
\hline Sample & chlorite & ullite & pyite & siderite & calcite & dolomite & plag-spar & K-opar & Dol/Cal & chlor/litite \\
\hline $91 \mathrm{JW}-2$ & 0 & 0 & 0 & 0 & 110 & 0 & 0 & 4 & 0 & 0 \\
$91 \mathrm{JW}-3$ & 0 & 9 & 9 & 9 & 4 & 0 & 0 & 0 & 0 & 0 \\
$91 \mathrm{JW}-5$ & 0 & 0 & 0 & 0 & 110 & 0 & 0 & 5 & 0 & 0 \\
\hline Average & 0 & 3 & 3 & 3 & 74.67 & 0 & 0 & 3 & 0 & 0 \\
Ratio of averages \\
\hline
\end{tabular}

Greyson Formation (Proterozoic) samples from Lombard thrust plate

\begin{tabular}{|c|c|c|c|c|c|c|c|c|c|c|}
\hline Semple & chlorite & illite & pyrite & siderite & calcite & dolomite & pleg-epar & K-opar & Dol/Cal & chlor/lite \\
\hline 93HFp3 & 4 & 12 & 0 & 0 & 0 & 0 & 12 & 0 & 0 & 0.33 \\
93HFp6 & 0 & 10 & 0 & 0 & 0 & 0 & 17 & 0 & 0 & 0 \\
\hline Average & 2 & 11 & 0 & 0 & 0 & 0 & 14.5 & 0 & 0 & 0.18 \\
Ratio of averages &
\end{tabular}

Newland Formation (Proterozoic) samples from Helena salient

\begin{tabular}{|l|c|c|c|c|c|c|c|c|c|c|}
\hline Sample & chlorite & Illite & pyrite & siderte & calcite & dolomite & plag-opar & K-opar & Dol/Cal & chlor/llite \\
\hline MPPC-3 & 9 & 4 & 0 & 5 & 11 & 57 & 5 & 7 & 5.18 & 2.25 \\
MPPC-4 & 12 & 5 & 0 & 0 & 0 & 72 & 5 & 0 & inf lorge & 2.40 \\
MPPC-6 & 22 & 8 & 3 & 6 & 0 & 14 & 7 & 7 & inf large & 2.75 \\
MPPC-8 & 8 & 3 & 0 & 0 & 7 & 100 & 3 & 4 & 14.29 & 2.67 \\
MPPC-9 & 19 & 8 & 0 & 4 & 0 & 54 & 6 & 5 & inf large & 2.38 \\
MPPC-10 & 6 & 0 & 0 & 0 & 100 & 13 & 0 & 0 & 0.13 & inf large \\
MPPC-16 & 7 & 4 & 6 & 0 & 0 & 83 & 0 & 0 & inf large & 1.75 \\
MPPC-17 & 7 & 6 & 5 & 5 & 74 & 14 & 0 & 0 & 0.19 & 1.17 \\
MPPC-22 & 10 & 6 & 5 & 5 & 0 & 51 & 6 & 8 & inf large & 1.67 \\
Perry-6P & 8 & 7 & 0 & 4 & 16 & 7 & 8 & 8 & 0.44 & 1.14 \\
\hline Average & 10.8 & 5.1 & 1.9 & 2.9 & 20.8 & 46.5 & 4 & 3.9 & 2.24 & 2.12 \\
\hline
\end{tabular}

Samples from Norcen Energy Resources UTP-Kimpton 1-11 borehole \#16, table J1 and Figure J1 Llthology lo predominately very dark gray dolomitic siltite and argllite.

\begin{tabular}{|l|c|c|c|c|c|c|c|c|c|c|}
\hline Sample & chlorite & illite & pyrite & slderite & celcite & dolomite & plag-epar & K-opar & Dol/Cal & chlornllite \\
\hline $8000 \mathrm{KR}$ & 10 & 14 & 9 & 4 & 15 & 43 & 5 & 4 & 2.87 & 0.71 \\
$9000 \mathrm{KR}$ & 12 & 5 & 9 & 0 & 4 & 37 & 12 & 0 & 9.25 & 2.40 \\
$1000 \mathrm{KR}$ & 29 & 13 & 13 & 0 & 0 & 9 & 12 & 0 & inf large & 2.23 \\
$1100 \mathrm{KR}$ & 33 & 9 & 0 & 0 & 6 & 8 & 22 & 4 & 1.33 & 3.67 \\
$12300 \mathrm{KR}$ & 35 & 12 & 0 & 0 & 21 & 0 & 10 & 7 & 0 & 2.92 \\
$13100 \mathrm{KR}$ & 27 & 11 & 0 & 0 & 0 & 4 & 12 & 8 & inf large & 2.45 \\
$14100 \mathrm{KR}$ & 35 & 12 & 0 & 0 & 0 & 0 & 10 & 7 & 0 & 2.92 \\
$14750 \mathrm{KR}$ & 20 & 7 & 0 & 0 & 0 & 6 & 9 & 7 & inf large & 2.86 \\
\hline Average & 25.86 & 10.86 & 4.43 & 0.57 & 6.57 & 14.43 & 11.86 & 4.29 & 2.20 & 2.38 \\
Ratio of averages & \\
\hline
\end{tabular}




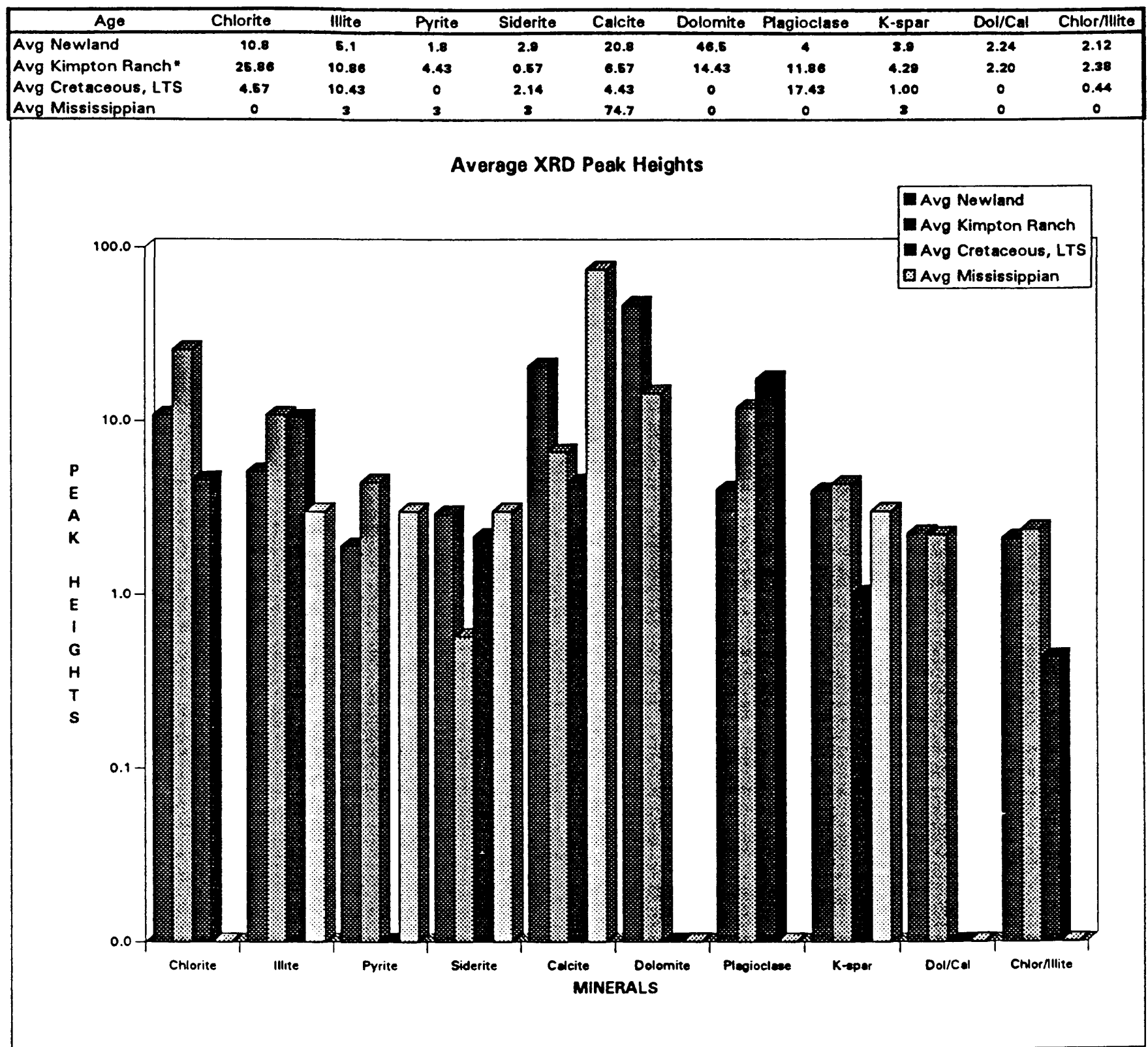

"Kimpton Ranch borehole samples are predominately very dark gray dolomitic siltite and argillite, probably Newland Fm.

Figure J2. Graphic representation of data shown in table J6. XRD relative peak heights were analysed and measured by George Desborough. "Avg Kimpton Ranch" represents average values of samples from Norcen Energy Resources UTP-Kimpton 1-11 borehole \#16, table J1 and figure J1; "Avg Cretaceous, LTS" represents average values of Cretaceous samples from the Lombard thrust sheet. 


\section{COAL RESOURCES OF THE HELEMA MATIONAL FOREST}

By C.W. Holmes

\section{GEOLOGY}

An examination was made for coal within the Helena National Forest because significant coal deposits are present to the northeast in the Great FallsLewistown coal field and to the south in the Lombard coal field (fig. K1). In addition, "coal" was reported (Whipple and others, 1987) in Tertiary lake deposits in area enclosed by the northwestern part of the Forest. Because rocks similar in age and character to those of the coal-bearing strata of the coal fields are present within the Forest, a two week survey was made over the most likely host areas in the Forest.

The Great Falls-Lewistown coal field, northeast of the Big Belt Mountains, contains discontinuous coal beds 3-6 ft thick that commonly grade into carbonaceous shales. The workable seams have relatively low ash content $(<10$ percent) with an average of 4.4 percent sulfur. A bed analyzed for sulfur and sulfur isotopes revealed a chemistry of a typical Lower Cretaceous/Upper Jurassic coal $\left(\delta^{34} S-10\right.$ to -20$)$.

The Lombard coal field, south of the Big Belt Mountains, is only about 6 square miles in area (fig. K1) and contains bituminous coal that has a high cooking capacity. The field has been neglected because its coal has a high ash and sulfur content--ash $=30$ percent, sulfur 8.2 percent. Coal seams 6 ft thick were previously mined but are tectonically distorted.

Observed rocks that are nearest in composition to coal include a carbonaceous shale northwest of Radersburg, in the southwestern part of the Elkhorn Mountains (fig. K1). This high-carbon shale (more than 2 percent total organic carbon) is similar to the carbonaceous shale in the Great Falls-Lewistown coal field and there is a possibility that extensive drilling and exploration could reveal some coal. However, this carbonaceous shale is associated with volcanic rocks and any coal present should be similar in character to the Lombard coal. No Tertiary coal was found during this survey. Near the northwestern part of the Forest, lake "deposits" that have a high carbonaceous appearance were found to be a soil horizon. The landowners in the region were surveyed and no one had ever seen rock resembling coal in the area.

In conclusion, concealed coal may lie beneath parts of the Forest and extensive drilling would be required to find it. Such deposits would be discontinuous and, for the most part, uneconomical.

\section{REFERENCE CITED}

Whipple, J.W., Mudge, M.R., and Earhart, R.L., 1987, Geologic map of the Rogers Pass area, Lewis and Clark County, Montana: U.S. Geological Survey Miscellaneous Investigations Series Map I-1642, scale 1:48,000. 


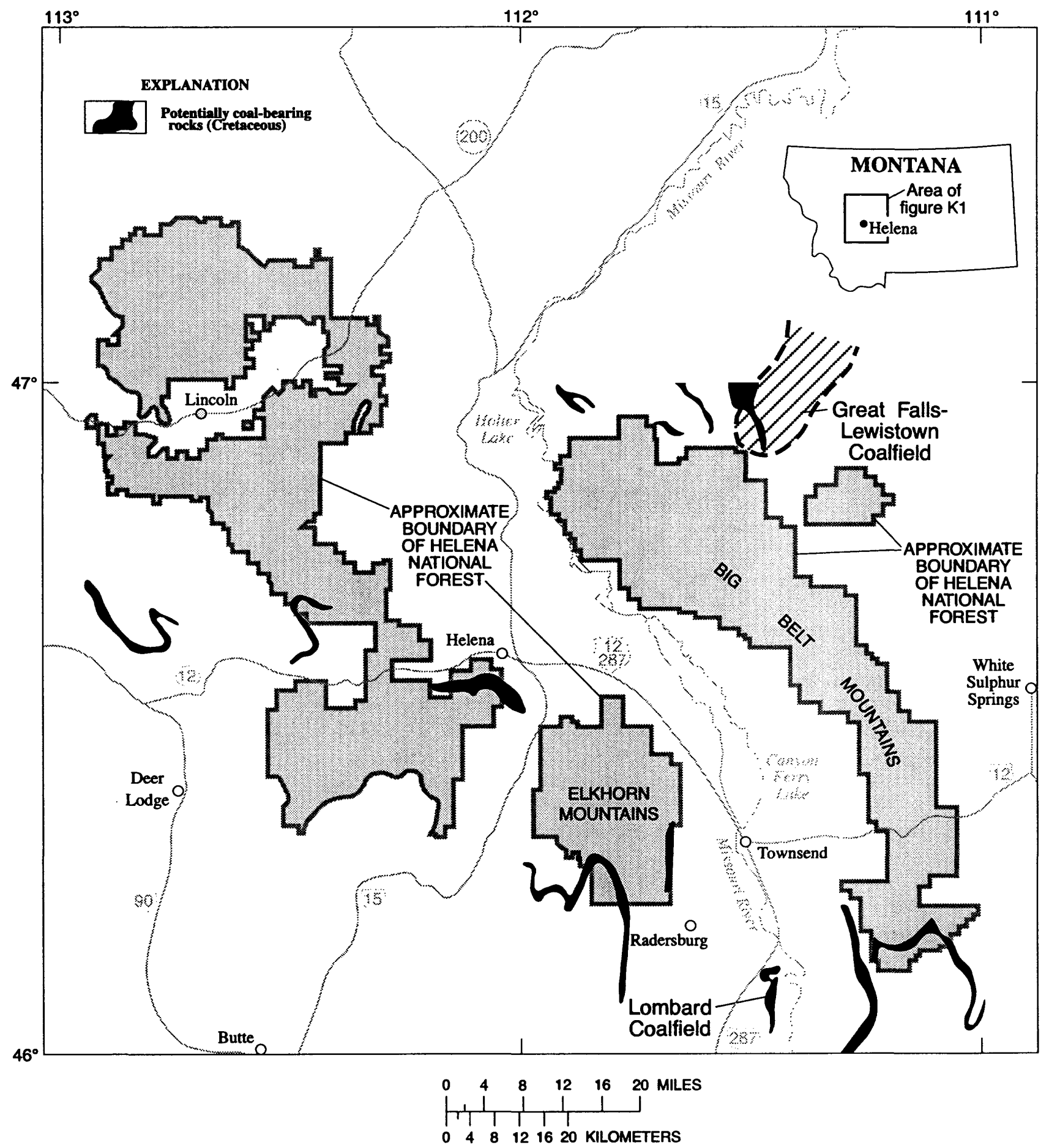

Figure K1. Map showing coal fields and potentially coal-bearing rocks in vicinity of the Helena National Forest. 


\section{A P P E Y D I $\mathbf{X}$}

TABLE OF CONTENTS

Digital data products

Chapters

Plates

Programs

References cited

Resource/reserve classification

Geologic time chart

\section{DIGITAL DATA PRODUCTS}

Many of the maps that appear on the plates of this report, and many of the figures that appear in the text, are available in digital form (CD-ROM disk) in the companion U.S. Geological Survey Open-File Report 96-683-B (Green and Tysdal, 1996). This appendix provides a listing of the digital products and programs ${ }^{1}$ that were used in their compilation. The data contained on the CD-ROM are designed to augment and enhance this hard copy Open-File Report by improving clarity of maps and figures through the use of color, and by providing finer detail to some of the black-and-white maps and figures. Some figures, particularly those of Chapter $C$ in which computer-generated pixels depict gradients of changing geophysical characteristics, are poorly displayed by the shades of gray on the paper version. On the computer, these gradations are shown more clearly and in greater detail when presented in color.

\section{Chapters}

\section{Chapter $\underline{A}$}

Adobe PDF and PostScript formats

Figure A1. Index map of Helena Naitonal Forest

\section{Chapter $\underline{B}$}

Adobe PDF and PostScript formats

Figure Bl. Map of major geologic features in western Montana

\section{Chapter $\underline{\mathrm{C}}$}

Adobe PDF and PostScript formats

Figure C1. Merged aeromagnetic anomaly map

Figure C2. Index map of aeromagnetic surveys

Figure C3. Bouguer gravity anomaly map

Figure C4. Terrace-magnetization map from aeromagnetic data

Figure C5. Terrace-density map of residual Bouguer gravity

Figure C6. Potassium aeroradioactivity map

Figure $C 7$. Uranium aeroradioactivity map

Figure C8. Thorium aeroradioactivity map

Figure C9. Uranium:Thorium aeroradioactivity map

Figure C10. Potassium:Thorium aeroradioactivity map 


\section{Chapter $\underline{D}$}

Adobe PDF and Hewlett-Packard HPGL2 formats

Figure D1. Map of geochemical study area

Figure D2. RASS outcrop sample sites

Figure D3. RASS and NURE stream-sediment samples sites

DBF format

Figure D5. NURE stream-sediment samples sites

Original geochemical data ( 4 data sets)

\section{Chapter $\underline{\mathrm{E}}$}

Microsoft EXCEL format

Table El. Location and descriptions of mines and prospects in Forest

\section{Chapter $F$}

Adobe PDF and PostScript formats

Figure F1. Map showing regions, subregions, and minding districts/areas

Figure F2. Map of the Elkhorn Mountains mining districts

Figure F3. Map of the Elkhorn Mountains, satellitic plutons

Figure F4. Table of sample sites

\section{Chapter $\underline{G}$}

Adobe PDF and PostScript formats

Figure G1. Simulated porphyry copper deposits

Figure G2. Simulated skarn gold deposits, not Elkhorn Mountains

Figure G3. Simulated skarn gold deposits, Elkhorn Mountains

Figure G4. Simulated polymetallic replacement deposits

Figure G5. Simulated Climax molybdenite deposits

Figure G6. Simulated hot-spring gold deposits

Figure G7. Simulated epithermal quartz-adularia vein deposits

Figure G8. Arsenic and antimony content of mineralized samples

Table G1. Grade-tonnage model

Table G3. Summarizes simulated metal

\section{Chapter J}

Adobe PDF and PostScripts formats

Figure J1. Map showing drill holes, sample sites, and petroleum plays

Chapter $\underline{\mathrm{K}}$

Adobe PDF and PostScript formats

Figure K1. Map showing coal fields and potentially coal-bearing rocks

\section{Plates}

ARC/INFO

Hewlett-Packard HPGL2, and Adobe PDF and PostScript formats

Map A. Geologic map of Helena National Forest

Map C. Map showing formations mainly composed of limestone and (or) dolomite

Map D. Map showing streams with reported placer operations

Map E. Map showing sample locations of placers sampled for analysis of gold 
Map K. Map showing permissive and favorable tracts for porphyry coppermolybdenum posits

Map L. Map showing permissive and favorable tracts for gold and tungsten skarn deposits

Map M. Map showing permissive tract for polymetallic replacement deposits

Map N. Map showing permissive tract for Climax molybdenum deposits

Map 0. Map showing permissive tract for York-type exhalative(?) gold deposits and Cretaceous gold-bearing vein deposits

Map P. Map showing permissive tract for hot-spring gold-silver deposits and quartz-adularia deposits

Map Q. Map showing permissive tract for sedimentary exhalative zinclead deposits

Map R. Map showing permissive and favorable tracts for redbed sedimenthosted copper deposits

Map showing terrane permissive and (or) favorable for gold deposits

Map showing drainage net of streams

Map showing Forest boundary

\section{EARTHVISION}

Hewlett-Packard HPGL2 and Adobe Acrobat formats

Map F. Map showing lead in stream sediments

Map G. Map showing gold in stream sediments

Map I. Geochemical/geophysical model for lead

Map J. Geochemical/geophysical model for gold

GSMAP

Boundaries of mining districts/areas, used in Maps B, D, E

\section{GSPOST}

Locations of mines and prospects, used on Map B

Locations of placer mines and prospects, used on Map D

Locations of sites of placer gold sampled for analysis, used on Map $E$

\section{Programs ${ }^{1}$}

Adobe, Acrobat, and PostScript are trademarks of Adobe Systems, Inc. ARC/INFO is a trademark of Environmental Systems Research Institute. EarthVision is a trademark of Dynamic Graphics, Inc. HPGL2 is a trademark of the Hewlett-Packard Company. IBM is a trademark of the International Business Machines Corporation. Microsoft is a trademark of the Microsoft Corporation.

Other trademarks and service marks are the trademarks and service marks of their respective companies.

GSMAP and GSPOST are programs written by Selner and Taylor (1993), published by the U.S. Geological Survey 


\section{REFERENCES CITED}

Green, G.R., and Tysdal, R.G., 1996, Digital maps and figures on CD-ROM for mineral and energy resource assessment of the Helena National Forest, west-central Montana: U.S. Geological Survey Open-File Report 96-683-B, 1 CD-ROM disk.

Selner, G.I., and Taylor, R.B., 1993, System 9. GSMAP, and other programs for the IBM PC and compatible microcomputers, to assist workers in the earth sciences: U.S. Geological Survey Open-File Report 93-511, 363 p., 2 disks.

\footnotetext{
${ }^{1}$ Any use of trade, product, or firm names is for descriptive purposes only and does not imply endorsement by the USGS. Although these data have been used by the USGS and have been successfully imported into data base programs, no warranty, expressed or implied, is made by the USGS as to how successfully or accurately the data can be imported into any specific application software running on any specific hardware platform.
} 


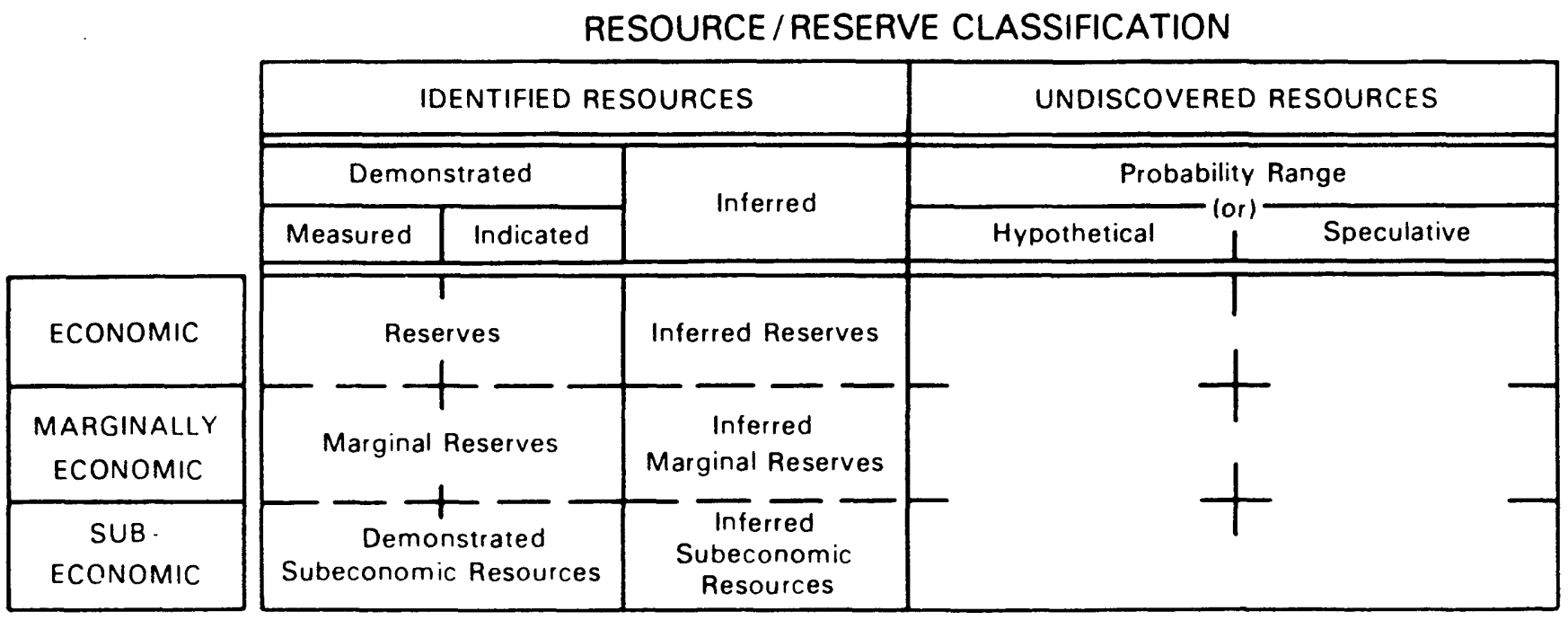

Major elements of mineral resource classification, excluding reserve base and inferred reserve base. Modified from U. S. Bureau of Mines and U. S. Geological Survey, 1980. Prificiples of a resource/reserve classification for minerals: U. S. Geological Survey Circular 831, p. 5. 
GEOLOGIC TIME CHART

Terms and boundary ages used by the U.S. Geological Survey, 1986

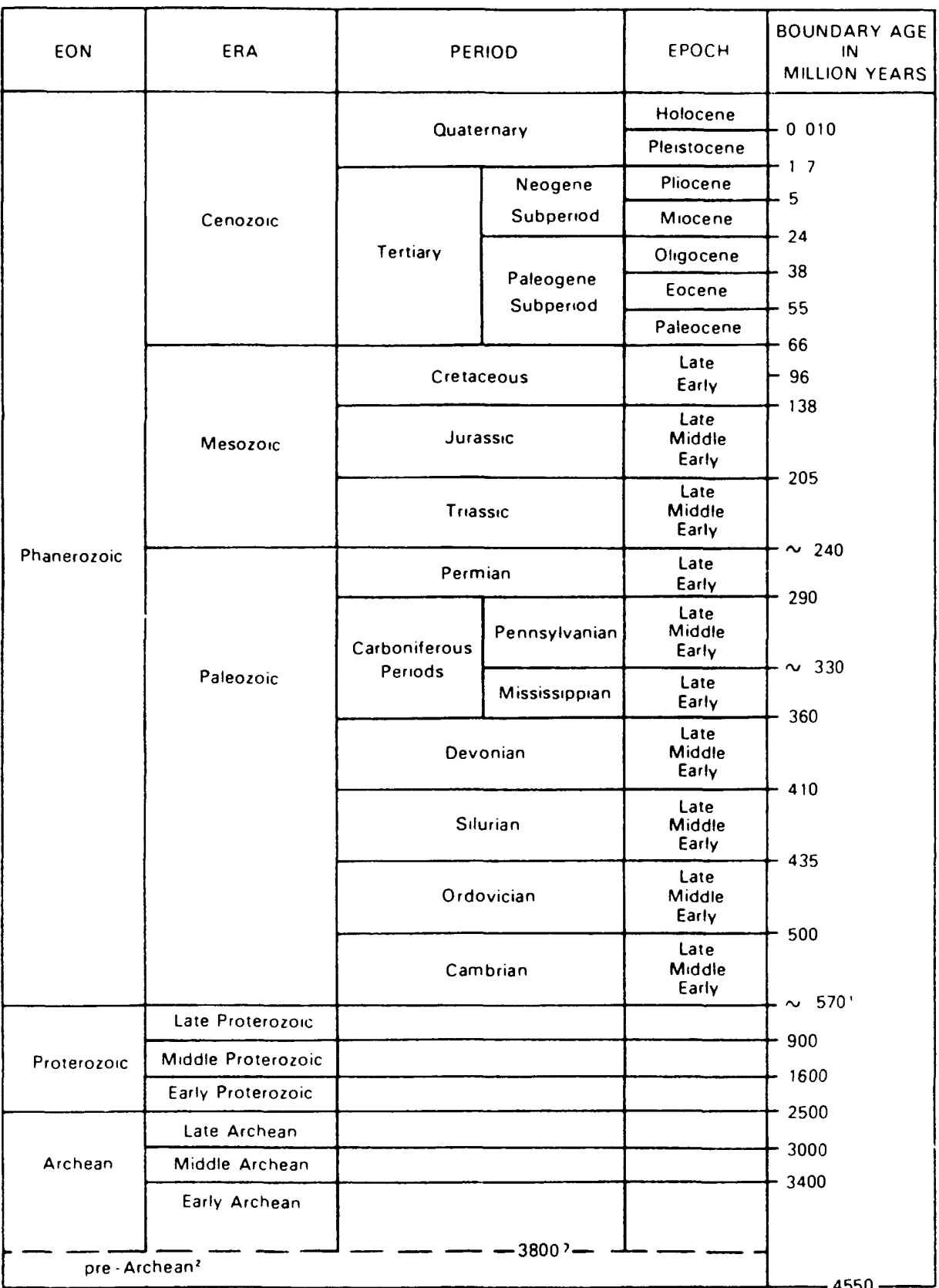

'Rocks older than $570 \mathrm{~m}$.y. also called Precambrian, a tıme term without specific rank

2 Informal time term without specific rank.

U S GOVERNMENT PRINTING OFFICE. 1987-773-038;40.036 REGION NO 8 